\title{
(Genetic) epidemiology as a tool to identify risk factors for emergence and persistence of illness in the functional psychoses
}

Citation for published version (APA):

van Os, J. J. (1995). (Genetic) epidemiology as a tool to identify risk factors for emergence and persistence of illness in the functional psychoses. [Doctoral Thesis, Maastricht University]. IPSER Foundation. https://doi.org/10.26481/dis.19951129jo

Document status and date:

Published: 01/01/1995

DOI:

10.26481/dis.19951129jo

Document Version:

Publisher's PDF, also known as Version of record

Please check the document version of this publication:

- A submitted manuscript is the version of the article upon submission and before peer-review. There can be important differences between the submitted version and the official published version of record.

People interested in the research are advised to contact the author for the final version of the publication, or visit the DOI to the publisher's website.

- The final author version and the galley proof are versions of the publication after peer review.

- The final published version features the final layout of the paper including the volume, issue and page numbers.

Link to publication

\footnotetext{
General rights rights.

- You may freely distribute the URL identifying the publication in the public portal. please follow below link for the End User Agreement:

www.umlib.nl/taverne-license

Take down policy

If you believe that this document breaches copyright please contact us at:

repository@maastrichtuniversity.nl

providing details and we will investigate your claim.
}

Copyright and moral rights for the publications made accessible in the public portal are retained by the authors and/or other copyright owners and it is a condition of accessing publications that users recognise and abide by the legal requirements associated with these

- Users may download and print one copy of any publication from the public portal for the purpose of private study or research.

- You may not further distribute the material or use it for any profit-making activity or commercial gain

If the publication is distributed under the terms of Article $25 \mathrm{fa}$ of the Dutch Copyright Act, indicated by the "Taverne" license above, 


\title{
(GENETIC) EPIDEMIOLOGY AS A TOOL TO IDENTIFY RISK FACTORS
}

\section{FOR EMERGENCE AND PERSISTENCE OF ILLNESS IN THE FUNCTIONAL PSYCHOSES}

\author{
PROEFSCHRIFT \\ ter verkrijging van de graad van doctor \\ aan de Rijksuniversiteit Limburg te Maastricht \\ op gezag van de Rector Magnificus, Prof. Mr. M.J. Cohen \\ volgens het besluit van het College van Dekanen, \\ in het openbaar te verdedigen \\ op woensdag 29 november 1995 om 16.00 uur
}

door

Johannes Jacobus van Os

geboren te Utrecht in 1960 
Promotores:

Prof. dr. M. W. de Vries

Prof. R.M. Murray, DSc (University of London, UK)

Beoordelingscommissie: Prof. dr. H.M. van Praag (voorziltter)

Prof. dr. A.P.W.M. Appels

Prof. dr. W. van den Brink (Universiteit van Amsterdam)

Prof dr. J. Jolles

Prof dr P.P.G. Hodiamont (Katholieke Universiteit Brabant)

IPSER FOUNDATION

PO Box 214

6200 AE Maastricht

tel -31-43-299770

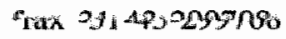


a 
$\therefore$

$\begin{array}{lllll}3 & + & \vdots & -\end{array}$

$\therefore \div \quad$

Bमta

sis 


\section{Table of Contents}

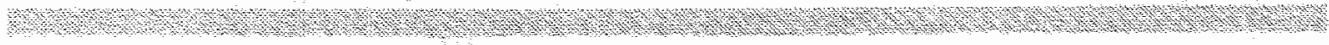


-4ate 


\section{Chapter 1.}

1.1

1.2

1.3

1.4

I. 4.1

1.4 .2

1.4 .3

1.5

1.5.1

1.5.1.1

1.5.1.2

1.5 .2

1.5.2.1

1.5 .2 .2

1.5.3

1.5.3.1

1.5.3.2

1.5.4

1.5 .4 .1

1.5 .4 .2

1.5 .5

1.5.5.1

1.5.5.2

1.5.6

1.5.6.1

1.5.6.2

1.6

1.7

Chapter 2.

2.1

2.1.1

2.1 .2

Chapter 3.

3.1

Chapter 4.

4.1

4.1.1

4.1 .2

4.1.3

\section{INTRODUCTION AND RATIONALE}

About this thesis $\ldots \ldots \ldots \ldots \ldots \ldots \ldots \ldots \ldots$. 3

Psychosis in the European context. ...............

Study overview .........., n........... 15

Heterogeneity in illness course and psychiatric symptomatology 19

Psychiatric symptoms and illness course . . . . . . . . . . 19

Diagnostic categories and illmess course ............ 21

Psychopathological dimensions and illness course ..... 26

Heterogeneity in illness course and risk factors for onset of functional psychotic illness $\ldots \ldots \ldots \ldots \ldots \ldots \ldots \ldots \ldots$

Ageing $\ldots \ldots \ldots \ldots \ldots \ldots \ldots \ldots \ldots \ldots \ldots \ldots$

Ageing and illness onset.

Ageing and illness course $\ldots, \ldots \ldots \ldots \ldots \ldots$.

Ethnic group $\ldots \ldots \ldots \ldots \ldots \ldots \ldots \ldots \ldots \ldots$

Ethnic group and illness onset ...............

Ethnic group and illness course . . . . . . . . . . . . .

Life events . . . . . . . . . . . . . . . . . .

Life events and illness onset . . . . . . . . . . . . . . 39

Life events and illness course ............... 47

Cerebral ventricle dimensions ................ 50

Cerebral ventricle dimensions and illness onset ..... 50

Cerebral ventricle dimensions and illness course .....

Childhood development $\ldots \ldots \ldots \ldots \ldots \ldots \ldots \ldots \ldots \ldots, 52$

Childhood development and illness onset ......... 52

Childhood development and illness course . . . . . . .

Familial morbid risk ................... $\quad 56$

Familial morbid risk and illness onset . . . . . . . $\quad 56$

Familial morbid risk and illness course ......... 57

Risk factors determining the heterogeneity of the tardive

dyskinesia outcome $\ldots \ldots \ldots \ldots \ldots \ldots \ldots \ldots$

Clinical studies of this thesis . . . . . . . . . . . . . . 63

\section{METHODOLOGICAL ISSUES IN FOLLOW-UP STUDIES}

Interpreting follow-up studies $\ldots \ldots \ldots \ldots \ldots \ldots \ldots . \ldots$

Natural history studies ..................... 79

Prediction of outcome studies $\ldots \ldots \ldots \ldots \ldots \ldots \ldots . . \ldots$

PSYCHOPATHOLOGY AS A RISK FACTOR FOR PERSISTENCE OF PSYCHOTIC ILLNESS; PREDICTIVE VALIDITY OF CATEGORICAL AND DIMENSIONAL REPRESENTATIONS

Psychopathological syndromes in the functional psychoses: associations with course and outcome ............ Psychol. Med., in press.

RISK FACTORS FOR EMERGENCE AND PERSISTENCE OF PSYCHOSIS

Ageing

Puberty and the onset of psychosis $\ldots \ldots \ldots \ldots \ldots$.

Schiz. Res. 10: 7-14, 1993

Gender, psychopathology, and development: from puberty

to early adulthood .......................

Schiz. Res. 14: 105-113, 1995

Ageing is a risk factor for non-affective, non-organic

psychosis in the elderly $\ldots . . . \ldots \ldots \ldots . . . .$.

Soc. Psychiarry \& Psychiat. Epid. 30: 16I-165. 1995. 
4.2

4.2 .1

4.3 .1

4.4

4.4.1

4.5

4.5 .1

4.5 .2

4.6

4.6 .1

Chapter 5 .
Ethnic group

Schizophrenia in ethnic minorities: clarification from the

1991 census $\ldots . . . \ldots \ldots \ldots \ldots \ldots \ldots \ldots \ldots . . . \ldots \ldots$

Psychol. Med. in press.

161

The incidence of mania: time trends in relation to gender

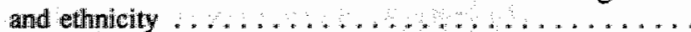

Soc. Psychiatry \& Psyehiat. Epid, in press

Evidence for good prognosis psyehosis in Afro-Caribbeans

in the UK . . . . . . . . . . . .

Br. Med, J, in press.

Life events

The influence of life events on the subsequent course of psychotic illness $\ldots \ldots \ldots \ldots \ldots \ldots \ldots \ldots \ldots \ldots$ Psychol. Med. 24: 503 513, 1994

Cerebral ventricle dimensions

Increased intra-cerebral CSF spaces predict unemployment and negative symptoms in psychotic illness:

a prospective study $\ldots \ldots \ldots \ldots \ldots \ldots \ldots$

Br. J. Psychiat. 166: 750-759, 1995

Neurodevelopment

Premorbid abnormalities in mania, schizomania, acute

schizophrenia and chronic schizophrenia ..........

Soc. Psychiatry \& Psychiar. Epid, in press.

Evidence for similar developmental precursors of chronic affective disorder and schizophrenia in a general population

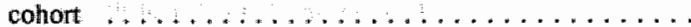

Submitted for publicationt

Genetic diathesis

Does farniliality predispose to both emergence and

persistence of psychosis? A prospective study . . . . . . .

Submitted for publication

\section{RISK FACTORS FOR THE TARDIVE DYSKINESIA OUTCOME}

Risk factors for tardive dyskinesia in a prospectiwely assessed sample of patients with recent onset psychosis . ........ Submitted for publication

Epilogue $\ldots \ldots \ldots \ldots \ldots \ldots \ldots \ldots \ldots \ldots$

Summary $\ldots \ldots \ldots \ldots \ldots \ldots \ldots \ldots \ldots \ldots \ldots \ldots \ldots \ldots \ldots \ldots$

Samenvatting $\ldots \ldots \ldots \ldots \ldots \ldots \ldots \ldots \ldots$

Acknowledgements

Curriculum Vitae

List of peer-refereed publications 


\section{Chapter 1: Introduction and Rationale}

(3) 


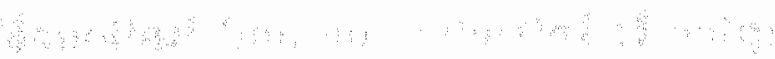

: 


\subsection{About this thesis}

"From this follows unavoidably the task so important for clinical and social psychiatry, o search them for symptoms, which from experience will permit predictions regarding the future course and outcome. This will, after all, be the question asked of ws"

Schneider, 1925 .

The aim of this thesis was to examine risk factors for emergence and persistence of psychotic illness.

In the first chapter, we will review this area in relation to i) international clinical practice, and ii) the international scientific literature, highlight issues requiring special attention, and formulate our hypotheses.

In the second chapter, the methodological issues pertaining to our area of interest are evaluated.

In the subsequent chapters, we will present the results of 13 studies to examine our hypotheses, and, in the epilogue, offer a synthesis of our findings. 


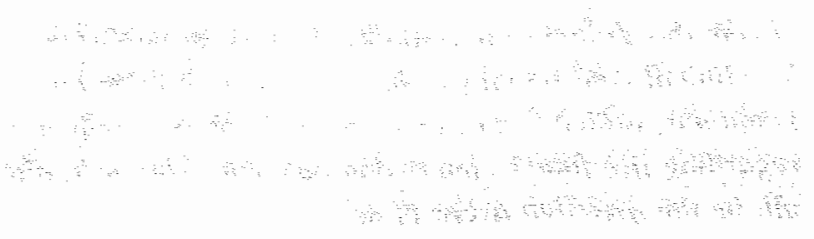

अे+ि

H.

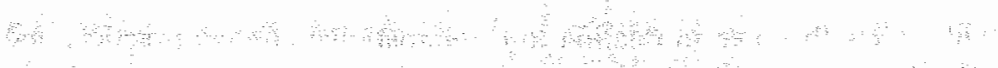

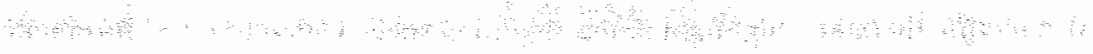

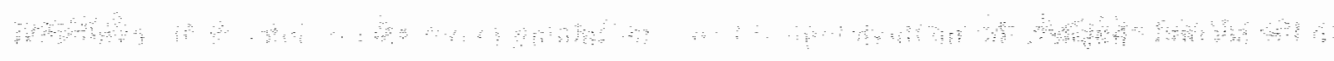

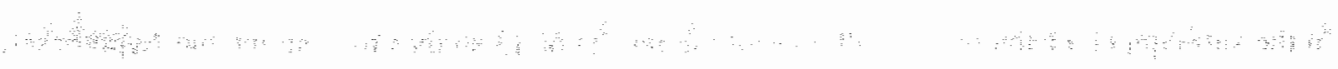




\subsection{Psychosis in the \\ European context}

\section{Heterogeneity as a function of assumptions versus heterogeneity as a function of scientific inquiry.}

More than in any other medical specialty, beliefs tend to fill the gaps of scientific knowledge in psychiatry, and these beliefs may to a large extent be determined by sociocultural and historical factors (Van Os \& Neeleman, 1995). Given the increasingly international arena of psychiatric practice within, for example, the European Union, perceptions of right and wrong associated with local convictions are coming more to the fore. It is conceivable that local convictions in psychiatry determine to a large degree, before anything else, how mental illness is diagnosed and treated, what the perceived priorities are in mental health, and what research efforts are needed to ensure progress in the field.

Local convictions take the form of a priori assumptions, which, unlike scientific hypotheses, are rarely -if ever- the subject of a scientific inquiry: the "truth", after all, is not falsifiable. Below, we will give a brief description of the current "schisms" and heterogeneity (Van Os et al, 1994) in especially European psychiatry, and how this state of affairs led us to formulate the questions, that constitute the basis of this thesis, with regard to the i) risk factors, ii) manifestations, and iii) outcome of psychotic disorders.

\section{A divided psychiatry: concepts of psychotic illness in Europe}

In the early nineties we conducted an attitudinal and epidemiological study in two European countries broadly representative of the more empirical (U.K.) and rational (France) orientations in psychiatry (Van Os et al, 1993). A random sample of 230 psychiatrists in these countries were asked to fill in a questionnaire, designed to elicit opinions on aetiology, diagnosis and management of (what they understood to be) schizophrenia. British and French psychiatrists showed prominent differences of opinion in 31 out of 38 statements. It transpired that British and French psychiatrists used markedly different diagnostic criteria and contrasting methods of treatment for schizophrenia. For example (Table 1), French psychiatrists reserved the label schizophrenia for disorders with onset before age 45 , a chronic course and poor outcome, with "dissociation" and "discordance" as the key symptoms. In the U.K., good outcome and late-onset cases tended to be included, as well as the delusional disorders. Schneider's first rank symptoms were considered core features.

These results show that there is disagreement among psychiatrists about one of most basic epidemiological concepts: what constitutes a case (for treatment, not of "disease")? As case definition is a starting point for all further efforts towards primary and secondary prevention, this state of affairs is disconcerting. The existence of more than one system of descriptive validity results in variation of what are seen to be the meaningful correlates such as cause, treatment and prognosis of psychiatric disorder.

For example, French psychiatrists favoured psychoanalytically orientated aetiological statements, such as absence of the paternal image and maternal ambivalence, whilst the British scored high on items postulating genetic causation. 
Table 1. Mean scores for British and French psychiatrists on questionnaire items about the detiology, manifestarions, outcome and treatment of "schizophrenia" (source: Van Os et al, 1993)

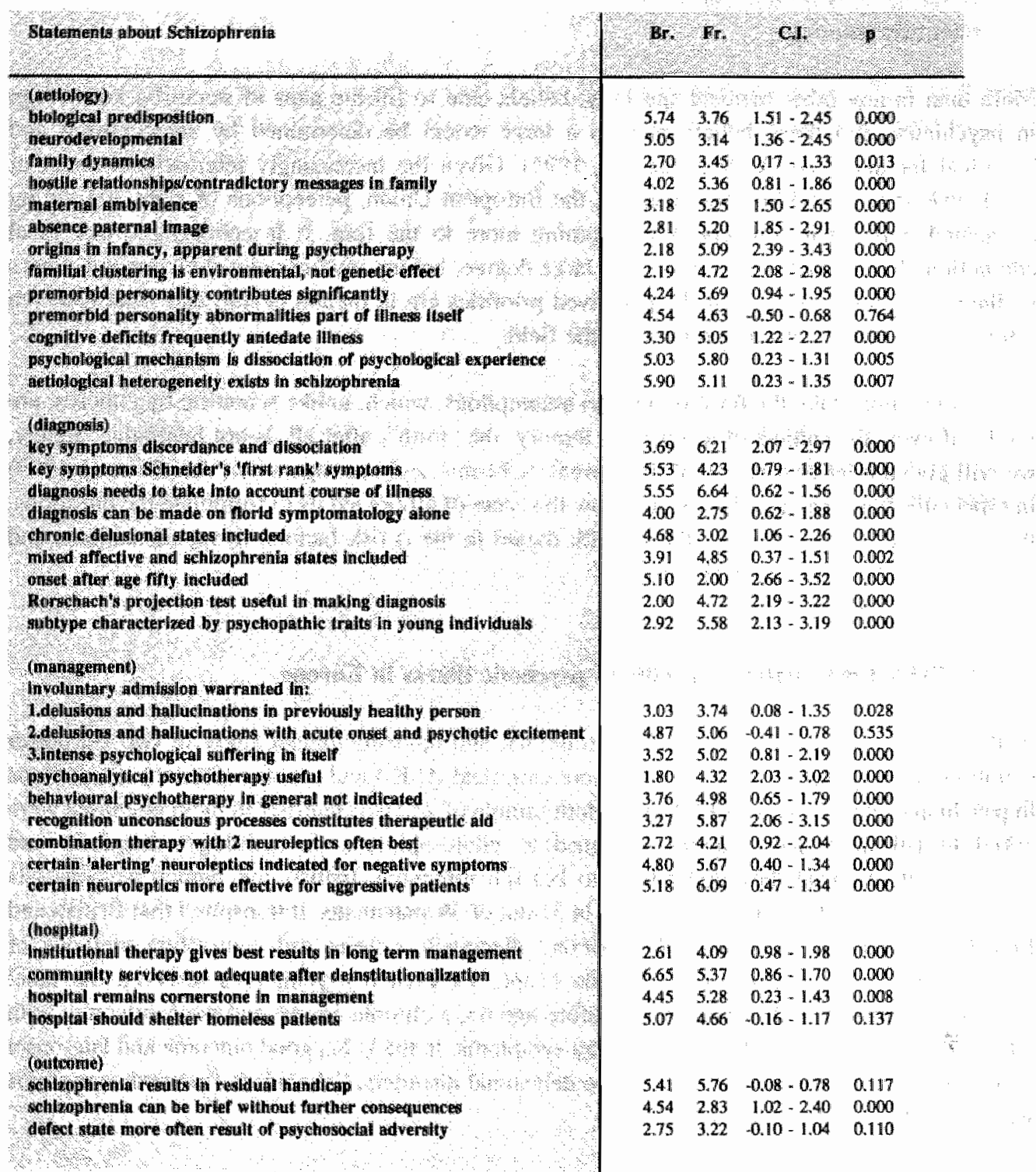

$\mathrm{Br}=$ British sarnile $(\mathrm{n}=92) ; \mathrm{Fr}=$ French Sample $(\mathrm{n}=69)$

C.I. $=95,0$ confidence inverwal of differences

Higher walues indicale greater agrement with statement

(there ass a seven poini scale between agreemenudisagrement)

In general, there were striking differences regarding the role of family dynamics and parental factors in the causation of schizophrenia, French psychiatrists giving much more importance to these than their British colleagues. Thus, not only is there disagreement about case definition and illness course, opposite views also exist regarding the aetiology of psychotic illness along the lines of a mature-nurture polemic. 
Different attitudes to management were also observed. The British sample disagreed with psychotherapeutic statements in favour of biological and behavioural theory. In France, psychiatrists saw a more important role for the hospital than in Britain; but the latter appeared more frustrated with the perceived inadequacy of community services. French psychiatrists had more distinctive indications for different anti-psychotics, and probably as a consequence showed a preference for treating patients with two anti-psychotic agents, which was disapproved of by the British.

Having established such large differences in what lies at the heart of epidemiological psychiatry (ie what constitutes a case for treatment?), we also studied the administrative incidence of schizophrenia in the two countries. Similar, large, Anglo-French discrepancies were encountered. Before age 45, rates of schizophrenia were much higher in France, but much lower after that age, especially in women. In England, there was a sharp increase after age 65 years, whereas in France rates continued to fall (figure 1).

Fig. I Male and female first admission rates for schizophrenia by sex and age group. England and France, 1973-82. (Source: Van Os et al. 1993)

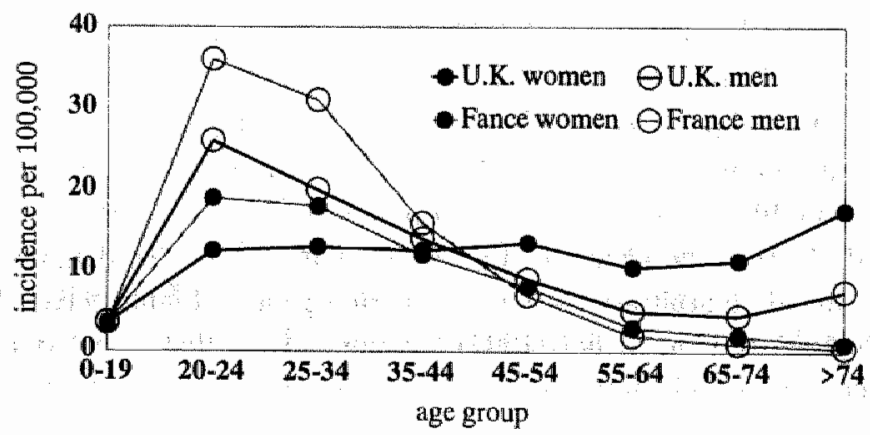

Examination of longitudinal trends in age-adjusted rates in both sexes revealed that in France, especially in men, rates were increasing over the second half of the period, whereas in England rates were falling throughout the period (figure 2).

We subsequently showed that it is likely that i) diagnostic divergence made an important contribution to the contrasting administrative incidence rates in the two countries, and ii) diagnostic drift had occurred in both countries in opposite directions, explaining the differences in longitudinal trends in the administrative incidence of schizophrenia (Van Os et al, 1993).

In summary, different concepts regarding i) case definition, ii) presumed aetiological factors and the role of environmental and genetic influences, iii) course and outcome, are associated with differences in the treatment and epidemiology of major mental disorders. Surely, the scientific evidence on which these concepts are based needs to be scrutinised?

It could be argued that French and English psychiatry lie at opposite ends of the European continuum, and that differences between other European countries would be less conspicuous. We think this is at best only partly true: As there remains a large degree of non-savoir as to what constitutes the causes and best treatment of psychiatric disorders, there are many examples 
of how the local cultural climate influences the way in which psychiatric professionals attempt to fill the gaps (Van Os \& Neeleman, 1994a).

Fig. 2 Age adjusted male and female first admission rates for schizophrewia.

England and France, 1973-82 (Source, Van Os et al, 1993)

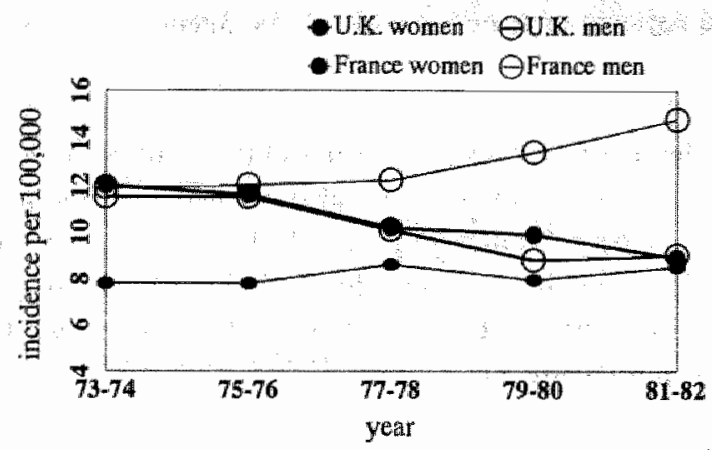

For example, the lack of reliable markers for psychiatric diagnoses has contributed to the continued use of "nationalistic" diagnostic categories, such as the Scandinavian "reactive psychosis" and the French "bouffee delirante". In the United States, a series of rather ephemeral (Zimmerman, 1988) diagnostic systems has seen the light, which may reliably serve economical, administrative and inter-clinician communicative purposes, but which, as far as scientific validity is concerned, have been likened to "ex cathedra pronouncements by expert committees" by some (Brockington et al, 1991). For example, the decision to define the diagnostic category of schizophrenia in terms of an arbitrary chronicity criterion yields a highly selected patient group. Reviewing the DSM-IV system, Sedler (1994) has pointed out that "what is missing....is any recognition that the prima facie self-evidence of given categories is largely a function of a conceptual/perceptual synthesis whose condition of possibility have been historically determined".

Local beliefs also tend to govern the administration of particular treatments. Modified electroshock treatment in, for example, psychotic depression has fallen into comparative disrepute in countries like the Netherlands and Germany, whereas in the U.K., Denmark, France and Bulgaria it is much more widely used (Schornagel \& Assman, 1992; Kirov, 1995). Psycho-analytical theory (revolving around "unconscious" conflicts) is popular among mental health workers in many continental countries, such as France, Germany, and Switzerland (also in the aetiology and treatment of psychosis), whereas behavioural principles are favoured in, for example, the U.K. (Van Os \& Neeleman, 1994a). There are intemational differences in the indications and perceived therapeutic properties of medications such as anti-psychotics. For example, Dollfus and Van Os (1995), demonstrated a $4000 \%$ (sic) difference between two European countries in the officially advised maximum dosage of a popular antipsychotic. Similarly, a curious North-South divide in the annual per capita exposure to benzodiazepine tranquilizers is apparent (figure 3). The German practice to treat relatively mild neurotic disorders on an in-patient basis in costly "psycho-somatic" clinics contrasts with efforts elsewhere to treat these conditions in the community and to reserve hospital admission mainly for psychotic patients. 
The highly heterogeneous descriptions of psychiatric morbidity associated with adverse migration and displacement in Europe needs to be further examined. A survey of Turkish workers conducted in the Netherlands suggested neurotic illnesses, associated with worries about family at home, are common (Sayil, 1984), whereas another survey showed high rates of schizophrenia in immigrants from Suriname and Morocco (Selten \& Sijben, 1994). In the U.K., similarly high rates of schizophrenia have been reported in Afro-Caribbean migrants (Harrison et al, 1988), whereas in France African migrants often are labelled as presenting with bouffée delirante (Johnson-Sabine et al, 1983). Workers in Germany and Denmark showed that there were yet other modes of presentation in immigrants from different backgrounds (Jensen et al, 1989; Lazardis, 1987). "National" attitudes may also be reflected in, for example, quality of mental health legislation, and, perhaps more importantly, the degree to which correct implementation is enforced. Most European countries, especially those within the EC, have now shed or amended their old 19th century mental health acts, but important differences exist. In countries like England and France, involuntary admission of psychotic patients is in practice a largely clinical decision, whereas in, for example, Austria, the Netherlands and parts of Germany the decision is ultimately made by a judge or his representative. This is an important difference, as in the latter three countries a legal appeal procedure is "built in", which in the Netherlands has been reported to result in quashing of involuntary admissions (requested by the clinician) in up to almost sixty percent of cases within on average five days of detention (Nijman et al, 1993).

Fig. 3 Per capita exposure to benzodiazepime tranquillizers

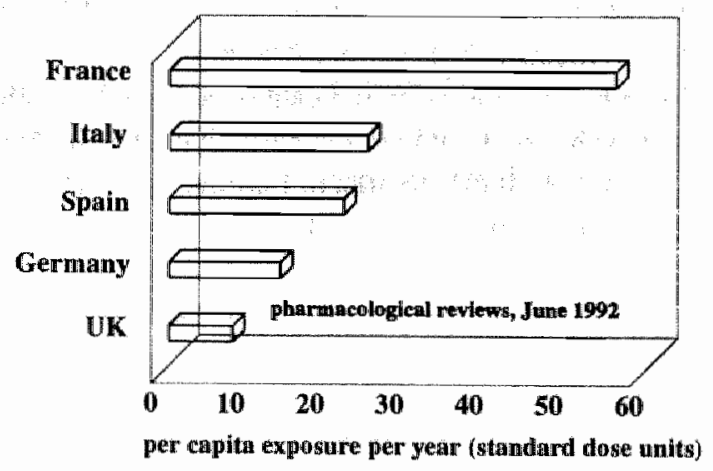

In view of the above, it is small wonder that currently conspicuous differences exist in the duration and content of psychiatric training across Europe. The actual national duration varies from 4 to 7 years, and in some training schemes the trainee is more a postgraduate student under continuous assessment, in others more an apprentice with extensive service commitments who has to pass a cross-sectional exit exam. Some training schemes require extensive psychotherapy training, others emphasize training in academic ability. Many countries in the European Union are forcing their system of training into the Procrustean bed of "Euro-training" and "Eurocertification", but such labels are unlikely to consolidate contrasting beliefs across Europe (Neeleman \& Van Os, 1994).

It is possible that the particular interests and ideas, transmitted during training of psychiatrists, even shape the way in which care is delivered, and to whom, within the context of the particular organization of the local health care system (eg National Health Service systems versus systems funded through social and private insurance schemes; Van Os \& Neeleman, 1994b). Dutch 
observers, commenting on British community provisions, noticed that it appeared better equipped to deal the with (the medical needs of) chronic psychotic patients, whilst the Dutch provisions were more geared toward the psychological (psychotherapeutic) needs of less disturbed patients (Hest \& Wolters, 1992; RLAGG, 1992). The same can be said for ambullatory services in other EU countries such as Belgium.

\section{A research mandate for the functional psychoses}

We conclude that widespread diversity exists, and is not restricted to isolated examples. Contrasts in attitudes and practice are not a bad thing. On the contrary: the challenge is to exploit the diversity, yet to overcome the parochial. The area is rich with aetiological, nosological, and prognostic assumptions that can be readily transformed into hypotheses for scientific exploration. Only in this way can the scientific evidence underlying national assumptions be scrutinised, and the validity for different treatment approaches established. Focussing on the functional psychoses, we summarize the material discussed above into four major areas to formulate questions and proposals that may advance our clinical knowledge on the risk factors, nosology and outcome of the functional psychoses.

i) the diagnostic assumption - National diagnostic constructs are, as we have shown, essentially a priori assumptions that may have a confusing effect on many areas of psychiatric practice. For example, much of the outcome literature on "schizophrenia" cannot be generalized because of idiosyncratic case definition (Angst, 1988), so that it is not known whether the trend towards improved prognosis in schizophrenia, observed in recent meta-analytic studies, is due to changing diagnostic criteria or better psychiatric treatment (Hegarty et al, 1994). As a direct result, we are still in the dark as to whether antipsychotic treatment improves the outcome (not just the relapse rate) of schizophrenia in the long term.

We suggest that the way forward is not to rely on poorly validated constructs as a basis for case selection for both treatment and clinical research. First of all, the presence or absence of psychosis may have a lot more face validity for initial selection, and allows for secondary investigation of interaction by diagnostic construct. Secondly, it has been argued cogently (eg Costello, 1992) that a much more profitable approach may lie in the investigation of the symptoms themselves, rather than our imperfect and invalid notions of how they should be combined. Clusters of correlated give rise to symptoms dimensions (as opposed to categories), which again appear to have more face validity as a basic construct from which to begin classification. Reluctance on the part of the psychiatric profession to deviate from the categorical assumption may be related to (see also Kendell, 1993) its natural conservatism, innumeracy (psychologists have exploited the area gainfully), and the general medical stratagem to think in dichotomies, rather than in dimensions (Pickering, 1968).

ii) the "aetiological" assumptions - Historically, psychiatrists have been quick to translate evidence of statistical association into evidence of causality. Koch's postulates were enthusiastically applied to psychiatric disorders, in terms of a requirement for complete specificity in a unique and unconfounded cause (eg "life events", or "genes"). Although with the advent of the epidemiology of chronic diseases the need for new postulates was recognized (Susser, 1985) thinking about cause and its recognition in psychiatry has not nearly been as 
productive as, for example, in cardiovascular medicine. As discussed above, aetiological assumptions in psychiatry tend to cluster around "constitutional" versus "environmental" factors, exemplified by, for example, the term reactive psychosis", which is much more frequently diagnosed in the Scandinavian countries than elsewhere in Europe. However, for many of the factors that are considered of aetiological importance, it is not known whether they are truly causal factors, indicators of risk, modifiers of risk, risk factors for chronicity rather than emergence of disorder (or both), an artefact, or even illness manifestations. For instance, socalled life events "causing" reactive psychosis-may i) be an independent cause of psychosilis; ii) be the manifestation of a genetically determined "hazard proneness" in psychotic individuals and their first degree relatives; ii) interact with genetic vulnerability to increase risk of psychosis; iw) be a non-specific protective factor against chronicity; v) be the result of a "search for meaning" on the part of the patient; vi) be the result of early symptomatic behaviour.

The Koch's postulate analogy is further reinforced by the tendency to dichotomize continuous risk variables, so that a continuous risk factor is often conceived of as an all-or-non phenomenon (eg "family history of schizophrenia", "poor premorbid adjustment", "enlarged cerebral ventricles"s").

Given the popularity of aetiological postulates calling for specific and unconfounded causes, the search sofar has been especially for discrete subtypes of illness with independent causes, and paradigms have been developed for examining, for example, hypothesized discrete genetic and environmental causation (Murray et al, 1985; Lewis et al, 1987). As demonstrated above, psychiatrists in European countries apparently hold contrasting beliefs on what is nature and what is nurture in the causation of schizophrenia. A more likely, but also more complicated, scenario is that of gene-environment interaction, where neither genes nor environment are necessary or sufficient to bring about illness; the excess risk conferred by their interactive effect, however, can, either alone or in combination with other factors.

A more recent issue in the search for aetiology has been the suggestion that there are specific ethnic vulnerabilities to certain disorders, such as schizophrenia in UK Afro-Caribbeans and in Dutch Surinamers, and bouffée délirante in French African immigrants. As we have seen, UK schizophrenia (and in all \|likelihood the Dutch concept of schizophrenia as well) may include both good-outcome and poor-outcome disorders, whereas bouffee délirante is associated with a better outcome compared to the French schizophrenia construct. So what is the significance of such reports in relation to Europe's growing migrant populations? Are such individuals at increased risk of specific psychiatric disorders, and/or are they at risk of a particular outcome? Is the increase in risk spurious, eg related to administrative under-enumeration of the ethnic minority population, or can it be explained by, for example, differences in socio-economic status?

iii) the outcome assumption - Prediction of outcome is of fundamental importance, as knowledge about factors influencing illness course allow us to formulate measures aimed at secondary prevention. We have demonstrated above that assumptions relating to illness course are usually linked to diagnostic concepts and therefore dichotomized according to local tenet: disorder $\mathrm{X}$ has good outcome and disorder $\mathrm{Y}$ poor outcome, and specific risk factors can distinguish between the two. Mean differences in outcome are widely interpreted as validating diagnostic dichotomizations, but prediction of group membership on the basis of the validating variable is, 
in the rare cases where it has been examined, poor. One way forward is, using the agnostic approach toward initial case selection advocated above, to examine carefully which factors modify course of illness, ie to approach mental disorder from a strictly longitudinal perspective, rather than rely on cross-sectional constructs of mental disorder.

The longitudinal perspective can shed light on the diagnostic and aetiological issues discussed above: course and outcome in the functional psychoses can be linked to initial illness manifestations and antecedent illness risk factors. Diagnostic heterogeneity should be examined from a longitudinal perspective, relating clusters of cases (diagnostic categories) and clusters of symptoms (symptom dimensions) to subsequent course of illness, in an attempt to identify the most parsimonious solution of classification with the highest predictive validity. Heterogeneity of risk should also be examined from a longitudinal perspective. For example, is ethnic group a risk factor for onset of "schizophrenia", a risk factor for "good-outcome schizophrenia", or a non-specific protective factor against chronicity in the functional psychoses? If risk factors, for example those representing "constitutional" versus those representing "environmental" risk dimensions, can be shown to have associations in opposite directions with illness course, then the validity of such risk dimensions would be strengthened considerably.

iv) the methodological assumption - Psychiatric research is famed for its contradictory results. Even the numerous investigations using measurements which increasingly obviate the need for subjective judgement (and therefore generate less error), such as magnetic resonance imaging and cerebral blood flow studies have yielded inconsistent results over more than a decade of research (Chua \& McKenna, 1995). One of the reasons is that the technological advances appear to have far outstripped basic methodological advances. For example, observational and intervention studies reported in the field of epidemiology are characterised by extensive deliberations surrounding the choice of controls, sample size, alternative explanations in the data, effect modification, and potential systematic errors that may have influenced the results in certain ways. In psychiatry, however, the same type of research developments have been characterised more by continuing technological and statistical innovation and relabelling, rather than the more duller aspects of chance, bias, confounding and interaction, to the extent that the more rigorous application of the epidemiological method in itself may well lead to rather exciting findings in the same old data. The apparent assumption that clinical progress can be achieved by the application of ever more sophisticated technological and statistical tools without paying at least as much heed to the more basic epidemiological principles, may open the way for the use of hypotheses as over-valued ideas that are allowed to drive the data in the desired direction (Gur \& Gur, 1995). Epidemiology is an ideal tool to examine assumptions in psychiatry.

\section{This thesis: less thesis, more epidemiological groundwork}

In conclusion, the divergence in psychiatric concepts can be summarized in epidemiological dialect as follows: i) what is a case (for treatment)? ii) what are the risk factors for becoming a case and how do they interact with each other?, and iii) what are the risk factors for chronicity? The aim of this thests was to examine these issues, from a longitudinal perspective and using classical and genetic epidemiology as tools. 
i) We addressed the first question (what is a case for treatment?) by examining categorical (clusters of cases) and dimensional (clusters of symptoms) representations of psychopathology in unselected samples of individuals with functional psychotic illness, and establishing their comparative predictive validity.

ii) We also examined areas of risk, broadly divided into "constitutional" (cerebral ventricle dimensions, premorbid social and neurological development, and familial morbid tisk of psychosis) "reactive" (life events) and demographic (migrant status, ageing) dimensions, in relation to i) emergence of psychopathological phenomena, ii) chronicity of these phenomena. We also examined relationships between risk factors, notably between life events and genetic predisposition; we tried to develop a simple research paradigm that would allow us to demonstrate gene-environment interaction. Finally, we examined the specificity of risk factors in relation to categorical and dimensional representations of psychopathology.

In the second part of this chapter, we will place these issues in context with regard to the current scientific literature, and formulate hypotheses which will be examined in chapters 3-5. Finally, we will attempt to integrate the literature review and our findings from a clinical perspective, and propose an empirical model of heterogeneity in the functional psychoses. 
मित

an

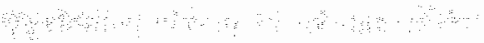
a

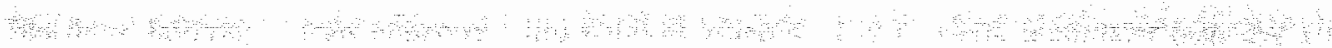

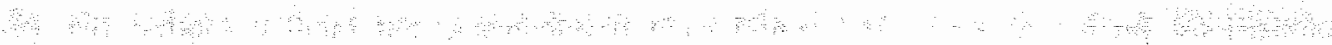

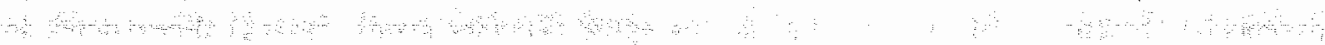

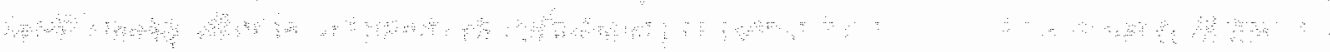

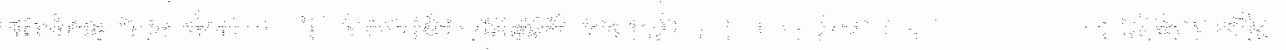

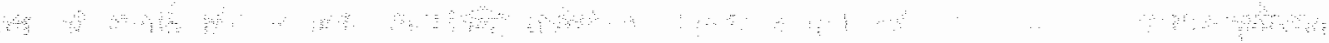
Has $\therefore \quad \because \quad 3 \quad-4$ 


\subsection{Study overview}

\section{THE LONGITUDINAL PERSPECTIVE}

There is evidence that most of the variability in the course of schizophrenia takes place in the first five to ten years of the illness (Bleuler, 1972; Ciompi, 1980; Huber et al, 1980; McGlashan, 1986, 1988; Breier et al, 1991) and that there is considerable heterogeneity in outcome (Bleuler, 1972), the prediction of which often eludes even experienced clinicians (Giel et al, 1984). From the point of view of public health, the identification of factors that mediate the variability in course creates scope for secondary prevention by targeting groups with high risk of continuing social and/or clinical disability. Furthermore, factors determining heterogeneity in course and outcome can also shed light on the nature and classification of psychotic illnesses and their underlying mechanisms, as outcome arguably is one of the most important criteria by which to establish the validity of any diagnostic concept (Kendell, 1989). In the absence of an aetiologically validated cllassification of the mental disorders, elucidation of the factors predicting treatment response and outcome in the longer term may constitute the next best thing.

In this study, three strategies were used to detect patterns in outcome heterogeneity in the functional psychoses. These strategies focussed on three areas of outcome prediction (figure 4): i) the relationship between risk factors for illness onset and outcome; ii) the relationship between the illness manifestations themselves and outcome; iii) the relationship between both antecedent risk factors and illness manifestations on the one hand, and the organic outcome of tardive dyskinesia on the other.

Fig. 4. Three strategies to examine outcome heterogeneity in the functional psychoses

\begin{tabular}{|c|c|c|c|}
\hline & $\begin{array}{l}\text { To examine risk } \\
\text { factors for onset of } \\
\text { illness, and their } \\
\text { relevance for illness } \\
\text { course }\end{array}$ & $\begin{array}{l}\text { To determine whether } \\
\text { psychopathological } \\
\text { features predict illness } \\
\text { course }\end{array}$ & $\begin{array}{l}\text { To determine which } \\
\text { factors predict organic } \\
\text { outcomes }\end{array}$ \\
\hline $\begin{array}{l}\text { ANTECEDEN } \\
\text { RISK }\end{array}$ & & & \\
\hline $\begin{array}{l}\text { ONSET } \\
\text { PSYCHOPAT }\end{array}$ & LOGY & & \\
\hline
\end{tabular}

These three areas were chosen because of the discrepancy between the clinician's need to identify risk factors for chronicity, and the lack of guidance offered by conflicting assumptions described in the previous section, and a contradictory literature discussed in more detail below. 


\section{Illness manifestations and outcome.}

In clinical practice, it is important to use the study of symptomatology and course of illness to identify sub-categories of patients whose prognosis can be reliably shown to differ in various respects. As discussed below, many studies have investigated the relationship between either diagnostic categories (a priori symptom constellations) and individual symptoms or groups of symptoms on the one hand, and course and outcome on the other. Recently, there has been renewed interest in a dimensional representation of symptomatology in schizophrenia and other functional psychoses, which may be a useful tool in the study of the genetic and pathophysiological processes underlying schizophrenia and other psychiatric disorders, and their treatments (Liddle et al, 1987a \& b, Liddle et al, 1992; Dworkin et al, 1988; Van Praag et al, 1987, Van Praag et al, 1990). Furthermore, a dimensional representation of the functional psychoses seems more compatible with the flexible and dimensional way in which treatments are prescribed in clinical practice: no treatment is disorder specific (eg antipsychotic and antidepressant treatments), and in practice treatments for different typological constructs largely overlap, althongh on average they may differ (Johnstone et al, 1988). We investigate i) whether a dimensional representation can be validated using course and outcome as the validating criterion, ii) how it relates to psychiatric treatments, iii) how it relates to the traditional typological approach, iv) whether the predictive power of the dimensional approach exceeds that of the traditional categorical approach to classification in psychiatry; if there are one or more psychopathological dimensions scored along a continuum, one would expect their predictive validity to be greater than for categories deternined by arbitrary cut-offs within the distribution of scores, because the latter approach to classification results in loss of information (figure 5); and v) the specificity of risk factors for psychosis with regard to categorical and dimensional representations of psychopathology.

Fig. 5. Predictive validity: categorical and dimensional representations of psychopathology

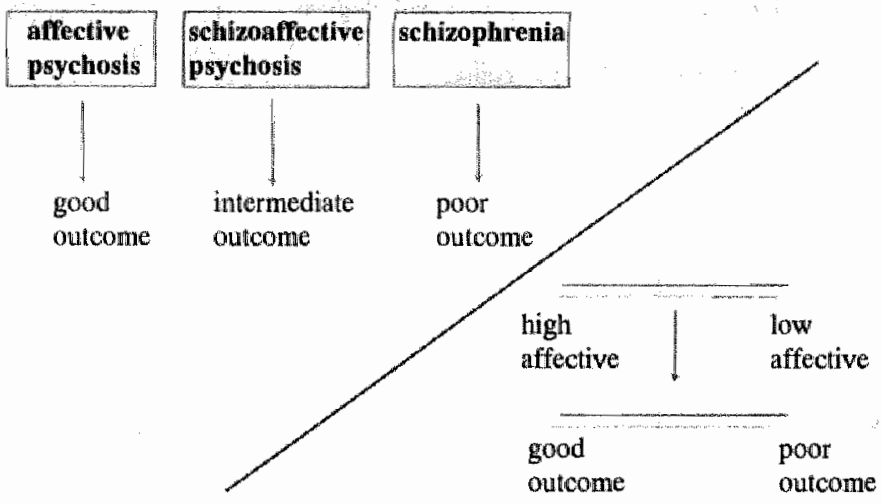

\section{Outcome heterogeneity and risk factors for emergence and persistence of illness.}

Can the study of "high-risk" groups be used to identify sub-categories of patients whose prognosis can be reliably shown to differ in various respects? The research on risk factors for onset (emergence) of psychosis has been conducted largely separately from research on risk for chronicity (persistence). Furthermore, the determinants of course of illness have been neglected 
compared to the determinants of onset of disease. For example, there have been more than 100 controlled comparisons of cerebral ventricle dimensions in relation to onset of disease, but there have been only 6 studies that examined the question whether ventricle enlargement is associated with course of illness, and most of these six were of poor quality (reviewed in chapter 4.4 .1 ), The situation is similar for risk factors such as life events, ethnic group, ageing, premorbid developmental deviance, and familial morbid risk of psychosis Undoubtedly, the lack of follow-up research in this area is associated with the high cost of such investigations, and the comparatively "dirty" (confounded) area of outcome. However, it is important to study course of illness in high risk groups. First of all, primary prevention in psychiatry is not feasible at present, whereas secondary prevention is something that all psychiatrists practice on a daily basis. Much more information is needed to further enhance our efforts for secondary prevention, and the study of prognosis in high-risk groups is useful in this regard. Furthermore, the finding that the same risk factor is implicated in both onset and outcome of a disease, in such a way that it can be differentiated from other high-risk groups, is suggestive of the existence of discrete major effects (this is not the same as assuming discrete subtypes), and may constitute an important contribution towards elucidating the heterogeneity of underlying mechanisms or possibly even aetiollogy of functional psychotic illness (figure 6).

Fig. 6: Risk factors for emergence of illness also predict persistence of illness.

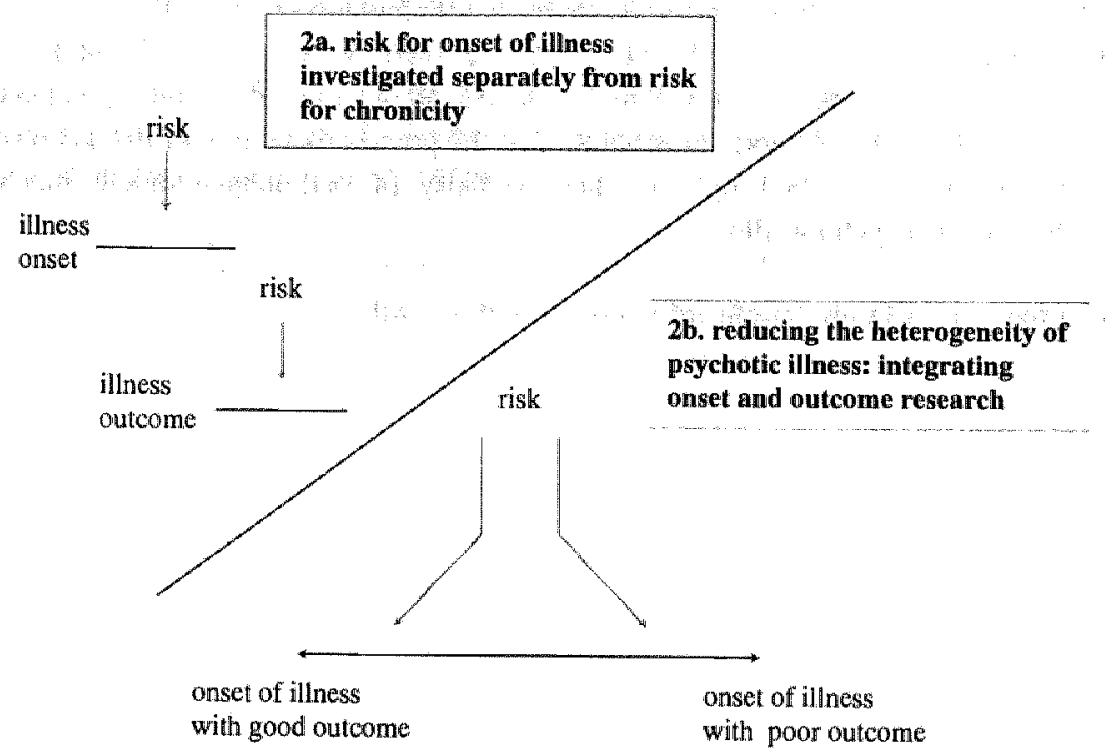

Onset wulnerability does not necessarily equate with persistence vulnerability. For example, two individuals affected with tuberculosis caused by the same infective agent may have widely different course of illness for a variety of factors unrelated to the causal agent such as genetically mediated variation in response to pharmacologic treatment. Similarly, in psychotic illness, sex has a powerful effect on outcome (Bardenstein \& McGlashan, 1990, but is not thought to increase the risk of onset of schizophrenia (Castle \& Murray, 1991). For other factors, however, there is a suggestion that onset vulnerability and persistence vulnerability overlap. There is, for example, the widely held and popular, but controversial, notion that certain risk factors can discriminate prospectively between good outcome, "reactive" psychoses and poor outcome, 
"constitutional" psychoses (Robins \& Guze, 1970). This postulation still awaits confirmation. We therefore investigate the predictive value of risk factors thought to be most important in this regard (age, ethnic group, life events, cerebral ventricle dimensions, neurodevelopment, and familial morbid risk of psychosis).

Because the literature is skewed towards risk factors of emergence of illness, we will present little additional data on the relationship between the above risk factors and illness onset, if the literature review indicates that such an association has been well established. In some instances; however, we will also investigate risk factors in relation to emergence of illness, rather than in relation to its persistence. For example, whether or not ethnic group confers an increased risk for schizophrenia remains controversial, and whether or not ageing is a risk factor for psychosis in the elderly is speculated upon, but has never been established.

\section{Prediction of the organic outcome of tardive dyskinesia}

The study of relatively unusual, but disabling, outcomes, indicative of organic brain dysfunction is necessary to develop strategies to prevent their occurrence. Furthermore, it may be possible to identify a subgroup of patients who, possibly by virtue of a developmental brain defect, are at high risk of developing such outcomes. Evidence is accumulating that an illness characterised by negative symptoms (Liddle \& Barnes, 1993; Brown \& White, 1992) and cognitive deterioration (Waddington, 1986) is associated with such an outcome: the neurological syndrome of tardive dyskinesia (TD). We will demonstrate that the prospective study of the TD movement disorder, may be used to shed light on heterogeneity of pathophysiological mechanisms underlying functional psychotic illness.

The three strategies will now be put into context with regard to the scientific literature. 


\subsection{Heterogeneity in illness course and psychiatric symptomatology.}

\subsection{1 psychiatric symptoms and illness course}

Instead of trying to demonstrate associations between outcome and a priori notions of diagnostic categories as is usually the case, one can try to obtain fundamental evidence from the symptoms thernselves (Grayson, 1986), whether it be observer-elicited, or possibly self-reported at the experiential level using qualitative methods (DeVries \& Delespaul, 1989). In its simplest form, such research examines associations between individual symptoms or a group of symptoms and outcome. There is some evidence suggesting that presenting symptoms in the functional psychoses are related to the subsequent outcome of these disorders. Frank Fish $(1962 ; 1974)$ used to distinguish between positive and negative symptoms, a notion which has become an influential differentiation in schizophrenia research, both on the objective and subjective level of experience (Selten $e t a l, 1993$ ). There is a suggestion that negative symptoms may be moderately associated with poor outcome in schizophrenia. Robins \& Guze (1970) reviewed 9 follow-up studies from around the world, and concluded that "striking emotional blunting" and a "hebephrenic picture", which would now be regarded as negative symptoms by many (Andreasen, 1982), tended to be associated with a poor prognosis. Later studies have in general confirmed these findings (PogueGeile, 1989; Fenton \& McGlashan, 1991). These studies have been very influential, and Robins' and Guze's (1970) suggestion to conceive of good outcome schizophrenia as a separate disease entity, to be distinguished from illness with prominent negative symptoms, poor outcome and other features, lies at the heart of various proposals for subtyping schizophrenia (Crow, 1980; Murray et al, 1988; Carpenter et al, 1988).

The issue of whether positive symptoms such as delusions and hallucinations are associated with course and outcome is still unresolved. There seems to be little doubt (Harrow et al, 1990) that the presence of psychosis in affective disorder, especially mood-incongruent psychotic symptoms and Schneiderian first rank symptoms (Schneider, 1959), predict poor outcome (Lee \& Murray, 1988; Coryell et al, 1990 a \& b; Tohen et al, 1990; Tohen et al, 1992); similarly, many studies agree that the presence of "typical" schizophrenic symptoms in schizo-affective disorder, either concurrent or in the absence of affective features, does not bode well for the future (Brockington et al, 1980a \& b; Harrow \& Grossman, 1984; Samson et al, 1988; Grossman et al, 1991; McGlashan \& Williams, 1990).

However, the findings in schizophrenia are extremely inconsistent. Some studies found a positive association with outcome for some Schneiderian symptoms, and a negative association for others (Bland \& Om, 1980a; Bland, 1982). Other studies failed to find any effect of more typical positive schizophrenic symptoms on outcome (Carpenter et al, 1978; Pope \& Lipinski, 1978; Brockington et al, 1978; Shepherd et al, 1989; Fenton \& McGlashan, 1991), whereas yet others did find an effect indicating poorer outcome (Eitinger et al, 1958; Stephens \& Astrup, 1963; Cloninger et al, 1985; Huber et al, 1980; Prentky et al, 1980; Kay \& Murrill, 1990; Endicott et $a l_{x}$ 1986; Kendell et al, 1979; Leff et al, 1992; Pogue-Geile \& Harrow, 1985). Finally, some studies also found an effect indicating poorer outcome, but only in patients receiving optimal neuroleptic treatment (Breier et al, 1991), or older patients (Prudo \& Blum, 1987), or only in the prediction of long term outcome (McGlasgan, 1986), or in the case of persistent schizophrenic symptoms (Shepherd et al, 1989). 
Symptoms as catatonita and thought disorder also reportedly have prognostic significance in schizophrenia (e.g. Stephens \& Astrup, 1963; Bland \& Orn, 1980b; Tsuang \& Winokur, 1974; Tsuang et al, 1981; Carpenter \& Strauss, 1991), and whille there is a traditional attribution of better prognosis in the presence of an index affective component (reviews by Robins \& Guze, 1970; McGlashan, 1988), some influential studies have reported informative null-findings (Shepherd et al, 1989, Carpenter et al, 1978).

With the possible exception of negative symptoms, the literature on the subject of symptomatology and outcome remains inconclusive. Furthermore, the literature on negative symptoms is limited as it only focusses on schizophrenia, variably defined, and may be confounded by sex and/or length of illness, which are rarely taken into account. There may be many reasons for the discrepancies between studies examining the association between positive symptoms and outcome, such as differences in sample size, differences in case sellection, failure to take into account sex as a confounding factor, chance findings due to multiple testing with a range of individual symptoms; and differences in outcome measures. Selection bias may be one of the main factors involved. For example, studies with an index sample of mostly chronic patients with more psychomotor poverty (e.g. Fenton \& McGlashan, 1991) are likely to find an association between negative symptoms and outcome, but not between positive symptoms and outcome. Studies like the five year follow-up of the International Pilot Study of Schizophrenia (IPSS), that included 807 more widely defined schizophrenia cases and a small proportion of patients with affective psychosis of varying illness duration, and were thus more likely have a mix of negative and positive psychopathological syndromes (Leff et al, 1992), found a modest effect of schizophrenic positive symptoms on type of illness course. The fact that one of the IPSS centres was not able to show such an effect (Carpenter et al, 1978), may be related to the small numbers used in that study. Finally, studies that included a group of patients with any functional psychosis (Kendell et al, 1979; Cloninger et al, 1985) did find an effect on outcome of schizophrenic positive symptoms. In general, the effect of positive symptoms in these studies was confined to measures of course and persistence of psychosis, and was less apparent on social outcome.

In summary, there is a large body of literature on the relationship between psychiatric symptoms and prognosis in the functional psychoses. Some trends emerge, especially for psychotic symptoms and typical schizophrenic symptoms to predict poorer prognosis in the affective and schizo-affective disorders respectively, and for negative symptoms to predict poor prognosis in schizophrenia. However, interpretation of studies is made difficult because there are many potential symptoms to be tested, high rates of type I and type II errors will inevitably lead to conflicting results. More important, however, is that associations between symptoms and illness course are usually examined within the constraints of the various diagnostic approaches, which, given the uncertain validity of traditional psychiatric disorders (Costello, 1992), will lead to bias because the both the predictor variable (symptoms) and the criterion variable (course and outcome) are restricted to a narrow range. Within any diagnostic category, the narrow symptomatic and outcome range will restrict variability and thus impede detection of effects (Streiner \& Norman, 1989). Pope and Lipinsky (1978) in one of the most comprehensive reviews, concluded that "most so-called schizophrenic symptoms, taken alone and in cross section, have remarkably little, if any, demonstrated validity in determining diagnosis, prognosis and treatment response in psychosis....this compromises both clinical treatment and research". 


\subsection{2 diagnostic categories and illness course}

Since the discovery that there were important national differences in the concepts of psychiatric disorders such as schizophrenia (Cooper et al, 1972, WHO, 1973, Saugstad \& Odegaard, 1983), and the discovery that even experienced psychiatrists from similar professional backgrounds could not achieve satisfactory interrupter agreement (Kreitman et al, 1961), the main focus has been on improving reliability of psychiatric diagnoses. Even though important international differences continue to exist (Van Os et $a l, 1993$ ), standardized interviews used by trained. individuals can now yield impressive diagnostic reliability, and enhance the replicability of scientific studies. In addition, instead of description of paradigm cases with a certain set of symptoms as the standard, the focus is now on operational criteria to define diagnostic categories. The two most recent international systems using operational criteria are ICD-10 (WHO, 1992) and DSM-IV (APA, 1994), the latter one, together with its predecessors, being the most influential psychiatric work on classification ever produced One of the radical innovations of these systems, especially the DSM-III and subsequent series, is that the combined diagnostic categories are mutually exclusive and jointly exhaustive. In other words, if there are 100 diagnostic categories, only 99 are independent, the 100th being what the other 99 are not. Such universal coverage is commendable, but introduces at the same time problems regarding the validity of the categorical approach. Reliability and validity of a set of diagnostic categories have a rather enigmatic relationship. For example, given the existence of a certain psychiatric disorder, increased reliability will per definition benefit the validity of the disorder. If life events in the three weeks before onset of illness are a risk factor for a certain psychiatric disorder, but not for any other mental illness, than the demonstration of the specificity of life events as a risk factor is clearly highly dependent on the reliability of the criteria setting it apart from the rest of the disorders. Unfortunately, however, the relationship does not necessarily hold in the other direction. Given the uncertainty of the existence of distinct psychiatric disorders, high reliability in systems of universal coverage may, if anything, decrease the validity of the multitude of mutually exclusive and jointly exhaustive categories, the perils of which may not have been fully appreciated (Van Praag, 1992).

In comparison to the efforts directed at improving the reliability of psychiatric diagnoses, attempts to improve the validity of diagnostic categories have been relatively few and far apart. Robins \& Guze (1970), Klerman and colleagues (1984), and Kendell (1989) drew attention to the problem of clinical validity and discussed ways of actually establishing the vallidity of psychiatric syndromes. Validation of psychiatric syndromes has proven to be a problematic fiell, as the various ways in which the validity of a syndrome might be established (Robins \& Guze, 1970) have yielded ambiguous results. For example, even the most sophisticated statisticall techniques are not able to distinguish cases of schizophrenia from those of manic-depressive disordler on the basis of their phenomenology (Kendell \& Gourlay, 1970; Kendell \& Brockington, 1980), and no sharp demarcation between the two syndromes can be drawn on genetic grounds (reviewed by Taylor, 1992). Similarly, although the literature is far from conclusive (not least because researched less carefully), factors such as life events, ethnic group, ageing,

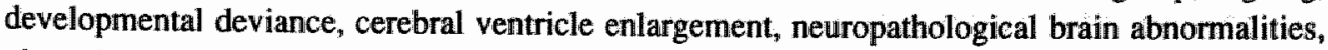
obstetric complications, increased $\mathrm{D} 2$ receptors in the basal ganglia, abnomal eye-tracking movements and increased latency of the P300 wave may equally well operate to increase the risk of affective disorders (Bebbington et al, 1993; Jones et al, 1994a; Done et al, 1994a, 1994b; Rogers, 1990; Harrison et al, 1988; Crow et al, 1994; D'Haenen et al, 1994; Van Os et al, 1995; 
Galdos et al, 1993; Galdos \& Van Os, 1995; Brown et al, 1986; Blackwood et al, 1987; Lewine et al, 1995).

Although psychologists distinguish a variety of different forms of validity, in psychiatry validity has very much come to be equated with predictive validity, ie the possibility of making a statement about the prognostic and therapeutic implications of a disorder (Kendell, 1989) importance of predictive validity first of all owes much to the imability of establishing an aetiological validating criterion in psychiatry (although some progress has been made - see Murray et al, 1985), which in medicine is accepted as the yard stick of the validity of any classification. However, even an aetiologically validated classification derives much of its usefulness from the fact that allows clinicians to make statements about outcome and response to treatment with a high probability of being correct. Therefore, in the absence of an aetiologically validated classification, a diagnostic classification with high predictive validity concerning course and treatment response is perhaps for practical purposes not markedly inferior to a truly aetiologically validated classification, or at least arguably constitutes the next best thing. Secondly, the impontance of course and outcome as a validating criterion stems from the attempt by Kraepelin to validate syndromes using clinical course, which proved so successful that our modern diagnostic classifications are still heavily influenced by Kraepelin's original ideas. On the basis of their differences in course and outcome, Kraepelin distinguished manicdepression and dementia praecox, the former characterised by good outcome, the latter by a deteriorated illness course (Kraepelin, 1919).

The finding that affective illness has on average a better outcome than schizophrenia has been replicated numerous times, but together with the apparent confirmation of the predictive validity of the basic distinction between affective and schizophrenic psychoses, a number of findings have appeared in the literature which show the need to reexamine the underlying assumption that our diagnostic categories can be separated on the basis of long term outcome.

- The conventional wisdom is that schizophrenia and manic depressive psychosis are separate "disease entities", each with a specific pattem of symptoms and course, and with distinct causes waiting to be discovered. However, it has been demonstrated that there is considerable variation in the course and outcome of schizophrenia, with up to a third of individuals making a good social and symptomatic recovery (Bleuler; 1972, Ciompi;, 1980; Shepherd et al, 1989), also in studies applying the more restrictive DSM-III criteria (Harding et al, 1987). Kraepelin, in the fifth edition of his Psychiatrie, ein Lehrbuch für Studierende und Ärzte (1919), already acknowledged that a substantial proportion (around $15 \%$ ) of his own patients with dementia praecox eventually recovered from the defect state. Conversely, it is by no means clear that patients with affective disorder have an invariably good prognosis; more than $60 \%$ of patients with psychotic depression or mania are psychosocially impaired at 5-year follow-up (Coryell, 1990a \& b). Up to a third of patients with bipolar affective disorder have poor social outcome (Clayton, 1981). Follow-up studies of patients with depressive disorders seen in psychiatric settings have shown that only a quarter will recover from an index episode and remain well for more than ten years thereafter, whereas around $15 \%$ of patients will have a continuous illness course without remission (Piccinelli \& Wilkinson, 1994). Similar rates of chronicity have been reported for community samples (Sargeant et al, 1990) and in widely different cultures 
(Thornicroft \& Sartorius, 1990). Lee \& Murray (1988) conducted a follow-up study of a series of 89 hospitalised depressed patients, and found that less than a fifth had remained entirely well and more than $40 \%$ of first admission cases had "very poof outcome". McGlashan (1984) reported that two-thirds of a group of 163 schizophrenia patients, but also one third of a group of 44 patients with unipolar affective disorder had poor outcome. From these data, it can be inferred that there is apparently considerable overlap between affective disorders and schizophrenia as far as outcome is concerned.

- From the perspective of predictive validity, further doubt is thrown upon the old categorical distinction between schizophrenia and affective psychoses by studies examining illness course of so-called intermediate forms of illness, such as schizoaffective illness (Kasanin, 1933). This term, recognized by both ICD-10 and DSM-IV, is used to denote those patients that display both schizophrenic and affective symptoms either simulltaneously or sequentially. A number of carefully conducted studies have shown that the illness course of such patients is also intermediate, ie more benign than in schizophrenia, but worse than in affective disorders. For example, Tsuang \& Dempsey (1979), in a 30 to 40 year longitudinal study, showed that outcome for a group of 85 patients exhibiting both affective and schizophrenic features was significantly poorer compared to a group of 325 patients with affective disorders, selected by the criteria of Feighner and colleagues, and significantly better than in a group of 200 schizophrenia patients. Hawk and colleagues (1975) described a study that included patients with at least one psychotic symptom, and examined the relationship between outcome and diagnosis. These authors found that the mean outcome in the (DSM-II) cohort with schizophrenia was poorer than in the nonschizophrenic patients. Coryell and colleagues (1990 a \& b) showed that affective psychotic disorders have a less disabling course than schizo-affective disorders; Grossman and collaborators (1991) showed that, overall, schizophrenia patients had poorest outcome, followed by schizo-affective patients, bipolar manic, and unipolar depressive patients. Similar findings were reported by Brockington and colleagues (1980a, 1980b), Marneros and collaborators (1989, 1990) and Maj \& Perris (1990), whereas a review by Samson ef al (1988) came to a similar conclusion. In general, longer term retrospective studies have yielded similar results (reviews by Harrow \& Grossman, 1984; Samson et al, 1988). There is further evidence that acute "atypical" "unspecified", "schizophreniform", "reactive" and "delusional" psychoses are more related to affective disorder than to schizophrenia, and occupy a position somewhere between affective and schizo-affective psychosis in terms of outcome, sex distribution, family history and incidence (Robins \& Guze, 1970; Fowler, 1978; Murray \& O'Callaghan, 1991; Susser \& Wanderling, 1994; Tsuang el al, 1976).

Therefore, there is in fact little to suggest that diagnostic categories can be separated (validated) on the basis of their outcome. In fact, several approaches can be taken to examine the null hypothesis that diagnostic categories in the functional psychoses cannot be separated on the basis of their outcome. Kendell and Brockington (1980) described a method of detecting discontinuity between one syndrome and another, based on the relationship between symptomatology and outcome. They argued that assessing the relationship between symptomatology and a second, 
independent, variable was likely to yield a more powerful method to demonstrate discontinuity between one syndrome and another. These authors examined the relationship between a score on a linear function, expressing the variation in symptomatology between typical schizophrenia and typical manic depressive illness (ie the variation in symptom pattems was reduced to a single linear dimension), and the patients' scores on eight different indices of outcome. The linear function score was available from a previous study by the same authors, using discriminant function analysis, a multivariate statistical technique (Everitt \& Dunn, 1991) used to disprove the null hypothesis that a linear discriminant function of $p$ variables (eg symptomatology or outcome variables) do not discriminate between groups $D 1$ and $D 2$ (eg affective and schizophrenic diagnoses). Their sample included 57 patients with schizophrenia, 4 with paranoid psychosis, and 73 with affective psychosis, and the mean follow-up period was 6.5 years. None of their analyses produced a non-linear relationship between symptomatology and any of the outcome measures. Such a non-linear relationship would have supported the notion of a discontinuity between affective and schizophrenic psychoses, and the absence of non-linearity may indicate that such a discontinuity between the affective and schizophrenic psychoses simply does not exist.

These issues are illustrated in figure 7 (after Kendell \& Brockington, 1980): a mean difference between two syndromes on a continuous outcome measure does not validate the existence of two separate disorders (figure 7a), as the underlying rellationship between symptomatology, expressed a linear continuum, and outcome can be either linear (indicating absence of discontinuity between the two syndromes; figure 7b) or non-linear (compatible with discontinuity and therefore supporting the validity of both syndromes as distinct entities; figure $7 \mathrm{c}$ ).

Fig. $7 a$ Usial means of illustrating the relationship between symptomatalogy and outcome (after Kendell \& Brockintgton, 1980)

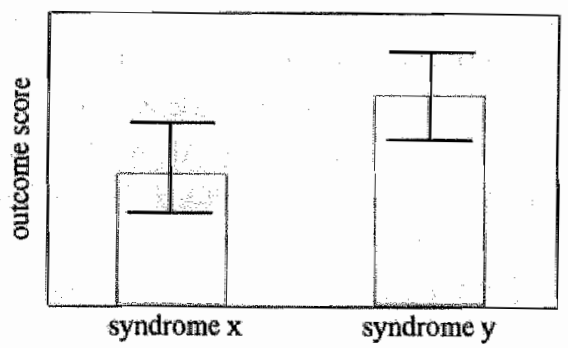

Brockington and colleagues (1991) conducted discriminant analyses using 30 variables, mostly variables on psychopathology and course of illness, in a sample of 302 psychotic patients with mixed affective and non-affective diagnoses. Their results showed that, for schizophrenia, the distribution of scores on the discriminant function was not bimodal. Rather, a skewed unimodal distribution was found, indicative of a schizodepressive continuum. Although it was found that bipolar disorder did emerge as a distinct grouping, the authors commented that a definition of bipolar disorder purely in terms of manic symptoms is unsatisfactory because it is known that some patients with the manic-depressive diathesis never have typical manic episodes. The one study using discriminant analysis that reported evidence of bimodality, suggesting that schizophrenia is a discrete disorder, will not be discussed here, as no outcome variables other than number of admissions to hospital were included in the discriminant analysis (Cloninger, 1985).Furthermore, the findings have been questioned (Grayson, 1986), and it has been cogently 
argued that the data were in fact more compatible with a skewed unimodal, rather than a bimodal distribution (Brockington et al, 1991).

Fig. 7 Relationship between symptomatology and owcome when symptomotology is comtated to a linear variable

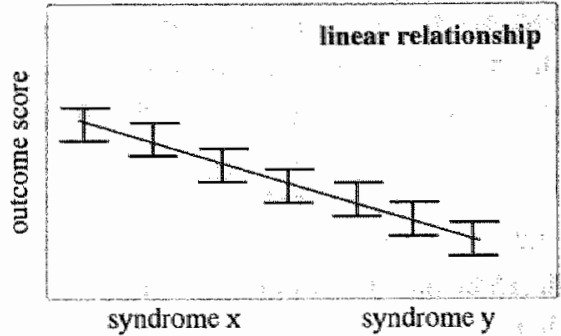

The weight of the evidence from these studies appears to indicate that analysis of outcome measures cannot produce subgroups confirming the existence of discrete diagnostic categories.

Fig. $7 c$

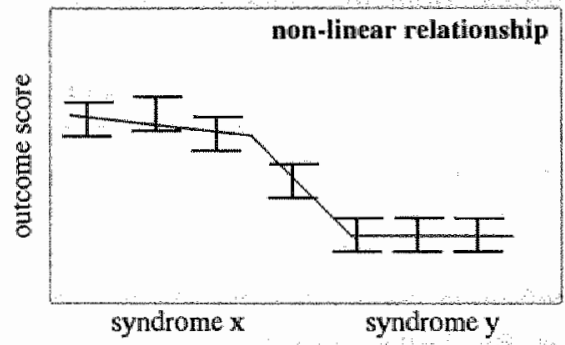

Of course, results from any of the above multivariate analytical procedures must be interpreted carefully. The choice of variables to be used for the discriminant analyses, for example, is cruciall, and slight variation in variable choice may give strikingly different results. Equally important is choice of subjects; exclusion of intermediate cases between two groupings may lead to spurious confirmation of the existence of two categories. Furthermore, many different techniques exist for the same basic multivariate analytical procedure, yielding findings which are not necessarily comparable.

A slightly different and possibly more robust technique was used by Johnstone and colleagues (1992) in the Northwick Park Functional Psychosis study. These authors followed-up threehundred and twenty-six consecutively admitted patients with functional psychotic illness after 2.5 years, and used polychotomous logistic regression in an attempt to identify a subgroup of independent variables (historical, clinical, mental state and outcome variables) which can be used to classify the patients into one of four diagnostic categories. Ordered polychotomous logistic regression yields odds ratios (OR) which are a single test statistic that summarizes the differences between the groups. The parameter of the ordered logistic regression is conceptually very similar to that in ordinary dichotomous logistic regression (McCullagh, 1980). In the study by Johnstone and colleagues, however, most groups of wariables failed to generate models that were successful in discriminating between diagnostic categories. These findings concur with those in the studies discussed above. 
The objection that can be brought up against these studies, is that the results may have been confounded. For example, the prevalence of female sex is likely to gradually decrease from affective to schizoaffective and schizophrenic disorder. As female sex is associated with a more benign course of ilness (Bardenstein \& McGlashan, 1990), the finding of a trend in the association between degree of affective symptomatology and outcome could be explained by sex rather than by diagnosis. In chapter 3.1 we will therefore examine whether i) there is a linear trend in the association with diagnosis, ordered from acute, affective disorder through to insidious, non-affective disorder, and outcome, and ii) whether this association is confounded by sex or chronicity.

In summary, a large body of literature has demonstrated that while on average outcome in the affective psychoses is better than in schizophrenia, there is a large degree of overlap. Furthermore, outcomes appear to be ordered, in that more affective symptomatology and more acute type of onset predicts better outcome. These findings are suggestive of an illness continuum predictive of an outcome continuum, and may indicate that mean differences in outcome between disorders should not be taken as evidence of the validity of the categorical classification, but rather as evidence of the inability to distinguish separate disorders on the basis of their outcome. In ather words, from the point of view of predictive validity, psychotic disorders may occupy different positions on an illness continuum, and not embody separate disease entities. In fact, a more appropriate way to go about the problem of validation in the functional psychoses may be to actively try to validate a psychopathological representation where individuals are assigned to a position along a linear continuum of one or more axes or dimensions.

\subsection{3 psychopathological dimensions and illness course}

In the previous section it was pointed out that what in fact appears to have been validated is a continuum rather than a typology of psychopathology: an illness continuum predicting an outcome continuum. As the categorical approach to psychiatric diagnosis cannot accommodate such evidence of continuity, and multiple testing for associations between a range of correlated individual symptoms and outcome is problematic, identification of a linear continuum of psychopathology along one or more axes or dimensions may constitute a more profitable approach.

There may exist a discrepancy in clinical psychiatry between what we teach and what we preach. Although, on average, psychiatric treatments and clinical outcomes in the functional psychoses differ from each other as a function of diagnostic category, there is a large degree of overlap, and no treatment or clinical outcome is disorder-specific. Conceptualisation of psychotic disorders as provided by influential taxonomic systems, however, is strictly typological and portrayed as mutually exclusive diagnostic categories.

The "usefulness" argument of the typological approach postulates that while not ideal, this strategy facilitates communication between clinicians and formulation of predictions regarding course and outcome. While this is certainly true, the usefulness argument is in practice often equated with the validity argument, which postulates that typological constructs really represent fundamentally different concepts. Much of this confusion is historical, in that, as discussed 
above, most of the typological constructs had been conceived and accepted as "valid" long before the issue of validity became pivotal, and it has since been perpetuated by a largely conservative professional group.

This apparent confusion between the "usefulness" and the "validity" arguments is crucially important. If typologcial constructs were trully valid, then so would be the current practice to identify efficacious treatments and factors important for secondary (and perhaps primary in the long term) prevention: separately for each typology. Nearly all the major treatment trials and observational studies report on typologically "homogeneous": samples. However, if it is demonstrated that a different form of classification is more valid, than a different strategy to identify efficacious treatments and prevention strategies may also be called for. In other words, the validity of our classification of psychotic disorders directly reflects on the validity of our attempts to identify treatments and factors related to primary and secondary prevention.

The major strategy towards authenticating psychiatric classification systems has been to try to confirm the validity of the existing approach, rather than to try to find a system with superior validity. We have proposed that, in the absence of an aetiological validating criterion in the functional psychoses, a classification validated by course and outcome may constitute the next best thing. Reviewing the evidence with regard to the predictive validity of typologies in the functional psychoses, we concluded that the comparative study of course and outcome of illness has failed to show that typological constructs are fundamentally different concepts. The evidence reviewed above, however, suggests that a linear continuum from acute/affective psychosis to insidious/non-affective psychosis, may correspond to a continuum of outcome: carefully conducted studies indicate that diagnostic categories, when ordered along a continuum ranging from affective, acute onset psychosis to insidious onset, non-affective psychosis, are predictive of progressively poorer outcome. In other words, what may be more valid is not the individual diagnostic categories, but the order in which they best reflect the linear continuum of one or more underlying psychopathological dimensions.

The major competitor to the typological approach to classification is one where individuals are assigned positions onto one or more continuous axes or dimensions. The main advantages of such a system is that they do not rigidly force clinicians to declare limits where none may exist, and that they are extremely adaptable, as they can be transformed into any number of categories and back again.

With the introduction of factor analysis by Spearman around the turn of the century a tool was available which allowed the discovery of basic dimensions of, for example, personality. Moore (1929) was one of the first to apply the technique to psychiatric symptoms, and with the increasing number of rating scales of psychiatric symptoms and the introduction of powerful computers, the field has been developed ever further (eg Eysenck, 1955), often in a context of heated discussions about the relative merits of typological and dimensional modells. In a more general form, the categorical/dimensional argument is frequently encountered in epidemiological studies. For example, the serum cholesterol concentration can be expressed as a continuum, but individuals may also be allocated to groups with "high" and "low" cholesterol concentration. Kendell (1993) points out that in psychiatry, there is no absolute preference for either, and that the choice of classification depends on the sort of issue one wants to address. One important advantage of dimensional models, however, is that it avoids arbitrary dichotomization leading 
to loss of information. For example, there may be little a priori knowledge of what constitutes a "high" cholesterol group and what a "Jow" group, and by placing a cut-off somewhere in the distribution, the point of variation that is most relevant to other biological processes may get "lost" in one of the two categories. We believe that, as far as the study of heterogeneity of course and outcome is concerned, a dimensional representation of psychopathology may be more informative than apological one, as outlined below.

A modest body of work has been dedicated to the study of clusters of correlated symptoms, identified using multivariate techniques such as principal component analysis and factor analysis. Many of the earlier studies (eg Everitt et al, 1971; Fleiss et al, 1971; Spitzer et al, 1967) applied such methods attempting to validate traditional psychiatric syndromes, using a range of symptoms and signs and a mix of diagnostic categories. More recently, much use has been made of dimensional models of psychopathology in schizophrenia research. The focus in this area has been not so much on nosological issues, but rather on the relationship between psychopathological dimensions and underlying pathophysiological processes within the diagnostic category of schizophrenia. Although the precise nature and numbers of symptoms clusters remains controversial, a number of studies have replicated the results of Liddle (1987a), who reported three clusters of symptoms consisting of i) negative features such as affective flattening and poverty of speech; (ii) positive features such as delusions and hallucinations; and iii) an entity relating to "disorganization" of behawiour and including incoherent speech and incongruity of affect (Brown \& White 1992; Peralta et al, 1992; Malla et al, 1993; Arndt et al, 1991; Mortimer et al, 1990; Van der Does et al, 1993). These syndromes of schizophrenia have in turn been examined in order to establish whether they show differential patterns of associations with neuropsychological and biological variables (eg Liddle et al, 1987b; Brown \& White; 1992; Liddle et al, 1992). While the results appear to provide some support for the validity of the three syndrome model, caution should be exercised. First, factor anallysis is a rather treacherous tool, that generates results which may be more of a reflection of the selection of items for analysis and the selection of patients, rather than evidence of a true underlying natural structure of symptomatology. Many studies have made use of instruments which are either more or less concept driven, such as the SANS and the SAPS (Andreasen, 1982; Andreasen, 1984), or rather limited in scope, such the BPRS (Lukoff et al, 1986) and the Krawiecka scale (Krawiecka, 1977), or a very much reduced list of items of a comprehensive instrument such as the Present State Examination (Wing et al, 1974). In other words, inclusion of scales specificallly designed to rate one or more groups of symptoms which are highly correlated, or reduction of more comprehensive instruments to resemble such scales, will not surprisingly return a "factor" resembling such a group of symptoms. Secondly, the cross-sectional nature of the research associating such symptom dimensions makes it often unclear how the results should be interpreted. For example, claims have been made that differential patterns of cerebral blood flow, associated with psychomotor poverty, reality distortion, and disorganization, reflect differential abnormalities of brain function underlying each of the three syndromes (Liddle, 1992). However, a more mundane explanation is that each group of symptoms generates different levels of activity in different locations of the brain. Furthermore, the different pattern of blood flow may be the results of confounding by (a number of) third variables, such as attention, age, or length of illness.

In summary, the study of dimensional representation of psychopathology initially took place against the background of a debate on the relative merits of dimensional versus typological 
models of psychopathology. With the advent of highly influential comprehensive typological diagnostic systems of high reliability (but uncertain validity) the debate appeared to have faded somewhat into the background. Recently, the study of psychopathological dimensions has been used extensively again in schizophrenia research, in an attempt to link heterogeneity in psychopathology to heterogeneity in the processes underlying schizophrenia. However, the vagaries of multivariate statistical procedures and cross-sectional study designs make results difficult to interpret. We propose a study (chapter 3.1) combining the issues of the heterogeneity of disease processes, and the dimensional/typological debate, through i) using course and outcome as a validating eriterion for different symptom dimensions in the functional psychoses, and ii) a comparison of the predictive validity of typological and dimensional representations of psychopathology. 
$\therefore \quad \cdots \quad \therefore \quad: \quad \cdots$

$\cdots$

A $\quad \cdots \quad \cdots$

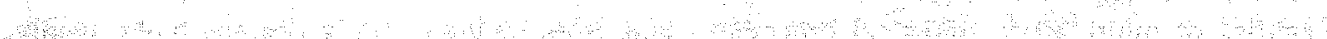

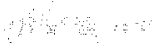

मा ख

$\therefore \quad \cdots$ $\therefore \therefore \therefore$ 


\subsection{Heterogeneity in illness course and risk factors for onset of functional psychotic illness}

\subsubsection{AGEING}

\subsubsection{Ageing and illmess onset}

There is important variation in the age-specific incidence of the functional psychoses. In Kraepelin's well-known series of 1054 cases of dementia praecox, he noted that before the age of 10 , the disorder was extremely rare, and that the prevalence rose rapidly after puberty. He also noted that "a not inconsiderable number of cases still reach development in the fourth, fifth and even sixth decade", and the male to female ratio changed dramatically with age, an excess of men being apparent before the age 40 , to make way for a female excess after that age. He also reported an interesting fluctuation in the male to female ratio around puberty: before the age of 10 , the male to female ratio was 2.3 , dropping to 1 between the ages of 10 and 15 , to rise again to 1.9 in the next quinquennium. Kraepelin concluded about this fluctuation that "there will certainly be a temptation here to think of sexual development, which is earlier in the woman" (Kraepelin, translated by M. Barclay, 1919, pp 230-231).

All these findings have since been replicated and further examined, with the exception of two aspects, which we believe are crucially important in determining the relationship between ageing and onset of psychosis.

Neurodevelopment and psychosis - First, the fluctuation in the male to female ratio suggests, as noted by Kraepelin, that maturational processes influence the emergence of psychosis. This fluctuation is readily interpretable in the light of modern neurodevelopmental theories of schizophrenia and related psychoses, which postulate that early abnormalities in the brains of pre-schizophrenic individuals give rise to symptoms 15-30 years later, when important maturational processes take place (Murray \& Lewis, 1987; Saugstad, 1989a \& b; Weinberger, 1987; Feinberg, 1982/83). This theory provides a plausible conceptual framework, but remains speculative, and it is difficult to provide direct evidence. In chapters 4.1 .1 and 4.1.2, we attempt to provide a more direct link between neurodevelopment and onset of psychosis. If puberty underlies the events that initiate schizophrenia, as some neurodevelopmental theories predict, then, since puberty in girls declares itself at a younger age than in boys, onset of psychosis around puberty should follow this trend. To examine the hypothesis that the differential onset of puberty in boys and girls results in earlier onset of psychotic symptoms in girls, we examined data regarding first admissions to mental illness hospitals in both England and France. In order to examine diagnostic and psychopathological issues and to establish the relationship between menarche and onset of psychosis, we also studied a sample of adolescents, referred to a regional adolescent unit in South London.

Neurodegeneration and psychosis - In spite of Kraepelin's observations, psychiatrists have traditionally been reluctant to accept that disorders identical to early-onset schizophrenia appear in old age. Depending on local assumptions, warious categories were used to accommodate these disorders which, as a result, have remained comparatively underinvestigated and of uncertain status, especially outside the UK. For example, until the advent of DSM-III-R, schizophrenia in the United States simply did not exist after the age of 45 , and in France this still remains the case. In other countries, the term "paraphrenia" is used in clinical practice. More recently, 
however, these disorders have been increasingly brought back from obscurity. An important epidemiological aspect concerns the possible relation of the ageing process and emergence of psychosis. If schizophrenia is a neurodevelopmental disorder, the link between early brain defect and maturational processes becomes increasingly tenuous with advancing age. Indeed, a more plausible explanation is the suggestion that primary organic brain dysfunction underlies these disorders in old age (Murray et al, 1992), thus setting them apart from the neurodevelopmental disorders. Our descriptive epidemiological work on data from the UK and France (Van Os et al, 1993; see figure 1 above) showed that in the UK, where the term schizophrenia is applied across the whole age range, is suggestive of such a relationship between organic brain dysfunction (degeneration) and psychosis, as the administrative incidence of schizophrenia was rising after the age 60 . In chapter 4.13 , we conduct an analytical study to further examine the relationship between ageing and onset of psychosis.

\subsubsection{Ageing and illness course}

The earlier the onset of the illness, the poorer the prognosis. Such an association has been shown for all psychotic disorders, such as schizophrenia (eg Stoffelmayr et al, 1983; Moller et al, 1982; Kolakowska et al, 1985; Gilbert et al, 1995), schizoaffective disorder (Mameros et al, 1988; del Rio Vega \& Ayuso-Gutierrez, 1990), and affective disorder (Brodaty et al, 1993; Winokur et al, 1993). Although some studies reported inconclusive findings (eg Coryell et al, 1990a \& 1990b; McGlashan, 1986; Huber et al, 1980), we have not been able to locate a study reporting that older age of onset was associated with unfavourable illness course, and it likely that sample selection can explain the discrepancy of the results. For examplle, age of onset was not an important predictor of outcome in the Chestnut Lodge follow-up study (McGlashan, 1986), but there was little variation in the age of the sample at baseline, as patients were mostly young and from upper social class. The association between age of onset and prognosis has face validity, as the impact of illness is much more damaging in an individual who has not yet completed the full social and physical maturational process, especially in illnesses which often run a chronic course, such as psychotic disorders. As early age of onset in schizophrenia is also commonly associated with negative symptoms, poor premorbid functioning and familial clustering of psychotic illness (Murray et $a,, 1992$ ), it is clear that age of onset may be an important confounder in examining relationships between many predictor variables and outcome. In this thesis, the role of age of onset as a confounder will therefore be considered where necessary:

\subsubsection{ETHNIC GROUP}

\subsubsection{Ethnic group and illness onset}

Defining ethnic group - Sex can act as a proxy for hormonal, genetic, psychological or social factors in epidemiological studies, and social class for anything from life-style to education. Associations with such variables may therefore be difficult to interpret. The term "ethnicity", however, is even more heterogeneous, and unfortunately has taken on the meaning of both race and ethnicity, the former term (as a term) being largely discredited in scientific research since the second world war. Race is a construct of the biological sciences, as a means of division of mankind on the basis of physical characteristics. It is used to define a group which in the past 
shared a common gene pool in which genetic differentiation from other populations has occurred. While this sort of division may perhaps have face validity, it has been pointed out that, for example, no single race can be discriminated from another on the basis of its genetic traits (Cooper, 1984). On the other hand, race as a construct has its use in clinical medicine, for example in the process of diagnostic deliberations leading to the recognition of sickle cell disease in patients of African origin (Hill, 1989). It has been pointed out, however, that the construct of race is now considered of much more use for the study of social determinants rather than of biological determinants of disease (Senjor \& Bhopal, 1994).

Ethnicity is a social construct, implying shared social background, often shared experience of migration, shared culture leading to a prevailing sense of common identity, and a common language or treligious tradition (Cooper, 1984; Allen, 1990). As such, its use in the biological sciences is confusing, as findings relating to the social construct of ethnic group are often presented as evidence that an underlying biological risk factor is operating (McKenzie \& Crowcroft, 1994). The prevailing view in the UK at present is that ethnicity is essentially a matter of self-perception. This principle, for example, lies at the heart of the 1991 OPCS Census classification of ethnicity. For the purposes of scientific research, however, this system has only limited value, as it is liable to considerable fluctuation (one third of subjects changed their selfassigned ethnicity over a period of two years in one study (Leech, 1989)). Furthermore, it has proven very difficult to understand, define and conceptualize ethnic identity (Phinney, 1990). On the other hand, the term "ethnicity" has also more and more come to replace the discredited term of "race" in scientific research, which tends to confuse things even further.

There have therefore been calls to establish international acceptable principles for the classification and description of ethnic groups (Bhopal et al, 1991; Senior \& Bhopal, 1994). As yet, no such system of classification exists. For the purpose of our research, therefore, we have relied on a classification of ethmicity mainly based on appearance, country of birth and appearance and country of birth of parents. We do not claim that this method of classification necessarily produces entirely homogeneous groups, but we believe that it is useful to start with broad categories based on region of origin before attempting to propose more specific (and much smaller and difficult to handle statistically) categories. For the purpose of defining these broad categories, the method is very adequate to describe the three main ethnic groups (European white, Afro-Caribbean, African) in the Camberwell catchment area, where our studies were conducted. In addition, we pay attention to the possible confounders that may operate in examining relative risks between ethnic groups, such as social class.

Ethnic group and onset of illness - Over the last 20 years, a considerable amount of academic activity has generated a body of empirical work showing that rates of schizophrenia in AfroCaribbean migrants and their offspring in the U.K. are higher than those in Non-Afro-Caribbean individuals (Harrison et al, 1988; Castle et al, 1991; Wessely et al, 1991). Recent investigations have reported rate ratios as high as 18, for some (Harrison, 1990) a reason to point out the role of migrant studies in aetiological inwestigations, for others (Sashidharan, 1993) ground to denounce "nineteenth century race science". The argument is complicated by reports of equally high first contact rates of schizophrenia in Dominica (Kay, 1992), whilst first admission (which is likely to underestimate true incidence) rates in Jamaica may be much lower than those reported for Afro-Caribbeans in the U.K. (Hickling, 1991). Given the potential scientific and social consequences of these findings, a particular carefull review of the methodological problems 
associated with determining rates of schizophrenia in Afro-Caribbean individuals is warranted. Rather than reviewing all the existing studies themselves, in this section we will focus on issues of methodology, because all studies have similar problems in this area. As rates are made up of a numerator and a denominator, we will consider possible bias and confounding for each separately. In addition, as most of the current focus in ethnic minority research is on schizophrenia in Afro-Caribbeans, on the basis of which some quite specific hypotheses were generated, we will examine the specificity of these findings both in terns of ethnic group and diagnostic category.

\section{OVERESTIMATION OF THE NUMERATOR?}

- Diagnostic reliability - The introduction of operationalised criteria, such as the RDC and DSM-III, has greatly increased the reliability of the diagnosis of schizophrenia. Some (mostly case register based) studies, however, applied operational criteria retrospectively to case note material (Castle et al, 1991; Wessely et al, 1991; Carpenter \& Brockington, 1980; Bagley, 1971; McGovern \& Cope, 1987), and thus are still dependent on what a variety of different clinicians chose to record over sometimes very lengthy periods. Applying operational criteria to prospectively gathered data in service based studies is clearly preferable, but will inewitably result in small numbers (Harrison et al, 1988; King et al, 1994), jeopardizing the statistical stability of the results.

Other studies (Selten \& Sijben, 1994; Glover, 1989; Cochrane \& Bal, 1989) relied on national, routine data sources, such as the Mental Health Enquiry and the Dutch National Case Register, which coded diagnoses according to the ICD, which was not operationalised, and therefore tended to be unreliable. Although unreliability in itself may not bias the results (Adelstein et al, 1968), it cannot be excluded that diagnostic reliability varies as a function of ethnicity, in which case it could render the results invalid. Furthermore, observer bias of junior and more senior doctors, involved in the ICD codings, cannot be excluded, especially as reports of high prevalence of psychiatric morbidity in Afro-Caribbean immigrants started to appear only years after the influx of large numbers of such individuals in the 1950s and 1960s (Kiev, 1963; Gordon, 1965). However, it has been shown that U.K. psychiatrists, at least in more recent times, are reluctant to make a routine diagnosis of schizophrenia in AfroCaribbeans (Lewis et al, 1990), which, if anything, would tend to bias the numerator, resulting in lower rates in Afro-Caribbeans. Furthermore, the predictive validity of a diagnosis of schizophrenia in Afro-Caribbeans has recently been validated in the UK (Sugarman, 1992).

- Diagnostic validity - Unfortunately, all psychiatric diagnoses, including schizophrenia, are relatively ill defined and poorly validated (Costello, 1992; Kerr \& McClelland, 1991). The use of operationalised criteria, such as the RDC and DSM-III may increase reliability, but have equally poor validity. Many authors have raised the possibility that the label schizophrenia may be inappropriately applied to Afro-Caribbean patients, who in fact have an acute, more benign, and transitory illness (Littlewood \& Lipsedge, 1981). However, this is not supported by various outcome studies, that have shown 
that there may be no differences in the course and outcome of schizophrenia between Afro-Caribbean and Caucasian patients (Sugarman, 1992; Harvey et al, 1990b), one study actually reporting a more deteriorated course of illness in Afro-Caribbeans (Prudo \& Blum, 1987).

- Behavioural toxic factors - Cannabis use may be a possible explanatory factor in all studies comparing rates in Afro-Caribbeans and Caucasians. Even though AfroCaribbean schizophrenia patients admitted more often to cannabis use than their Caucasian counterparts in one study (Harvey et $a l, 1990 \mathrm{~b}$ ), the available evidence appears to be against the existence of a cannabis induced, independent, self-limiting: schizophrenia-like illness ("cannabis psychosis"; Thomicroft et al, 1992), which could lead to misclassification and inflation of the numerator. Although cannabis may be an independent risk factor for chronic schizophrenia (Andreasson et al, 1987), and high rates in Afro-Caribbeans could possibly be partly explainable in terms of differential use of cannabis, it is very unlikely that such a mechanism is the sole cause for the observed excess, as a recent prospective study, although limited by small numbers, found no differences in cannabis use in a sample of incident psychotic patients (King et $a l, 1994)$.

- Pathways to care - Admission based studies, whether retrospective or prospective (Carpenter Brockington, 1980; Glower, 1989; Harvey et al, 1990b), run the risk of exaggerated numerators for Afro-Caribbean individuals. A combination of a higher threshold to newer forms of community care, and a higher social visibility of disturbed behaviour leading to involuntary admission may give rise to higher admission rates in Afro-Caribbeans. Workers in Birmingham identified 6\% Afro-Caribbeans in the general popullation in community they served, yet the proportion among all patients admitted compulsorily to the psychiatric hospital was 37\% (McGovern \& Cope, 1987). As pointed out earlier, the same does not hold for non-psychotic disorders, for which consumption of traditional psychiatric services by Afro-Caribbeans tends to be lower. The one existing (matched) case-control study (Wessely et al, 1991) in this area, while elegantly avoiding the problem in estimating the denominator, used non-psychotic controls, which may, for the reasons described above, have resulted in a biased ratio of discordant pairs. Another study by the same group, however, focussed on first contact cases, and was able to show that admission bias in admission based studies is unlikely to have led to spurious results (Castle et al, 1991).

\section{UNDERESTIMATION OF THE DENOMINATOR?}

- What is the size of the Afro-Caribbean population? - Many studies have relied rather uncritically on inaccurate data. The 1981 Census provided ethnicity of "head of household". The proportion of "persons usually resident in private households", by ethnicity of "head of household", is presumed to yield a correct estimate of the size of the Afro-Caribbean population, including children. In this way, there is clearly scope for misclassification. Whereas for cases, misclassification would lead to a drop in the rate ratio, under-assignment of Afro-Caribbean status in the denominator would inflate the rate ratio. To overcome this, workers have used material from the Labour 
Force Survey, in which people were asked to give their self-perceived ethnic origin. However, almost a million refused to answer this question; 1.8 million confessed to being Asian or West Indian, but little is known about responses for half such populations born in Britain. Anecdotal evidence is increasing that the pools from which cases and denominator populations are drawn from may be different. For example, the numbers of schizophrenia cases in the ethnic minority groups may have been exaggerated by the effect of young, vulnerable ethnic minority individuals, drifing into deprived inner city areas with a more stable (and heaithy) white population. As these cases have no equivalent denominator pool to be related to (because they are unlikely to participate in any form of official registration), it could be argued that they should be excluded from the analyses to avoid numeratordenominator bias.

On the face of it, it seems unlikely that the size of the Afro-Caribbean population has been underestimated by (reported rate ratios of) 4-18 times. On closer inspection, however, it is clear that this greatly depends on the age group from which individuals may be missing. As it is likely that missing individuals are young men, most at risk of schizophrenia, rate ratios may be disproportionably affected.

The 1991 UK Census data may bring improvement, but care must be taken to correct for underenumeration. The Census Validation Survey will not be able to establish how many of the substantial number of persons missed by the Census are Afro-Caribbean. Sex ratios by ethnicity are extremely suspect. For example, according to 1991 Census

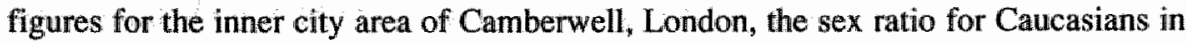
the age group 15-44 is 0.94, and for Afro-Caribbeans 0.69 (own calculations), highlighting the possibility of a large number of young men being differentially missed by the Census. In addition, according to Siegel (1974), under-reporting in the US Census may be in the region of 4 to $18 \%$ in Black men, compared to 1 to $4 \%$ in White men, and there is anecdotal evidence of a similar ethnic discrepancy in the 1991 British Census.

- Age structure of the Afro-Caribbean population - Schizophrenia is essentially a disorder of young people, although there is increasing evidence that many cases occur in old age, especially in women (Castle \& Murray, 1993). As the Afro-Caribbean population is likely to have a more pyramidal age-sex structure compared to the rectangular shape of the native population, this will clearly bias rates of schizophrenia. Furthermore, many epidemiological studies exclude late-onset cases (Castle \& Murray, 1993), which will lead to a further increase in the rate ratio. Age standardisation in the studies reviewed here has been crude (Harrison et al, 1988), if present at all (Harvey et $a l, 1990 \mathrm{~b})$. Only Castle et al (1991), in a retrospective, case-note, and catchment area based study of first contact cases reported standardisation to the 1964 population structure. However, the width of the age bands used was not reported, so that the possibility of residual confounding cannot be excluded.

- Social Class - Schizophrenia is overrepresented among the lower social classes (Eaton, 1980), but there is no convincing evidence that onset of schizophrenia and socioeconomic status are associated (Jones et al, 1994b; Wiersma et al, 1983), so that it is 
unlikely that the reported ratio measures could be confounded by social class. Still, it can be argued that the onus should be on the researchers to show that no such confounding mechanism exists in the study of ethnicity and schizophrenia incidence. No study, however, was able to stratify by socio-economic status. Although some of the best studies involved small, supposedly uniformly deprived areas (Harrison et al, 1988; Castle et al, 1991; Wessely et al, 1991), a 10\% sample from the 1991 Census in Camberwell, where two of these studies were completed, indicates that even within such a small area, Afro-Caribbean economically active residents aged 16 and over are $1.8(95 \%$ CI: $1.7-1.9)$ times more likely to be unemployed than their Caucasian counterparts and $2.1(95 \%$. CI 1.7-2.6) times more likely to belong to social classes 4 and 5 (own calculations). It can be argued that the above magnitude of the association of the confounder (social adversity) with the exposure under investigation (ethnicity) is not nearly high enough to be able to explain rate ratios in the order of 4-18. On the other hand, social class and unemployment may be only imprecise proxies for the latent 'real' deprivation factors associated with disease, the odds of which could be many times higher than those for lower social class and unemployment alone. Again, however, is should be stressed that there is no good evidence linking social class and onset of schizophrenia (Jones et al, 1994b; Wiersma et al, 1983).

\section{SPECIFICITY OF FINDINGS}

Similar to the findings in the areas of neuroimaging and life event research, evidence is emerging that the findings are not specific to schizophrenia. For example, of the few reports that included affective illness, all found similar elevated rates among members of ethnic minority groups (Leff et al, 1976; Harrison et al, 1988; Hemsi, 1967). There is also a growing awareness that, compared to the group of European whites, high rates may not be confined to the Afro-Caribbean population (Rwegerella, 1977; Selten \& Sijben, 1994; King et al, 1994).

In conclusion, compared to cerebral ventricle dimensions and life events, the evidence supporting an association between ethnic group and onset for psychosis is weaker. Therefore, before considering the relation between ethnic group and course and outcome, we will first focus on ethnic group in relation to incidence of psychotic illness. Although across studies comparing the rate of schizophrenia between white and Afro-Caribbean populations, strength and consistency in the reported elevated rate ratios stand out, there is similar consistency in unresolved methodological problems throughout the literature, the effect of which on the reported associations is invariably to inflate the numerator or deflate the nominator, and thus to inflate the rate ratio, with the likely exceptions of misclassification of Afro-Caribbean status in schizophrenia cases, and the apparent reluctance on the part of U.K. psychiatrists to apply a schizophrenia diagnosis to Afro-Caribbean cases. Cannabis use, social cllass, and differential pathways to care could all theoretically lead to an inflated rate ratio, but, as discussed above, it is unlikely that these factors have contributed to the consistently elevated rate ratios across studies. More important issues are the differential mobility and ways to correct for underenumeration of ethnic minorities in official data. As both these factors can theoretically be corrected for by adjusting the denominator, we will attempt in chapter 4.2 .1 
to calculate rates for various ethnic groups with revised denominator values, based on the 1991 OPCS Census data. Furthermore, the specificity of the findings both in terms of diagnosis and ethnic group remains underinvestigated. In chapter 4.2 .2 we will further address and examine these issues.

\subsubsection{Ethnic group and illness course}

The research on illness course and ethnic group has been motivated by two issues. First, the study of illness course may shed light on the possibility of misdiagnosis of ethnic minority patients by "ethnocentric" white psychiatrists (Littlewood \& Lipsedge, 1981). However, as discussed above, misdiagnosis is unlikely to have contributed to the observed high rates among ethnic minorities. Secondly, the study of illness course may shed light on the observation that patients in developing countries may have a better prognosis than their counterparts in the industrialised world (Sartorius et al, 1986). This WHO study used longitudinal measures of course, and it was found that patients in developing countries more often had a mild or intermediate course of illness, which was not explained by differences in mode of onset alone. Although these findings, and other studies reporting such differences between countries, must be viewed with caution (Lin \& Kleinman, 1988), they provide nevertheless a basis for hypothesis testing in future studies.

There have been some follow-up studies comparing outcome in different ethnic groups (Sugarman, 1992; McGovern \& Cope, 1994; Prudo \& Blum, 1987; Harvey et al, 1990b). These studies thave either found no differences or worse outcome for individuals of Afro-Caribbean

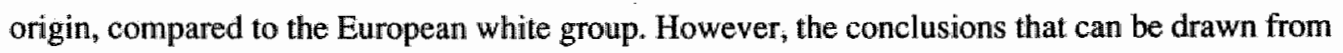
these studies are limited. Cooper showed that social class is associated with course and outcome in schizophrenia (Cooper, 1961). Unfortunately, none of the follow-up studies adjusted for confounding by level of education and socio-economic status, which may in fact have obscured the fact that the Afro-Caribbean group has better outcome. Two studies were retrospective (Birchwood er al, 1992; Harvey et al, 1990b); and none included longitudinal measures of course. Two studies reported higher readmission rates for Afro-Caribbean subjects (Birchwood et $a l, 1992 ;$ McGovern et al, 1994; Leff et al, personal communication), but this is not a good measure of clinical outcome, especially in view of the high rates of pollice contact in ethnic minority patients, leading to subsequent readmission (McGovem et al, 1994; Perera et al, 1991). In chapter 4.2.3, we report the findings of our own 4-year follow-up study of 191 subjects. In this study, we also address the issue of how ethnic groups are defined, and how definition of ethnicity actually affects the results. The link between migration and psychosis is well established (Ödegaard, 1932). We therefore also examine to what extent experience of migration may explain any effect of ethnic group on course and outcome of illness (McKenzie \& Crowcroft, 1994). 


\subsubsection{LIFE EVENTS}

\subsubsection{Life events and illness onset.}

A purely "social" cause for psychotic illnesses is unlikely. However, the influence of social factors in relation to the onset and relapse of schizophrenia has been demonstrated times and again, and influential investigators into the biological basis of schizophrenia have contended that any aetiological model must account for the relationship between stress and schizophrenia (Weinberger, 1987; Murray et al, 1988). Early observations included the high rate of schizophrenia onsets in recruits entering military service, especially in the first month (Steinberg \& Durell, 1968), and the high exacerbation rate of patients exposed to too much pressure in rehabilitation programmes (Wing et al, 1964). While the situations in these correlational studies have face validity with regard to the concept of "stress", the further study of stress in relation to onset of psychosis requires an operationalisation of the concept. Furthermone, the concept of stress has developed more and more towards an interactive model, whereby the external "stressful" circumstances interact with the individuals cognitive set and particular resources. In other words, stress must be measurable, taking into account the diverging perceptions and reactions that may occur in different individuals in response to a similar event. The recording of a series of events, commonly referred to as "life events", occurring before onset of illness thas become one of the most important scientific paradigms in the study of the relationship between stress and onset of psychiatric illness. The area of measurement of life events in relation to onset of illness is fraught with methodological problems, a full discussion of which is beyond the scope of this work. Bebbington (1988) listed 9 requirements for the study of life events in schizophrenia, and concluded that no study had met all requirements (Table 2). The most important requirements possibly are that i) there should be an objective rating of the degree to which events were independent of illness factors (ie reversed causality: the illness brought about: the event and not the other way round), and ii) there should be an objective rating of the impact of events, given that two persons may have entirely different experiences with regard to the same event. Some other requirements will be discussed in more detail below.

\section{Table 2. Requinrements for life event studies in schizophrenia} (from Bebbington, 1988)

Standardized method of symptomatic assessment

Standardized nethod of case definition

Limitation to cases where it is possible to date onset accurately

Onset defined as move from effectively sympton free state to a psychiotic one

Structured collection of events from a defined period before onset

Precide dating of evenis to identifying the salient period of effect

Objective ratings of the inpact of events

Objective rating of the degree to which events are independent actions of the subject that might have been due to emergency of ilhess

An appropriate control group

In spite of these methodological difficulties, the results of 7 studies reviewed by Bebbington (1988) were suggestive of an excess of stressful life events in the three weeks before onset of schizophrenic illness. Notably, the findings of a large WHO collaborative study (Day et al, 1987) were interesting, as the study sample included 386 cases with a variety of functional psychotic 
ilnesses from nine catchment areas around the world. No suitable control groups were collected, but the findings are suggestive as in all the centres events tended to cluster in the three weeks before onset, and to a lesser degree in the 3 weeks before that. The association between illness onset and events was not specific for schizophrenia. More recently, support for an association between life events and illness onset has come from three studies which introduced important methodological improvements. Two of the three studies employed a design involving the prospective assessment of patients who had recovered from a known episode of illness, comparing the frequency of events in a set period preceding onset with analogous periods in the same patient not followed by relapse, and with the mean event rate in non-relapsing patients (eg Ventura et al,1989; Malla et al, 1990) Both found that events were commoner in periods preceding relapse of psychosis. The longitudinal design in these two studies was particularly suitable to circumvent the problem of recall bias, or the "search for meaning", whereby individuals with psychiatric illness attempt to give meaning to their illness by linking them to past events which would otherwise perhaps have been considered insignificant subjectively. The other advantage is that the patients can be used as their own controls, which obviates the problem of confounding. The Camberwell Collaborative Psychosis Study (Bebbington et al, 1993) was unique, in that it met all the 9 requirements set out above and included a wide range of psychotic illnesses. Again, it was shown that the greatest discrepancy between cases and community controls was for the more severe events in the combined category of "independent" and "possibly independent" events (see chapter 4.3.1) in the three months immediately prior to onset. Furthermore, the findings were not confined to any particular diagnostic category.

Although the association between life events and illness onser is not specific to any particular disorder within the functional psychoses, there is some evidence that the association is stronger at the affective end of the disorder-spectrum. For example, Paykel (1978) calculated the relative risk to express the magnitude of the association between life events and onset of disorder. He found that the risk of developing depression increased sixfold in the six months after experiencing markedly threatening life events. However, the comparable increase in risk for schizophrenia was two- to fourfold: In our own study (Bebbington et al, 1993), we found that the risk of developing psychotic depression was increased sevenfold, compared to a fivefold increase for schizophrenia. Brown (Brown et al, 1973) used another estimate, the 'brought forward time" and reported similar results.

Because of the consistency of the findings, the temporal relationship and the plausibility of stress as a "destabilizing" mechanism, much thought has been give to the possible aetiological importance of life events. In view of the dearth of knowledge on the mechanisms and aetiology of the functional psychoses, much of the theorising on this issue has been necessarily simplistic, but at least has provided researchers with some refutable working hypotheses. All theories have to take into account the joint effects of genetic and environmental factors, given the undoubtedly genetic contribution to affective disorder and schizophrenia. We will consider interactive, additive and independent gene-environmental models and their predictions regarding life events and familial morbid risk of illness, which is the subject of one of the investigations in this thesis.

Genetic and environmental independent effects - Possibly the simplest model is to assume that there is a dichotomy of risk: onset of illness is either related to stress or to familial factors (indicative mostly of genetic predisposition rather than influences of the shared environment). This would be an example of the discrete subtype model, where both environmental and genetic 
factors are thought to be sufficient in their own right to induce lliness (eg Murray er $a l, 1985$ ), which is illustrated in table 3 below:

Table 3. Expected risk of illness wnder discrete subrype model of gene-enviromment materaction

\begin{tabular}{|c|c|c|}
\hline (5) & rolmerability & no vulnerability \\
\hline $\begin{array}{l}\text { life event } \\
\text { no wife event }\end{array}$ & $\begin{array}{l}\text { high } \\
\text { high }\end{array}$ & $\begin{array}{l}\text { low } \\
\text { high }\end{array}$ \\
\hline
\end{tabular}

Under this model, one would expect low rates of illness in the relatives of sporadic (ie nongenetic, in this case life event-induced) cases. A study by Pollitt (1972) is often quoted in support of such a distinction. This author reported a much higher morbid risk of depression $(21 \%)$ in the relatives of probands whose depressive illness was not preceded by stressful events, than in probands whose illness developed in response to severe physical stress (6-12\%). However, subsequent work involving far more sophisticated measurement of possible stressors did not concur (Brown \& Harris, 1978); notably, in a comparison between depressive patients with and without a family history of major depression, Perris and colleagues (1982) failed to find differences between the two groups in the number of stressful events prior to the episode. In the Camberwell Collaborative Depression Study (McGuffin et al, 1988 a \& b), 83 families were ascertained through depressed probands. The relatives of the probands whose illness was preceded by stressful life events or chronic difficulties had slightly higher lifetime rates than the relatives of probands whose onset of illness was not preceded by such events. In the same sample (Bebbington et al, 1988) it was demonstrated that onset of the more severe "endogenous" type of depressive illness was not independent of antecedent life stress, and therefore not different from non-endogenous, milder types of depression in that respect. Perhaps even more surprisingly, it was demonstrated that, instead of the expected inverse relationship between reactivity to stress and familial loading, both life events and depressive disorder appeared to be familial, suggesting that event-related depression is something which befalls hazard-prone rather than stress-susceptible individuals (McGuffin et al, 1988 a \& b). Work from the Zurich Cohort Study (Ernst et al, 1992) has demonstrated an excess of reported life events in pre-depressed individuals in a comparison with normal controls. Interestingly, this difference remained constant over many years, suggesting depressed individuals are indeed more "hazard-prone" "A twin study involving more than 800 pairs reported similar results (Kendler et al 1991 ): a model in which resemblance in the number of life events in twins was due only to genetic factors could be rejected, but the results were consistent with a substantial familiallenvironmental effect. Genetic factors appeared to play a role in dependent, but not independent life events. Plomin (1994) has argued that life events, traditionally considered as an environmental variablle, are subject to considerable genetic influence. Analogous to the findings reported by Kendler and colleagues (1991), twin data from Plomin (1994) suggested that heritability estimates for life events were higher for (but did not appear to be confined to) events befalling the self as opposed to events happening to other persons.

This lack of evidence for discrete subtypes is not confined to life events; Roy and Crowe, in a more general review of the evidence supporting such models, concluded that there was little to support the sporadic-familial distinction, and even some of those who were important initial 
advocates of the model as a research strategy (Lewis et $a l_{*} 1987$ ), came to the same conclusion (Lewis, 1990).

Genetic and environmental interactive effects - The so-called arousal theories of schizophrenia postulate that patients with schizophrenia have an abnormal response to stress, ie they react with psychotic symptoms. Or, in another version, they do not only react abnormally, but they are also abnormally sensitive to stress (Norman \& Malla, 1993a). Within the arousal theory framework, several authors have proposed "vulnerability-stress" models (Norman \& Malla, 1993b), which accommodate both pre-existing vulnerability and reaction to stress, postulating that the likelihood of developing schizophrenic symptoms is due to the interaction between biologically mediated vulnerability and the amount of stress experienced. The vulnerability-stress model assumes that neither genetic predisposition nor environmental factors such as life events are in themselves sufficient to bring about illness, but that the increase in risk conferred by their interaction can (Table 4).

Table 4. Expected risk of illness under interactive model of gene-environment interaction.

\begin{tabular}{|l|ll|}
\hline & vilnerability & no vulnerability \\
Wife event & high & low \\
no life event & low & low
\end{tabular}

Zubin \& Spring (1977) proposed a continuously distributed biological liability to schizophrenia on one axis, and the degree of stress on the other (figure 8), the highest risk being conferred by elevated scores on both axes. Interaction between genes and environment may involve genes altering the sensitivity to a predisposing environment, or environments altering the penetrance of a given gene. The nature of the interaction between life events and genetic factors is likely to involve a genetical control of sensitivity to the environment: genetic factors increase the risk for illness in part by modifying the individual's sensitivity to the illness-inducing effect of stressful life events (Kendler \& Eaves, 1986; Kendler et al, 1995). Assuming independent life events and genetic factors are not correlated (ie there is no genetic control of exposure to the environment - which is likely to be the case for at least the independent life events (see above), but are randomly distributed across the population, it would be expected that most people with schizophrenia would present by virtue of their biological predisposition in the face of low to moderate levels of stress (Norman \& Malla, 1993b). This concurs with what Brown \& Harris called a "triggering" role for life events in schizophrenia. These authors, on the bases of their own findings (Brown et al, 1973; Brown \& Harris, 1978), postulated that dispositional factors play the larger part in the aetiology of schizophrenia, and that life events associated with low to moderate levels of stress merely precipitate illness.

A smaller number of individuals, however, may develop illness as a result of very high levels of stress in combination with only little biological vulnerability. This latter group would constitute the "reactive psychoses" and it can be argued that such reactive illnesses will have a better prognosis than illness associated with "constitutional" factors (see below). 
Because, under the interactive model, the environmental exposure greatly magnifies the influence of a given genetic predisposition or vice versa, the prediction with regard to familial morbid risk in probands with and without the environmental exposure would be that relatives of exposed probands would have higher rates of illness.

Fig. 8 Possible relationship between vulnerability, stress, and onset of schizophrenia (after Zubin \& Spring, 1977)

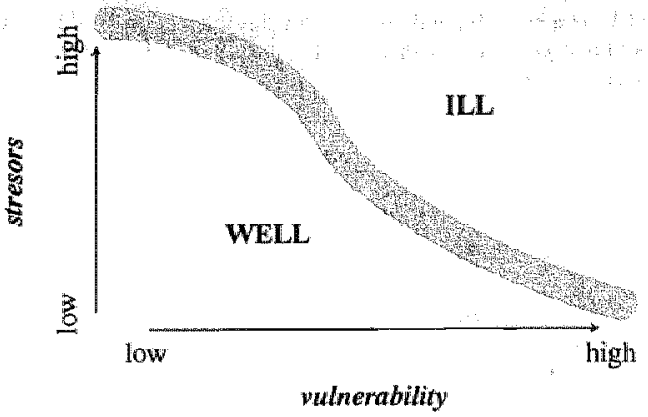

Thus, given a frequent exposure and a moderately prevalent genotype, the rate of illness in the monozygotic co-twins of exposed probands will be higher than for the co-twins of unexposed probands. This, however, is not always true. A weak interactive effect would not yield a higher rate of illness in the relatives. It follows, however, that if a genetic and an environmental factor are examined jointly and a higher rate of illness in the relatives can be demonstrated, then an interactive mechanism is much more likely than independent or additive gene-environment effects. However, failure to demonstrate such a relationship does not exclude interaction. We have explored the mathematical reasoning underlying these statements in table $5 \mathrm{a}$ and $5 \mathrm{~b}$. Assuming multiplicative rather than additive risk models provides the strongest support for the supposition that higher rates of illness will occur in the relatives of probands exposed to life events.

Three studies examined for possible gene-environment interaction; two of these yielded findings which would support such a mechanism. Phelan et al (1991) interviewed 1523 employees of a major US corporation. Because of the large sample size, life event assessment did not include contextual threat nor independence of abnormal behaviour ratings, and family history on first degree relatives was assessed through the proband interview. Depending on the type of life event, both positive and negative interaction with family history of depression was found, suggesting genetic factors both increase and decrease an individuals susceptibility to the depression-inducing effect of life events. The number of statistical tests was very large, however, and some of their findings may have been due to chance. In a further report on the study, already mentioned above, of female-female twin pairs by Kendler and colleagues (1991), some ingenious evidence was provided for an interaction between life events and onset of depression.In twins considered at lowest genetic risk (monozygotic twin, co-twin unaffected), the probability of onset of major depression per month was predicted to be $0.5 \%$ and $6.2 \%$, respectively, for those unexposed and exposed to a severe life event. 


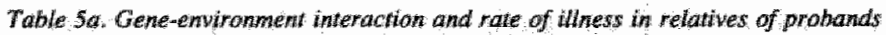
exposed to the predisposing enviromonent

We wish to demonstrate that, given an enwronmental and a genetic risk factor, the risk of lilness in the relatives is higher in probands exposed to the predisposung environment if there is gene-enwironment intercction, whereas the reverse is true if the effects of genes and prextisposing environment are addithe (ie relatives of exposed probands will have lower rates of ilness).

The firt $2 \times 2$ table describer the risk of developing illness in relation to an environmental risk factor (E) and a genetic risk factor as indieated presence of illness in the first degree relatiwes (Fi), E+ indicates a predisposing environment, and $\mathbb{E}$ - denotes a pirotective environmenl. The risks follow an adduve nodel; "al "being the baseline risk in the absence of both risk factors" "b" and "c" are the main effects of FH and E, respectively, and "d" denotes the interaction, the the excess risk associated with the combination of $\mathrm{E}$ and FH. Under the additiwe gene-environment model, $d=0$; ander the interactive model $d>0$. The following constratints apply: $a, b, c, d y d$; and $a+b+c+d \leq 1$

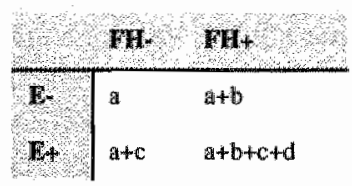

The second $2 \times 2$ table describes the frequencies with which the factors $\mathrm{E}$ and FH occur in the population. $\mathrm{E}$ and FH are not correlated. The marginal totals of FUlt and FH-; as well as those of $\mathrm{E}+$ and $\mathrm{E}$, sum to unity.

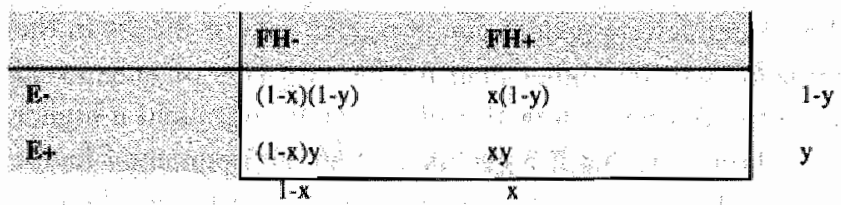

Given the frequencies of the risk factors, and the magnitude of the risk they confer, we can calculate the frequency distribution of the different combination of risk factors among the cases in the population.

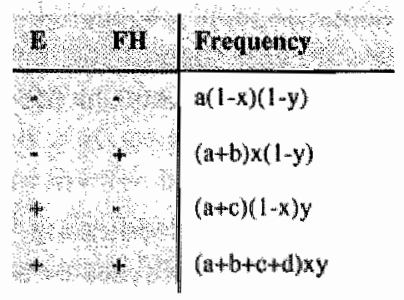

Thus, the probability of being a case with a positive family listory, given a protective environment:

$P(F H+1 E-)=(a+b) \times(1-y))((a+b) x(1-y)+a(1-x)(1-y))=(a+b) x /((a+b) x+a(1-x))$

and the probability of being a case with a positive family history. given a predisposing environment:

$P(F H+1 E+)=(a+b+c+d) x y /((a+c)(1-x) y+(a+b+c+d) x y)=(a+b+c+d) x)((a+b+c+d) x+(a+c)(1-x))$

Thus, the prediction that $\mathrm{P}(\mathrm{BH}+1 \mathrm{E}+\mathrm{)})>\mathrm{P}(\mathrm{FH}+\mathrm{I} \mathrm{E}-)$ can be written as.

$(a+b+c+d) x /((a+b+c+d) x+(a+c)(1-x))>(a+b) x /((a+b) x+a(1-x)) \Rightarrow(a+b+c+c) x+(a+c)(1-x) /(a+b+c+d) x<(a+b) x+a(1-x) /(a+b) x$ $\Rightarrow 1+(a+c)(1-x) /(a+b+c+d) x<1+a(1-x) /(a+b) x \Rightarrow(a+c)(1-x)(a+b) x<a(1-x)(a+b+c+d) x \Rightarrow(a+c)(a+b)<a(a+b+c+d) \Rightarrow$ $a^{2}+a c+a b+b c<a^{2}+a b+a c+a d \Rightarrow b c<a d \Rightarrow a d>b c \Rightarrow d>b c a$

Given the following risks: $a=0,05 ; b=0,05 ; c=0.05$, it follows $a d b c$ is true for interaction lerms $d>0.05$, ie only interaction of moderate strength is neded in order to show the effect of increased risk of illness the relatives. However, absence of increased risk in relatives does not exclude interaction. With additive effects, in the absence of interaction ( $d=0$ ), ad $>$ be can never happen. Thus, higher rates of illness in the relatives is specific for gene-environment interaction, but absence of higher rates in the relatives does not exclude interaction. 


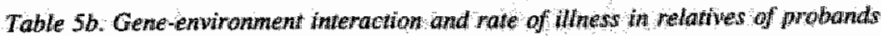
exposed to the predisposing environment"

We wish to demonstrate that, given an enwironmental and a gemetic nisk factor the risk of lliness in the nelatives is higher in probands exposed to the predisposing environment if there is gene-environment inferaction, wheneas the reverse is true if the effects of genes and predisposing environment are additive (ie telatives of exposed probands will have hower rates of ill ness).

The first $2 \times 2$ table describes the risks of developing iliness un ralation to an environmental risk factor (E) and a genetic risk factor as indicated presence of ullness in the first degree relatives (FH) $\mathrm{E}+$ indicates a predisposing environment, and $\mathrm{E}$ - denotes a protective environment. The risks follow a multiplicative model, "a" being the baseline risk in the absence of both risk factors, "b" and "c $c$ "are the main effects of FH and $\mathrm{E}$, respectively, and " $\mathrm{d}$ " denotes the interaction, ie the excess risk associated with the combination of $\mathrm{E}$ and $\mathrm{FH}$. Under the multiplicative gene-environment model $\mathrm{d}=0$; under the interactive model, $\mathrm{d} \times 0$. The following constraints apply: $a \geq 0 ; b, c, d \geq 1$; and abcd $\leq 1$.

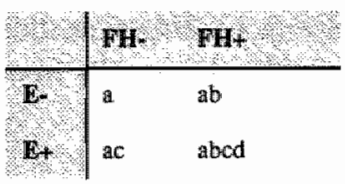

The second $2 \times 2$ table describes the frequencies with which the factors $E$ and FH accur in the poptation. $E$ and $F H$ are thot correlated. The marginal totals of FH+ and FH-, as well as those of E+ and E-, surn to unity.

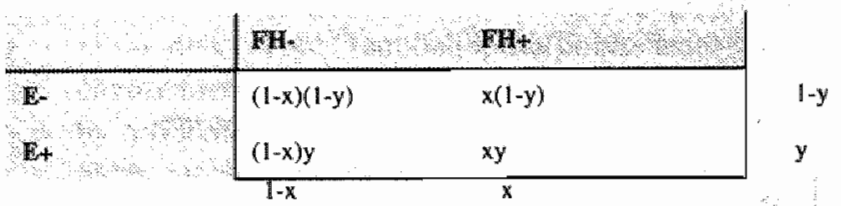

Given the frequencies of the risk factors, and the magnitude of the risk they confer, we can calculate the frequency distribution of the different combination of risk factors among the cases in the population.

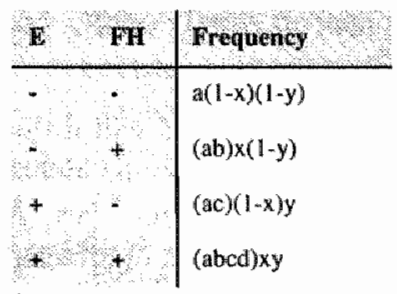

Thus, the probability of being a case with positive family history given a protective environment:

$P(F H+1 E-)=(a b) \times(1-y) /((a b) x(1-y)+a(\|-x)(1-y))=(a b) x /((a b) x+a(1-x))$

and the probability of being a case with a positive familly history, given a predisposing environment:

$P(E H+1 E *)=(a b c d) x y /(a c(\|-x) y+(a b c d) x y)=(a b c d) x) /(a b c d x+(a c)(1-x))$

Thus, the prediction that $\mathrm{P}(\mathrm{FH}+\mid \mathrm{E}+\mathrm{H})>\mathrm{P}(\mathrm{FH}+\mid \mathrm{E}-)$ can be written as:

$(a b c d) x /(a b c d) x+(a c)(1-x))>(a b) x /((a b) x+a(1-x)) \Rightarrow b d x /(b d x+(1-x))>a b x /(a b x+a(1-x)) \Rightarrow$ $b d x+(1-x) / b d x<(a b x+a(1-x)) / a b x \Rightarrow 1+(1-x) / b d c<1+a(1-x) / a b x \Rightarrow(1-x) a b x<b d x(a(1-x) \Rightarrow$ $x(1-x)<d x(1-x)$

This is always true for $d>1$. Thus, interaction between multiplicative risk factors is always accompanied by higher rates of illness in the relatives. 
In those at highest genetic risk (monozygotic twin, co-twin affected), these risks were $1.1 \%$ and $14.6 \%$, respectively. Evidence for statistical interaction was provided in regression analyses (Kendler et $a l, 1995$ ). Although not without weaknesses (eg contextual threat and independence of life events were not assessed), the twin design of this study was particularly suitable to examine the issue of gene-environment interaction. The Camberwell Collaborative Depression Study undoubtedly employed the most sophisticated method in assessing the occurrence of life events, as well as their contextual threat and independence of abnormal behaviour of the subject; all available first degree relatives of 86 depressed probands were interviewed. The authors reported that, contrary to the prediction of the gene-environment interaction model, individuals at high familial risk for depression appeared to be less sensitive to the illness-inducing effect of life events than individuals at low genetic risk (McGuffin et al, 1988 a \& b). However, this association was only weak and non-significant, and in apparent conflict with other results from the study.

Genetic and environmental additive effects - Under this model, life events, genetic factors and other predisposing influences from the "aetiological pool" act in an additive fashion to produce an increase in the liability to develop schizophrenia, disease occurring when an indiwidual's cumulative vulnerability exceeds a certain threshold (Table 6).

Table 6. Expected risk of illness under additive model of gene-environment interaction.

\begin{tabular}{|c|c|c|}
\hline & Yulmerability & no volnemability \\
\hline $\begin{array}{l}\text { Iffe event } \\
\text { no life event }\end{array}$ & $\begin{array}{l}\text { high } \\
\text { medium }\end{array}$ & $\begin{array}{l}\text { medium } \\
\text { low }\end{array}$ \\
\hline
\end{tabular}

Under this assumption, individuals with the more extreme exposures to life events will differ quantitatively with regard to familial morbid risk from individuals with low exposure: the higher the liability due to environmental factors, the lower the risk of illness in the relatives. A hypothetical example would be to compare the risk of illness in the identical co-twin of affected twins who i) where exposed to the predisposing environment, and ii) who were exposed to the protective environment. The prediction would be that the rate of illness in the co-twins of individuals exposed to the protective environment would be much higher than for those exposed to the predisposing environment. Contrary to the interaction model discussed above, the increase in risk conferred by exposure to life events is the same regardless of degree of genetic predisposition.

In conclusion, life events are a risk factor for psychiatric disorder; the association appears more prominent for affective disorder than for schizophrenia. In affective disorder, the evidence as regards the aetiological mechanism of life events in relation to genetic factors does not favour a discrete subtype model of independent environmental and genetic causation, and there is some evidence supporting a gene-environment interaction relationship. It is not known whether the same applies to (affective and non-affective) psychotic illness. In chapter 4.3.1, we report findings on the relationship between life events and familial morbid risk in functional psychoses spanning the psychopathological continuum. The hypothesis to be tested with regard to life events and familial morbid risk will be that of the discrete subtype model/additive model (ie 
probands with life events will have lower familial morbid risk) versus the interactive model (probands with life events will have higher familial morbid nisks. Thus, evidence for higher rates of illness in the relatives of probands exposed to life events would be compatible with the geneenvironment interaction model.

\subsubsection{Life events and illness course}

Some of the evidence that "reactive" illness is associated with good outcome has already been alluded to above: Under all the gene-environment models, a better prognosis in more "reactive" illness might be expected because

- a relationship between stressful life events and onset of illness has been demonstrated for both schizophrenia (Bebbington, 1988; Norman \& Malla, 1993b) and affective disorder (Paykel, 1969; Brown \& Harris, 1978), but appears to be stronger in affective disorder (which has a mone favourable prognosis);

- although both mild and severe forms of schizophrenic and affective illness tend to run in families (ie are "familial"), the proportion of the liability due to genes rather than environment appears to differ between mild and severe forms of disorder, as indicated by differences in concordance rates between monozygotic (MZ) and dizygotic (DZ) twins, and variation of morbid risk of illness in the relatives of patients. For example, twin studies of "endogenous", severe depressive illnesses have consistentlly shown that familial aggregation of such disorders can be explained by a genetic, rather than an environmental component (Gershon et al, 1976; Bertelsen et al, 1977; McGuffin \& Katz, 1986). In twin studies of less severe "meurotic" depression; however, family environment rather than genes explain most of the variation in liability (Slater \& Shields, 1969; Torgersen, 1986). Although suggestive, these findings need to be interpreted cautiously; for example, family studies conducted by McGuffin and colleagues (1988a), and Andreasen and collaborators (1986) showed that finding any differences in morbid risk of illness in relatives was heavily dependent on the definition of "endogenous". A similar phenomenon, however, can be observed in the schizophrenic disorders. For example, Kallmann (1938) found that the relatives of typical, "nuclear" schizophrenia patients were more often affected than are the relatives of those suffering from less severe or "peripheral" schizophrenia. Some later studies have found that the rate of schizophrenia in family members appears to be higher if the proband's illness is characterized by features such as early onset, poor premorbid adjustment and affective flattening, all of which are indicative of poor outcome (Farmer et al, 1983; Sham et al, 1994). Again, however, these findings meed to be interpreted cautiously, as for example Farmer and colleagues (1987) showed that broadening the phenotype of schizophrenia to include affective disorder with mood incongruent delusions produces a rise in the $\mathrm{MZ} / \mathrm{DZ}$ concordance ratio (ie makes it a more "genetic" form of schizophrenia), whereas affective symptoms in schizophrenia are traditionally associated with better outcome. Similarly, there is some evidence that female probands may have higher rates of illness in their first degree relatives (eg Goldstein, 1990), whereas female sex also is associated with a less deteriorated illness course. 
It can thus be inferred that while an environmental influence in the onset of affective and schizophrenic disorders is well established, there is also some evidence that the more severe disorders are more "genetic" and the milder disorders more "environmental", but the literature in this area remains controversial. If the severe disorders are indicative of a higher genetic diathesis, then one would expect that poorer course and outcome in the functional psychosis are associated with a higher familial risk for psychosis in the relatives. (This theme will be further examined in section 1.5.6.2, where we will review the literature on the relationship between familial morbid risk and outcome in the functional psychoses).

Another, more direct, source of proof for the supposition that life events predict a less deteriorated illness course is to be found in the outcome literature. Indeed, the statement that schizophrenic illness preceded by life events is associated with a more benign course of illness can be encountered in many major psychiatric textbooks (eg Kendell \& Zeally, 1993). Early authors interpreted this as an argument for the validity of a discrete-subtype model: Robins and Guze (1970) concluded that good prognosis schizophrenia, associated with precipitant stress and acute onset, probably was not mild schizophrenia, but a different illness entity, reminiscent of the concept of "reactive psychosis", especially popular in the Scandinavian countries (Strömgren, 1985).

Some of the earliest studies reporting an association between premorbid stress and good outcome of psychotic illness were by Philips (1953) and Zubin (1961), but their study measures were relatively arbitrary and difficult to replicate Vaillant (1964), reviewing the outcome literature in schizophrenia in the introduction to his 1964 prospective study, remarked that "prediction [of outcome] should be based upon rellatively objective criteria". He introduced seven prognostic criteria about which he commented "no criterion is so self-evident that different raters could agree uniformly on its presence or absence in every single patient. Nevertheless, determination of these criteria requires no special skill and these are criteria that have been found consistently by many investigators to be correlated with favourable schizophrenia prognosis". This author found, in a sample of 100 schizophrenia patients, and this was replicated by Stephens and colleagues in a similar study (1966), that the prognostic factor with one of the highest correlation coefficients with illness remission was presence of precipitating factors at baseline assessment; however, it was not clear whether this association was confounded, as presence of precipitating factor correlated highly with other significant predictor variables, such as "acute onset" and being "nonschizoid". In spite of his call for objectivity in assessing predictors of outcome, neither the study by Vaillant, nor the one by Stephens and colleagues used any particular methodology in the assessment of precipitating factors, and presence or absence was scored either from casenotes or according to the admitting doctor's clinical impression.

Nevertheless, Vaillant's and Stephens' finding generated considerable interest, and were replicated partly by the WHO international follow-up study of schizophrenia (1979). The results of the twoyear follow-up of 906 patients (67\% with schizophrenia) in 9 psychiatric centres worldwide showed that absence of precipitating stress was among the best five past history predictors of overall two-year outcome in PSE-Catego schizophrenia patients. As in the study by Vaillant, "absence of precipitating stress" was associated with poorer outcome. However, evidence of this association was apparent only if study site was included in the analyses, and occurring amidst a multitude of statistical tests with many predictor variables. Moreover, the identification of stressful events was left essentially to the rater's clinical discretion. Separate studies from two 
of the centres (Washington and London) participating in the WHO international follow-up study reported similar findings at two year (Strauss \& Carpenter, 1974) and five-year (Prudo \& Blum. 1987) follow-up, but shared the same shortcomings in the assessment of "precipitating stress". Similarly, Wallis (1972) found an association between premorbid stress and less subsequent time spent in hospital in 512 male schizophrenia patients, but his concept of stress was rather overinclusive, encompassing aspects of personality, such as obsessionality and psychopathy, and alcohol misuse. Furthermore, premorbid stress was not related to other dimensions of outcome, and all stress-related experiences were derived retrospectively from case-note material.

Serban (1975) questioned Vaillant's findings, and reported negative results in his own study on predictors of readmission (including premorbid stress) over a two year period in a sample of 419 mainly chronic schizophrenia patients. This echoes less well-known earlier negative findings from a number of other authors (Cole et al, 1954, Johanson, 1958). In the study by Cole, severity of precipitating stress was associated with outcome in a sample of 486 psychotic patients (54\% with schizophrenia), but after adjustment for possible confounders the association disappeared, indicating that precipitating stress was not an independent risk factor for outcome.

We are thus left in a state of some confusion, almost certainly the consequence of the methodological drawbacks shared by these investigations. Rater bias cannot be excluded because outcome was not rated blind to exposure status. Secondly, no attempt was made to develop a reliable rating system to rate events, and to objectively rate the degree to which events were independent of actions of the subject that might have bene due to the emergence of illness (Brown \& Harris, 1978). This latter factor is also known as reversed causality; a patient may for example lose his job or have conflicts with his partner because of his emerging illness, but later attribute his relapse to the loss of his job or marital conflict (see also above). The absence of a reliable system to rate events other than by clinical impression is also important. For example, Brockington et al (1991) showed that the kappa for rating the presence of premorbid stress between a group of clinicians was only 0.32 , indicating very poor interrater reliability. Thirdly, onset was not defined as a move from an effectively symptom free state to a psychotic one. This is important, as conceptually for stress to be associated with onset (and subsequent recovery) of illness, it must be shown that there was a real change in the clinicall state of the patient. Readmission to hospital, though suggestive, is not necessarily related to onset or relapse of disease. Similarly and fourthly, no distinction was made between datable and non-datable onsets. Non-datable onsets should be treated with care in any study relating life events to onset and subsequent outcome of illness, as the exact relationship between stress and onset cannot be established in the absence of a definable and datable onset. If investigators rate cases with insidious onset or uncertain onset automatically as life event megative, they may erroneously conclude that insidious/uncertain onset cases were not precipitated by premorbid stress simply because they are uncertain about the onset. Therefore, associations found between premorbid stress and good outcome may be explained either by the distinction between insidious (often associated with early onset and poor outcome; Murray \& O'Callaghan, 1991) and acute onset (with or without life events), or by the absence or presence of premorbid stress in those cases with non-insidious onsets (see chapter 4.3.1). Fifthly, no rating of the impact of events was available. For example, loss of a job may be a source of severe stress to one, but a minor nuisance or even a source of comfort to another. Sixthly, the period prior to illness over which the events were measured fluctuated widely between studies or were not specified; and lastly, outcome ratings were rarely ascertained blind to exposure status. 
Harder and colleagues (1981) paid attention to the possibility of reversed causality in their study design, and the independence of premorbid stress as a risk factor. They prospectively ascertained outcome in 111 first-admission schizophrenia patients (subdivided in three groups of competing definitions of schizophrenia) having assessed at baseline the presence of "life-events stress"' in the previous year with a modified version of the Social Readjustment Rating Scale by Holmes and Rahe (1967). They excluded items that "were judged to be very likely consequences of the disorder", and excluding events that were "obviously the result of the patient's pathological condition". They also weighted the level of stress for recency of occurrence in relation to disease onset. They found that life-events stress was associated with better outcome for two definitions of schizophrenia, but not for the DSM-II definition of the disorder. This variability of the findings in relation to definition makes them difficult to interpret, but they remain suggestive:

In summary; it is difficult to find a study in the literature reporting negative findings as regards the relationship between life events and subsequent better illness prognosis. There are genuine reasons, however, to be cautious because of the many methodological shortcomings and the possibility of significant publication bias. Publication bias is a recognized phenomenon, i.e. studies with statistically significant results are more likely to be published than those finding no difference between the groups studied. It has been found that publication bias is especially relevant to observationali studies on exposures which are difficult to measure and which carry a small relative risk (Easterbrook et al, 1991), Over the last ten years, the methodology for measuring life events thas improved considerably (Bebbington, 1988), but has been applied uniquely to the study of risk mediated by life events for onset of psychosis; not its prognostic implications. In chapter 4.3.1, we describe a study which examines the relationship between life events and course of illness, in a follow-up of the Camberwell Collaborative Psychosis Study (Jones et at, 1993).

\subsubsection{CEREBRAL VENTRICLE DIMENSIONS}

\subsubsection{Cerebral ventricle dimensions and illness onset}

That the mean size of the lateral cerebral ventricle is on average larger in schizophrenia patients than in well controls is widely accepted. Andreasen and colleagues (1990a) reviewed some 50 studies involving blind controlled comparisons of the ventricle/brain ratio using CT-scanning, and concluded that the evidence of enlargement in schizophrenia patients was convincing, as around $75 \%$ of case-control comparisons revealed statistically significant differences. It has, however, been pointed out that there is a paradoxical trend for the more recent CT studies to find smaller and smaller differences between cases and controls (Horn \& McManus, 1992).

Many follow-up studies have concurred in showing that these changes in cerebral ventricle size are not progressive. In the absence of i) gliosis in the brains of schizophrenia patients (Crow et $a l, 1989$ ) and ii) a simple inverse relationship between ventricular size with surrounding regions in patients with schizophrenia in most studies (Kelsoe et al, 1988), it is possible that the determinants of ventricle enlargement operate early in fetal life (Murray et al, 1988), as gliosis in response to brain tissue damage does not occur before birth, and foetal brain tissue expansion is associated with a decrease in the ventricle to brain volume (ie enlarged ventricles may represent incomplete brain maturation). 
Magnetic resonance imaging (MRI) has been both confirming and extending the CT-findings (eg Degreef et al, 1992). However, such work is often complicated as instead of reporting on differences in size of the whole ventricular system or whole brain volume and examining for interactions with specific regions, a multitude of comparisons are conducted for each region separately in usually rather small samples. Furthermore, many CT and MRI stuclies have continued to struggle with the question on how to define "abnormally large" ventricles. In fact, this problem can be obviated by avoiding arbitrary dichotomization and treat cerebral ventricle size as a continuous risk factor. Thus, we were able to establish (Jones et al, 1994a) that there is a linear trend in the association between lateral ventricle size and onset of schizophrenia, which indicates that risk is not confined to a subgroup with very lange ventricles, but rather that ventricular enlargement is best conceived as a continuous risk factor: the larger the ventricles, the larger the risk of schizophrenia.

There is little evidence that these findings are specific to schizophrenia. Rather, instead of the $75 \%$ positive case control VBR comparisons reported by Andreasen (1990a) for schizophrenia samples, the proportion in the much smaller number of $\mathrm{CT}$ studies on affective disorder appears to be closer to $50 \%$ (Andreasen et $a l, 1990 \mathrm{~b}$; Jones et $a l, 1994 \mathrm{a}$;), which is still considerably higher than could be expected by chance if there were no differences between cases and controls, and certainly not evidence for specificity of the finding to schizophrenic psychosis. Furthermore, studies involving patients with affective disorder in particularly suffered from small sample sizes, and therefore some of the negative studies are not readily interpretable as measures of effect and confidence intervals were not provided (see below; eg Rossi et al, 1987; Weinberger et al, 1982). Indeed, the studies with the largest samples reported a significant difference between cases and controls (Andreasen et $a l, 1990 \mathrm{~b}$; Dolan et $a l, 1985$ ). Of interest is the study by Harvey and colleagues (1990a), who attempted to define a separate subgroup of patients on the basis of the VBR, in a sample of 72 patients with functional psychosis and 50 healthy controls. The results of their admixture analysis showed that functional psychotic patients had in generall a higher mean VBR than controls, but it was not possible to identify any particular patient subgroup. The more recent MRI literature is similarly inconclusive with both positive and "negative" (ie inconclusive) results in comparisons between (small numbers of) affective disorder cases and controls (eg Coffey et al, 1993; Coffman et al, 1990; Johnstone et al, 1989; Swayze et al, 1990).

A possible reason for the divergence in the proportion of positive findings in calse-control studies with samples of schizophrenia and affective patients is that differences between affective disorder patients and controls are either smaller than between schizophrenia patients and controlls, or occur in a more restricted area. Our own recent study provides support for both propositions (Jones et $a l, 1994 \mathrm{a}$ ). For example, it was found that there was no large or significant difference in lateral ventricle volume between affective disorder cases and controls. However, the odds ratio (OR) for linear trend across the three tertiles of third ventricle maximum area was $2.2(95 \%$ confidence interval: 1.3-3.8) in the comparison between schizophrenia cases versus controls, compared to $1.8(95 \% \mathrm{Cl}: 1.1-2.8)$ for affective psychosis cases versus controls (an OR of unity would have indicated no difference between cases and controls). The results from a study by Weinberger and colleagues (1982) provide support for the supposition that affective cases do differ from controls, but less than schizophrenia cases. The data in this study showed that there was a gradual increase in VBR from normal control subjects $(n=26$; mean VBR $=2.9)$ to affective disorder cases ( $n=23$; mean VBR $=3.8$ ), schizophreniform disorder cases $(n=35$; mean= $5.3)$, and chronic schizophrenia cases $(n=17$; mean $V B R=6.0)$. These findings demonstrate that 
there may in fact be a trend along a continuum from acute, affective Ilness to chronic, nonaffeetive Iliness in the association between enlarged ventricle size and onset of mental illness, similar to the trend in tendency to chronicity shown above in section 1.4 .2 and in chapter 3.1 . Therefore, the use of a dimensional representation of psychopathology may be productive in the field of neuroimaging. and may shed light on some of the inconsistencies in the literature.

\subsubsection{Cerebral ventricle dimensions and illness course}

Compared to the vast amount of literature on the relationship between cerebral structure and onset of psychosis, the number of studies trying to elucidate the relationship between cerebral structure and illness course is negligible and difficult to interpret, as illustrated in chapter 4.4.1.

Neverthelless, the notion that enlarged ventricles in schizophrenia predict poor outcome is often advocated, and usually based on cross-sectional findings relating VBR or other measures to current levels of symptomatology, age of onset, premorbid adjustment, or "course" of illness prior to the scamning procedure (eg Johnstone et al, 1989; Pandurangi et al, 1988). The usefulness of this approach may have been overestimated, and the potential for bias and confounding been underestimated (see chapter 4.4.1). Especially the evidence that enlarged veritricles are associated with negative symptoms is weak (Farmer et al, 1987), and in our own study, in which particular attention was paid to the possibility of confounding (Jones et al, 1994a), no large or significant associations were found between cerebral ventricle dimensions and illness chronicity, early age of onset or premorbid social functioning (all indicators of poor outconie), Given the fact that influential researchers have postulated some of their theories on the assumption that cerebral ventricle dimensions are associated with illness course (Crow, 1980; Murray et al, 1992), there is a need for truly prospective studies to clarify this issue. In chapter 4.4.1, we will examine the relationship between cerebral ventricle size on the one hand, assessed by CT-scanning, and course and outcome on the other, in a large sample of patients with functional psychotic illness.

\subsubsection{CHILDHOOD DEVELOPMENT}

\subsubsection{Childhood development and illness onset}

There is now a wealth of evidence to support Kraepelin's (1919) earlier observation that preschizophrenic individuals often show subtle developmental abnormalities. Earlier studies used so-called "follow-back" designs, collecting data from, for example, school records and child guidance clinics, and reported mainly on abnormalities in social development. Pre-schizophrenic children were found to have impaired peer relationships, be socially isolated, and display withdrawn and anti-social behaviour (Offord \& Cross, 1969; Watt, 1978; Robins et al, 1966; Cannon et al, 1995a). All these studies are prone to selection bias, but a recent study investigating childhood social adjustment in pre-schizophrenic individuals in a population based, historical cohort study of the National Child Development Survey 1958 cohort replicated these earlier results (Done et al, 1994a \& b), as did a Swedish cohort study (Malmberg et al, 1995). 
In addition, a number of reports using increasingly sophisticated methodological designs, have indicated that pre-schizophrenic children have lower IQ scores than normal controls or their siblings. These findings cannot be attributed to a subgroup of cases with very low IQ scores, as two population-based, historical cohort studies have now convincingly shown that there is a linear trend in the association between later schizophrenia and $\mathrm{IQ}$ scores in childhood and adolescence: the lower the IQ score, the higher the risk of later schizophrenia (Jones et al, 1994).

Interesting new data have now been published regarding subtle abnomalities in early motor and language development. High risk studies prospectively follow the development of children born to schizophrenic mothers. One of the best known high risk studies (Fish et al, 1992) reported that early abnormalities on fine and gross motor developmient predicted later schizophrenia-spectrum disorders in adulthood. Another approach was used by Walker and colleagues (1994), who showed that blind raters watching family "home movies" depicting pre-schizophrenic children and their siblings, could distinguish the child destined to develop the disease, on the basis of its motor behaviour, such children showing more abnormalities. Jones and colleagues (1994), using an historical cohort approach found that a more representative sample of pre-schizophrenic children were at increased risk of delayed motor milestones and language development, compared to normal controls.

Thus, there is little doubt that disturbed premorbid motor, cognitive and social development in childhood are risk factors for later schizophrenia. However, there is evidence that premorbid abnormalities are also present in other psychiatric disorders, although possibly in a lesser degree. Crow and colleagues (1994) reported childhood neurological abnomnalities in both ICD schizophrenic and non-schizophrenic psychotic individuals in the 1958 cohort of the UK National Child Development Study, and the same group (Done et al, 1994a) reported high levells of social maladjustment in pre-neurotic children (but not pre-affective psychotic children) in this cohort, as well as premorbid cognitive deficits in both pre-affective psychotics and pre-neurotics (Done et al, 1994b). Rogers (1990), using data from the 1946 British birth cohort, showed that childhood social abnormalities predicted adult affective disorder outcomes; and Cannon and collaborators (1995b) reported similar findings for bipolar disorder. Depressed children have high rates of depression and alcoholism in both first and second degree relatives (Puig Antich $e t$ al, 1989), which in turn has been associated with elevated rates of psychomotor and language delay (Harian, 1989).

The question arises what these various premorbid abnormalities actually constitute, and how the apparent lack of diagnostic specificity, but varying degree of severity in different diagnostic groups, should be interpreted. In general, premorbid developmental deviance may, for example, i) be an early sign of the disorder, ii) be on the pathway between cause and manifestation of adult mental disorder, iii) be an indicator of risk, without actually mediating it (a "trait marker"), iv) a mediator of risk (contributory causal factor), v) a predisposing factor (needing a second factor to form a sufficient cause), vi) a factor potentiating predisposition (eg interacting with genetic predisposition), vii) be an endophenotype, ie a marker of disease transmission in families, viii) be a risk factor for chronicity (severity), as well as onset of mental disorder (ie the higher the exposure, the more severe the disease). At present, there is no clear indication which of these eight main possibilities is the correct one, but some are more falsifiable than the others, and not all are mutually exclusive. In order to test the hypothesis that premorbid abnormalities are early signs of the disorder, one would i) need to have detailed knowledge about the manifestations of 
the disorder from the very start of the pathogenesis of the illness process, which at present is clearly not possible. Furthermore, if premorbid abnomalities are illness manifestations then our current measurements must be severely lacking in sensitivity and specificity, as many normal controls present with the same premorbid characteristics as measured by our current instruments, and in many schizophrenia patients no such characteristics are picked up. In fact, as long as the pathogenesis of psychotic illness and its relation to the ensuing manifestations are not known and measurable, it may be wery difficult to test the above hypotheses i to $\mathrm{v}$.

Hypotheses vi-viii are more readily falsifiable. As we have seen above, twin studies are particularly suitable to test hypotheses involving interaction between premorbid abnormalities and genetic predisposition, but, as far as we are aware, this has not been investigated. One study. however, reported that higher familial risk in the relatives was associated with poor premorbid functioning (Foerster ef al, 1991). As shown in table 5a \& 5 b shown earlier, this may indicate interaction of risk factors. However, such an explanation cannot account for the fact that the rate of developmental deviance is increased in the (as yet) unaffected offspring of individualls with schizophrenia (Fish et al, 1992), and such findings are in fact more in support of hypothesis vii above.

The specific argument that these premorbid abmormalities are also predictors of chronicity in the functional psychoses (hypothesis viii) can be more easily the object of a scientific investigation, as detailed below.

\subsubsection{Childhood development and illness course}

If premorbid abnormalities are predictive of chronicity in the functional psychoses one would expect that i) premorbid adjustment predicts poor outcome; ii) diagnostic categories that are ordered allong their reported tendency towards chronicity, are also ordered in the frequency with which patients have displayed premorbid developmental deviance; iii) the type of developmental abnormalities will not differ between the poor outcome groups of various diagnostic categories. The literature offers some support for item iii), as discussed above in section 1.5.5.1., in that cognitive, social and motor abnormalities have been shown not to be specific for schizophrenia, although the evidence is not as compelling in the non-schizophrenic disorders (but then it also has been researched to a much lesser degree). However, this issue has not been investigated in terms of chronicity. For example, do groups of poor outcome affective patients have the same premorbid abnormalities as schizophrenia patients, defined in terms of chronicity (as in DSM-III \& DSM-IV)? This question will be addressed in chapter 4.5.2.

The literature offers more guidance with regard to item i), namely that premorbid maladjustment predicts poor outcome. Stoffelmayr (1983) reviewed the early generation of studies relating premorbid adjustment and outcome in schizophrenia, and concluded not only that the two were very strongly related, but also that the strength of the association was largely consistent across studies, most variation being due to random error. However, premorbid adjustment in most of the earlier studies was not assessed in a structured way, from sources of widely varying relliability, and always retrospective. Later studies used better instruments, such as the Premorbid Adjustment Scale (PAS; Cannon-Spoor et al, 1982) which assesses premorbid social and role functioning during childhood, early adolescence, late adolescence and adulthood, but premorbid 
assessments remained retrospective, with considerable scope for both observation and recall bias; especially in the case of such a remote exposure. For example, in two long-term ontcome studies of chronic (at baseline) schizophrenia patients, one in a rural area state hospiral with patients of low economic status, the other in a private institution with patients of mostly privileged backgrounds, found that being a "Joner" or not having "skills \& interests" (Fenton \& McGlashan, 1987), and a "poor premorbid adjustment" (a dichotomized item derived from the PAS score; Childers and Harding, 1990) predicted poorer outcome after up to 30 years, although the study by Fenton \& McGlashan suggested that the effect was largest in the first decade of follow-up (McGlashan, 1986). Similar findings were reported by Kolakowska et al (1985). The accuracy of assessment of premorbid traits in patients who were already chronic at base line must be questioned. Two more recent studies examined schizophrenia patients at their first onset and prospectively examined premorbid adjustment and course of illness. Rabiner et al (1986) reported that continual remission after up to one-year follow-up was associated with scores on the Premorbid Asocial Adjustment Scale in a group of 36 schizophrenia patients, such that poorer adjustment predicted less remission. Mayerhoff et al (1994), reported that in a group of 70 first onset patients, premorbid adjustment measured with the PAS was associated with evidence of Carpenter's "deficit" syndrome at 6-12 months follow-up.

The study of Werry and colleagues (1991) stands out, as the subjects were incident and representative cases of adolescent-onset psychosis (DSM-III schizophrenia $(n=30)$ and bipolar disorder $(n=23)$ ) collected in a defined catchment area, in whom premorbid adjustment could be much more reliably assessed in view of their young age at baseline assessment (mean 13.9 years), and the availability of parental informants. Abnormal premorbid adjustment, using DSMIII-R major divisions of personality disorder on a four-point severity scale, was the best predictor for poor five-year outcome in both schizophrenia and bipolar illness cases. Diagnostic misclassification did not account for these findings.

This latter study suggests that the association between premorbid adjustment and outcome may not be specific to schizophrenia. Indeed, there is very good evidence suggesting that premorbid abnormalities are associated with more chronicity in a range of illnesses. One of the findings in the Cologne Study (Deister \& Marneros, 1993), was that an asthenic/low self-confidence personality was among the few of many potential predictor variables that was significantly assaciated with 28 -year outcome in a group of 106 patients with carefully diagnosed affective disorder. In a 1-3.8 years follow-up of a sample of 242 consecutive referrals with DSM-III defined unipolar depressive episode, Brodaty and colleagues (1993) reported that poor premorbid personality, measured at baseline using both an index of DSM personality disorder and an Eysenck Personality Inventory predicted poorer global outcome. Interval between onset and treatment and receipt of treatment and premorbid neuroticism accounted for $55 \%$ of the variance on length of the episode in a sample of fifty-five patients with primary major depression, followed up from onset of illness until recovery (the median length of episode was one year; Scott et al, 1992). The discipline of psychology usually has a much less dogmatic approach towards diagnostic classification: Harder and colleagues (1990) followed a representative sample of 145 incident, catchment area cases over a period of two years, including a wide range of neurotic, affective and psychotic diagnostic categories. Premorbid adjustment, as measured by the Philips Premorbid Adjustment Scale, and social class were the best predictors of functioning level and overall clinicall status at two years, regardless of diagnostic category. Duggan and colleagues (1990) followed a series of 89 depressed patients given two personality tests in the 
mid-sixties and accorded a score on a neurotic-psychotic continuum (DI). High neuroticism score on the Eysenck Personality Inventory predicted poor overall outcome and chronicity, and high psychotic scires on the DI were also associated with poor long-term outcome,

The studies by Opjordsmoen (1989, Links and colleagues (1990) and Vega \& Ayuso-Gutierrez (1990) were methodologically weak, but all reported associations between premorbid adjustment and outcome in unipolar/schizo-affective psychoses, borderline personality disorder, and schizoaffective psychosis respectively.

The best studies to date include those by Coryell and colleagues, who reported biannual assessments of 103 patients with depressive and schizo-depressive psychosis (Coryell et al, 1990a), and 70 patients with manic and schizo-manic psychosis (Coryell et al, 1990b): A rating of adollescent friendship patterns derived from the SADS- $L$ was strongly associated with persistent delusions in all groups, indicative of poorer outcome. It was not clear, however, to what extent this predictor was independent of other risk factors for persistence of delusions.

In summary, premorbid adjustment is associated with both onset and chronicity of the functional psychoses. The evidence for the former relationship is stronger, as some of the best studies included detailed premorbid assessments in the areas of motor, cognitive and social development: Studies reporting associations with outcome had to rely on rather unreliable retrospective assessments, with scope for significant recall bias. Furthermore, it is not clear whether the same type of premorbid developmental deviance is associated with both onset and chronicity. In chapters 4.5 .1 and 4.5 .2 , we will examine the issue of chronicity in relation to premorbid adjustment using i) a historical cohort approach with accurate exposure measurements, and ii) a proxy approach, using proxies for premorbid adjustment that can be more reliably measured. The issue will also be briefly examined in chapter 4.6.1.

\subsubsection{FAMILIAL MORBID RISK}

\subsubsection{Familial morbid risk and illness onset}

Family, twin, and adoption studies have established that the familial cliustering of schizophrenia and related psychoses is due to genetic, rather than shared familial-environmental factors. With a morbid risk of $10 \%$ for broadly defined schizophrenia in the first degree relatives of schizophrenia probands, versus $1 \%$ in the general population, the genetic component is undoubtedly strong, and this would seem one of the most important clues we have to its aetiology (Gottesman \& Bertelsen, 1991). The genetic contribution to schizophrenia and other psychoses is therefore well established, but one issue, rellevant to this thesis, remains contentious: definition of the phenotype. As in the previously reviewed areas of psychosis research, genetic research is similarly bedeviled by the lack of clear separation of the psychoses by classical symptoms into well-defined clusters, and the existence of forms of illness which are inconveniently "intermediate" between the classical affective and schizophrenic syndromes. On balance (reviews by Taylor, 1992; and Crow, 1990), genetic research has failed to uphold the Kraepelinian distinction between affective and schizophrenic psychoses, as a number of large studies, though by no means all studies, have shown that i) the morbid risk of mania and depression is higher in the first degree relatives of schizophrenia probands than in controls, and 
ii) the morbid risk for schizophrenia is higher in relatives of affectively ill probands. For example, in the Iowa 500 study, it was reported by Tsuang and collaborators (1980) that the morbid risks for bipolar affective disorder, assessed through personal and approximate interviews; in the first degree relatives of schizophrenic, manic and depressed probands were $2.4 \%, 5.3 \%$ and $3 \%$ respectively, compared to only $0.3 \%$ in the first degree relatives of control subjects. In the same sample, a later analysis by Kendler et al (1985) also demonstrated a significantly higher risk for schizoaffective disorder in the first degree relatives of schizophrenia probands. Conversely, the Iowa 500 study also found that the risks for schizophrenia in the first degree relatives of schizophrenic, manic and control probands were $5.5 \%, 3.2 \%$ and $0.6 \%$ respectively. Gershon and colleagues (1988) reported that the morbid risks for bipolar and unipolar disorder in the first degree relatives of schizophrenic subjects were 2-3 times greater than those of the relatives of normal controls (bipolar: $2.2 \%$ vs $0.8 \%$; unipolar: $14.7 \%$ vs $6.7 \%$ ). Although a number of other large and carefully conducted studies failed to show such overlap in terms of morbid risks of affective and schizophrenic psychoses in the first degree relatives of schizophrenic and affective probands respectively (Baron et al, 1985; Coryell \& Zimerman, 1988; Gershon et al, 1975; Baron et al, 1982; Maier et al, 1993), the above findings cannot be reasoned away, and no study to date has shown that schizoaffective disorder breeds true, especially schizodepressive disorder. One explanation for the discrepant findings may lie in an important distinction with regard to the affective non-affective dichotomy and famillial homotypia: the presence of psychosis in affectively ill probands. For example, although Kendler and colleagues initially (1985) failed to find evidence for an excess of schizophrenia in the relatives of manic-depressive probands, the results changed dramatically when analyses focussed on the first-degree relatives of probands with psychotic affective disorder (Kendler $e t$ al, 1986), in that there was an excess risk of schizophrenia in these relatives ( $4.3 \%)$, which even surpassed the risk for schizophrenia in the relatives of schizophrenia probands (3.7\%). In another study relating to the same sample, they also reported that the morbid risk for schizophrenia in the relatives of bipolar patients with psychosis was significantly elevated at $2.5 \%$, compared to $1 \%$ in control probands. Similar findings were reported in the Roscommon family studly (Kendler et al, 1993), which was one of the very few studies to use an epidemiologic approach to proband sampling. The risk for schizophrenia as determined by life-table analyses was significantly higher in the relatives of probands with schizo-affective disorder $(6.7 \%)$, other non-affective psychosis $(5.1 \%)$, and psychotic affective illness $(2.8 \%)$, compared to the morbid risk in relatives in control probands $(0.5 \%)$. These authors concluded that their findings suggested "that the familial liability to schizophrenia is, at least in part, a liability to develop psychosis". Therefore, the study of familial clustering as a risk factor is best carried out in samples of broadly defined psychotic (including affectively psychotic) patients and their first degree relatives. This strategy has been employed in chapter 4.6.1.

\subsubsection{Familial morbid risk and illness course}

The study of familial risk of psychosis in relation to outcome of psychotic disorders can shed light on various issues. For example, the "degeneration" theory, which originated in France in the 19th century, would predict that "hereditary" forms of psychotic illness would have a poor prognosis compared to "acquired" illness (see also section 1.5.3.2). On the other thand, more recent theory has proposed that "environmental" forms of schizophrenia are characterised by brain abnormalities, negative symptoms and poor treatment response and prognosis (Weinberger, 
1984; Murray et $a l, 1985$ ). Also, the finding that severity (assuming chronicity is a marker of severity) of illness fluctuates with the degree of familial morbid risk strongly suggests that there are mild, less genetic and severe, more genetic forms of the disorder. Such an observation is difficult to explain under the assumption that only one gene is the source of resemblance between relatives, and is more compatible with, for example, a model of either the action of polygenes regulating penetrance of a single gene, or a phenotype resulting from the additive effect of multiple genes at different loci or a multifactorial model (ie polygenic plus environmental effects). For example, differences in outcome in relation to genetic loading are readily accomnodated in terms of a multifactorial liability/threshold model (Reich et $a l, 1972$, 1979; McGuffin et al, 1987). Under this model, the existence of two thresholds is postulated, lying at different points of a continuum of liability. Individuals lying beyond the first threshold will develop a milder "broad" form of illness, whereas those lying beyond a second threshold will display a more severe, "narrow" form of the disorder. As the first degree relatives of psychotic patients have a risk of around ten times of developing the disorder, their mean liability under the multifactorial threshold model will be shifted upward. Reich and colleagues (1979) have demonstrated that, under "isocorrelational" circumstances, the multifactorial threshold model will predict that the mean liability of relatives of narrow cases with more severe illness is higher than that of broad cases with milder illness (figure 9).

\section{Fig. 9 Two threshold multifactorial madel}

\section{THRESHOLD 1}

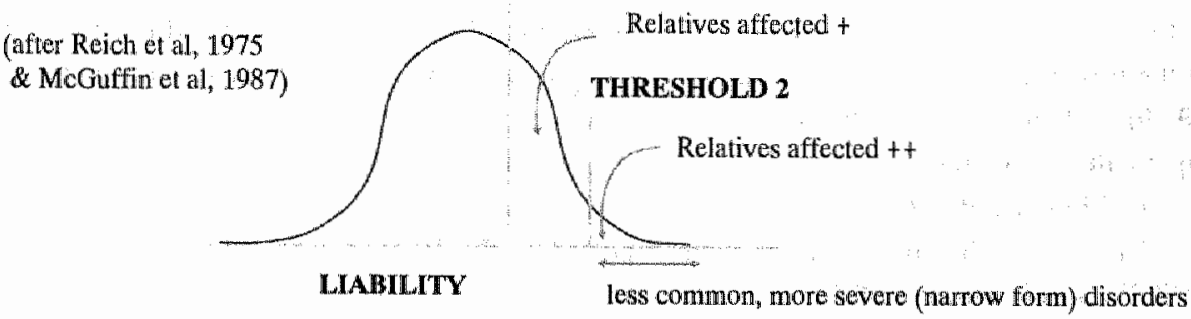

common, milder (broad form) disorders

Almost all studies relating outcome to familial morbid risk in the functional psychoses have used a simple "family history positive versus family history negative" approach, but failed to take into account that the number of relatives and their age might vary as a function of outcome. For example, differences in fertility may exist between the families of good outcome and poor outcome patients with schizophrenia, such that the relatives of good outcome schizophrenia patients may be more numerous and younger than the families of poor outcome schizophrenia cases. Kendler and Tsuang (1988), provided some empirical evidence for such a systematic difference. The result of the age and family size factor is twofold: younger age in the relatives of good-outcome patients would decrease the familial morbid risk compared to the relatives of poor-outcome patients; but the larger size of the families of good-outcome patients would increase the familial morbid risk for schizophrenia compared to that in the relatives of poor-outcome probands. Therefore, the studies in this area are very difficult to interpret, some reporting a positive association between familial loading and poor outcome (eg Vailant, 1964; McGlashan, 1986), some no association (eg Vaillant, 1962; Bleuler, 1972), and only one very early study reporting a negative association (Bleuler, 1931). 
Two outcome studies took into account some of the confounding factors stuch as age, sex and family size, in examining the association between familial morbid risk and illness course. Fowler and colleagues (1972) compared 28 good and 25 poor prognosis schizophrenia patients and interviewed their rellatives mostly blind to proband outcome status. Of the 126 first degree relatives in the poor prognosis group, 13 had schizophrenia; in the good prognosis group the prevalence of schizophrenia was much lower ( 5 out of 137 ; risk ratio $=0.6 ; 95 \% \mathrm{CI}=0.5-0.9$ our calculations from the data provided). The risk for affective disorder (bipolar and unipolar depressive disorders) was lower in the poor outcome group, although this just failed to reach statistical significance ( $R R=0.4 ; 95 \%$ CI $0.2-1.1$ - our calculations from the data provided). Although this study adjusted for the number of relatives, no adjustment was made for age and sex of the relatives; thus, confounding by age might have operated in producing spurious results. The largest study using adjusted morbid risk data to date was by Kendler \& Tsuang (1988), who examined outcome and familial psychopathology in a sample of 253 DSM-III patients with schizophrenia and their 723 first-degree relatives. Family history was viewed from a cohort perspective, where relatives were the focus of the analysis, and the relationship to either a goodoutcome or a poor-outcome proband became the exposure. Morbid risk of non-affective psychotic disorder was higher in the relatives of poor-outcome probands, more so at short-term outcome than at long-term outcome, although for both periods this failed to reach statistical significance $(p=0.16$ and $p=0.66$ respectively). Morbid risk of affective illness was higher in relatives of good outcome patients both at short term and long term follow-up $(p=0.09$ and $p=0.05$ respectively). The major problem with this study was that information on outcome was lost by dichotomizing outcome into good and poor outcome categories, and that short term outcome (arguably the most sensitive measure) was assessed retrospectively from very old casenote material. Furthermore, the cohort approach makes it wery difficult to adjust for important possible confounding factors in the association between family history and outcome, such as age at onset and sex, which may be associated with both outcome and family history of schizophrenia (Sham et al, 1994).

In conclusion, there is some evidence that more "genetic" forms of schizophrenic illness have poorer outcome, but also that liability to affective illness in schizophrenia produces better outcome. This latter finding is somewhat odd, in wiew of the fact that it has been reported that bipolar patients with a positive family history of mania (assessed through interview with the relatives) or schizomania also have a poorer clinical outcone, as assessed by the number of episodes (Winokur et al, 1993). Further, indirect, evidence that more "genetic" forms of affective illness have poorer outcome (just as in schizophrenia) is provided by twin studies, in which it has been shown that familial aggregation of "endogenous", severe depressive illnesses can be explained by a genetic, rather than an environmental component (Gershon et al, 1976; Bertelsen et al, 1977; McGuffin \& Katz, 1986). The problem of investigating separate diagnostic categories of uncertain walidity appears to be reflected in the uncertain and contradictory results. For example, investigating the relationship between outcome and schizophrenia which has already been defined in terms of chronicity (as in the DSM series) will hamper detection of such an association.

Therefore, the possibility that familial loading for schizophrenia predicts poor outcome, and reports that affective illness predicts good outcome in schizophrenia and poor outcome in affective illness need further investigation and clarification. In chapter 4.6 .2 , these issues are 


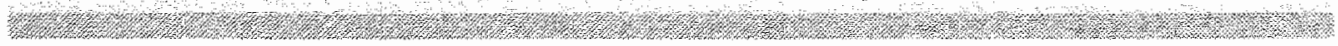

further examined, using a broad sample of psychotic patients, and using an improved analytical approach to establish familial loading for psychiatric disorder. 


\subsection{Risk factors determining the heterogeneity of the tardive dyskinesia outcome}

Tardive dyskinesia is characterised by repetitive involuntary movements of the tongue, lips and face (such as lip smacking or chewing movements), and by abnormal choreifom movements of the trunk and limbs. The risk factor research in tardive dyskinesia has always, to a certain extent, been polarized by competing views about the role of antipsychotic medication in bringing on abnormal movememts. One the one hand, there is evidence to suggest that antipsychotic drugs have a major role in the aetiology of tardive dyskinesia (TD; Jeste \& Wyatt, 1981; Kane \& Smith, 1982), and some research definitions even require long periods of exposure to antipsychotic medication as a criterion for the diagnosis of tardive dyskinesia: One the other hand, there are well documented reports of moderately prevallent cases of spontaneous dyskinesias from the pre-antipsychotic area, which, together with more contemporary observations of spontaneous dyskinesias, suggest that the primary risk factor may be psychiatric disease, rather than the interventions associated with it (Crow et al, 1983; Crow, 1994; Fenton et al, 1994; Owens et al, 1982; Waddington et al, 1990). The latter view is supported by the fact that there is no strong evidence linking duration and/or dose of antipsychotics to the emergence of TD. It is classically considered an "idiosyncratic" drug reaction, rather than one that will inevitably appear if only length and dose of antipsychotic medication are long respectively high enough.

A range of other factors have been reported to be important mediators of risk, such as age, sex, psychiatric diagnosis and organic brain dysfunction (Jeste \& Caligiuri, 1993; Bames, 1987).

One way out of this polarizing state of affairs is the view that abnormal movements may be the result of the co-action of disease and its interventions, or -more likely- an interaction between the two, as implied by some authors. For example, there have been some reports linking emergence of TD to negative symptoms and cognitive deterioration, which would appear to indicate that deterioration of the psychotic illness process itself is involved in bringing on abnormal movements (Liddle et al, 1993).

The literature, however, is controversial. For example, it seems odd that female patients should be at higher risk of developing TD (Yassa \& Jeste, 1992), when female patients with schizophrenia have been shown to have a less deteriorated course of illness (and therefore less need for antipsychotic medication), less negative symptoms, and less evidence of structural brain abnormalities (Castle \& Murray, 1991; Bardenstein \& McGlashan, 1990; Andreasen et al, 1986; Bogers et al, 1990). We propose that the following factors contribute to these controversies:

- Most samples are selected for their continuing exposure to antipsychotic medication, and therefore for their chronicity. Samples selected on the basis of prolonged exposure to only one possible risk factor are likely to yield biased estimates. Furthermore, selection on the basis of chronic exposure to antipsychotics cannot answer the question of whether or not antipsychotics and length of antipsychotic treatment are related to TD, as there may be too little variation in exposure to, and length of, antipsychotic treatment. 
- TD is most often defined as a discrete condition by more or less arbitrary dichotomization of continuous abnormal movement scores (Kane et al, 1986; Chouinard et al, 1986; Glazer et al, 1991; Waddington et al, 1986), the Abnormal Involuntary Movement Scale (AIMS; Guy, 1976) being most frequently used. Such an approach facilitates analyses and interpretation of data, but ignores the fact that there is important variation in the phenomenon along an unbroken scale of severity. Some studies (Waddington et al, 1990, O'Hara et al, 1993) include analyses on both dichotomous and continuous outcome measures, but then differences between the two are difficult to interpret.

- Many studies report statistical significance as measured by $P$-values, without readily interpretable quantification of effect sizes that can be compared across studies (Waddington et al, 1990; Liddle et al, 1993; O'Hara et al, 1993). As $P$-values depend on both sample size and effect size, "negative" reports from such studies are difficult to evaluate.

- Unless the focus is on a comprehensive set of risk factors, the possibility that results are confounded cannot be excluded. For example, sex, age, cumulative exposure to antipsychotics, psychiatric diagnosis, affective symptoms, negative symptoms, cognitive impairment and misuse of alcohol and drugs have all been reported as risk factors for TD (Jeste \& Caligiuri, 1993). However, many of these variables are likely to be associated with each other, especially age, sex and affective symptoms, and while each may turn out to be a "risk factor" when considered separately, only one or two may actually be independent primary risk factors when all are considered together:

- Most of the work is on cross-sectional surveys of prevalent, and therefore older, patients with long term exposure to antipsychotics. The possibility of reversed causality cannot be excluded for some putative mediators of risk. For example, severe TD may lead to affective symptoms, or alcohol and drugs may be taken in an attempt to alleviate distress associated with TD. It is further possible that both TD and crosssectional "risk factors" are all manifestations of the same underlying disease process. In prospective studies, interpretation of findings may also be difficult. For example, the presence of negative or affective symptoms at baseline may "predict" later onset of TD because they are associated with negative and affective symptoms at the time of measurement of TD (the "true" risk factors).

To clarify such problems of temporal interpretation, a combined prospective and cross-sectional approach is necessary. Waddington and colleagues (1990) drew attention to the importance of establishing the temporal order of events with regard to experience of risk and outcome of TD. These authors found that at baseline, there was no difference in cognitive function between patients who did and did not go on to develop TD. However, "those who went on to develop TD showed a selective deterioration in their cognitive function over the time period in which their involuntary movements emerged".

In chapter 5.1, we will examine associations between fixed and variable exposures, and the outcome of tardive dyskinesia. Notably, we will attempt to replicate the previous findings discussed above, which suggested that an illness characterised by negative symptoms and cognitive deterioration is more likely to be complicated by disabling abnormal movements. 


\subsection{Clinical studies of this thesis}

We report the results of a number of prospective cohort studies (chapter 3 ; chapter 4 , sections $4.2 .3,4.3 .1,4.4 .1$ and 4.6 .1 ; chapter 5 ), four case-register incidence studies (chapter 4 , sections 4.1.1.4.1.3,4.2.1,4.2.2), one case-register risk study (chapter 4, section 4.5.1), one cross-sectional survey (chapter 4, section 4.1.2) and one historical cohort study (chapter 4, section 4.5.2). These studies were set up to test the following broad hypotheses concerning risk factors for emergence and persistence of psychotic illness, and to the nosological issue of "case" definition:

- We predicted that the predictive validity of (overlapping) dimensional representations of psychopathology in the functional psychoses would be greater than for (mutually exclusive) traditional categorical representations. Categorical representations of psychopathology, when ordered approximately along the axes of these dimensions, in fact show significant linear trends in their associations with measures of course and outcome, indicating that thus ordered they occupy different positions on an illness continuum corresponding to progressively more severe illness.

- Our prediction was that i) puberty would be associated with onset of psychosis, and ii) in the elderly, increasing age would be a risk factor for onset of psychosis.

- We further hypothesized that the high rates of psychotic illness observed in UK AfroCaribbeans would be genuine, but that the surplus risk might be explainable in terms of an excess of good-outcome psychosis.

- We expected to find contrasting outcomes in high risk groups: good outcome in lifeevent related illness, and poor outcome in illness associated with enlarged cerebral ventricles, poor premorbid adjustment, and high familial morbid risk of severe psychosis. We hypothesized that these associations would vary as a function of baseline psychopathology, reflecting the above discussed findings that the association between life events and illness onset is stronger in the affective psychoses, whereas the association between cerebral ventricle dimensions, premorbid maladjustment and familial morbid risk and illness onset is more apparent in the non-affective psychoses.

- We predicted that the organic outcome of tardive dyskinesia would be associated with poor outcome illness.

The order of the studies is not by study sample. We chose to present issues to do with psychiatric nosology first, followed by demographic risk factors (age, ethnic group), "social" risk factors (life events), and constitutional risk factors (cerebral ventricle dimensions, neurodevelopment, genetic diathesis). Finally, we investigate the tardive dyskinesia outcome. 


\section{REFERENCES}

Adelstein, A.M., Downham, D., Stein, Z. \& Susser, M. (1968) The epidemiology of mental illness in an English city. Social Psychiatry, 3, 47-56.

Allen, R. E. (ed) (1990) Concise Oxford Dictionary, 8th edition. New York: Oxford University Press. American Psychiatric Association (1994) Diagnostic and Statistical Manual of Mental Disorders (4th edn) (DSM-IV) Wahington, DC APA.

Andreasen, N.J. (1982) Negative versus positive schizophrenia: definition and validation. Archives of General Psychiatry, 36, 1325-30.

L4., (1984) Scale for the Assessment of Positive Symptoms. liowa City, IA University of IOWA.

-. Nasrallah HA, Dunn V, et al. Structural abnormalities in the frontal system in schizophrenia: a magnetic resonance study. Archives of General Psychiatry 1986; 43: 136-1.44.

.......... Scheftner, W, Reich, T. et al (1986). The validation of the concept of endogenous depression: a family study approach. Archives of General Psychiatry, 43, 246-251.

_....... N.C., Swayze, V.W., Flaum, M., Yates, W.R. Arudt, S., McChesney, C. (1990) Ventricular enlargement in schizophrenia evalluated with computed tomographic scanning Archives of General Psychiatry, 47, 1008 1015.

...... Swayze, V.W. Flaum, M., Alliger, R., Cohen, M. (1990) Ventricular abnomalities in affectiwe disorder: clinical and demographic correlates. Archives of General Psychiatry, 47, 893-900.

Andreasson, F., Alleback, P., Engstrom, A. \& Rydberg, U. (1987) Cannabis and schizophrenia. A longitudinal study of Swedish conscripts. Lancet, $543,1483-1485$.

Angst, J. (1988) European long-term follow-up studies of schizophrenia. Schizophrenia Bulletin, 14, 501-513.

A.rndt, S., Alliger, R.J. \& Andreasen, N.C. (1991) The construction of positive and negative symptoms: the failute of a two dimensional model. British Journal of Psychiarry, 58, 317-322.

Bagley, C. (1971) Mental illness in immigrant minorities in London. Iournal of Biosocial Science, 3, 449-459.

Bardenstein, K. McGlashan, T. (1990) Sex differences in affective, schizoaffective, and schizophrenic disorders. A review. Schizophrenia Research, 3, 159-72.

Baron, M. Gruen, R., Kane., J. \& Asnis, L. (1985) Modern research criteria and the genetics of schizophrenia. American Journal of Psychiatry, 142; 697-701.

-.-..., Gruen, R. Asnis, L. \& Kane, L. (1982) Schizo-affective illness, schizophrenia and affective disorders: morbid risk and genetic transmission. Acta Psychiatrica Scandinavica, 65, 253-262.

Barnes, T. (1987) The present status of tardive dyskinesia and akathisia in the treatment of schizophrenia. Psychiatric Developments, 4, 301-319.

Bebbington; P. \& Kuipers, L. (1988) Social influences on schizophrenia. In: Schizophrenia, the major issues (Eds. P. MoGuffin \& P. Bebbington). Heinemann: Oxford.

-.... Wilkins, S, Jones, P.B., Foerster, A., Murray, R., Toone, B. \& Lewis, S. (1993) Life events and psychosils. Initial results from the Camberwell Collaborative Psychosis Study. British Journal of Psychiatry, $162,72-79$.

Bertelsen, A. Harvald, B. \& Hauge, M. (1977). A Danish twin study of manic-depressive disorders. British Journal of Psychiatry, 130, 330-51.

Bhopal, R., Philmore, P. \& Kholi, H.S. (1991). Inappropriate use of the term "Asian": ann obstacle to ethnicity and health research. Journat of Public Health Medicine, 13, 244-46.

Birchwood, M., Cochrane, R., MacMillna, F., Copestake, S., Kucharska, J. \& Carris, M. (1992) The influence of ethnicity and family structure on relapse in first-episode schizoprhenia: British Journal of Psychiatry, $161,783-790$.

Blackwood, D., Whalley, L.J., Christie, J., Blackbuum, I.M., St Clair, D., McInnes, A. (1987) Changes in auditory P3 event-related potential in schizophrenia and depression. British Journal of Psychiatry, 150, 154160.

Bland, R. \& Orn, H. (1980a) Schizophrenia: Schneider's first rank symptoms and outcome. British Journal of Psychiatry, 137, 63-68.

- a (1980b) Prediction of long term outcome from presenting symptoms in schizophrenia. Journal of Clinical Psychiary, 41, 85-88.

-1982) Predicting the outcome in schizophrenia. Canodian Journal of Psychiatry, 27, 52-62.

Bleuler, M. (1972) Die Schizoplurenen Geistesstörungen im lichte langjäriger Kranken-und Familiengeschichten. Stuttgart: G. Thieme.

Bleuler, E. (1931) A contribution to the problem of herediry among schizophrenics. Journal of Nervous and Mental Diseases, 74, 393-467.

Bogerts, B., Ashtari, M., Degreef, G. et al. Reduced temporal limbic structure volumes on magnetic resonance images in first episode schizphrenia. Psychiarry Research: Neuroimaging. 35, 1-35. 
Breier, A, Schreiber, J, Dyer, J. \& Pickar, D. (1991) National Institute of Mental Health longitudinal study of chronic schizophrenia. Prognosis and predictors of outcome. Archives of General Psychichtry, 48, 239-246.

Brockington, I, Kendell, R.E. \& Leff, I. (1978) Definitions of schizophrenia concordance and prediction of outcome. Psychological Medicine, 8, 387-398.

I, Kendell, R., Wainwright, S. (1980a). Depressed patients with schizophrenic or paranid sympioms. Psychological Medicine, 10, 665-675.

-.w....., Wainwright, S. Kendell, R. (1980b). Manic patients with schizophrenic or paranoid symptoms. Psychological Medicine, 10, 73-83.

-., Roper, A., Copas, J., Buckley, M., Anrade; C., Wigg. P. et al. (1991) Schizophrenia, bipolar disorder and depression. A discriminant analysis, using 'lifetime' psychopathology ratings. British Jownal of Psychiatry, 159, 485-494.

Brodaty, H., Harris, L., Peters, K., Wilhelm, K., Hickie, I. \& Boyce, P. al al (1993) Prognosis of depression in the elderly. British Jounal of Psychiatry, 163, 589-596.

\& Harris, T.O. (1978) Social Origins of Depression. London: Oxford Uniwersity Press:

- \& Peto, J. (1973) Life events and psychiatric disorders. Part 2: Nature of causal link. Psychological Medicine, 3, 159-76.

Brown, R., Colter, N., Corsellis, $N$. et al (1986) Postmortem evidence of of stmuctural brain changes in schizophrenia. Archives of General Psychiarry, 43, 36-42.

Brown, K. \& White, T. (1992) Syndromes of schizoplurenia and some clinical correlates. British Journal of Psychiatry, 161, 317-322.

Cannon, M., Kargin, M., Jones, P.B., Hollis, C. \& Muray, R.M. (1995a) Predictors of adult psychos is in children who present to a child psychiatric clinic. Schizophrenia Research, 15, 190.

-...., Jones "P.B., Gilvarry, K., McKenzie, K., Rifkin, L. \& Murray, R.M. (1995b) Patterns of childhood social development in schizophrenia, bipollar disorder and normal controls. Schizophrenia Research, $15,190$.

Cannon-Spoor, E., Potkin, S. \& Wyatt, RJ. (1982) Measurement of premorbid adjustment in chronic schizophrenia. Schizophrenia Bulletin, 8, 470-484.

Carpenter, L. Brockington, I. (1980) A study of mental illness in Asians, West Indians, and Africans living in Manchester. British Journal of Psychiatry, 201-206.

Carpenter, W.T., Heinrichs, D.W, \& Wagman, A.M.I. (1988) Deficit and non-deficit forms of schizophrenia: the concept. American Iournal of Psychiatry, $145,578-583$

-....., Bartko, J. Strauss, J. \& Hawk, A. (1978) Signs and symptoms as predictors of outcome" a repont for the international pilot study of schizophrenia. American fournal of Psychiarry. 135, 940 45.

- Strauss, J. (1991) The prediction of outcome in schizophrenia IV: 11 year follow-up of the Washington IPSS Cohort. Journal of Nervous and Mental Disease, 179, 517-525.

Castle D, Wessely $S$, Der G, Murray, $\mathbb{R}$. The incidence of operationally defined schizophrenia in Camberwell, 1965 1984. British Joumal of Psychiatry, 1991; 159; 790-794.

\& Murray, R.M. (1991) The neurodevelopmental basis of sex differences in schizophtrenia. Psychological Medicine, $21,565-575$.

Murray, R.M. (1993) The epidemiology of late-onset schizophrenia. Schizophrenia Bulietin. 4, 691699.

Childers, S.E. Handing, C.M. (1990) Sex, premorbid social functioning, and long-ternn outcome in DSM-HII schizophrenia. Schizophrenia Bulletin, 2, 309-317.

Chouinand $G_{3}$. Annable $L$, Mercier $P$, et al. A five year follow-up study of tardive dyskinesia. Psychopharmacological Bulletin 1986; 22: 259-263.

Chua, S.E., \& McKenna, P.J. (1995) Schizophrenia-a brain disease? A critical review of structural and functional cerebral abnormalitiy in the disorder. British Journal of Psychiatry, 563-583.

Ciompi, L. (1980) The natural history of schizophrenia in the long term. British Journal of Psychiatry, 136, 413. 420.

Clayton, PJ. (1981) The epidemiology of bipolar affective disorder. Comprehensive Psychiatry, 22, 31-43,

Cloninger, C., Martin, R., Guze, S. C Clayton, P. (1985) Diagnosis and prognosis in schizophrenia. Archives of General Psychiatry, 42, 15-25.

Cochrane, P. \& Bal, C. (1989) Mental hospütall admission rates of immigrants to England: a comparison of 1971 and 1981. Social Psychiatry and Psychiatric Epidemiology, 24, 2-11.

Coffey. C., Wilkinson, W., Weiner, R. et al (1993) Quantitative cerebral anatomy in depression: a controlled magnetic resonance imaging study. Archives of General Psychiotry, 1993, 50, 7-16.

Coffman, J.A., Bornstein, R., Olson, S., Schwarzkopf, S. \& Nasrallah, H. (1990), Cognitive impairment and cerebral structuure by MRI in bipolar disorder. Biological Psychiatry. 27, 1188-96.

Cole, M.E., Swensen, C.H. \& Pascal, G.R (1954). Prognostic significance of precipitating stress in mental illness. Fournal of Consulting Psychology, 18, 171-175. 
Cooper, J.M., Kendell, R.E.y Gurland, B.J., Sharpe, L., Copeland, I. \& Simon, R. (1972) Psychiarric diagnosis in New York and London. Maudsiey Monograph 20. Londion: Oxford University Press.

Cooper, B. (1961) Sowial class and prognosis in schizophtenia. Part I and Part II British Journol of Preventive and Social Medicine, 15,17-41.

Cooper, $R$. A note on the biological concept of race and its application in epidemiological research. American Heart Journal, $108,715-23$.

Coryell, W., Keller, M. Lavori, P., ef al (1990a) Affective syndromes, psychotic features, and prognosis. I: depression. Archives of General Psychiatry, 47, 651-657.

L. - et al (1990b) Affective syndromes, psychotic features, and prognosis. Il: mania. Archives of General Psychiatry, 47, 658-662.

-...... \& Zimmerman, M. (1988) The heritability of schizophrenia and schizoaffective disorder. Archives of General Psychiatry, 45, 323-327.

Costello, C. (1992) Research on symptoms versus research on syndromes. Arguments in fawour of allocating more research time to the study of symptoms. British Journal of Psychiarry, 160, 304-308.

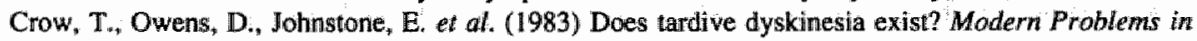
Pharmacopsychiatry, 21, 206-219.

...m....... Syndromes of schizophrenia (1994). British Journal of Psychiatry, 165, 724-27.

........ Done, D.J. Sacker, A. (1994) Neurological abnormality in children who develop psychosis in adulthood. Schizophrenia Research, 11, 96 :

........, (1980). Molecular pathology of schizophrenia: more than one disease British Medical Journal, 280, 6668

m.n.m..... Ball, J., Bloom, S.R. et al (1989) Schizophrenia as an anomaly of development of cerebral asymmetry. Archives of General Psychiatry, 46, 1145-1150.

-n....., (1990) The continuum of psychosis and its genetic origins: the 65 th Maudsley Lecture. British Journal of Psychiatry, 156,788-797.

Day, R., Nielsen, J.A., Korten, A., Emberg, G., Dube, K.C., Gebhart, J. et al (1987) Stressful life events preceding the acute onset of schizophrenia: a cross-national study from the World Health Organization. Culture, Medicine \& Psychïatry, 11, 1.23-205.

Degreef, G. Ashtari, M., Bogerts, B. et al (1992) Volumes of ventricular system subdivisions measured from magnetic resonance images in first-episode schizophrenic patients. Archives of General Psychiatry, $49,531-7$.

Deister, A. \& Marneros, A. (1993) Predicting the long-term outcome of affective disorders. Acta Psychiatrica Scandinavica, 88, 174-177.

DeVries, M. W. \& Delespaul, P.A.E.G. (1989) Time, context and subjective expeirences in schizophrenia. Schizophrenia Bulletim, 15, 233-244.

D'Haenen, H., Bossuyt, A. et al (1994) Dopamine D2 receptors in depression measured with single photon emission computed tomography. Biological Psychiarry, 35, 128-132.

Dolan, R., Calloway, S., Mann A. ef al (1985) Cerebral ventricular size in depressed subjects. Psychological Medicine, 15, 873-88.

Dollfus, $S$ \&an $\mathrm{Os}_{3}, \mathrm{~J}$, (1995) How psychiatrists prescribe anti-psychotics in the UK and France. British Journal of Psychiatry (letter), 166, 263-264.

Done, D.J., Crow, T.F., Johnstone, E.C. Sacker, A. (1994a) Childhood antecedents of schizophrenia and affective illness: social adjustment at ages 7 and 11. British Medical Journol, 309, 699-703.

-..m......... Sacker, A.\& Crow, T.J. (1994b) Childhood antecedents of schizophirenila and affective illiness: intellectual performance at ages 7 and 11. Schizophrenia Research, 11, 96-97.

Duggan, C.F., Lee, A.S. \& Murray. R.M. (1990) Does personality predict long-term outcome in depression? British Jourmal of Psychiatry, 157, 19-24.

Dworkin, R.H., Lenzenweger, M., Moldin, S., Skillings, G. \& Levick, S. (1988). A Multidimensional approach to the genetics of schizophrenia. American Jowrnal of Psychiatry, 145, 1077-1083.

Easterbrook, P.J., Berlin, J.A., Gopalan, R. \& Mathews, D. (1991) Publication bias in clinical research. Lancet, $337,867-72$.

Eaton, W.W. (1980) A formal theory of selection for schizophrenia. American Journal of Sociology, 86, 149-158.

Eitinger, L., Laane, C.V. Langfeldt, $G_{n}$ (1958) The prognostic value of the clincial picture and the therapeutic value of physical treatment in schizophrenia and the schizophreniform states. Acta Psychiatrica et Neurologica Scondinavica, 33, 33-52.

Endicott, J., Nee. J., Cohen, J., Fleiss, J. \& Simon, R. (1986) Diagnosis of schizophrenia. Prediction of sthort term outcome. Archives of General Psychiarry $43,13-19$.

Everitt, B. \& Dunn, G. (1991) Applied Multivariate Data Anolysis. Edward Amold: London.

Gourlay, A......... Kendell ${ }_{n}$ R.E. (1971) An attempt at validation of traditional psychiatric syndromes 
cluster analysis. British Journol of Psychiatry, $119,399-412$.

Eysenck, H.J (1955) Psychiatric diagnosis as a psychological and statistical problem. Psychologicol Reports, 1 , 3-17.

Farmer, A. Jackson, R., McGuffin, P. \& Storey, P. (1987) Cerebral ventricular enlargement in chronic schizophreniat consistencies and contradictions. British Journal of Psychiatry, $150,324,330$.

-. McGuffin, P. \& Gottesman, 1.L. (1987). Twin concordance for DSM-III schizophrenla scrutinizing the validity of the definition. Archives of General Psychicatry, 44, 634-41.

-....... McGuffin, P. Spitznagel, E. (1983) Heterogeneity in schizophnenta a cluster analytic approach. Psychiatry Research, 8, 1-12.

Feinberg, I. (1982/83) Schizophrenia: caused by a fault in programmed synaptic elimination during adolescence? Jowrnal of Psychiatry Research, 17, 319-34.

Fenton, W. Wyatt, R. \& McGlashan, T. (1994) Risk factors for spontaneous dyskinesia in schizophrenia. Archives of General Psychiatry, 51, 643-650.

-....... W.S. McGlashan, T.H. (1987) Sustained remision in drug-free schizophnenics. American Journal of Psychiatry, 144, 1306-1309.

-....-... W. \& McGlashan, T (1991) Natural history of schizophrenia subtypes. II. Positive and negative symptoms and long-term course. Archives of General Psychiatry, 48, 978-986.

Fish, F. (1962) Schuzaphremia. Wright: Biristol.

(1974) Climical Psychopathology (revised by M. Hamilton). Wright, Bristol.

Fish, B., Marcus, J., Hans, S.L., Auerbach, J. \& Pendue, S. (1992) Infants at risk for schizophrenia: Sequelae of a genetic neurointegrative defect. Archives of General Psychiatry, 49, $221-235$.

Fleiss, J., Gurland, B. \& Cooper, J. (1971) Some contributions to the measurement of psychopathology . Brivish Journal of Psychiarry, 119, 647-656.

Foerster, A. Lewis, S.W., Owen, M. Murray, R.M. (1991) Low birth weight and a family history of schizophrenia predict poor premorbid functioning in psychosis. Schizophrenia Research, 5, 13-20.

Fowler, R.C. (1978) Remitting schizophrenia as a variant of affective disorder. Schizophrenia Bulletin, 4, 68-77. - McCabe, M., Cadoret, R, Winokur, G. (1972). The validity of good prognosis schizophrenia. Archives of General Psychiatry, 26, 182-186.

Galdos, P. \& Van Os, J: (1995) Sex, Psychopathology, and development: from puberty to early adulthood. Schizophrenia Research, 14, 105-112.

......, Van Os, J. \& Murray, R.M. (1993). Puberty and the onset of psychosis. Schizophrenia Research, 10, 7-14.

Gershon, E.S., Bunney, W., Leckman, J. et al (1976). The inheritance of affective disorders": a review of data and hypotheses. Behaviour Genetics, 6, 226-71.

- Mark, A., Cohen, N., Belizon, N. Baron. M., Knobe, K.E. (1975) Transmitted factors in the morbid risk of affective disorders: a controlled study. Jaurnal of Psychiatry Research, 12, 283-299.

Giel,R., Wiersma, D., de Jong, P.A., Slooff,C.G. (1984) Prognosis and outcome in a cohort of patients with non-affective functional psychosis. European Archives of Psychiary and Neuralogical Sciences, 234, 97-101.

Gilbert, P., Harris, J., McAdams, L.A. \& Jeste, D.V. (1995) Neuroleptic withdrawal in schizophrenic patients. Archives of General Psychiatry, 49, 221-235.

Glazer, W., Morgenstern, H. \& Doucette, J. (1991) The prediction of chronje persistent versus intermittent tardive dyskinesia. British Journal of Psychiatry, 1991; 158: 822-828.

Glover, G. (1989) The pattern of psychiatric admission of Caribbean-born immigrants in London. Social Psychiatry and Psychiatric Epidemialogy, 24, 49-56.

Guy W. (1976) ECDEU Manual for Psychopharmacology, pp 534-537. Washington, DC: DHEW.

Goldstein, J., Farone, S., Weij, P. et al (1990) Sex differences in the familial transmission of schizophrenia. British Journal of Psychiatry, 156, 819-825.

Gordon, E.B. (1965) Mentally ill West Indian immügrants. British Journal of Psychiatry, 111, 877-887.

Gottesman, I., and Bertelsen, A. (1991) Schizophrenia: classical approaches with new twists and provocative results. In: The New Genetics of Mental Illness (Eds. P. McGuffin \& R. M. Murray) pp 85-98. Oxford: Butterworth-Heinemann.

Grayson, D. (1986) assessment of evidence for a categorical view of schizophrenia. Archives of General Psychiatry, 712-713.

Grossman, L., Harrow, M., Goldberg, J. \& Fichtner, C. (1991) Outcome of schizoaffective disonder at two longterm follow-ups: comparisons with outcome of schizophrenia and affective disorders. American Jourrial of Psychiatry, 148, 1359-1365.

Gur, R. \& Gur, R. (1995) Hypofrontality in schizophrenila: RIP. Lancet, 345, 1383-1384.

Harder, D., Greenwald, D., Ritzler, B., Strauss, J., Kokes, R. \& Gift, T. (1990). Prediction of outcome among 
adult psychiatric first-admissions, Journal of Climicail PSychology, 46, 119-129.

- Gift, T.E. Strauss, J.S., Ritzler, B.A. \& Kokes, RF (1981) Life events and two-year outcome in schizophrenia. Jowrnal of Consulting and Clinical Psychology, 49, 619-626.

Harding, CM., Brooks, G.W. Ashikaga, T, Strauss, J.S. \& Breier, A. (1987), The Vermont longitudinal study of persons with severe mental illiness. American Journal of Psychiarry, 144, 718-726 \& 727 735.

Harian A (1989) Dewelopmental delay in of fspring of parents with affective disorder and depnession: psychosocial sequels or a constitutional state? Acta Paedopsychiatrica, 52, 287-97.

Hanison, G., Owens, D, Holton, A, et al (1988) A prospective study of severe mental disorder in AfroCaribbean patients. Psychological Medicine, 18, 643-657.

- Searching for the causes of schizophremia the role of migrant studies Schizophrenia Bulletin, 1990 ; 16: $663-671$.

Harrow, M. Groseman, L. (1984) Outcome in the schizoaffective disorders: a critical review and reevaluation of the literature. Schizophrenia Bullevin, 10,85-108,

-min-, Goldberg, J., Grossman, L. \& Meltzer; H. (1990) Outcome in manic disorders A naturalistic follow-up study. Archives of General Psychiatry, 47, 665-671.

Harvey 1., McGuffin, P., Williams, M. \& Toone, B. (1990a) The ventricle-brain ratio (VBR) in functional psychoses: an admixture analysis. Psychiatry Research. Neuroimaging, 35, 61-69.

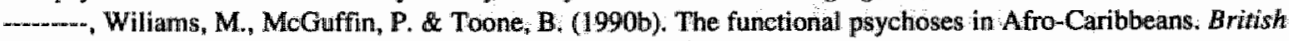
Journal of Psychiatry, 157, 515-22.

Hawk, A, Carpenter, W. \& Strauss, J. (1975) Diagnostic criteria and five-year outcome in schizophrenia. Archives of General Psychiatry, 32, 343-347.

Hegarty, I,D, Baldessarini, R., Tohen, M, Watemaux, C, \& Oepen, G. (1994). One hundred years of schizophrenia: a meta-analysis of the outcome literature. American Journal of Psychiatry, 151, 1409-1416.

Hemsi, L.K. (1967) Psychiatric Morbidity of West-Indian immigrants. Social Psychiatry, 2, 95-100.

Hest, F.P. \& Wolters, R.J. (1992) Deeltijdbehandeling in Engeland en Wales. Tijdschrift voor Psychiatrie, 32, $354-361$.

Hickling, F. (1991) Psychiatric hospital admission rates in Jamaica, 1971 and 1988. British Journal of Psychiatry, $159,817-821$.

Hill, A.V. Molecullar markers of ethnic groups. In: Ethnic Factors in Health and Disease (Eds J.K. Cruickshank \& D.G. Beevers). London: Wright, pp 25-31.

Holmes, T.H. \& Rahe, R.H. (1967) The social readjustment scalle Journal of Psychosomatic Research, 11, 213218.

Horn, J. \& McManus, I.C. (1992) Ventricular enlargement in schizophrenia. British Journal of Psychiatry, 160 , $687-697$.

Huber, G., Gross, G., Schulttler, R. \& Linz, M. (1980) Longitudinal studies of schizophrenic patients. Schizophrenia Bulletin, 6, 592-605:

Jensen, S., Schaumburg, E., Leroy, B., Larsen, O., Thorup M. (1989) Psychiatric care of refugees exposed to vilolence. Acta Psychiatrica Scandinavica, 80: 125-31.

Jeste, D. \& Wyatt, R. (1981) Changing epidemiology of tardive dyskinesia. An overview. American Joumal of Psychiany, 138, 297-309.

...... Caligiuri MP (1993). Tardive dyskinesia. Schizophrenia Bulletin, 19, 303 315.

Johanson, E.A. (1958) a study of schizophrenia in the male. Acta Psychiarica Scandinavica, 125, 1.56 (suppl.).

Johntson-Sabine, E., Mann, A.; Jacoby ef al (1983) Bouffeé délirante: an examiration of its current status. Psychological Medicine, 13,771-78.

Johnstone, E, Owens, D., Crow, T.J., Frith, C.D., Alexandropolis, K., Bydder, G. \& Colter, N. (1989) Temporal lobe structure as determined by nuclear magnetic resonance in schizophrenia and bipolar affective disorder. Journal of Neurology, Newrosurgery, and Psychiatry, 52, 736-741.

-........, Frith, C., Crow, T., Owens, D., Done, D., Baldwin, E. \& Charlette, A. (1992) The Northwick Park 'functional' psychosis study: diagnosis and outcome. Psychological Medicine, 22, 331-46.

- . E. C. Crow, T.J., Frith, C.D. \& Owens, D.G. (1988) The Northwick Park "functional"psychosis study: diagnosis and treatment response. Lancet, July 19th, 1988.

Jones, P "Harvey. I. Lewis, S. Toone, B., Van Os, J., Williams, M. \& Murray, R.M. (1994a). Cerebral ventricle dimensions as risk factors for the functional psychoses. Psychological Medicine, 24, 995-1011.

-n-.-m.n.-, Rodgers B, Murray R, \& Marmot M (1994b) Child devellopmental risk factors for adult schizophrenia in the British 1946 birth cohort Lancet, 344, 1398-402.

- P.B., Bebbington, P., Foerster, A., Lewis, S, Murray, R., Russel, A., Sham, P., Toone, B. \& Wilkins, S. (1993) Premorbid social underachievement in schizophrenia. Results from the Camberwell Collaborative Psychosis Study. British Journal of Psychiatry, 162, 65-71.

Kallmann, F.J. (1938) The Genetics of Schizophrenia. New York: J.J. Agusta. 
Kane, J. \& Smith, J. (1982) Tardive dyskinesial prevalence and risk factors, 1959 to 1979 . Archives of Cenercal Psychiairy, 39, 473-481.

-..., Woerner $M_{3}$ Borenstein $M$, et al (1986). Integrating incidence and prevalence of tardiwe dyskinesia. Psychopharmacology Bulletin, 22, 254-258.

Kasanin, J. (1933) The acute schizoaffective psychoses. Americam Journal of Psychiatry, 90, 97-126.

Kay, S. \& Murrill, L. (1990) Predicting outcome of schizophrenia: significance of symptom profiles and outcome dimensions. Comprehensive Psychiatry, 31, 91-102.

Kay, R.W. (1992) Prevalence and incidence of schizophrenia in Afro-Caribbeans. British Jourmat of Psychiatry, $160,421$.

Kelsoe, J.R.; Cadet, J.L., Pickar, D., Weinberger, D.R. (1988) Quantitative neuroanatomy in schizophrenia. Archives of General Psychiarry, 45, 533-41.

Kendell, R.E., \& Zealley, A. K. (Eds) Diagnosis and classification. In: Companion to Psychiatric Studies, fifth edition, pp 277-295. London: Churchill Livingstone.

2....... (1989) Clinical validity. Psychological Medicine, 19, 45-55.

, R. \& Gourlay, J. (1970) The clinical distinction between the affective psychoses and schizophrenia. British Journal of Psychiatry, 117, 261-266.

- - Brockington, I.F. (1980) The identification of disease entities and the relationship between schizophrenic and affective psychoses. British Journal of Psychiatry, 137, 324-331.

......... Brockington, I. \& Leff, J. (1979) Prognostic implications of six alternative definitions of schizophrenia. Archives of General Psychiatry. 35, 25-31.

Kendler, K.S., Neale, M., Heath, A., Kessler, R. \& Eaves, L.J. (1991). Life events and depressive symptoms: a twin study perspective. In: The New Genetics of Mental Iliness (Eds. P. McGuffin \& R.M. Murray). Heinemann: Oxford.

……, Gruenberg, A.M. \& Tsuang. M.T. (1985) Psychiatric illness in first-degree relatiwes of schizophrenic and surgical control patients: a family study using DSM-III criteria. Archives of General Psychiasry, 42, 770= 779 .

- (1986) A DSM-III family study of the non-schizophrenic psychotic disorders. American Journal of Psychiatry, 143, 1098-1105.

...-.... Tsuang. M.T. (1988) Outcome and familial psychopathology in schizophrenia. Archives of General Psychiatry, 45, 338-346.

_-_-_- Kessler, R., Walters, E., MacLean, C., Neale, M., Heath, A. \& Eaves, L. (1995) Stressful life events, genetic liability, and onset of an episode of major depression in women. American Jourmal of Psychiatry. $152,833-842$.

---_--, \& Eaves (1986) Models for the joint effect of genotype and environment on liability to psychiatric illness. American Journal of Psychiatry, 143, 279-289.

-..n-..-.., McGuire, M., Gnuenberg, A.M., O'Hare, A., Spellman, M. \& Walsh, D. (1993) The Roscommon family study. J. Methods, diagnosis of probands, and risk of schizophrenia in relatives. Archives of Generai Psychiatry, 50, 527-540.

Kerr, A. \& McClelland, H. (Eds) (1991) Concepts of Mental Disorder: a Continuing Debate. Gaskell: London.

Kiev, A. (1963) Beliefs and delusions of West Indian immigrants to London. British Jourmal of Psychiatry. 109, 356-363.

King, M., Coker, E, Leavey, G., Hoare, A. Johnson-Sabine, E. (1994) Incidence of psychotic illness in London: a comparison of ethnic groups. British Medical Journal, 309, $1115-9$.

Kirov K. (1995) Bulgarian psychiatry: development, ideas, achievernents. History of Psychiatry, in press.

Klerman, G.L., Vaillant, G.E., Spitzer, R. \& Michels, R. (1984) Debate on DSM-III. American Journal of Psychiatry, 141, 1126-1127.

Kolakowska, T., Williams, A.O., Ardern, M., Reveley, M.A., Jambor, K., Gelder, M.G. \& Mandelbrote, B.M. (1985) Schizophrenia with good and poor outcome. I: early clinical features, respontse to neuroleptics and signs of organic dysfunction. British Journal of Psychiatry, 1.46, 229-246.

Kraepelin, E. (1919) Dementia Praecox and Paraphrenia (translated by M. Barclay) pp 230-231. Edinburgh: Churchill Livinstone.

Krawieck@, M., Goldberg. D. \& Vaughan, M. (1977) A standardized psychiatric assessment for rating chronic psychotic patients. Acta Psychiatrica Scandinavica, 55, 299-308.

Kreitman, N., Sainsbury, P., Morrisey, J., Towers, J. \& Scrivener, J. (1961) The reliability of psychiatric assessment: an anallysis. Journal of Mental Science, 107: 887-908.

Lazardis K. (1987) Psychiatrische Erkrankungen bei Ausländern - Hospitalisations - und nationalitătsspezifische Inzidenz. Nervenarzt 58 : 250-255.

Lee, A. \& Murray, R. (1988) The long term outcome of Maudsley depressives. British Journal of Psychiatry, $153,741-751$. 
Leff, J., Fisher, M. \& Bertelsen, A. (1976) A cross nationall study of mania: British Journal of Psychiatry, 129. $428-42$.

-..-na-., Sartorius, N., Jablensky, A., Korten, A. \& Emberg, G. (1992) The International Pilot Study of Schizophrenia: five year follow-up findings. Psychological Medicine, 22, 131-145.

Lewine, R., Hudgins, P., Brown, F., Caudle, J. \& Risch, S.C. (1995) Differences in qualitatiwe brain morphology findings in schizophrenia, major depression, bipolar disorder and normal volunteers,Schizophremia Research, $15,253-259$.

Lewis, S., Revelley, A.M, Reveley, M.A., Chitkara, B. \& Muray, R.M. (1987) The farmilial/sporadic distinction as a strategy in schizophrenia research. British Journal of Psychiatry, 151, 306-313.

- L_- (1990) Computerized tomography in schizophrenia: 15 years on. British Journal of Psychiatry, 157 (suppl): 16-24.

Lewis L, Croff-Jeffreys C, David A. Are British psychiatrists racist? British Journal of Psychiatry, 1990; 157: 410-415.

Liddle PF, Barnes TRE, Speller J and Kibel D. Negative symptoms as a risk factor for tardive dyskinesia. British Journal of Psychiatry 1993; 163: 776-780.

-.....-m., (1987a) The symptoms of schizophrenia. A re-examination of the positive-negative dichotomy. British Journal of Psychiatry, 151, 145-51.

_....... (1987b) Schizophrenic syndromes, cognitive performance and neurological dysfunction. Psychological Medicine , 17, 49-57.

w.w....., Friston, K., Frith, C., Hirsch, S., Jones T. Frackowiak, R. (1992) Patterns of cerebral blood flow in schizophrenia. British Journal of Psychiatry, 160, 179-186.

Lin, K. \& Kleinman, A. (1988) Psychopathology and clinical course of schizophrenia: a cross-cultural perspective. Schizophrenia Bulletin, 14, 555-567.

Littlewood, R. \& Lipsedge, M. (1981) Acute psychotic reactions in Caribbean-born patients. Psychological Medicine, $11,303-318$.

Lukoff, D., Nuechteriein, K. \& Ventura, J. (1986). Manual for the expanded brief psychiatric rating scale (BPRS). Schizophrenia Bulletin, 12, 594-602.

Maier, W., Lichtermann, D., Minges, J., Hallmayer, J., Heun, R., Benkert, O,, Levinson, D.F. (1993) Continuity and discontinuity of affective disorders and schizophrenia. Results of a controlled family study. Archives of General Psychiatry, 50, 871-83.

Malmberg, A.K., Lewis, G., David, A., Allebeck, P. \& Brandt, L. (1995) Premorbid personality, social behaviour and schizophrenia: a prospective cohort study. Schizophrenia Research, 15, 194.

Manley, B. (1986) Multivariate Statistical Methods. Chapman \& Hall: London.

Maj, M. \& Perris, C. (1990) Patterns of course in patients with a cross-sectional diagnosis of schizoaffective disorder. Journal of Affective Disorders, 20, 71-77.

Malla, A., Cortese, L., Shaw, T.S. G Ginsberg, B. (1990) Life events and relapse in schizophrenia: a one year prospective studly. Social Psychiarry and Psychiatric Epidemiology, 25, 221-224.

-...- Norman, R.M.G., Williamson, P. Contese, L. \& Diaz, F. (1993). Three syndrome concept of schizophrenia. A factor analytic study. Schizophrenia Research, 10, 143-150.

Marneros, A., Deister, A, Rohde, A., Steinmeyer, E.M., Jünemann, H. \& Fimmers, R. (1988) Long-term course of schizoaffective disorder. European Archives of Psychiatry and Neurological Sciences, 237, 264-275.

...-...-, Deister, A., Rohde, A., Steinmeyer, E.M. \& Jinemann, H. (1989) Long-term outcome of schizoaffective and schizophrenic disorders: a comparative study. European Archives of Psychiatry and Neurological Sciences, 238, 118-125.

................ (1990). Psychopathological and social status of patients with affective, schizophrenic and schizoaffective disorders aftyer Jong-term course. Acta Psychiarrica Scandinavica, 82, 352-358.

Mayerhoff, D.I., Loebel, A.D., Alvir, J. (1994) The deficit state in first-episode schizophrenia. American Journal of Psychiarry. 151, 1417-1422.

McCullagh P. Regression models for ordinal data. Journal of the Rayal Statistical Society 1980; 42: 109-142.

McGlashan, T. (1984) The Chestnut Lodge follow-up study II. Long term outcome of schizphrenia and the affective disorders. Archives of General Psychiatry, 41, 586-601.

-... (1986) Predictors of shorter-, medium- and longer-term outcome in schizophrenia. American Journal of Psychiatry, 143, 50-55.

-.-. Carpenter, W. Bartko, J. (1988) Issues of design and methodology in long-term follow-up studies. Schizophrenia Bulletin, 14, 569-574.

- Williams, P. (1990) Predicting outcome in schizoaffective psychosis. Journal of Nervous and Mental Disease. $_{178,518-520}$

McGovem, D., \& Cope, R. (1987) First psychiatric admission rates of first and second-generation Afro-

Caribbeans. Social Psychiatry, 22, 139-149. 
Hemmings, $P_{2 *}$ Cope, R. \& Lowerson, A. (1994) Long-term follow-up of young Afro-Caribbean Britons and white Britons with a frist admission diagnosis of schizophrenia. Social Psychiatry and Psycthiarric Epidemiology, 29, 8-19.

McGuffin, P. Katz, R. (1986) Nature, nurture and affective disorders. In: Recent advances in the biology of affective disorder (Eds. J. Deakin \& H: Freeman). British Journal of Psychiatry Special Publication. Headley Bros: Ashford.

-.-, Katz, R., Aldrich, J. et al (1988a) The Camberwell Collaborative Depression Study I. British Sournal of Psychiatry, 152, 766-774.

-.-.- Katz, R. \& Bebbington, P. (1988b) The Camberwell Collaborative Depression Study III. British Journal of Psychiatry, 152, 775-782.

-..--, Farmer AE, Harvey I. A polydiagnostic application of operational criteria in psychotic illness: development and relliability of the OPCRIT system. Archives of General Psychiatry, 1991;48: 764-770.

McGuffin, P., Farmer, A. \& Gottesman, I. (1987) Is there really a split in schizophrenia? The genetic evidence. British Journal of Psychiatry, 150, 581-592.

McKenzie, K.J. \& Crowcroft, N.S. (1994) Race, ethnicity, culture, and science. British Medical Journal, 309, 286-87.

Moller, H.J., von Zerssen, D., Werner-Eillert, K. \& Wuschner-Stockheim, M. (1982) Outcome in schizophrenic and similar paranoid psychoses. Schizophrenia Bulletim, 8, 99-108.

Moore, T.V. (1929) The empirical detemination of certain sydromes underlying praecox and manic-depressive psychoses. American Journal of Psychiatry, 9. $719-738$

Montimer, A., Lund, C. \& McKenna, P. (1990) The positive-negative dichotomy in schizophrenia. British Journal of Psychiarry, 157, 41-49.

Murray, R.M., Lewis, S.W. \& Reveley, A.M. (1985) Towards an aetiological classification of schizophrenia. Lancet, i, 1023-1026.

-- \& Lewis, S.W. (1987) Is schizophrenia a neurodevelopmental disorder? British Medical Joumal, 295, 681-682.

---.-, Lewis, S.W., Owen, M.J., et al (1988) The neurodevelopmental origens of dementia praecox. In Schizophrenia: the Major Issues (eds P. Bebbington \& P. McGuffin) pp 90-107. Oxford: Heinemann.

- \& O'Callaghan, E. (1991) The congenital and adult-onset psyehoses: Kraepelin lost, Kraepelin found. In Concepts of Mental Disorder, a Continuing Debate (Eds A. Kerr \& H. McClelland) pp 48-65. London: Gaskell.

-...-, O'Callaghan, E., Castle, D, et al (1992) a neurodevelopmental approach to the classification of schizophrenia. Schizophrenia Bullerin, 18, 319-332.

Neeleman, J. \& Van Os, J. (1994). The feasability of a psychiatric common market - British and continental approaches to postgraduate psychiatric training. Psychiatric Bulletin, 18, 193m196.

Nijman H, a Campo J, Ravelli D. (1993) Inbewaringstellingen; kort maar krachtig? Tijdschrift voor Psychiatrie, $35,58-66$.

Norman, R. \& Malla, A. (1993a) Stressful life events and schizophrenia II: Conceptual and methodological issues. British Journal of Psychiatry, 162, 166-174.

-........, \& Malla, A. (1993b) Stressful life events and schizophrenia I: A review of the research. British Journal of Psychiatry, 162, 166-174.

Offord, D. \& Cross, L. (1969) Behavioural antecedents of adult schizophrenia; a rewiew. Archives of General Psychiarry, 21, 267-283.

O'Hara, P., Brugha, T. . Lesage, A. \& Wing, J. (1993) New findings on tardive dyskinesia in a conmunity sample. Psychological Medicine, 23, 453-465.

Odegaard, $\emptyset$. (1932) Emigration and insanity: a study of mental disease among Norwegian-born population in Minnesota. Acta Psychiatrica et Neurologica Scandinavica, suppl. 4.

Owens, D.G.C., Johnstone, E.C., and Frith, C.D. (1982) Spontaneous involuntary disorders of movement. Anchives of General Psychiatry. 39, 452-461.

Pandurangi, A.K., Bilder, R., Rieder, R., Mukherjee, S. \& Hamer, R. (1988) Schizophrenic symptoms and deterioration. Relation to computed tomographic findings. Journal of Nervous and Mental Disease, 176, 200-206.

Paykel, E. S. (1974) Recent life events and clinical depression. In: Life Stress and MIness (Eds E. Gunderson \&. Rahe). Charles Thomas: Springfield, pp 134-163.

$$
253 .
$$

Peralta, V., De Leon, J. \& Cuesta, M. (1992) Are there more than two syndromes in schizophrenia? a critique of the positive-negative dichotomy. British Journal of Psychiatry, 161, 335-343. 
Perera, R, Owens, D \& Iohinstone, E. (1991) Disabilities and circumstances of schizophrenic patients- a followup study. VII Ethnic aspects. A comparison of three matched groups. British Journal of Psychiatry, 159, Suppl. 13, 40-42.

Perris, H., von Knotring, Lu \& Perris, C. (1982) Genetic vulnerability for depression and life events. Neuropsychobiology, 8, 241-247.

Phelan, J, Schwartz, J.E., Bromet. E.J., Dew, M., Parkinson, D., Schulberg, H., Dunn, L., Blane, H. \& Curtis, E. (1991) Work stress, family stress and depression in professional and managerial employees. Psychological Medicine, 21, 999-1012.

Phililps, L. (1953) Case history data and prognosis in schizophrenia, Journal of Nervous and Menral Disease, $117,515-525$.

Phinney, I.S. (1990) Ethnic identity in adolescents and adults: review of research. Psychological Bulletin, 108, 499-514.

Piccinell, M. \& Wilkinson, G. (1994) Outcome of depression in psychiatric settings. British Journal of Psychiatry, 164, 297-304.

Pickering, G.W. (1968) High Blood Pressure. (2nd edition) Churchill, London.

Pogue-Geile, M. Harrow. M. (1985) Negative symptoms in schizphrenia: their Jongitudinal course and prognostic significance. Schizophrenia Bulletin, 11, 427-439.

- - (1989) The prognostic significance of negative symptoms in schizophrenia. British Journal of Psychiatry, 155 (suppl. 7) 123 127.

Pollitt, J. (1972) The relationship between genetic and precipitating factors in depressive Ilness. British Journal of Psychiatry, 121, 67-70.

Pope, H. \& Lipinski, J. (1978) Diagnosis in schizophrenia and manic depressive illness. Archives of General Psychiatry, $35,811-828$.

Prentky, R., Lewine, R., Watt, N. \& Fryer, J. (1980) A longitudinal study of psychiatric outcome: developmental wariables vs, psychiatric symptoms. Schizophrenia Bulletin, $6,139-148$.

Prudo, R. \& Blum, H.M. (1987) Five-year outcome and prognosis in schizophrenia: a report from the London field research centre of the International Pilot Study of Schizophrenia. British Journal of Psychiatry, 150, 345-354.

Puig Antich J, Goetz D, Davies M, Kaplan T, Davies S, Ostrow L, Asnis L, Twomey J, Iyenqar S, Ryan ND (1989) A controlled family history of prepubertal major depressive disonder. Archives of General Psychiatry, $46,406-18$.

Rabiner, C., Wegner, J. Kane, J. (1986) Outcome study of first episode psychosis, I: relapse rate after 1 year. American Journal of Psychiatry, 143, 1155-115i8.

Regionaal Instituut voor Ambulante Geestelijke Gezondheizorg (RLAGG, 1992) RIAGGs in Cijfers, Amisterdam: NVAGG.

Reich, T., James, J.W. \& Morris (1972) The use of multiple thresholds in determining the the mode of transmission of semi-continuous traits. Annals of Human Genetics, 36, 163-184.

........, Cloninger, C., Wette, R. \& James, J. (1979) The use of multiple thresholds and segregatrion analysis in analysing the phenotypic heterogeneity of multifactorial traits. Annals of Human Generics, 42, 371-389.

- Guze, S.B. (1975) The multifactorial model of disease transmission I: description of the model and its use in psychiatry. British Jaurnal of Psychiatry, 127, 1-10.

del Rio Vega, J.M. \& Ayuso-Gutierrez, J.L. (1990) Course of schizoaffective psychosis: a retrospective study. Acta Psychiatrica Scandinavica, 81, 534-537.

Robins, L.N. (1969) Deviant children grown up. Baltimore, Williams \& Wilkins.

Robins, E. Guze, S.B. (1970) Establishment of diagnostic validity in psychiatric illness: its application to schizophrenia: American Journal of Psychiatry, 126, 983-987.

Rogers, B. (1990) Behaviour and personality in childhood as predictors of adult psychiatric disorder. Journal of Child Psychology and Psychiatry, 3, 393-414.

Rossi, A., Stratta, P., Petruzzi, C. et al (1987) A computerized tomographic study in DSM-III affective disorders. Journal of Affective Disorders, 12, 259-262.

Roy, M.A. \& Crowe, R. (1994) Validity of the familial and sporadic subtypes of schizophrenia. Amierican Journal of Psychiatry. $151,805-814$.

Rwegellera, G.G.C. (1977) Psychiatric morbidity among West-Africans and West-Indians living in London. Psychological Medicine, 7, 317-329.

Samson, J, Simpson, J. \& Tsuang, M. (1988), Outcome studies of schizoaffective disorders. Schizophrenic Bulletin, 14, 543-554.

Sargeant, K., Bruce, M., Florio, L. \& Weissman, M. (1990) Factors associated with 1-year outcone of major depression in the community. Archives of General Psychiatry, 47, 519-526.

Sartorius, N., Jablensky, A., Korten, A., Emberg, G., Anker, M., Cooper, J.E. \& Day, R. (1986). Early 
manifestations and first-contact incidence of schizophrenia in different cultures A preliminary repont on the initial evaluation phase of the WHO Collaborative study on Determinants of Outocne of Severe Mental Disorders. Psychological Medicine, 16, $909-928$.

Sashidharan. S.P. AfromCaribbeans and schizophrenia: the ethnic vulnerabilty hypothesils re-examined. International Review of Psychiatry, 1993, 5:129-144.

Saugstad,L.F. \& Odegard,O. (1983) Persistent discrepancy in international diagnostic practice simce 1970. Acta Psychiatrica Scandinavica, 68,501-10

..m. (1989a) Age at puberty and mental Illness. Towards a neurodewelopmental aetiology of Kraepelin's endogenous psychoses. British Journal of Psychiatry, 155, 536-44

. (1989b) Social class, mamiage, and fertility in schizophrenia Schizophuenia Bullerin, 15. 9-43. Sayil, I. (1984) Psychiatric problems of Turkish workers in Holland. Iniermational Journal of Social Psychiatry, 30: 267-273.

Schneider, K. (1959) Clinical Psychopathology. Translated by M.W. Hamilton. London: Orune \& Stratton.

Schneider, K. (1925) Wesen und Erfassung des Schizophrenen. Zeitschrif fur die gesamte Neurologie whd Psychiatrie, 99, 542-547.

Schomagel, W., Assmann, V. (1992) Pleidooi voor een rumer indicatiegebied van electroconvulsie therapie. Tijdschrift woor Psychiatrie, 34: 131-135.

Scott, J., Eccleston, D. Boys, R. (1992) Can we predict the persistence of depression? British Iowinal of Psychiatry, 161, 633-637.

Sedler, M.J. (1994) Foundations of the new nosology. The Journal of Medicine and Philosophy, 19, 219-238.

Selten, J.P., Sijben, N.E., van den Bosch, R.J., Onnloo-Visser, J. \& Warmendam, H. (1993) The subjective experience of negative symptoms: a self-rating scale. Comprehensive Psychiotry, 34, 192-7.

_..._. Sijben, N. (1994) First admission rates for schizophrenia in immigrants to the Netherlands. The Dutch National Register. Social Psychiarry and Psychiaric Epidemiology, 29, 71-77.

Serban, $G_{\text {. }}$ (1975) Relationship of mental status, functioning, and stress to readmission of schizophrenics. British Journal of Social and Clinical Psychology, 14, 291-301.

Sham, P., Jones, P., Russel, A., Gilwarry K., Wilkins, S., Foerster, A., Bebbington, P., Lewis, S., Toone, B. \& Murray, R. (1994) Age at onset; sex, and familial psychiatric morbidity im schizophrenia. Report from the Camberwell Collaborative Psychosis Study. British Journal of Psychiatry 165, 466-473.

Shepherd, M, Watt, D., Falloon, I, et al (1989) the natural history of schizophreniat a five year follow-up study of outcome and prediction in a representative sample of schizophrenics. Psychological Medicine, monograph suppl 15 .

Siegel J.S. (1974) Estimates of coverage of the population by sex, race and age in the 1970 census. Demography, 11: 1-23.

Slater, E. Shields, J. (1969) Genetical aspects of anxiety. In: Studies of Anxiety; British Journal of Psychiatry Special Publication no. 3. Headlley Bros: Ashford.

Spitzer, R., Fleiss, J. Endicott, J. \& Cohen, J. (1967) Mental status schedule. Archives of general psychiary, 16, 479-493.

Steimberg, H. Durell, J. (1968) A stressful situation as a precipitant of schizophrenic symptomis: an epidemiological study. British Journal of Psychiatry, 114, 1097-105.

Stephens, J. \& Astrup, C. (1963) Prognosis in "process" and "non-process" schizophrenia: American Jowrital of Psychiatry, 119. 945-953.

-.n-n.w... Astrup, C., Mangrum, J.C. (1966) Progmostic factors in recovered and detteriorating schizophrienics. American Jowrnal of Psychiary, $122,1116-1121$.

Stoffelmayr, B., Dillavou, D. \& Hunter, J. (1983) Premorbid functioning and outcome in schizophrenial" a cumulative analysis. Jowrnal of Conculting and Clinical Psychology, 51, 338-352.

\& Carpenter, W. (1974) The prediction of outcome in schizophrenia. Archives of General Psychiatry, $31,37-42$.

Streiner, D.L. \& Noman, G.R. (1989) Validity, In: Health Measurement Scales, pp 106-125. Oxford: Oxford Medical Publications.

Strömgren, E. (1985) World-wide issues in psychiatric diagnosis and classification and the Scandinavian point of view. In Mental disorders, Alcohol and Drag Relared Problems. Amsterdam: Excerpta Medica.

Sugamman, P.A. Outcome of schizophrenita in the Afro-Caribbean community (1992), Social Psychiarry of Psychiatric Epidemiology, 27, 102-5.

Susser, M. (1985) Epidemiology in the United States after World War II: the evolution of technique. Epidemiological Review, 7, 147-77.

Susser, E. Wanderling, J. (1994) Epidemiology of nom-affective acute remitting psychosis vs schizophrenia. Archives of General Psychiatry, 51, 294-301.

Swayze, V., Andreasen, N.C., Alliger, R., Ethardt, J.C. \& Yuh, W. (1990) Structural brain abnomalities in 
bipolar mood disorder: ventricular enlargement and focal signal hyperintensities, Archives of General Psychiatry, 47, 1054-1059.

Taylor, M. (1992) Are schízophrenia and affective disorder related? A selective literature review. Americam Journal of Psychiatry, 149, 22-32.

Thompson, P. \& Melizer, H. (1993) Positive, negatiwe, and disorganisation factors from the schedule for affective disorders and schizophrenia and the present state examination British Journal of Psychiatry, $163,344351$.

Thornicroft, G. \& Sartotius, N. (1993) The course and outcome of depression in different cultures: 10 year follow-up of the WHO collaborative study on the assessment of depressive disorders Psychological Medicine, 23, 1023-1032.

...m........ (1990) Cannabnis and psychosis: is there epidemiological evidence for an associaiton? British Journal of Psychiarry, 157, 25,33.

Tohen, M., Watemaux, C. Tsuang, M. (1990) Outcome in Mania. Archives of General Psychiatry, 47, 11061111.

.........., Tsuang. M. Goodwin, D. (1992) Prediction of outcome in mania by mood-congruent or moodincongruent psychotic features. American Journal of Psychiatry, 149, 1580-4.

Torgersen, S. (1986) Genetic factors in moderately severe and mild affective disorders. Archives of General Psychiatry, 43, 222-6.

Tsuang, M.T. \& Dempsey, G.M. (1979) Long term outcome of Major Psychoses II. Schizoaffective disorder, compared with schizophrenia, affective disorders, and a surgical control group; Archives of General Psychiatry, 36, 1302-1304.

......-... \&inokur. G. (1974) Criteria for subtyping schizophrenia. Clinical differentiation of hebephrenic and paranoid schizophrenia. Archives of General Psychiatry, 31, 43-4.7.

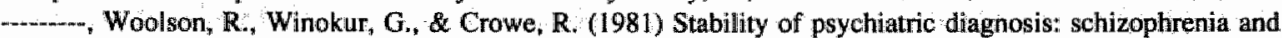
affective disorders followed up over a 30- to 40-year period Archives of Geneval Psychiatry, 38, 535-539.

-m-m.m... Dempsey, G.M. \& Rauscher, F. (1976) A study of "atypical schizophrenia"; Archives of General Psychiatry, 33, 1157-1160.

-....... Winokur, G. \& Crowe, R.R. (1980) Morbid risks of schizophrenia and affective disorders among first degree relatives of patients with schizophrenia, mania, depression, and surgical conditions. British Jourhal of Psychiatry, 137, 497-504.

Vaillant, G.E. (1964) Prospective prediction of schizophrenic remission. Archives of Gentral Psychiatry, 11, 509. 518 .

-mm (1962) The prediction of recovery in schizophrenia. Journal of Nervous and Mental Diseases, 135, 534-543.

Van der Does, A.J., Linszen, D.H., Dingemans, P.M., Nugter, M. A. \&. Scholte, W.F. (1993) A dimensional and categorical approach to the symptomatology of recent onset schizophrenia. Journal of Nervous and Mental Disease (in press).

Van Os, J., Galdos, P., Lewis, G., Mann, A. \& Bourgeois, M. (1993) Schizophrenia sans frontierest concepts of schizophrenia among French and British Psychiatrists. British Medical Journal, 307, 489-492.

............, Galdos, P. Lewis, $\mathbf{G}_{i, k}$ Mann, A. \& Bourgeois, M. (1994), Schisms in Earopean Psychiatry In: Psychiarry in Europe: Developments and Directions, London: Gaskell

wan.m.- Neeleman, J. (1994a) Mental illness in Europe. British Medical Jowmal, 309, 1218-1222.

........... \& Neeleman, J. (1994b). European Union Government legislation affecting psychiatric practice. Psychiatric Bulletin, 18, 390-394.

-.-men-, Neeleman, J. (1995), Europe and psychiatric ethics. Psychiatric Bulletin, 19, 1-3.

-w...m.... Jones, P. Lewis, G. , Wadsworth, M., \& Murray, R.M. (1995), Evidence for simillar developmental pnecursors of chronic affective illness and schizophrenia in a general population birth cohort. (submitted, Archives of General Psychiatry).

Van Praag, H.M., Kahn, R.S., Asnis, G.M. et al (1987) Denosologization of biological psychiatry or the specificity of 5-HT disturbances in psychiatric disordens. Journal of Affective Disorders, 13, 1-8.

............ Asnis, G.M., Kahn, R.S. et al (1990) Nosological tunnel vision in biological psychiatry. A plea for a functional psychopathology. Annals of the New York Academy of Science, 600, 501-10.

....m..., (1992) Make believes in psychiatry or the perils of a progress. New York: Brunner Mazel.

Ventura, $\mathbf{J}_{*}$, Nuechterlein, K.H., Lukoff, D. \& Hardisty, J.P. (1989) A prospective study of stressful life events and schizophrenic relapse. Jounal of Abnormal Psychology, 98, 407-411.

Waddington, J.L., and Youssef, H.A. (1986) An unusual cluster of tardive dyskinesia in schizophrenia: association with cognitive dysfunction and negative symptoms. American Journal of P.5ychiatry, 143, 1162165

-......., Youssef, H.A., Kinsella, A. et al (1990). Cognitive dysfunction in schizophrenita followed up over 5 
years, and its longitudinal relationship to the emergence of tardiwe dyskinesia. Psychological Medecine. 20. $835-842$.

Walker. EF. \& Lewine, R.J. (1994) Prediction of adult-onset schizophrenia from childhood home movies of the patients. American Journal of Psychiatry $147,1052-1056$.

Wallis, G. Stress as a predictor in schizophrenia. British Jownat of Psychiatry, 120, 375-384.

Watt. N.F. (1978) Patterns of childhood social development in adult sichizophrenics. Archives of General Psychiatry, 35, 160-166.

Weinberger, D.R. (1984) CAT scan findings in schizophrenia: Speculation on the meaning of it all. Joirnal of Psychiatry Research, 18, 477-490.

-2..... Delisi, L., Perman, G. et al (1982) Computed tomography in schizophreniform disorder and other acute psychiatric disorders. Archives of General Psychiaty. 39, 778-783.

(1987) Implications of nomal brain development for the pathogenesis of schizophrenia. Archives of General Psychiary, 44, 660-669.

Werry, J.S., McClellan, J.M. \& Chard, L. (1991) Childhood and adolescent schizophrenic, bipolat, and schizoaffective disonders: a clinical and outcome study. Journal of the American Acodemy of Child and Adolescent Psychiatry, 30, 457-465.

Wessely S, Castle D, Der G, Murray, R. Schizophrenia and Afro-Caribbeans: A case controll study. British Journal of Psychiatry, 1991; 159: 795-801.

Wiersma, D., Giel, R., De Jong, A. et al (1983) Social class and schizophrenia in a Dutch cohort. Psychological Medicine, 13, 141-50.

Wing, J.K., Monck, E., Brown, G. \& Carstairs, G. (1964) Morbidity in the community of schizophrenic patients discharged from London mental hospitals in 1959. British Journal of Psychiarry, 110, 10-21.

.-.--, Cooper, J.E. Sartorius, N. (1974) The Measurement and Classification of Psychiatric Symptoms. Cambridge University Press, Cambridge.

Winokur, G., Coryell ,W., Keller, M., Endicott, J., Akiskal, H. (1993) A prospective follow-up study of patients with bipolar and primary unipolar affective disorder. Archives of General Psychiatry 50, 457-465.

World Health Organization (1973) Report of the international pilot stwdy of schizoprhenia, vol 1. Geneva: WHO. , (1992) The 1CD-10 Clasification of Mental and Behavioural Disorders. Genewa: WHO.

Yassa R, Jeste DV (1992). Sex differences in Tardive Dyskinesia: a Critical review of the literature. Schizophrenia Bulletin, 18,701-715.

Zimmerman, M. (1988) Why are we rushing to publish DSM-IV? Archives of General Psychiatry. 45, 11351138 .

Zubin, J. and Spring, B. (1977) Vulnerability: a new view of schizophrenia. Journal of Abnormal Psychology, $86,103-126$.

, (1961) A biometric approach to prognosis in schizophrentia. In (P. Hoch \& J. Zubin "eds) Comparative Epidemiology of the Mental Disorders. New York: Grune \& Stratton. 
as

औ

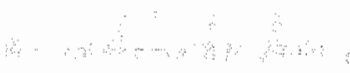

$\therefore \quad \vdots \quad \vdots \quad-\quad$

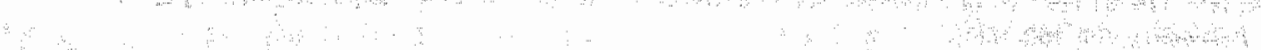

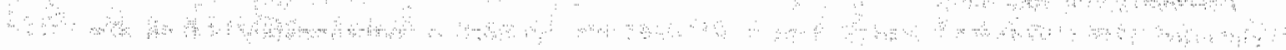

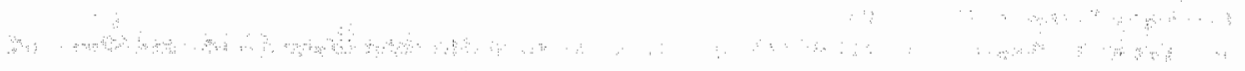

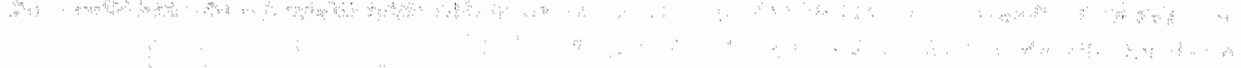

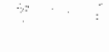




\section{Chapter 2: Interpreting Follow-up Studies}

Cr: 


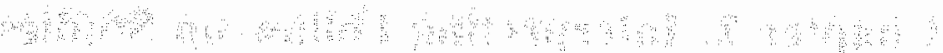




\subsection{Interpreting Follow-up Studies}

Follow-up studies in schizophrenia mainly serve the following two purposes:

- they shed light on the natural history of the disorder (natural history studies).

- they identify factors that modify the risk of chronicity and/or treatment response (prediction of outcome studies).

However, follow-up investigations are "dirty". A multitude of factors influence illness course, few of which can be collected, let alone controlled for, by the investigator. Results are therefore easily confounded. Course and outcome are highly dependent on sample selection and attrition, and both generalisability and validity of the findings may be jeopardised. No time is therefore wasted in trying to have an understanding of these factors.

\subsubsection{NATURAL HISTORY STUDIES}

Interpretation of the many natural history studies is often difficult because of a myriad of factors that are crucial in determining aspects of the results (Vaillant, 1978; Tsuang et al, 1979; Bleuler, 1972, 1978; Bland, 1982; Westermeyer \& Harrow, 1988; Bartko et al, 1988; McGlashan et al, 1988; Breier, 1988; Shepherd et al, 1989; An der Heiden \& Krumm, 1991; Harding, 1994).

\section{Defining the schizophrenia syndrome}

A recent review of the international literature, in which 320 follow-up studies from 1895 to 1992 were surveyed, found that outcome was significantly better in patients diagnosed according to systems with broad criteria or undefined criteria $(46.5 \%$ and $41.0 \%$ were "improved" respectively), as compared to systems with narrow criteria (27.3\% improved). A decline in the reported rate of favourable outcomes over the past decade was also ascribed to shifts in diagnostic criteria (Hegarty et al, 1994). This study highlights the fact that the results of all schizophrenia follow-up studies are highly dependent on the diagnostic construct used For example, follow-up studies using "intuitively" defined schizophrenia according to ICD-9 or the older "broad" concept of DSM-II, on average yield prognostically more favourable results than those using the more restrictive criteria of the later DSM-series (Westermeyer \& Harrow, 1984). The use of DSM criteria for schizophrenia in follow-up studies has been criticised (Angst, 1988), on the ground that they select a type of disorder that is already defined in terms of chronicity (ie the six months duration criterion). Thus, an element of circularity is introduced: poor outcome disorders have poor outcome. The use of different diagnostic criteria also has an important influence on the prevalence of important predictors of outcome, which, if results are presented uncontrolled, further complicates the picture. For example, the skewed sex distribution (excess of male patients) found in samples of subjects with "six-month" DSM-III/IV schizophrenia will

\footnotetext{
'Parts of this chapter will appear as: $J$. van $O s, P$. Wright and $R$. Murray: risk factors for emergence and persistence of psychosis: an empirical model of outcome heterogeneity. In. Baillière's Clinical Psychiatry: International Practice and Research (Eds M. Waller \& D. van Kammen). London: Baillière Tindall.
} 
be much attenuated in other, less restrictive, schizophrenia constructs (Castle et al, 1993). As female sex is associated with better outcome (see below), the relatively unfavourable outcome in DSM schizophrenia as compared to the ICD construct is likely to reflect, at least in part, sex differences in the course of schizophrenia.

Within the DSM series, there is likely to be further variation in course and outcome of the schizophrenia construct as criteria are being changed repeatedly. The influence of these changes on results of schizophrenia research has not been investigated, each new version of the DSM series being treated by many as the most "valid" update in psychiatric classification. The frequent boundary changes, however, are likely to have a subtle, but possibly important, influence on the comparability and generalisability of research results from one DSM generation to the other.

Of course, the real problem is that the validity of the variably defined schizophrenia construct remains uncertain. From the scientific point of view, it is unwise to start with the "splitting" approach if the diagnostic boundaries remain arbitrarily defined and are subject to frequent boundary changes. Indeed, an American commentator, reviewing the long-term outcome literature, concluded that "until significant progress is made in reducing the heterogeneity of schizophrenia, its criteria should en in the direction of inclusiveness" (McGlashan, 1988). A more useful approach, therefore, employed by several investigators, is to start out with a "lumping" approach, and to examine for interaction of effects with diagnostic category. For example, in the lowa 500 series, all cases of functional psychosis were included, and outcome was compared between various DSM-III categories (Tsuang \& Dempsey, 1979) which yielded evidence for an intermediary outcome for schizoaffective disorder, compared to schizophrenia and affective disorder. Another approach is to try to investigate, within a group of patients with functional psychosis, measures of course and outcome in relation to clusters of related symptoms (ie symptom dimensions), rather than clusters of cases (ie diagnostic categories). For example, it may be profitable to compare the predictive power of recently identified symptom dimensions (eg Liddle et al, 1992) with that of diagnostic categories.

The issues pertaining to diagnostic variation are summarised in figure 1. By including samples with a wide range of clinical expression of psychotic illness, the outcome variance found will be greater. The advantage of this approach is that within-sample clinical heterogeneity, in terms of either symptom dimensions or diagnostic categories, can be examined more easily than by starting out with narrowly defined cases of schizophrenia at the extreme end of the psychopathological "continuum". As research strategies are turming increasingly towards investigation of neural mechanisms of symptom dimensions (eg Liddle et al, 1992; McGuire et al, 1993), inclusion of more representative samples (ie over the whole continuum of psychosis) also appears the more rational approach.

Furthermore, in the previous chapter we demonstrated that quantitative, but not qualitative differences in outcome between acute, affective and insidious, non-affective psychotic disorders have been described. What appears most "valid" is a psychopathological continuum of one or more symptom dimensions. From the scientific point of view, a "lumping" approach in selecting cases for epidemiological research makes sense. 


\section{Assessing course and outcome}

Strictly speaking, the term outcome is misleading, as it implies that there is such a thing as an endpoint in the illness process, whereas in actual fact there is a continuous dynamic adaptation between individual, illness, and the environment. Thus, the endpoint implied by the term outcome refers to that of the study rather than the illness course. Here, outcome is used simply to denote the result of the research assessment that took place somewhere during the course of a patient's illness.

Fig. I Relationship berween diagnosis, outcome and sex. Restrictive diagnostic constructs of schizophrenia have less outcome variability, poorer average outcome and a higher male to female ratio than less restrictive constructs. Subtle changes in DSM diagnostic criteria affect outcome comparability across DSM generations.

\section{DIAGNOSTIC RANGE}

affective psychosis non-affective psychosis

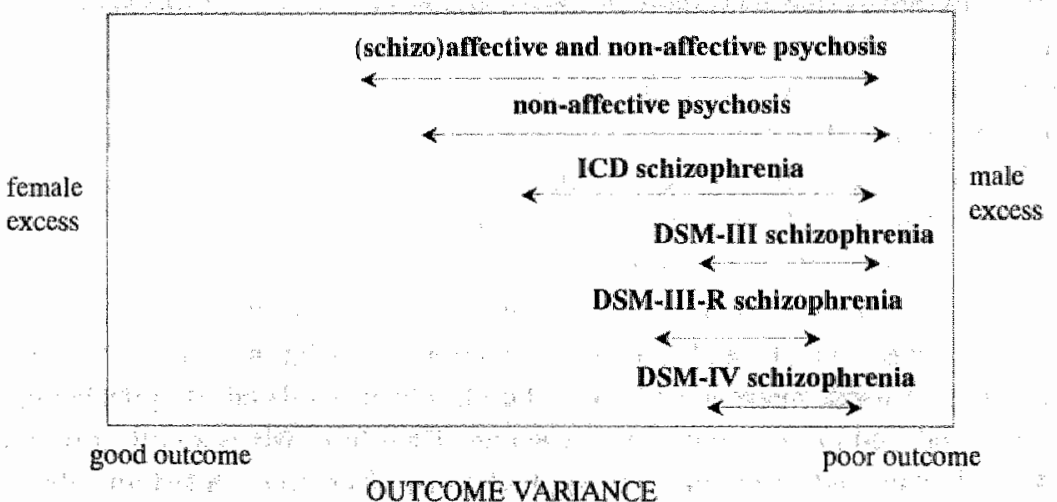

In general, outcome studies include a baseline assessment and one or more follow-up assessments. Ideally, the baseline assessment should precede the follow-up assessment, ie the study should be prospective. Similarly, serial assessments are desirable so as to be able to build an accurate picture of the patient's iliness course. For example, Coryell and colleagues followed a sample of patients over five years using biannual assessments (Coryell et al 1990). Very few studies, however, fulfill the criteria of prospective and serial assessments. For example, very long-term follow-up studies (with some exceptions - eg Bleuler, 1978), have to depend on retrospective baseline assessments, and serial assessments require complicated statistical analyses. Usually, studies report a baseline assessment and a single follow-up assessment, which consists of cross-sectional and/or retrospective longitudinal assessments. Cross-sectional assessments measure the patient"s current state during the follow-up interview, whereas retrospective longitudinal measurements rate, for example, the time spent in hospital or the usual severity of symptoms over a specified period prior to the interview.

In general, the longer the follow-up period, the less representative a single cross-sectional followup assessment is likely to be of the patient's illness course, just as a single measurement of temperature is unlikely to tell us much about temperature developments over the last 20 years. Longitudinal assessments have more validity with respect to illness course, but are heavily dependent on the quality of the source of information for the retrospective rating exercise, and 
the longer the follow-up period, the more contamination by error in the data. Follow-back assessments covering periods of more than 5 years are likely to be imprecise and require increasingly crude distinctions in order to give some degree of accuracy of variabillity in course. Instruments have been developed to rate course longitudinally, such as the WHO life chart; which offers a semi-operationalised assessment of social and clinical aspects of ilness course (WHO, 1992, see below). For both cross-sectional and Iongitudinal assessments, informant accounts are crucially important (McGlashan, 1984; An der Heiden \& Krumm, 1991).

It is recognised that outcome of psychiatric illness is multidimensional and difficult to conceptualise in one summary outcome measure (Brown, 1966, Kenniston et al, 1971, Strauss \&. Carpenter, 1978). Kenniston and colleagues (1971) drew attention to the fact that, in the same patient, outcome may vary sharply as a function of the outcome measure used: patients may continue to work in spite of severe psychotic symptoms, or alternatively they may be quite isolated while only presenting with mild residual paranoid ideas. Therefore, the use of "global" outcome measures combining different independent dimensions should be avoided. The usual approach has been to define "social" and "clinical" outcomes. Three caveats exist in the adoption of this approach. Firstly, if the outcome measures used are not based on validated instruments, the replicability of results will be jeopardised. Secondly, whille the multidimensional approach may reflect clinical reality, the scientific evaluation of putative risk factors for a deteriorated illness course is being made more complex, as multiple testing with a variety of outcome measures is necessary with the inherent problem of false positive results (Type I errors). Thirdly, the decision to define "socilal" and "clinical" outcomes is arbitrary, and when a variety of outcome measures is used, many are likely to be closely interrelated, despite being classified under various social and clinical outcome measures. Therefore, when a collection of outcome measures is used, data reduction may be applied to produce true underlying dimensions of outcome (Van Os et al, 1995a).

\section{Assessing "recovery" or "remission".}

The use of dichotomized terms such as recovery or remission is perhaps questionable. The psychotic illness process seen in clinical practice is more suggestive of fluctuation along a continuum of illness severity, in comparisons both between and within patients. It may therefore be more useful to rank patients on ordered categories of operational measures of illness severity, rather than force a decision about recovery by placing an arbitrary cut-off resulting in loss of information. Bleuler (1978) defined four levels of symptom severity: most severe, medium severe, less severe and cured. It should be noted that cured included those states where psychotic symptoms were still present, although only detectable on detailed psychiatric examination. In Bleuler's 23-year follow-up study, $20 \%$ of patients were classified as cured. Care should be taken to compare seemingly similar outcomes across studies. The Iowa 500 follow-up study (Tsuang et al, 1979) reported psychiatric status at 30-40 year follow-up as no symptoms, some symptoms and incapacitating symproms. The proportion of patients with no symptoms was $20 \%$, similar to Bleuler's study. However, in the Iowa 500 series a rating of no symptoms really meant absence of psychiatric symptoms, and certainly did not include patients with psychotic signs.

The latter example illustrates the limited comparability between studies. In general, measures of course and outcome tend to differ from study to study, and much would be gained if a "minimal 
set" (Marengo, 1994) of course parameters were used consistently in the literature lnstruments developed by the WHO (1992) provide semi-operational, multi-dimensional (social, clinical) measures for course and outcome, which have high face validity. For example the WHO (1992) modified Bleuler's four categories of symptom severity to give a rating of usual severity of symptoms over a limited period (eg two years). Similarly, definitions are provided for course type, which are rated as episodic, convinuous, neither episodic nor continuous and newer actively psychotic in this periad. (Table 1).

Table 1: Course type to be rated over a two-year follow-up period as defined in the WHO life chart (WHO, 1992)

episodic discrete episodes (none longer than 6 MONTHS) with clear periods of remission' in between: Ai least one remission lasted 6 months or more:

continuous - psychotic* over most of the period If any remissions, these were brief (none longer than 6 months).

neither episodic nor continuous - e.g. longest psychotic episode was 12 months and longest remission was 9 months.

never actively psychotic in this period

* definitions are also provided for "remission" and "psychotic"

Often measurements of service use are presented, such as time spent in hospital and number of readmissions, which are taken to reflect clinical course and illness severity. Such measures of service use are valuable for economic analyses, but it is not possible to disentangle the administrative, clinical and social factors that determine hospital use (Garmezy, 1970). Better measures include the time patients live independently, le outside institutions and supported accommodation (including care provided by relatives at home), or the relapse rate, regardless of readmission.

In a recent one year follow-up study, a combination of time to first remission and level of remission has been successfully used, using clinical trial instruments such as the Clinical Global Impressions scale (Lieberman et al, 1993). This group introduced a major improvement in outcome methodology, by introducing a standardised antipsychotic treatment protocol over the follow-up period, thus in fact controlling for treatment administered over the follow-up period (see below). However, the term "treatment response" to denote outcome variance (Liebeman et $a l, 1993$ ) is slightly misleading, as variation in outcome may onlly be partly related to variation in treatment response. For example, their finding that brain pathomorphology predicted level of remission might also have been found if no treatment had been administered at all. The separation of variation due to treatment response and to the underlying illness process itself would require the inclusion of a placebo-medicated control group, which is not feasible because of ethical reasons. 


\section{Length of follow-up}

The length of follow-up varies from one or two years (Johnstone et al, 1990; Jablensky et al, 1992, Lieberman et al, 1993) to two or more decennia (Ciompi \& Müller, 1976; Ciompi, 1980; Huber ef al, 1980, Harding et al, 1987; McGlashan, 1984. The greatest variability in illness is to be found in the first 5-15 years of illness (Strauss \& Carpenter, 1977, Bleuler, 1972, 1978), after which less fluctuation in course can be expected, although substantial changes very late in the course may not be infrequent (Bleuler, 1978; Ciompi \& Müller, 1976; Harding et al $l_{4}$ 1987). Furthermore, with the progression of time, a large group of patients becomes increasingly impaired with each subsequent episode; $35 \%$ of patients developed such states of increasing impaiment in the first five years of illness in a carefully conducted study of a sample of 49 representative incident cases of schizophrenia (Shepherd et al, 1989). Longer term studies suggest that this percentage further increases over the next 10 years, but McGlashan (1988) has pointed out that the deterioration then tends to "bottom out", and is in fact not infrequently followed by a phase of late improvement (Vaillant, 1978; Engelhardt, 1982; Harding et al, 1987; Ciompi \& Müller, 1976; Bleuler, 1972, 1978), This state of affairs is depicted in figure 2 after Breier and colleagues (1991; 1992).

It follows that it is of crucial importance to the results at what point in the illness course the cohort's baseline assessment takes place and for how long the patients are followed up. For example, a follow-up over five years during the "plateau" phase will reveal little fluctuation; a follow-up very late in the illmess may actually show improvement. It also follows that too large a fluctuation of length of follow-up and/or of duration of illness prior to baseline assessment is not to be recommended. If, for example, some patients are first admissions followed for 5 years and others, within the same sample, are patients with 10 year duration of illness who are followed for 15 years, the resulting mix of follow-up epochs and levels of chronicity will make it very difficult to meaningfully interpret the results unless the sample is stratified in subgroups of chronicity and length of follow-up.

Fig. 2 Hypothesized "common" illness course trajectory showing initial stepwise decline with each episode (1). followed by a "plateau" phase (2), and late improvement.

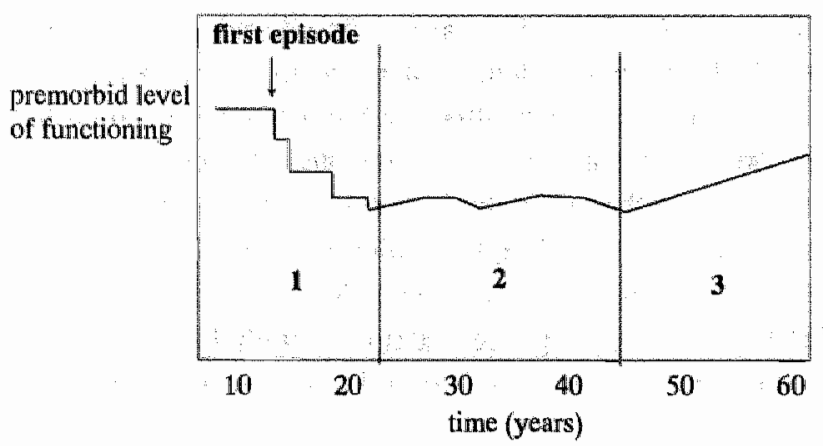

\section{Controls or no controls?}

It is often difficult to compare outcomes across studies, especially natural history studies. For example, being married, having friends or employment are all heavily dependent on the 
characteristics of the source population. If employment is included among the outcome measures (which it often is), than global outcome will be worse in a study population drawn from an area with high rates of unemployment. Furthermore, it is conceivable that longitudinal wariation in psychiatric symptomatology reflects similar, age-related variation in psychological well-being in the normal population. Therefore, results should ideally be compared with a group of psychiatric and non-psychiatric controls. One well-known study (Tsuang \& Dempsey, 1979) compared schizophrenic, schizoaffective and affective patients with a group of surgical controls. Thus, outcomes in psychiatric patients could be compared with population baseline values; the rate of "good" outcome in controls was between $84 \%$ and $89 \%$, depending on the outcome measure used. These issues are further illustrated in table two, which summarises some of the literature on long term outcome of first admissions (thus keeping constant length of illness), selected on the basis of criteria discussed above, between the beginning of the century and 1990. It appears that there are as many outcome measures as there are outcomes studies. Nevertheless, some important conclusions can be drawn:

- Whatever the year, place, length of follow-up, attrition rate and diagnostic criteria used, there is significant variability in long term outcome.

- Although there is significant variability, there is no "cure" for the majority of patients. Illness may often get progressively worse early in the course of the disorder; however, a substantial proportion of patients may experience some late improvement as discussed earlier.

- There is a high degree of mortality in patients, most of which, after the decline in tuberculosis earlier this century, is due to suicide - between $10-13 \%$ of all patients (Roy, 1982; Caldwell \& Gottesman, 1990).

\section{Sample selection and generalisability of results}

The vast majority of studies rely on hospital admission as a selecting criterion. In the case of schizophrenia, this means missing around $10 \%-20 \%$ of subjects who will never be admitted, and who might systematically differ on important variables associated with long term outcome. For example, female patients may be underrepresented, given that women have a less deterioratted illness course, and for that reason may be more often treated on an out-patient basis (see also Walker \& Lewine, 1994). Studies conducted in private hospitals select individuals from the higher socio-economic classes and/or particular ethnic groups, which also affects generalisability, and results from such institutions do not necessarily apply to all schizophrenic patients. Arbitrary age cut-offs may also jeopardise generalisability (eg Ciompi \& Muller, 1976; Jablensky et al, 1992).

The issue of generalisability is also important in relation to the length of illness prior to baseline assessment. Some long-term outcome studies followed-up patients whose baseline condition was of chronic, severe and persistent illness necessitating very lengthy periods of hospitalisation, residing in a rural area (eg Harding et al, 1987) or selected for high socio-economic status (eg McGlashan, 1984). The findings on the natural history of schizophrenia presented in such studies are only generalisable to similar populations selected for severe disability, area of residence or socio-economic status. For example, in Ciompi \& Muller's (1976) study of first admission patients with schizophrenia, followed for an average of 37 years, around $25 \%$ of 228 cases had an undulating course type and an outcome of mild or recovered. 


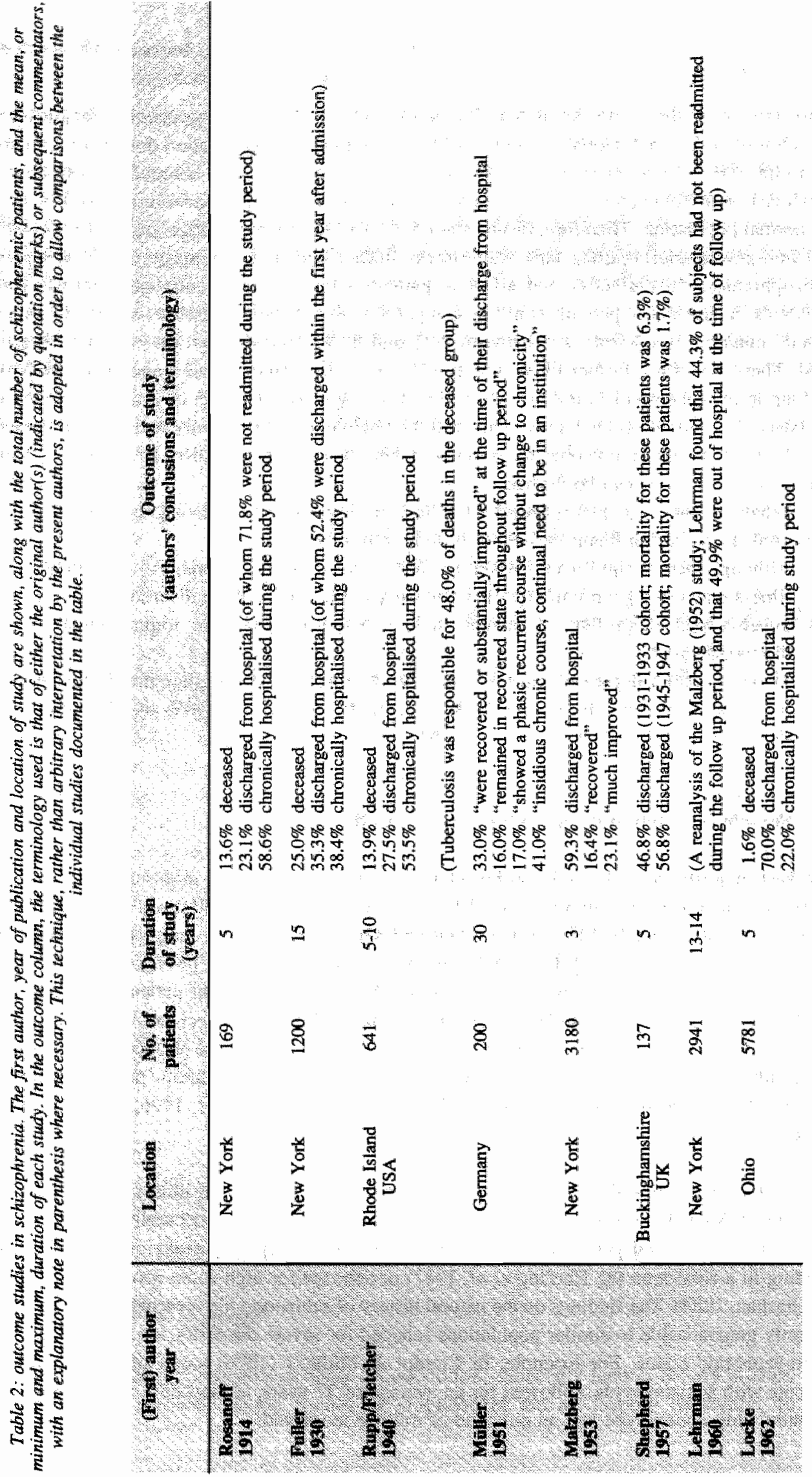




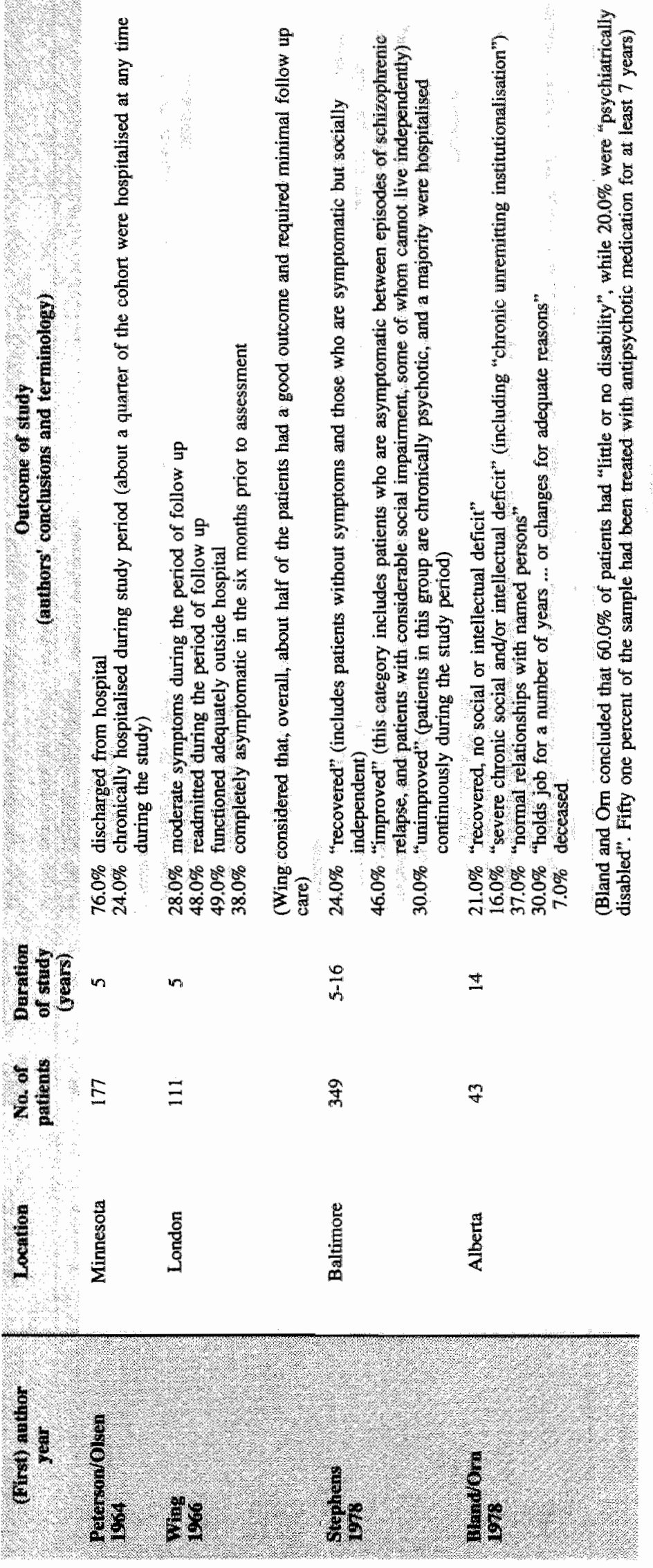


In the Vermont Longitudinal Research Project, however, which followed 82 schizophrenic patients who were already severely disabled at baseline for 20-30 years, this percentage was only $7 \%$. Conversely, the proportion of patients with a more chronic type of course with moderate or severe outcome were $10 \%$ and $38 \%$ in the Swiss and the American samples respectively (Harding, 1988).

In order to be representative, subjects should ideally be incident cases drawn from a defined geographical area. For example, the WHO Determinants of Outcome Study attempted to identify all cases of non -organic psychosis making a first lifetime contact with a helping agency in twelve areas, and followed 1379 such patients for a period of two years (Jablensky et al, 1992).

In any cohort study, whether retrospective or prospective, the ascertainment of outcome data requires that all subjects are followed into the future, to determine whether they develop the outcome of interest. The major source of bias in cohort studies is failure to obtain adequate information on every study participant, or to collect it in different proportions for those exposed and non-exposed to an important determinant of outcome. For example, differential attrition of individuals with insidious onset of illness and negative symptoms will result in a spuriously high proportion of recovered patients at follow-up; differential attrition of individuals of a particular social class or ethnic group may mean that results are only generalisable to a limited group of patients. To a degree, the likely effect of attrition can be investigated by comparing those who did and did not participate in the study on important baseline variables.

\subsubsection{PREDICTION OF OUTCOME STUDIES}

Prediction of outcome requires careful baseline assessment of predictor variables (exposures). Prospective studies are therefore preferable, with the exception of simple or immutable predictor variables such as age, sex and diagnosis, which can usually be assessed reliably retrospectively. Studies should be carried out blind to index data to minimise the risk of observer bias.

\section{Specific hypotheses versus general searches for predictors}

Studies on prediction of outcome may focus on specific risk factors for chronicity, such as brain abnormalities (eg Williams et $a l, 1985$ ), or try to find predictors among many variables collected at baseline (eg McGlashan, 1986a \& b). The first approach has some advantages. First, in this way a specific scientific question can be answered. Second, the study can be designed in such a way that information on essential confounders is simultaneously collected, and the most appropriate type of analysis, yielding adjusted estimates of effect size for the specific predictor can be conducted (Van Os et al, 1994, 1995 a \& b).

\section{Validity of results}

We discussed above the effect of attrition on the generalisabillity of results in follow-up studies establishing the natural history of disease. Although differential attrition of, for example, female patients may affect generalisability of the study, the study may still be valid for male subjects. 
In analytic cohort studies, however, where specific hypotheses on associations between predictor variables and outcome are tested, the validity of the results may be seriously affected by losses to follow-up if attrition is related independently to both the predictor variable and other risk factors for the outcome under study. Assume, for example, that male sex is associated with increased risk of braîn abnormality (Lieberman et al, 1992, Castle \& Murray, 1991). If, in a study examining the relationship between brain abnormalities and outcome, those lost to followup have i) more brain abnormalities, and ii) independent of any brain abnormality, better outcome because they are more often of female sex, than a biased estimate of the association between brain abnormality and outcome will result.

\section{Baseline associations or predictors of outcome?}

Risk factors for poor social or clinical outcome may in fact be repetitions of associations that were already evident at baseline; these should therefore where possible be adjusted for to assess the true prospective character of risk. For example, evidence of developmental delay in schizophrenic patients may predict high levels of negative symptoms at follow-up, but if a similar association was present at baseline assessment, the statement that developmental delay predicts subsequent negative symptoms (ie at follow-up) can only be verified by adjusting for baseline level of negative symptoms.

\section{Effect sizes}

Rarely are risk factors for poor outcome reported in terms of effect size and confidence intervals, yet if high risk groups are to be targeted for secondary prevention, this is obviously an important issue. The usual practice of reporting only $P$-values has its disadvantages, as $P$-values are dependent on both sample size and effect size, with the result that negative findings are difficult to evaluate. Therefore, it is preferable to report both a measure of effect and an informative measure to evaluate the role of chance such as the range within which the true magnitude of the effect lies with a certain probability (confidence interval).

Throughout this study, we will distinguish between risk and causality. These two terms are often confused, such that risk is equated with causality in the absence of reasonable evidence to support such claims. We will consider risk as a factuall indication of statistical association, and will speak of causality in terms of judgement, based on criteria as proposed by Hill (1965). Most of this work is on heterogeneity of risk, and we do not necessarilly equate that with aetiological heterogeneity. For example, the pleiotropic effects of one or more genes could well result in two illnesses with markedly different risk profiles, but the same underlying cause. In the absence of valid means to directly establish the cause of psychosis, examining heterogeneity in risk is a useful first approach towards the elucidation of the primary and secondary prevention of psychotic illnesses. These issues will be further discussed in the epilogue. 


\section{Confounding}

Altemative explanations need to be considered in reporting associations. For example, women are more likely to experience and/or report life events and, in schizophrenia, may be less likelly to have brain abnomalities (Bebbington et al, 1993, Castle \& Murray, 1991). Therefore, any association between life events or brain abnomalities on the one hand and course and outcome on the other may be confounded by sex. In fact, there is good evidence that sex, social class and ethnicity are all associated with outcome (Bardenstein \& McGlashan, 1990, Cooper, 1961; McKenzie et al, 1995). These three variables are likely to be associated also with many biological and social predictor variables, so that a potential confounding mechanism needs to be adjusted for (figure 3).

Fig. 3 Sex may comfound many predictor-outcome relationships. Depicted are two popular notions, namely that brain abnormalities and life events are associated with a more and a less deteriorated illness course respectively, However, both associations may be confounded by sex.
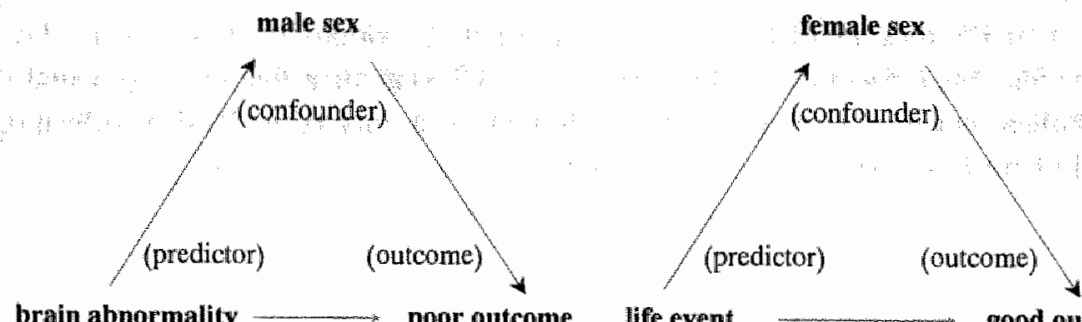

brain abnormality

poor outcome

life event

good outcome

Duration of illness before first treatment may predict poorer outcome (Crow et al, 1986; Jablensky et al, 1992; Loebel et al, 1992). However, none of the studies examining this issue were able to examine the effect of social support, social class or ethnicity (likely to be associated with decreased or delayed access to service and also associated with outcome). An often made mistake is to adjust for confounders only if statistical significance in the association with either the outcome or the predictor variable can be demonstrated. Confounders, however, should be selected and adjusted for a priori, on the basis of their potential confounding mechanism, not on statistical significance (Clayton \& Hills, 1993). As statistical significance is based on both effect size and sample size, smaller samples are paradoxically much less likely to suffer from confounding if the criterion is based on statistical significance!

In order to show the importance of confounding by basic demographic variables such as sex and social cllass, we will briefly review the outcome literature with regard to these two variables: sex and socio-economic status.

- sex - The issue whether or not sex is associated with outcome is of crucial importance (Seeman, 1986; Goldstein, 1988; Childers \& Harding, 1990). The sexes are usually combined in a single outcome distribution. There is, however, evidence that outcome may be different across the sexes in first or recent onset representative samples drawn from a defined area. In such 1-8 year follow-up studies, women have been found to, for example, have a more favourable course pattem, to live more often independently, be readmitted less, spend less time in a psychotic state, be less socially impaired at 
follow-up, and to spend more time in remission (Salokangas, 1983, Angermeyer et al, 1990, Shepherd et al, 1989, Jablensky et al, 1992, Navarro et al, 1995, Goldstein, 1988). The findings are consistent across cultures, and do not appear to be diagnosisspecific, or related to a particular diagnostic construct of schizophrenia (Navarro et al, 1995, Bardenstein \& McGlashan, 1990, Goldstein, 1988; Angermeyer ef al, 1990). Effect sizes range from men being nearly two times more likelly to have more than mild socilal impairment at follow-up and being half as likely to experience a complete remission (Shepherd et $a$, , 1989 , our calculation from data provided), being three times more likely to have a non-remitting illness course, and living independently $20 \%$ less over the follow-up period (Navamo et al, 1995).

The question arises whether the differences in outcome can be explained by a third variable associated both with sex and outcome, such as substance abuse, age of onset, premorbid adjustment and prevalence of brain abnormality (Castle \& Murray, 1991), or whether the differences are intrinsic to being female. "The lack of diagnostic specificity for the sex-outcome association points in this direction. For example, it may be that in the normal population the life course of men is similarly more often dysfunctional compared to women. If this is the case, then the divergence between the sexes in outcome distribution is immutable and, consequently, separate norms for each sex should be used in reporting outcomes, similar to immutable sex differences in lifeexpectancy at a given age. Alternatively, both intrinsic and confounding mechanisms may contribute to the sex differences in schizophrenia outcomes.

There is evidence that at least a proportion of the differences in outcome variance can be explained by a third variable. Angermeyer and colleagues (1990) reported that adjustment for age and marital status reduced the effect of sex, although it did not nullify it. Navarro et al (1995) specifically examined the issue of alternative explanations in a sample of 166 patients with recent onset functional psychotic illness, followed for an average of for 4 years. Women had better outcome, regardless of diagnosis. Adjustment for age of onset and diagnosis (females more often presenting with good outcome affective psychosis) reduced the associations between sex and a range of outcome measures by about $20 \%$, but did not nullify it: Adjustment for family history, obstetric complications and cerebral ventricle size assessed with $\mathrm{CT}$ scanning, did only marginally affect the association. Premorbid functioning did not reduce the association, concurring with Goldstein's (1988) finding that premorbid functioning reduced the association between sex and five-year outcome by not more than $13 \%$. The variable that explained most of the sex-outcome association was clinical expression of illness at baseline. Between $20 \%$ and $60 \%$ (depending on the outcome measure) of the sex-outcome associations was explained by dimensions of symptomatology identified by multivariate analysis (Van Os et al, 1995a), especially a dimension of negative symptoms and insidious onset of illness, on which women scored lower than men.

In summary, the finding that women have a less deteriorated illness course is consistent across cultures, diagnostic construct and measures of outcome used. Some of the variation in outcome can be explained by differences in anamnestic variables such as age of onset, marital status and premorbid adjustment, but also differences in 
clinical expression of the illness itself account for the divergent sex outcome distributions. It cannot be excluded that similar associations between sex and life course exist in the general population, associated with, for example, sex differences in emotional expression or socialisation. Thus, the question whether women have different ilness characteristics or whether being female confers a relative protection againgt deteriorated life course in sickness and in health must be resolved by controlled studies. Whatever the reason, the association of sex with both outcome and with many of the other exposures used to predict illness course implies that confounding by sex should be adjusted for in many instances when testing hypotheses about biological and social predictors of outcome.

- socio-economic status - Lower social class appears to be associated with poorer outcome in schizophrenia. Cooper (1961), followed a sample of 219 first admission cases within a defined geographical area in the UK and examined various measures of course and outcome in relation to social class. There was a linear trend in the association between social class and time spent in hospital in the first two and five years of illness Patients of lower social class were less likely to recover, less likely to respond to rehabilitation, and more likely to be readmitted. Similar findings emerged form a large study in the USA (Myers \& Bean, 1968). Three decennia later in the UK, Mckenzie and colleagues (1995) reported similar findings in the Camberwell Collaborative Psychosis Study which followed 166 subjects of recent onset psychosis over four years. These authors found that, over three levels of socioeconomic status, the risk of non-remitting illness over four years follow-up increased by a factor 2.4 with each level of social class; the risk of non-recovery over the follow-up period similarly increased with a factor 2 . In this study, the association between social class and outcome was not diagnosis-specific within the functional psychoses. Indeed, similar findings have been reported in a sample of 248 bipolar patients (OConnell et al, 1991).

Given the public health importance of an association between class and outcome, it is surprising that there has been a great decline in interest in this issue over the last 15 years (Cohen, 1993). Furthermore, socio-economic status is likely to confound many associations between outcome and social or biological exposures, yet its effect is rarely controlled for.

\section{Treatment}

Treatment is an important factor in prediction of outcome studies. In follow-up studies which examine the natural history of the disorder, treatment may have an important influence on the results. "Treatment is a major uncontrolled variable in any "naturalistic"' study, but some (Keller et al, 1983) have argued cogently that treatment becomes an outcome in itself, as it is prescribed in response to the patient's condition and the severity of the underlying illness process, which may not be fundamentally altered by any treatment. In follow-up studies which examine prediction of outcome, the influence of treatment is only important if it is associated both with outcome and, independently, with the exposure under investigation. This may occur under two conditions: i) clinicians prescribe treatment differentially, and ii) patients respond to/comply with 
treatment differentially. This, however, is unlikely. First of all, clinicians are often not aware of life events as elicited by careful research interviews (or cerebral ventricle dimensions as assessed by CT-scan or premorbid adjustment as measured by the Premorbid Adjustment Scale), and secondly, clinicians (fortunately) tend to prescribe according to the patient's actual need rather than their expectations as to prognosis. For example, in the case of two patients with a similarly severe illness, the clinician would not discharge the one patient who had experienced a life event and keep the other in hospital. Both patients would be discharged only if they had recovered sufficiently. With regard to the second argument that treatment response or treatment compliance would vary with the degree of exposure to a risk factor, this is not really an alternative explanation, but would merely show the mechanism by which the risk factor influences outcome. In other words, treatment response/compliance would lie on the causal pathway between exposure and outcome. In the studies of this thesis, which were carried out in a maturalistic setting with treatments being administered in a uniform way in one catchment area over a fouryear period, we follow Keller (1983) in considering treatment as an outcome variable, rather than one determining outcome. 


\section{REFERENCCS}

Angermeyer M, Kuhn, L. \& Goldstein, JM. (1990) Gender and the course of schizophnenia: differences in treated outcomes. Sichizophrenu Bulletin, 16, 293-307.

Angst, J. (1988) European long-tem follow-up studies of schizophrenia. Schzophrena Bulletin, 14, $501-513$.

An der Heiden, W, K Kumm, B. (1991) The course of schizophrenia same remarks on a yet unsolved problem of retrospective data collection, European Archives of $P$ sychiany and Clinical Neurasciences, 240,303-306,

Bandenstein, K, MoGlashan, T (1990) Gender differences in affective, schizoaffective, and schizophrenic disorders. A review Schizophrenta Research, 3, 159-72.

Bartko, J, Carpenter, W, \& McGlashan, T. (1988) Statistical issues in long-term followup studies. Schizophrenia Bulletin, 14,575-587

Bebbington, P., Wilkins, S, Jones, P. B., Foerster, A., Murray, R, Toone, B \& Lewis, S, (1993) Life events and psychosis. Initial results from the Camberwell Collaborative Psychosis Study. British Journal of Psychiatry, 162, 72-79.

Beiser, M, Iäcono, W. G, Enckson, D. (1989) Temporal stability in major mental disorders. In: The walidity of psychiatric diagnosis (Eds. Robins LN, Barrett JE) New York: Rawen Press, pp. $77-98$.

Bland, R.C. \& Om, $\mathrm{H}$, (1978) 14 year outcome in early schizophrenia. Acta Psychiatrica Scandinavica, 58, 327. 338.

Bland, R.C. (1982) Predicting the outcome in schizophrenia. Canadian Journd of Psychiatry, 27, 52-62.

Bleuler, M. (1972), Die Schizophrenen Geistesstörungen im lichte langjäriger Kranken - und Familiengeschichten. Stuttgart: G. Thieme.

Breier, A. Small sample studies: unique contribution for large sample outcome studies. Schizophrenia Bulletin, 14, 589-593.

Breier, A., Schreiber, J.L., Dyer, J. et al (1992) Course of illness and predictors of outcome in chronic schizophrenia: implications for pathophysiology. British Journal of Psychiatry, 161 (suppl. 18) 38-43.

Brown, G.W., Bone, M., Dalison, B. \& Wing, J.K. (1966) Schizophrenia and social care. London: Oxford University Press.

Caldwell, C.B. \& Gottesman, I.I. (1990) Schizophrenics kill themselves to: a rewiew of risk factors for suicide. Schizophrenia Bulletin, 16, 571-589.

Castle \& Murray, R. (1991) The neurodevelopmental basis of sex differences in schizophrenia. Psychalogical Medicine, 21, 565-575.

Castle, D., Wessely, S. \& Murray, R (1993). Sex and schizophrenia: effects of diagnostic stringency, and associations with premorbid variables. British Journal of Psychiatry, 162, 658-664.

Childers, S. \& Harding, C.M. (1990) Gender, premorbid functioning, and long-term outcome in DSM-III schizophrenia. Schizophrenia Bulletin, 16, 309-318.

Ciompi, L. \& Müller, C. (1976) Lebensweg und Alter der Schizophrenen. Eine katamnestische Langzeitstudie bis ins Senium. Berlin-Heidelberg-New York: Springer.

Ciompi, L. (1980) The natural history of schizophienia in the long term. British Jownal of Psychiatry, 136, 413420.

Clayton, D. Hills, M. (1993) Confounding and standardization. In: Statistical Modełs in Epidemiology, pp 133140.

Cohen, C.I. (1993) Powerty and the course of schizophrenia: implications for research and policy. Hospital and Community Psychiatry, 44, 951-958.

Cooper, B. (1961) Social class and prognosis in schizophrenia. Part I and Part II. Britisti Jourmal of Preventive and Sacial Medicine. 15, 17-41.

Coryell, W., Endicott, J. Keller, M. (1990) Outcome of patients with chronic affective disorder: a five year follow-up American Jourmal of Psychiatry, 147, 1627-1633.

Crow, T.J., MacMillan, J.F., Johnson, A.L. \& Johnstone, E.C. (1986) The Northwick Park study of first episodes of schizophrenia. II: A randomised controlled trial of prophylactic neuroleptic treatment. British Journal of Psychiatry, 148, 120-127.

Engelhardt DM, Rosen B, Feldman J, Engelhardt JAZ (1982) A 15 year follow up of 646 schizophrenic outpatients. Schizophrenia Bulletin, 8, 493-503.

Fuller, R. G. (1930) Expectation of hospital life and outcome for mental patients on first admission. Psychiatric Quarterly, 4, 295-323.

Garmezy, N. (1970) Process and reactive schizophrenia: some conceptional issues. Schizophrenia Bulletin, 1, 3074.

Gift, T.E., Strauss, J.S., Kokes, R.F.y Harder, D.W. Ritzler, B.A. (1980) Schizophrenia: affect and outcome. American Joumal of Psychiatry, 137, 580-585. 
Goldstein, J M, (1988), Sex differences in the course of schizophrenia, Amertican Jow nal of Psychiatry, 145, 684689

Grossman, L, Harrow, M, Goldberg, \& \& Fichtner, C (1991) Ontcome of schizoaffective disorder at two longtem follow-ups comparisons with outcome of schizophrenia and affective disorders. American Jownal of Psychiatry, 148,13591365 .

Haas, GL, Glick, ID, Clarkin, I.F, Spencer, IH, Lewis, A.B. (1990). Sex and schizophrenia outcome: a clinical trial of an inpatient family intervention. S6hizophenia Bulletin, $16,277-92$.

Harding, C.M., Brooks, G.W. Ashikaga, T, Strauss, J, Breier A. (1987) The Vermont longitudinal study of persons with severe mental illness, I methodology, study sample and overall status 32 years later. American Jounal of Psychiatry, $144,718-726$.

Harding, C.M (1988) Course types in schizophreniat an analysis of European and American Studies. Schizophremia Bullerin, 14, 633-642.

Harding C.M. 1994, An examination of the complexities in the measurement of recoivery in severe psychiatic disorders. In: Schizophrenia. Exploring the Spectrum of Psychosis (pp 153-170). New York: Wiley \& Sons.

Hegarty, ID, Baldessarini, $\mathbb{R}$. Tohen, $M$, Waternaux, C. \& Oepen, $G$. (1994). One hundred years of schizophrenia: a meta-analysis of the outcome literature. American Jowrnal of PSychiatry, 151, 1409-1416.

Hill, A. (1965) The environment and disease:association or causation? Journal of the Royal Sochety of Medicine, $58,295-300$

Huber, G., Gross, G., Schüttler, R. \& Linz, M. (1980) Longitudinal studiss of schizophrenic patients. Schizophrenia Bulletin, 6, 592-605.

Jablensky, A., Sartorius, N., Ernberg, G. et al (1992) Schizophrenia: manifestations, incidence and course in different culture. A world health organizaiton ten-country study. Psychological Medicine, suppl. 20.

Johnstone, E.C., MacMillan J.F., Frith, C.D., Benn, D.K. \& Crow, T.J. (1990) Further investigation of the predictors of outcome following first schizophrenic episodes. British Journal of Psychilatry, 157, 182189.

Kane, J.M., Rifkin, A, Quitkin, F. Nayak, D. \& Ramos-Lorenzi, J. (1982) Fluphenazine vs, placebo in patients with remitted, acute first episode schizophrenia. Archiwes of General Psychiatry, 39, 70-73.

Keller, M.B., Lavori, P.W., Endicott, J, Coryell, W, Klemian, G.L. (1983) "Double depression": two-year follow-up. American Journal of Psychiatry, 140, 689-694,

Kenniston, K, Boltex, S \& Almond, R. (1971) Multiple criteria of treatment outcome. Journal of Psychiatry Research, 8, 107.

Lehrman, N.S. (1960) A state hospital population five years after admission: a yardstick for further evaluative comparison of follow up studies. Psychiatric Quarterly 34,658-681.

Liddle, P., Friston, K., Frith, C., Hirsch, S, Jones T. \& Frackowiak, R. (1992) Patterns of cerebral blood flow in schizophrenia. British Journal of Psychiatry, 160, 179-186.

Lieberman, J., Jody, D., Geisler, S., Vital-Herne, J., Alvir, I.M., Walsleben, J. \& Woemer, M.G. (1989) Treatment outcome of first episode schizophrenia. Psychopharmacology Bulletin, 25, $92,96$.

Lieberman, J.A., Alvir, J. Woerner, M. Degreef, G, Bilder, R.M., Ashtari, M. et al (1992) Prospective study of psychobiology in first-episode schizophrenia at Hillside hospitall. Schizophrenia Bullevin, 18, 351-371.

Lieberman, J., Jody. D., Geisler, S., Alwir, J., Loebel, A., Szymanski, S, et al (1993) Time courre and biologic comelates of treatment response in first episode schizophrenia. Archives of General Psychiatry, 50, 369-376.

Locke, B.Z. (1969) Outcome of first hospitalisation of patients with schizophrenia. Public Health Reports, 77, $801-805$.

Malzberg, B. (1953) Rates of discharge and rates of mortality among first admissions to the New York civil state hospitals (3nd paper). Mental Hygiene, 37, 619-654.

Marrengo, J. (1994) Classifying the courses of schizophrenia. Schizophrenia Bulletin, 20, 519-535.

McGlashan, T. (1984) The Chestnut Lodge follow-up study L. Follow-up methodology and study sample. Archives of General Psychiatry, 41, 573-585.

McGlashan, T. (1986a) Predictors of shorter-, medium- and langer-term outcome in schizophrenia. American Jowrnal of Psychiatry, 143, 50-55.

McGlashan T.H. The prediction of outcome in chronic schizophrenia. IV (1986b) The Chesnut Lodge Follow-up study. Arch. Gen. Psychiatry 43, 167-176.

MoGlashan; Carpeniter, W. \& Bartka, J. (1988) Issues of design and methodology in long-term follow-up studies. Schizophrenia Bulletin, 14, 569-57.4.

McGlashan, T.H. \& Bardenstein, K.K. (1990) Gender differences in affective, schizoaffiective, and schizophrenic disonders.

Schizophrenia Bulletin, 16, 319-29.

McGlashan, 1988. A selective review of recent North American long-term follow-up studies of schizophrenia. Schizophrenia Bulletin, 14, 515-541. 
McGuire, P.K., Shah, G.M. Murray, R.M. (1993) Increased blood flow in Broca's area during auditory hallucinations in schizophrenia. Lancet ii, 703-6.

McKenzie, K., Van Os, J., Fahy, T., Jones, P., Harvey, I. Toone, B., \& Murray, R.M. (1995) Evidence for good prognosis psychosis among UK Afro-Caribbeans. British Medical Journal, in press.

Muller, V. (1951) Katamnestiche Erhebungen über den Spontanverlauf der Schizophrenie. Monatsschrift für Psychiatrie-Neurologie, $122,257-276$.

Myers, IKK Bean, LL (1968) A decade later a follow-up of social class and mental illness. New York: Wiley:

Navarro, F., Van Os, J., Jones, P. \& Murray, RM. (1995) Explaining sex differences in outcome in the functional psychoses (submitted manuscript).

O'Connel, Mayo, J., Flatow, L., Cuthbertson, B, \& O'Brien, B.E. (1991). Outcome of bipolar disonder on longterm treatment with lithium. British Journal of Psychiatry, 159, 123-129.

Peterson, D.B. \& Olson, G.W. (1964) First admitted schizophrenics in drug era Archives of General Psychiarry, $11,137-144$ :

Rabiner, C., Wegner, J. \& Kane, J. (1986) Outcome study of first episode psychosis, I: relapse rate after 1 year. American Journal of Psychiatry, 143, 1155-1158.

Regier, D.A., Myers, J.K., Kramer, M. et al (11984) The NIMH epidemiologic catchment area program: historical context, major objectives and study population characteristics. Archives of General Psychiatry, 41, 934-941.

Rosanoff ${ }_{i}$ A.J. (1914) A statistical study of prognosis in insanity. Journal of the American Medical Association, $62,3-6$.

Roy, A. (1982) Sücide in chronic sehizophrenia. British Journal of Psychiatry, 141, 171-177.

Rupp, C. \& Fletcher, E.K. (1940) A five to ten year follow up studly of 6411 schzzophrenic cases. American Journal of Psychiatry, 96, 877-888.

Salokangas, R.K.R. (1983) Prognostic implications of the sex of schzophrenic patients. British Journal of Psychiatry, 142, 145-151.

Salokangas, R.K.R.,\& Stengard, E. (1990). Sex and short-term outcome in schizophrenia. Schizophrenia Research, 13, 333-345.

Scottish Schizophrenia Research Group (1989) The Scottish first episode schizophrenia study: V. Two year follow up. Acta Psychiatrica Scandinavica, 80, 597-602.

Seeman, M.V. (1986). Current outcome in schizophrenia: women vs men. Acta Psychiatrica Scandinavica 73, 609-617.

Shepherd, M. (1957) A study of the major psychoses in an Englisht county. London: Oxford University Press.

Shepherd, M., Watt, D., Falloon, I., et al (1989) the natural history of schizophrenia: a five year follow-up study of outcome and prediction in a representative sample of schizophrenics. Psychological Medicine, monograph suppl 15.

Stephers, J., Astrup, C., Mangrum, J.C. (1966) Prognostic factors in recovered and deteriorating schizophrenics. American Journal of Psychiatry, 122, 1116-1121.

Stepheris, J. (1978) Longterm prognosis and follow up in schizophrenia. Schizophrenia Bulletin, 4, 25-47.

Strauss, J.S. \& Carpenter, W. (1977) Prediction of outcome in schizophrenia III: five-year outcome and its predictors: Archives of General Psychiatry, 34, 159-163.

Strauss, J. S. Carpenter, W. (1978) The progmosis of schizophrenia: rationale for a multidimensional concept. Schizophrenia Bulletin, 1978, 56-67.

Teschke, G.C. (1978) Die Schizophrenen Geistesstörungen im lichte langjäriger Kranken - und Famillengeschichten by Manfred Bleuler. Schizophrenia Bulletin, 4, 48-55.

Tsuang, M.T. \& Dempsey, G.M. (1979) Long term outcome of Major Psychoses II. Schizoaffective disorder, compared with schizophrenia, affective disorders, and a surgical control group. Archives of General Psychiatry, 36, 1302-1304.

Tsuang, M.T., Woolson, R.F., Fleming, J.A. (1979) Long term outcome of major psychoses. Archives of Gieneral Psychiatry, 36, 1295-1301.

Vaillant, G.E. (1964) Prospective prediction of schizophrenic remission. Archives of General Psychiatry, 11, 509518.

Vaillant, G.E. (1978) Prognosis and the course of schizophrenia. Schizophrenia Bulletin, 4, 20-24.

Van Os, J., Fahy, T., Bebbington, P., Jones, P., Wilkins, S., Sham, P. et al. (1994) The influence of life events on the subsequent course of psychotic illness. Psychological Medicine, 24, 503-513:

Van Os, J., Falhy, T., Jones, P., Harvey, I., Sham, P., Lewis, S., Williams, M., Toone, B \& Murray, R. (1995) Psychopathological syndromes in the functional psychoses: associations with course and outcome.

Psychological Medicine, in press. 
Van Os, J. T. Fahy, P. Jones, I. Harvey, S. Lewis, M. Williams, B. Toone, R. Murray (1995b). Increased intracerebral CSF spaces predict unemployment and negative symptoms in psychotic illness: a prospective study. British Journal of Psychiatry, 166, 150-159.

Walker, E.F. \& Lewine, R.J. (1993) Sampling biases in studies of gender and schizophrenia. Schizophrenia Bulletin, 19, 1-7

Westermeyer, J.F. \& Harrow, M. (1984) Prognosis and outcome using broad (DSM-II) and narrow (DSM-III) concepts of schizophrenia. Schizophrenia Bulletin, 10, 624-637.

Westermeyer, J.F. \& Harrow, M. (1988) Course and outcome in schizophrenia. In: Handbook of Schizophrenia (Eds. M.T. Tsuang \& J.C. Simpson), Ratterdam: Elseviers:

Williams, A.O. Reveley, M.A., Kolakowska, T., Andem, M. \& Mandelbrote, B. (1985) Schizophrenia with good and poor outcome. II. Cerebral ventricular size and its clinical significance. British Journal of Psychiatry. $146,239-246$.

WHO (1992) WHO coordinated multi-center study on the course and outcome of schizophrenia. WHO: Geneva.

Wing JK (1966) Fiwe year outcome in early schizophrenia. Proceedings of the Royal Society of Medicine, 59, $17-18$.

World Health Orgamization (1979) Schizophrenia: an International Follow-up Study. Chichester: Wiley. 
a * :

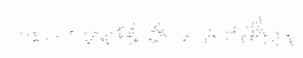

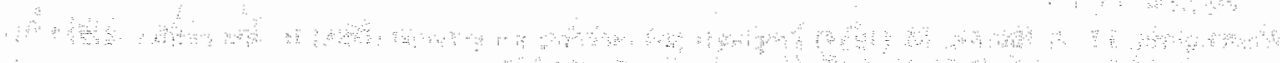

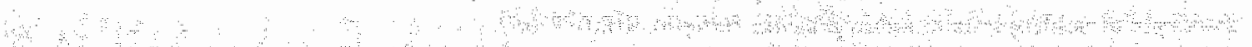

and

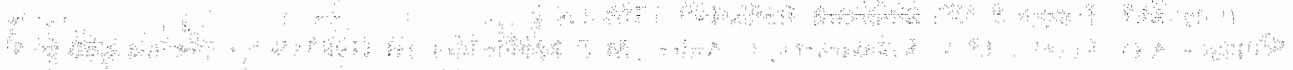
se " a $\therefore$ a

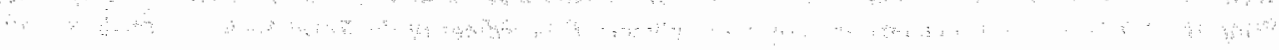

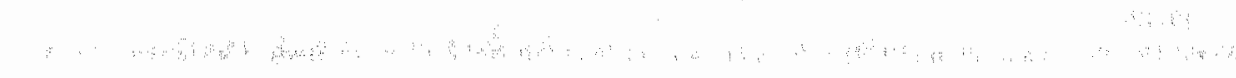


Chapter 3: Psychopathology as a risk factor for persistence of psychotic illness; predictive validity of categorical and dimensional representations 


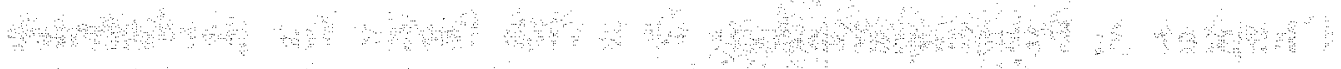

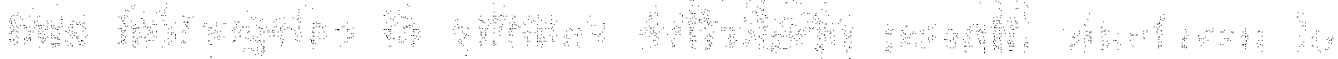

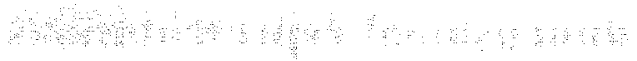




\subsection{Psychopathological syndromes}

In the functional psychoses: associations with course and outcome

\section{SUMMMARY}

The aim of this study was to identify underlying dimensions of psychopathology in a cohort of patients with functional psychosis of recent onset, and to examine their prognostic value. Factor analysis of the psychopathological features of 166 consecutively admitted patients with functional psychosis of recent onset revealed seven psychopathological dimensions, explaining $63 \%$ of the variance. Five of these seven syndromes bore differential associations with subsequent treatment and illness course, independent of $i$ ) associations with DSM-IIIR diagnosis, ii) associations with other prognostic factors and iii) associations with the baseline values of outcome variables. The most striking associations were shown for an early and insidious onset syndrome with affective flattening, which predicted a more disabled course of illness on 3 of 4 outcome dimensions, and which was commoner in males and single individuals. A second syndrome, characterised by bizarre behaviour, inappropriate affect, catatonia, and poor rapport showed similar, slightly less striking, associations with illness course as well as with poor premorbid social functioning. A third syndrome, characterised by positive psychotic symptoms was to a lesser degree associated with poorer outcome, whereas a fourth syndrome distinguished by manic symptomatology predicted a more benign illness course. A fifth syndrome identified by lack of insight predicted more time in hospital and admission under a section of the Mental Health Act over the follow-up period.

A further finding was that dimensional representations of psychopathological features were considerably more useful than categorical representations (DSM-III-R and ICD 10) as predictors of illness course and treatment decisions.

\section{INTRODUCTION}

The categorical approach to psychiatric diagnosis ignores the fact that considerable overlap may exist between different diagnostic categories. It has been suggested (Liddle, 1987a; Hirsch, 1991) that examination of more restricted psychopathological syndromes which spread over several diagnostic groups may be a more profitable approach for research, and cumulative evidence from published findings indicates that at least three dimensions of psychopathology may characterize schizophrenia (eg Liddle, 1987a; Mortimer et al, 1990; Peralta et al, 1992).

Psychopathological syndromes, usually identified through multivariate statistical techniques, require external validation. Studies relating subsets of clinical phenomena to neuropsychological (Liddle 1987b, Brown \& White, 1992) and biological (Liddle et al, 1992) variables have provided some support for the validity of a three syndrome construct in schizophrenia. However, the interpretation of cross-sectional associations between psychopathological dimensions and 
other variables is difficult, especially as regards the temporall relationship between two variables in samples of mostly chronic schizophrenic patients. It is therefore desirable to relate dimensions of psychopathology prospectively to follow-up data. Such an approach may also be helpful in clarifying to what degree psychotic symptoms are predictive of subsequent course and outcome of illness, as published findings are generally inconsistent. While a case could be made for negative symptomatology as a modest predictor of outcome in schizophrenia (Pogue-Geile, 1989; Fenton \& McGlashan, 1991), the issue of whether the same applies to other symptoms is still unresolved. For example, some studies found a positive association with outcome for some Schneiderian symptoms, and a negative association for others (Bland \& Orn, 1980, Bland, 1982). Other studies failed to find any effect of more typical positive schizophrenic symptoms on outcome (Carpenter et al, 1978; Pope \& Lipinski, 1978; Brockington et al, 1978; Shepherd et al, 1989, Fenton \& McGlashan, 1991), whereas yet others did find an effect indicating poorer outcome (Stephens \& Astrup, 1963; Cloninger et al, 1985; Huber et al, 1980; Prentky et al, 1980; Kay \& Murrill, 1990; Endicott et al, 1986; Kendell et al, 1979; Leff et al, 1992). Similarly, in spite of the traditional attribution of better prognosis in the presence of an index depressive component (reviewed by McGlashan, 1988), some well known studies have reported informative null findings (Shepherd et al, 1989, Carpenter et al, 1978).

Recent work on symptom dimensions has examined samples of schizophrenic patients , but in view of the doubtful validity of current diagnostic criteria (Costello, 1992), it may be useful to study a sample of patients selected on the sole basis of the presence of psychotic symptoms, so as to avoid having to make initial assumptions about the relationship between symptoms and signs.

The choice of symptoms to be included in the multivariate analyses used to identify psychopathological dimensions is crucial. The outcome of factor analytical procedures using concept-driven instruments like the SANS and the SAPS will resemble these underlying concepts, especially if other symptoms that form an integral part of schizophrenia, such as affective symptoms, are excluded. A range of signs and symptoms should therefore be included.

In this study, we wished to examine prospectively the possible association between psychopathological syndromes and i) course and outcome of psychotic illness, and ii) treatment variables over the follow-up period, using a range of signs and symptoms to define psychopathological dimenisions and using a range of contemporary diagnostic categories of psychotic illness. We also wished to explore whether any observed association with course and outcome could be explained by i) associations between psychopathological syndromes and baseline values of outcome variables (eg an association between a psychopathological syndrome and unemployment at follow-up could disappear after adjustment for unemployment at baseline), and ii) associations with known predictors of outcome, such as level of premorbid functioning, marital status and gender, hypothesizing that psychopathological syndromes would be associated with outcome measures independently of these.

We further wished to examine whether a dimensional representation of psychotic symptomatology would prove a more powerful predictor of outcome than current categorical classification systems (DSM-IIIR and ICD-10), hypothesizing that the former would do better than the latter. 
It is now customary to measure outeome of illness along different dimensions, and some previous studies have relied on outcome dimensions based on cross-sectional measures at followup (Strauss \& Carpenter, 1978). However, many chance events may influence cross-sectional findings. We therefore included measures of both (longitudinal) course and (cross-sectional) outcome": in order to construct dimensions of illness course and outcome.

\section{METHOD}

\section{Cohort and baseline assessments}

A cohort of 337 consecutive admissions (including a small proportion of out-of-catchment-area tertiary referrals) to two South London hospitals, with at least one psychotic symptom according to the RDC (Spitzer et all, 1978) occurring in clear consciousness, were extensively investigated, and those that had had onset of illness within 5 years (mean:2.2 years) of index assessment. $(n=191)$ were followed up after 4 years (mean length of follow-up: 47 months ; S.D $=14.0$; with deaths excluded: $S . D .=12.5$ ).

Baseline assessments were generally completed within three days of admission and included the Present State Examination (Wing et al, 1974), and demographic ratings. Socio-economic status at birth or during early childhood was assessed using the Registrar General's classification of paternal occupation. Ethnicity was classified as white European, Afro-Caribbean, or other ethnic group.

Age at onset was defined as appearance of first symptoms of psychosis, and duration of illness: was defined as the difference between age at index assessment and age of onset. Premorbid adjustment was measured with the Philips scale social adjustment section (1953), based on maternal interviews, conducted by a rater who was blind to the proband's characteristics including diagnosis. The Phillips scale is a simple 7 point ordered categorical scale ranging from "a leader...." to "no desire to be with playmates, peers...from early childhood". "The Operational Criteria Checklist for Psychotic Illness (OCCPI; McGuffin et al, 1991) was completed for all patients for the time up to index assessment to give index diagnosis. The OCCPI check-list is based on phenomenological descriptions in the Present State Examination, and includes signs and symptoms used in a wide range of operational definitions of psychiatric conditions (ICD, RDC, DSM-IIIR, etc). The computer program OPCRIT (McGuffin et al, 1.991) was used to analyze the OCCPI data. Psychopathological data were derived from detailed cross-sectional mental states at index assessment, on the basis of the PSE, and from the patients' case records. PSE interviews had been conducted by three experienced clinicians who had received PSE-training at the same centre. Thus, psychopathology ratings used in the analyses (see below) were derived from both cross-sectional interview and more longitudinal case-note assessments (Brockington et al, 1992).

\section{Follow-up}

The following instruments were used to provide cross-sectional outcome measures: Global Assessment of Functioning-symptomatology (GAF-s; WHO 1992), derived from the Global Assessment Scale (Spitzer et al, 1975), WHO/Disability Assessment Schedule (DAS; Jablensky,et 
al, 1980), Iager Negative Symptom Rating Scale (Iager, 1985), Hamilton Depression Scale (HAMD; Hamilton, 1960) and the PSE.

To assess course of illness and associated disabilities longitudinally, we used a slightly modified version of the Life Chart instrument from the Multi-Center Study on the Course and Outcome of Schizophrenia (WHO, 1992). The life chart assesses course of illness in a number of ways, using clear definitions for all ratings. Course type was rated as episodic (no episode longer than 6 months), continuous (no remission longer than six months), neither episodic nor continuous, and not psychotic in this period (Appendix). Type of remission was coded as "mainly complete", "mainly incomplete" and "mixed". A "usual severity of symptoms" rating was made to indicate the symptomatic level of the patient during most of the follow-up period. Ratings were severe (1), moderate (2), mild (3) or recovered (4). The amount of time spent in a psychotic state was also rated, as were parasuicidal acts and instances of assault. A rating was also given as to whether there had been clear evidence of negative symptoms (as defined by the lager scale) over the follow-up period. In addition, the life chart rates the proportion of the follow-up period spent unemployed (time in institutions not counted; full time students and full time housewives were rated as employed), living independently, in hospital, in prison (of any duration, including being held in custody at the police station), or without accommodation ("vagrant").

Data on psychological and pharmacological interventions over the follow-up period were also obtained. Psychotherapy was defined as having regular sessions with a mental health worker, dedicated specifically to treatment with psychotherapeutic techniques.

Variables measuring time, for example, time spent in hospital, time unemployed, etc, were expressed as the proportion of the length of the individual follow-up period.

In the great majority of cases, multiple sources of information were used for follow-up assessments, and, where possible and with the subject's permission, general practitioners, family members, spouses, hospital and hostel staff, case notes, etc., were consulted (median number of informants: 2 ; range: $0-3$ ).

\section{Analyses}

Analyses were performed using the STATA software (release 3.1, STATA corporation, 1993).

- identifying symptom dimensions:

Initial unrotated factors were extracted with principal component analysis (Manley, 1986) on the correlation matrix of the OCCPI checklist, reduced to 20 main items (see Table 2). Factors with an eigenvalue greater than 1 were then subjected to varimax rotation. Finally, regression factor scores were produced for each case for subsequent analyses. Factor loadings smaller than 0.5 were omitted. The OCCPI checklist contains a number of items which are not taken into account in the OPCRIT diagnostic classification algorithms (e.g. criteria for substance misuse, family history, and demographic characteristics), and these were not included in the factor analysis. Two items that were a combination of symptoms and signs, e.g. 'delusions and hallucinations for one week" were also excluded. 
As we wished to validate the outcome of the factor analysis with independently collected. data on course and outcome and premorbid functioning, items relating to these in the OCCPI checklist were excluded (three items relating to course of illness and three items about premorbid adjustment). Thus, a comprehensive list of affective and psychotic symptoms and signs remained, including age of onset and type of onset. Manic and depressive symptoms were each entered as the sum of the ratings on the individual items for mania and depression. Of the symptoms and signs included, the median proportion with non-zero (zero indicating absence) values was $43 \%$ (range: $16 \%-90 \%$ ).

\section{- identifying outcome dimensions}

Factor analysis of the correlation matrix of 21 measures of course and outcome (see Table 3; median proportion of non-zero values: $51 \%$; range: $7 \%-92 \%$ ), was undertaken in a similar fashion as for the OCCPI data. However, instead of calculating factor scores we selected two or three outcome measures from each factor identified to be included in the analyses (total: 9 scales). This was done so as to have a range of more readily interpretable clinical outcome measures, while taking into account the dimensional nature of the data. The decision to include these scales was made a priori, on the basis of similar scales having been included in previous research reports, or on the basis of their face validity for clinical practice (see below). The selection included DAS, Iager scale, usual level of negative symptoms over follow-up period, usual symptom severity, course of illness, time in hospital, time living independently, unemployment and employment status at follow-up.

\section{- hypothesis testing}

Multiple regression analysis, yielding regression coefficients (B), was used to adjust the means of continuous outcome variables. Variables were transformed to remove skewness where appropriate, and residuals were examined for evidence of non-linearity. In addition, where significant associations were found, squared terms were fitted to examine whether there was evidence for departure from linearity. Some outcome variables were transformed into dichotomous variables if they inclined towards two categories. For example, course of illness was transformed into "continuous" and "non-continuous". Furthermore, some variables were severely skewed, tending towards a half-normal distribution (presence-absence), in which case dichotomization was considered more appropriate (eg time in psychotherapy). Logistic regression, yielding odds ratios (OR), was used to account for confounding with dichotomous dependent variables.

Syndrome scores (which were orthogonall, ie uncorrelated) were entered jointly in the equations as the explanatory variables. In examining which of the psychopathological syndromes were associated with the six dimensions of course of illness, a conservative approach to effect estimation was chosen, and to reduce the number of tests, only outcome dimensions explaining more than $10 \%$ of the variance were modelled in the anallyses. For pairs of outcome measures covering a dimension, isolated associations were considered positive either if significant at the $p \leq 0.01$ level, or if significant at the $p$ $\leq 0.05$ level together with an association at the $\mathrm{p} \leq 0.05$ level in the other measure of that same dimension. In the case of three outcome measures covering an outcome dimension, the same applied, except that one association at the $\mathrm{p} \leq 0.01$ level had to be accompanied by at least a trend at the $\mathrm{p} \leq 0.1$ level for another measure of that dimension. 
In comparing the contribution of sets of variables to a model, $F$ (for continuous outcomes) or $\chi^{2}$ (for binary outcomes) tests of linear restrictions were applied to the most recently estimated model, testing that the coefficients of a set $x_{1} \ldots \ldots . x_{n}$ expllanatory variables was jointly zero. Also used for this purpose were the multiple correlation coefficient from multiple regression models, as an index of the goodness of fit of the model, and the positive predictive value (PPV) from the logistic regression classification table, as an index of the proportion of cases with a certain outcome that are correctly classified on the basis of the independent variables in the model (the conventional cutpoint of $P=0.5$ was used to produce the classification matrices).

All analyses were adjusted for sex, catchment area status and duration of illness (which was also strongly correlated with number of previous admissions: $r=0.7, p<0.001$ ) as these variables may be related both to psychopathology and, independently, to illness outcome.

\section{RESULTS}

One hundred and ninety-one patients met our inclusion criteria; adequate follow-up data were obtained on $166(87 \%)$ over a period of 18 months. Seven patients had committed suicide, and two had died of other causes. One-hundred and fifty-three patients were interviewed personally, 4 over the telephone, and of the patients not interviewed, high-quality information was available on a further 13. Three patients refused; some information was available on 14 patients (one of whom had died), but was deemed not complete enough for inclusion in the analyses; 8 patients could not be traced:

Compared to the 166 follow-up subjects, the 25 patients not followed tup were very similar in age, sex, ethnicity, index diagnosis, paternal social class, and age and type of onset (Table 1).

\section{Cohort characteristics}

Of the 166 follow-up subjects, $59(36 \%)$ were female, and the mean age at baseline assessment was 26.4 years (range: $16-50 ; \mathrm{SID} .=6.5$ ). At baseline assessment, 75 subjects $(45 \%$ ) had a DSM-IIIR diagnosis of schizophrenia or schizophreniform disorder, 43 (26\%) of affective psychosis (depressive and bipolar psychoses), and 12 (7\%) of schizo-affective psychosis. Thirtysix $(22 \%)$ had a diagnosis of schizophreniform disorder, delusional disorder or unspecified functional psychosis. White Europeans comprised $53 \%$ of the cohort.

The mean duration of illness prior to baseline assessment was 2.2 years (S.D.: 2.0); 70 subjects (42\%) were first admissions. There were no significant differences in duration of illness between males and females (range of means: 2.0-2.2), ethnic groups (range of means: 2.0-2.5), or social classes (range of means: 1.8-2.4), nor in age of onset of first psychotic symptoms between the ethnic $(\mathrm{F}=0.6 ; \mathrm{p}=0.6)$ and social groups $(\mathrm{F}=0.002 ; \mathrm{p}=1.0)$. 
Table 1*. Characteristics of patients not followed-up in companison with follow-tup siample

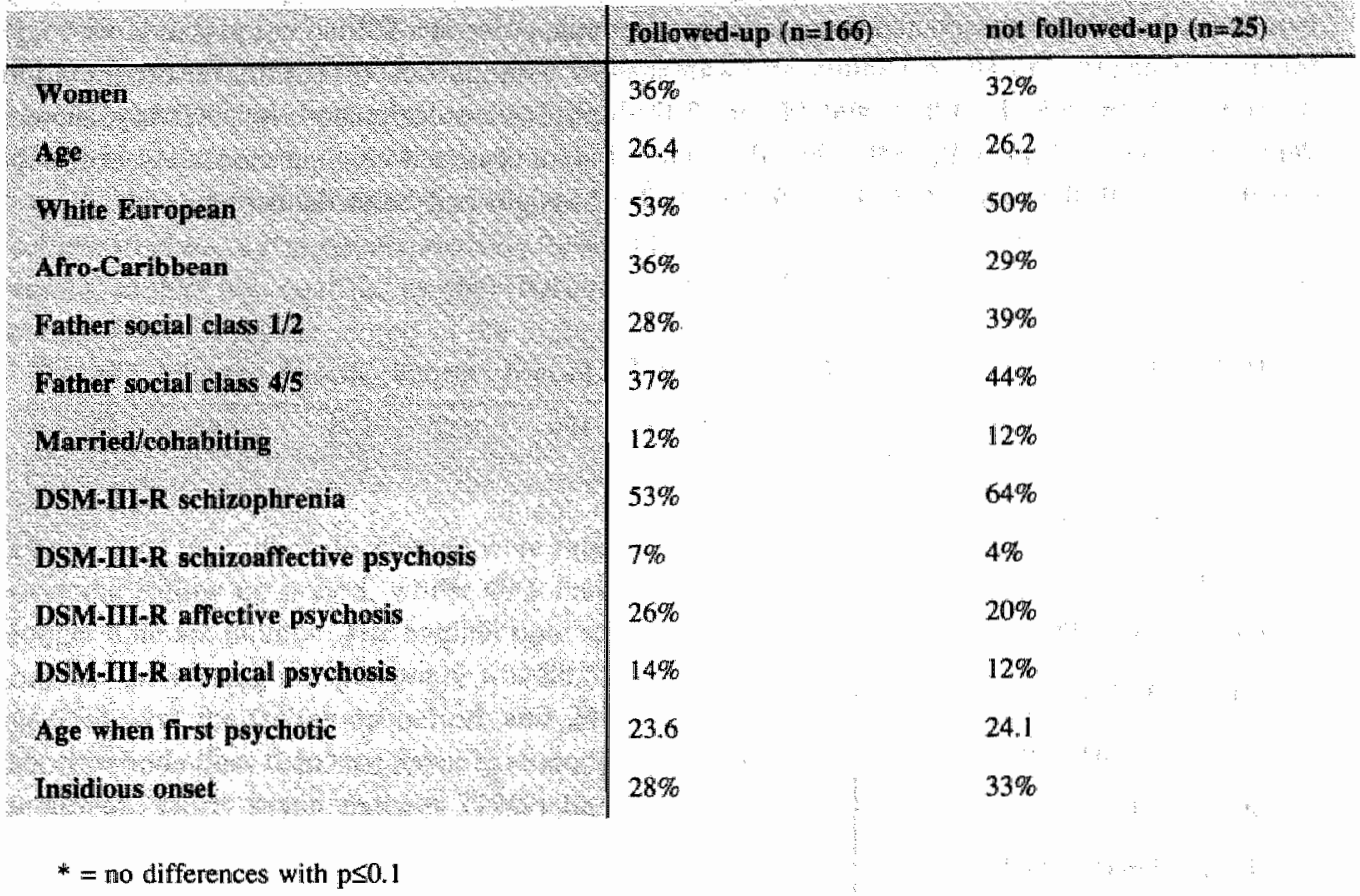

Men and women showed the expected (Castle \& Murray, 1991) difference in age of onset (males: 22.7 years; females: 25.2 years; $t=-2.3 ; p=0.03$ ). Similarly, the mean age of onset was highest for the affective psychoses (25.9 years), and lowest for schizophrenia (21.7 years), with schizo-affective and other psychoses in between ( 23.2 and 24.9 respectively). These findings are further evidence of non-differential follow-up and random inclusion at baseline assessment.

Compared to local district patients, patients referred from elsewhere were more likely to be White European ( $76 \%$ versus $44 \% ; \mathrm{p}<0.01)$, and to be from parental class 1 and $2(47 \%$ versus $21 \%$; $p<0.01$ ). There were no sex differences between the two groups (district: $36 \%$ females, elsewhere: $35 \%$ ), but in the non-district group schizophrenic patients were somewhat overrepresented ( $57 \%$ versus $41 \%$; $\mathrm{p}=\mathrm{NS})$.

\section{Factor analysis of OCCPI items}

The factor analysis of the 20 OCCPI items yielded a pattern of segregation onto 7 factors, explaining $63 \%$ of the variance (Table 2). The first factor had heavy loadings in bizarre behaviour, catatonia, inappropriate affect and difficult rapport; the second in bizarre delusions, passivity phenomena, thought interference and hallucinations; the third in mania, grandiose delusions and relation between affective and psychotic symptoms; the fourth in early and insidious onset, and blunting of affect; the fifth in symptoms of depression and depressive delusions; the sixth in lack of insight; and the seventh in other delusions (mainly persecutory delusions and delusions of reference). 
Factors one, two and four bear some resemblance to Liddle"s (1987a) psychopathological syndromes in chronic schizophrenia (disorganisation, reality distortion, and psychomotor poverty syndromes) and were designated inappropriate-catatonia, delusions-hallucinations, and insidiousblunting respectively. These slightly awkward names were chosen to avoid overinterpretation through terminology. Factor 3 , and factors 5 through 7 were designated, respectively, mania, depression, lack of insight, and other delusions. Factor scores were approximately normally distributed, with the exception of the factors lack of insight and other delusions.

Table 2. Factor analysis of OCCPI items

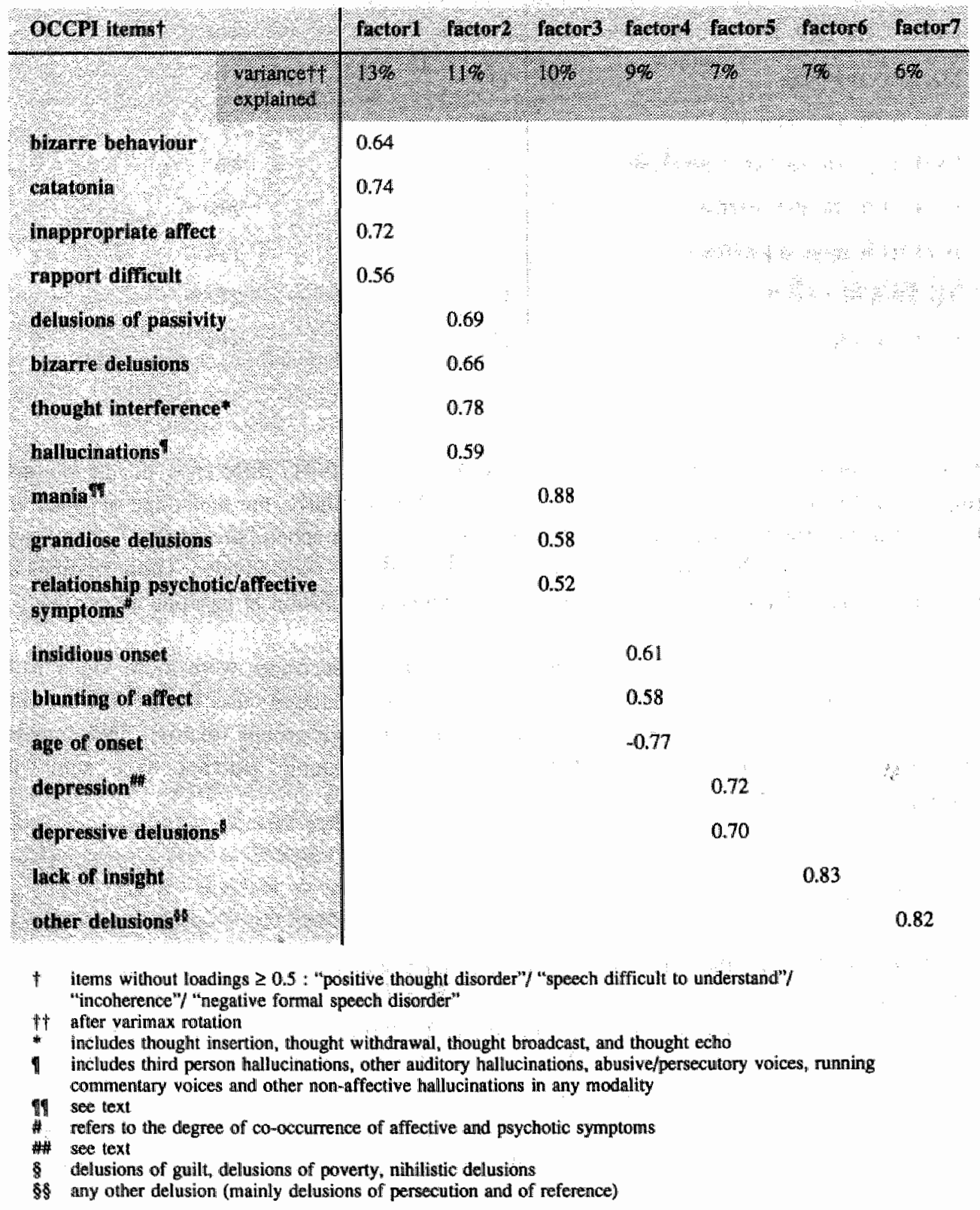


Mean factor scores differed significantly (ANOVA: $\mathrm{p} \leq 0.01$ ) among the 4 DSM-IIIR diagnostic categories described above except for the dimensions lack of insight $(p=0.3)$ and other delusions $(\mathrm{p}=0.4)$ Analyses with ICD-10 categories gave similar results. Of course, the association of factor scores with DSM-IIIR diagnostic categories is not surprising, as the criteria to make up the individual diagnoses were used in the factor analysis. However, examination of the ranges of the factor scores in each diagnostic category indicated considerable intra-group variation and overlap between categories. For example, even in the DSM-IIIR category of affective and schizoaffective psychosis, respectively $23 \%$ and $25 \%$ of subjects had insidious-blunting syndrome scores in the top quartile of the range of scores of the whole cohort; similarly, $17 \%$ of schizophrenic subjects scored in the highest quartile of the mania syndrome.

\section{Course and outcome}

Fourty-nine percent of the sample were rated as having a usual symptom severity of "recovered" over the follow up period, whilst $40 \%$ had had a continuous illness course without remissions longer than six months. The median time spent in hospital was $9.1 \%$ (range. $0 \%-100 \%$ ), and the median time living independently was $88.6 \%$ (range: $0 \%$-100\%). Eleven patients (7\%) had been vagrant over the follow-up period, and $24(15 \%)$ had been in prison; most of these had spent short periods (less than two days) in custody; only a minority had been imprisoned for more than a month. Thirty-seven patients (23\%) had shown self-harm behaviour, including completed suicide in seven.

\section{Outcome dimensions}

Factor analysis of the 21 outcome measures used in the study yieldled 6 factors (Table 3), which accounted for $73 \%$ of the variance. The factors were designated: 1. severity of illness; 2 . negative symptoms and social disability; 3. unemployment; 4. time living independently; 5 . vagrancy and imprisonment; 6. depression and self-harm. As explained above, two or three scales from components explaining more than $10 \%$ of the variance were selected to be included in the analyses (see Table 4).

\section{Associations with illness course}

After adjustment for confounding variables, significant associations between the insidiousblunting syndrome and 3 of the 4 outcome dimensions was apparent, all indicative of a more disabled course of illness (Table 4). Similarly, the inappropriate-catatonia syndrome was associated with a more disabled course in terms of disability-negative symptoms and unemployment, whereas the Delusions-hallucinations syndrome was associated with the illness severity dimension of outcome. The mania syndrome as associated with a more benign illness in terms of negative symptoms and illness severity, whereas the depression syndrome was not associated with any of the outcome measures. The other delusions syndrome predicted, analogous to the delusions-hallucinations syndrome, a higher levell of illness severity over the follow-up period. The confounding variables in general reduced the observed associations by only a tiny amount, and only in some instances were the regression coefficients reduced by around $20 \%$. 
Additional adjustment for DSM-IIIR diagnosis, using either two categories (schizophrenic/nonschizophrenic) four categories (affective, schizoaffective, atypical, schizophrenic) or six (depressive, bipolar; schizodepressive, schizobipolar, schizophrenic, atypical) reduced the associations only by a very small amount, with the exception of the association between the mania syndrome and the DAS score, where the regression coefficient was more than halved. This indicates that associations between psychopathological syndromes and outcome were not simply reflections of diagnostic differences. Fitting squared terms of significant predictor variables did not provide strong evidence for departure from linearity.

Table 3. Factor analysist of autcome measures

\begin{tabular}{|c|c|c|c|c|c|c|}
\hline Outcome measure & factorl 1 & froctor 2 & fretors & factort & factors & tactoro \\
\hline oxplanod & 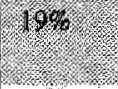 & $15 \%$ & 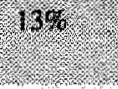 & 1276 & 86 & 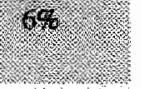 \\
\hline Time psychotic & -0.86 & $\cdots$ & $x$ & 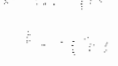 & 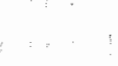 & 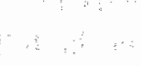 \\
\hline Type of remission & 0.63 & & & 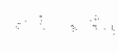 & $3 \ldots$ & \\
\hline Usual severity of symptoms & 0.78 & 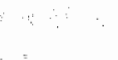 & $\because \quad \%$ & $\cdots$ & $\therefore$ & $\because \cdots$ \\
\hline Course type & 0.84 & & & 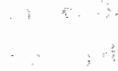 & 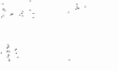 & \\
\hline GAr-symptoms & 0.74 & & & & & \\
\hline Inger scale & & 0.87 & & & & \\
\hline DAs: & & 0.80 & & & & \\
\hline DAS-globalt & & 0.75 & & & & $\therefore$ \\
\hline Negative symptoms over follow-up & & 0.78 & & & & \\
\hline Time unemployed & & & 0.83 & +8 & & 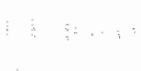 \\
\hline Unemployment due to mental illness & & & & $\therefore \cdots$ & $\cdots$ & \\
\hline Employed at follow ap & & & -0.73 & : : & & \\
\hline Thine in hospital & & & & 0.86 & & \\
\hline Milme living independenty & & & & -0.81 & 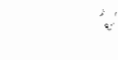 & \\
\hline Assaultatlveness & & & & 0.61 & & \\
\hline Imprisonment & & & & $\because$ & 0.84 & \\
\hline Vagrancy & & & & $\therefore$ & 0.85 & 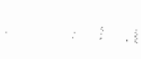 \\
\hline MAMD,seore & & & & & 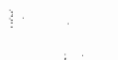 & 0.50 \\
\hline Self harm/sulcide & & & & $\cdots$ & & 0.67 \\
\hline
\end{tabular}

As all factor scores had approximately zero means and unit variance, and the range of the factor score values was in general in the order of 4 or $\mathbf{5}$ standard deviations, it is possible to make an 
estimate of the effect size over the range of values of the psychopathological syndromes, ie the difference between extremes. Thus, as the delusions-hallucinations syndrome factor score increased by one unit (ie one standard deviation), the odds of having a non-remitting course of illness increased multiplicatively by 1.8 . Over 4 standard deviations, individuals in the highest category of delusions-hallucinations syndrome scores were $1.8^{4}$ or 10.5 times more likely to have a non-remitting illness course than the lowest category. Similar arithmetic applies to the exponentiated regression coefficient of $\log$ transformed continuous variables (Iager scalle weighted score, DAS-score, time in hospital, time living independently). For example, over the range of approximately four standard deviations of the lack of insight score, individuals in the highest category were $\left(e^{0.26}\right)^{4}$ or 2.8 times more likely to have spent a given time in hospital. For untransformed continuous variables (time unemployed) the regression coefficient is the additive increase with one unit change in the predictor variable. Thus, over four standard deviations of the insidious-blunting score, the difference in time unemployed over the follow-up period was $4 \times 8.2$ or $32.8 \%$.

\section{Relationship to other predictors of outcome and baseline associations}

The observed associations between psychopathological syndromes and course and outcome could have been mediated by factors that are known to have an influence on the course of psychosis; such as gender, premorbid social adjustment and baseline marital status. Syndrome scores bore differential associations with some of the above variables at a conservative level of significance ( $p<0.01)$. Male and single patients scored significantly higher on the insidious-blunting syndrome (gender: $t=3.3, p=0.001$; single status: $t=4.7, p<0.001$ ), whereas premorbid social adjustment (higher score indicating poorer adjustment) was significantly associated with the inappropriatecatatonia syndrome $(r=0.26, p=0.003$ ). However, adjusting for these factors in the relevant equations in general only attenuated the previously reported associations between psychopathological syndromes and outcome by a very small amount.

Similarly, we adjusted for baseline values of outcome measurements in order to examine whether the associations between psychopathological syndromes and outcome measures really were simply a repetition of associations that might already have been present at baseline. For example, associations with negative symptoms were adjusted for negative symptoms as defined by the PSE at baseline (affective flattening, self neglect, slowness); associations with usual symptom severity for PSE total score at baseline; associations with unemployment for employment status at baseline; and associations with time living independently for weeks spent as a psychiatric inpatient before baseline assessment. Again, despite some very significant effects of baseline measures, the strength and significance level of previously reported associations between psychopathological syndromes and outcome measures changed generally by only a small amount. For example, weeks spent as an in-patient before baseline assessment was significantly associated with time in hospital and time living independently over the follow-up period (both: $p<0.001$ ), but the associations with lack of insight remained unchanged $(B=0.25$ and $B=0.22$ respectively, both $\mathrm{p}<0.05$ ). Similarly, unemployment at baseline was significantly associated with unemployment at follow-up ( $\mathrm{p}=0.001)$, but the association with the insidious-blunting syndrome remained unchanged $(O R=0.4, p=0.004)$. These findings confirm that psychopathologicall syndromes are true predictors of outcome. 


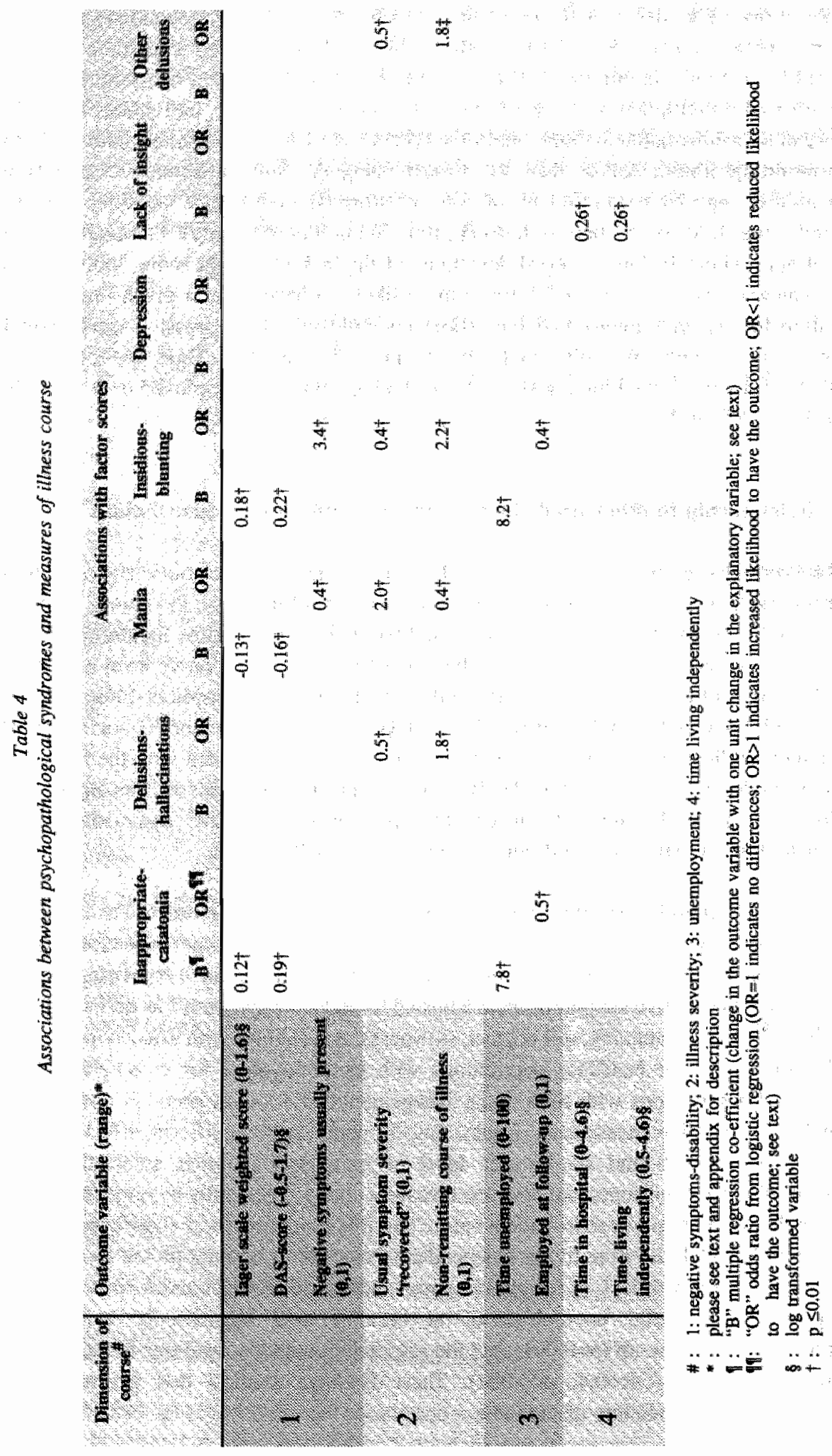




\section{Associations with treatment}

The following interventions over the follow-up period were modelled with the psychopathological syndromes as predictor variables" time on antipsychotics, having received anti-depressants, lithium or psychotherapy over the follow-up period, having been in rehabilitation, or having been admitted under a section of the Mental Health Act (all binary variables, except time on antipsychotics). Apart from gender, catchment area status and duration of illness, diagnosis (schizophrenic and non-schizophrenic psychoses) was also controlled for. Conservatively, only associations at the $1 \%$ significance level or lower are reported. Each of the syndromes bore contrasting associations with one or more treatment modalities, with the exception of the other delusions syndrome and the insidious-blunting syndrome. The inappropriate-catatonia syndrome was negatively associated with psychotherapy $(\mathrm{OR}=0.14$; $p<0.009$ ), and treatment with anti-depressants $(\mathrm{OR}=0.5 ; \mathrm{p}=0.008)$, whereas the depressive syndrome was positively associated with anti-depressant treatment $(O R=1.8 ; p=0.006)$. Both the manic and the depressive syndrome were associated with lithium treatment (manic syndrome: $O R=4.3 ; p<0.001$; depressive syndrome: $O R=2.2 ; p=0.001$ ), whereas the delusions-hallucinations syndrome predicted more time on antipsychotics $(B=7.4 ; p=0.01)$. Lack of insight predicted admission under a section of the Mental Health Act over the follow-up period (OR=1.8; $\mathrm{p}=0.008$ ). Adjusting for DSM-IIIR diagnosis using four or six categories instead of just schizophrenic and non-schizophrenic psychoses tended, if anything, to further enhance these associations with treatment variables, rather than reduce them.

\section{Categories and dimensions}

Which approach -dimensional or categorical-is most useful in predicting course and outcome? One way to examine the relative contributions of categorical and dimensional systems is to examine in the statistical analyses the effect of each after controlling for the other. Six DSM-IIR diagnostic categories of psychosis (depressive, bipolar, schizo-depressed, schizo-manic, schizophrenia, atypical) were compared with the seven psychopathological syndromes. First, for each outcome and each treatment variable, those psychopathological syndromes or DSM-IIIR categories (with the schizophrenic category as the baseline) that showed associations at at least the $10 \%$ significance levell were identified. Secondly, each outcome measure was modelled, entering jointly the syndromes and categories previously identified, as well as the confounding factors. Thirdly, the relative contribution of each set of variables -syndromes and categories-, was assessed through tests of linear restriction as described earlier. The analyses revealed highly significant ( $\leq 0.01$ ) contributions of psychopathological syndromes to all nine outcome and all but one of the six treatment models, over and above the contributions of DSM-IIIR diagnostic categories. In contrast, however, diagnostic categories were only significantly associated with the DAS score, and with employment status at follow-up. This indicates that psychopathological syndromes are considerably better predictors of illness course and treatment over the follow-up period than diagnostic categories. Also when the diagnostic categories and psychopathological syndromes that contributed at the $10 \%$ significance level were each considered separately as sets of independent variables (without inclusion of confounders) in models of outcome and treatment variables, a similar result was apparent: psychopathological syndromes explained consistently more of the variance of continuous dependent variables than diagnostic categories, and had consistently higher positive predictive values derived from the logistic regression classification 
table of binary dependent variables (Table 5). Analyses with ICD-10 diagnostic categories yielded a similar pattern of results.

\section{DISCUSSION}

Our results demonstrated that some psychopathological syndromes were strongly associated with illness course and treatment over the follow-up period. These associations were independent of DSM-IIR diagnosis and known predictors of outcome, and were not simple repetitions of associations already present at baseline. Syndromes were better predictors of outcome and treatment than diagnostic categories. We will first discuss to what extent these findings may be due to bias, confounding, or the vagaries of factor analytical procedures.

\section{Methodological issues}

Factor analysis in effect groups variables rather than subjects (Dunn, 1993); for the purpose of outcome prediction this approach would appear valid, as the clinician is concerned with individual characteristics in the patient that have prognostic relevance. Our factor analysis on outcome measures was conducted mainly to summarize the data and facillitate interpretation of the analyses, and the chosen names for the factors, though subjective, appear to have high face validity with regard to the variables that loaded onto the factors. On the other hand, the model of the factor analyses of the OCCPI items was taken to represent 'true' dimensions of psychopathology. We were therefore careful in the choice of names for the factors; cumbersome though names as insidious-blunting and inappropriate-catatonia may be, they reduce the danger of misleading conceptualization through statistical procedures. As factor analytical techniques express relationships between only those symptoms and characteristics that the investigators have fed into the analysis, there is not necessarily a link between "real" underlying psychopathological syndromes, and the syndromes identified by factor analysis. For instance, inclusion of variables relating to premorbid social adjustment will yield, perhaps not surprisingly, a factor "premorbid personal-social relationships" (Lenzenweger $e t a l, 1991$ ), and inclusion of a more comprehensive range of symptoms than just the SANS or the SAPS leads to a rapid increase of the number of factors (Everitt et al, 1971; Fleiss et al, 1971; Spitzer et al, 1967). Our approach was to include a comprehensive range of affective and non-affective symptoms and signs considered relevant in a variety of diagnostic systems (of which it is reasonable to assume that they have some validity with regard to psychotic syndromes), using illness course and treatment variables to validate the outcome of the factor analysis of psychopathological symptoms. This is not necessarilly the best strategy, but at least has a chance to substantiate the factor analytical findings:

The correlation between two variables can yield very inflated estimates in the case of variables with a very high or a very low incidence, and the validity of any cluster of variables must be checked against their distribution. Problems are revealed by factors with large loadings on only one skewed variable, in which case the "factor" may not represent a dimension, but rather a subgroup of patients who display that symptom within the sample as a whole (Maxwell, 1971, 1972). 
Table 5 Comparison of predictive wallding of dimensional and categorial representations of psychopathology

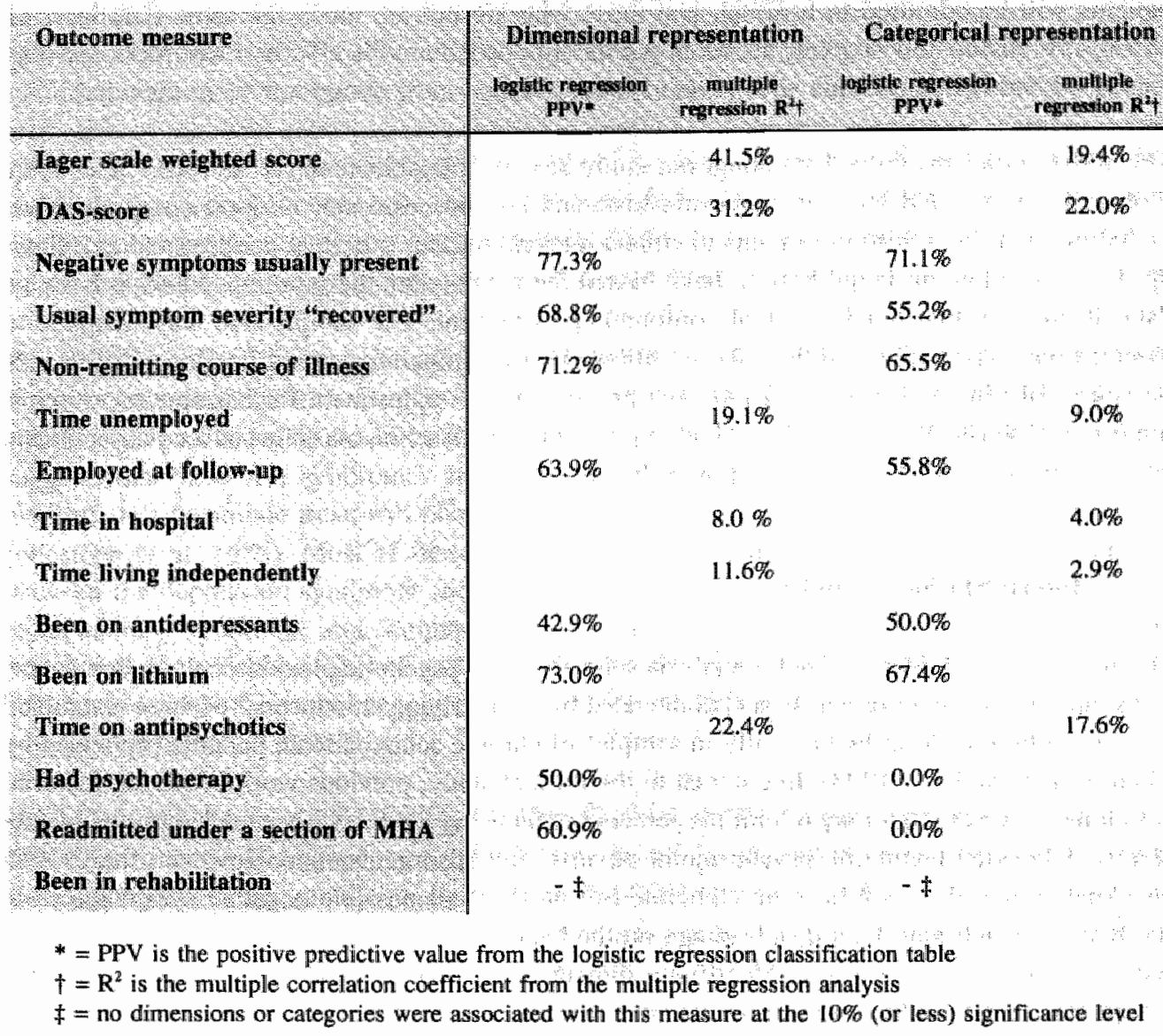

In the present study, the factors representing 'insight' and 'other delusions' are therefore questionable (the prevalence of lack of insight and other delusions was $79 \%$ and $80 \%$ respectively), but it should be pointed out that for the purpose of this investigation -prediction of outcome- the distinction between dimension and subgroup is not crucial. From the clinical perspective, both are relevant if they are associated with illness course and/or treatment.

The fact that only one rater collected follow-up information obviates problems related to interrater reliability, but may be a disadvantage if the one rater rates everything wrong. However, even if there were such a measurement error, it is highly unlikely to have varied as a function of baseline psychopathological syndromes, and would therefore not have led to spurious associations. Similarly, any systematic differences between the three PSE raters cannot have occurred as a function of outcome.

Multiple statistical testing may lead to chance findings. Therefore, in examining the associations between syndrome scores and measures of course, we chose for a conservative interpretation of $P$-values throughout. 
Our results are onily generalizable to the population of patients with psychotic illness who i) have relatively recent onset of ilness, and ii) who are admitted to hospital. While most schizophrenic patients will be admitted to hospital, and those who are not are much the same (Castle et al, 1993) this distinction is likely to be important for patients with affective disorders, as only depressed and manic patients with severe illness and psychotic symptoms were included.

The above criticisms notwithstanding, our study has considerable strengths as well. Cases were prospectively selected without diagnostic bias, and baseline assessments were comprehensive, including interviews with informants to collect relevant measures both at baseline and at followup. Loss to follow-up is unlikely to have biased the results, and the rater was blind to baseline data. It unlikely that much residual confounding is present. The sample size was adequate to examine our hypotheses, and the analyses allowed for a comparative quantification of effect size on course of illness. The issue of outcome prediction after adjustment for baseline measures is important (Johnstone $e t a l, 1989$ ) and attempts to include baseline measures as a covariate have, as far as we are aware, not been reported before.

\section{Interpretation of findings}

Factor analytical findings - Factor analysis suggested that psychotic phenomenology in a cohort of recent onset psychotics was best characterised by 7 underlying structures; 3 of these resernbled factorial solutions described recently in samples of chronic schizophrenic patients (reviewed by Thompson \& Meltzer, 1993). In contrast to the present study, previous work inwolving factorial solutions of symptomatology of chronic schizophrenia using the SANS has tended to yield fairly clearly delineated factors of "psycho-motor poverty' and 'disorganisation'. However, the OCCPI checklist simply does not have an elaborate inventory of all possible negative symptoms as in the SANS, which may be a disadvantage on the hand, but an advantage on the other as it will not 'bias' the factor analysis in identifying dimensions in a set of (highly correlated) negative symptoms. It is therefore perhaps even more convincing that the negative symptoms that were recorded in the OCCPI yielded a pattern of covariation that is comparable with previous work in chronic schizophrenia. For example, previous studies have found that the symptoms of 'blunting of affect' and 'inappropriate affect' were among the best discriminators between the psycho-motor poverty and disorganisation syndromes (Brown \& White, 1992; Thompson \& Meltzer, 1993) and in this respect the insidious-blunting syndrome and the inappropriatecatatonia syndrome clearly resembled factor results of other studies. Furthermore, thought disorder ('alogia") has been found to be a poor discriminator between these two factors (Brown \& White, 1992; Thompson \& Meltzer, 1993), which is also in keeping with our own results, as this variable did not load onto any of the factors. However, the fact that neither positive nor negative thought disorder had substantial loadings on any factor in this study may also be related to the relative insensitivity of the PSE (and OCCPI) in the area of observable speech and behaviour, as symptoms in these domains are only rated when present in fairly severe form. Lack of insight, manic and depressive syndromes have also been described previously (Everitt $e t$ al, 1971; Fleiss et al, 1971; Spitzer et al, 1967). Soni and colleagues (1992), have pointed out that the SANS and the SAPS, frequently used in the more recent factor analytic studies, exclude affective items and can therefore not detect an affective syndrome. As noted earlier, two of the syndromes (lack of insight and other delusions) may not be true dimensions of symptomatology, and the conservative interpretation is that they represent subgroups of patients within the 
psychotic sample, who tend to have certain relatively rare symptoms (or do not display very frequent symptoms).

Ilness course and treatment - Overall, of the seven syndromes, the depression and other delusion syndromes appeared weakest in terms of external validation. The depression syndrome was not predictive of outcome and there was overlap in terms of treatment associations with the mania syndrome. The other delusions syndrome was not associated with any treatment modality, and overlapped with the delusions-hallucinations syndrome in predicting outcome. There is therefore reason to doubt the validity of two separate affective and two separate positive symptom syndromes, and a more parsimonious latent structure may be more appropriate.

The remaining five syndromes were associated differentially with medical interventions and illness course over the follow-up period, independent of DSM-MIR diagnosis and associations with known predictors of outcome. The associations with treatment over the follow-up period suggests that these five syndromes are related to hypothetical variables, on the basis of which medical staff formulate treatment plans, over and above the usual diagnostic considerations (cf Johnstone et al, 1988). Most of these were relatively straightforward, such as the association between the depression syndrome and antidepressant and lithium treatment, and the delusionshallucinations syndrome and antipsychotic treatment. The negative association between the inappropriate-catatonia syndrome and psychotherapeutic and anti-depressant treatments is more difficult to interpret and would suggest that in practice these interventions are considered 'contratindicated' (May et al, 1981).

Although some syndromes were rather simillar in their profile of predicting outcome, they were dissimilar in their associations with treatment and other predictor variables. For example, both the inappropriate-catatonia syndrome and the insidious-blunting syndrome were predictive of negative symptoms and unemployment, but only the inappropriate-catatonia syndrome had strong negative associations with psychotherapy and antidepressant treatment, and only the insidiousblunting syndrome was additionally predictive of greater illness severity over the follow-up period. Furthermore, the insidious-blunting syndrome was associated with male sex and single status, whereas the inappropriate-catatonia syndrome was associated with poor premorbid social adjustment.

Lack of insight was associated both with more time spent in hospital, as with increased likelihood of being admitted under a section of the Mental Health Act. Our findings concur with previous reports linking lack of insight to poor compliance and poor outcome (Soskis \& Bowers, 1969; McGlashan et al, 1981; Bartko et al, 1988; McEvoy et al, 1989; Buchanan, 1992), and support the view that insight is an important concept in the functional psychoses (David, 1990).

The manic syndrome was the only one associated with a more favourable outcome. This is in agreement with the now traditional attribution of better prognosis in the presence of an index affective component. In view of the lack of discrimination between depressive and manic syndromes by treatment variables, the strict separation between manic and depressive symptom clusters as outcome predictors may not be justified. 
Categortes and dimensions - Whatever the ultimate model of psychotic illness: dimensional, categorical or both (Kendell, 1975; Everitt et al, 1971), the present study would suggest that a dimensional representation of psychosis is more useful for prognostic purposes, and also more cllosely related to treatment decisions in the management of functional psychotic illnesses, implying psychiatrists are already thinking dimensional in clinical practice. Typical 'schizophrenic syndromes, such as the insidious-blunting syndrome, associated with malle gender and single status, and the inappropriate-catatonia syndrome, associated with poor premorbid social functioning; were in fact not confined entirely to the category of schizophrenia. Such overlap is important, and suggests that, for example, developmental deviance and characteristic gender differences associated with the diagnostic category schizophrenia, may be equally well conceived as risk factors for certain psychopathological syndromes that cross conventional diagnostic boundaries (Murray \& O'Callaghan, 1991). 
APPENDIX 1.

\section{COURSE TYPE IN THIS PERIOD.}

In estimating duration of an episode that began before this period but extended into the period, include the time before this period.

- episodic - discrete episodes (none longer than 6 MONTHS) with clear periods of remission in between. At least one remission lasted 6 months or more.

- continuous - psychotic over most of the period. If any remissions, these were brief (none longer than 6 months)

- neither episodic nor continwows - e.g., longest psychotic episode was 12 months and longest remissuon was 9 months.

- never actively psychoric in this period:

USUAL SEVERITY OF SYMPTOMS. (Categories are modified from M. Bleuler.)

Select the rating that best describes MOST of the period. If no rating applies to more than half of the period, select the one with the longest duration.

- mild - behawior was generally nomal and illness was not immediately obvious in conversation, although definite psychotic symptoms persisted.

- moderate - consistently showed signs of health in spite of marked psychotic symptoms, e.g, could speak with reasonable clarity on some occasions; could work or care for others to some extent; marked psychotic symptoms may be avert or suggestive (see definitions).

- severe - could not carry on a coherent conversation; could not work without close supervision; required constant care.

- recovered - onlly residual or no psychotic sympionis.

\section{PSTCHOTIC}

\section{A, OVERT SIGNSS AND SYMPTOMS}

- hallucinations or pseudohallucinations (in any modality);

- delusions;

- marked thought and speech disorder (e.g.n. incoherence, irrelevance, thought blocking. neologisms, incomprehensibility of speech) other than simple retandation or acceleration;

- marked qualitative psychomolor disorder (e.g., negativism, mutism or stupor, catatonic excitement; constrained attitudes or unnatural postures maintained for long periods) other than retardation or acceleration of movements;

- bizarre or grossly inappropriate behavior (e.g. talking to self or giggling to self, acts incomprehensible to others, loss of social constraints, etc.,

\section{B. SUGGESTIVE SIGNS AND SYMPTOMS}

- extrene reduction or loss of interests, initiative and drive, leading to serious deterioration of the performance of usual activities and tasks;

- extrene social withdrawal;

- severe excitement, purposeless destructiveness or aggression (frequent episodes or continuous);

- episodes or persistent states of overwhelming fear or severe anxiety:

- gross and persistent self-neglect.

\section{REMSSION}

A state following a psychotic episode, in which none of the symptoms and signs requined for rating a psychotic episode are present. The absence of psychotic symptons and signs qualifies as a remission only if it lasts for four weeks or more. Two varieties of remission will be distinguished:

(A) COMPLETE REMISSION: the subject is virtually symptom-free and shows his/her usual premorbid personality;

(B) INCOMPLETE REMISSION: the subject is no longer psychotic (in the sense defined abowe) but shows any combination of: (i) residual symptoms (i.e. "traces" of the symptoms and signs which were present in the psychotic episode but which in no way would justify a rating of a contimuing psychotic episode); (ii) non-psychotic symptoms, e.g., non-specific neurotic or mild affective disturbances which are not traits of the premorbid personality; (iii) personality change, tge, accentuation of premorbid personality traits or emergence of new traits; change or loss of interests; stable alterations of expressive and communicative behavior; decline of intellectual performace.

\section{COMMUNITY LIVING NDEPENDENT OF PROFESSIONAL SUPERVISION}

Independent community liwing includes all living arangements other than an institution (e.g., hospital, jail), a supervised residence, and homeless/vagrant. Thus, it includes living alone, living with friends "living with family, and any other non-institutional domicile. 


\section{APPENDIX $2{ }^{2}$}

In chapter 1, we discussed the evidence that diagnostic categories, when ordered approximately along an acute/affective-insidious/non-affective continuum, predict progressively poorer outcome, although it was noted that this association may be confounded In this chapter, we demonstrated that higher scores along the axes of negative symptom dimensions, positive symptom dimensions, and a lack of illness awareness dimension on the one hand, and lower scotes along the axis of affective syndromes on the other, also predicted progressively poorer outcome. We would therefore expect that the above mentioned order of diagnostic categories reflects the linear continuum of one or more of these dimensions. We have already implied, guided by previous reports on this subject, that diagnostic categories, thus ordered, reflect different positions along an acute/insidious and an affective/non-affective continuum.

To further examine these issues, it is necessary to demonstrate that i) the association between ordered diagnostic categories and outcome is not confounded by gender and duration of illness, which are both associated with psychopathological features and outcome; and ii) that ordered diagnostic categories in fact reflect the linear continuum of one or more important psychopathological dimensions.

We hypothesized that, even after adjustment for potential confounders, the effect of diagnostic categories would not only lie in the between-category contrasts they provide, but also in the linear trend they exhibit in their association with outcome when ordered along these an acute/affective and insidious/non-affective continuum ${ }^{3}$. To test this hypothesis, we compared both contrasts between diagnostic categories, and linear trends across diagnostic categories, ordered along the acute/affective and insidious/non-affective dimension. Thus, the sequence was from DSM-III-R affective psychosis (bipolar and unipolar psychoses), atypical psychosis (unspecified psychosis, schizophreniform psychosis and delusional disorder), schizo-affective psychosis and schizophrenia (as the baseline; Table 1). This order was determined by the published findings discussed in chapter 1 .

Linear trends between DSM-III-R diagnosis in the associations with all outcome measures were evident. Although much of the trend was due to the difference between affective psychosis and the schizophrenia baseline, a fairly gradual change in odds ratios and regression coefficients was evident for most outcome measures. Adjustment for gender and illness duration prior to baseline assessment in general reduced the parameters by only a tiny amount.

Having established that the association between ordered diagnostic categories and outcome is not confounded, we wished to examine to what degree this order reflects the linear continuum of one or more psychopathological dimensions. Mean values of the sums of the negative syndromes (insidious-blunting and inappropriate-catatonia), positive syndromes (delusions-hallucinations and other delusions), and affective syndromes (mania and depression), as well as the insight syndrome, were plotted by ordered DSM-III-R categories (Fig. 1). With the exception of the

\footnotetext{
${ }^{2}$ This appendix was not included in the original article

${ }^{3}$ Kendell. R.E. \& Brockington, I.F. (1980) The identification of disease entities and the relationship berween schizophrenic and affective psychoses. British Journal of Psychiatry, 137, 324-331.
} 
insight syndrome, this revealed fairly cllear trends in the expected directions, most of it again due to contrasts between the schizophrenic and affective categories, but also with significant contributions of the intermediate categories.

As discussed in chapter 1, previous investigations have been unable to separate diagnostic categories in the functional psychoses on the basis of their outcome profille, In addition, an outcome order has been established from affective/acute psychosis to schizoaffective and schizophrenic psychoses. The validity of such an order was supported by the fact that it effectively "summarized" the linear continua of several underlying psychopathological dimensions, which were shown to be strongly associated with course and outcome. If predictive validity is to be the yardstick for the usefullness of classification, then ordered diagnostic categories may be better thought of as occupying different positions on an illness continuum, rather than as constituting valid separate disease entities.

Fig. I DSM3R typologies in relation to psychopathological dimensions

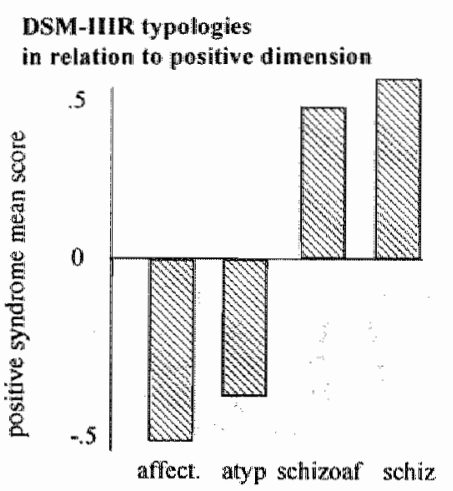

DSM-IUIR typologies

in relation to affective dimension

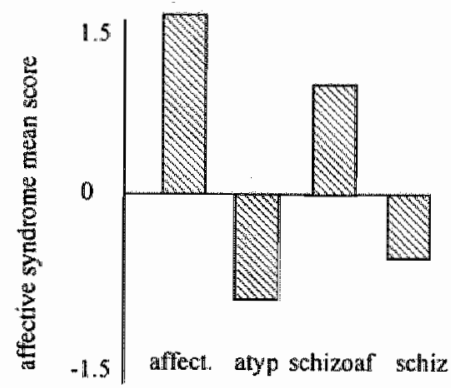

DSM-IIIR typologies

in relation to negative dimension

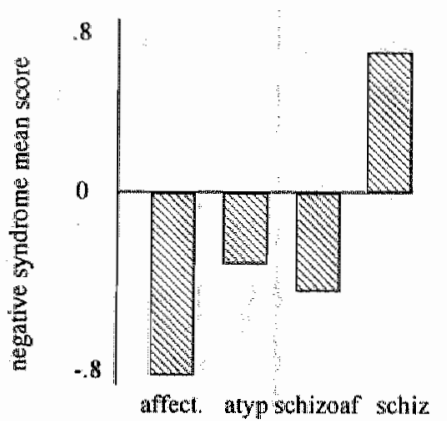

DSM-IIIR typollogies in relation to log insight dimension

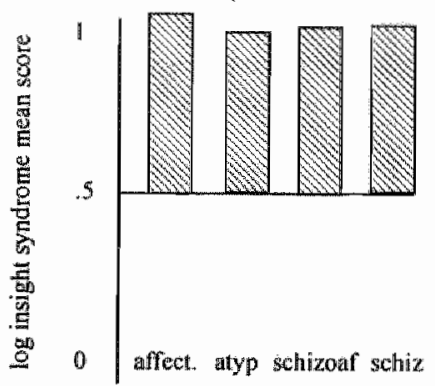




\section{REFERENCES}

Barko, G, Herczeg, I. Zador, G. (1988) Clinical symptomatology and drug compliance un schizophrenic patients. Acta Psychiatrica Scandinawica, 77, 74-76.

Bland, R. Om, H. (1980) Schizophrenia Schneider's first rank symptoms and outeome, British Journal of Psychiatry, 137, 63-68.

-2._1982) Predicting the outcome in schizophrenix. Canadian Jownal of Psychiarry, 27, 52-62.

Brockington, I. Kendell, R.E. Leff, J. (1978) Definitions of schizophrenia: concordance and prediction of outcome. Psychological Medicine, 8, 387-398.

_._. Roper, A., Edmunds, E., Kaufman, C. \& Meltzer, H. (1992) A longitudinal psychopathological schedule. Psychological Medicine, 22, 1035-1043.

Brown, K. \& White, T. (1992) Syndromes of schizophrenia and some clinical correlates. Brinsh Journal of Psychiatry, 161, 317-322.

Buchanan, A. (1992) A two-yeat prospective study of treatment compliance in patients with schizophrenia. Psychological Medicine, 22, 787-797.

Carpenter, W., Bartko, J., Strauss, J. \& Hawk, A. (1978) Signs and symptoms as predictors of outcome: a report for the intemational pilot study of schizophrena. American Journal of Psychiatry, 135, 940-45.

Castle \& Murray, R. (1991) The neurodevelopmental basis of sex differences in schizophrenia. Psychological Medicine, 21, 565-575.

_._.-..... Phelan, M., Wessely, S. \& Murray. M. (1993) Which schizophrenics are not admitted at first psychiatric contact? An analysis of 484 patients. Schizophrenia Research, $9(2,3), 130$.

Cloninger, C., Martin, R., Giuze, S. \& Clayton, P. (1985) Diagnosis and prognosis in sichizophrenia: Archives of General Psychiatry $42,15-25$.

Costello, C. (1992) Research on symptons versus research on syndromesi. Arguments in favour of allociting more research time to the study of symptoms. British Journal of Psychiatry, 160, 304-308.

David, T. (1990) Insight and Psychosis. British Journal of Psychiatry, 157, 774-775.

Dunn, G., Sham, P. \& Hand, D. (1993) Statistics and the nature of depression. Psychological Medicine, 23,871 889.

Endicott, J., Nee, J., Cohen, J., Fleiss, J. Simon, R. (1986) Diagnosis of schizophrenia: Prediction of short term outcome. Archives of General Psychiatry, 43, 13-19.

Everitt; B., Gourlay, A. \& Kendell, RE: (1971) An attempt at validation of traditional psychiatric syndromes by cluster analysis. British Journal of Psychiatry, 119, 399-412.

Fenton, W. \& McGlashan, T. (1991) Natural history of schizophrenia subtypes. Il. Positive and negative symptoms and long-term course. Archives of General Psychiatry, 48, 978-986.

Fleiss, J., Gurland, B. \& Cooper, J. (1971) Some contributions to the measurement of psychopathology. British Journal of Psychiatry, 119, 647-656.

Hamilton, M. (1960) A rating scale for depression. Journal of Nevrology, Neurosurgery and Psychiatry, 23, 56 62.

Hirsch, $S_{\text {. }}$ (1991) Affective symptoms in schizophrenia, basic. prodromal and residual: implications for a functional approach to diagnosis. In: Concepss of Mental Disorder: a Continuing Debate (Eds. A. Kert \&

H. McClelland). Gaskell: Londion.

Huber, $\mathrm{G}_{\text {w, }}$ Gross, G., Schüttler, $\mathbb{R}_{\text {. \& }}$ Linz, M. (1980) Longitudinal studies of schizophrenic patients. Schizophrenia Bulletin, 6, 592-605.

Iager, A.C., Kirch, D.G. \& Wyatt, R.J. (1985) A negative symptom raling scale. Psychiatry Resecarch 16, 27-36.

Jablensky, A., Schwartz, R. \& Tomov, T. (1980) WHO collaborative study of impairments and disabilities associated with schizophremic disorders. A preliminary communication. Objective and methods. Acta Psychiatrica Scandinavica, stippl. 285, 152-163.

Johnstone, E.C., Crow, T.J., Frith, C.D. \& Owens, D.G. (1988) The Northwick Park "functional" psychosis study: diagnosis and treatmer.t response. Lancet, July 19th, 1988.

Kay, S. \& Murrill, L. (1990) Predizting outcome of schizophrenia: significance of symptom profiles and outcome dimensions. Comprehensive Psychiarry, 31, 91-102.

Kendlell, R. The role of diagnosis in psychiary. Oxford: Blackwell, pp 134.

, R., Brockington, $\mathbb{1 1}$. Leff, J. (1979) Prognostic implications of six altematiwe definitions of schizophrenia. Archives of General Psychiatry, 35, 25-31.

Leff, J., Sartorius, N., Jablensky, A., Korten, A. E Ermberg, G. (1992) The International Pillot Studly of Schizophrenia: five year follow-up findings. Psychological Medicine, 22, 131-145.

Lenzenweger, M., Dworkin, R., Wethington, E. (1991) Examining the underlying structure of schizophrenic phenomenology: evidence for a three-process model. Schizophrenia Bulletin, 17, 515-524. 
Liddle, P. (1987a) The symiptoms of schizophrenia. A re-examination of the positive-riegative dichotomy. British Journal of Psychiatry, 151, 145-51.

- (1987b) Schizophrenic syndromes, cognitive performance and neurological dysfunction. Psychological Medicine, 17, 49-57.

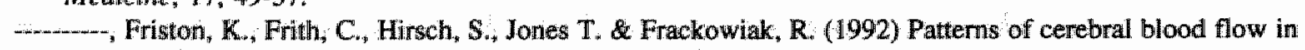
schizophrenia. Briths Jowrnal of Psychiarry, 160, 179-186.

Marley, B. (1986) Multivariate Statistücal Methods. Chapman \& Hall: London.

May, P. Tuma, $H_{\text {, Dixon; }}$ W. (1981) Schizophrenia, a follow up study of the results of five forms of treatment. Archives of General Psychiary, 38, 776-784.

Maxwell, A.E. (1971) Multivariate statistical methods and classification problems. British Jowrnal of Psychiarry, $119,121-7$.

- A.m. E. (1972) Difficulties in a dimensional description of symptomatology. British Journal of Psychiatry, $121,19-26$.

McEvoy, J., Freter, $\mathrm{S}_{*}$ Everett, $\mathrm{G}_{*,}$ (Geller, $J_{*,}$ Appelbaum, Apperson, J. et al (1989) Insight and the clinical outcome of schizophrenic patients. Journal of Nervous and Mental Disease, 177, 48.51.

McGlashan, T. (1981) Does attitude toward psychosis nelate to outcome? American Journal of Psychiatry, 138, $797-801$.

.2.-.... (1988) A selective review of necent North American long-term followup studies of schizophrenia. Schizophrenia Bulletin, 14, 515 542.

McGuffin, P., Farmer, A.E \& Harwey, L. (1991) A polydiagnostic application of operational criteria in psychotic illness: development and reliability of the OPCRIT system: Archives of General Psychiatry;48, 764-770. Mortimer, A., Lund, C. \& McKenna, P. (1990) The positive-negaqtive dichotomy in schizophrenia. British Jowrmal of Psychiatry, $157,41-49$.

Murray, R.M. \& O'Callaghan, E. (1991) The comgenital and adult-onset psychoses: Kraepelin lost, Kraepelin found. In Concepts of Mental Disorder, a Continuing Debate (Eds A. Ker \& H. McClelland) pp 48-65. London: Gaskell.

Peralta, V., De Leon, J. \& Cuesta, M. (1992) Are there more than two syndromes in schizophrenia? a critique of the positive-negative dichotiomy. British Journal of Psychiatry, 161, 335-343.

Philips, L: (1953) Case history data and prognosis in schizophrenia. Journal of Nervous and Mental Diseases, $117,515-525$.

Pogue-Geile, M. (1989) The prognostic significance of negative symptoms in schizophrenia. British Journal of Psychiatry, 155 (suppl. 7) 123-127.

Pope, H. L Lipinski, J. (1978) Diagmosis in schizophrenia and manic depressive illness. Archives of General Psychiatry, 35, 811-828.

Prentky, R., Lewine, R., Watt, N.\& Fryer, J. (1980) A longitudinal study of psychiatric outcome: developmental variables vs. psychiatric symptoms. Schizophrenia Bulletin, 6, 139-148.

Shepherd, M., Watt, D., Falloon, $I_{*}$ \& Smeeton, N. (1989), The natural history of schizophnenia: a five-year follow-up study of outcome and prediction in a representative sample of schizophrenics. Psychological Medicine, Monograph Suppl. 15.

Soni, S., Hollis, S., Reed, P. \& Musa, S. (1992) Syndromes of schizophrenia on factor analysis. British Journal of Psychiatry, $161,860-861$.

Soskis, D. \& Bowers, M. (1969) The schizophrenic experience: a follow-up study of attitude and posthospital adjustment. Journal of Nervous and Mental Disease, 149, 443-449.

Spitzer, R., Fleiss, J. Endicot, J. \& Cohen, J. (1967) Mentall status schedule. Archives of general psychiatry, 16; $479-493$.

Gibbon, M Endicott, I. (1975) The Global Assessment Scale (GAS). New York State Psychiatric Institute: New York NY.

-mandicott, J. \& Robins, E. (1978) Research diagnostic criteria: rationale and reliabillity. Archives of General Psychiatry, 35, 773-782.

ST:ATA Comporation (1993) STATA Reference Manual: Release 3.I, 6th Ed. College Station:TX.

Stephens, J. A Astrup, C. (1963) Prognosis in "process" and "non-process" schizophrenia. American Journal of Psychiatry, 119, 945-953.

Strauss, J.S. \& Carpenter, W.T. (1978) The prognosis of schizophrenia rationale for a multidimensional concept. Sichizophrenia Bulletin, 4, 56-67.

Thompsom, P. \& Meltzer, H. (1993) Positive, negative, and disorganisation factors from the schedule for affective disorders and schizophrenia and the present state examination. British Journal of Psychiatry, 163, 344-351.

Wechsler, D. (1955) Wechsler Adult Intelligence Scale: Manual: Psychological Corporation: New York.

WHO (1992) WHO coordinated multi-center study on the course and outcome of schizophrenia. WHO: Geneva. 


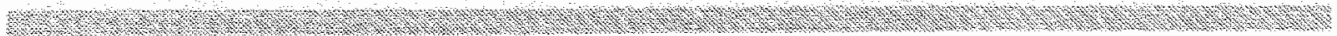

Wing. J.K. Cooper, J.E. \& Sartorius, N. (1974) The Measurement and Classification of Psychuatric Symptoms. Cambridge Uniwersity Press, Cambridge. 
$\therefore$

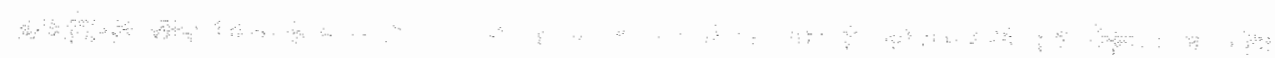


Chapter 4: Risk Factors for Emergence and Persistence of Psychosis 


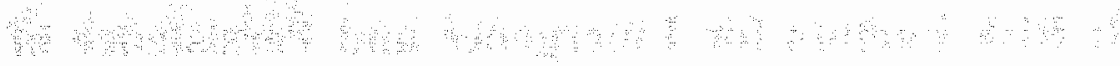




\subsubsection{Puberty and the onset of psychosis}

\section{SUMMARY}

According to the neurodevelopmental hypothesis of schizophrenia, maturational events in the brain at puberty interact with congenital defects to produce psychotic symptoms. As girls reach puberty at a younger age than boys, we predicted that (i) women would show earlier onset of psychotic illness arising around puberty, and (ii) onset of psychosis in women would be related to menarche. Analysis of epidemiological data regarding admission to psychiatric units in a) England over the period 1973 - 1986, b) France over the period 1975-1980, as well as examination of 97 psychotic adolescents referred to an adolescent unit over a 14 year period, supported both these propositions.

\section{INTRODUCTION}

"Then what a change in the mental activity of the brain does the period of puberty cause. Looking at the matter from the combined point of view of physiologists and psychologists, we must connect the new development of the affective faculties, the new ideas, the new interests in life, the new desires and organic cravings, the new delight in certain sort of poetry and romance, with a new evolution of function in certain parts of the brain that had lain dormant before" (Thomas Clouston, 1892).

Why do the characteristic delusions and hallucinations of schizophrenia seldom occur before adolescence? Several recent explanations have been proposed along the lines originally put forward by Clouston in his discussion of "developmental insanity". According to these, late developmental events in the brain underlie the emergence of psychotic symptoms (Feinberg 1982/83; Weinberger, 1987; Murray et al, 1988; Saugstad, 1989). Because early adolescence is a period of profound endocrine changes, many have speculated that the process of puberty triggers the maturational events that produce psychosis; indeed, Kraepelin (1909) already considered that menarche and onset of psychosis were related.

Puberty occurs in the growing boy or girl as the gonads change from the infantile to the adult state, but there are marked sex differences in the timing of its principal manifestations. The adolescent growth spurt begins in girls at about the age of 10.5, and in boys at about 12.5 years of age (Marshall \& Tanner, 1986). There are smaller differences in the age at which the first secondary sex characteristics appear, boys' genitalia begin to develop four months to 1 year later than girls' breasts (Taranger \& Karlberg, 1976; Taranger et al, 1976; Largo \& Prader, 1983a, 1983b). The steepest rise in testosterone concentrations in boys occurs between about 12 and 14 years of age; in girls the most dramatic rise of estradiol is between 11 and 13 years of age (Preece, 1986).

If puberty underlies the events that initiate schizophrenia, as some neurodevelopmental theories predict, then, since puberty in women declares itself at a younger age than in men, onset of psychosis around puberty should follow this trend. To examine the hypothesis that the 
differential onset of puberty in men and women results in earlier onset of psychotic symptoms in girlls, we examined data regarding first admissions to mental illness hospitals in both England and France. In order to examine diagnostic issues and to establish the relationship between menarche and onset of psychosis, we also studied a sample of adolescents, referred to a regional adolescent unit in South London.

\section{EPIDEMIOLOGICAL STLUDIES}

\section{a) England}

Figures were obtained from the Department of Health regarding first admissions for schizophrenia and paranoia (I.C.D. 295 \& 297) to psychiatric hospitals and units in England over the period 1973-1986. As shown in fig. 1, the first admission rates show a dramatic increase from the age of fifteen years in both sexes; this increase is more accentuated in men.

Fig. I First admission rates of schizophrenia and paranoia per 100,000 person-years by age group. England 1973-1986

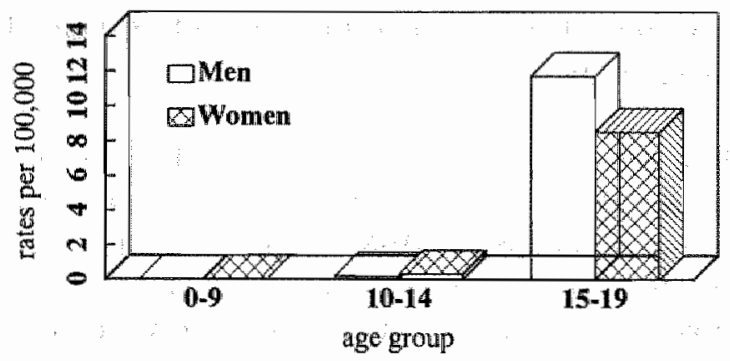

To further examine sex differences, we calculated male to female ratios of first admission rates in consecutive age groups, as shown in fig. 2 . Between ages 10 and 14 years, the rate ratio is 0.85 , indicating a slight, non-significant, female excess $(\mathrm{z}=0.50, \mathrm{p}=0.3,95 \%$ C.I. $0.62-1.17)$. This is followed by a sharp increase in the male to female ratio in each quinquennium until the age of 25 years, and a more gradual decline afterwards. To examine whether the rate ratio shows real variation between age strata, we tested the hypothesis that the ratios in different strata were the same as their pooled summary estimate. The Mantel Haenszel test of the heterogeneity of the rate ratio (Rothman, 1986) across the strata 10-14 years and $15-19$ years, revealed that the observed difference of ratios was highly significant $\left(\chi^{2}=10.39, p<0.01\right)$.

\section{b) France}

Several authors have shown that different diagnostic criteria for schizophrenia produce differences in the ratio of male to female patients (Lewine, 1984; Castle et al, 1991). The above epidemiological findings might therefore be due to the particular diagnostic habits of U.K. psychiatrists, and might not exist in a country where different diagnostic criteria are applied. Therefore, we also examined epidemiological data from France. In this country, a distinction is made between "schizophrenies chroniques" on the one hand, and "bouffée délirante" on the other 
(INSERM, 1969). Schizophrenia is regarded as a chronic syndrome, with onset mostly before the age of 30 (Pichot, 1982, 1984); similarities between the French concept and schizophrenia as defined in DSM-III have been noted (Kellam, 1989).

Fig. 2 Male to female ratios of first admission rates per 100,000 person years by age growp.

England $1973-86$

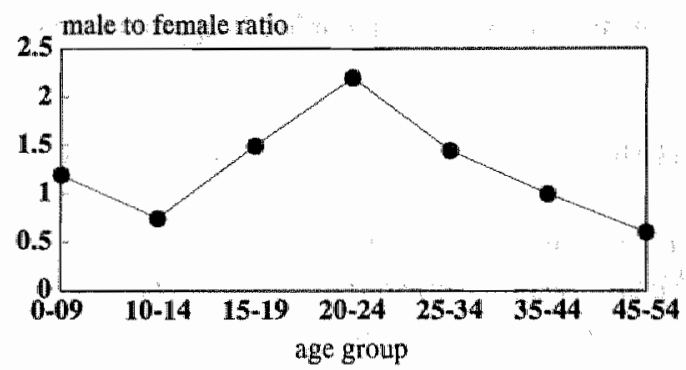

Bouffée delirante refers to acute short-lived psychosis, and may correspond to either acute schizophrenia, affective or other psychosis in ICD-9 (Johnson-Sabine et al, 1983).

Figures were obtained from the Institut National de la Santé et de la Recherche Medicale in Paris concerning first admissions for schizophrenia (INSERM code 02) to state mental illness hospitals and units in France over the period 1975 - 1980. As shown in figure 3, the trend in male to female ratios across age strata is virtually identical to that in England.

\section{CLINICAL STUDY}

Subjects referred between 1976-1990 to the regional adolescent unit at the Bethlem Royal Hospital in South London were selected for study. Nearly all had been in-patients in the unit, which has a flexible male to female bed ratio. Parental and family interviews form a routine part of the multidisciplinary assessment. All medical, nursing, social work, and occupational therapy notes of subjects whose discharge summary indicated the presence of any psychotic symptom (i.e delusion, hallucination, thought disorder or at least two of the following: catatonia, incongruity of affect, blunted affect) were scrutinized by PG. All correspondence and accessory information was also examined. The quality of note-taking in the Bethlem Royal Hospital is high, with considerable standardization of history taking (Institute of Psychiatry Training Committee, 1973).

Fig. 3 Male to female ratios of first admission rates per 100,000 person years by age group. France 1975-80

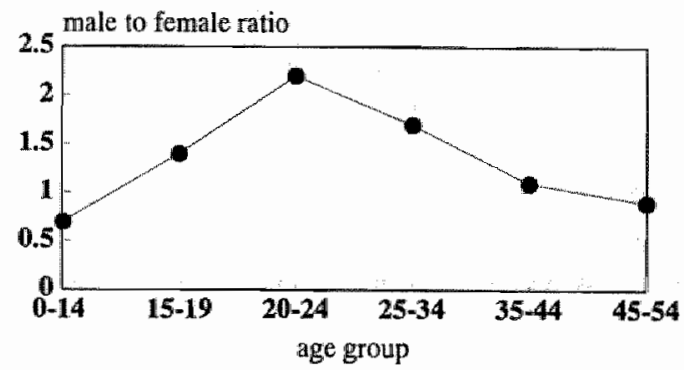


Psychotic symptoms were defined as indicated by Wing ef al (1974), and were rated on a three point scale where 0 indicated that the symptom was absent, 1 that it was doubtfully present or was minimal in severity, and 2 that it was definitely present. Based on all available information, an estimate was made of the age of onset of the disorder. Onset was defined as the emergence of psychotic symptoms that were described with sufficient clarity to rate as 2 . Similarly, the age of first contact with psychiatric services for psychosis was also determined. Age of menarche for girls, defined as onset of menstruation, as recorded in the notes was ascertained.

\section{Sample characteristics}

One hundred-and-three subjects (age range 12-18) with psychotic symptoms were identified. Of these, 6 were excluded because of gross organic pathology. The final sample thus consisted of 49 men and 48 women. $53 \%$ of the sample were Caucasian, 35\% Afro-Caribbean, and $12 \%$ of other ethnic origin.

The ICD discharge diagnoses of the 97 subjects are shown in Table 1. There were no significant differences between men and women as regards ethnicity, presence of mental retardation, or ICD diagnosis, although more women had a diagnosis of affective psychosis. Only two subjects, a boy and a girl, were described in the medical notes as prepubertal.

Table 1. ICD-9 diagnosis by sex in 97 psychotic adolescents

\begin{tabular}{|c|c|c|c|c|}
\hline ICD 9 Diagnosis & Men & & Women & \\
\hline Schizophrenia & 26 & & 24 & 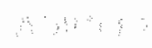 \\
\hline Schino-afiective disorder & 7 & & 8 & \\
\hline Bipular disorder & 1 & & 4 & \\
\hline Major depression & 0 & $\therefore$ & 2 & : \\
\hline Other Psychosis & 15 & & 10 & \\
\hline Total & 49 & & 48 & \\
\hline
\end{tabular}

Age of onset

The mean age of onset of psychotic symptoms was significantly higher for men (mean=14.8, S.D.=1.3) than for women (mean=14.1, S.D. =1.1, $\mathrm{p}=0.006$ ). Age of first contact with services for psychotic symptoms was also significantly higher for men $(p=0.016)$. Breakdown by diagnostic category shows that this is largely due to a sex difference in age of onset which was present in the schizophrenic group, but not in the non-schizophrenic group (table 2). These findings were independent of definition of age of onset (table 2).

To facilitate comparison with our epidemiological samples, we divided subjects with a diagnosis of schizophrenia or schizo-affective disorder into two groups, one with onset before the age of fifteen and one age 15 or older, for each of the two definitions of onset. 
Table 2. Mean age of onset by sex and diagnostic category

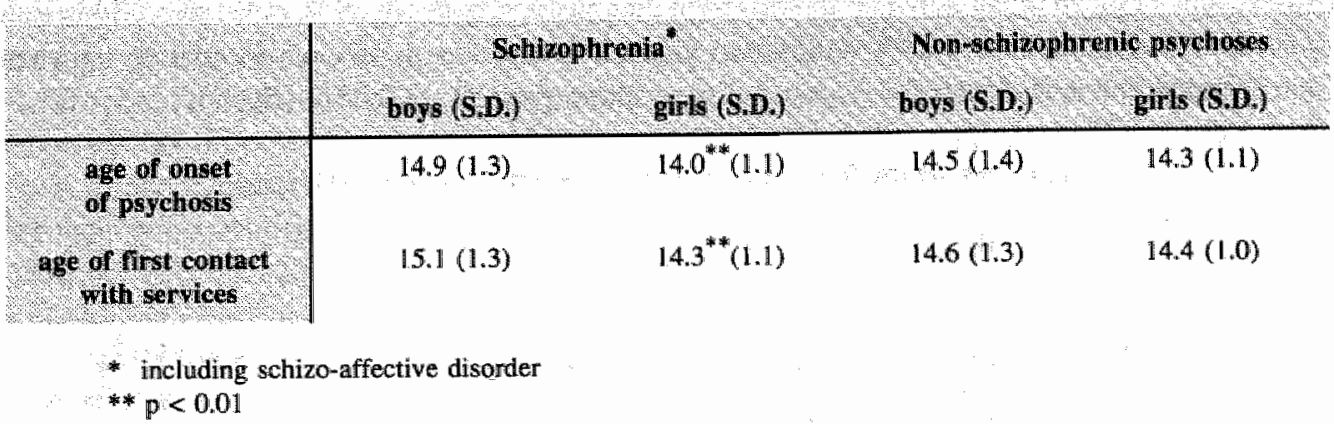

We then compared the numbers of men and women in each group in a $2 \times 2$ design, using the Chi-square statistic with continuity correction to test for significance. There were significantlly more girls in the early onset group (table 3 ).

Table 3. Onset of psychotic symptoms by sex and age group in the group of schizophrenic subjects

\begin{tabular}{|c|ccc}
$\begin{array}{c}\text { age of onset } \\
\text { psychosis }\end{array}$ & men & women & $\chi^{2}$ \\
$(\mathbf{p})$
\end{tabular}

(* = including schizo-affective disorder)

These findings might be biased in that boys, because of later onset of puberty, could remain longer than girls under the care of child psychiatric services rather than those for adolescents. We therefore examined records of admissions to the Maudsley children department with a psychotic discharge diagnosis (ICD 295, 296, 297, 298 and 299) over a five year period, and found only two boys and one girl aged 11 or older, who had presented with non-organic psychosis as defined in our inclusion criteria. None had been referred to the adolescent unit in the first instance. All three were under the age of 13 , and reanalysis of our data, taking into account these admissions to the child psychiatric department (based on the assumption that over fourteen years there would have been 6 male and 3 female admissions with psychosis), showed no significant difference from the original results. It is therefore unlikely that our sample was biased in this sense.

\section{Menarche}

In those girls in whom both the age of menarche and age of onset of psychotic symptoms was recorded in the notes (schizophrenia $n=20$, other diagnoses $n=12$ ), the mean delay between 
menarche and onset of psychotic symptoms was one year, four months. There were no significant differences in mean delay between the different diagnostic groups. In $59 \%$, onset was within one year after menarche and in $88 \%$ within two years. There was a significant correlation between age at menarche and onset of first psychotic symptom $(r=0.54, p=0.001)$ (fig. 4).

Fig. 4 Onset of psychosis and menarche: scatterplot and linear regression

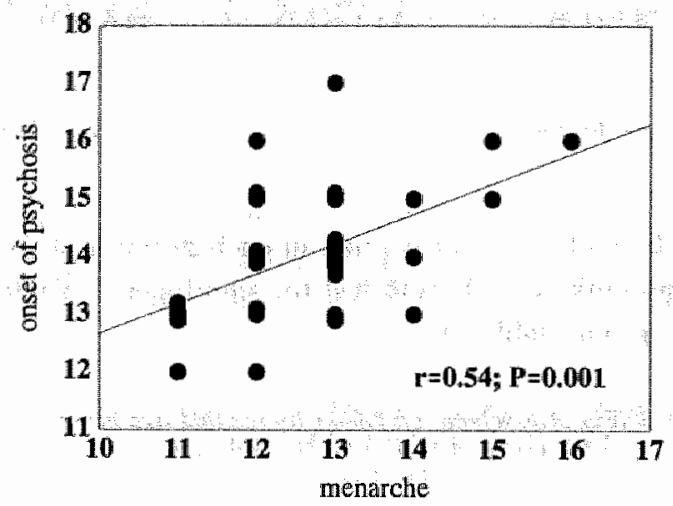

\section{DISCUSSION}

\section{The findings}

Examination of first admission rates of individuals who received a diagnosis of schizophrenia or paranoia in England over a 13 year period demonstrated a slight, non-significant female excess in the age group 10-14 years, followed by a growing male surplus as rates rose sharply with increasing age. There was a highly significant change in the male to female ratio across the age strata 10-14 years and 15-19 years. These findings were replicated in France. Furthermore, we found that the majority of women in our clinical sample had onset of psychosis within one year after menarche, and that the two were significantly related.

It appears, therefore, that there is no male excess in schizophrenia around puberty, and although our evidence is not definitive, it is suggestive of earlier onset in girls. This conclusion, however, is seemingly at odds with the well replicated finding that men manifest schizophrenia at an earlier age than women (Lewine et al, 1981; Loranger, 1984; Angermeyer \& Kühn, 1988). Comparison of age distribution curves for schizophrenia consistently show a higher proportion of young men and an excess of older women (Castle \& Murray, 1991). Sex distribution curves, however, are not isomorphic and some girls do manifest schizophrenia in adolescence. Because of the small numbers of very early onset cases in most studies, trends regarding sex differences around the time of puberty are likely to be obscured, and to date no study has addressed this issue specifically. From the child psychiatric literature, however, it can be inferred that very early onset cases do not necessarily follow the adult pattern. Although many authors have described an excess of boys among schizophrenics with onset in earlier childhood (e.g. Green et al., 1984; Russel et al., 1989), there appears to be a trend towards an increasing proportion of female patients near puberty. For instance, in the study of Green et al (1984) the male to female ratio in subjects with DSM-III schizophrenia was 7:1 for the age range 6-8 years but fell 
to $1: 1$ between $9-11$ years. Reanalysis of data from Gillberg (Gillberg, 1986; personall communication, 1992), regarding a population-based survey of all teenagers (age range 13-19) treated as in-patients for operationally defined psychotic disorders, demonstrates that for schizophrenia there was a significantly earlier age of onset in girls. Bettes \& Walker (1987) showed that in a sample of 1084 children aged 5-18 years with a psychotic diagnosis (nearly $90 \%$ schizophrenia), there was a dramatic increase in positive symptoms such as delusions and hallucinations in girls between ages $9-10$ and $11-12$ years, whereas boys showed this increase between ages 11-12 and 13-14 years. These findings corroborate our results.

Our epidemiological data allowed us only to analyze for sex differences in 5 year age groups, and we were not able to produce age distribution curves for both sexes based on data over single years. The change in the sex ratio over quinquennia, however, has been used in many previous studies to demonstrate differential age of onset for men and women in schizophrenia $(\mathrm{e} . \mathrm{g}$ Lewine et al, 1981; Loranger, 1984). Our clinical sample was not epidemiologically based, but it is difficult to conceive how bias could operate in, for instance, very young female schizophrenics being admitted to hospital, but not their male counterparts.

It would appear that the dramatic rise in psychotic symptomatology around puberty takes place earlier in women than in men, followed by a substantial male preponderance later in adolescence.

\section{Interpretation}

The temporal association between puberty and onset of psychosis begs the question of what the exact nature of this relationship might be. Little is known about the relation between puberty and various developmental processes that may precipitate psychosis. Some authors (Feinberg 1982/83; Saugstad, 1989), citing evidence that the devellopmental regression of excessive interneuronal contacts persists into the second decade of life (Huttenlocher, 1979), have suggested that schizophrenia may arise as the result of errors in this process of maturational reorganization of the human brain. The reduction in synaptic density, however, starts well before puberty, and it is therefore unlikely that this process is triggered by, for instance, sex hormones (Feinberg, 1990). Others (Weinberger, 1987; Murray et al, 1988) advanced the theory that this process of synaptic pruning, or other developmental changes during pubescence, may allow latent, congenital defects in neurodevelopment to become manifest. Animal studies have provided a model of how a fixed congenital lesion could remain relatively unapparent until the age of sexual maturation, and then have a profound impact on behaviour (Goldman \& Alexander, 1977). Apart from synaptic pruning, a number of other late developmental events could provide a "permissive" environment for the latent defect to be uncovered during the adolescent period. For instance, myelination in the corticolimbic circuitry during late adolescence may serve to trigger psychotic illness (Benes, 1989), and sex hormones possibly affect myelination (Timiras, 1972).

The course of the development of the brain"s dopamine systems led Finch \& Morgan (1987) to speculate that asynchrony in dopamine receptor ageing might cause schizophrenia. The finding of Wong et al (1984), that dopamine receptors in the striatum and frontal cerebral cortex showed a different pattern of decline in men and women may also be relevant in this regard. The role of sex hormone secretion in adolescence has been considered by many authors. For instance, estrogen may have a direct effect on synaptic structure (Olmos et al, 1987). Hruska \& Silbergeld 
(1980) reported an enhancement of striatal dopamine response by the female sex hormone; a sudden surge of gonadal steroids could set of a chain of chemical events in predisposed individuals, resulting in illness. However, a potent anti-dopaminergic activity of estrogens has also been described (Raymond et al., 1978), and female sex hormones may thus act as a protective factor in schizophrenia by raising the valnerability threshold for psychosis in women (Seeman, 1985; Häfner et al, 1991). If estrogen acts as a biological modulator, its protective effect might not be established until late adolescence, as secretion of the gonadotrophins and principal gonadal steroids in its regular cyclical pattern will not be established immediately after menarche; only $14 \%$ of menstrual cycles are ovulatory in the first year postmenarche, with $50 \%$ ovulatory by 3 years (Apter, 1980). This would mean that before completion of the changes associated with puberty, girls are susceptible to psychosis, and that afterwards they are protected for some time, whereas in boys puberty does not have such a protective effect. Our finding of an excess of girls in early adolescence, followed by a male surplus after the age of 15 is, therefore, compatible with such an estrogen protection effect.

Another interpretation to consider is that psychological and biological changes associated with puberty may cause a subgroup of girls, who are genetically predisposed, to develop a psychotic illness. For instance, stress or hormonal changes associated with menarche may act as a precipitant. Fluctuation of hormone levels has been implicated in the development of puerperal psychosis (Kumar \& Brockington, 1988), and several surveys have demonstrated an increase in the number of admissions for psychotic disorders during the para-menstrual phases of the menstrual cycle (4-5 days before and during the onset of menses) (e.g. Targum et al, 1991).

More research is needed in order to clarify the temporal relationship between puberty and the onset of psychosis. Much could be learned from study of morbid risk of psychosis in relatives of subjects with very early onset psychosis. A number of non-invasive techniques have been used to assess brain maturational processes, such as magnetic resonance imaging (Jernigan et al, 1990), ellectroencephalography (Feinberg, 1982/83) and evoked potentials (Lafrenière et al, 1990). Exploration of the functional significance of these maturational processes in relation to the onset of psychosis could provide a challenging arena for the study of brain-behaviour relationships. 


\section{REFERENCES}

Angermeyer, M. \& Kühn, L. (1988) Gender differences in age at onset of schizophrenia. European Archives of Psychiaury and Neurological Seiences, 237, 351-364.

Apter, D. (1980) Senum steroids and pituitary hormonies in female puberty: A partly longitudinal study. Clinical Eindocrinology, 12, 107-120.

Benes, F.M. (1989) Myelination of cortical-hippoccampal relays during late adolescence. Schizophrenia Bulletin, $15,585-593$.

Bettes, B.A. \& Walker, E. (1987) Positive and negative symptoms in psychotic and other psychiatrically disturbed children. Journal of Child Psychology and Psychiarry, 28, 555-568.

Castle, D.J. \& Murray. R.M. (1991) The neurodevelopmental basis of sex differences in schizophrenia. Psychological Medicine, 21, 565-575.

-_._--, Wessely S., Der, G., et al (1991) Is operationally defined schizophrenia more common in men? Paper presented at the International Congress on Schizophrenia Research, Tuscon : April 21-25.

Clouston, T. (1892) In Clinical Lectures on Mental Diseases, 3rd edition. London: J. \& A. Churchill.

Feinberg, I., Thode, H.C. Ir, Chugani, H.T, et al (1990) Gamma distribution model describes maturational curves for delta wave amplitude, cortical metabolic rate and synaptic density. Journal of Theoretical Biology, $142,149-61$.

-., (1982/83) Schizophrenia: caused by a fault in programmed synaptic elimination during adolescence? Journal of Psychiatric Research, 17, 319-34.

Finch, C.E. \& Morgan, D. (1987) Aging and schizophrenia: a hypothesis relating asynchrony in meural aging processes to the manifestations of schizophrenia and other neurological diseases. In Schizophrenia and Aging (eds. N.E. Miller \& D. Cohen) pp 97-108. New York: Guilford.

Gillberg, C. (1986) Teenage psychoses- epidemiology, classification and reduced optimality in the pre-, peri- and neonatal periods. Joumal of Child Psychology and Psychiatry, 27, 87-98.

Goldman, P.S. \& Alexander, G.E. (1977) Maturation of prefrontal cortex revealed by local reversible cryogenic depression. Nature, 267, 613-615.

Green, W.H., Campbell, M., Hardesty, A.S, et al (1984) A comparison of schizophrenic and autistic children. Journal of the American Academy of Child Psychiatry, 23, 399-409.

Hruska, R.E., \& Silbergeld, E.K. (1980) Increased dopamine receptor sensitivity after estrogen treatment using the rat rotation model. Science, $208,1466-1468$.

Huttenlocher, P.R. (1979) Synaptic density in human frontal cortex-development changes and effects of aging. Brain Research, 163, 195-205.

Institut National de la Santé et de la Recherche Médicale, Section Psychiatrie (1969) Classification Française des Troubles Mentaux. Bulletin de l'Institut National de la Santé et de la Recherché Médicale, 24, supplément no. 2.

Institute of Psychiatry Training Committee. (1973) Notes on Eliciting and Recording Clinical Information. Oxford: Oxford University Press.

Johnson-Sabine, E.C., Mann, A.H., Jacoby, R.J., et al (1983) Bouffee delirante: an examination of its current status. Psychological Medicine, 13,771-778.

Kellam, A.P.M. (1989) French empirical criteria for the diagnosis of non-affective non-organic psychoses. Comparison between the critera suggested by Professors Pull and Pichot and those of DSM-III-R. British Journal of Psychiatry, 155, 153-159.

Koh, T. \& Eyre, J. (1988) Maturation of the corticospinall tracts assessed by electromagnetic stimulation of the motor cortex. Archives of Disease in Childhood, 63, 1347-1352.

Kraepelin. E. (1909) In Psychiatrie, ein Lehrbuch für Studierende und Ärtzte (achte Auflage). Leipzig: Verlag von Johiann Ambrosius Barth.

Largo, R. H. \& Prader, A. (1983a) Pubertal development in Swiss boys. Helvetica Paediatrica Acta, 38, 211-228. , \& Prader . A. (1983b) Pubertal development in Swiss girls. Helvetica Paediatrica Acta, 38, 229-243.

Lewine, R., Straus, J. \& Gift, E. (1981) Sex differences in age at first hospital admission for schizophrenia: fact or artefact. American Journal of Psychiatry, 138, 440-444.

-.-.-. Burbach, D. \& Meltzer, H. (1984) Effect of diagnostic criteria on the ratio of male to female schizophrenic patients. American Journal of Psychiatry, 141, 84-87.

Loranger, A.W.(1984) Sex difference in age at onset of schizophrenia. Archives of General Psychiatry, 41, 157161.

Marshall, W.A. \& Tanner, J.M. (1986) Puberty. In Human Growth vol II (eds F. Falkner \& J.M. Tanner) pp. 171-209. New York: Plenurn Press. 
Miller, K. Hormberg, $W$, \& Lenard, H. (1991) Magnetic sfimulation of motor cortex and nerve roots in children. Maturation of cortocimotoneuronal projections. Electroencephelography and Clinical Neurophysiology, 81. $63-70$.

Murray, R. M. Lewis, S.W. Owen, M.J, et al (1988) The neurodevelopmental origens of dementia praecox. In Schiophrenas the Major lssues (eds P. Bebbington \& P. MeGuffon) 90 90-107. Oxford: Heinemann.

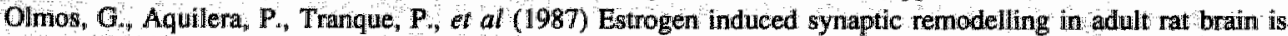
accompanied by the reorganization of newronal membranes, Brain Research, $425,57-64$.

Pichor, $\mathrm{P}$ (1982) The diagnosis and classification of mental disorders in French speaking countries background, current vew and comparison with ohter nomenclature. Psychological Medicine, 12, 475-492

(1984) The French approach to claissification Brith Jownal of Psychiatry, 144, 113118.

Preece, M,A. (1986) Prepubertal and pubertal endocrinology. In Human Growth vol II (eds F. Falkner \& J.M. Tanner) pp: 211-224. New York: Plenum Press.

Raymond, V, Beaulieu, M, Labrie, $F$, et al (1978) Potent antidopaminergle activity of estradiol at the pituitary level of prolactin release. Science, $200,1173-1175$.

Rothman, KJ: (1986) Madern Epidemiology. Boston: Little, Brown and Company.

Russel, A. T., Bott, L. \& Sammons, C. (1989) The phenomenology of schizophrenia occurring in childhood. Jourtwal of the American Academy of Child and Adolescent Psychiotry, 28, 399-407.

Saugstad, L.F. (1989) Social Class, Marriage, and fertility in schizophrenia. Schizophrenia Bulletin, 15, 9-43.

Seeman, MV (1985) Sex and schizophrenia. Canadian Journal of Psychiatry, 30, 313-315.

Taranger, $J$ \&arlberg, P. (1976) The somatic development of children in a Swedish urban community: VII. Graphic analysits of biological maturation by means of maturograms Acta Paediatrica Scandinavica (Suppl). 258, 136-146:

-, Lichtensteitn, H. \& Svennberg-Redegren, I. (1976) The somatic development of children in a

Swedish urban community: VI, Somatic pubertal development, Acta Paediatrica Scandinavica (suppl), 258, 121-135.

Timiras, P. (1972) Physiologic changes at adolescence. In Developmental Physiology and Aging (ed. P.S. Timiras) pp 364-374. New York: Macmillan

Weinberger, D.R (1987) Implications of nomal brain dewelopment for the pathogenesis of schizophrenia. Archives of General Psychiatry, 44, 660-669.

Wing, JK, Cooper, J.E. Sartorius, N. (1974) The Measurement and Classification of Psychiatric Symptoms. London: Cambridge University Press.

Wong, D.F., Wagner, H.N., Dannals, R.F. el al (1984) Effects of age on dopamine and serotonin receptors measured by positron tomography in the living human brain. Science, 226, 1393-1396.

Yakovlev, P.I. \& Lecours, A.R. (1964) The myelogenetic cycles of regional maturation of the brain. In Regional Development of the Brain in Early Life (Ed. A. Minkowski) pp 3-70. Boston: Blackwell Scientific Publications. 


\subsubsection{Gender, psychopathology, and development: from puberty to early adulthood}

\section{SUMMARY}

We tested the hypothesis that the expression of schizophrenic psychopathology is dependent on the stage of adolescent development. The study had a retrospective design, using high-quality case-note material of cases of schizophrenia at first admission. One-hundred-and-eighty patients with onset of illness between age 11 and age 21 years were included. Sexual delusions were more apparent in women (OR=3.6; 95\% CI 1.6-8.0), but otherwise no gender differences in the frequency of a range of positive symptoms were apparent. There was evidence that the age at which positive symptoms first appeared differed between men and women. The frequency of typical, "first rank", schizophrenic symptoms such as auditory hallucinations, passivity phenomena and thought interference, increased linearly with age in male patients, but did not vary with age in their female counterparts. The likelihood of displaying delusional beliefs such as persecutory delusions, explanatory delusions, delusions of reference and grandiose delusions increased with age in both sexes, but the association was stronger in men. The observation that typical schizophrenic symptoms in male patients are relatively uncommon during early adolescence, but increase in frequency as they grow older, could be explained by the later manifestation of puberty and associated maturational processes in boys.

\section{INTRODUCTION}

A developmental influence on the psychopathology of psychotic disorders in childhood and adolescence has been described by several authors. Psychotic symptoms tend to be relatively crude and poorly elaborated at an early age, compared with those described in older subjects. (Kolvin et al, 1971; Russel et al, 1989; reviews by Tsiantis et al, 1986; Werry, 1992). However, comparisons using detailed psychopathological measures over an extended age range have been lacking, and while the disparities in psychopathology between childhood and adolescent schizophrenia on the one hand, and adult schizophrenia on the other are considered quantitative rather than qualitative by some (Werry, 1992), these differences have not yet been subject of evaluative research.

The neurodevelopmental hypothesis of schizophrenia postulates that developmental events in the brain underlie the emergence of psychotic symptoms (Feinberg 1982/83; Weinberger, 1987; Murray et al, 1988; Saugstad, 1989). Because early adolescence is a period of profound maturational changes, many have speculated that the process of puberty triggers the events that produce psychosis. Such theories are compatible with the massive increase in the incidence of psychotic conditions seen around and after puberty, and with highly significant changes in the sex ratio around puberty, indicating that age of onset figures are closely mirrored by the timing of maturational processes (Kraepelin, 1919; Bettes \& Walker, 1987; Galdos et al, 1993a; Galdos et al, 1993b). We wished to investigate whether psychotic illnesses arising in this period of life are associated with a particular pattern of psychopathology. We hypothesized that positive symptoms would become more frequent with increasing age, and that men, analogous to gender 
differences in the timing of puberty, would 'lag behind' women in the development of positive schizophrenic symptoms between early adolescence and early adulthood.

\section{METHOD}

\section{Sample}

Subjects were incident cases (first admissions) of psychosis, consecutively referred to hospital to i) the adolescent unit, and ii) the adult units. Both the adolescent and the adult samples have been described before in detail (Galdos et al, 1993a; Harvey et al, 1990; Jones et al, 1993). Briefly. the adolescent sample consisted of subjects (age range: 11-18 years) referred between 1976-1990 to the regional adolescent unit at the Maudsley and Bethlem Royal Hospital in South London, selected if their discharge summary indicated the presence of any psychotic symptom (i.e. delusion, hallucination or thought disorder) in clear conseiousness. Adult cases of similarly defined functional psychosis, aged 16 to 60 years, who had taken part in a previous study (Harvey et al, 1990; Jones et al, 1993), were drawn from two cross-sectional samples of consecutive patients, admitted to the Maudsley and Bethlem Royal and King s College Hospital in South London.

All medical, nursing, social work, and occupational therapy notes of all adolescent patients, and of patients from the adult sample with onset of illness up to age 21 years, were scrutinized. Thus, the examined age of onset range was from age 11 to age 21 years. This age range was taken to include the period of the most important developmental processes of possible relevance to the onset of psychosis, such as typical pubertal changes (Marshall \& Tanner, 1986), myelination in the corticolimbic circuitry during late adolescence (Benes, 1989), and developmental regression of excessive interneuronal contacts persisting into the second decade of life (Huttenlocher, 1979).

All correspondence and accessory information was also examined. The quality of note-taking in the Maudsley and Bethlem Royal Hospital and King's College Hospital is high, and histories and mental states are collected using a semi-structured interview (Institute of Psychiatry Training Committee, 1973).

Psychotic symptoms were defined as indicated by Wing et al (1974), and were rated on a three point scale where 0 indicated that the symptom was absent, $\mathbb{1}$ that it was doubtfully present or was minimal in severity, and 2 that it was definitely present. In the analyses, only a rating of 2 was considered valid. Based on all available information, an estimate was made of the age of onset of the disorder. Onset was defined as the emergence of psychotic symptoms that were described with sufficient clarity to rate as 2 .

\section{Diagnosis}

The Operational Criteria Checklist for Psychotic Ilness (OCCPI; McGuffin et al, 1991) was completed for all patients. The OCCPI check-list is based on phenomenological descriptions in the Present State Examination (Wing et al, 1974), and covers a wide range of operational 
definitions of psychiatric conditions. The computer program OPCRIT (McGuffin et al, 1991) was used to analyze the OCCPI data. DSM-III-R diagnoses were grouped into affective psychoses (manic, bipolar and depressive psychoses), schizophrenia (including schizophreniform psychosis and schizo-affective psychosis), and atypical psychoses (any other psychosis). Reliability of DSM-III-R OPCRIT diagnosis between the two raters was tested on a random sample of 20 subjects. Kappa was satisfactory at 0.69 for DSM-III-R diagnoses.

\section{Psychopathology}

In order to reduce the amount of information provided by the individual psychotic symptoms, symptoms were grouped together into syndromes, according to the PSE Syndrome Checklist (Wing et al, 1974), with some modifications. Only positive symptoms which occurred with a frequency of $10 \%$ or higher were included in the analyses.

Thus, the following syndromes were identified: i) auditory hallucinations; ii) passivity phenomena; iii) thought interference (thought withdrawal, thought broadcasting, thought insertion); iv) explanatory delusions and fantastic delusions (delusions involving hypnotism, physical forces, alien forces, fantastic themes); v) sexual delusions (including delusions of pregnancy); vi) delusions of reference (delusions of reference and misidentification); vii) persecutory delusions; viii) grandiose delusions (including religious delusions); ix) depressive delusions (delusions of guilt, hypochondriasis, catastrophe, nihilistic delusions). The kappa for these 9 syndromes, using the same random sample of twenty subjects, was high (mean: 0.86 ; range:0.73-1.00).

\section{Analyses}

Analyses were conducted with the EGRET statistical software (version 0.26.6, Statistics and Epidemiology Research Corporation, North Carolina, U.S.A.). "The nine psychopathollogical syndromes were the binary response variables, and associations with sex and age of onset (as five consecutive age of onset groups) were examined in logistic regression models. First, age of onset and gender were entered together, so that the effect of each could be examined while adjusting for the other. Odds ratios and $95 \%$ confidence intervals for the explanatory variables were calculated from the result of the logistic regression (Breslow \& Day, 1980). Subsequently, the model was extended with gender by age of onset interactions, and tested for significance using the likelihood ratio statistic. Stratified analyses were performed if there was evidence of significant interaction, using classical epidemiological methods, such as those of Mantel and Haenszel (1959). Kappa's were calcullated with the SPSS for Windows software (Norusis/SPSS INC., 1990). 


\section{RESULTS}

\section{The sample}

The sample consisted of 180 subjects (97 from the adolescent unit; 83 from the adult units); 106 $(59 \%)$ were male, and 74 (41\%) female. Two subjects in the adolescent sample were described as pre-pubertal. One hundred and fourty-seven subjects ( $82 \%$ ) had a diagnosis of broadly defined schizophrenia (men: $60 \%$ ), $20(11 \%)$ of affective psychosis, and $13(7 \%)$ of delusional disorder and atypical psychosis (percentage men un non-schizophrenic psychoses. $52 \%$ ).

Mean age of onset tended to be lower in the schizophrenic psychoses (16.0 years; $\mathrm{SD}=2.6$ ) compared with the other psychoses $(17.2 ; \mathrm{SD}=2.7$ in both other categories, $F=2.7 ; p=0.07)$. Mean age of onset was 16.8 (SD: 2.5 ) in male subjects, and 15.5 (SD: 2.7$)$ in women $(t=3.4 ; p=0.001$ ). Possible explanations for this discrepancy in age of onset have been discussed previously (Galdos et al, 1993a). There was no evidence that earlier age of onset in women was confined to any particular diagnostic category (ANOVA sex by diagnosis interaction: $F=0.13 ; p=0.9$ ).

The mean difference between actual age at admission and age at onset (henceforth called illness duration) was small at 0.49 years (SD 0.9 years); the median value was 0.0 . There were no differences in illness duration between men and women $(t=0.09 ; p=0.9)$ or diagnostic categories $(F=0.5 ; p=0.7)$, nor was illness duration associated with age of onset $(r=0.03 ; p=0.7)$.

\section{Psychopathology in schizophrenic patients}

In the first instance, analyses were confined to the group of schizophrenic patients only (Table 1). The likelihood of displaying delusional syndromes, except depressive and sexual delusions, increased with age. Evidence of an association with gender was only present in sexual delusions, women more frequently displaying this symptom.

Evidence of significant interaction was present in typical schizophrenic symptoms such as auditory hallucinations, passivity phenomena, and thought interference. Subsequent stratified analysis

revealed that there was a strong linear trend in the association with these symptoms in men, but not in women (Table 2).

\section{The problem of diagnostic inaccuracy in adolescence}

One of the problems in examining psychopathology in adolescence is the well known interchangeability between schizophrenic and affective features (Zeitlin, 1983), associated with unexpected responses to lithium in individuals diagnosed as schizophrenic (Horowitz, 1977). Furthermore, clinicians are not infrequently faced with "atypical" presentations, that are not clearly schizophrenic, but nonetheless are associated with severe disability and requirement of long-term care (Steinberg, 1985). In view of the above factors, misdiagnosis is common, and especially in younger adolescents some time has to pass before the diagnosis can be established (Zeitlin, 1983; Steinberg, 1985; Werry, 1991). Therefore, we repeated the comparisons between 
adult and adolescent patients, using all patients with a diagnosis of functional psychosis. The results thus obtained were similar: significant associations were found between age of onset and the delusional syndromes, with the same exception of sexual delusions (significant association with gender) and depressive delusions (no association with either sex or age of onset).

Table 1, Psychopathological syndromes and associanons with sex and age of onsel: (age $11-21$ years)

\begin{tabular}{|c|c|c|c|c|c|c|}
\hline \multirow[t]{2}{*}{ Psychopathological syndromes } & \multicolumn{2}{|r|}{ sext } & \multicolumn{2}{|c|}{ age or onsett } & \multicolumn{2}{|c|}{$\begin{array}{l}\text { sex by age of } \\
\text { onsel } \\
\text { interaction lorm }\end{array}$} \\
\hline & on* & $95 \%$ CI & $0 \mathrm{OR} *$ & $95 \% \mathrm{Cl}$ & LRST & p raline \\
\hline auditory hallucinations & 1.3 & $(0.6-2.7)$ & 1.3 & $(0.9-1.8)$ & 4.9 & 0.03 \\
\hline passivity phenonena & 1.0 & $(0.5-2.3)$ & 1.2 & $(0.9-1.7)$ & 3.9 & 0.05 \\
\hline thought interference & 1.1 & $(0.5-2.2)$ & 1.4 & $(1.0-1.9)$ & 3.5 & 0.06 \\
\hline explanatory delusions & 1.2 & $(0.5-2.8)$ & 2.2 & $(1.5-3.1)$ & 0.1 & 0.8 \\
\hline sexual delusions & 3.6 & $(1.6-8.0)$ & 12 & $(0.8-1.6)$ & 0.7 & 0.4 \\
\hline delusions of reference & 1.3 & $(0.6-2.6)$ & 1.6 & $(1.2-2.2)$ & 1.3 & 0.3 \\
\hline persecutory delisions & 0.9 & $(0.4-1.9)$ & 1.6 & $(1.2-2.2)$ & 1.2 & 0.3 \\
\hline grandiose delusions & 0.7 & $(0.3 \times 1.6)$ & 1.5 & $(1.0-2.1)$ & 0.5 & 0.5 \\
\hline depressive delusilons & 0.8 & $(0.3-1.9)$ & 1.2 & $(0.8-1.7)$ & 0.02 & 0.9 \\
\hline
\end{tabular}

\$: Sex and age of onset were entered together in the logistic regression equations; thus, the effect of each was examined while controlling for the ofher.

* : $\mathrm{OR}=$ odds ratio; eg $\mathrm{OR}=1.3$ means women were 1.3 times more likely to have displayed the symptom:

**: $\mathrm{OR}=$ odds ratio; $\mathrm{eg} \mathrm{OR}=1.3$ means that with each unit in age of onset ( 5 consecutive age groups between ages $11-21)$ the odds of having the symptom increased with a factor 1.3.

$\dagger$ : Likellihood ratio statistic on $1 \mathrm{df}$.

Again, significant sex by age of onset interactions in the same direction were apparent for hallucinations $(p=0.045)$, passivity phenomena $(p=0.01)$ and thought interference $(p=0.02)$. In addition, sex by age of onset interactions approaching statistical significance now also became apparent for persecutory delusions $(p=0.07)$ and delusions of reference $(p=0.08)$. Stratified analysis for these also revealed a significant trend with increasing age in men (persecutory delusions: $p=0.001$; delusions of reference: $p=0.001$ ), but not in women (persecutory delusions: $\mathrm{p}=0.6$; delusions of reference: $\mathrm{p}=0.5$ ). A post-hoc stratified analysis in the schizophrenic category revealed a similar gender discrepancy for both persecutory delusions (men: $p=0.002$; women: $p=0.2$ ) and delusions of reference (men: $p=0.003$; women: $p=0.3$ ).

Although no significant gender by age of onset interactions could be demonstrated in the other delusional syndromes, a stronger age trend tended to be present in male patients. For example, the linear trend in the association between age of onset and presence of explanatory delusions was significant at the $p<0.001$ level in men, but at the $p=0.02$ level in women. However, no such discrepancy was detectable in depressive delusions, whereas in sexual delusions only an association with gender was apparent. 
Table 2. Association between firt rank symptoms and age of onset, by sex.

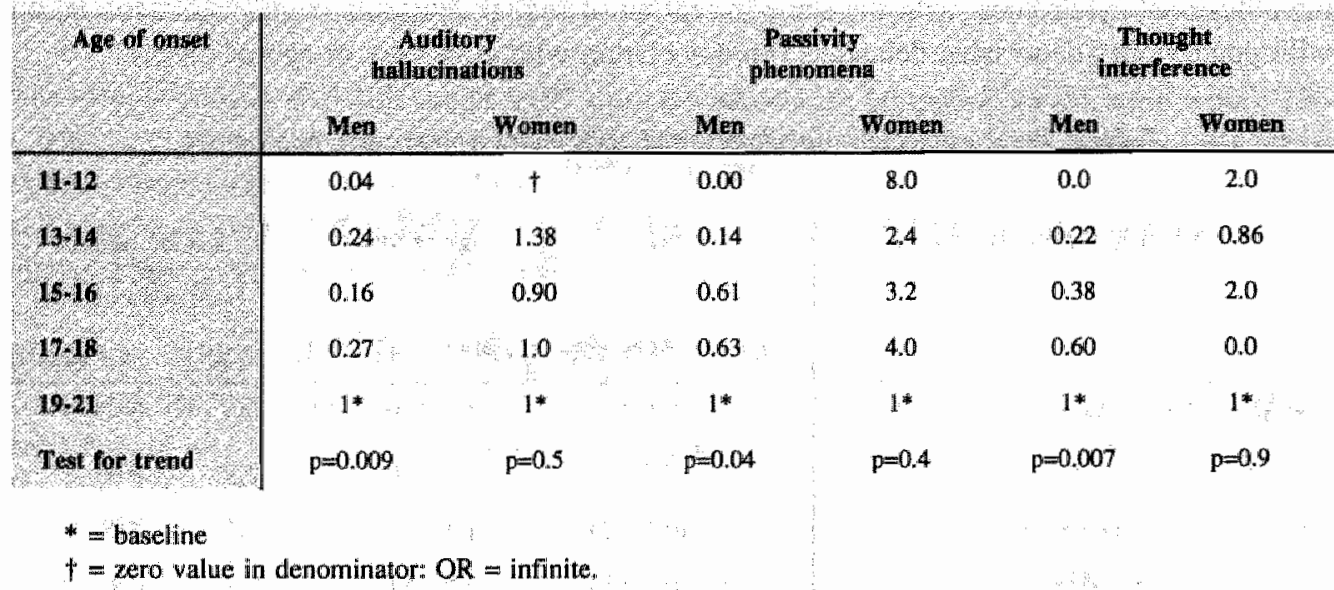

The findings in the combined diagnosis group were not the result of a "confounding" effect of diagnosis, as the parameter estimates changed only by a trivial amount when diagnosis was controlled for in the regression equations.

\section{DISCUSSION}

We found that, with the exception of sexual delusions and depressive delusions, the likelihood of displaying a range of delusional syndromes and typical schizophrenic experiences increased linearly with age from early adolescence to early adulthood. The association was stronger in men, especially for typical schizophrenic phenomena, where no age trend was apparent in women.

\section{Methodological issues}

Retrospective case-note studies are by definition suspect, as they rely on data collected by many different persons with different levels of experience. However, in our study, the most important case-note data were from the hand of psychiatric registrars belonging to the same rotational scheme, who had been trained to conduct the same semi-structured interviews (Institute of Psychiatry Training Committee, 1973). In the training scheme, there has been the same emphasis on positive symptoms, as defined by the PSE, over the period under investigation. Furthermore, if a systematic bias was operating in our data collection, this would be the same for early and later age of onset groups, as well as for men and women. It cannot, therefore, explain the fact that, for typical schizophrenic symptoms, age of onset differences were found in men, but not in women.

Substance misuse is unlikely to have played a role in this study, as only cannabis misuse is possibly sufficiently prevalent among patients in this part of South London to cause significant effects. Subjects with a toxic psychosis due to cannabis would have been excluded from the 
study, and for the drug to have a confounding effect on the reported associations, it would have to be strongly associated independently with psychopathology, age of onset and gender, which: is unllikely.

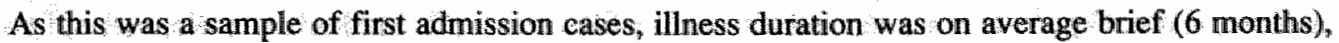
and not associated with either diagnostic category, age of onset, or gender. As actual age at admission corresponded closely to age of onset, the results are not obscured by the separate effects of actual age at admission and age of onset of psychosis.

We conclude that our findings cannot be explained away as artefactual, although we do not dispute the fact that additional data on intelligence and education would have improved the study (see below).

\section{Interpretation of findings}

It has been suggested that brain maturational processes around puberty are the anatomical substrate of the transition to the adult capacity for problem solving and abstract thinking (Feimberg, 1982/83; 1990; Goldman-Rakic; 1987), It is likely that the occurrence of positive symptoms of psychosis in adolescence is related to these maturational processes. The adolescent growth spurt begins in girls at about the age of 10.5, and in boys at about 12.5 years of age (Marshall \& Tanner, 1986). Thus, the later manifestation of puberty and associated maturational processes in boys, could explain why typical schizophrenic ("first rank", Schneider, 1959) symptoms in early adolescence were relatively sparse, as seen in the more immature pattem of psychopathology of prepubertal psychosis, followed by an increase in the frequency of these symptoms through subsequent age of onset groups. In a previous publication (Galdos et al, 1993a), we provided epidemiologic evidence of a temporal relationship between puberty and the onset of psychotic symptoms, with girls showing an earlier onset than boys between the ages of 11-14 years, to be followed by a marked excess of boys among those with onset later in adolescence. The present results are compatible with an association between maturational processes in adolescence and the manifestation of symptoms of schizophrenia. Our findings concur with those of Bettes \& Walker (1987), who showed a first significant increase in positive symptoms in girls between the ages of $9-10$ and $11-12$ years, whereas boys showed this increase between the ages of 11-12 and 13-14 years.

It is now well established that, even before the appearance of typical delusions and hallucinations, many schizophrenic patients display subtle behaviourall, cognitive and motor developmental abnormalities when compared with their peers (Offord \& Cross, 1969; Rutter, 1984; Aylward et al, 1984; Jones \& Murray, 1993). This suggests that developmental events during adolescence interact with some pre-existing abnormality to produce psychotic symptoms (Weinberger, 1987; Murray et al, 1988), rather than an abnormality in the maturational process itself provoking these phenomena, as some authors have proposed (Feinberg, 1982/3; Saugstad, 1989).

Modern neuropsychological theories of schizophrenia have also considered developmental aspects of the formation of typical symptoms. For example, Frith (1994) discusses the work of Shultz and colleagues (1980), showing that in early childhood subjects could not distinguish between 
an intended movement of the lower leg and the reflexive movement initiated by a gentle tap of the knee. This suggests a lack of awareness of intentions, without which children cannot distinguish between intentional and accidental responses. It can be argued that development of intentional awareness is necessary in order to experience passivity phenomena, in that, although pathologically, experiences are recognised as not willed ("made"). Similarly, development of an awareness of the belliefs and intentions of others may be necessary for the formation of, for example, persecutory delusions, as they involve false inferences about the intentions of other people. Frith (1994) discusses experiments showing that in early childhood subjects cannot appreciate that others have false beliefs, and that more complicated tasks assessing beliefs in others cannot be achieved until late childhood:

In the delusional syndromes, it would appear that there is also a more straightforward relation with age, in that some delusions simply depend on age-specific knowledge. For example, explanatory delusions involving mechanisms such as $\mathrm{X}$-rays and electricity require knowledge that depends on educational progress rather than on maturational processes per se. Such a general education effect may explain why, in our study, delusional syndromes showed a stronger age effect (effect of education), and less interaction with gender (indicating an association with biological maturation) than symptoms classically considered experiences rather than beliefs, such as auditory hallucinations, passivity phenomena and thought interference. For example, a young patient might be able to explain that he feels controlled, without elaborating on commonly described mechanisms, such as electronic devices or telepathy. As these sort of explanatory delusions are arguably the most dependent on education, one would expect these to be strongly correlated with age. Our data support this notion, as explanatory delusions showed the strongest association with age, compared to the other psychopathological syndromes.

The fact that we were not able to formally examine associations with education, intelligence and verbal ability, all of which are relevant in this regard (Tsiantis et al, 1986), must be considered a limitation to our study. We feel that it is unlikely, however, that important differences in these variables would have existed between boys and girls.

Differences in age of onset are one of the most robust findings in schizophrenia research (Castle \& Murray, 1991). A substantial proportion of schizophrenic patients display typical delusions and hallucinations before the age of twenty; between the ages of 15 and 19 years, nearly twice as many boys than girls have the onset of the disorder (Galdos et al, 1993a). From our results it follows that differences in the psychopathology among male and female schizophrenics may be confounded by variation in the age of onset of the sample, if adolescent patients are included. Samples containing more early onset cases have a greater chance of finding gender differences (more positive symptoms in women) than samples with a later onset of illness. This may explain some of the inconsistencies in the literature on gender-related differences in the psychopathology of schizophrenia; some studies have reported an increased frequency of positive symptoms such as persecutory delusions in women (Goldstein \& Link, 1988; Forrest \& Hay, 1971), whilst others reported negative findings, or results in the opposite direction (Shtasel et al, 1992; McGlashan \& Bardenstein, 1990). However, the issue of gender differences in the frequencies of specific "first rank" symptoms (Schneider, 1959), such as passivity phenomena, was not addressed in these studies, and figures on the proportion of early onset cases are in general not available. 
In conclusion, the comparative study of the psychopathology in psychotic patients from childhood through adulthood may shed light on the association between cerebral development and symptom expression, as well as on the underlying neuropsychological mechanisms of symptom formation. Gender differences in psychopathology may be confounded by the developmental modification of clinical presentation. 


\section{REFERENCES}

Aylward $\mathbb{E}_{w}$ Walker $\mathbb{E}_{\text {, Bettes }}$ B. (1984) Intelligence in schizophrenia: meta-analysis of the research, Schizophrenia Bulletin, $10,430-459$

Benes, F.M. (1989) Myelination of cortical-hippoccampal relays during late adolescence. Schizophrenia Bulletin, 15, 585-593.

Bettes, B.A. and Walker, E. (1987) Positive and negative symptoms in psychotic and other psychiatrically disturbed children. Journal of Child Psychology and Psychiatry, 28, 555-568.

Breslow, N. \& Day, N. (1980) Statistical Methods in Cancer Research, Vol 2. Lyon: WHO.

Castle, D.J. and Murray, RM. (1991) The neurodevelopmental basis of sex differences in schizophrenia. Psychological Medicine, 21, 565-575.

Feinberg, I., Thode, H.C. Jr, Chugani, H.T, et al (1990) Gamma distribution model describes maturational curves for delta wave amplitude, cortical metabolic rate and synaptic density. Journal of Theoretical Biology, 142, 149-61.

2....... (1982/83) Schizophrenia: eaused by a fault in programmed synaptic elimination during adolescence? Journal of Psychiatry Research, 17, 319-34.

Forrest, A. and Hay, A. (1971) Sex differences and the schizophrenic experience. Acta Psychiaticra Scandinavica, 47, 137-149.

Frith, C. (1994) Theory of mind in schizophrenia. In : The Neuropsychology of Schizophrenia (Eds. A. David \& J. Cutting). Hove: Lawrence Erlbaum Associates.

Huttenlocher, P.R. (1979) Synaptic density in human frontal cortex-development changes and effects of aging. Brain Research, 163, 195-205.

Galdos $P_{n}$, Van Os, J. and Murray, R. (1993a) Puberty and the onset of psychosis. Schizophirenia Research, 10 , $7-14$.

-....., Van Os, J., Takei, N., and Murray, R. (1993b) Puberty and the onset of psychosis. Schizophirenia Research, 10, 279-280.

Goldman-Rakic, P. (1987) Development of cortical circuitry and cognitive functions. Child Development, 58 , 601-622.

Goldstein, J. and Link, B. (1988) Gender and the expression of schizophrenia. Journal of Psychiatry Research, $22,141-155$.

Green, W.H., Campbell, M., Hardesty, A.S., et al (1984) A comparison of schizophrenic and autistic children. Journal of the American Academy of Child and Adolescent Psychiatry, 23, 399-409.

Harwey, I. Wiliams, M., McGuffin, P. and Toone, B. (1990). The functional psychoses in Afro-Caribbeans. British Journal of Psychiatry, 157, 515-22.

Horowitz ${ }_{*},(1977)$ Lithium and the treatment of adolescent manic depressive illness. Diseases of the Nervous System, 38, 480-483.

Institute of Psychiatry Training Committee. (1973) Notes on Eliciting and Recording Clinical Information. Oxford: Oxford University Press.

Jones, P.B., Bebbington, P., Foerster, A. Lewis, S., Murray, R., Russel, A., Sham, P., Toone, B. and Wilkins, S. (1993a) Premorbid social underachievement in schizophrenia. Results from the Camberwell Collaborative Psychosis Study. British Jowrmal of Psychiarry, 162, 65-71.

Kolvin, I., Ounsted, C., Humphrey。 M. and McNay, A. (1971) Studies in the childhood psychoses: II. The phenomenology of childhood psychoses. British Journal of Psychiatry, 118, 385-395.

Kraepelin, E. (1919) Dementia Praecox and Paraphrenia (translated by M. Barclay) pp 230-231. Edinburgh: Churchill Livinstone.

Mantel, N. \& Haenszel, W. (1959) Statistical analysis of data from the retrospective study of disease. Journal of the National Cancer Institute, 22, 719-748.

Marshall, W.A. and Tanner, J.M. (1986) Puberty. In Human Growth vol II (eds F. Falkner and J.M. Tanner) pp. 171-209. New York: Plenum Press.

McGlashan, T. and Bardenstein, K. (1990) Gender differences in affective, schizoaffective, and schizophrenic disonders. Schizophrenia Bulletin, 16, 319-329.

McGuffin, P., Farmer, A.E. Harvey, I. (1991) A polydiagnostic application of operational criteria in psychotic illness: development and reliability of the OPCRTT system. Archives of General Psychiatry, 48, 764-770.

Norusis, M.J./SPSS INC. (1990) SPSS/PC ${ }^{+}$Statistics 4.0. Chicago: SPSS Inc.

Offord DR and Cross LA. (1969) Behavioural antecedents of adult schizophrenia. Archives of General Psychiatry, 21, 267-283.

Russel, A. T., Bott, L. and Sammons, C. (1989) The phenomenology of schizophrenia occurring in chillhood. Journal of the American Academy of Child and Adolescent Psychiatry, 28, 399-407.

Rutter M. (1984) Psychopathology and development: 1.Childhood antecedents of adult psychiatric disorder. 
Australian and New Zealand Journal of Psychiary, 18: 25-234.

Schneider, K. (1959) Clinical Psychopathology. Translated by M.W. Hamilton. London: Grune and Stratton.

Shultz, T.R., Wells, D. and Sarda, M. (1980) The development of the ability to distinguish intended actions from mistakes, reflexes and passive movements. British Journal of Social and Clinical Psychology, 19, 301-310.

Shtasel, D., Gur, R., Gallacher, F., Heimberg, C. and Gur, R. (1992) Gender differences in the clinical expression of schizophrenia. Schizophrenia Research, 7, 225-231.

Steinberg, D. (1985) Psychotic and other severe disorders in adolescence In Child and Adolescent Psychtatry, Modern Approaches (eds M. Rutter and L Hersow) pp 567-584. Oxford Blackwell Scientific Publications.

Tsiantis, J., Macri, I. and Maratos, O. (1986) Schizophrenia in children, a review of European research. Schizophrenia Bulletin, 12, 101-119.

Werry, J.S. (1992) Child and adolescent (early onset) schizophrenia: a review in light of DSM-III-R. Journal of Autism and Developmental Disorders, 22, 601-624.

Werry, J.S., McClellan, J. and Chard, L. (1991) Childhood and adolescent schizophrenic, bipolar, and schizoaffective disdorters: a clinical and outcome study. Journal of the American Academy of Child and Adolescent Psychiatry, 30, 457-465.

Wing, J.K., Cooper, J.E. and Sartorius, N. (1974) The Measurement and Classification of Psychiatric Symptoms. London: Cambridge University Press.

Zeitlin, H. (1983) The Natural History of Psychiatric Disorder in Childhood. MD Thesis, University of London. 
-

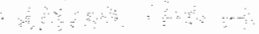

B. $\quad \cdots \quad \because \quad \cdots$

a

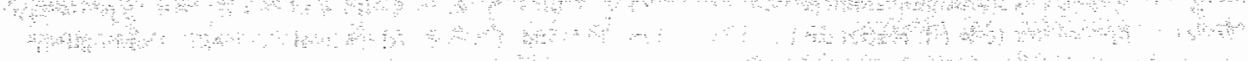

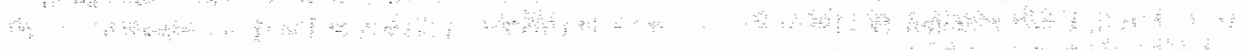
$\therefore \quad \therefore \quad$ - $\quad \therefore \quad$ - $\quad$ -

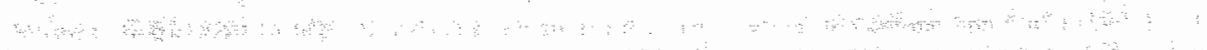

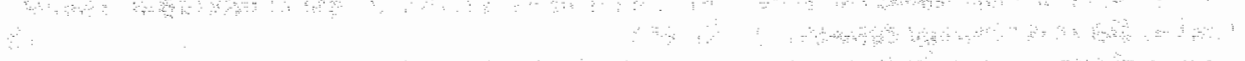

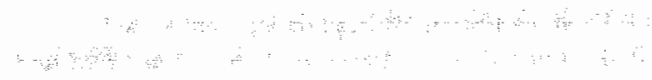




\subsubsection{Increasing age is a risk factor \\ for psychosis in the elderly}

\section{SUMMARY}

We examined the association between ageing and administrative incidence rate of late onset (after age 59) non-organic, non-affective psychosis in two samples of patients aged 60 years or older, who were first admitted to hospital in i) the Netherlands between 1978 and $1992(n=8010)$, and ii) nine Regional Health Authorities in England and Wales $(n=1777)$ between 1976 and 1978. There was a linear trend in the association between increasing age and first admission rates for non-organic, non-affective psychosis in the elderly, after adjustment for the possible confounding effects of time trend and gender, corresponding to an $11 \%$ increase in the incidence with each five year increase in age. These observations support a connection between degenerative brain processes and onset of non-affective psychosis in the elderly.

\section{INTRODUCTION}

Non-affective non-organic psychoses in the elderly are more common in females and typically present as a paranoid delusional illness, often accompanied by hallucinations. Some symptoms associated with early onset illness, such as formal thought disorder, catatonia and affective flattening, are uncommon (Roth, 1955; Pearlson, 1989 et al, 1989; Howard et al, 1993). We have argued that it is premature to regard early and late-onset schizophrenia (onset after the age of 45 or 60 years) as phenotypically homogeneous (Howard et al, 1993), and that to consider the two groups as separate may enhance our understanding of their aetiology (Castle \& Murray, 1993). For example, in late onset schizophrenia, morbid risk of illness in relatives is lower (Kay, 1959; Kay \& Roth, 1961; Post, 1966; Castle \& Howard, 1992), and both sensory deficits (Cooper, 1976; Prager \& Jeste, 1993) and social isolation are thought to contribute to aetiology (Kay \& Roth, 1961; Kay et al, 1976). A substantial proportion of patients have subtle neuropsychological impairments (Naguib \& Levy, 1987) which do not progress to dementia (Hymas et al, 1989). The relation of these deficits to those found in early-onset schizophrenia (Foerster $e t$ al, 1991) is unclear.

Both physiological and pathological age-related changes may contribute to the aetiology and expression of the late-onset psychoses. Surprisingly, however, is has never been established whether or not age is a genuine risk factor (Berrios, 1992). DHSS data (1985) on admissions to NHS mental hospitals and units in England and Wales showed an age-related increase in first admission rates for (ICD9) schizophrenia and paranoid states, the rates rising from 8.7 per 100,000 in the 65-74 age group, to 14.5 per 100,000 in the 75+ age group. According to data presented by Castle \& Murray (1993) the incidence of DSM-III-R schizophrenia in the elderly may increase with age, but the authors did not further explore this issue. Tien (1991) showed an age-related increase of hallucinations in a community sample using trained lay interviewers, but no differentiation was made between organic and non-organic conditions. Thus, there is some

'J. van Os, R. Howard, N. Takei R. Murray (Sacial Psychiarry and Psychiatric

Epidemialogy, 30, 161-165) 
indication that the incidence of non-affective non-organic psychosis in the elderly is in fact agerelated, as is dementia (Bickel \& Cooper, 1994).

We wished to establish whether, within the elderly as a whole, age is a risik factor for late-onset psychotic syndromes, using i) a large, national data set covering psychiatric admissions to mental hospitals and units in the Netherlands and ii) a similar, but smaller data set from nine Regional Health Authorities in England and Wales. We hypothesized that i) the incidence of non-affective non-organic psychosis would increase with age, and ii) that the age-related increase in risk would be evident in both males and females.

\section{METHOD}

Data on psychiatric admissions of individuals aged 60 years and older were obtained from the Stichting Informatie Gezondheidzorg in the Netherlands (SIG; Foundation for Information on Health Care). The SIG keeps records on admissions to institutions for in-patient psychiatric care in the Netherlands, with the exception of admissions to psychiatric wards of general hospitals. These wards represent fewer than $10 \%$ of psychiatric beds, and as the great majority of incident psychotic cases will be admitted to general psychiatric hospitals, few cases are missed by the SIG register (Selten \& Sijben, 1994). The SIG collects information on a standard form, completed by the physician in charge of the patient; if, amongst others, the entries "ICD9 diagnosis", "age" or "sex" have not been completed, the SIG returns the form, to ensure that complete information will be obtained. The SIG register has been running since 1970, and reached a constant and satisfactory level of national data collection in 1978 (Selten \& Sijben, 1994). The data presented here refer to four-digit ICD-9 discharge diagnoses of all first admissions between 1978 and 1992.

Four digit ICD diagnoses by age are not routinely available in the U.K. on a national level. We had, however, in a previous study (Takei et al, 1994) collected such data from 12 Regional Health Authorities in England and Wales. Complete data on first admissions were available for three consecutive years (1976, 1977 and 1978) for nine of the 12 Regional Health Authorities.

Yearly population data for the period 1978-1992 were obtained from the Centraal Bureau voor de Statistiek (CBS) in the Netherlands, and the Office for Population Censuses and Surveys (OPCS) in the U.K. for 1976-1978.

Non-affective non-organic psychosis in the elderly may be coded to any of the following diagnostic categories: schizophrenia (ICD9 295.0 to 295.9), paraphrenia (ICD9 297.2), paranoid states (ICD9 297.0 297.1 297.8 and 297.9), and non-affective other psychosis (ICD9 298.2 to 298.9). These categories were therefore included in the sample, whilst subjects with a second or third ICD diagnosis of organic psychotic condition (ICD 290 to 294) were excluded from the sample. All these functional psychotic disorders will henceforth be referred to as "psychosis". The diagnostic net was cast as wide as possible, since the presentation of (apparently) functional psychosis in the elderly can be extremelly heterogeneous, falling into a range of diagnostic categories. Thus, the results could not be confounded by time trends in diagnostic fashion over the study period (Almeida et al, 1992), or by clinicians' tendency to increasingly apply certain diagnostic categories, such as paraphrenia, with increasing age of their patients. 


\section{Analyses}

Incidence rates in the Netherlands over the period under investigation were calculated for individuals aged 60 years or ollder ( $n=8010$ over 15 years) in five year age bands. First, age and sex-specific rates were calculated for each of 15 years over the study period (1978-1992), and depicted in graph form, connecting the median values of the age-specific rates, thus allowing for visual inspection of the degree of variation of rates over the years: Comection of the median incidence rate values reduced the effect of years with extreme outliers. Because of the limited size of the sample from the nine Regional Health Authorities in England and Wales ( $\mathrm{n}=1777$ over three years), especially among the very old, the cumulative three-year age- and sex-specific incidence rates were calculated for these data, with an upper age cut-off of 75 years and older.

Secondly, to provide statistical support for the observations, a test for trend for incidence ratios was performed using Poisson regression (Stata, version 3.1), always including the person-year data as the "offset". In Poisson regression, the logarithm of the rate is the "response" variablle, and the effects of a range of 'explanatory' variables on the rate can be examined. The exponentiated regression coefficient for a given explanatory variable equals the rate ratio, i.e. the rate in the "exposed" group divided by the baseline rate. A series of models was fitted with time (measured in years between 1978 and 1992, to allow for a time trend of incidence rates in the Dutch data), gender and age as the explanatory variables. Time was fitted by including linear, quadratic, cubic and quartic terms, and examining which polynomial was sufficient to model the long term variation in incidence rates (in fact, a combination of a linear and a quadratic term was adequate). Next, age and sex were included in the model as indicated below. Age by gender interaction terms were fitted to examine whether there was significant heterogeneity in the effect of age at the two levels of gender.

\section{RESULTS}

The incidence of psychosis showed a significant increase with age in both countries (Fig. 1), rising from around 10 cases per 100,000 person-years in the age group 60-65 years, to just over 25 cases per 100,000 person years in the age group $90+$ years in the Netherlands. The test for trend for rate ratios, adjusting for time trend and gender, using Poisson regression, was significant at the p<0.001 level, and corresponded to a $11 \%$ increase in the incidence with each of the seven age groups in the Netherlands (IRR:1.11;95\% Cl:1.09-1.13). In the Dutch data, there was a significant age by gender interaction $(\mathrm{p}=0.02)$, indicating that the association with age was slightly stronger in males (IRR: $1.15 ; 95 \%$ CI:1.11-1.18) than in females (IRR: 1.10; 95\% CI: $1.08-1.12$ ).

In the U.K., the incidence rose from around 10 cases per 100,000 person-years in the age group $60-65$, to around 19 per 100,000 person years in the age group 75 years and over. The test for trend for incidence rate ratios was similarly significant at the $p<0.001$ level, and corresponded to a $29 \%$ increase $(95 \% \mathrm{Cl}: 24 \%-35 \%$ ) with each of the four age groups (Fig. 2). There was no evidence for an age by gender interaction in the U.K. data $(p=0.2)$. 
In both countries, the incidence of psychosis was higher in females, controlling for age and time trend, the rate ratio being $1.6(95 \% \mathrm{Cl}: 1.5-1.7)$ in the Netherlands, and $1.6(95 \% \mathrm{CI} 1.4-1.8)$ in the U.K. There was also a significant time trend indicating that the incidence of non-affective psychosis in the elderly in the Netherlands had decreased slightly with time.

Fig. 1 hichence of non-affective psychosis th the elderly, the Netherlands 1978-92.
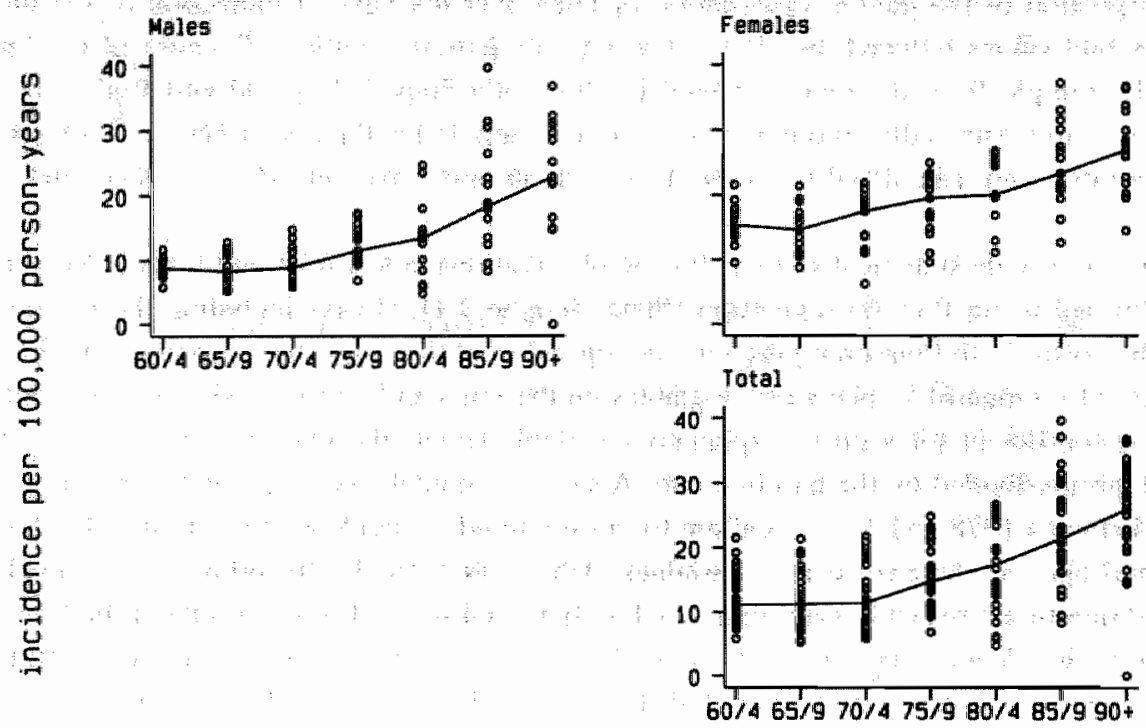

agegroup

Fig. 2 Incidence of non-affective psychosis in the elderly. nine regions in England and Wales, 1976-78
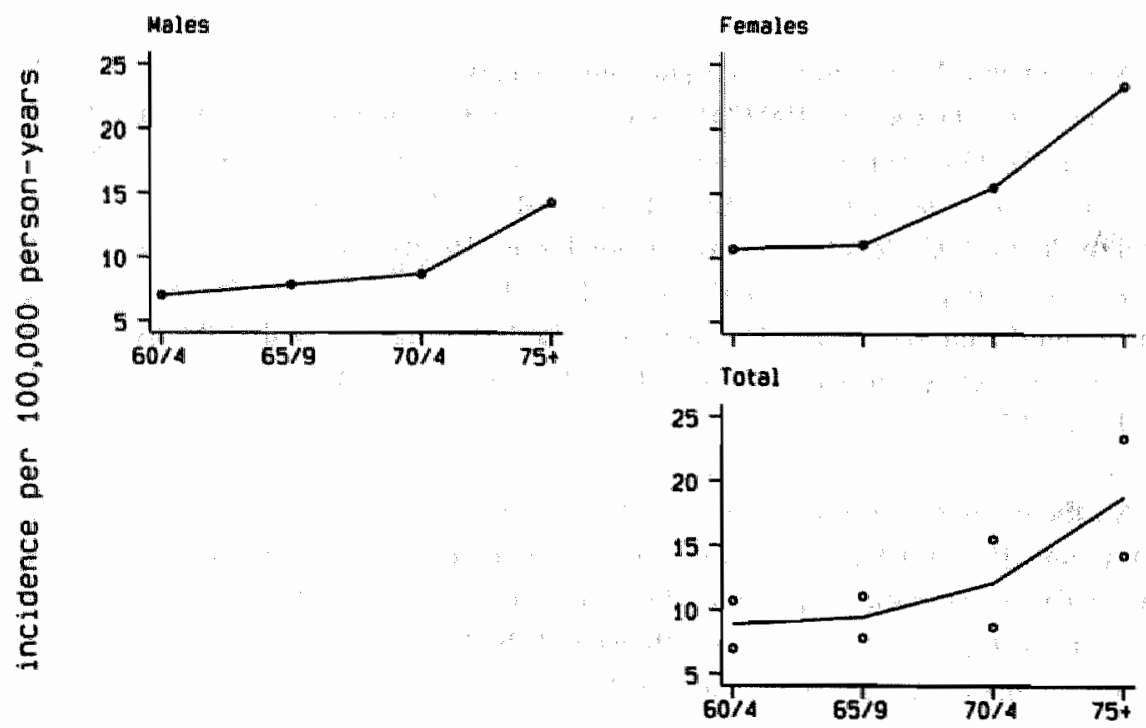


\section{DISCLSSION}

\section{Methodological Issues}

An obvious interpretation of our findings is that a number of cases of organic psychosis, such as cases of Alzheimer's disease (AD) with delusions and/or hallucinations, may have been mistakingly included among the patients with "non-organic, non-affective psychosis".

Since increasing age is associated with an increase in the incidence of $\mathrm{AD}$ (Bickel \& Cooper, 1994), inclusion of such patients could be suggested as a possible reason for the apparent rise in the most elderly individuals. Those cases of psychosis, however, which the clinician suspected to be due to organic pathology, as indicated by a first, second or third ICD diagnosis of organic psychotic condition, were excluded from the sample, indicating that at least at the time of discharge no obvious organic symptomatology was apparent (see also below).

Our incidence rates were determined from hospital admissions and are therefore probably an underestimation of the true incidence of schizophrenia and non-affective psychosis in the elderly. However, our data are a more accurate estimate of the incidence of treated psychosis in old age, and as such are valuable, in view of the dearth of incidence data on these conditions (Castle \& Murray, 1993). If admission bias is to explain our findings, one would have to postulate an age related increase in the probability of being admitted to a psychiatric hospital with non-affective psychotic illness. On the one hand, such a bias might exist because of age-related risk factors favouring hospital admission, such as loss of spouse or other support, and physical illness. On the other hand, with increasing age individuals are also more likely to live in a supported environment, especially in the Netherlands, which has one of the highest levels of provision of supported living arrangements for the elderly in Europe. Furthermore, elderly patients are less likely to be admitted to hospital because of perceived risk to other persons. Castle \& Murray (1993), in a study of all first-contact patients with non-affective psychosis in Camberwell, over the period 1965-1984, found that individuals with late onset illness were equally likely to be treated on an in-patient basis as the younger patient group.

The UK data are quite old, and admission policies, bed capacity and other potentially important factors may have changed since 1978. Although we cannot be certain that results would be similar in a contemporary sample, it should be pointed out that many of these changes were also introduced in other European countries (Van Os \& Neeleman, 1994), and in the Dutch data, adjustment for such a period effect did not attenuate the association with age.

\section{Interpretation of findings}

The evidence we present above suggests that ageing is a risk factor for non-affective non-organic psychosis in the 7th to 10th decade of life. Our finding of a "dose-response" relationship between age and incidence of psychosis may be considered evidence of causality (see Hill, 1964), and suggests that an age-rellated aetiological factor is operating. While we have argued above that it is unlikely that our findings can be explained solely on the basis of inclusion of cases of early $\mathrm{AD}$, it is nevertheless possible that, at least in a proportion of cases, a relationship between late onset non-affective psychosis and degenerative brain disease does exist. For 
example, Hymas and colleagues (1987) showed that late paraphrenics were not only impaired on several cognitive functions compared to healthy age-matched controls, but also that, in spite of improvement of psychotic symptomatology, these patients showed a further cognitive decline during a, on average, 3.7 years follow-up period. However, onlly two patients were thought to have developed a dementia during the follow-up period. These authors conjectured that there was an organic substrate for late paraphrenia, associated with relatively subtle and slowly progressive cerebral changes. There is also evidence that at least one third of patients with late onset schizophrenia and related disorders demonstrate a variety of brain pathologies, in excess of the proportion of normal controls with such

abnormalities (reviewed by Lesser et al, 1993).

We also replicated previous findings that the incidence of late-onset psychosis is higher in females. Howewer, our modest rate ratio of $1.6 \mathrm{in}$ both the U.K. and the Netherlands seemingly contrasts with previously described female to male ratios of around $8-10$ for similar disorders in old age (Castle \& Howard, 1992). Our study, however, focussed on incidence rate ratios, whereas previous reports focussed on prevalent cases as seen in clinical practice, without relating these to the source population. 


\section{REFERENCES}

Almeida, O. Howard, R. Forstl, H. and Levy, R. (1992) Should the diagnosis of late paraphrenia be abandoned? Psychological Medicine, 22, 11-14.

Berrios, G.E. (1992) Psychotic symptoms in the elderly: concepts and models. In: Delusions and Hallucinations in Old Age (Eds C. Katona \& R. Levy) pp 3-14. London: Gaskell.

Bickel, H. and Cooper, B. (1994) Incidence and relative risk of dementia in an urban elderly population: findings of a prospective field study. Psychological Medicine, 24, 179-192.

Castle, D.J. \& Murray, R.M. (1993) The epidemiology of late-onset schizophrenia. Schizophrenia Bulletin, 4. 691-699.

-..... and Howard, R. (1992) What do we know about the aetiology of late-onset schizophrenia? European Psychiatry, 7, 99-108.

Cooper. A.F. (1976) Deafness and psychiatric illness. British Journal of Psychiatry, 129, 216-226.

DHSS (Department of Health and Social Security, 1985) Mental Health Statistics. HMSO, London.

Foerster, A., Lewis, S.W., Owen, M. \& Murray, R. (1991) Premorbid personality in psychosis:effects of sex and diagnosis. British Journal of Psychiatry, 158, 171-176.

Hill, A. The environment and disease:association or causation? Journal of the Royal Society of Medicine 1965 ; 58: $295-300$.

Howard, R, Castle, D. Wessely, S. and Murray R. (1993) A comparative study of 470 cases of early and lateonset schizophrenia. British Journal of Psychiatry. 163, 352-357.

Hymas, N. Naguib, M. \& Levy, R. (1989) Late paraphrenia -a follow-up study. International Journal of Geriatric Psychiatry, 4, 23-29.

Kay, D. (1959) Observations on the natural history and genetics of old age psychoses: Stockholm material 19311937 (abridged) Proceedings of the Royal Society of Medicine, 52, $791-794$. --, Cooper, A. Garside, R. and Roth, M. (1976) The differentiation of paranoid from affective psychoses by patients' premorbid characteristics. British Journal of Psychiatry, 129, 207-215.

- . \& Roth, M. (1961) Environmental and hereditary factors in the schizophrenia of old age ("late paraphrenia") and their bearing on the general problem of causation in schizophrenia. Journal of Mental Science, 107, 649-686.

Lesser, I., Miller, B., Swartz, J., Boone, K., Mehringer, M. \& Mena, I. (1993) Brain imaging in late-life schizophrenia and related psychoses. Schizophrenia Bulletin, 4, 773-782.

Naguib, M. \& Levy, R. (1987) Late Paraphrenia-neuropsychological impairment and structural brain abnormalities on computed tomography. International Journal of Geriatric Psychiatry, 2, 83-90.

Pearlson, G. Kreger, L. Rabins, P. Chase, G. Cohen, B. Wirth, J. Schlaepfer, T. and Tune L. (1989) A chart review study of late-onset and early-onset schizophrenia. American Journal of Psychiatry, 146, 1568-1574.

Post, F. (1966) Persistent Persecutory States of the Elderly. Pergamon: Oxford.

Prager, S. \& Jeste, D. (1993) Sensory impaiment in late-life schizophrenia. Schizophrenia Bulletin, 4, 755-772.

Roth, M. (1955) The natural history of mental disorder in old age. Journal of Mental Science, 101, 281-301.

Selten ${ }_{1}$ J.P. \& Sijben, N. (1994) First admission rates for schizophrenia in immigrants to the Netherlands. The Dutch National Register. Social Psychiatry and Psychiatric Epidemiology, 29, 71 i-77.

Takei, N., Sham, P., O'Callaghan, E., Glover, G. \& Murray, R. (1994) Prenatal influenza and schizophrenia: is the effect confined to fernales? American Journal of Psychiatry, 151, 117-119.

Tien, A.Y. (1991) Distributions of hallucinations in the population. Social Psychiatry and Psychiatric Epidemiology, 26, 287-292.

Van Os, J. \& Neeleman, J. (1994) Mental illness in Europe. British Medical Journal, in press. 
का.

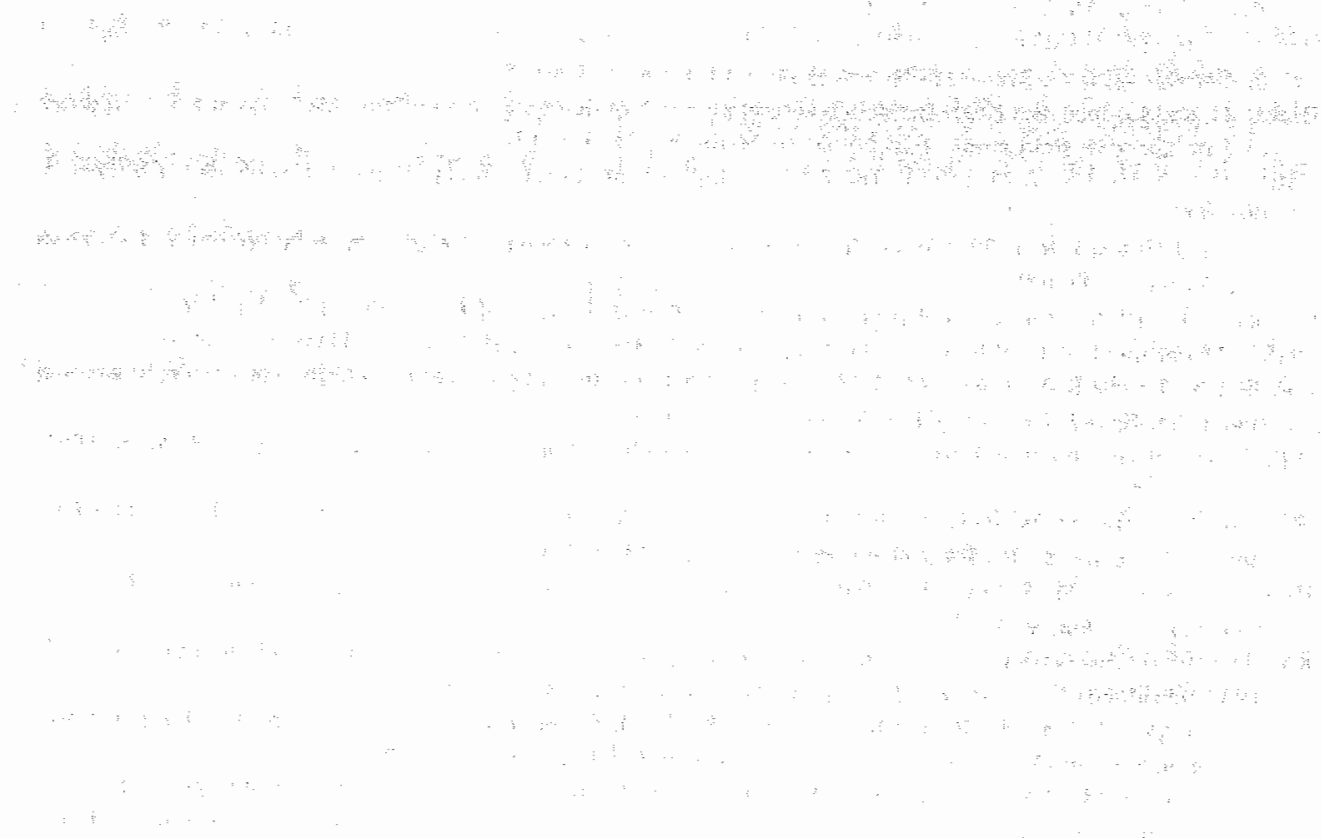




\subsubsection{Psychotic illness in ethnic minorities: Clarification from the 1991 UK census}

\section{SUMMARY}

Age and sex-adjusted first admission rates for operationally-defined schizophrenia and other nonaffective psychosis in different ethnic groups were calculated over the period 1988-1992 in a defined catchment area, in South London. Standardized rates for schizophrenia, corrected for ageand gender-related under-reporting in the 1991 census, and a $20 \%$ underestimate of the size of the ethnic minority populations in the area, were not only higher in the Afro-Caribbean group (SMR:3.1; 95\% CI: 2.0-4.7), but also in the African group (SMR: 4.2; 95\% CI: 2.8-6.2). It was further found that higher rates were not specific to schizophrenia. These findings suggest that some common factor associated with ethnic minority membership is important in bringing on an excess of psychotic illness.

\section{INTRODUCTION}

Harrison (1990) has reviewed incidence studies of schizophrenia amongst Afro-Caribbean migrants to the UK, and concluded that rates of this disorder are high amongst such individuals. The excess is not due to misdiagnosis (Harrison et al, 1988; Lewis et al, 1990; Castle et al, 1991; Wessely et al, 1991), or to admission bias, as studies that selected first contact cases rather than first admission cases reported similar findings (Bebbington et al, 1981; Harrison et al, 1988; Castle et al, 1991; Wessely et al, 1991). Although much of the current focus has been on schizophrenia in the Afro-Caribbean population, there is some evidence that the excess is not specific to schizophrenia (Harrison et al, 1988; Leff et al, 1976), and not specific to the AfroCaribbean population (Rwegellera, 1977).

However, valid epidemiological evidence has been lacking, because of the absence of reliable figures on the size and age structure of ethnic minority populations; this has been a source of much criticism (Burke, 1989; Sashidharan, 1993; Jablensky, 1993). The 1991 census is the first to include comprehensive data on the ethnic composition of the general population, providing a unique opportunity to assess accurately the incidence of both schizophrenia and other pychosis in not only Afro-Caribbean and white groups, but also in other ethnic comparison groups. We investigated first admission rates for operationally-defined schizophrenia and other psychosis in the deprived inner-city area of Camberwell over the period 1988-1992, employing the 1991 census data to establish denominators for Afro-Caribbean, African and white groups, who together constitute more than $90 \%$ of the population in the area.

\section{METHODS}

\section{Sample}

A list was generated from the Maudsley/Bethlem Royal Hospital computerised admission index 
of all first admission patients from Camberwell, South London, between 1988 and 1992, aged 16 and over, who received a discharge diagnosis of "schizophrenic psychosis" (including schizoaffective type; ICD codes 295.0-295.9), "paraphrenia" (ICD 297.2), or "other non-organic psychosis" (ICD 298.1-298.9). This broad sample was chosen to avoid the possibility of missing any patients who had been inappropriately labelled.

Although we only examined in-patients, evidence from a previous study (Castle et al, 1991) indicated that, ower the period 1965-1984, the proportion of in-patient and out-patient contacts with schizophrenia in Camberwell had remained stable; furthermore, there was no ethnic bias in the proportion of patients receiving in-patient or out-patient treatment, both in the group of patients with $\mathrm{RDC}$ schizophrenia $(\mathrm{OR}=1: 0 ; 95 \% \mathrm{Cl}=0.5-2.0)$ and with any $\mathrm{ICD}$ non-affective psychosis as defined above (OR=0.9; $95 \% \mathrm{CI}=0.6-1.5)$.

\section{Diagnosis}

Case notes were rated using the OCCPI checklist. The OCCPI checklist is based on phenomenological descriptions in the Present State Examination, and includes signs and symptoms used in a wide range of operational definitions of psychiatric conditions (ICD, RDC, DSM-IIIR, etcc McGuffin et al, 1991). Although the raters of the OCCPI checklist were not blind to ethnicity, the computer program OPCRIT, which generates diagnoses of a range of operational systems for psychotic illnesses (RDC, DSM-III, DSM-III-R, etc; McGuffin et al, 1991), was used to analyze the OCCPI data blindly. The three authors who rated cases (JvO, DC, NT) took part in a reliability study of OPCRIT. We chose to examine RDC diagnosis of schizophrenia ${ }_{4}$ for which the mean Kappa for the three pairs of raters was 0.74 .

\section{Demographic data}

Other information systematically obtained for all individuals included: sex, ethnicity, and country of birth of both patient and parents. "Ethnicity" categories were White, Afro-Caribbean, African, and "Other". Ethnicity was based on both on the patient's description as well as on appearance and place of birth of both the subject and his parents. These data were directly rated from the case records; checks on date of contact; date of birth and country of origin were made from the front-sheets of the case-notes. In a previous study involving Maudsley case notes using the same methodology (Castle et al, 1991), checks on ethnicity ratings on a subset of 34 patients, using data from previous direct-interview studies involving these patients, found no erroneous ratings.

Patients who were living outside the catchment area at the time of first admission were excluded. Similarly, checks were made on first contact status; subjects who had had in-patient or outpatient contact with psychiatric services prior to the episode were excluded.

\section{The 1991 census}

Demographic data concerning the general population of Camberwell were supplied by the Office of Population Censuses and Surveys (OPCS). Data from the 1991 census (100\% samples) were 
used, and population estimates for the previous and subsequent years (1988-1990, 1992) were interpolated. There are a number of problems with the census, that are unlikely to be fully resilved by the Census Validation Survey. We therefore attempted to adjust for these in a conservative manner, i.e. biased towards the mull hypothesis of no differences in rates of schizophrenia between the white and other ethnic groups. Ethnic groups in the Census include a sizeable group "other Blacks", which comprises both Afro-caribbean, African and other individuals (eg "Black British"; OPCS, personal communication, 1993). As it was not possible to further divide this group, it was added in its entirety to both the Afro-Caribbean and African populations in the denominator for the calculation of rates.

A second issue is that of sex-related under-enumeration among the younger age-groups in the census (OPCS, 1992). Whilst females outnumbered males in all three ethnic groups under investigation, the ratio of males to females in the population aged between 15 and 44 years was 0.94 for the white group, 0.87 for the African group, and 0.69 for the Afro-Caribbean group. A simple correction was therefore made, such that the number of Afro-Caribbean and African males in the age-group 15 to 44 was increased to yield a sex ratio of unity (effectively increasing the denominator in the age-groups most at risk for schizophrenia, and thus reducing rates for schizophrenia; see Appendix).

According to Siegell (1974), under-reporting in the US census may be in the region of 4 to $18 \%$ in black men, compared to 1 to $4 \%$ in white men. There is anecdotal evidence of a similar ethnic discrepancy in the 1991 British census. We therefore applied, in addition to the above adjustments, a general $20 \%$ under-reporting correction for the Afro-Caribbean and African groups, which again would tend to decrease incidence rates (Appendix).

\section{Analyses}

Five-year rates for RDC schizophrenia and other psychosis were calculated, based on the census figures for the population at risk in Camberwell, ie those aged 16 years and over. Unadjusted rates were compared using the rate ratio (RR). Indirect standardization was used to adjust for age and sex: the expected number of cases in each stratum of the study population (ethnic minority groups) was calculated by multiplying the stratum-specific rates of the standard population (white population) by the number of person-years of the study population in that category; differences in adjusted rates were expressed as the standard mortality ratio (SMR). Three broad age bands were used $(16-34,35-54,55+)$. Exact confidence limits were calculated where the observed number of cases (d) was less than 10 ; for $d \geq 10$ approximate confidence intervals are given (Breslow \& Day, 1981; Rothman, 1986).

\section{RESULTS}

\section{Rates for first contact, first admission schizophrenia}

A total of 110 patients with first contact, first admission non-affective psychosis were identified; case notes could be located for all subjects, and 79 fulfilled RDC diagnostic criteria for schizophrenia (Table 1). The remaining 31 patients were labelled "other psychosis". There were 
no significant differences in the proportion of patients receiving a schizophrenic diagnosis in the different ethnic groups $\left(\chi^{2}=0.1 ; \mathrm{d} f=3 ; \mathrm{p}=0.9\right)$. The distribution of cases over the five consecutive years was rather similar in the main ethnic groups: white group: 12,11,8,11,2; Afro-Caribbean group: $4,8,10,6,1$, African group: $9,6,6,8,3$.

Table 1. Diagnostic distribution of first contact, first admission cases of non-affective psychosis by ethnicity. Camberwell 1988-1992.

\begin{tabular}{|c|c|c|}
\hline Whanic group & ICO non-afiective psychosisf & RDC schizophrenia (\%) \\
\hline White & 44 & $30(68 \%)$ \\
\hline Mroclaribbean & -1 & $22(76 \%)$ \\
\hline African & 32 & $23(72 \%)$ \\
\hline Other & $\because$ & $4(80 \%)$ \\
\hline Total & 110 & $79(72 \%)$ \\
\hline
\end{tabular}

† "includes ICD "schizophrenic psychoses" (295.0-295.9), "paraphrenia" (297.2), and "other non-organic psychoses" (298.1-298.9)

The total five-year incidence of schizophrenia, using unadjusted census figures, was 79 per 515,626 person-years, yielding a five-year rate of 15.3 per 100,000 person-years $(95 \%$ CI: 12.3 19.1). The number of schizophrenic individuals of "other ethnicity" $(n=4)$ was deemed too small and heterogeneous to yield meaningful measures of effect, and were not included in the comparative analyses. Rates for schizophrenia, unadjusted for sex, age and under-enumeration were: i) white group: 7.8 per 100,000 person-years (95\% CI: 5.4-11.1); ii) Afro-Caribbean group: 34.4 per 100,000 person-years (95\% CI: 22.6-52.3); iii) African group: 53.2 per 100,000 personyears (95\% CI: $35.5-80.1$ ), yielding unadjusted rate ratios of $4.4(2.4-7.3)$ and $6.8(4.3-10.9)$ respectively. Adjusting for age, gender and under-enumeration yieldied SMR's of 3.1 (2.0-4.7) and $4.2,(2.8-6.2)$ respectively (Table 2$)$. Thus, adjustment for age, sex and under-enumeration reduced both measures of effect by 30-40\%. The SMRs for RDC schizophrenia were high both in the age-group 16-34 years, as well as in the group aged 35 to 54 years, although the confidence intervals in the latter were wider due to the small number of subjects (Table 2).

We also compared rates of "other psychosis", adjusting for age, sex and under-enumenration as described above. The SMR for the Afro-Caribbean and African groups combined (in view of the small numbers) versus the white group was $2.4(1.4-3.8)$.

\section{DISCUSSION}

Standardized rates for operationalised schizophrenia were higher not only in the Afro-Caribbean group, but also in the African group, after correction for age and gender related under-reporting in the census data, and a $20 \%$ underestimate of the size of the Afro-Caribbean and African populations. Higher rates, however, were not specific to schizophrenia. Before discussing these findings further, we will first consider some important methodological issues. 
Our rates represent an underestimate of the real incidence of schizophrenia, as we selected cases on the basis of admission to hospital only However, the unadjusted rate of 1.5 per 10,000 person years is close to that reported by Cooper and colleagues (1987). Furthermore, the main focus was on between-group differences in rates, and, as explained above, data from our previous study (Castle et al, 1991) demonstrate that it is unlikely that an ethnic bias in admission policy has contributed to the results. Although we cannotexclude differential pathways to care for the white group and other ethnic groups, it is unlikely that any pathway bias would have lead to spurious results, because the great majority of incident cases of schizophrenia are eventually admitted to hospital (Cooper et al, 1986).

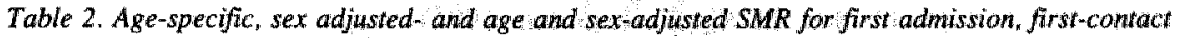
schizophrenia by ethnicity "1988-1992, after correction for estimates of age and gender-related under-reporting in the Census and a 20\% underestimate of the size of the Afro-Caribbean and African populations*.

\begin{tabular}{|c|c|c|c|c|c|c|c|}
\hline \multirow[t]{2}{*}{ Agegroup } & \multirow{2}{*}{$\begin{array}{l}\text { White group } \\
\text { Observed } \\
\text { cuses }\end{array}$} & \multicolumn{3}{|c|}{ Afro Caribbean group } & \multirow[b]{2}{*}{$\begin{array}{l}\text { Observed } \\
\text { caces. }\end{array}$} & \multicolumn{2}{|c|}{ African group } \\
\hline & & $\begin{array}{l}\text { Observed } \\
\text { cases }\end{array}$ & $\begin{array}{l}\text { Expected } \\
\text { cases. }\end{array}$ & sMr & & $\begin{array}{l}\text { expected } \\
\text { cases }\end{array}$ & SMR \\
\hline 16.34 & 18 & 18 & 6.0 & $3.0(1.9-4.8)$ & 22 & 5.0 & $4.4(2.9-6.6)$ \\
\hline $35=4$ & 3 & 3 & 0.6 & $5.0 .(1.01-14,4)$ & 1. & 0.4 & $2.5(0.1-14.2)$ \\
\hline 554 & 9 & 1 & 0.6 & $1.7(0.1-10.2)$ & 0 & 0.1 & 0 \\
\hline $16.55 \%$ & 30 & 22 & 7.2 & $3.1(2.04 .7)$ & 23 & 5.5 & $4.2(2.8-6.2)$ \\
\hline
\end{tabular}

* includes the whole category "other Blacks"

Although it has been claimed that is is unlikely that there are ethnic biases in rates of underenumeration (Teague, 1993), this conclusion was based on a comparison with Labour Force Surveys, which are likely to show the same ethnic biases as the Census (Burke, 1989). As we have shown, under-enumeration of ethnic minorities is likely to be a real problem in inner city areas, especially as those missing are in the age range most at risk for schizophrenia. We believe our corrections not to be excessive.

The category "other psychosis" is, of course, not a homogeneous entity. It was used however, solely to examine whether the same ethnic differences in rates applied to another group of nonschizophrenic psychoses.

Ethnicity for cases was not ascertained in the same manner as for the population used in the denominator, as in the latter self-reporting in repsonse to Census questions about ethnicity was the source of information. Such a numerator-denominator bias could lead to an underestimation of the size of the ethnic minority populations in relation to the cases in the numerator. We have demonstrated, however, that our results are quite robust to possible under-enumeration. Furthermore, inclusion of the the entire "other black" group will have minimized this problem. The investigators were not blind to ethnicity in rating the OCCPI checklist, but diagnosis was made "blind" by a computer programme. Furthermore, rates were high in ethnic minority groups regardless of diagnosis. 
In a previous study in the same area, we reported an association between onset of schizophrenia and lower social class (Castle et al, 1993). Social class may therefore have confounded our results. We were not able to adjust for socio-economic status as we did not have aecess to age by sex by class by ethnicity Census data. A $10 \%$ Census sample, however, showed that AfroCaribbean economically active residents aged 16 and over in Camberwell were 1.8 (95\% CI: 1.71.9) times more likely to be unemployed than their white counterparts and 2.11 (95\% Cl 1.7-2.6) times more likely to belong to social classes 4 and 5 . For the African group, the odds were 2.2 (2.1-2.4) and 2.5 (1.9-3.3) respectively. Although suggestive, these differences are small compared to the reported SMRs of schizophrenia; if social class were to account for the whole difference in rates between the white and black groups, the effect size of class would need to be around 4-5 times greater that the SMR (Rothmian, 1986).

The numbers of schizophrenic cases in the ethnic minority groups may have been exaggerated by the effect of large numbers of young vulnerable ethnic minority individuals drifting into deprived inner city areas with a more stable (and healthy) white population. This factor may especially apply to African migrants, who, compared to the Afro-Caribbean population, have a less stable population base in Camberwell. However, in both ethnic minority groups, first admission rates were elevated also in the older age-group of 35 to 54 year olds, which makes it unlikely that differential mobility has significantly contributed to the results.

Accepting the limitations arising out of the inability to control satisfactory for socio-econommic status and differential mobility, and the different methods used to ascertain ethnicity for cases and for the general population, the results do support the notion of higher incidence rates of schizophrenia among the Afro-Caribbean and African populations in Camberwell, South London, the "true" rate ratios, compared to the white group, lying between 2.0-4.7 and 2.8-6.2 respectively. However, elevated rates were not specific to schizophrenia, and the previous focus on Afro-Caribbean patients also appears too selective. This has important implications for various specific explanations that have been put forward, such as the use of cannabis (McGovem \& Cope, 1987), and biological theories (Wessely, 1991; Eagles, 1991). Whilst these factors may play a role, a non-specific increase in psychosis among various groups of ethnic minorities would appear to indicate that common sources of stress on members of ethnic minorities, such as cultural adjustment, discrimination, impact of migration and racism, are also important determinants. 


\section{APPENDIX}

Example of correction for gender-related ond general under-enumeration in the 1991 Census, shown for the group Afro-Caribbean males and "orher Black" males in Camberwell

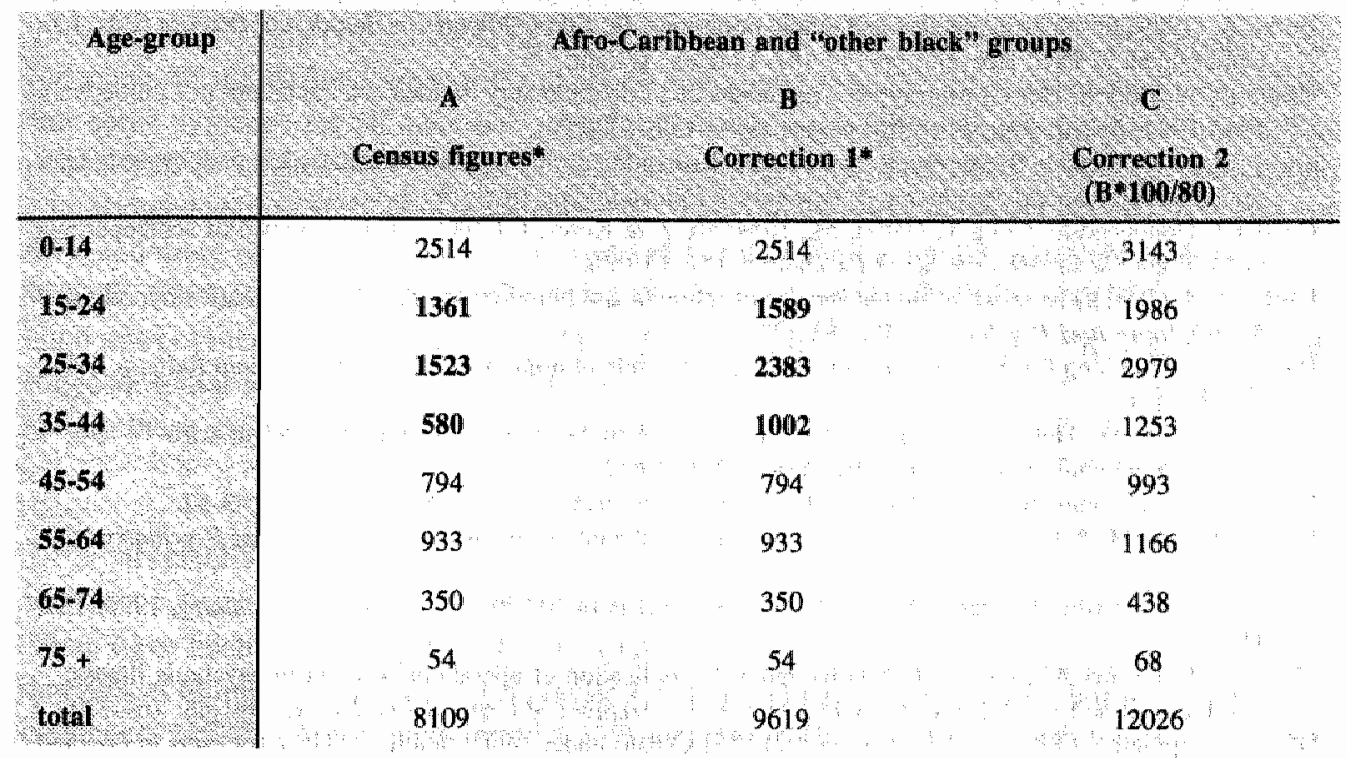

* bold figures point towards a discrepancy in the gender distribution, and subsequent corrections, which consisted of an increase of the number of males in the age-groups 15-44, such that the sex ratio in these age-groups was unity (see text). 


\section{REFERENCES}

Bebbington P, Hury I, Tennant C. Psychiatric disorders in selectied immigrant groups in Camberwell. Social Psychiatry, $1981,16,43-51$.

Breslow N.E \& Day NE. (1987) Statistical Methads in Cancer Research, Vol 1, ppt68-72. Lyon: WHO.

Burke A, 1989 ) Pyychatric practice and ethinic minorities. In, Ethnic Factors in Health and Disease (Eds. IK. Cruickshank \& $10, \mathrm{G}$ Beevero) pp $179-187$ London, Whigh:

Castle D, Wessely S, Der G, Murray, R. The incidence of operationally defined schizophrenia in Camberwell, 19651984 British Joumal of Psychiatry, 1991, $159,790794$.

$\mathrm{C}_{-}$, Seott K, Wessely S, Mumay, R. Does social deprivation during gestation and early life predispose to later schizophrenia? Social Psychiatry \& Psychiatic Epidemiology, 1993, 28, 1 -4.

Cooper J, Goodhead D, Cralg I, Harris, M, Howards, J, \& Korer, J. (1987). The incidence of schizophrenia In Nottingham. Brinish Journal of Psychiatry, 151:619-26.

Eagle, JM. (1991) The relationship between schizophrenia and immigration. Are there altemative hypotheses? British Journal of Psychiawy, 159, 783-789.

Harrison $\mathrm{G}$, Searching for the causes of schizophrenia: the role of migrant studies schizophrenia Bulletin, 1990 ; 16 66 63-671.

Owens, D., Hollon, A et al (1988) A prospectiwe study of severe mental disorder in AfroCaribbean patients. Psychological Medicine, 18, 643-657.

Jablensky, A. The epidemiology of schizophrenia Curent Opinion in Psychiatmy, 1993; 6. 43-52.

Leff, J, Fisher, M. Bertelsen. A. (1976) A cross national study of mania. British Journal of Psychiatry, 129, $428-42$.

Lewis L, Croft-Jeffreys C David A. Are British psychiatrists racist? British Journal of Psychiatry, 1990; 157: 410.415.

McGuffin P, Fammer AE Harvey I. A polydiagnostic application of operational criteria in psychotic illmess. development and reliability of the OPCRTT system. Archives of General Psychiatry, 1991;48: 764-770.

Office of Population Censuses \& Surveys (1992) 1991 Census under-enumeration: guidance for users in England and Wales, Census Newsletter, 24, 9-10.

Rothman K Modern Epidemiology Boston, Little, Brown and Company, 1986.

Rwegellera ${ }_{n}$ G.G.C (1977) Psychiatric morbidity among West-Africans and West-Indians living in London. Psychological Medicine, 7, 317-329.

Sashidharan, SP. Afro-Caribbeans and schizophrenia: the ethnic vulnerability hypothesis ne-examined. International Review of Psychiatry, 1993; 5: 129-144.

Siegel JS. Estimates of coverage of the population by sex, race and age in the 1970 census. Demography, 1974; 11: $1-23$.

Teague, A. (1993) Ethnic group: first results from the 1991 census. Population Trends, 72, 12-17.

Wessely S, Castle D, Der G, Murray, R. Schizophnenia and Afro-Caribbeans: A case control study. British Journal of Psychiatry, 1991; 1159: 795-801. 


\subsubsection{The incidence of mania: \\ Time trends in relation to gender and ethnicity}

\section{SUMMARY}

There are conflicting reports about possible changes in the incidence of mania. First contact rates for mania in the defined area of Camberwell between 1965 and 1984 were established. There was some evidence for an increase in the first contact rate of mania, especially in women. This rise may be associated with the influx into Camberwell of individuals of Afro-Caribbean origin, who showed significantly higher rates than the white group (adjusted rate ratio: $3.1 ; 95 \%$ CI 1.4 6.9 ), and more often displayed mixed manic and schizophrenic symptomatology (risk ratio. 2.2 ; 95\% CI 1.1 4.3). The incidence of mania has not decreased, and may actually have increased. High rates of mental illnes among members of ethnic minorities are not specific to schizophrenia, suggesting that a risk factor common to both manic and schizophrenic illness is more prevalent among these groups.

\section{INTRODUCTION}

There have been conflicting reports about changes in the incidence of mania over the last few decades. A number of studies have suggested increased incidence rates for the illness. Parker and colleagues (1985), for instance, found a 35\% increase in first admission rates for mania between 1967 and 1977 in New South Wales, which they suggested was due to clinicians using this label more after the introduction of lithium for bipolar disorder. Similarly, Kendell and co-workers (1993) noted an increase in first admission rates for mania in women between 1971 and 1989 in Edinburgh. However, in England and Wales, Der and colleagues (1990) reported a decline in first admission rates for manic depressive psychosis between 1952 and 1986, without a compensatory increase in any other diagnostic category. A similar decline in first admission rates has also been found for manic depressive psychosis in France, especially in women, in the absence of a concurrent increase in other psychiatric diagnoses (Van Os et al, 1993). The latter two studies, however, did not distinguish between depression and mania, and none of the above studies could exclude the possibility of bias due to changing diagnostic habits, fluctuating proportions of "false" first admissions (i.e. patients labelled "first admission" who in effect had had previous psychiatric treatment elsewhere), or alterations in admission policies over the years (Kendell et al, 1993).

In a previous study (Castle et al, 1991), we reported that the incidence of operationally defined schizophrenia in the area of Camberwell, South London, rose over the period 1965-84. One reason for the disparity between this finding and a number of reports of a decline in first admission rates in the U.K. for schizophrenia over the same period (Der et al 1990; Eagles \& Whalley, 1985; Kendell et al, 1993), lay in the changes in the ethnic composition of Camberwell over the two decades from the mid-1960s, specifically the increase in the proportion of AfroCaribbeans. Evidence has accumulated that Afro-Caribbean individuals living in the U.K. have a higher risk of developing schizophrenia than do their white counterparts (Rwegellera, 1977;

'J. van Os, N. Takei, D. J. Castle, S. Wessely, G. Der, A. MacDonald \& R.M. Murray (Sacial

Psychiatry \& Psychiatric Epidemiology, in press) 
Dean et al, 1981; MoGovem \& Cope, 1987; Harrison et al, 1988; Castle et al, 1991; Wessely et al, 1991). Is this increased risk confined to schizophrenia? Leff and colleagues (1976), examining the annual incidence of mania in Camberwell, found that first generation AfroCaribbeans (i.e. Caribbean-born) had higher rates than did the indigenous whites. Within the affective disorder spectrum, the increase in relative risk for Afro-Caribbeans in the U.K may be confined to mania; thus, Bebbington and collaborators (1981) noted that mean age-corrected admission rates for mania were more than three times higher in first generation Afro-Caribbeans than in U.K.-born subjects, but if all affective disorders were combined no clear ethnic difference was discemible. Der and Bebbington (1987) reported similar results. However, there are a number of difficulties with these studies. The study by Leff and colleagues (1976) was the only one to use operational criteria for mania, but the sample included only hospital admissions. All studies excluded manic cases with schizophrenic "first rank" symptoms, and none included AfroCaribbean individuals born in the U.K. (second generation), who instead were incorporated in the group of individualls "born in the U.K." together with whites and other ethnic groups; in some of the studies, the number of Afro-Caribbean individuals identified was very small (eg only 6 in the study by Leff et al, 1976). Furthermore, an initial presentation bias may be operating. Previous studies did not include those individuals with a first presentation of depression who might subsequently have developed manic episodes, and there may exist ethnic differences in the proportion of manic patients who will present with previous depressive episodes (Makanjuola, 1985).

If indeed there is an increased risk for both mania and schizophrenia in Afro-Caribbean individuals in the U.K., this would have important implications both for psychiatric service provision and for theories of aetiology. For example, a variety of social and biological factors have been suggested as explanations for the increased risk of schizophrenia in Afro - Caribbeans (Harrison, 1990; Wessely, et al 1991). If the increased risk were not confined to schizophrenia, but were also evident in mania, this could suggest that the excess in incidence is determined by a risk factor common to both mania and schizophrenia, rather than by a specific biological risk factor for schizophrenia.

The aims of this study, then, was to explore time trends in the incidence rate for operationally defimed mania in a delimited area, in relation to ethnic group. In view of the above mentioned evidence indicating gender differences in time trends in the incidence of mania, rates for men and women were also compared.

\section{METHOD}

\section{Sample}

The Camberwell Cumulative Psychiatric Case Register (Wing \& Hailey, 1972) provides a comprehensive list of all persons from the area of Camberwell in South London who had their first contact with the psychiatric services between 1964 and 1984. A print-out was generated from the Register, of all first-contact patients between 1965 and 1984 who received a register diagnosis of "mania" or "hypomania" (equivalent ICD codes 296.0, 296.2, 296.4), "schizophrenic psychosis" (ICD codes 295.0-295.9), including schizoaffective type (ICD 295.7), "paraphrenia" (ICD 297.2) or "other nonorganic psychosis" (ICD 298.1-298.9). This broad sample was chosen 
to avoid the possibility of missing any patients who had been inappropriately labelled, and to allow for variation in diagnostic habits.

\section{Diagnosïs}

In a previous study, case notes of patients with an ICD diagnosis of schizophrenia and related conditions (including "schizo-affective disorder", "paraphrenia", and "atypical psychoses") had been collected by DC and SW (Castle et al, 1991), and rated using the Operational Criteria Checklist for Psychotic IIIness (OCCPI) and the OPCRIT computer program, which generate a range of operational definitions for psychotic illnesses (RDC, DSM-III, DSM-III-R, etc; McGuffin et al, 1991). For the purpose of the current study, schizophrenia was defined according to the RDC criteria of Spitzer and colleagues (1978). Inter-rater reliability, based on 50 cases rated by both DC and SW, was good (kappa $=0.82$ for RDC diagnoses). For the present study, cases from this sample with an RDC diagnosis of mania or schizomania were identified.

Patients with a Register diagnosis of mania or hypomania were not rated using the OCCPI checklist, but were given a single RDC diagnosis (Spitzer et al, 1978) by two raters (NT and JvO). Two categories of RDC manic disorder were distinguished: i) mania, ii) mania with schizomania. Inter-rater reliability for cases with a Register diagnosis of mania was assessed on a random subset of 15 case records which were rated by NT and JvO; kappa for RDC diagnoses was 0.78 .

The two authors who rated the manic cases (NT and JvO), and one of the authors who rated cases of schizophrenia and related disorders (DC), took part in a reliability study of OPCRIT. The mean Kappa for the three pairs of raters for RDC diagnoses of schizophrenia and affective psychosis was 0.74 (range: $0.70-0.76$ ).

\section{Demographic data}

Other information systematically obtained for all individuals included: sex, age at first contact, date of birth, and ethnicity and country of birth of both patient and parents. Ethnicity ratings were based on appearance, country of birth of subject and country of birth of the parents. "Ethnicity" categories were white, Afro-Caribbean, African, Asian, and "other", while "country of birth" categories were UK and Eire, West Indies (Caribbean), Asia, Africa, and "other". These data were directly rated from the case records; checks on date of contact, date of birth and country of origin were made from the front-sheets of the case-notes and the Camberwell Register itself. Checks on ethnicity ratings were made on a subset of 34 patients, using data from previous direct-interview studies involving these patients; no erroneous ratings were found. Patients who were living outside the catchment area at the time of first contact but had erroneously been included in the Register, were excluded. Similarly, checks were made on first contact status to preclude bias due to inclusion of "false" first contact cases (see above).

Demographic data concerning the general population of Camberwell were supplied by the Office of Population Censuses and Surveys (OPCS). Data from the 1961, 1971, and 1981 censuses (100\% samples) were used, and population estimates for the intermediate years were interpolated. 
The censuses did not record ethnicity as such, but "country of origin" was recorded, and the 1981 census also included "country of origin of head of household".

\section{Analyses}

An adjustment was made for the missing notes, by ascertaining the percentage of rated patients (by sex and/or ethnicity where necessary) in each cohort with a register diagnosis of (hypo)mania or schizophrenia and related conditions, who fulfilled RDC criteria for mania or mania with schizomania, this proportion was then added to the total in each category (these corrections resulted in only minor changes).

Incidence rates for RDC mania and mania with schizomania were calculated, based on the census figures for the population of Camberwell, directly standardized to the 1964 age and sex structure. Changes in rates over time were analysed using a test for trend. A simple linear regression model was used, with the log rates as the dependent, and the four time periods (see below) as the independent variable. A $P$ value was obtained from the slope of the regression line, assuming an additive model. The magnitude of the association between incidence rate and an exposure variable was expressed as the rate ratio. Confidence intervals for rate ratios were calculated according to Rothman (1986). Associations between binary variables were expressed as the risk ratio (Rothman, 1986).

\section{RESULTS}

\section{Sample characteristics}

There were 701 patients on the register in the appropriate categories. Case records were available on $625(89 \%)$, of whom 80 were excluded because of psychiatric contact before 1965 , the patient not being a resident of the psychiatric catchment area, or because there was an obvious organic basis to the illness. The notes were of insufficient quality to rate in 8 patients, and these were counted as missing in further calculations.

Patients were allocated to four time cohorts, according to their date of first contact, namely 1965-69 (first cohort), 1970-74 (second cohort), 1975-79 (third cohort), and 1980-84 (fourth cohort). The number of patients in each cohort for whom notes were missing was $31(20 \%), 28$ $(18 \%), 11(7 \%)$, and $6(4 \%)$. The greater proportion of missing notes in the first and second cohorts is due to a number of these notes having been destroyed because of lack of storage space at one of the local hospitals. There is no reason to suspect that this introduced any systematic bias; specifically, there were no significant differences in the proportion of men, or those born outside the U.K, between subjects with missing and available notes. Table 1 shows the number of individuals who fulfilled RDC criteria for mania with schizomania across the four cohorts $(n=106)$, the great majority of whom $(89.4 \%)$, had been inpatients at first contact. Incidence rates were callculated for mania and mania with schizomania. There was a trend for an increase in the rates of both over the period under study. 
Table 1. Numbers of individuals fulfuling RDC criteria for mania with schizonana, by cohort, and onmal incidence rates for mania and manio with schizomanita, standardized to the 1964 age and sex structwre.

\begin{tabular}{|c|c|c|c|c|}
\hline 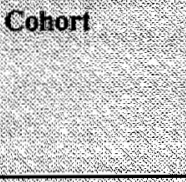 & 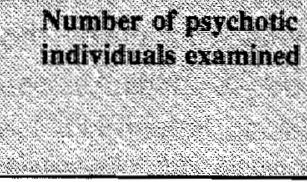 & 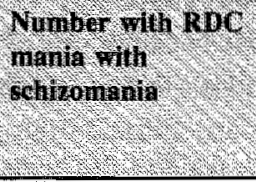 & 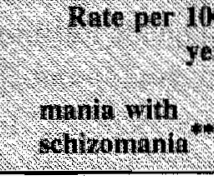 & 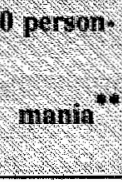 \\
\hline 196569 & 156 & $23(15 \%)$ & 2.8 & 1.7 \\
\hline $1970 \% 7$ & 152 & $25(16 \%)$ & 3.4 & 20 \\
\hline 197570 & 163 & $30(18 \%)$ & 43 & 3.8 \\
\hline 198084 & 154 & $28(18 \%)$ & 4.1 & 3.4 \\
\hline Test for trend & & & $p=0.09$ & $p=0.1$ \\
\hline
\end{tabular}

* Numbers are adjusted for missing notes, according to the percentage of individuals (in parenthesis) with a register diagnosis of "schizophrenia', "paraphrenia", 'mania" or 'other mon-organic psychosis', who fulfilled RDC criteria for mania or schizomania.

* for definitions of mania and mania with schizomania, see text

\section{Incidence of mania by gender}

Analyses of age-adjusted rates for RDC-mania with schizomania by gender (Table 2) revealed that the overall incidence rates were similar in both sexes across the four cohorts. The rate for mania with schizomania was between 2.5 to 4.9 per 100,000 person-years. Rates for mania with schizomania tended to increase in both sexes, but this increase only reached statistical significance for women; the findings for mania were similar.

Table 2. Annual age-adjusted rates (rate ratios) of RDC mania with schiromania per 100,000 person-years, by sex

\begin{tabular}{|c|c|c|c|}
\hline Golort & \multicolumn{3}{|c|}{$\begin{array}{l}\text { Anuil rates (rate rathos) of ADC mania with achinomania per } \\
100,000 \text { person-years by sex }\end{array}$} \\
\hline & \multicolumn{3}{|c|}{ mania with schizonrania } \\
\hline & women & men & rate ratio $(95 \% \mathrm{Cl})$ \\
\hline 106669 & 3.0 & 2.5 & $1.2(0.4-3.7)$ \\
\hline 19707 & 3.6 & 3.2 & $1.1(0.4-2.7)$ \\
\hline $19767 \%$ & 3.8 & 4.9 & $0.8(0.4-1.8)$ \\
\hline $19004 \%$ & 4.4 & 3.8 & $1.2(0.5-2.7)$ \\
\hline Test roo trend & $\mathrm{p}=0.02$ & $\mathrm{p}=0.3$ & \\
\hline
\end{tabular}




\section{Incidence of mania by ethnicity}

In order to investigate any effect of ethnicity on our results, we established, among the total number of individuals fulfilling RDC criteria for mania with schizomania, the proportion of both Caribbean-born $(n=22)$ and UK-born Afro-Caribbeans $(n=28$; Table 3$)$.

Table 3 . Proportion of all indiwiduals fulfiling RDC criteria for mania with schizomania, by country of birth and ethricity

\begin{tabular}{|c|c|c|}
\hline Cohort & $\begin{array}{l}\text { Individuals born } \\
\text { in the West Indies }(n=22)\end{array}$ & $\begin{array}{l}\text { First- and second } \\
\text { generation Arro-Cariblieans }(n=28)\end{array}$ \\
\hline 1965.69 & $20 \%$ & $21 \%$ \\
\hline $1970-74$ & $23 \%$ & $24 \%$ \\
\hline 1975.79 & $14 \%$ & $21 \%$ \\
\hline 1080.84 & $25 \%$ & $37 \%$ \\
\hline
\end{tabular}

Census data for Camberwell show that the proportion of the population born in the West Indies increased from $2.5 \%$ in 1961 , to $4.9 \%$ in 1971 , and $6.6 \%$ in 1981 . The 1981 census also classifies residents by the birthplace of the head of the household. This can be used as a rough estimate of the proportion of the total population who were ethnic Afro-Caribbeans (i.e. those born in the West Indies, and those bom in the U.K. combined); the figure for 1981 was $11.5 \%$ Afro-Caribbean. To avoid error through possible underestimation of the size of the AfroCaribbean population, all denominator data for the group born in the West Indies and the AfroCaribbean group were corrected for a $10 \%$ underenumeration in all the analyses comparing ethnic groups (Tables 4 and 5).

It is clear (Table 3) that the proportion of Caribbean-born individuals among manic subjects is 8 times higher in the first cohort, and 3-5 times higher in the subsequent cohorts than the proportion in the general population of Camberwell. In the later years of the study, a similar excess is seen for the Afro-Caribbean group as a whole (i.e. both Caribbean-born and U.K.-born).

Using the census data, corrected as described above, we calculated rates for RDC mania with schizomania by country of birth (Table 4). The rate of mania for "all individuals born in the West Indies" was significantly greater than that for "all individuals borm in the U.K.", across all four time bands. Of course, in the later years of the study an increasing proportion of the general population in Camberwell were ethnic Afro-Caribbeans born in the U.K. ; this probably accounts for the decline in the rate ratio for "country of birth" from the second to the fourth cohort. The limitations of the census data allow us to estimate rates by ethnicity as such only for the final five years of the study (again, "country of birth of head of household" data were used). Table 4 shows that for 1980-84, the rate of mania for all ethnic Afro-Caribbeans was four times that for all other ethnic groups combined (the great majority of whom would be white).

Among all patients with mania, individuals in the Afro-Caribbean group were significantly more likely to fulfil criteria for "schizomania" than those in the white group (risk ratio: $2.2 ; 95 \% \mathrm{CI}$ : 1.1-4.3). Comparison of rates for mania (i.e. with exclusion of schizomanic disorder) in the 1980-84 cohort, by ethnicity, revealed a decrease in the rate ratio from 4.1 for mania with 
schizomania to $3.0(95 \%$ CI: $1.3-7.4)$.

As the exact age structure of the Afro-Caribbean population in Camberwell over the period under investigation is not known, but is likely to have contained relatively few individuals in the older age groups compared to the white population, we attempted to correct for this effect. In our sample, age of onset distribution curves for Afro-Caribbeans and "all other ethnicities" were similar in form, but the range in Afro-Caribbeans was 16-64 years, and 16-88 years in the "all other ethnicities" sample. We therefore calculated, for the 1980-84 cohort, rates for "all other ethnicities" with onset before 65 , assuming that all individuals in the general population in Camberwell aged 65 and over were white, to obtain a more conservative estimate of rate ratios between Afro-Caribbeans and "all other ethnicities" in the under 65s. With this correction, individuals of Afro-Caribbean origin showed rates of mania between 3.1 (mania with schizomania; 95\% CI: 1.4-6.9), and 2.2 (mania; 95\% CI: 0.9-5.6) times that of their white counterparts.

Table 4. Five year rates (rate ratios) of RDC mania with schizomania per 100,000 person years, by country of birth*.

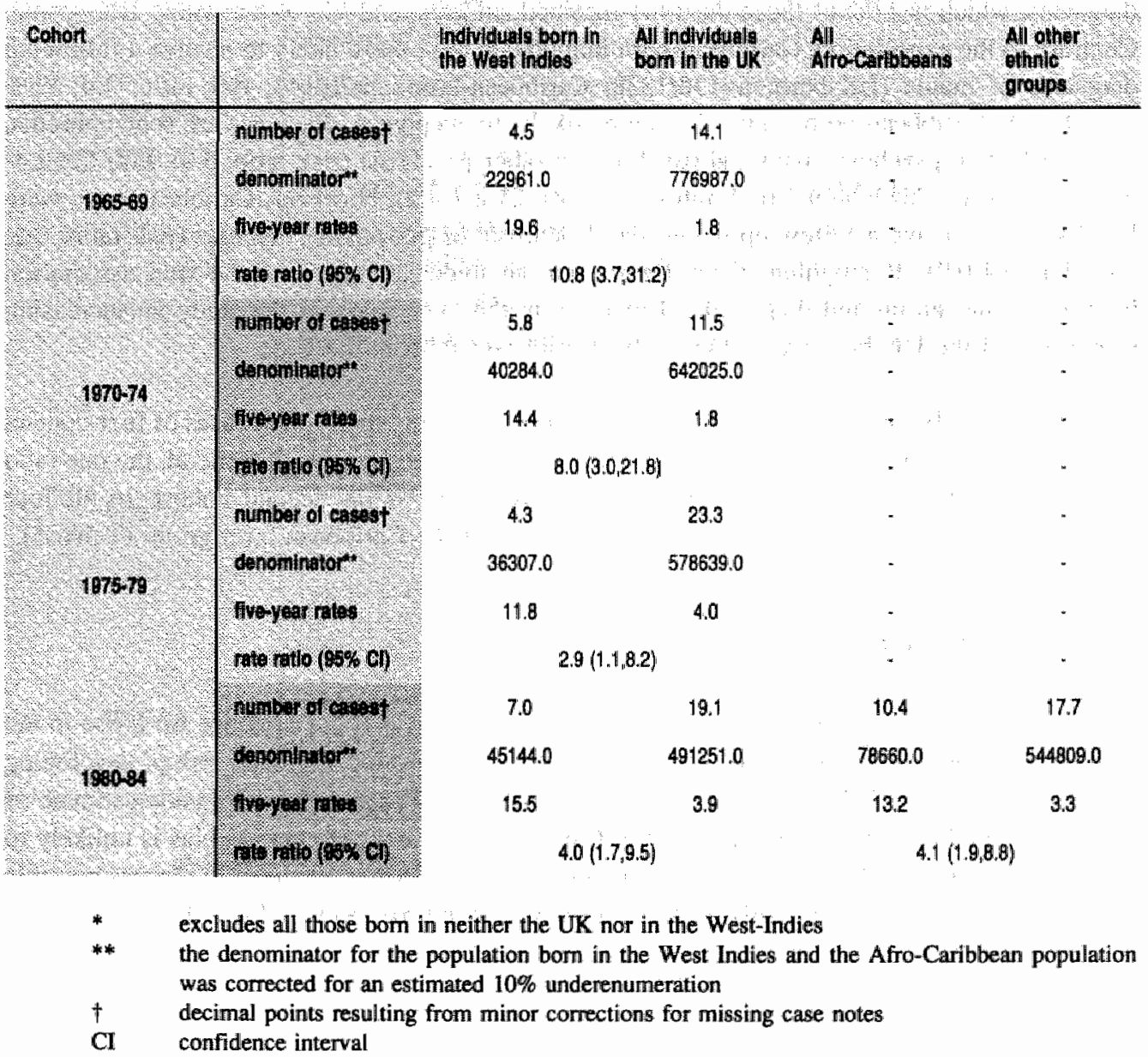




\section{Initial presentation bias?}

As pointed out above, one reason for the high rates of mania in Afro-Caribbeans may lie in differences of illness presentation, Afro-Caribbean bipolars possibly presenting more often with initial manic episodes, and bipolar whites with initial depressive episodes To examine the possibility of differential change of diagnosis, we conducted a search for all Maudsley patients on the Camberwell Case Register who had i) an initial first contact diagnosis of depression during the period 1965-1984, and ii) whose country of birth was either the UK ( $n=2454 ; 91 \%$ ) or the Caribbean ( $\mathrm{n}=239,9 \%$ ). This group of initial depressive contacts (IDC) was subsequently matched with computerized records of all discharges (including data on ICD discharge diagnosis and country of birth) to the Maudsley hospital between 1970 and 1993, yielding a follow-up range for IDC cases from 5 to 28 years. This yielded matches with 2441 in-patient episodes (subjects could be matched more than once if they had multiple admissions), corresponding to 1481 subjects. This group of 1481 had the same distribution of Caribbean-borm and UK-born subjects (Caribbean-born: $n=143 ; 10 \%$; UK-born: $n=1338$; 90\%) as the whole IDC group, indicating an equal probability for both groups of being matched. Of the 1481 cases, a total of 335 patients $(23 \%)$ had diagnoses in a non-depressive category, 158 of whom had a manic diagnosis, which is $11 \%$ of those the 1481 matched subjects, and $6 \%$ of the whole IDC group. Contrary to the expectation, Caribbean-born individuals were more likely to receive a follow-up diagnosis of mania (UK-born: $n=138 / 1338$; Caribbean-born: $n=20 / 143$; risk ratio: 1.4; 95\% CI:0.9-2.1). Caribbean-born were also more likely to receive a diagnosis of schizophrenia, schizo-affective psychosis, paranoid psychosis or other psychosis (risk ratio: $3.9 ; 95 \%$ CI: 2.46.3), especially schizophrenia (risk ratio: $4.7 ; 95 \%$ CI 2.7-8.2). However, Caribbean-born were less likely to receive a follow-up diagnosis of neurosis or personality disorder (risk ratio: 0.6 ; 95\% Cl: $0.4-0.9$ ). If anything, these figures are an underestimation of the true association between ethnic group and diagnostic change, as in the later years of the study an increasing proportion of the UK-born were ethnic Afro-Caribbeans born in the U.K.

Adding the new figures of bipolars initially presenting as depressives to the cases of first-contact mania with schizomania, we recalculated the rates in both ethnic groups. In general, the rate ratio was only affected by a small amount, with the exception of the second cohort. In all four cohorts, however, rates remained significantly higher for the Caribbean-born group (Table 5).

\section{DISCUSSION}

Over the twenty-year period from 1965 we found that there was some evidence for a rise in the incidence of mania in Camberwell. The Afro-Caribbean group was at greater risk of developing mania (rate ratio $=3.1 ; 95 \%$ CI 1.4-6.9), after adjustment for age and a $10 \%$ underestimate of the size of the Afro-Caribbean population in Camberwell. Initial presentation bias is unlikely to have affected our results. Before considering these findings further, we need to discuss possible methodological flaws in our study, some of which thave already been addressed. 


\section{Methodological issues}

The use of first-contact rather than first-admission patients avoids the possibility of bias due to changes in admission policies over the years. Although it is theoretically possible that general practitioners in Camberwell have changed their pattern of referral of manic patients to psychiatric services, the available evidence in the U.K. suggests that almost all patients with severe mental illness are referred to psychiatric services (Cooper et al, 1987). Furthermore, even if such a change was operating, it would probably result, if anything, in a lower proportion of such patients being referred to hospital in more recent years (see Prince \& Phelan, 1990).

We assessed the widest feasible range of diagnoses on the register, and it is unlikely that we omitted any significant number of manic patients.

Table 5. Five-year rates (rate ratios) of first contact $R D C$ mamia with schizomania, and first admission mania after initiol depressive episodes per 100,000 person years, by country of birth*

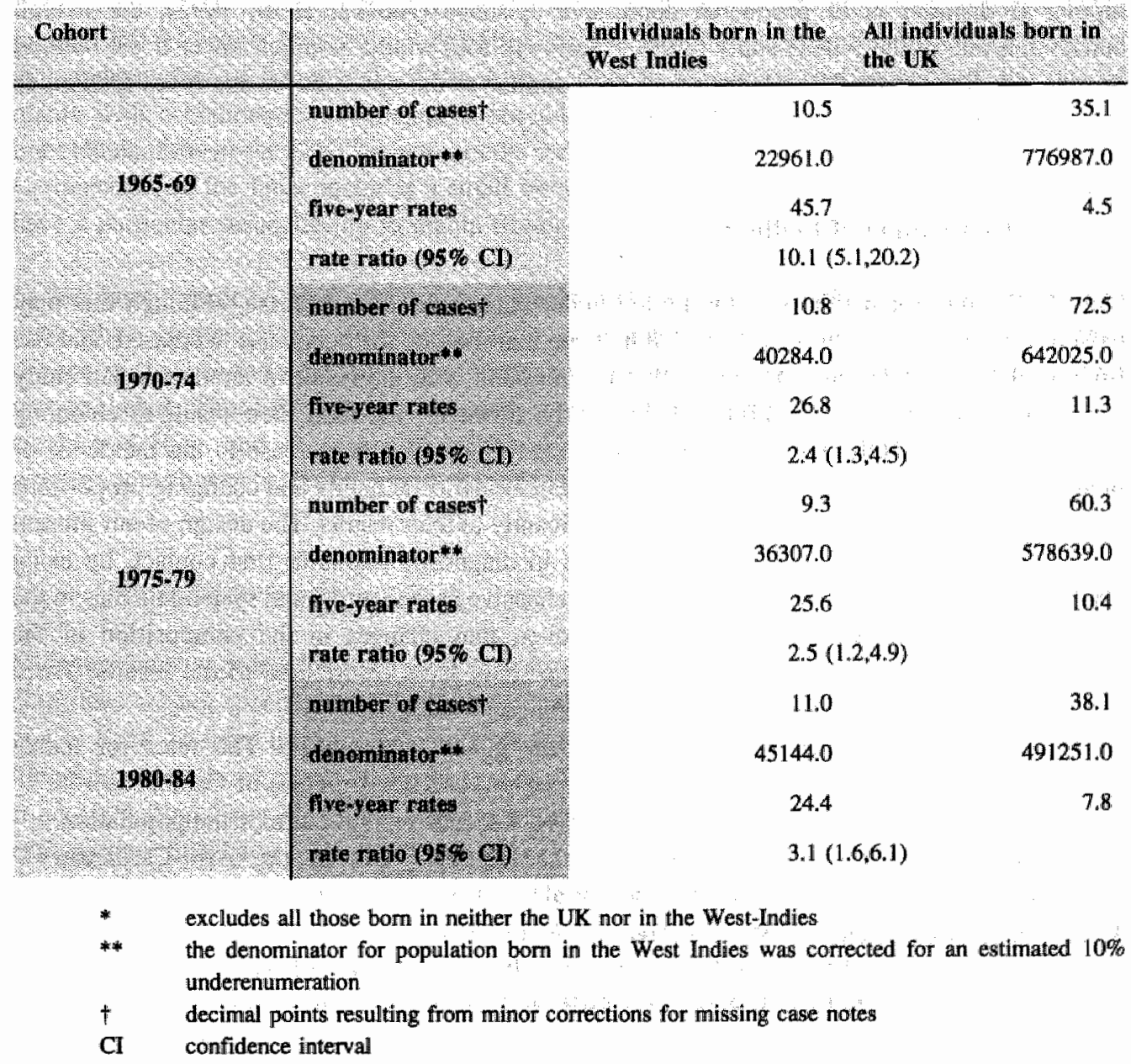


We have shown that exclusion of bipolar patients with first episodes of depression is unlikely to have led to spurious results. Although diagnostic change from depression to mania was only evaluated for cases on the register who had subsequent admissions to hospitall, $90 \%$ of our first contact sample with manic disorder were inpatients, so that few manic patients with initial depressive episodes would have been milssed. Our follow-up period to asses diagnostic change was long enough, as most of the conversion from depression to bipolar disorder takes place in the first five years (Akiskal et al, 1995). The overall rate of change from depression to bipolar disorder in our study must be somewhere between $6 \%$ (of initial first-contact depressives, a substantial portion of which wauld have moved out of the area before suffering an affective relapse) and $11 \%$ (of those initial depressives who remained in the area and had further contact with services). This estimate is similar to figures of the recent NIMH 11 -year follow-up study of major depressive disorder, which found that $3.9 \%$ of subjects developed (manic) bipolar I disorder, and $8.6 \%$ (hypomanic) bipolar II disorder over the ensuing years (Akiskal et al, 1995). Given that many hypomanic episodes occuring in bipolar II disorder are unlikely to be diagnosed as such (eg mild hypomania of a few days' duration), and the predominant clinical picture in bipolar II disorder is of protracted depressive episodes (Akiskal et al, 1995), the overall conversion rate in the NIMH study $(3.9 \%+8.6 \%=12.5 \%)$ would be much lower if the method of case detection in the NIMH study had been similar to the one used in this investigation, as many bipolar II cases would have been missed.

\section{Interpretation of findings}

Overall, the rate for mania over the period under study tended to increase, although this may have arisen by chance. Our results do not indicate that the rate for mania has decreased, and our finding of a significant increase in women is consistent with the result of another recent study (Kendell et al, 1993). The authors of this study, however, ascribed their result to changing diagnostic habits, while the authors of another study reporting an increase in the incidence of mania (Parker et al, 1985) suggested that changes in diagnostic habits and changing proportions of "false" first admissions had contributed significantly to their results. The design of our present study, however, precluded bias due to changes in diagnostic habits at first contact, by using standardized operational criteria based on comprehensive case records, and systematic bias in the missing notes is unlikely. It is possible however, that changes in the composition of the population of Camberwell over the period of the study have contributed to the results. Three major factors could have a bearing on these findings.

- Changes in the age and sex structure of the general population. The rates for mania shown in tables 1 and 2 are standardized to the age and sex structure of Camberwell in 1964, thus obviating any effect of the changing age and sex structure of the population. We also "corrected" conservatively for the relative youth of the Afro-Caribbean population in Camberwell, and this did not alter our results significantly.

- Changes in the socioeconomic structure of the population. It has been reported that mania is evenlly distributed between the social classes in Camberwell (Leff et al, 1976; Der \& Bebbington, 1987). Furthermore, as Castle and colleagues (1991) noted, the minor changes in the socioeconomic structure in Camberwell over the period under study could not account for the magnitude of the changes in the incidence of major psychiatric disorders in Camberwell. Thus, this is unlikely to be a major factor in our study. 
- Changes in the ethnic composition. Our finding of an increased risk for mania in AfroCaribbean individuals is in agreement with earlier reports (Leff et al, 1976; Bebbington, 1981; Der \& Bebbington, 1987) of a relative risk of four-six times in Caribbean-born individuals compared to U.K.-born individuals.

It is not possible to determine exactly, however, whether high rates in the Afro - Caribbean group were the main determinant of the apparent increase in the incidence of mania over the study period. Because of the inadequacies of the census data, it is difficult to give precise rates of mania by ethnic grouping, and by both ethnic grouping and gender. On the basis of our earlier estimate that Afro-Caribbeans comprised $11.5 \%$ of the population in $1981^{\text {w we calculated a rate }}$ for mania with schizomania of 3.3 per 100,000 person-years for all non Afro-Caribbeans between 1980 and 1984. Table 4 shows that the rate of mania with schizomania was 1.8 per 100,000 person-years for the general population for 1965-69. At this time there would have been very few UK-born Afro-Caribbeans in the population entering the age at risk for mania, as the major influx from the West Indies was in the 1950s and 1960s. The rate of 1.8 for "all individuals born in the U.K." in the 1965-69 cohort was lower than the rate of 3.3 for "all non-Afro-Caribbeans" in the 1980-84 cohort (rate ratio: $1.8 ; 95 \%$ Cl: $0.9-3.7 ; \mathrm{p}=0.09$ ). Thus, this difference suggests that, independent of the growth of the Afro-Caribbean population in Camberwell, the rate of mania with schizomania was rising. It should be noted, however, the 1965-69 rate for "all individuals born in the U.K." is not strictly comparable to the 1980-84 rate for "all non-AfroCaribbeans", as the latter includes a small number of immigrants from Africa who might also have a particular susceptibility to mania (Rwegellera, 1977).

In the entire sample, Afro-Caribbean individuals, and especially women, were more likely than their white counterparts to fulfil criteria for schizomania and, correspondingly, there was a decline in the rate ratio for "all Afro-Caribbeans" versus "all other ethnicities" if schizomania was excluded. Thus, Afro-Caribbeans with mania more frequently displayed "schizophrenic" symptomatology, and an excess of mixed affective and schizophrenic states, particularly in women, contributed to the increased risk of mania in Afro-Caribbeans.

\section{Conclusions}

Most previous work has focussed on schizophrenia in the Afro-Caribbean population. However, high rates are not specific to schizophrenia, and the previous focus on Afro-Caribbean patients is also likely to have been too selective, as several studies have reported high rates of mental illness in a variety of ethnic minority groups (Rwegellera, 1977; Selten \& Sijben, 1994; King et al, 1994; Van Os et al, 1995). This has important implications for some of the more specific explanations that have been put forward, such as the use of cannabis, and certain biological theories (McGovern \& Cope, 1987; Harrison, 1990; Wessely, 1991). Whilst these factors may play a role, a non-specific increase in psychosis among various groups of ethnic minorities may indicate that common sources of stress on members of ethnic minorities, associated with sociocultural adjustment and the impact of migration, are also important determinants. 


\section{REFERENCES}

Akiskal, H.S., Maser, J. D. \& Zeller, PJ et al (1995) Switching from 'unipolar' to bipolar II. Archives of General Psychiatry, S2, 114123 .

Bebbington, P., Hurry, J. \& Tennant, C. (1981) Psychiatric disorders in selected immigrant groups in Camberwell. Social Psychiatry, 16, 43-51.

Castle, D. Wessely $\$$. Der, G et al (1991) The incidence of operationally defined schizophrenia in Camberwell, 1965-1984. British Journal of Psychiatry, 159,790-794.

Cooper, J., Goodhead, D., Craig, T. et al (1987) The incidence of schizophrenia in Nottingham. British Journal of Pyychiatry, 151, 619-626.

Dean, $G_{6}$, Walsh, $D_{*,}$ Downing, $H_{*}$, et al (1981) First admissions of native-born and immigrants to psychiatric hospitals in South-East England 1976. British Journal of Psychiatry, 139, 506-512.

Der, G. \& Bebbington, P. (1987) Depression in inner London: a register study. Social Psychiatry, 22, 73-84.

L__, Gupta, S. \& Murray, RM (1990) Is schizophrenia disappearing? Lancet, 335, $513-516$.

Bagles, J. \& Whalley, L. (1985) Dechine in the diagnosis of schizophrenia among first admissions to Scottish mental hospitals from 1969-1978. British Jownal of Psychiatry, 146, 151-154.

Harrison, G., Owens, D., Holton, A et al (1988) A prospective study of severe mental disorder in AfroCaribbean patients. Psychological Medicine, 18, 643-657.

(1990) Searching for the causes of schizophrenias the role of migrant studies. Schizophrenia Bulletin, $16,663-671$.

Kendell, R. Malcolm, D. \& Adams, W. (1993) The problem of detecting changes in the incidence of schizophrenia. British Journal of Psychiatry, 162, 212-218.

King, M., Coker, E, Leavey, G., Hoare, A. Johnson-Sabine, E. (1994). Incidence of psychotic illness in London: a comparison of ethnic groups. British Medical Journal, 309, 1115-9.

Leff, J., Fisher, M. \& Bertelsen, A. (1976) A cross national study of mania British Journal of Psychiatry, 129, $428-42$.

Makanjuola, R.O. (1985) Recurrent unipolar manic disorder in the Yoruba Nigerian: further evidence. British Journal of Psychiarry, 147, 434-437.

McGiuffin, P. Farmer, A. \& Harvey, I. (1991) a polydiagnostic application of operational criteria in studies of psychotic illness: development and reliability of the OPCRIT systern. Archives of General Psychiatry, 48, $645-650$.

McGovern, D., \& Cope, R. (1987) First psychiatric admission rates of first and second-generation AfroCaribbeans: Social Psychiatry, 22, 139-149.

Parker, G., O' Donnell, M. \& Walters, S. (1985) Changes in the diagnosis of the functional psychoses associated with the introduction of lithium. British Journal of Psychiatry, 146, $377-382$.

Prince, M. \& Phelan, M. (1990) Trends in schizophrenia. Lance, 335, 851-852.

Rothman, K. (1986) Modern Epidemiology. Boston, Little, Brown and Company.

Rwegerella, G. (1977) Psychiatric morbidity annong West Africans and West Indians liwing in London. Psychological Medicine, 335, 851-852.

Selten, J.P. \& Sijben, N. (1994) First admission rates for schizophrenia in immigrants to the Netherlands. The Dutch National Register, Social Psychiatry and Psychiarric Epidemiology, 29, 71-77.

Spitzer, R. Endicott, J. \& Robins, E. (1978) Research diagnostic criteria: rationale and reliability. Archives of General Psychiatry, 35, 773-782.

Van Os, J., Galdos, P., Lewis, G., Mann, M. \& Bourgeois, M. (1993), Schizophrenia sans frontières. British Medical Journal, 307, 489-492

Van Os, J. Castle, D. Takei, N., Der, G., \& Murray, R. (1995a) Schizophrenia in ethnic minorities: clarification from the 1991 census. Psychological Medicine, in press.

Wessely, S., Castle, D., Der, G. et al (1991) Schizophrenia and Afro-Caribbeans: A case control study. British Journal of Psychiatry, 159, 795-801.

Wing, J. \& Hailey, A. (1972) Evaluating a community psychiatric service: the Camberwell Register, 1964-1971. Londion: Oxford University Press. 


\subsubsection{Evidence for good prognosis psychosis in Afro-Caribbeans in the UK'}

\section{SUMMARY}

In view of reports of high incidence of psychotic illness in certain ethnic groups, we wished to compare course and outcome of psychotic illness between two groups of Afro-Caribbean ( $n=53$ ) and British-born White individuals ( $\mathrm{n}=60)$, in a cohort study of consecutive admissions, followed-up for 4 years. Subjects were patients admitted to two South-London hospitals with a recent-onset psychotic illness, and the main outcome measures were: illness course, self-harm, social disability, hospital use and treatment variables, adjusted for socio-economic origin. The Afro-Caribbean group spent more time recovered over the follow-up period, (adjusted $O R=5.0$; $95 \% \mathrm{Cl}=1.7-14.5$ ), were less likely to have had a continuous illness (adjusted $\mathrm{OR}=0.3 ; 95 \%$ $\mathrm{CI}=0.1-0.8$ ), were less at risk of self-harm (adjusted $\mathrm{OR}=0.2 ; 95 \% \mathrm{CI}=0.1-0.8$ ), and less likely to have been prescribed antidepressant treatment (adjusted $\mathrm{OR}=0.3 ; 95 \% \mathrm{CI}=0.1-0.9$ ). There were no differences in hospital use, but the Afro-Caribbean group had more involuntary admissions (adjusted $\mathrm{OR}=8.9 ; 95 \% \mathrm{CI}=2.1-35.6$ ), and more imprisonments over the follow-up period (adjusted $\mathrm{OR}=9.2 ; 95 \% \mathrm{CI}=1.6-52.3$ ). Afro-Caribbean psychotic patients in the $\mathrm{UK}$ have more good outcome psychotic illness. The combination of high incidence and less deteriorated illness course in this ethnic group may be due, at least in part, to excess exposure to precipitants in the social environment.

\section{INTRODUCTION}

An increased incidence of affective and non-affective psychosis in the UK has been demonstrated in Afro-Caribbeans (Leff et al, 1976; Harrison, 1988; Wessely et al, 1991; King et al, 1994; Van Os et al, 1995a, 1995b). A number of cross-cultural studies have shown that the prognosis of psychotic disorders such as schizophrenia is better in nonindustrialised countries (Key-Ming \& Kleinman, 1988). Some researchers have questioned whether Afro-Caribbeans in the UK might have a similarly good prognosis and whether the increased incidence of psychosis in this group might be due to an excess of good prognosis illness (Littlewood \& Lipsedge, 1981).

Follow-up studies set to test this hypothesis have not shown better outcome for AfroCaribbeans when compared to British-born Whites or White Europeans (McGovern \& Cope, 1994, Sugarman, 1992; Harvey et al, 1990; Birchwood et al, 1992). However, this failure may have been due to a number of methodological flaws. The studies have not controlled for social class or age of onset of illness though lower socio-economic status and early age of onset are related to poorer outcome (Cooper, 1961; Robins \& Guze, 1970). As UK AfroCaribbeans are of lower socio-economic status and have earlier age of onset illness than their White British peers (Van Os et al, 1995a; Harvey et al, 1990), these differences could mask any better prognosis if they are not controlled for. 
Some studies were retrospective (Birchwood et al, 1992; Harvey et al, 1990), and all relied on cross-sectional clinical information gained at the time of follow-up, rather than on both crosssectional and longitudinal clinical data from the whole follow-up period (McGlashan et al, 1988). Often, outcome measures were reported which are related to service accessibility and the social environment as well as to the clinical state of the patient, such as hospital admissions and use of the Mental Health Act. Such outcomes may tell us little about clinical illness course.

The studies have all had limited power because of small numbers of patients. None has prospectively investigated self-harm, even though this is an important enough outcome to be a health of the nation target and the literature on non-psychotic samples suggests that the AfroCaribbean group are less at risk of this outcome (Burke, 1976; Merrill \& Owens, 1987; Raleigh \& Balarajan, 1992). It is therefore essential to examine whether the same holds for patients with psychosis, who are at very high risk of suicide and parasuicide.

There are anthropological arguments against using Western concepts such as schizophrenia on people from other cultures (Littlewood \& Lipsedge, 1981). Furthermore, the current practice of studying single diagnostic categories of questionable validity may introduce selection bias; especially as the excess risk for psychosis in the Afro-Caribbean group has not been shown to be disorder-specific (Harrison et al, 1988, King et al, 1994; Van Os et al, 1995a \& 1995b).

We report here a prospective study of psychosis comparing the course and outcome in UK AfroCaribbeans and British-born Whites. Cross-sectional assessments of outcome were supplemented by longitudinal measures of course over the whole follow-up period. Multiple sources of information were used. Self harm, socio-economic status and treatment differences were also assessed as was the effect of age of onset.

\section{METHOD}

A cohort of individuals with functional psychosis were drawn from two cross-sectional series of consecutive patients admitted to 2 south London Hospitals $(\mathrm{n}=337$; including some out-ofcatchment-area tertiary referrals). Sampling procedures were virtually identical in both (Jones et al., 1993; Harvey et al, 1990). Hospital admissions aged 16-60 were screened and recruited if delusions, hallucinations or formal thought disorder as defined by the Research Diagnostic Criteria of Spitzer et al (1978), were present in clear consciousness. At least one first degree relative was contacted for information in order to obtain corroborative information.

Patients whose illness had started within five years of recruitment were selected for the 4 year follow-up study ( $n=191$ ). Thus, a sample was obtained of relatively recent onset of illness, avoiding the fallacy of mixing follow-up epochs, which has a confounding effect (McGlashan; 1986).

\section{Socio-demographic and baseline assessments}

The Registrar General's classification of paternal occupation at birth was used to assess socioeconomic status in early childhood. Place of birth and place of parents birth were used as proxy 
variable's to define different "ethnic" groups. The patient was asked their place of birth and their parents' place of birth. Those patients who were white skinned, who were born in the UK and whose parents were born in the UK comprised the White British-born group and those who had both parents bom in the Caribbean constituted the Afro-Caribbean group.

Age of onset of illness was defined as age at which psychotic symptoms first emerged, duration of illness was defined as the time between age of onset and follow-up. The Operational Criteria Checklist for Psychotic Illness (OCCPI; McGuffin et al, 1991) was completed on all patients for the time up to the baseline assessment to give a diagnosis using computerized algorithms. Psychopathological data were derived from detailed cross-sectional mental states at base-line assessment, on the basis of the Present State Examination (Wing et al, 1974) and from the patients records.

\section{Follow-up}

The method and rationale for the follow-up procedures and outcome measures have been described in detaill elsewhere (Van Os et al, 1995c \& 1995d). Briefly, follow-up data were collected by $J v O$, blind to all index data. Because it has been suggested that outcome of psychiatric illness should be seen as a multidimensional concept (Strauss \& Carpenter, 1978), a factor analysis was conducted of all 21 outcome ratings to identify different clinical and social outcome domains (Van Os et al, 1995c). The domains identified were: 1. negative symptoms/social disability; 2 . severity of illness course; 3 . time living independently; 4. unemployment; 5. imprisonment and vagrancy; and 6. depression/self-harm. Instead calculating factor scores, the meaning of which is difficult to appreciate in relation to clinical practice, thirteen a priori chosen outcome measures were identified to represent these different outcome domains (Van Os et al, 1995c; Table 1). Instruments used were the Iager Scale for the assessment of negative symptoms (Iager, 1985), the WHO Disability Assessment Schedule (Jablensky et al, 1980), the Hamilton Depression Scale (Hamilton, 1960), and a modified version of the WHO life chart (WHO, 1992a), which assesses longitudinally employment, independent living and hospitalization, self harm, and treatments received. It also assesses severity of illness course, using clear definitions for all ratings. Course type was rated as episodic (no episode longer than 6 months), continuous (no remission longer than six months), neither episodic nor continuous, and not psychotic in this period. A "usual severity of symptoms" rating indicates the symptomatic level of the patient during most of the follow-up period. Ratings were severe, moderate, mild or recovered. Self-harm included all attempts at self-harm, regardless of the outcome (ie both parasuicide and completed suicide were included).

Data on six areas of treatment over the follow-up were collected. Treatments were: time on antipsychotic, anti-depressant and mood stabilising medications over the follow-up period; time in psychotherapy; and time in rehabilitation (Table 1).

Multiple sources of information were used for the follow-up assessments and where possible and with the subject's permission, general practitioners, family members, spouses, hospital and hostel staff and case notes were consulted (median number of informants: 2; range: $0-3$ ). 
Table I. Ethnic group and i) dimensions of course and outcome, scales used to cover these, and ii) treatment variables.

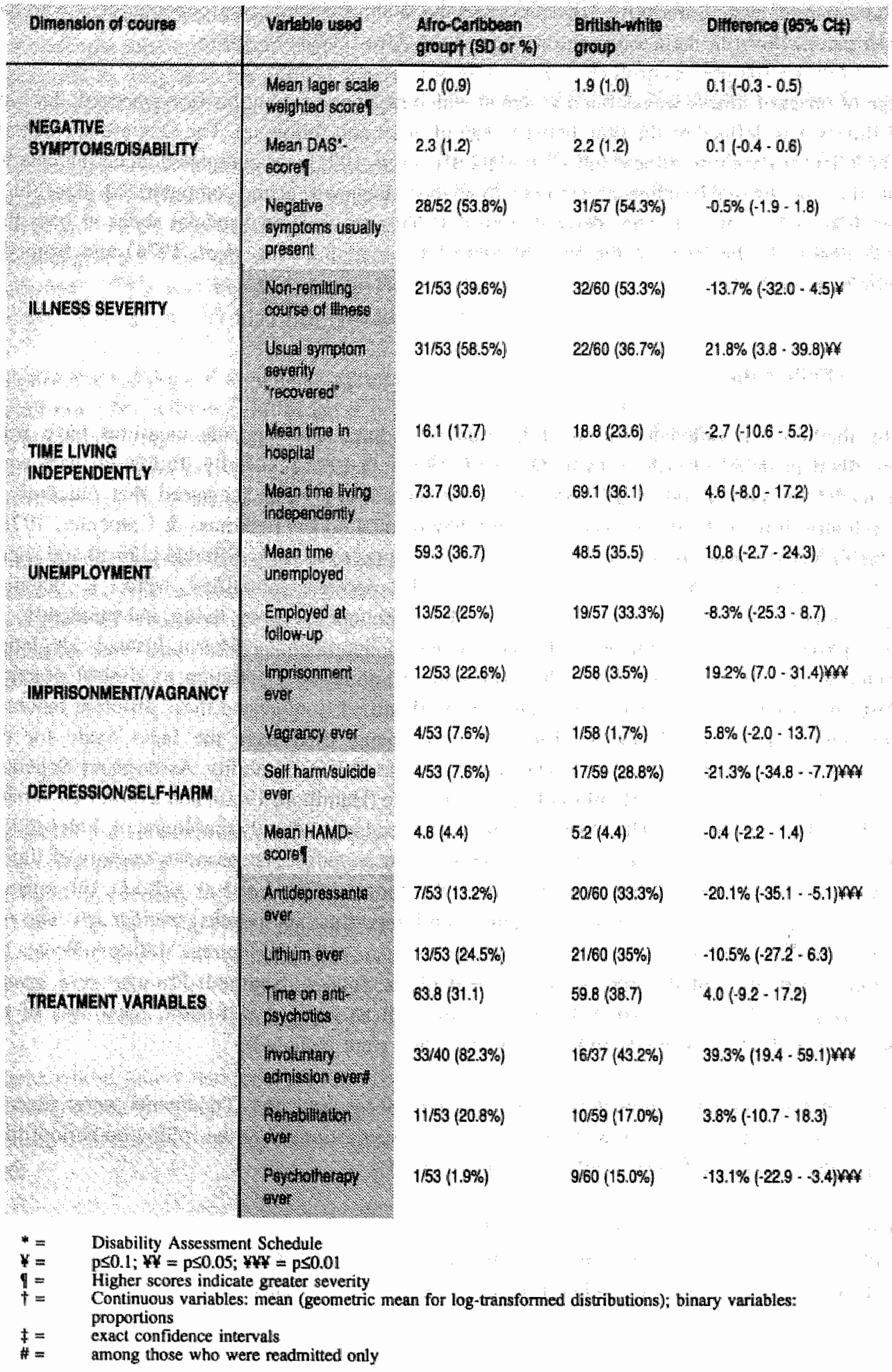




\section{Analyses}

An in depth discussion of the analyses and transformation of variables has been given elsewhere (Van Os et al, 1995c \& 1995d). The means for continuous variables, and the proportions for binary variables were compared between the two ethnic groups. Means were adjusted using multiple regression, and proportions using logistic regression, yielding odds ratios Variables measuring time, for example, time spent in hospital, time unemployed, etc, were expressed as the proportion of the length of the individual follow-up period Some outcome variables were transformed into dichotomous variables if they inclined towards two clinically meaningful categories using the modal value as the cut-off. This has been described in detail in a previous study (Van Os et al, 1995c). For example, for continuous vartables such as time in psychotherapy, and time on anti-depressants, which had severely skewed distributions (the majority having had no such interventions), dichotomization was considered more appropriate, the cut-off being zero values. Similarly, course of illness was transformed into "continuous" and "non-continuous", and usual symptom severity in "recovered" and "non-recovered".

\section{RESULTS}

\section{Sample}

Of the 191 individuals, follow-up data were available for $166(87 \%)$ of these 166,53 were in the Afro-Caribbean group and 60 in the British-born white group. All further analyses refer to these two groups. The Afro-Caribbean group was of lower socio-economic origin, and length of follow-up was slightly longer in the Afro-Caribbean group (Table 2). There was no ethnic bias in attrition (Van Os et al, 1995c \& 1995d).

\section{Associations with ethnicity}

Of the 19 statistical models comparing outcome and treatment between the two ethnic groups, significant differences were apparent in six.

There were large differences between the two groups in three outcome dimensions: AfroCaribbean individuals were more likely to be rated as "recovered" over the follow-up period, and there was a trend to have had more often a non-continuous illness course. The Afro-Caribbean group were less likely to have displayed evidence of self-harm, and were more likely to have been imprisoned (Table 1).

Three differences in treatment were apparent: Afro-Caribbean individuals were less likely to have received psychotherapy and anti-depressant treatment, and were more likely to have been admitted involuntarily over the follow-up period (Table 1). 


\section{Adjustment for confounding factors}

Adjustment for age of onset and childhood social class revealed substantial negative confounding by these variables in that the magnitude of the associations between ethnic group and measures of severity of course doubled after accounting for their effects. Associations between ethinic group and imprisonment and involuntary admission over the follow-up period were similarly affected. Additional adjustment for catchment area status, DSM-III-R diagnosis, gender and length of illness even further increased the magnitude of the associations (Table 3 ).

Table 2. Sample characteristics at baseline

\begin{tabular}{|c|c|c|}
\hline 10 & Arro Caribbean group $(n=53)$ & British-white group $(n=60)$ \\
\hline fernale gender & $16 / 53(30.2 \%)$ & $19 / 60(31.7 \%)$ \\
\hline parental class 12 & $5 / 49 \cdot(10.2 \%)$ & $20 / 57(35.1 \%)$ \\
\hline parental class 3 & $15 / 49(30.6 \%)$ & $23 / 57(40.4 \%)$ \\
\hline prarental class $4 \mathrm{~s}$ & $29 / 49(59.2 \%)$ & $14 / 57(24.6 \%) *$ \\
\hline age of onset ( jears) & 22.8 & 24.3 \\
\hline duration of liness (years) & 2.8 & 3.1 \\
\hline schiopphrenin & $27 / 53(51 \%)$ & $27 / 60(45 \%)$ \\
\hline schizoalfective disor der & $3 / 53(5.6 \%)$ & $5 / 60(8.3 \%)$ \\
\hline affecthe psychosis. & $15 / 53(28.3 \%)$ & $12 / 60(20 \%)$ \\
\hline itypical psychosits? & $8 / 53(15.1 \%)$ & $16 / 60(26,7 \%)$ \\
\hline length of rollow up (munths) & 49.2 & $43.9 * *$ \\
\hline
\end{tabular}

After adjustment for class and age of onset the Afro-Caribbean group was 0.3 times less likely to have had a continuous illness course and 5.0 times more likely to have had a usuall symptom severity of "recovered". The odds of self harm and having been prescribed anti-depressants in the Afro-Caribbean group was decreased by a factor 0.2 and 0.3 respectively; the risk of imprisonment and involuntary admission ower the follow-up period was increased by a factor of 8.2 and 6.2 respectively. The effect of socio-economic origin on these variables was in the opposite direction for the clinical outcome measures. Social class was significantly associated with both course of illness and usual symptom severity, such that over three levels of socioeconomic origin (I/II, III, and IV/V) the risk of continuous illness increased multiplicatively by a factor 2.4 with each level, and the probability of a usual symptom severity of "recovered"" decreased by a factor of 0.5 with each level (Table 4 ).

There were no large or significant interactions with DSM-III-R diagnosis, and differences were similar for individuals from the Afro-Caribbean group born outside or inside the UK. However, raw effects were stronger in the group of non-schizophrenic psychoses. For example, the odds ratio for recovery was $6.4(95 \% \mathrm{CI}: 1.6-25.5)$ in the non-schizophrenic psychoses, versus 2.4 (95\% CI: $0.6-9,3)$ in schizophrenia. Similarly, the protective effect for self-harm was more evident in the non-schizophrenic psychoses (OR: $0.1 ; 95 \% \mathrm{CI}: 0.01-0.64$; schizophrenia: $\mathrm{OR}=0.4$; 95\% CI: $0.1-2.0$ ). 
Table 3. Associations between ethic growp and illness course and effect of confounding factors

\begin{tabular}{|c|c|c|c|c|}
\hline 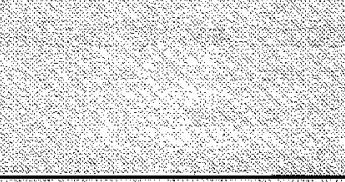 & $\begin{array}{l}\text { Arow Garibbean rs } \\
\text { While } \\
\text { (unadjusted) }\end{array}$ & 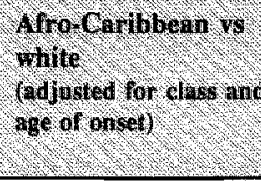 & & 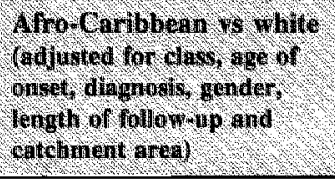 \\
\hline Continuous ilhess course & $0.6(0.3-1.2)$ & $0.3(0.1 \cdot 0.8)$ & $\because$ & $0.2(0.1-0.8)$ \\
\hline $\begin{array}{l}\text { Usual symptom severity } \\
\text { recovered }\end{array}$ & $2.4(1.1-5.2)$ & $5.0(1.7-14.5)$ & & $7.2(2.0-25.7)$ \\
\hline $\begin{array}{l}\text { Imprisonment over follow } \\
\text { ip period }\end{array}$ & $8.2(1.7-38.6)$ & $9.2(1.6-52.3)$ & & $18.5(2.2-155.2)$ \\
\hline $\begin{array}{l}\text { Prescribed arifi- } \\
\text { depressants } \\
\text { over follow wp period }\end{array}$ & $0.3(0.2-0.8)$ & $0.3(0.1-0.9)$ & 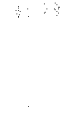 & $0.3(0.1-0.9)$ \\
\hline $\begin{array}{l}\text { Gelf harm orer follow ip } \\
\text { perlod }\end{array}$ & $0.2(0.1-0.7)$ & $0.2(0.1-0.8)$ & & $0.2(0.1-0.7)$ \\
\hline $\begin{array}{l}\text { limvolunitary admission } \\
\text { over follow-mp period }\end{array}$ & $6.2(2.2-17.6)$ & $8.9(2.1-35.6)$ & & $9.0(2.6 \times 31.3)$ \\
\hline $\begin{array}{l}\text { Psychotheraps over } \\
\text { follow-up period }\end{array}$ & $0.1(0.01-0.9)$ & $0.2(0.02-1.4)$ & & $0.2(0.01-1.6)$ \\
\hline
\end{tabular}

Table 4. Associations between childhood social class and illness course

\begin{tabular}{|c|c|c|c|}
\hline & 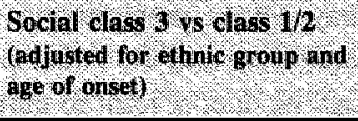 & 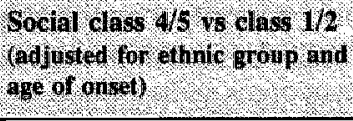 & 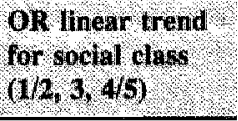 \\
\hline $\begin{array}{l}\text { Continunous illiness } \\
\text { courot }\end{array}$ & $2.5(0.8-8.6)$ & $5.8(\mathbb{1} .5-22.1)$ & $2.4(1.2-4.6)$ \\
\hline $\begin{array}{l}\text { Usliah sy mptom } \\
\text { otwerity recoverted }\end{array}$ & $0.3(0.1-1.0)$ & $0.2(0.1-0.8)$ & $0.5(0.3-0.96)$ \\
\hline
\end{tabular}

\section{DISCUSSION}

Our findings give a necessarily complex picture to the outcome of psychosis in Afro-Caribbeans. when compared to British-born Whites. There is better prognosis with regards to symptoms, course of illness and para/suicide, there is poorer prognosis with regards to social and service dependent outcomes such as the use of sections of the Mental Health Act and time spent in jail.

\section{Methodological Issues}

Chance is an unlikely explanation for our findings. Face to face interviewing meant the rater was not blind to ethnic status, but the mix of poorer and better outcomes found in the Afro-Caribbean group is not suggestive of systematic bias. This is the first study to take into account possible alternative explanations, such as socio-economic origin. The better outcome of psychosis in AfroCaribbeans was shown using longitudinal data but not cross-sectional data. A similar discrepancy 
was noted in another study (Van Os et al, 1994), and this phenomenon is likely to be related to the high degree of misclassification associated with cross-sectional outcomes: a single measure of temperature is unlikely to be representative of temperature fluctuations over the last four years.

The comparatively large changes in the ethnicity parameters in statistical models (more than $200 \%$ in some instances) after adjusting for socio-economic origin and other variables, shows that ethnicity as a statistical concept is extraordinarily confounded, and should lead one to be very cautious in interpreting "ethnic" differences, our own included.

\section{Interpretation of findings}

No previous outcome study comparing ethnic groups with psychosis in the UK has used longitudinal measures. Moreover, the better prognosis shown by our longitudinal data does not become quite apparent until differences in social class are allowed for in the analysis. Previous studies may have failed to show the relatively good prognosis for Afro-Caribbeans with psychosis that we found because of the confounding effect of social class. Lower social class predicts poorer outcome in schizophrenia (Cooper, 1961) and social class is a well known confounding variable in comparisons between ethnic groups (McKenzie \& Crowcroft, 1994).

The link between social adversity and prognosis is not straightforward. Though lower social class predicts poorer outcome from established illness, the presence of environmental precipitants ("life events") predict better prognosis (Vaillant, 1964; Van Os et al, 1994). The social pressures associated with ethnic minority membership may act as precipitating factors for psychosis (Creed, 1995; King et al, 1995). Both the high incidence and the better prognosis of psychosis in Afro-Caribbeans that we have demonstrated may be due, at least in part, to an excess of such illness with social precipitants.

Despite favourable symptom and course indices, Afro-Caribbeans in London are still more likely to be admitted under a section of the Mental Health Act, and to spend similar amounts of time in hospital as British-bom White patients. They are also more likely to be in jail during the follow-up period. When the diagnosis of schizophrenia is taken into account, Afro-Caribbeans are even more likely to have the Mental Health Act used at admission or to be in jail. The difference in use of the Mental Health Act and prison services has been demonstrated in previous studies (eg Perera et al, 1991) but our study suggests that these variables may not be related to the clinical state of the patient. More likely, it reflects differing degrees of engagement with psychiatric services.

The WHO outcome study found a better prognosis for people from developing countries but showed that this was not due to an excess of brief illnesses of good prognosis, regardless of diagnosis (WHO, 1992). Robins and Guze, in an influential paper (1970), differentiated good outcome schizophrenia from illness with poor outcome, and postulated that the former was associated with severe premorbid stress, and the latter with constitutional factors. Our findings also indicate that better outcome was evident in the Afro-Caribbean group. We suggest that the risk profiles of psychosis may be different in our two groups, and postulate an excess of socially mediated illness in the Afro-Caribbean group. 
There have been a number of hypotheses: to explain the underlying mechanisms for better prognosis in developing countries. It has been postulated that the poorer outcome may be because of poorer social support, poorer opportunities for employment, greater stigma with regards to the diagnosis of mental illness and greater survival of more vulnerable people with neurological abnormalities who later go on to suffer from schizophrenia (WHO, 1992). We did not find differences in the social environment which could explain the findings. In fact, the AfroCaribbean group fared better clinically even in the face of continuing social adversity. There is no evidence that there is less stigma in the Afro-Caribbean community compared to the British White community with regards to mental illness. Speculation that the better prognosis may be due to family support and better opportunities for employment (WHO, 1992b) were not supported by this study.

There is some indirect evidence that there may have been psychopathological differences during the follow-up period between the groups. It has been postulated that depression in AfroCaribbeans is not recognized, which could explain the lower likelihood of being prescribed antidepressant treatment in Afro-Caribbeans (Elk et al, 1986). However, the increased risk of para/suicide in British-born Whites suggests that higher rates of treatment with antidepressants may have been related to genuine differences in the prevalence of depressive disorder. The WHO outcome study also showed that depressive symptoms were more prevalent in people with schizophrenia in developed countries (WHO, 1992b). Similar ethnic differences may exist in the proportion of manic patients who will present with previous depressive episodes Makanjuola (1985), found that among the Yuruba in Nigeria 55\% of patients could be classified as suffering recurrent mania, whilst in Westem societies combinations of manic and depressive episodes is much more common than unipolar manic disorder (Perris, 1966).

This is the first prospective study to show ethnic differences in para/suicide in a psychotic sample, and concurs with work showing such differences in other samples (Burke, 1976; Merrill \& Owens, 1987; Raleigh \& Balarajan, 1992). However, there is a suggestion that rates of parasuicide may be increasing in Afro-Caribbeans to the level of the British White population (Merrill \& Owens, 1987), which needs to be investigated further. 


\section{REFERENCES}

Binchwood. M., Cochrane, R, MacMinla, F., Copestake, S., Kucharska, J. \& Carris, M. (1992) The influence of ethricity and family structure on relapse in first-episode schizoprhenia. British Journal of Psychatry, 161 , $783-790$.

Burke, A.W. (1976) Socio-cultural deteminarits of attempted suicide among West-Indians in Birmingham: ethnic origin and immigrant status. British Journal of Psychiatry, 129, 261-266.

Cooper, B. (1961) Soclal class and prognosis in schizophrenia. Part I and Part II British Journal of Preventive and Social Medicine, $15,17-41$.

Gik, $R_{n}$, Dickman, BJ \& Teggin, A.F. (1986) Depression in schizophreniat a study of prevalence and treatment. British Journal of Psychiatry, 149, 228-9.

Hamilton M. (1960) A rating scale for depression. Journal of Neurology, Newrosurgery and Psychiatry, 23, 5662 .

Harder D.W. Gift, T.E., Strauss, J.S., Ritzler, B.A. \& Kokes, R.F. (1981) Life events and two-year outcome in schizophenia. Jourmal of Cansulting and Clinical Psychology, 49.619-626.

Harrison, $\mathrm{G}_{3}$, Owens, $\mathrm{D}_{n}$, Holton, A. er al (1988) A prospective study of severe mental disorder in AfroCaribbean patients. Psychological Medicine, 18, 643-657.

Harvey, I, Wiliams, M., McGuffin, P. \& Toone, B. (1990). The functional psychoses in Afro-Caribbeans. British Jowrial of Psychiary, 157, 515-22.

Iager, A.C., Kirch, D.G. Wyatt, R.J. (1985) A negative symptom rating scale Psychiatry Research 16, 27-36.

Jablensky, A. Schwartz, R. \& Tomov, T. (1980) WHO collaborative study of impairments and disabilities associated with sehizophrenic disordlers. A preliminary communication. Objective and methods. Acta Psychiatrica Scandinavica, suppl. 285, 152-163.

Jones, P.B., Bebbington, P., Foerster, A., Lewis, S., Murray, R., Russel, A., Sham, P., Toone, B. \& Wilkins, S. (1993) Premorbid social underachievement in schizophrenia. Results from the Camberwell Collaborative Psychosis Study, British Journal of Psychiatry, 162, 65-71.

King, M. Coker, E., Leavey, G., Hoare, A. Johnson-Sabine, E. (1994) Incidence of psychotic illness in London: a comparison of ethnic groups. British Medical Journal, 309, 1115-9.

Leff, J., Fisher, M. Bertelsen, A. (1976) A cross national study of mania. British Journal of Psychiarry, 129 , $428-42$.

Keh-Ming, L. \& Kleinman, A.M. (1988) Psychopathology and clinical course of schizophrenia: a cross cultural perspective. Schizophremia Bulletin, 14, 555-567.

Littlewood, R. \& Lipsedge, M. (1981) Acute psychotic reactions in Caribbean-born patients. Psychological Medichine, 11,303-318.

Makanjuola, R.O. (1985) Recurrent unipolar manic disorder in the Yoruba Nigerian: further evidence. British Journal of Psychiatry, 147, 434-437.

McGlashar, T.H. (1986) Predictors of shorter-, medium-and longer-term outcome in schizophrenia, American Journal of Psychiatry, 143, 50-55.

_....... Carpenter, W. Bartko, J. (1988) Issues of design and methodology in long-term follow-up studies. Schizophrenia Bulletin, 14, 569-574.

McGovern, D., Henmings, P., Cope, R. Lowerson, A. (1994) Long-term follow-up of young Afro-Caribbean Britons and white Britons with a frist admission diagnosis of schizophrenia. Social Psychiatry and Psychiatric Epidemiology, 29, 8-19.

McGuffin, P., Farmer, A.E. \& Harvey , 1. (1991) A polydiagnostic application of operational criteria in psychotic illiness: development and reliability of the OPCRIT system. Archives of General Psychiatry, 48, 764-770.

McKenzie, K.J. \& Crowcroft, N.S. (1994) Race, ethnicity, culture, and science. British Medical Jourmal. 309 , $286-87$.

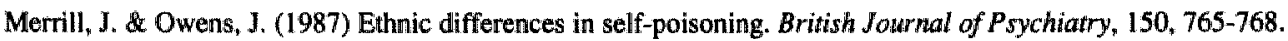

Perera, R., Owens, D. Wohnstone, E. (1991) Disabilities and circumstances of schizophrenic patients- a followup study. VII: Ethnic aspects. A comparison of three matched groups. British Journal of Pisychiatry, 159 , Suppl. 13,40-42.

Perris, C. (1966) A study of bipolar manic-depressive and unipolar recurrent depressive psychosis. Acta Psychiatrica Scandinavica, 42, suppl. 194

Raleigh, V. Balarajan, R. (1992) Suicide levels and trends among immigrants in England and Wales. Health Trends, 24, 91-94.

Robins, E. Guze, S.B. (1970) Establishment of diagnostic validity in psychiatric illness: its application to schizophrenia. American Journal of Psychiatry, 126, 983-987.

Spizer, R., Endicott, J. \& Robins, E. (1978) Reseanch diagnostic criteria: rationale and reliability. Archiwes of General Psychiatry, 35, 773-782. 
Strauss, J.S. \& Carpenter, W. (1978) The prognosis of schizophrenia: rationale for a multidimensional concept. Schizophrenia Bulletin, 1978, 56-67.

Sugarman, P.A. Outcome of schizophrenia in the Afro-Caribbean community (1992). Sacial Psychiatry d Psychiatric Epidemiology, 27, 102-5.

Vaillant, G.E. (1964) Prospective prediction of schizophrenic remission. Archives of General Psychiatry, 11, 509518.

Van Os, J., Fahyy, T., Bebbington, P., Jones, P., Wilkins, S., Sham, P. el all. (1994) The influence of life events. on the subsequent course of psychotic illness. Psychological Medicine, 24, 503-513.

J. Castle, D. Takei, N., Der, G., \&urray, R. (1995a) Schizophrenia in ethnic minorities: clarification from the 1991 census. Psychological Medicine, in press:

, Takei, N., Castle, D., Wessely, S., Der, G. \& Murray, R. (1995b) The incidence of mania: time trends in relation to gender and ethnicity (submitted manuscript)

-, J., Fahy, T., Jones, P., Harvey, I., Sham, P., Lewis, S., Williams, M., Toone, B. \& Murray, R. (1995c) Psychopathological syndromes in the functional psychoses: associations with course and outcome: Psychological Medicine, in press.

-.-.... J. T. Fahy, P. Jones, 1. Harvey, S. Lewris, M. Williams, B. Toone, R. Murray (1995d). Increased intracerebral CSF spaces predict unemployment and negative symptoms in psychotic illness: a prospective study. British Journal of Psychiatry, 166, 750-759

Wessely S, Castle D, Der G, Murray, R. Schizophrenia and Afro-Caribbeans: A case control study. British Journat of Psychiatry, 1991; 159: 795-801.

WHO (1992) WHO coordinated multi-center study on the course and outcome of schizophrenia. WHO: Geneva.

Wing, J.K. Cooper, J.E. \& Sartorius, N. (1974) The Measurement and Classiffication of Psychiatric Symptoms. Cambridge University Press, Cambridge. 


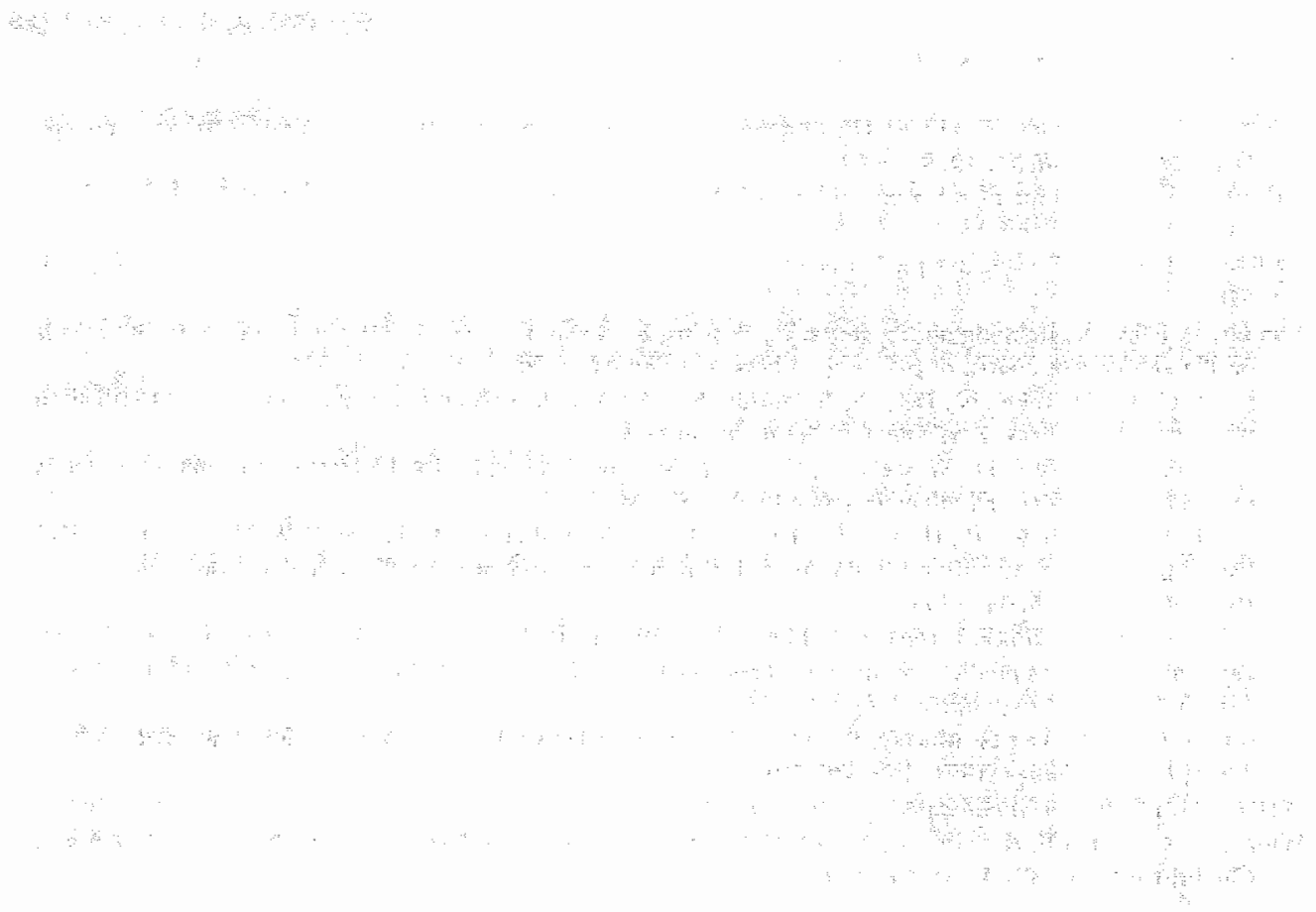




\subsubsection{The influence of life events on the subsequent course of psychotic illness'}

\section{SUMMARY}

Fifty nine psychotic patients with acute onset of illness, who had been interviewed about their experience of stressful life events before the episode, were followed up for an average of 42 months. Thirty patients (51\%) had experienced a stressful life event in the three months immediately before onset $(\mathrm{EV}+), 29$ had not ( $\mathrm{EV}-$ ).

In patients with an RDC diagnosis of affective disorder or unspecified functional psychosis the presence of stressful life events was associated subsequently with milder symptom severity, less time spent in hospital, more treatment for depressive symptoms and less for psychotic symptoms. In schizophrenia, differences were less apparent, but patients with event associated episodes had less need of anti-psychotic maintenance medication over the follow-up period and tended to have spent more time in complete remission. EV+ schizophrenic subjects also had higher morbid risk for schizophrenia in their first degree relatives, and tended to be female and to have less typical symptoms than EV-schizophrenic patients.

Patients with an insidious onset of illness were more likely to have a diagnosis of schizophrenia, and had poorer outcome than patients with acute illness onset, regardless of diagnostic category

\section{INTRODUCTION}

Over the last twenty years, a large body of research has examined the relationship between adverse life events and the relapse of illness in individuals with psychosis, mainly schizophrenia (Bebbington et al, 1993a). Life events research is beset with methodological obstacles (reviewed by Bebbington, 1987), particularly when it involves psychotic illness as it must be restricted to those cases where onset is relatively recent, clear-cut, and capable of accurate dating. Results; therefore, remain inconclusive. Three recent studies $_{*}$, however, employed a design involving the prospective assessment of patients who had recovered from a known episode of illness, comparing the frequency of events in a set period preceding onset with analogous periods in the same patient not followed by relapse, and with the mean event rate in non-relapsing patients (e.g Ventura et al. 1989; Malla et al., 1990; Jolley, Cramer \& Hirsch, pers. comm., 1990). All found that events were commoner in periods preceding relapse of psychosis.

Is the course of psychotic disorders that follow stressful life events different from those without significant preceding events? Some prospective studies have suggested that psychosis preceded by severe life events, may have a more benign course of illness (Vaillant, 1964; Strauss \& Carpenter, 1974; Prudo \& Blum, 1987). However, these studies had serious methodological shortcomings concerning the assessment of precipitants (raters were not blind, and there was no objective rating of the impact of events), and failed to take into account the effect of confounding factors in the statistical analyses. 
In Scandinavia, much emphasis is placed on the concept of reactive psychoses, which are said to have paranoid, depressive, or confusional symptoms (see Strömgren, 1985; Cooper, 1986), differentiating them from typical schizophrenic presentations. In spite of its widespread acceptance, however, it appears that the concept of 'reactive psychosis', i.e. psychotic reactions in 'nomal' individuals after severe stress, followed by complete recovery, has yet to be validated by empirical data. The reactive psychosis concept assumes what Brown and colleagues (1973) called a formative causal role, where events are of fundamental significance in the causation of the condition, and by implication much more important than other factors, for instance, inheritance. The alternative is that in psychotic illnesses life events 'merely' trigger onset or relapse in constitutionally susceptible individuals (Brown $e t a l, 1973$ ). If this were so, the course of il]ness thereafter should be the same as in patients whose onset or relapse was not 'brought forward' by life events, but who have the same predisposition to severe mental illness.

Here, we use the follow-up of the Camberwell Collaborative Psychosis study (Jones et al, 1993; Bebbington $e t a l, 1993 b)$, to establish the relationship of life events to phenomenology, outcome, and familial psychiatric morbidity. We hypothesized that life events would have a formative' role, and thus that i) phenomenological differences would be apparent between those psychotic subjects who had, and those who had not, experienced a life event prior to onset of illness, ii) life-event related illness would be associated with decreased familial morbid risk for psychosis, and iii) the presence of stressful life events before the onset of psychosis would be associated with a more benign course.

\section{METHOD}

\section{Index cases and baseline assessments}

The Camberwell Collaborative Psychosis study is a prospective investigation of familial, developmental, and social aspects of 195 patients consecutively admitted to the Maudsley, Bethlem Royal and Dulwich North hospitals with functional psychosis between March 1987February 1988, and October 1988 - August 1989. The overall rationale for the study has been described previously (Jones et $a l, 1993$ ), as were details of sampling and method. The chosen age range was from 16 to 50 years, and the primary criterion for inclusion in the study was the presence of delusions, hallucinations, or thought disorder in clear consciousness, as defined in the Research Diagnostic Criteria (Spitzer et al., 1975).

Subjects were interviewed within three days of admission. The baseline assessment included detailed demographic and clinical information, neuropsychology, neuroimaging and premorbid functioning. The Operational Criteria Checklist for Psychotic Illness (OCCPI; McGuffin et al, 1991) was completed for all patients for the time up to index assessment (index RDC diagnosis). The OCCPI clieck-list is based on phenomenological descriptions in the Present State Examination (Wing et al, 1974), and covers a wide range of operational definitions of psychiatric conditions. The computer program OPCRIT (McGuffin et al, 1991) was used to analyze the OCCPI data. Psychopathological data were derived from detailed cross-sectional mental states at index assessment on the basis of the Present State Examination (PSE), 9th Edition (Wing et al., 1974). In addition, the PSE data were analysed with the CATEGO programme, providing 38 syndrome scones. PSE syndrome profiles were constructed for all subjects: syndromes were 
recorded as present if given a rating of 2 or above by CATEGO. Syndrome scores for the categories organic impairment, subcultural delusions and doubful interview were not included in the analyses. Every effort was made to obtain case records containing information on all admissions before index assessment.

\section{Information on relatives}

The procedure for collecting information on first degree relatives thas been described elsewhere (Foerster et al, 1991 a \& b; Sham et al, 1994). Information was obtained from three sources: i) the proband's medical records, ii) the proband"s mother and iii) the medical records of relatives with a psychiatric history. Mothers were interviewed according to the Family History - Research Diagnostic Criteria (FH-RDC, Andreasen et al, 1986), after permission to do so was obtained from the proband. The FH-RDC criteria were applied blind to information on the proband.

\section{Life events}

The recording and rating of life events has been described in detail in a previous paper (Bebbington et al., 1993b). Briefly, individuals with a datable onset of psychotic symptoms were interviewed for a history of events during the six months prior to onset, rated according to the contextual rating of threat technique developed by Brown and Harris (1978). Datable onset was defined as an abrupt change, with the emergence of florid symptoms (delusions, hallucinations, passivity experiences and disorders of the form, flow, or sense of possession of thought) over a two-week period. Onset included transitions from a symptom free state or neurotic symptoms only ("true" onsets), as well as from residual symptoms (exacerbations). Where florid psychotic symptoms emerged from a short lived neurotic prodrome, the onset was dated to the beginning of the latter. Where there had been a background of persistent neurotic symptoms, onset was chosen as the point of emergence of the psychotic symptoms. Events were rated along a four point scale, a rating of 1 indicating marked threat, 2 moderate threat, 3 mild threat, and 4 no threat. In practice, events were analyzed mainly by using a category combining the ratings ' 1 ' and ' 2 ', and a category containing events rated ' 3 ', the main role of events rated ' 4 ' being to allow the event-history to be over-inclusive. A second rating was of the degree to which the event was apparently imposed upon subjects and could not be regarded as arising from symptomatic behaviour; events were thus divided into those that are logically independent, possibly independent, and probably dependent. In our previous study (Bebbington et al, 1993b), we found the greatest discrepancy between cases and community controls for the more severe events (i.e. rating 1 or 2 ) in the combined category of independent and possibly independent events in the three months immediately prior to onset. In the present study, therefore, a history was considered positive for stressful events if a subject had experienced this type of event prior to onset of illness.

\section{Follow-up}

Of the 195 patients, those whose first psychotic symptoms had appeared within five years of index assessment were selected for the 4 year follow-up study. Thus, a sample was obtained with 
relatively recent onset, avoiding the confounding effect of mixing follow-up epochs (Carpenter \& Kirkpatrick, 1988, McGlashan, 1986). Patients were traced and contacted with permission from their responsible medical officer. An effort was made to interview subjects in the commumity, and to avoid in-patient assessments where possible, as these would unfavourably bias cross-sectional outcome measures. The rater (JvO) was blind to all data collected at index assessment.

The following instruments were used to provide cross-sectional outcome measures: Global Assessment of Functioning-symptomatology (GAF-s; WHO 1992), derived from the Global Assessment Scale (Spitzer et al, 1975), Disability Assessment Schedule-social disability (DAS-s; Jablensky et al, 1980), Tager Negative Symptom Rating Scale (lager, 1985); Hamilton Depression Scale (HAMD, Hamilton, 1960); Abnormal Involuntary Movement Scale (AIMS; Guy, 1976); and the Present State Examination (Wing et al, 1974).

To assess course of illness and associated disabilities longitudinally, we used a slightly modified version of the life chart instrument from the Multi-Center Study on the Course and Outcome of Schizophrenia (WHO, 1992). The life chart assesses course of illness in a number of ways, using clear definitions for the rater to base his ratings on. Course type was rated as episadic (no episode longer than 6 months), continuous (no remission longer than six months), neither episodic nor continuous, and not psychotic in this period (Appendix). A usual severity of symptoms rating was made to indicate the symptomatic level of the patient during most of the follow-up period Ratings of severe and moderate referred to the presence of marked and obvious psychotic symptoms, whereas ratings of mild or recovered indicated that behaviour was generally normal and psychosis not immediately apparent, or absent (Appendix). In addition, the life chart rates the proportion of the follow-up period subjects had been in hospital, in a psychotic state, and in complete remission. Data on pharmacological and psychotherapeutic interventions over the follow-up period were also obtained. Psychotherapy was defined as regular sessions with a mental health worker, specifically dedicated to treatment by means of psychotherapeutic techiniques. A second OCCPI was completed for the patients for the time up to follow-up (follow-up RDC diagnosis):

In general, multiple sources of information were used for follow-up assessments, and where possible general practitioners, family members, spouses, hospital and hostel staff etc., were interviewed with the subject's permission.

\section{Analyses}

Analyses were performed using the SPSS-PC software (Norušis/SPSS INC, 1990). Proportions were compared using the chil squared test with Yates' correction, or the two-tailed Fisher exact test where appropriate. Student's t-test was used to test differences in means. Multiple regression analysis was used to adjust for confounding factors. Variables were transformed to remove skewness where appropriate, and residuals were examined for any non-linearity. Some variables that were severely skewed and tended towards a categorical distribution (i.e. presence or absence) were treated as dichotomous variables ("continuous" illness course, AIMS score). The logistic regression model was used for binary dependent variables; odds ratios (OR) and $95 \%$ confidence intervals (CI) were calculated from the result of the logistic regression. 
The lifetime risks of schizophrenia and affective disorder in first degree relatives were estimated by the method of Strömgren (1935; Slater Cowie, 1974), using age of onset distributions derived from Mental Health Enquiry data on 12 regions in England and Wales (Takei et al, 1992) to define the period of risk.

\section{RESULTS}

One hundred and thirty one patients met the inclusion criteria for the follow-up study. Of these, $50(38 \%)$ had not been eligible for life event assessment because of an insidious, non-datable onset (39) or because onset had occurred more than twelve months prior to assessment (11). Of the remaining 81 subjects who had presented with datable onsets in the twelve months prior to assessment, $70(86 \%)$ were interviewed about their history of events. Follow-up data were available on $59(84 \%)$ of these 70 patients, 3 of whom had committed suicide. Compared to the 59 follow-up subjects, the 11 patients not followed up were very similar regarding sex, premorbid adjustment, ethnicity, index diagnosis, presence of life events, or age of first contact, the largest difference being in ethnic composition (proportion Caucasians in follow-up group: $66 \%$; in group lost to follow-up: $55 \%, \chi^{2}=0.2 ; p=0.7$ ).

Of the 39 patients who had presented with an insidious onset of illness, the majority had an index RDC diagnosis of schizophrenia (68\%). Male sex was significantly more common in the insidious onset group, and also within the combined group of schizo-affective and schizophrenic subjects $\left(\chi^{2}=4.2 ; p=0.04\right)$. After controlling for in patient status at follow-up, sex, ethnicity and RDC diagnosis, negative symptom scores at follow-up were significantly higher in the insidious onset group ( $B=0.7 ; p=0.001)$, as were DAS-s scores $(B=0.7 ; p=0.02$ ), indicating more social disability.

\section{Characteristics of the follow-up sample}

Of the 59 follow-up subjects, 34 (58\%) were male. At index assessment, 13 subjects $(22 \%)$ had a RDC diagnosis of affective psychosis, 21 (36\%) of schizo-affective psychosis, $20(34 \%)$ of schizophrenia and $5(8 \%)$ of unspecified functional psychoses. Caucasians comprised $66 \%$ of the sample, Afro-Caribbeans $24 \%$, and other ethnic groups $10 \%$. The mean length of follow-up was 42 months (range=10-61; S.D. $=10$ ), mean symptom severity over the follow up period 3.3 (S.D. $=0.95$ ). Patients had spent on average 17\% (S.D. 20) of the follow-up period in hospital, $58 \%$ of the period (S.D. 34) on anti-psychotics, and $13 \%$ of the period (S.D. 27) on antidepressants. Mean GAF-s score at follow-up was 53 (S.D. 21), and mean DAS-s score 1.2 (S.D. 1.1). Twelwe subjects $(20 \%)$ were in-patients at the time of follow-up.

\section{Clinical presentation at index assessment and life events}

Of the 59 patients followed-up, $30(51 \%)$ had experienced severe events prior to onset $(\mathrm{EV}+)$, 29 had not (EV-). There were more femalles in the EV+ group, and more subjects who were inpatients at the time of follow-up, these differences being non-significant. Slight differences existed in ethnic composition and index RDC diagnosis distribution, and the $\mathrm{EV}+$ and $\mathrm{EV}$ - 
groups were very similar regarding premorbid adjustment, the proportion of "true" onsets, age of first psychiatric contact and at index assessment, and the number of previous admissions (Table 1).

Table 1. Characteristics of patherts with and whithout stressful life events prior to onset of illness:

\begin{tabular}{|c|c|c|}
\hline 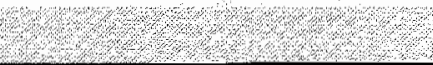 & EVt & $\mathrm{BV}$ \\
\hline Number & 30 & 29 \\
\hline Temiles & 16 & 9 \\
\hline Cauicasians & 21 & 18 \\
\hline Oiher ethnicities & 9 & $11:$ \\
\hline $\begin{array}{l}\text { Premorbid adjustmient scale, } \\
\text { childhood (mean) }\end{array}$ & 12.4 & 11.4 \\
\hline $\begin{array}{l}\text { Premorbild adjust ment scalle, } \\
\text { adolescence (mean) }\end{array}$ & 15.1 & 15.8 \\
\hline Age of onset psychosis & 24.2 & 24.0 \\
\hline proportion in-patient at follow-up & $30 \%$ & $10 \%$ \\
\hline proportion "true" onsets & $85 \%$ & $79 \%$ \\
\hline Age at index adinussion & 26.0 & 27.2 \\
\hline $\begin{array}{l}\text { Nimber of previous admiksions } \\
(5 \text { or more rated as } 5)\end{array}$ & 1.9 & $\cdots:$ \\
\hline RDC-schizophrenia & 9 & 11 \\
\hline RDC-schizo-depressive & 5 & 4 \\
\hline RDC-schizo-manichipolar & 5 & 7 \\
\hline RDC-depression & 3 & 1 \\
\hline RDC-maniabipolar & 5 & 4 \\
\hline RDC unspecified psychosis & 3 & 2 \\
\hline
\end{tabular}

* No differences with $\mathrm{p} \leq 0.05$

In the group of patients with an index diagnosis of schizophrenia, differences were apparent in the frequencies of the 38 individual CATEGO syndromes between EV+ and EV-subjects (Fig. 1). EV- subjects tended to display more symptoms in the categories NS (nuclear syndrome: EV+: 22\%; EV - 64\%; two-tailed Fisher's exact test: $\mathrm{p}=0.09$ ) and NP (non-specific psychosis: EV+: 11\%; EV-: 64\%; two-tailed Fishers's exact test: $\mathrm{p}=0.03$ ). No such differences were apparent between EV+ and EV- subjects in the group of patients with affective and atypical psychoses. 
Fig. I PSE syndrames in EV+ and EV-schizoplinentic subjects

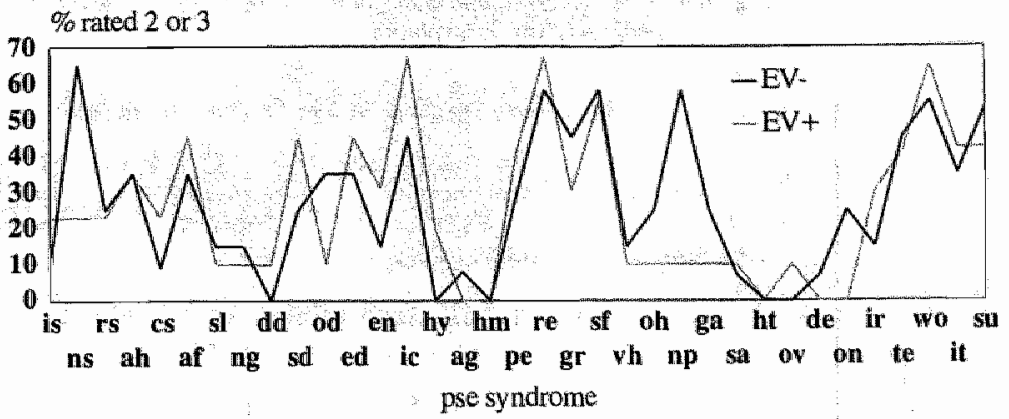

The majority of EV+ subjects in the schizophrenic group were female (56\%), versus $27 \%$ in the $\mathrm{EV}$ - group $(\mathrm{p}=0.3)$. The same trend was apparent in the non-schizophrenic group ( $52 \%$ and $33 \%$ respectively; $p=0.4$ ).

\section{Family history and life events}

In the schizophrenic group (Table 2), the lifetime morbid risk estimate of schizophrenia was higher in the first degree relatives of EV+ probands $(22.8 \%)$ than those of EV-probands $(0 \%$; two-tailed Fisher exact test: $\mathrm{p}=0.05$ ). In the non-schizophrenic group, no such differences existed between relatives of EV+ and EV- probands. In neither group (Table 3 ) were the lifetime risk estimates of affective disorders (depression, mania, bipolar and schizo-affective disorder) significantly different between first degree relatives of $\mathrm{EV}+$ and $\mathrm{EV}$ - probands. Only two first degree relatives had a diagnosis of unspecified functional psychosis, both probands were in the group of $E V+$, non-schizophrenic subjects. The difference in morbid risk for unspecified functional psychosis between $\mathrm{EV}+$ and $\mathrm{EV}$ - subjects in this group was not significant.

Table 2. Liferime risk of FH-RDC schizophtenia in first degree relatives of RDC schizophrenic and RDC non schizophrenic probands in relation to characteristic of proband

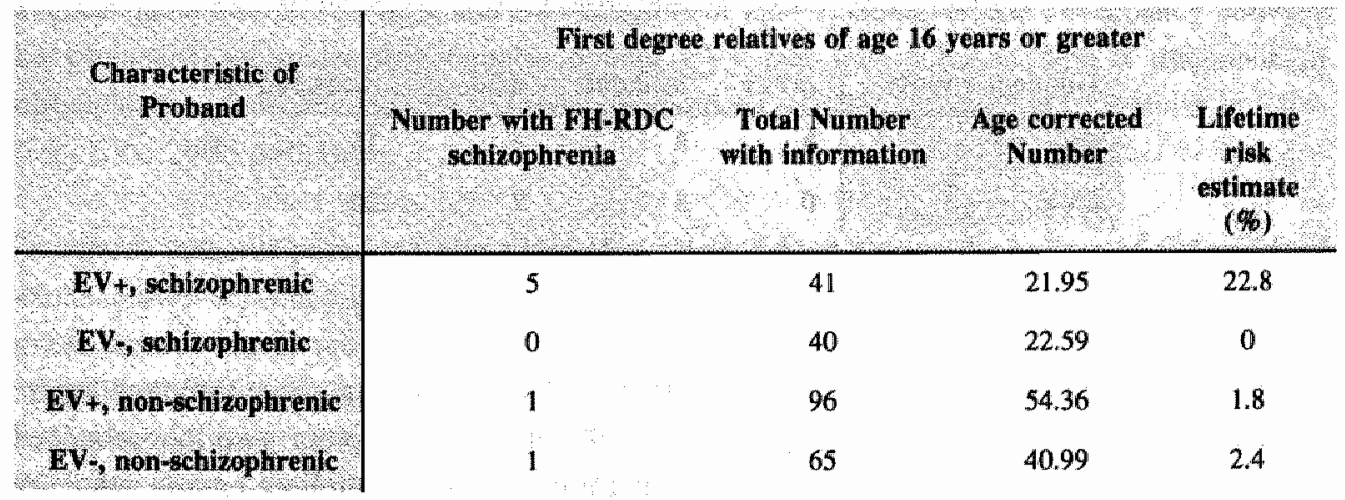

significant differences: $E V+$ schizophrenic ws EV- schizophrenic, $p=0.05$ (two-tailed Fisher exact test) 
Table 3. Lifetime risk of FH-RDC affective disorder (schizo-affective disorder, depression, mania and bipolar disorder) in first degree relatives of RDC schizophevic and RDC non-schizophrenic probands in relation to characteristic of proband

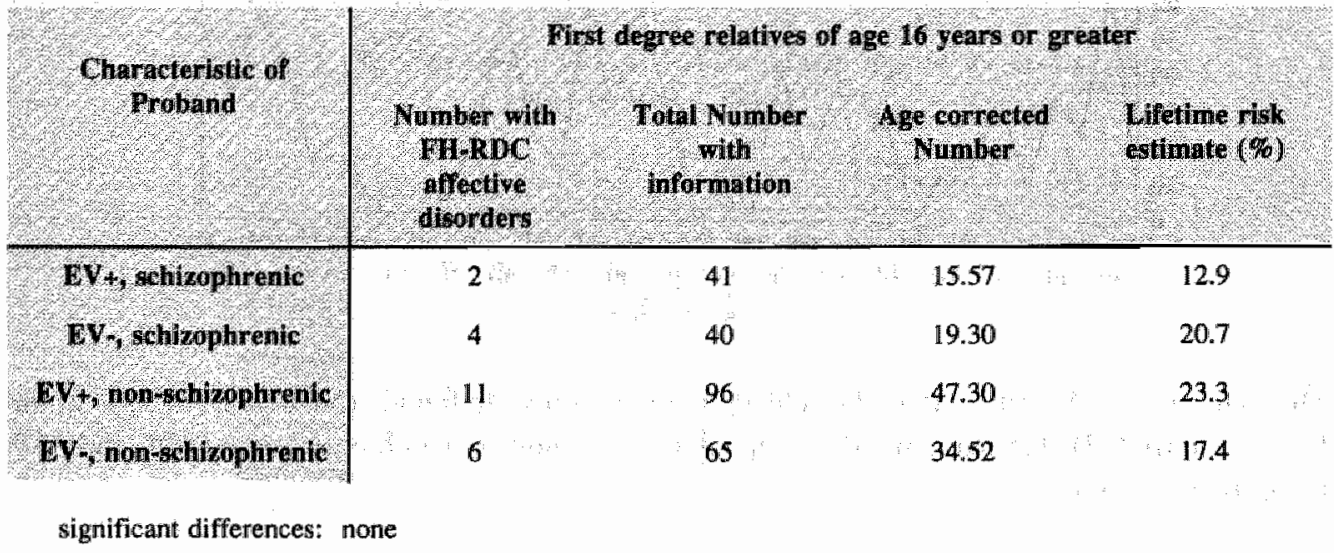

\section{Outcome}

$\mathrm{EV}+$ subjects had better longitudinal outcome in that they showed significantly milder symptom severity over the follow-up period compared to EV-subjects (Table 4). EV+ subjects also spent less time in hospital, less time on anti-psychotics, and more often scored zero on the AIMS at follow-up (Table 5). There were trends at the 10\% significance level for EV+ subjects to have less often a "continuous" illness course, and to have been less time on anti-depressant medication. Differences in time spent in a psychotic state, time spent in complete remission the number of admissions, and the receipt of psychotherapy were, although not significant at trend level, in the same direction (EV+ subjects showing a more benign illness course and more often receiving psychotherapy).

Table 4. Usual symptom severity over follow-up periad in subjects with and without stressful events prior to onset of illness.

\begin{tabular}{|c|c|c|c|c|}
\hline syrppton aryority & correre & noderite & inila & recovened \\
\hline ov. & 3 & 8 & 3 & 15 \\
\hline ave & 0 & 3 & 7 & 20 \\
\hline
\end{tabular}

(Test for trend: $\chi^{2}=4.6 ; p=0.03$

No clear differences could be shown in GAF-s scores, DAS-s scores, negative symptom scores or HAMD scores. The predictive effect of life events on usual symptom severity remained significant after correction for possible confounders (in-patient status at follow-up, sex, ethnicity and index-RDC diagnosis) in a multiple regmession model $(B=0.5 ; p \leq 0.05)$. In order to obtain a risk estimation of this effect, we modelled usual symptom severity as a binary variable (moderate/severe versus mild/recovered), which revealed that EV+ subjects were 8.1 times more likely (95\% CI: 1.2-53) to have had a symptom level of mild/recovered over the follow-up 
period. Significant effects of life event status, after controlling for confounding factors, were also found for time in hospital ( $\mathrm{B}=-0.7 ; \mathrm{p} \leq 0.01)$, AlMS score $(\mathrm{OR}=10 ; 95 \% \mathrm{Cl}: 1.7-50)$, and time on antidepressants $(\mathrm{B}=15.1 ; \mathrm{p} \leq 0.05)$.

Breakdown of index-RDC diagnoses into schizophrenic and non-schizophrenic psychoses (atypical, affective and schizo-affective presentations) showed that the effect of life events on outcome measures was not the same in the two groups (Table 5). Among patients with an index diagnosis of non-schizophrenic psychosis, after adjustment for confounding factors, EV+ subjects were 10.2 (95\% CI: 1.2-88) times more likely to have had a symptom severity rating of mild/recovered, and 10 (95\% CI: 2.5-100) times more likely to score zero on the AIMS. They had also spent less time in hospital $(B=-1 ; p \leq 0.01)$, and there was a trend to have spent more time on anti-depressants $(\mathrm{B}=17.1 ; \mathrm{p}=0.09)$.

In the schizophrenic group, no effect of life events on symptom severity could be shown. However, EV+ subjects, after correction for confounding factors, had received less antipsychotics over the follow-up period $(B=-34.9 ; p=0.04)$.

In view of the differences in morbid risk between the two groups regarding family history of schizophrenia, which could affect outcome measures, the presence or absence of family history was added into the regression equation as a binary variable. This reduced the effect of life event status on time on anti-psychotic medication to a trend at the $10 \%$ significance level $(\mathrm{p}=0.1)$.

\section{Outcome measures and follow-up-RDC diagnoses}

Seven subjects with an index diagnosis of schizophrenia were given a diagnosis of schizoaffective disorder at follow-up, and one subject with schizo-affective disorder received a diagnosis of schizophrenia at follow-up. These changes in diagnosis did not affect our findings conceming morbid risk; among patients with a follow-up diagnosis of schizophrenia, EVsubjects had a morbid risk of 0 , and EV+ subjects of $22.4 \%$. However, the discrepancy between the proportion of males and females among EV+ and EV- subjects with a diagnosis of schizophrenia $(n=14, E V+=7, E V-=7)$ became more accentuated (proportion of females in $E V+$ : $71 \%, \mathrm{EV}-\mathrm{:} 29 \%$; $=0.3)$.

As regards outcome measures, reanalysis of our data did not change the pattem of our previous results. However, after adjusting for confounding factors, $\mathrm{EV}+$ subjects in the non-schizophrenic group were 7 times more likely to have received psychotherapy (95\% CI: 1-51.2). After controlling for confounding factors, including family history, life event status in the schizophrenic group now predicted less time on anti-psychotic medication $(B=-36.1 ; p=0.04)$. The mean time of the follow-up period spent in complete remission was $28 \%$ in the EV+group, and $0 \%$ in the EV-group. Before entering confounding factors into the regression equation, there was a borderline significant effect of life events on time in complete remission $(B=0.77, p=0.06)$. The addition of sex, ethnicity, in-patient status and family history into the equation did not affect the regression coefficient $(B=0.77, p=0.2)$. 
Table 5. Outcome measures in patients with and withat stresiful life events prior to onset of illiness:

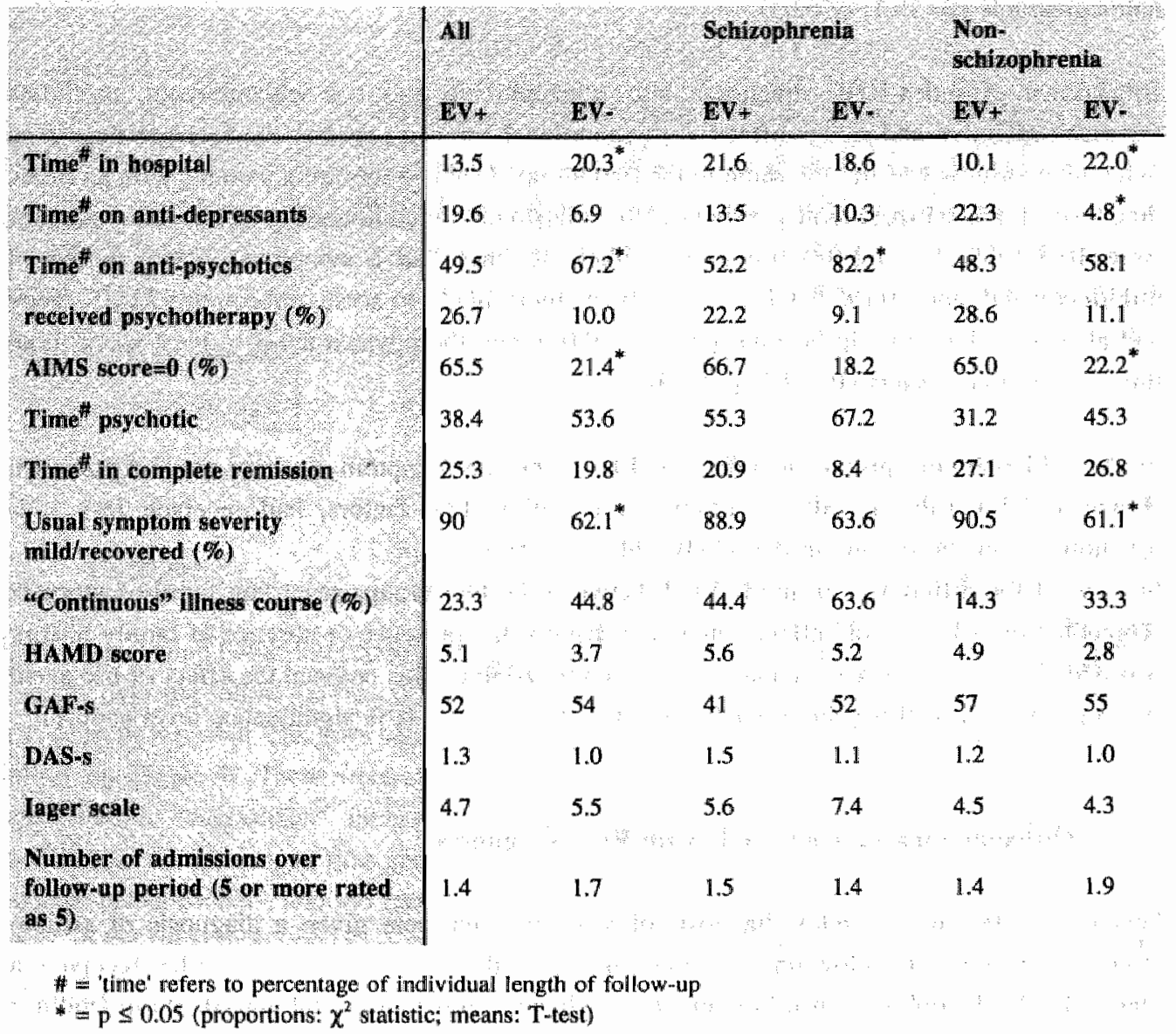

\section{DISCUSSION}

\section{Methodological Issues}

The link between life events and the onset of psychosis can only be studied where it is possible to date onset accurately. Cases of insidious onset were therefore excluded from our original life event study (Bebbington et $a_{l}, 1993 \mathrm{~b}$ ). The fact that patients with insidious onsets showed a male predominance, less affective symptomatology and a poorer outcome makes it likely that our life event sample was biased towards acute onset, good-outcome psychotic disorders. Among those with a datable onset, the proportion of females tended to be higher in the EV+ category. This may indicate that female psychosis is more reactive in nature, but could also be a reflection of the fact that normal females also report more life events, including events at the 1 or 2 severity level (Bebbington et al, 1993b). 
The frequency of the major psychotic disorders is low, so that a precise estimate of familial rates of psychiatric disorders requires a very large sample size. Our sample size is rather small for this purpose, especially when the probands are subdivided. Our main interest, however, was not to estimate absolute morbid risks, but differences. Where these were found, they were quite marked. We acknowledge that the significant difference between $\mathrm{EV}+$ and $\mathrm{EV}$ - schizophrenic subjects must be interpreted cautiously; there were no affected relatives in the EV-group, producing a morbid risk estimate of zero, and making it impossible to calculate confidence intervals by standard methods. The null hypothesis cannot therefore be confidently rejected, and a larger sample is likely to yield a more attenuated difference in familial morbid risk between EV+ and EV- schizophrenics.

The small sample size, especially when examining subgroups, jeopardized the statistical stability of our analyses. An indication of this is the sometimes very wide confidence intervals of the odds ratios. Type II errors are likely to have occurred, for example, in examining differences between EV+ and EV- schizophrenic subjects on PSE nuclear syndrome (NS) frequency and gender distribution. A difference of $42 \%$ in nuclear syndrome frequency is hardly without potential practical or theoretical significance. If the true percentages in the two groups were $22 \%$ and $64 \%$, our sample size would only have $27 \%$ power to reject the null hypothesis at the $\mathrm{p} \leq$ 0.05 significance level. The fact that such a large difference was not statistically significant could therefore be due to a Type H error. A sample size of 40 (instead of 20 ) would have given a significant result at the 2 percent significance level. The same applies to the multivariate analyses; although, for example, the effect of life events on time on anti-depressants was not significant at the 5 percent level in the group of non-schizophrenic psychoses, the regression coefficient $(B=17.1)$ indicated that the effect was in fact more marked than the (statistically significant) effect in the group of schizophrenic and non-schizophrenic psychoses combined $(\mathrm{B}=15.1)$.

Follow-up assessments were not completed on $16 \%$ of the index sample. The follow-up rate, however, is high by comparison with other studies, and bias testing revealed no differences in relevant variables between the two groups.

\section{Interpretation of findings}

In our view, the main findings cannot be explained away as artificial, although the morbid risk data should be interpreted cautiously. Our results suggest that the presence of stressful life events does have implications for the prognosis of psychotic disorders, but the relationship between life events and subsequent course differs between diagnostic categories.

In the group of patients with non-schizophrenic psychoses, events were associated with better longitudinal outcome measures, such as less severe levels of symptomatology and less time hospitalised, regardless of whether the index- or the follow-up RDC diagnosis was used. The fact

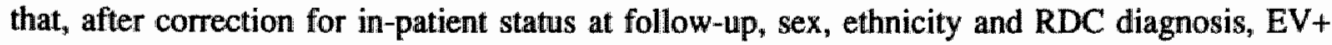


patients in the non-schizophrenic group received more anti-depressant medication and had llower AIMS scores, does appear to indicate that they were treated more for depressive and less for psychotic symptoms. This might explain some of the differences in longitudinal outcome measures we found between the two groups, as the outcome of psychotic disorders has been shown to be related to the degree of affective symptomatology present (Tsuang \& Dempsey, 1979, Pope et al, 1980). We did not find an inverse relationship between life events and familial loading, which is consistent with the results of similar investigations in samples of patients with depressive disorder (Bebbington et $a l_{,} 1991$, McGuffin et al, 1991). This may indicate that, in affective psychoses, genetic and environmental risk factors act through different, unrelated, pathways.

In the schizophrenic group, however, there was, contrary to our initial second hypothesis, a significant positive correlation between morbid risk for schizophrenia in first degree relatives and presence of life events. EV + subjects also tended to display less typical symptomatology at index assessment, and when the follow-up RDC diagnoses (presumably the most valid) were applied, it appeared that $E V+$ subjects had received significantly less anti-psychotic medication, and had spent more time in complete remission.

The association of life events with both genetic predisposition and a more favourable course in the schizophrenic group seems at odds with the popular notion that a family history of schizophrenia predicts poorer outcome. However, the only studies (Kendler \& Hays, 1983; Kendler \& Tsuang, 1988) examining the relationship between family history of psychiatric disorder and outcome in schizophrenia that did not suffer from important methodological limitations common to most studies in this field, failed to find a significant relationship between any dimension of outcome and risk in relatives, either for schizophrenia or for a broader category including all non-affective psychotic disorders. Similar results were reported from several wellknown, large-sample European outcome studies (Bleuler, 1978; Ciompi, 1980; Huber $e t$ al, 1980). Furthermore, our sample consisted of relatively good-outcome, acute onset cases, and it is possible that affected relatives had a similar, comparatively benign schizophrenic illness.

The morbid risk findings in the group of schizophrenic patients disagree with our initial hypothesis, but are perhaps less puzzling when viewed in the light of the suggestion by Brown and colleagues (Brown er al, 1973; Brown and Harris, 1978) that life events are related in different ways to the onset of schizophrenia and affective disorder. As mentioned before, these authors distinguished two types of causal role: a 'formative' role, where events are of fundamental aetiological significance, being more important in the causation of the condition than dispositional factors; and a 'triggering' role, where dispositional factors play the larger part, and life events merely aggravate a pre-existing (genetic) tendency. They suggested that the former was more characteristic of affective illness, and the latter of schizophrenia. This is consistent with our finding of an increased morbid risk for schizophrenia in the relatives of EV+ schizophrenic probands. A more 'formative' role of life events in affective psychoses versus a more "triggering' role in schizophrenia is also consistent with the marked effect of events on 
course of illness in the non-schizophrenic psychoses, which contrasted with the modest effect in schizophrenia.

The role of life events in relation to the onset and course of psychotic illness, however, is likely to involve not only an interplay between life events and genetic predisposition, but also other biological risk factors (Jones et al, 1994). The relationship between insidious onset, acute onset, gender and aetiological factors also requires further clarification. Our findings suggest that, compared with acute onset cases, insidious onset is associated with male sex and poor outcome in terms of negative symptoms and disability. The fact that EV+ schizophrenics received less antipsychotic medication over the follow-up period, tended to spend more time in complete remission, were less likely to be male and to show the nuclear schizophrenia syndrome (i.e. Schneiderian symptoms), might suggest that they lie at the opposite end of the schizophrenia spectrum from the insidious onset group; course of illness in EV+ schizophrenics may be more similar to that seen in affective psychotics. These findings are compatible with the proposal of Murray et al (1991) that schizophrenia should be divided into a severe -and predominantly maleneurodevelopmental type, and a milder form, commoner in females, which is aetiologically related to affective disorder (Fowler, 1978; Lewine, 1985). 


\section{APPENDIX}

\section{COURSE TYPE IN THIS PEAIOD.}

In estimating duration of an episode that began before this period but extended into the period, include the time before this period:

- ephodic dilerete episodes (none longer than 6 MONTHS) with clear periods of remission in between At least one remission lasted 6 months or more.

- continuous - psychotic over most of the perilod If any remissions, these were brief (none longer than 6 months).

- neither episodic nor conninuous - eg, Jongest psychotic episode was 12 months and longest remission was 9 months:

- never actively pychotic in this pertiod.

USUAL SEVEAITY OF SYMPTOMS. (Categortes ane modified from M. Blleuler.)

Select the ratimis that best describes MOST of the period, If no rating applies to more than half of the period, select the one with the Iongest duration.

- mild - behawior was generally normal and illness was not immediately obvious in conversation, although definite psychotic symptomis persisted.

- moderane - consistently showed signs of health in spite of marked psychotic symptoms, e.g. could speak with reasonable clarity on some occasions" could work or care for others to some extent; marked psychotic symptoms may be overt or suggestive (see definitions).

- : severe - could not carry on a coherent conversation; could not work without close supervision; required constant care.

- recovered - only residual or no psychotic sympioms.

\section{PSYCHOTIC}

\section{A. OVERT SIGNS AND SYMPTOMS}

- hallucinations or pseudohallucinations (in any modality):

- delissions:

- marked thought and speech disorder (e.g., incoherence, irrelevance, thought blocking, neologisms, incomprehensibility of speech other than simple retardation or acceleration;

- marked qualitatiwe psychomotor disorder (e.g., negativism, mutism or stupor; catatonic excitement; constrained attitudes or unnafural postures maintained for long periods) other than retardation or acceleration of movements;

- bizarre or grossly imappropriate behavior (e.g., talking to self or giggling to self, acts incomprehensible to others, loss of social constraints, etc.).

\section{B. SUGGESTINE SIGNS AND SYMPTOMS}

- extreme reduction or loss of interests, initiative and drive, leading to serious deterioration of the performance of usual activities and tasks;

- extreme social withdrawal;

- severe excifement, purposeless destructiveness or aggression (frequent episodes or continuous);

- episodes or persistent states of overwhelming fear or severe anxiety:

- gross and persistent self-neglect.

\section{REMISSION}

A state following a psychotic episote, in which none of the symptoms and signs required for rating a psychotic episode are present. The absence of psychotic symptoms and signs qualifies as a remission only if it lasts for four weeks or hore. Two varieties of remission will be distingtished:

(A) COMPLETE REMISSION: the subject is virtually sympiom-free and shows his/her usual premorbid personality;

(B) INCOMPLETE REMISSION" the subject is no longer psyehotic (in the sense defined above) but shows any combination. of: (i) residuall symptoms (i.e, "traces" of the symptoms and signs which were present in the psychotic episode but which in no way would justify a rating of a continuing pisychotic episode); (ii) non-psychotic symploms, e.g., non-specific neurotic or mild affective disturbances which are not traits of the premorbid personality; (iii) personality change, e.g., accentuation of premorbid. personality traits or emergence of new traits; change or loss of interests; stable alterations of expressive and communicative. behavior; decline of imtellectual performace.

\section{COMMUNITY LIVING INDEPENDENT OF PROFESSIONAL SUPERVISION}

Independent community living includes all living arangements other than an institution (e.g., hospital, jail), a supervised residence, and homeless/vagrant. Thus, it includes living alone, living with friends, living with family, and any other non-institutional domicile. 


\section{REFERENCES.}

Andreasen, N. C., Rice, J. P., Endicott, J., Reich, T. \& Coryell, W. (1986) The family history approach to diagnosis. How useful is it? Archives of General Psychiarry "43, 421-429.

Bebbington, P. (1987) Psychosocial etiology of schizophrenia and affective disorders. In Psychiatry revised edin (Ed. R. Michels). Philladelphia: Lippincott.

_._., Katz, R. \& McGuffin, P. (1991) The famillial aggregation of affective disorders: relation to symptom severity and social provocation. In The new genetics of mental illness (Eds. P. MoGuffin \& R. Murray) pp 130-145 . Oxford: Butterworth-Heinemann.

-_.n-..., Walsh, C. \& Murray, M. (1993a) Environmental and biological causes of functional psychosis. In Basic Issues in Psychopathology (Ed. C.G. Costello) pp 238-270. New York: Guilford Press.

. psychosis. Initial results from the Camberwell Collaborative Psychosis Study. British Journal of Psychiarry, $162,72-79$.

Bleuler, M. (1978) The schizophrenic disorders: long term patient and fomiliy studies, Clemens S. (translator). New Haven: Yale University.

Brown, G. W., Harris, T.O., \& Peto, J. (1973) Life events and psychiatric disorders. Part 2: Nature of causal link. Psychological Medicine, 3, 159-76.

_......-, \& Harris, T. O. (1978) Social Origins of Depression. London: Tavistock.

Carpenter, W.T. \& Kirkpatrick, B. (1988) The heterogeneity of the long-term course of schizophrenia. Schizophrenia Bulletin, 14, 645-652.

Ciompi, L. (1980) Catamnestic long-term study on the course of life and aging of schizophrenics: Schizophrenia Bulletin, 6, 606-618.

Cooper, B. (1986) Mental disorder as a reaction: the history of a psychiatric concept. In Life Events and Psychiatric Disorder (ed. H. Katchnig). pp 1-32. Cambridge: Cambridge University Press.

Foerster, A., Lewis, S.W., Owen, M. \& Murray, R. (1991a) Premorbid personality in psychosis ieffects of sex and diagnosis. British Journal of Psychiatry, 158, 171-176.

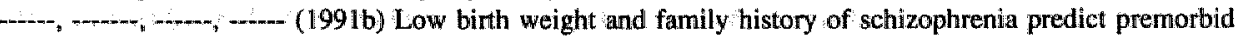
functioning in schizophrenia. Schizophrenia Research, 5, 13-20.

Fowler, R. (1978) Remitting schizophrenia as a variant of affective disorder Schizophremia Bulletin, 4, 69-77.

Gury,W. (1976) ECDEU Manual for Psychopharmacology, pp 534-537. Washington, DC: DHEW.

Hamilton, M. (1960) A rating scale for depression. Journal of Neurology, Neurosurgery and Psychiatry, 23, 5662.

Huber, G., Gross, G., Schüttler, R., \& Linz, M. (1980) Longitudinal studies of schizophrenic patients. Schizophrenia Bulletin, 6, 592-605.

Iager, A.C., Kirch, D.G. \& Wyatt, R.J. (1985) A negative symptom rating scale. Psychiatry Résearch 16, 27-36.

Jablensky, A., Schwartz, R. \& Tomov, T. (1980) WHO collaborative study of impaiments and disabilities associated with schizophrenic disorders. A preliminary communication. Objective and methods. Acta Psychiatrica Scandinavica, suppl. 285, 152-163.

Jones, P.B., Bebbington, P., Foerster, A., Lewis, S., Murray, R, Russel, A., Sham, P., Toone, B. \& Wilkins, S. (1993) Premorbid social underachievement in schizophrenia. Results from the Camberwell Collaborative Psychosis Study. British Journal of Psychiatry, 162, 65-71.

-.-, Van Os, J., Bebbington, P, Wilkins, S., Sham, P., Russel, A., Lewis, S., Toone, B. \& Murray, R. (1994) Life events and biological risk factors in the precipitation of psychosis. (paper presented at the Winter Meeting, Royal College of Psychiatrists, London, 1st to 4th February, 1994)

Kendler, K., \& Hays, P. (1983) Schizophrenia subdivided by the family history of affective disorder: A comparison of symptomatology and course of illness. Archives of General Psychiarry, 40, 951-955.

- $-\ldots$..... \& Tsuang, M. (1988) Outcome and familial psychopathology in schizophrenia. Archives of General Psychiatry, 45, 338-346.

--.--., K., Neale, M., Heath, A., Kessler, R. \& Eaves, L. (1991) Lifie events and depressive symptoms: a twin study perspective. In The new genetics of mental illness. (Eds. P. McGuffin \& R. Murray) pp 146-165.

Oxford: Butterworth-Heinemann.

Lewine, R. (1985) Schizophrenia: an amotivational syndrome in men. Canadian Journal of Psychiatry, 30, $316-$ 318.

Malla, A., Cortese, L., Shaw, T.S. \& Ginsberg, B. (1990) Life ewents and relapse in schizophrenia: a one year prospective study. Social Psychiatry and Psychiatric Epidemiology, 25, 221-224.

McGlashan, T.H. (1986) Predictors of shorter-, medium- and longer-term outcome in schizophrenia. American Journal of Psychiatry, 143, 50-55.

McGuffin, P., Farmer, A.E. \& Harvey, I. (1991) A polydiagnostic application of operational criteria in psychotic 
illness: development and reliabillity of the OPCRIT system. Archives of General Psychiatry,48, 764-770.

Murray, R.M. \& O'Callaghar, E. (1991) The congenital and adult-onset psychoses: Kraepelin lost, Kraepelin found. In Concepts of Mental Disorder, a Continuing Debate (Eds A. Kerr \& H. McClelland) pp 48-65. Londlon: Gaskell.

Norư̌s, MJ.SPSS INC: (1990) SPSS/PC ${ }^{+}$Statistics 40. Chicago. SPSS Inc.

Pope, H.. Lipinski, J.* Cohen, B. \& Axelrod, D. (1980) Schizo-affective disorder, an invalid diagnosis? A comparison of schizo-alfective disorder, schizophrenia, and affective disorder. American Journal of Psychiatry, 137, $921-927$

Prudo, R, \& Blum, H.M. (1987) Five-year outcome and prognosis in schizophrenia: a report from the London field research centre of the Intemational Pilot Study of Schizophrenia. British Journal of Psychiarry, 150, 345-354.

Sham, P., Jones, P., Russel, A, Gilvarry K, Wilkins; S.; Foerster, A., Bebbingtion, P., Lewis, S., Toone, B. \& Murray, R. (1994) Age at onset, gender, and famillial psychiatric morbidity in schizophrenia. Report from the Camberwell Collaborative Psychosis Study. British Journal of Psychiatry (in press).

Slater, E. \& Cowie, V. (1974) The Genetics of Mental Disarders. London: Oxfond Uniwersity Press.

Spitzer, R.L., Gibbon, M \& Endicott, J. (1975) The Global Assessment Scale (GAS). New York State Psychiatric Institute: New York NY,

Strauss, J. \& Carpenter, W. (1974) The prediction of outcome in schizophrenia. Archives of General Psychiatry, $31,37 \sim 42$.

Strörmgren, E. (1985) World-wide issues in psychiatric diagnosis and classification and the Scandinavian point of view. In Mental disorders, Alcohol and Drug Related Problems. Amsterdam. Excerpta Medica.

Strömgren, E. (1935) Zum Ersatz des Weinbergschen "abgekurzten Verfahrens" zugleich ein Beitrag zur Frage von der Erblichkeit des Erkrankungsalters bei der Schizophrenie. Zeitschrift für die gesamie Neurologie und Psychiatrie, 153, 784797.

Takei, N., O'Callaghan, E., Sham, P., Glover, G. \& Murray, R. (1992) Winter birth excess in schizophrenia: its relationship to place of birth. Schizophrenia Research, 6, 102.

Tsuang, M.T. \& Dempsey, G.M. (1979) Long term outcome of major psychoses II. Schizoaffective disorder, compared with schizophrenia, affective disorders, and a surgical control group. Archives of General Psychiatry, 36, 1302-1304.

Vaillant, G.E. (1964) Prospective prediction of schizophrenic remission. Archives of General Psychiatry, 11, 509: 5.18.

Ventura, J., Nuechterlein. K.H.. Lukoff, D. \& Hardisty, J.P. (1989) A prospective study of stressful life events and schizophrenic relapse. Jounal of Abnormal Psychology, 98, 407-411.

WHO (1992) WHO coordinated multi-center study on the course and outcome of schizophrenia. Geneva: WHO.

Wing, J.K. Cooper, J.E. \& Sartorius, N. (1974) The Measurement and Classification of Psychiatric Symptoms. Cambridge University Press, Cannbridge: 


\subsubsection{Increased intra-cerebral CSF spaces predict unemployment and negative symptoms in psychotic illness: \\ A prospective study'}

\section{SUMMARY}

Is has been suggested that cerebral ventricle dimensions are a risk factor for poor outcome in psychotic illness. A cohort of 140 patients with functional psychoses of recent onset who had undergone CT-scanning, were followed-up for an average of 46 months and assessed on six dimensions of course and outcome of illness. Left and right sylvian fissure volumes and, to a lesser extent, third ventricular volume predicted negative symptoms and unemployment over the course of the follow-up period, the latter association being mediated by poor cognitive functioning. There was a significant linear trend in risk over the sylvian fissure volume distribution in the cohort, and associations were especially evident in schizophrenic patients. No associations were found with global illness severity, duration of hospitalization, homelessness or affective symptomatology. These findings support the notion that cerebral ventricle dimensions are a continuous risk factor for some dimensions of outcome in the functional psychoses.

\section{INTRODUCTION}

A number of studies using computer assisted tomography (CT) or magnetic resonance imaging (MRI) have investigated a possible relationship between ventricle size and prognosis in schizophrenia; all but one suggested poorer outcome in patients with ventricular enlargement (Table 1). Most authors, however, expressed caution because of small sample size, bias due to loss to follow-up, retrospective baseline assessment, and CT-scanning at follow-up instead of at baseline. Furthermore, any association reported at follow-up might simply be a repetition of an association that was already present at baseline (eg associations between intracranial measures and negative symptoms and unemployment as suggested by some cross-sectional surveys (eg Andreasen $e t a l, 1982)$ ). Also relevant is the issue of statisticall analyses. Firstly, outliers and extreme values may affect results disproportionably, but evaluation of their effect is rarely mentioned in neuro-imaging research. It is therefore not clear whether positive findings can be accounted for by a subgroup of cases with large ventricles, or whether the increased risk is continuous. Secondly, in many studies, the ventricle to brain ratio (VBR) was used as a way to control for head size, based on the assumption that there is a linear relationship between head and ventricle size which does not differ between cases. This assumption is unjustified (Zatz \& Jernigan, 1983; Harvey et al, 1990a): the measure is prone to confounding by sociodemographic factors which may not only influence head and ventricle size in different ways, but also have an effect on course and outcome of psychotic illness. Sex, age, diagnosis, ethnicity, and socioeconomic status have all been found to determine intracraniall and ventricle size (Williams $e t$ al, 1985; Owens et al, 1985; Jones et al, 1994). These variables have also been shown to be associated with course and outcome in schizophrenia, indicating that they may confound any relationship between $\mathrm{CT}$ measures and outcome. 


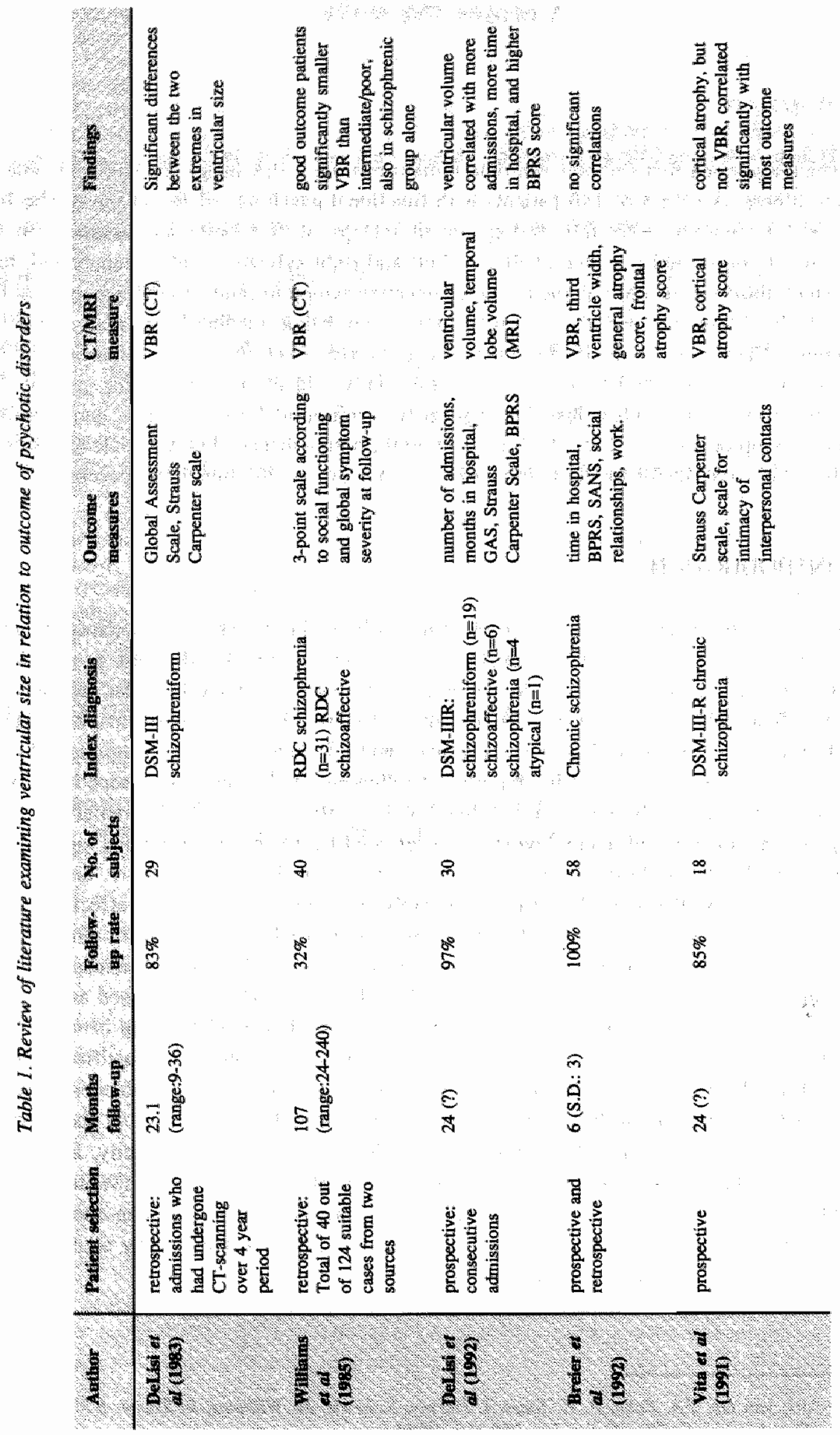


The present study aimed to overcome such methodological weaknesses. The possible relationship between course and outcome in the functional psychoses, and intra-and extra-cerebral CSF spaces, was investigated prospectively by following a cohort of consecutive admissions with functional psychosis of recent onset, who had undergone high resolution CT imaging. Analytical methods were used to examine possible confounding by sociodemographic characteristios and mixing of diagnostic groups, under the null hypothesis of no association between ventricle size and measures of course and outcome.

\section{METHOD}

\section{Cohort and baseline assessments}

The cohort and the assessment procedures have been described in detail elsewhere (Van Os et al, 1994, Van Os et al, submitted).. Briefly, 337 consecutive admissions to two South London hospitals, with at least one psychotic symptom according to the RDC (Spitzer et al, 1978) occurring in clear consciousness, were investigated (Jones et al, 1993; Harvey et al, 1990b). Those that had an onset of functional psychosis within 5 years (mean:2.2 years) of index assessment $(n=191)$ were followed up after an average of 4 years.

Baseline assessments included socio-economic status at birth or during early childhood using the Registrar General's classification of paternal occupation. Ethnicity was classified as white European, Afro-Caribbean, or other. Age at onset was defined as appearance of first symptoms of psychosis, and duration of illness as the difference between age at index assessment and age of onset. Patients were interviewed using the Present State Examination (Wing et al, 1974). Using both cross-sectional PSE data and case note material, the Operational Criterial Checklist for Psychotic Illness (OCCPI; McGuffin et al, 1991) was completed for all patients for the time up to baseline assessment and analyzed with the OPCRIT computer programme (McGuffin et $a l$, 1991) to give a DSM-III-R index diagnosis.

\section{Follow-up}

Follow-up data were collected by JvO, blind to all index data, including CT-measures. The following instruments were used to provide final cross-sectional outcome measures: Global Assessment of Functioning-symptomatology (GAF-s; WHO 1992), WHO/Disability Assessment Schedule (DAS; Jablensky, et al, 1980), Iager Negative Symptom Rating Scale (Iager, 1985), Hamilton Depression Scale (HAMD; Hamilton, 1960) and the PSE. Also rated at follow-up was employment status (students and full-time housewives rated as employed), and a measure of cognitive functioning: the Trail making test A and B (Reitan, 1958). This test was included to examine any possible mediating effect of cognitive functioning on predictors of outcome.

To assess course of illness and associated disabilities longitudinally over the follow-up period, we used a slightly modified version of the Life Chart instrument from the Multi-Center Study on the Course and Outcome of Schizophrenia (WHO, 1992). The life chart assesses course of illness in a number of ways, using clear definitions for all ratings. Thus, ratings were made of course type, type of remission, and usual severity of symptoms over the period of follow-up 
(Appendix). The amount of time spent in a psychotic state was also rated, as were parasuicidal acts. $A$ rating was also given as to whether there had been clear evidence of negative symptoms, as defined by the lager scale, over the follow-up period. In addition, the life chart rates the proportion of the follow-up period spent in independent living (i.e. not in an institution or supervised residence), in hospital, in prison (including police custody), or without accommodlation ("vagrant"), and the time spent unemployed whilst living outside institutions.

In order to reduce the number of outcome measures employed, six outcome dimensions were identified using factor analysis of all the above ratings. The rationale and method for this has been described elsewhere (Van Os et al, submitted). The dimensions were. 1. negative symptoms/social disability; 2 . severity of illness; 3 . time living independently; 4. unemployment; 5. imprisonment and vagrancy; and 6. depression/self-harm (Table 2).

\section{CT-Scanning}

This method is described in detail elsewhere (Jones et al, 1994). Axial CT-scans were all performed on a Siemens 9800 Scanner. Slices 1 cm thick, parallel to the floor of the anterior fossa and ascending to the vertex were analysed at an independent video console (IVC). Three raters, blind to other information on the subject, each rated an equal proportion of cases. Interrater reliability was assessed using around 100 measures by each rater, obtained from a sample of 10 patients (i.e. 10 structures were measured in each patient). The intra-class correlation was the Pearson product-moment correlation between the pairs of measurements in which each pair entered the calculation twice (i.e. as $\left\{X_{i 1}, X_{i 2}\right\}$ and also as $\left\{X_{i 2}, X_{i 1}\right\}$; see Dunn, 1989). Inter-rater reliability was high ( $>0.9$ and $p<0.05$ for each structure). Intracranial area was measured on each slice by summing all pixels in the range 0 to 100 Hounsfield units (HU; Hounsfield, 1973) within the skull. The brain/CSF boundary of the sylvian fissures, third and lateral ventricles was traced manually and the IVC required to sum pixels from 0 to $25 \mathrm{HU}$ within this area. Areas of the sylvian fissures, and the lateral and third ventricles were measured in all slices where they appeared. A lateral ventricular volume was constructed by adding together all the area measurements of its body, frontal horns, occipital horns and anterior horns (Penn et al, 1978). Sylvian fissure and third ventricle volumes were also calculated. Because of the difficulties associated with delineating superior and inferior limits of the third ventricle and sylvian fissures, area measures for these structures were also taken. For the third ventricle, a cross-sectional area taken from the slice through the middle of the ventricle was measured, whereas for the sylvian fissures the most superior slice, where the anterior horns were visible without the occipital horns or body of the lateral ventricle coming into view, were measured.

An estimate of the intracranial volume was made by summating the intracranial areas of four slices; the most superior slice on which the anterior horns alone were visible, the slice below this and the two slices above. These slices, yielding a truncated intracranial volume henceforth referred to as simply "intracranial volume", were chosen as i) the majority of the ventricular slices lay within them, and ii) the relevant data were available on all subjects. 
Table 2. Dimensions of course and outconte, and scalles used to cover these

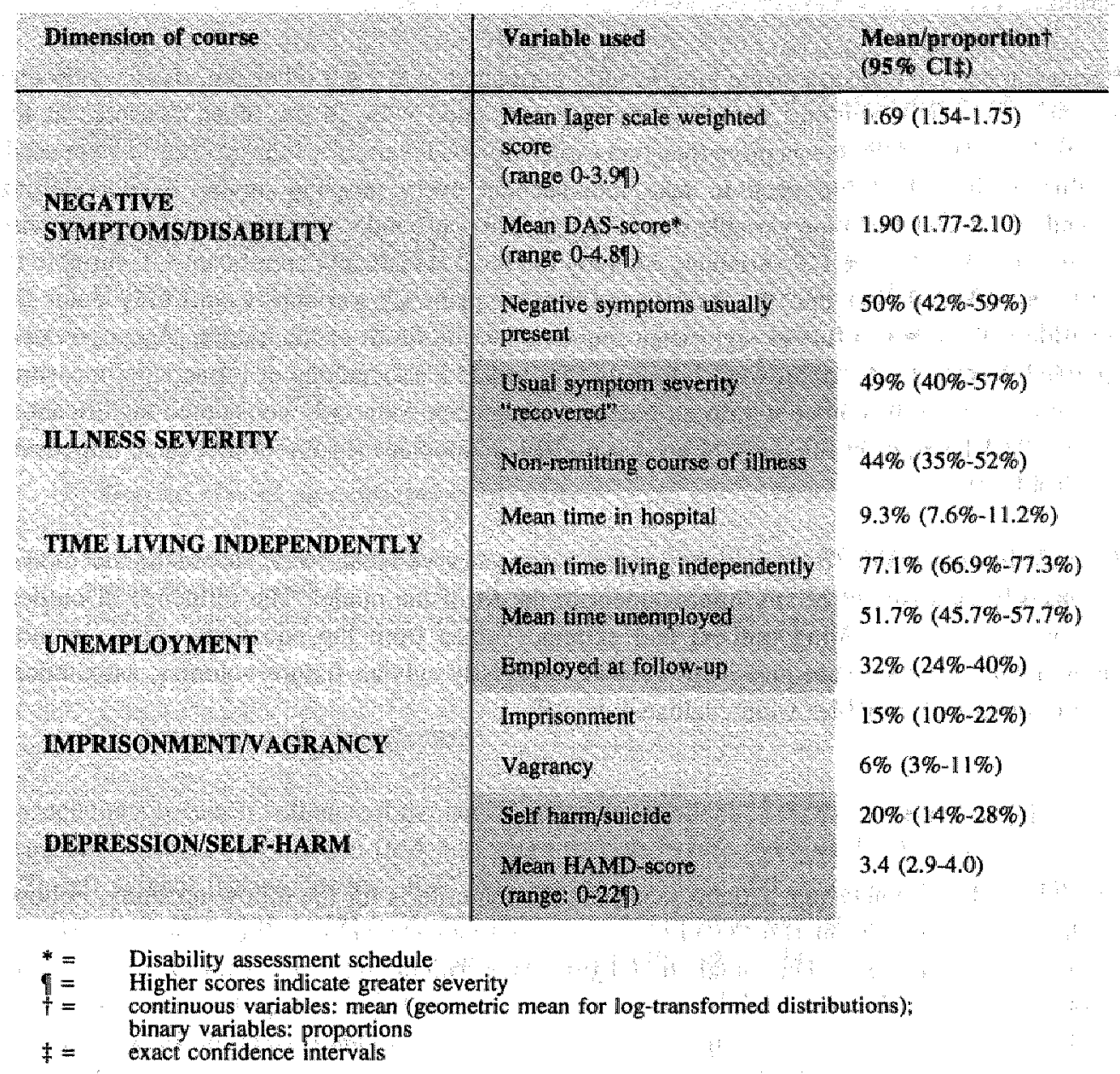

Sulcal area was measured, using the most superior slice that still contained lateral ventricular fluid, by tracing manually within the skull and cerebrum an annulus that contained the entire cortical outline and sulcal spaces. The IVC summed all pixels between 0 and $25 \mathrm{HU}$ inside this trace to give an estimated area of sulcal fluid. The boundaries of the interhemispheric fissure were traced on the same slice and measured similarly.

\section{Analyses}

Multiple regression analysis, yielding regression coefficients (referred to as " $\mathrm{B}$ "), was used to adjust for confounding variables in examining the association between illness course (the response variable) and CT measures (the exposure variables). Dependent variables were transformed to remove skewness where appropriate, and residuals were examined for evidence of non-linearity. Logistic regression was used to analyse dichotomous dependent variables to produce adjusted odds ratios (OR). Variables measuring duration, for example, time spent in 
hospital, time unemployed, etc, were expressed as a proportion of the individual follow-up period.

Because most CT-measures were significantly correlated with each other (eg, third ventricular and sylvian fissure volumes were significantly correlated with all the other measures at the p $<0.001$ level), and there was more than one explanatory CT-variable, step-up criteria were used, as this method does not need to take into account every possible model. The modelling procedure for each outcome variable was to fit possible confounders first, followed by a stepwise fitting procedure for the CT variables, whereby the most statistically significant CT variable (if any) was selected first into the model, and a subsequent CT variable would only enter the equation if there was a further significant improvement in the fit of the model. A conservative approach to effect estimation was chosen, in that isolated associations of intracranial measures with one measure of course within an outcome dimension were only considered significant at the $p \leq 0.01$ level, or at the $p \leq 0.05$ level if $p<0.1$ in another measure of that same dimension (see Table 2).

To exarnine departure from linearity, squared exposure variables were added to the model, followed by assessment of any improvement in the fit of the model. The influence of outliers was assessed by excluding outliers and extreme values from the equations. Also, as most intracranial measures were positively skewed, especially sylvian fissure volumes, associations with transformed variables were evaluated as well.

\section{RESULTS}

One hundred-and-ninety-one patients met the inclusion criteria for the follow-up study. Followup data were available on $166(87 \%)$ of these. Seven patients had committed suicide, and two had died of other causes. One-hundred and fifty-three patients were interviewed personally, of whom 4 over the telephone, and of the patients not interviewed, high-quality information was available on a further 13. On average, 1.5 (S.D. 0.8) sources of independent information, mostly through case-notes, mental health professionals and relatives, were also available for each subject interviewed.

Of the 166 subjects traced, 140 had consented to CT-scanning at index assessment. Compared with the 140 follow-up subjects with CT measures, the 51 patients not followed up or scanned were very similar on a range of baseline characteristics, suggesting non-differential loss to follow-up (Table 3).

\section{Characteristics of the sample scanned and followed-up}

Of the 140 subjects scanned and followed-up, $93(66 \%)$ were male, and the mean age at index assessment was 25.9 years. At this assessment, 64 subjects (46\%) had a DSM-III-R diagnosis of schizophrenia, $36(26 \%)$ of affective psychosis, $11(8 \%)$ of schizo-affective psychosis, and $29(21 \%)$ of schizophreniform psychosis, delusional disorder or unspecified functional psychosis. White Europeans comprised $54 \%$ of the sample. The mean length of follow-up was 46.4 months (range $=3-77 ;$ S.D. $=13.1$; range with deaths excluded: $26-77$ ). 
Table 3. Characteristics of patients lost to follow-up or excluded from $\mathrm{CT}$-study *

\begin{tabular}{|c|c|c|}
\hline Variable & Howgd hit & $\begin{array}{l}\text { not traced, not scaning, } \\
\text { or excluded }\end{array}$ \\
\hline DSM-III schizophrenia t & $62.1 \%$ & $54.9 \%$ \\
\hline DSMHI affective psychosis & 27.2 濰 & $25.5 \%$ \\
\hline DSM-II paranoulatypical psychosis & $10.7 \%$ & $19.6 \%$ \\
\hline Male sex & $66.4 \%$ & $60.8 \%$ \\
\hline White European & $53.6 \%$ & $50 \%$ \\
\hline Paternal socul class $4 / 5$ & $36.9 \%$ & $39.6 \%$ \\
\hline Mean age at inder ascessinent (years) & 25.9 & 27.4 \\
\hline Mean age of onset psychosis (years) & 23.2 & 25.3 \\
\hline
\end{tabular}

* no significant differences between the two groups (proportions: $\chi^{2}$ test with continuity correction; means: Student's t-test).

** refers to original index diagnosis; DSM-III-R diagnosis not availablle for subjects not followed up (Jones et al, 1993)

\section{CT measures and illness course}

Associations between volumes of the lateral ventricle (median: $18.5 \mathrm{~cm}^{3}$; range: $2.1-68.9$ ), third ventricle (median: 0.98 ; range: $0.14-3.5$ ), right sylvian fissure (median: $0.24 \mathrm{~cm}^{3}$; range: $0.00-$ 2.2), left sylvian fissure (median; $0.44 \mathrm{~cm}^{3}$; range: $0.00-3.6$ ), and areas of cortical sulcal fluid (median: $0.08 \mathrm{~cm}^{2}$; range: $0.00-4.22$ ), and inter-hemispheric area (median: $0.03 \mathrm{~cm}^{2}$; range: 0.00 1.96) on the one hand, and course and outcome on the other (see Table 2), were assessed through a regression analysis that controlled for diagnosis, sex, age ethnicity, childhood socio-economic status, head size (intra-cranial volume; median: 632; range: $370-772$ ), age of onset and duration of illness.

Of the 6 CT-measures, sylvian fissure volumes significantly improved the fit of the model of two outcome dimensions: greater volume of the left sylvian fissure predicted a longer proportion of the follow-up period spent in unemployment $(B=13.5 ; p=0.01)$, whereas the right sylwian fissure volume predicted unemployment at follow-up assessment $(B=2.8 ; p=0.01)$. Simillarly, greater right sylvian fissure volume was associated with presence of negative symptoms over the followup period $(B=2.2 ; p=0.007)$, and there was a trend for left sylvian fissure volume to predict more disability as measured by the DAS $(\mathrm{p}<0.1)$. Adding squared sylvian fissure volume terms did not further improve the fit of any of the models at even the $10 \%$ significance level.

Exclusion of cases with extreme values for right and left sylwian fissure volumes did not significantly alter the results; although in the time-spent-in-unemployment rating the llevel of significance in the association with left sylvian fissure volume dropped to $10 \%$, the value of the regression coefficient in fact decreased by only $5 \%$. It was possible to transform the skewed distributions of sylvian fissure volumes and the lateral and third ventricles to approximate normality; analyses with transformed exposure variables did not alter the pattern of the results. 
Therefore, in terms of risk, this means that, given that the ranges of the left and right sylvian fissure volumes were $0-3.6$ and $0-2.2$ respectively , one unit $(1.00)$ increase in sylvian fissure volume predicted 13.5 percent ( $95 \%$ CI: 3.4-23.6) more time of the follow-up period in unemployment, being 16.9 times $(95 \% \mathrm{Cl}: 1.9-142.9)$ more likely to be unemployed at followup, and 8.9 times (95\% CI: 1.8-43.3) more likely to have demonstrated negative symptoms over the follow-up period. Analyses with area measures, instead of volumes, for third ventricle and sylvian fissures yielded similar results.

These findings might represent baseline associations rather than true predictors of outcome. Therefore, analyses were repeated adjusting for baseline unemployment and baseline negative symptoms (PSE "affective flattening" syndrome). Despite very significant effects of these baseline measures, the effects of left and right sylvian fissure volumes were not attenuated in predicting unemployment over the follow-up period $(B=11.9 ; p=0.02)$, unemployment at followup $(\mathrm{OR}=17.5 ; \mathrm{p}=0.02)$ and negative symptoms over the follow-up period $(\mathrm{OR}=6.8, \mathrm{p}=0.02)$.

CT-measures were highly correlated, and the significant effect of sylvian fissure volume might obscure associations of similar strength (but with a slightly larger standard error) with other CTmeasures. To examine this, we repeated the stepwise regression analyses without sylvian fissure volumes, which revealed that, in the absence of sylvian fissure measures, third ventricular volume entered most of the equations, where a significant association was previously found, instead (negative symptoms over follow-up period: $p=0.03$; time spent in unemployment: $p=0.04$; employment status at follow-up: $\mathrm{p}=0.09$ ). Similarly, the effects of right and left sylvian fissure volumes were "interchangeable": if one was left out of the equation, the other was selected for inclusion in the model instead. However, if both sylvian fissure and third ventricular volume were left out of the equations, no significant association was observed between any of the remaining CT measures and any dimension of course and outcome, although associations were in the same direction.

In order to examine whether these effects were specific to schizophrenia, diagnostic-CT measure interaction terms were added with diagnostic categories collapsed into schizophrenic and nonschizophrenic psychoses (thus increasing the statistical power of the test for interaction). This yielded one interaction term of borderline statistical significance $(\mathrm{p}=0.06)$ in the equation with negative symptoms over the follow-up period as the dependent variable. Stratified analysis indicated that the association between negative symptoms and right sylvian fissure volume was much stronger in the schizophrenic psychoses (OR: 102.6; 95\% CI: 7.1-1486.0) compared to the non-schizophrenic psychoses (OR: $3.0 ; 95 \% \mathrm{CI}$ : 0.4-22.4).

In order to examine whether the effect of right and left sylvian fissure volume on unemployment measures was mediated by cognitive functioning, both parts of the Trails Test were modeled as described above for the measures of course and outcome. A significant association between left sylvian fissure volume and both test results was apparent, higher volumes predicting poorer performance (Trails $\mathrm{A}: \mathrm{B}=-1.8 ; \mathrm{p}=0.01$; Trails $\mathrm{B}: \mathrm{B}=-1.2 ; \mathrm{p}=0.01$; again, the effects of left and right fissure volumes were "interchangeable"). Furthermore, controlling for cognitive functioning (by adding Trails $B$ to the equation as an independent variable) significantly reduced the associations between right and left sylvian fissure volumes and the two measures of unemployment ( $p=0.3$ and $p=0.1$ respectively), but did not reduce the strength, nor the statistical significance of the association with level of negative symptoms over the follow-up period 
$(\mathrm{OR}=20.3,95 \% \mathrm{CI}=2.5-166)$. There was no evidence for interaction between DSM-III-R diagnosis and intracranial measures in the association with Trails test $A$ and $\mathbb{B}$.

\section{DISCUSSION}

There was a linear trend in the association between left and right sylvian fissure volumes on the one hand, and unemployment and presence of negative symptoms over the follow-up period on the other, over and above the effect of baseline status on these outcome variables. The association with negative symptoms was especially marked in schizophrenia. Poor cognitive functioning may mediate the risk of sylvian fissure volume on unemployment. No associations were found with the dimensions of symptom severity, time living independently, vagrancy/imprisonment, and depression/self harm. We will first consider to what extent our results can be explained by chance, bias or confounding.

\section{Methodological issues}

The retrospective clinical assessment of negative symptoms over the follow-up period may be more prone to misclassification than unemployment over the follow-up period and at follow-up. However, case note material and/or informants were usually available, and if misclassification of negative symptom status did occur, this is unlikely to have arisen as a function of size of CSF spaces, the rater being blind to all CT-data. Furthermore, the validity of this finding is supported by an association at the $10 \%$ significance level between sylvian fissure volume and the DAS, a measure of the same outcome dimension (negative symptoms/disability).

Measurement errors of, for example, sylvian fissure volumes were independent of the outcomes, as measurements were conducted at baseline. It is very unlikely that such non-differential misclassification of the exposure status will have lead to false assumptions about positive relationships; it may , however , have lead to false conclusions about lack of effect.

Multiple statistical testing may lead to chance findings, but we do not think that our results represent type I errors. Multivariate, step-wise regression models protect against a Type $\mathbb{I}$ error without increasing the Type II errors (Bartko et al, 1988). In examining the associations between intracranial measures and course of illness, we chose a conservative threshold at which to reject the null-hypothesis. In fact, the relatively large number of negative findings compared to previous work may relate to the tight control of confounding variables in the regression analyses.

In spite of these limitations, we feel our study has considerable strengths as well. It is the largest investigation addressing this subject, prospectively executed by rater who was blind to exposure data, and taking into account possible confounding factors, baseline values of outcome variables, the influence of extreme values, and the possibility of departure from linearity. 


\section{Interpretation of findings}

Sylvian fissure volume was more sensitive to future variation in course and outcome of psychotic iliness than other intracranial measures. The effect of sylvian fissure was not lateralized, as the effect of one was in fact "interchangeable" with the other. Our results are conisistent with the plethora of temporal lobe abnormalities reported in schizophrenia (e.g Bogerts et al, 1985; Suddlath et al; 1990). Furthermore, differences between patients and controls may be more marked for temporal-limbic structures than for any other region of the brain (Shenton et al, 1992). Third ventricular volume appeared the next best predictor of outcome, a finding that is congruent with our previous work (Jones ef $a l_{,}$1994), where third ventricular area was the strongest risk factor for schizophrenia and affective psychosis in a case-control study.

The absence of associations with time spent living independently (i.e. not in hospital or supervised residence), and symptom severity (course of illness and usual symptom severity), both arguably more related to positive than to negatiwe symptoms, suggests that $\mathrm{CT}$ measures are risk. factors for negative symptoms and disability rather than positive symptoms.

Associations between CT measures and cognitive impairment have been reported by many workers (e.g. Golden et al, 1980). It is possible, therefore, that cognitive impairment lies on the causal pathway between ventricular enlargement and the outcome of unemployment. This is supported by the finding of very significant associations between sylvian fissure volumes and a measure of cognitive functioning at follow-up, and by the fact that controlling for cognitive functioning greatly reduced the strength of the association with unemployment.

We have demonstrated that it is unlikely that diagnosis, sex, class, age, ethnic differences, duration of illness, age of onset and baseline status on outcome variables played a significant role in mediating the effect, and, in our previous study, no large or significant associations between CT-measures and obstetric complications or a family history of schizophrenia or other psychiatric illness could be demonstrated (Jones et al, 1994). An association between CT ventricle enlargement and poor response to treatment has been reported (Weinberger et al, 1980), but if this were the factor mediating the increased risk, one would expect an effect of CTmeasures on hospitalization and clinical outcome measures, rather than on unemployment and negative symptoms, which do not respond as well to anti-psychotic medications as positive symptoms. We therefore conclude that increased sylvian fissure volume is an independent risk factor for negative symptoms over the course of the illness, and of unemployment, the latter effect probably being mediated by cognitive functioning. There was no evidence of a sub-group of cases with very large ventricles to account for the findings; the increased risk was continuous over the population.

The absence of diagnostic interactions in the association between sylvian fissure volume and unemployment may indicate a lack of diagnostic specificity for this finding, but tests for interaction lack power, and effect modification by diagnostic category cannot be excluded, especially as the association between sylvian fissure volume and negative symptoms was largely confined to schizophrenic subjects. 


\section{APPENDIX 1.}

\section{COUASE TYPE IN THIS PERIOD.}

In estimating duration of an episode that began before this period but extended into the period, include the time before this period.

- episodic - discrete episodes (none longer than 6 MONTHS) with clear periods of remission in between. At least one remission lasted 6 months or more.

- continuous - psychotic ower most of the period. If any remissions, these were brief (none longer than 6 months).

- neither episodic nor continuous - e.g., longest psychotic episode was 12 months and longest remission was 9 months.

- never actively psychotic in this period.

USUAL SEVERITY OF SYMPTOMS. (Categonies are modified from M Bleuler.)

Select the rating that best describes MOST of the period. If no rating applies to more than half of the period, select the one with the longest duration.

- mild - behavior was generally normal and illness was not immediately obvious in conversation, although definite psychotic symptoms persisted.

- moderate - consistently showed signs of health in spite of marked psychotic symptoms, eg., could speak with reasonable clasity on some occasions; could work or care for others to some extent; marked psychotic symptoms may be overt or suggestive (see definitions).

- severe - could not carry on a coherent conversation; could not work without close supervision: required constant care.

- recovered - only residual or no psychotic symptorns.

\section{PSYCHOTIC}

\section{A. OVERT SIGNS AND SYMPTOMS}

- hallucinations or pseudohallucinations (in any modality):

- delusions;

- marked thought and speech disorder (e.g., incoherence, irnelewance, thought blocking, neologisms, incomprehensibility of speech) other than simple retardation or acceleration:

- marked qualitative psychomotor disorder (e.g., negativism, mutism or stupor; catatonic excitement" constrained attitudes or unnatural postures maintained for long periods) other than retardation or acceleration of mowements:

- bizarre or grossly inappropriate behavior (e.g., talking to self or giggling to self, acts incomprehensible to others, lass of social constraints, etc.).

\section{B. SUGGESTIVE SIGNS AND SYMPTOMS}

- extreme reduction or loss of interests, initiative and drive, leading to serious deterioration of the performance of usual activities and tasks;

- extreme social withdrawal;

- sewere excitement, purposeless destructiweness or aggression (frequent episodes or continuous);

- episodes or persistent states of overwhelming fear or sewere anxiety;

- gross and persistent self-neglect.

\section{REMISSION}

A state following a psychotic episode, in which none of the symptoms and signs required for rating a psychotic episode are present. The absence of psychotic symptoms and signs qualifies as a remission only if it lasts for four weeks or more. Two varieties of remission will be distinguished:

(A) COMPLETE REMISSION: the subject is wirtually symptom-free and shows his/her usual premorbid personality:

(B) INCOMPLETE REMISSION: the subject is no longer psychotic (in the sense defined above) but shows any combination of: (i) residual symptoms (i.e.., "traces" of the symptoms and signs which were present in the psychotic episode but which in no way would justify a rating of a continuing psychotic episode); (ii) non-psychotic symptoms, e.g. non-specific neurotic or mild affective disturbances which are not traits of the premorbid personality; (iii) personality change, e.g., accentuation of premorbid personality traits or emergence of new traits; change or loss of interests; stable alterations of expressive and communicative behavior; decline of intellectual performace.

\section{COMMUNITY LIVING INDEPENDENT OF PROFESSIONAL SUPERVISION}

Independent community living includes all liwing arangements other than an institution (e.g.n hospital, jail), a supervised residence, and homeless/vagrant. Thus, it includes living alone, living with friends, living with family, and any other non-institutional domicile. 


\section{REFERENCES.}

Andreasen, N.C., Olsen, S.A., Dennert, J. \& Smith, N. (1982) Ventricular enlargement in schizophrenia: relationship to positive and negative symptoms. American Journal of Psychiatry, $139,297$.

Bartko, J., Carpenter, W. McGlashan, T. (1988) Statistical issues in long-term follow-up studies. Schizophrenia Bulletin, 14, 575-587.

Bogerts. B., Meentz, $\mathbb{E}$, \& Schonfeldt-Bausch, R. (1985) Basal ganglia and limbic system pathology in sclizophirenia: a morphometric study of brain volume and shrinkage. Archives of General Psychiatry, 42, $784-791$.

Breier, A, Schreiber, J.L., Dyer, J. et al (1992) Course of illness and predictors of outcome in chronic schizophrenia: implications for pathophysiology. British Journal of Psychiatry, 161 (suppl. 18) 38-43.

DeLisi, L.E., Schwartz, C.C., Targum, S.D et al (1983) CT scan findings and outcome in acute schizophreniform disorder, Psychiatry Research, $9,169-171$.

L_- LE, Stritzke, P., Riordan, H. et al (1992). The timing of brain morphological changes in schizophrenia and their relationship to clinical outcome. Biological Psychiatry, 31, 241-254.

Dunn, G. (1989) Design and analysis of reliability studies: statistical evaluation of measurement errors, page 117. Edward Amold, London.

Golden, C.J, Moses, J, Zelazowski, R., Graber, B., Zatz, L., Horvath, T. \& Berger, P. (1980) Cerebral ventricular size and neuropsychological impairment in young schizophrenics. Archives of General Psychiatry, 37.619.

Hamilton, M. (1960) A rating scale for depression. Journal of Neurology, Neurosurgery and Psychiatry 23,56 62.

Harvey, I., Willians, M., Toone, B., Turner, S., Lewis, S. \& McGuffin, P. (1990a) The ventricular-brain ratio in functional psychoses: the relationship of lateral ventricular and total intracranial area, Psychological Medicine, 20, 55-62.

w.- Wiliarns, M., McGuffin, P. \& Toone, B. (1990b). The functional psychoses in Afro-Caribbeans British Journal of Psychiatry, 157, 515-22.

Iager, A.C., Kirch, D.G. \& Wyatt, R.J. (1985) A negative symptom rating scalle. Psychiatry Research 16, 27-36.

Jablensky, A., Schwartz, R. \& Tomov, T. (1980) WHO collaborative study of impairments and disabilities associated with schizophrenic disorders. A preliminary communication. Objective and methods. Acta Psychiatrica Scandinavica, suppl. 285, 152-163.

Jones, P.B., Bebbington, P., Foerster, A., Lewis, S., Murray, R., Russel, A., Sham, P., Toone, B. \& Wilkins, S. (1993) Premorbid social underachievement in schizophrenia. Results from the Camberwell Collaborative Psychosis Study. British Journal of Psychiatry, 162, 65-71.

-...-., Harvey, I., Lewis, S., Toone, B., Van Os, J., Williams, M. \& Murray, R. (1994) Cerebral ventricle dimensions as risk factors for schizophrenia and affective psychosis. An epidemiological approach to analysis. Psychological Medicine, 24, 995-1011.

McGuffin, P., Farmer, A.E. \& Harvey, I. (1991) A polydiagnostic application of operational criteria in psychotic illness: development and reliability of the OPCRIT system. Archives of General Psychiatry,48, 764-770.

Owens, D.G., Johnstone, E.C., Crow, T.J., Frith, C.D., Jagoe, J.R. \& Kreel, L. (1985) Lateral ventricular size in schizophrenia: relationship to the disease process and its clinical manifestations. Psychological Medicine, $15,27 \cdot 41$.

Penn, R.D., Belanger, M.G. \& Yasnoff, W.A. (1978) Ventricular volume in man computed from CAT scans. Annals of Neurology, 3, 216-223.

Reitan, R.M. (1958) Validity of the trail making test as an indicator of organic brain damage. Perceptual and Motor Skills $s_{\eta}, 271-276$

Shenton, M.E, Kikinis, R., Jolesz, F., Pollak, S., LeMay, M., Wible, C., Hokama, H., Martine, J., Metcalf, D., Coleman, M.v McCarley, R. (1992) Abnormalities of the left ternporal lobe and thought disorder in schizophrenia. New England Journal of Medicine, 327, 604-612.

Spitzer, R. Endicott, J. \& Robins, E. (1978) Research diagnostic criteria: rationale and reliability. Archives of General Psychiarry, 35, 773-782.

Suddath, R. Christison, G., Torrey, E.F., Casanove, M. Weinberger, D. (1990) Anatomical abnormalities in the brains of monozygotic twins discordant for schizophrenia. New England Journal of Medicine, 322, 789-794.

Vita, A. Dieci, M., Giobbio, G.M. et al (1991) CT scan abnormalities and outcome of chronic schizophrenia. American Journal of Psychiatry, 148, 1577-79.

Van Os, J., Fahy. T., Bebbington, P., Jones, P., Wilkins, S., Sham, P. et al (1994) The influence of life events on the subsequent course of psychotic illness. Psychological Medicine, 24, 503-513.

-_.-. J., Fahy, T., Jones, P., Harvey, I., Lewis, S., Toone, B. \& Murray, R. (1993) Psychopathological syndromes in the functional psychoses: associaiotns with course and outcome. (Psychological Medicine, 
in press)

Weinberger, D., Bigelow, L., Kleinman, J., Klein, S., Rosenblatt, J. \& Wyatt, R. (1980) Cerebral ventricular enlargement in chronic schizophrenia: an association with poor response to treatment. Archives of General Psychiatry, 37, 11-13.

WHO (1992) WHO coordinated multi-center study on the course and outcome of schizophrenia. Geneva: WHO.

Williams, A.O., Reveley, M.A., Kolakowska, T., Ardern, M. \& Mandelbrote, B. (1985) Schizophrenia with good and poor outcome. II. Cerebral ventricular size and its clinical significance. British Journal of Psychiatry, $146,239-246$.

Wing, J.K. Cooper, J.E. \& Sartorius, N. (1974) The Measurement and Classification of Psychiatric Symptoms. Cambridge University Press; Cambridge.

Zatz, L.M. \& Jemigan, T. (1983) The ventricle-brain ratio on computed tomography scans: validity and proper use. Psychiatry Research, 8, 207-214. 



\subsubsection{Premorbid abnormalities in mania, schizomania, acute schizophrenia and chronic schizophrenia ${ }^{i}$}

\section{SUMMARY}

We wished to examine the hypothesis that differences in outcome among affective and nonaffective psychoses are associated with differences in the degree of developmental deviance. We studied the case notes of 545 representing $88 \%$ of all patients with non-depressive functional psychoses, residing in a South London catchment area, who received their first psychiatric treatment between 1965-1984. Cases were classified according to the relative chronicity of their illness into four non-overlapping groups: mania, schizomania, acute schizophrenia, chronic schizophrenia. There was a linear trend in the association between illness chronicity and proxy measures of developmental deviance, such as premorbid unemployment, single status and poor academic achievement. Compared to individuals with mania, schizophrenic patients had a 3-6 times increased risk of premorbid abnormality. For patients with schizomania and acute schizophrenia, the risk was 1.5-3 times greater than for manic subjects. We conclude that the prevalence of premorbid abnormalities is highest among chronic schizophrenia, but similar disturbances also occur, to a lesser degree, in less disabling affective and non-affective psychotic disorders.

\section{INTRODUCTION}

There is a growing body of evidence that some chronic mental and physical disorders originate during child or possibly fetal development (Kraepelin, 1919; Fish, 1977; Watt, 1978; Barker et al, 1989, Barker \& Osmond, 1986, Fine et al, 1985). In line with the traditional Kraepelinian distinction between schizophrenia and manic-depressive psychosis, most of the psychiatric research on premorbid abnormalities has focussed exclusively on schizophrenic patients. More recently, however, attention has been drawn to the fact that a substantial proportion of patients with affective disorders have a similar chronic and disabling course as those with schizophrenia (Piccinelli \& Willkinson, 1994: Clayton, 1981). As both in affective disorder and in schizophrenia premorbid abnormalities are associated with poor outcome (Duggan et al, 1990; Stoffelmayr, 1983), there is reason to believe that in both disorders a (possibly similar) type of developmental abnomality is associated with chronic illness course in at least a proportion of cases. Recent studies have investigated neurobiological and neurodevelopmental hypotheses involving non-psychotic disorders, such as affective disorder, neurotic disorder, and personality disorder (Dolan, 1994; Hollander et al, 1990). Some evidence has emerged suggesting that individuals with affective disorders show subtle differences in cognitive and socio-behavioural development (Done et al, 1994; Rogers, 1990; Done et al, 1993; Hollis, 1994), similar to those found in samples of patients with schizophrenia. However, the degree of developmental deviance found in schizophrenia is certainly more conspicuous than that found in affective disorder (Foerster et al, 1991; Jones et al, 1993); similarly, in acute schizophrenia these premorbid abnormalities appear less prevalent than in the severe, early onset forms of the disorder (Foerster et al, 1991; Murray \& O'Callaghan, 1991). Thus, rather than making absolute distinctions 
between diagnostic categories, it may be useful to investigate the hypothesis that the degree of developmental deviance in psychiatric disorders varies as a function of the tendency of a particular disorder towards poor outcome and chronicity. Such a hypothesis would be compatible with the differences in the prevalence of childhood abnormalities between acute and chronic schizophrenia, and between schizophrenia and affective disorder.

We wished to examine the hypothesis that premorbid abnormalities may be found in both affective and non-affective psychotic disorders, and that risk of developmental deviance would reflect risk of poor outcome and chronicity of the disorder.

\section{METHOD}

\section{Sample}

The Camberwell Cumulative Psychiatric Case Register (Wing \& Hailey, 1972) provides a comprehensive list of all persons from the area of Camberwell in South London who had their first contact with the psychiatric services between 1964 and 1984. With the permission of the Maudsley Hospital ethics committee, a print-out was generated from the Register, of all firstcontact patients between 1965 and 1984 who received a non-depressive psychotic register diagnosis: "mania" or "hypomania" (equivalent ICD codes 296.0, 296.2, 296.4), "schizophrenic psychosis " (including schizo-affective type; ICD codes 295.0-295.9), "paraphrenia" (ICD 297.2) or "other nonorganic psychosis" (ICD 298.1-298.9). This broad sample was chosen to avoid the possibility of missing any patients who had been inappropriately labelled, and to allow for variation in diagnostic habits. As this is a study of risk, and not rates, no corrections are made for missing data.

\section{Diagnosis}

All the case notes of the subjects in this study were traced and scrutinized. Subjects were subsequently given a diagnosis on the basis of operationalised definitions of psychiatric illness.

In a previous study, case notes of patients with an ICD diagnosis of schizophrenia and related. conditions (including "schizo-affective disorder", "paraphrenia", and "atypical psychoses") had been scrutinized and rated by DC and SW (Castle et al, 1991), using the Operational Criteria Checklist for Psychotic Illness (OCCPI) and the OPCRIT computer program, which generate a range of operationall definitions for psychotic illnesses (RDC, DSM-III, DSM-III-R, etc; McGuffin et $\left.a l_{*}, 1991\right)$. Inter-rater reliability, based on 50 cases reted by both DC and SW, was good (kappa $=0.82$ for RDC diagnoses). Cases from this sample with an RDC diagnosis of mania or schizomania were also identified.

All the case notes of patients with a Register diagnosis of mania or hypomania were also scrutinized and rated with the OCCPI checklist by two of the investigators (NT and JvO), but only the OCCPI items necessary to make an RDC diagnosis (Spitzer et al, 1978) were rated. Inter-rater reliability for cases with a Register diagnosis of mania was assessed on a random subset of 15 case records which were rated by NT and JvO; kappa for RDC diagnoses was 0.78 . 
The two authors who rated the manic cases (NT and JvO), and one of the authors who rated cases of schizophrenia and related disorders (DC), took part in a reliability study of OPCRIT. The mean Kappa for the three pairs of raters for RDC diagnoses of schizophrenia and affective psychosis was 0.74 (range: $0.70-0.76$ ).

\section{Diagnostic comparisons}

In the comparison between diagnostic categories on premorbid, clinical and demographic variables, four non-overlapping groups of patients were defined. These included: i) RDC mania; ii) RDC schizomania; iii) "acute" schizophrenia; and iv) "chronic" schizophrenia. Chronic schizophrenia was defined as a condition characterised by typical delusions and/or hallucinations (Schneider, 1959), and meeting DSM-III-R (APA, 1987) criterion B (deterioration from premorbid levell of functioning), criterion $\mathrm{C}$ (schizo-affective and mood disorder ruled out), and criterion $D$ (continuous signs of disturbance for at least six months). Acute schizophrenia indicated a condition with typical delusions and/or hallucinations and DSM-III-R criterion C, but not meeting criterion $B$ and criterion $D$. The rationale for this division into acute and chronic schizophrenia was to create two schizophrenic syndromes, broadly corresponding to the British notions of acute and chronic schizophrenia (Van Os et al, 1993).

The four diagnostic categories were ordered according to their prognostic implications, based on the well established evidence (Westermeyer \& Harrow, 1988; Tsuang \& Dempsey, 1979) that i) affective psychoses (i.e. mania and schizomania) have a better outcome than non-affective schizophrenic psychoses (i.e. acute and chronic schizophrenia), and that ii) among the affective psychoses schizoaffective disorders have a worse prognosis and iii) schizophrenia defined in terms of deterioration from premorbid level of functioning and persistence of symptoms has a worse prognosis than acute schizophrenia without these signs. Thus, the order (from favourable to poor prognosis) was: mania, schizomania, acute schizophrenia, chronic schizophrenia.

\section{Demographic data}

Other information systematically obtained for all individuals included: sex, age at first contact, date of birth, and ethnicity and country of birth of both patient and parents. "Ethnicity" categories were white European, Afro-Caribbean, African, Asian, and "other", while "country of birth" categories were UK and Eire, West Indies (Caribbean), Asia, Africa, and "other". "These data were directly rated from the case records; checks on date of contact, date of birth and country of origin were made from the front-sheets of the case-notes and the Camberwell Register itself. Checks on ethnicity ratings were made on a subset of 34 patients, using data from previous direct-interview studies involving these patients; no erroneous ratings were found. Childhood social class was defined as paternal occupation as recorded in the notes (categorised into "unemployed", "manual work", "white collar", and "professional"). In a previous study (Castle et al, 1993) paternal occupation data from 20 randomly selected case records of the same sample were compared with information recorded on birth certificates, which routinely record such information. Despite a few minor dissimilarities (eg exact description of job), no errors were found. 
Patients who were living outside the catchment area at the time of first contact but had erroneously been included in the Register, were excluded. Similarly, checks were made on first contact status to preclude bias due to inclusion of "false" first contact cases (see above).

\section{Premorbid variables}

The following variables were used as indicators of premorbid social and academic development: academic achievement (no exams, CSE, O level, A level, and tertiary education); premorbid employment ("unemployed" $=6$ or more months of unemployment in the previous three years; "unstable" $=3$ or more jobs in the years prior to first contact; "stable"=one job in the years prior to first contact; "not on job market"); and marital status (married or living as married). Because of the small number of subjects in some diagnostic groups, premorbid variables were dichotomised ("unemployed", "no exams", "single") in the analyses.

\section{Analyses}

The main analyses used odds ratios to estimate the association between diagnostic category and other variables, calculated with the program EGRET (version 0.26.6, Statistics and Epidemiology Research Corporation, North Carolina, U.S.A.). All odds ratios refer to mania versus other diagnostic categories. Crude odds ratios, $95 \%$ confidence intervals and the test for trend for crude odds ratios were first computed. Odds ratios were adjusted with the logistic regression procedure; confidence intervals and tests for trend for adjusted odds ratios were calculated from the results of the logistic regression.

\section{RESULTS}

\section{Sample}

Out of 545 patients ( $88 \%$ of the total selection of 621 ), 71 had an RDC diagnosis of mania (males: 53\%), and 24 of RDC schizomania (males: 33\%). Ninety-seven patients met our criteria for acute schizophrenia (males: $50 \%$ ), and 195 subjects met the criteria for chronic schizophrenia (males: $55 \%$ ). Mean age of onset in males was 35.2 years for mania, 27.6 for schizomania (compared to mania: $p=0.2$ ), 34.5 for acute schizophrenia (compared to mania: $p=0.8$ ), and 33.2 for chronic schizophrenia ( $p=0.5$ ). In females, mean age of onset was 33.3 for mania, 35.6 for schizomania $(p=0.6), 36.3$ for acute schizophrenia $(p=0.5)$, and 50.4 for chronic schizophrenia $(\mathrm{p}<0.001)$

Data on sex, ethnicity and age of first contact with psychiatric services were available for all subjects, data on premorbid employment for $96 \%$, on premorbid academic achievement for $93 \%$, and on marital status for $95 \%$. 


\section{Premorbid variables}

We compared academic achievement, premorbid employment, and marital status between individuals with mania and with other diagnoses (Table 1), controlling for gender, age of onset and ethnicity. Because of the possible effect of social class background on education and age at marriage, patemal social class was also controlled for in the comparisons with academic achievement and marital status as the dependent variable. Compared to mania, the odds of being unemployed, single, or having left school without exams tended to increase with illness chronicity, although the trend was not monotonic or similar in magnitude for all measures.

The risk of premorbid abnormality in individualls with chronic schizophrenia was 3-6 times greater than for patients with mania, whereas for schizomanic and acute schizophrenic patients the increase in risk was 1.5-3 times. For marital status, differences between mania and chronic schizophrenia only became apparent after controlling for confounders. This was mainly due to the effect of age of onset in females: females with schizophrenia had much later age of onset and for that reason were more likely to be married at onset of illness, thus obscuring the difference between manic and schizophrenic individuals.

Employment and education variables are also subject to a cohort effect. Such an effect could have affected subjects with mania and schizophrenia differentially, as there was an excess of older schizophrenic females in our sample, who may not have had the same educational opportunities.

When year of birth was incorporated into the analysis, in order to test for a possible cohort effect, odds ratios differed only by a trivial amount, and the pattern of results remained the same.

\section{DISCUSSION}

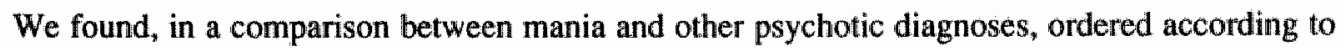
prognostic significance (iilness chronicity), that there was a linear trend in the association between illness chronicity on the one hand, and premorbid adjustment on the other. Schizophrenia defined in terms of deterioration and persistent psychopathology stood out especially* compared to mania, the odds of having poor premorbid functioning was between 3.0 and 5.6 times higher. However, could these findings be due to bias or confounding?

\section{Methodological issues}

A number of methodological issues have been discussed above. Elsewhere, we have discussed possible artefacts such as diagnostic bias, changing referral patterns, misclassification of first contact cases and missing case notes, demonstrating that these are unlikely to have influenced our results significantly (Castle et al, 1991). 


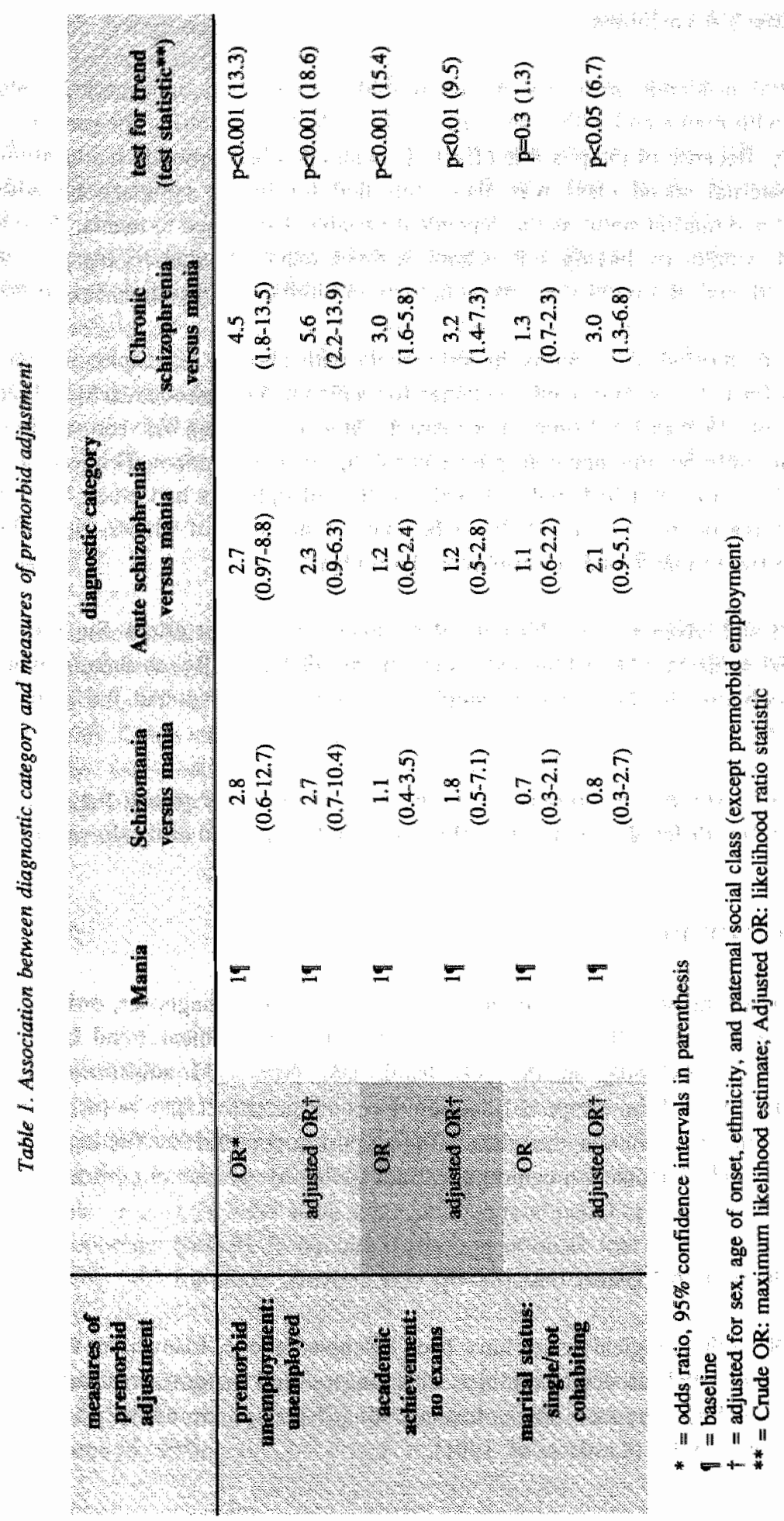


This was a study of first contact cases, ascertained irrespective of age, from a defined area, applying operationalised criteria to comprehensive case-note material. Thus, the sample can be expected to be representative of all schizophrenic and manic patients in Camberwell, avoiding conclusions being drawn about a group which has already been partlly-selected, especially with regard to age-of-onset, many studies not including late-onset cases. Although it is possible that mild cases of mania would not have come to psychiatric attention, this, if anything, would have reduced the reported associations as the more severe cases can be expected to show more evidence of developmental deviance. Changes in diagnostic practice will not have affected our results, as all cases were re-diagnosed using contemporary operationalised criteria, and we assessed the widest feasible range of diagnoses on the register.

The retrospective nature of our study is a limitation. Premorbid social and occupational functioning is difficult to ascertain reliably from case-records. However, we used relatively simple data such as academic achievement, employment and marital status to reduce error. We acknowledge that this approach has led to a certain degree of over-simplification of multi-facetted aspects of premorbid behaviour, only cases with the severest forms of premorbid abnormalities will have been detected. The premorbid variables used are probably. dependent on factors such as ethnicity, gender and social class, as well as age of onset of illness, but we were able to control for all of these in the analyses. It is likelly that academic achievement, employment and marital status are proxies of the same latent variable, premorbid adjustment, and results for all three concurred, thus enhancing the validity of our findings.

Despite the relatively large sample of cases, the incidence of a disorder such as schizoaffective mania and the prevalence of paternal unemployment were rare enough to jeopardize the statistical stability in some instances; as a consequence, confidence intervals for estimates were sometimes very wide.

\section{Interpretation of Findings}

The three different measures of premorbid adjustment we used discriminated, after controlling for social class, between relative severity of psychotic illness, the odds of having poorer premorbid adjustment rising progressively with illness severity. This is consistent with reports of subtle abnormalities, including cognitive changes (Aylward et al, 1984), and abnormal personality traits in childhood and adolescence (Walker \& Lewine, 1990), especially in patients with more severe forms of schizophrenia (Foerster et al, 1991). However, the increase in the odds ratios from mania to chronic schizophrenia was not monotonic in all measures, and much -but not all- of the trend was accounted for by the high values of the OR's in the chronic schizophrenic category. The conservative interpretation therefore is that, compared to other diagnostic categories, a severe, possibly "neurodevelopmental" type of chronic schizophrenia is especially, but not uniquelly, associated with developmental deviance. Similar premorbid abnormallities may be encountered in other psychotic syndromes, higher risk of schizoprenia-like chronic illness course being associated with increased likelihood of developmental deviance. 


\section{REFERENCES}

American Psychiatric Association (1987). Diagnostic and Statistical Manual of Mental Disorders, third edition, revised. Washinghton, APA.

Barker, D., Winter, P.D., Osmond, C., Margetts, B. \& Simmonds, S.J. (1989) Weight in infancy and death from ischaemic heart disease. Lancet 2, 577-580.

-n-..., D. \& Osmond, C (1986) Childhood repiratory infection and chronic bronchitis in England and Wales. Brinish Medical Journal, 293, 1271-1275,

Aylward, E. Walker, E. \& Bettes, B. (1984) Intelligence in schizophrenia: meta analysis of the research. Schizophrenia Bulletin: 10: 430-459.

Castle, D., Wessely, S., Der, G, Murray, R. (1991). The incidence of operationally defined schizophrenia in Camberwell, 1965-1984. British Journal of Psychiatry; 159: 790-794.

-......, Scott, K., Wessely, S., Murray, R. (1993) Does social deprivation during gestation and early life predispose to later schizophrenia? Sacial Psychiatry \& Psychiatric Epidemiology; 28: 1-4.

Clayton, P.J. (1981) The epidemiology of bipolar affective disorder. Comprehensive Psychiatry, 22, 31-43.

Dolan, M (1994). Psychopathy, a neurobiological perspective. British Journal of Psychiatry, 165, 151-160.

Done DJ, Crow TF, Johnstone EC \& Sacker A. (1994) Childhood antecedents of schizophrenia and affective illness: social adjustment at ages 7 and 11. British Medical Journal, 309, 699-703.

Done J, Sacker A \& Crow TJ (1993) Childhood antecedents of schizophrenia and affective illiness: intellectual performance at ages 7 and 11. Schizophrenia Research, 11, 96-97

Duggan CF, Lee AS, Murray RM (1990) Does personality predict long-term outcome in depression? British Journal of Psychiatry, 157, 19-24

Fine, P., Adelstein, A., Snowman, J., Clarkson, J. \& Evans, S. (1985) Long term effects of exposure to viral unfections in utero, British Medical Journal, 290, 509-511.

Fish, B. (1977) Neurobiological antecedents of schizophrenia in children. Archives of General Psychiatry, 34, $1297-1313$.

Foerster, A.n Lewis, S., Owen, M. ef al (1991) Premorbid personality in psychosis: effect of sex and diagnosis. British Jaurnal of Psychiatry, 158, 171-176.

Hollander, E., Schiffman, E, Cohen, B., Rivera-Stein, M., Rosen, W., Gorman, J. et al (1990). Signs of central nervous dysfunction in obsessive-compulsive disorder. Archives of General Psychiatry, 47, 27-32.

Hollis CP (1994) Developmental impairments in child and. adolescent-onset psychoses. Paper presented at the Winter Meeting, Royal College of Psychiatrists, London 1-4 February.

Jones, P.B., Bebbington, P., Foerster, A., Lewis; S., Murray, R., Russel, A., Sham, P., Toone, B. \& Wilkins, S. (1993) Premorbid social underachievement in schizophrenia. Results from the Camberwell Collaborative Psychosis Study. British Journal of Psychiatry, 162, 65-71.

Kraepelin, E. (1919) "Dementia Praecox and Paraphrenia". Translated by R. M. Barclay, section on Endogenous Dementias. Edited by George Robertson. Edinburgh: Livingstone.

McGuffin, P. Farmer, A. \& Harvey, I. (1991) A polydiagnostic application of operational criteria in studies of psychotic illness: development and reliability of the OPCRIT system. Archives of General Psychiatry: 48: $645-650$.

Murray, R.M. \& O'Callaghan, E. (1991) The congenital and adult-onset psychoses: Kraepelin lost, Kraepelin found. In Concepts of Mental Disorder, a Continuing Debate (Eds A. Kerr \& H. McClelland) London: Gaskell, pp 48-65.

Piccinelli M \& Wilkinson G (1994) Outcome of depression in psychiatric settings. British Journal of Psychiatry, 164, 297-304.

Rogers B. (1990) Behaviour and personality in childhood as predictors of adult psychiatric disorder. Jowrnal of Child Psychology and Psychiatry, 3, 393-414.

Schneider, K. (1959) Clinical Psychopathology. Translated by M.W. Hamilton. London: Grume \& Stratton.

Spitzer, R. Endicott, J. Robins, E. (1978) Research diagnostic criteria: rationale and reliability. Archives of General Psychiatry" 35: 773-782.

Stoffelmayr, B., Dillavou, D. \& Hunter, J. (1983) Premorbid functioning and outcome in schizophrenia: a cumulative anallysis. Journal of Conculting and Clinical Psychology, 51, 338-352.

Tsuang, M.T. \& Dempsey, G.M (1979). Long term outcome of major psychoses II. Schizoaffective disorder, compared with schizophrenim, affective disorders, and a surgical control group. Archives of General Psychiatry; 36: 1302-1304.

Van Os, J., Galdos, P. Lewis, G., Mann, A. \& Bourgeois, M. (1993) Schizophrenia sans frontières: concepts of schizophrenia among French and British Psychiatrists. British Medical Journal; 307: 489-492.

Walker, E Lewine, R. Prediction of adult onset schizophrenia from childhood home movies of patients. American Journal of Psychiairy 1990; 147: 1052-1056. 
Watt, N. (1978) Patterns of childhood social development in adult schizophrenics. Archives of Geveral Psychiarry, 44, 660-669.

Westermeyer, J. \& Harrow, M. (1988) Course and outcone in schizophrenia In: Handbovk of Schizophrenia, vol. 3 (Eds M. Tsuang J. Simpson) pp 205-244. Rotterdam: Elseviers.

Wing, J. \& Hailey, A. Evalwating a community psychiatric service: the Camberwetl Regüster, 1964 1971. London: Oxford University Press, 1972. 
a $\quad$ a

$+2$

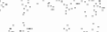

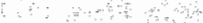

$\therefore \quad a$

$\therefore$ 


\subsubsection{Evidence for similar developmental precursors of chronic affective illness and schizophrenia in a general population birth cohort}

\section{SUMMARY}

In order to test the hypothesis that chronic and severe psychiatric disorder is associated with early developmental deviance, we examined associations between prospectively collected childhood data on the one hand, and chronic affective disorder and schizophrenia on the other, in a prospectively studied national British birth cohort of 5262 individuals born in the week March 3-9th 1946. Seventy-five cases (prevalence: $2.3 \%$ ) with chronic, severe affective disorder (CAD) were identified using a mental health survey at ages 36 and 43 . Thirty cases with schizophremia or schizoaffective disorder $(\mathrm{SZ})$, had been identified previously.

SZ cases and CAD cases showed the same developmental deficits, but significant interaction with gender was present in the latter. Attainment of motor milestones was later in female CAD cases, with a significant linear trend over the population distribution ( $\mathrm{OR}=1.5 ; 95 \% \mathrm{Cl}: 1.1-2.2)$, followed by greater risk of speech defects between the ages of 6 and 15 years $(O R=3.6 ; 1.8-7.5)$.

At ages 8,11 and 15 years, educational test scores differentiated between CAD cases and controls, such that the lower the test scores, the more likely children were to develop later CAD: again effects were more evident in women. At ages 13 and $15, \mathrm{CAD}$ cases were more likelly to be rated "persistently sad and gloomy" by their teachers (OR's $2.7 \& 2.5 ; p<0.05$ ), and as "apathetic" by the medical examiner between the ages of 6 and 11 years $(\mathrm{OR}=5.0 ; 95 \% \mathrm{Cl}: 1.7$ 14.4).

A comparison between the 75 subjects with CAD and a much larger group of subjects with less severe affective illness, revealed that the above behavioural, developmental and cognitive risk factors all differentiated the CAD group from those with less severe illness.

By age 43 years; men were more likely to have developed schizoplirenia $(\mathrm{OR}=1.8 ; 0.9-3.9)$, whereas women were more at risk of developing $\mathrm{CAD}(\mathrm{OR}=2.6 ; 1.6-4.3)$. The were few qualitative differences between CAD and SZ cases in the associations with developmental variables and later psychiatric disorder. However, effects tended to be greater for schizophrenia than for CAD. The results suggest that early social, cognitive and motor deficits are either the early manifestation of a unitary syndrome with poor outcome and gender-related pathoplastic effects on symptomatology, or the early manifestation of a common predisposition to severe psychiatric illness, with other factors determining the type of adult disorder.

\section{INTRODUCTION}

Early devellopmental deviance is a risk factor for later schizophrenia (Jones et al, 1994a), but the specificity of this finding remains in doubt, as there is a comparative lack of prospective research 
involving other diagnostic constructs (Rogers, 1990a \& 1990b). Some evidence has emerged, however, suggesting that individuals with affective disorder and non-schizophrenic psychosis show neurological abnormalities and subtle differences in cognitive development, similar to those found in patients with schizophrenia (Crow et al, 1994; Done et al, 1994).

There is evidence that childhood developmental deficit is not only a risk factor for the emergence of psychotic illness, but is also an important risk factor for illness persistence. A large body of work has demonstrated that pre-psychotic developmental deviance (mostly in the social domain) predicts poor outcome in schizophrenia (review by Stoffelmayr, 1983). This association is not specific to schizophrenia, as multiple studies have converged in showing a similar relationship in unipolar, bipolar and schizoaffective disorders (Deister \& Marneros, 1993; Brodaty et al, 1993; Scott et al, 1992; Harder et al, 1990; Duggan et al, 1990). For example, Coryell and colleagues (1990a) reported that good adolescent friendship patterns were strongly associated with recovery in a sample of 129 patients with chronic affective disorder (depression, mania, schizoaffective disorder), in a five-year prospective outcome study.

If attenuated early development is a risk factor for emergence and persistence of both affective illness and schizophrenia, one would expect that patients with affective illness show less developmental deficits than their schizophrenic counterparts, as affective disorder patients, on average, have a less deteriorated illness course. Also, as schizo-affective disorders have an outcome which is intermediate between affective illness and schizophrenia, one would expect their level of developmental deficit to be similarly intermediary (Tsuang \& Dempsey, 1979; Grossman et al, 1991). In a previous study of a population-based sample of 387 patients with non-depressive functional psychoses, we studied proxy measures of developmental deviance, such as poor academic achievement, pre-psychotic unemployment, and single status (Van Os et al, 1995a). Compared with individuals with mania, chronic schizophrenic patients had a 3-6 times increased risk of reported pre-psychotic deficits. For patients with schizomania and acute schizophrenia, the risk was 1.5-3 times greater than for manic subjects. These findings suggest that the degree of developmental deficit does indeed vary as a function of (chronicity of the) disorder, manic patients having the lowest risk of pre-psychotic deficits and the best outcome, chronic schizophrenic patients having a high risk of both pre-psychotic deviance and poor outcome, with schizomania and acute schizophrenia somewhere in between. These findings, however" may be distorted by retrospective assessment of remote exposures, using measures of pre-psychotic functioning that were necessarily crude. Furthermore, illness severity may differentially influence recollection of early events.

In summary, pre-psychotic developmental deviance appears to be a risk factor for both emergence and chronicity of illness in schizophrenia. Some evidence, however, suggests that i) this finding may not be specific to schizophrenia ${ }_{4}$ and ii) the degree of early deficit is associated with illness severity; patient groups with poor outcome carry the highest risk of attenuated development, regardless of diagnostic category. Thus, one prediction is that, compared with schizophrenic patients, patients with chronic affective disorder should have similar childhood deficits of similar severity, while those with less severe affective illness should not.

Data from the 1946 national birth cohort in the UK, assessed on 20 occasions over four decades, allowed us to investigate prospectively the relationship between developmental risk factors and i) severe, chronic affective disorder, ii) schizophrenia, in a population sample. A previous study 
(Rogers, 1990 a \& b), using material from this cohort, found that some, mainly envinonmental and behavioural-personality, factors in childhood were predictors of adult scores on a short version of the Present State Examination (PSE; Wing et al, 1974) measuring anxiety and depression. New material on mental health from a further contact with the cohort members, using an instrument similar to the PSE, enabled us to identify a group of survey members with severe, chronic affective disorder. Furthermore, we were now ablle to exclude recently identified subjects with severe mental illness, such as schizophrenia and schizoaffective psychosis (Jones et al, 1994a), and use these subjects as an additional informative comparison group. We hypothesized that, as children, both individuals with chronic affective disorder and individuals with schizophrenia would exhibit developmental deviance, ie would have shown, in childhood, i) an excess of behaviours and signs indicative of disturbed social development; ii) developmental. delay both early in childhood and later in adolescence; and iii) diminished cognitive abillity. We also predicted that, within the group of affective disorder, iv) these risk factors would be specific to the more severe and chronic forms of affective disorder (ie with chronic course and/or presenting with manic or psychotic symptoms).

\section{METHOD}

The MRC National Survey of Health and Development is an ongoing longitudinal study of a stratified sample of 5362 subjects (men: $52.5 \%$ ) born in Great Britain during the week 3-9th March 1946. Data were collected on 11 occasions at intervals of no longer than two years until the age of sixteen, and on 9 thereafter, the most recent contact occurring in 1989 at the age of 43. Details of these assessments have been described extensively elsewhere (Atkins et al, 1981; Wadsworth, 1991). Because the findings for the schizophrenia cases have been reported previously (Jones et al, 1994a; Jones et al, 1995), resullis for that disorder here are presented briefly for comparative purposes.

\section{Case definition}

A. chronic affective disorder - Case definition and definition of the risk set involved three criteria that were based on i) measures of both chronicity and severity of affective disorder, collected over two consecutive assessments at ages 36 and 43 , and ii) identification of subjects with schizophrenic or schizo-affective psychoses:

- Chronicity

Among those who had answered questions about a variety of chronic health problems, 174 subjects were identified who had reported both in 1982 and in 1989 that they had suffered "nervous or emotional trouble or persistent depression all or most of the time" (1982); and that they had suffered "recurrent nervous or emotional trouble or depression" (1989). These 174 subjects represented $28.8 \%(174 / 605)$ of the number of subjects in the risk set (see below) who had reported such problems in at least one of the interviews.

- Severity.

The severity criterion was defined conservatively as having a score of 5 or higher on either the index of definition scale of the Present State Examination (PSE), short version of which was administered in 1982 (Rogers \& Mann, 1986), and which included all PSE neurotic and affective features (including manic symptoms), or the equivalent 
score on a similar instrument administered in 1989. The instrument used in 1989 consisted of a simple twenty item structured interview on key symptoms of anxiety and depression, based upon those in the PSE. Because the distribution of the total scores had the same non-normal shape for both instruments (an approximately half-normal distribution, with the peak at zero and positively skewed), and total scores correlated with each other (Spearman rank correlation $=0.35 ;, p<0.0001$ ), the cut-off for the severity criterion in the 1989 instrument was placed at the same percentile (96.3\%) as the median total PSE score in the PSE ID cut-off category of 5.

- Absence of non-affective psychosis.

Subjects previously identified as having a diagnosis of schizophrenia or schizo-affective disorder at any level of certainty (Jones et al, 1994a) were excluded from becoming a case.

Cases met criteria one, two and three. The condition of the subjects who met all three criteria $(n=75)$ will henceforth be referred to as chronic affective disorder (CAD). CAD cases represented $12.6 \%$ of the 596 subjects who met either the severity criterion or had admitted to chronic nervous trouble on at least one occasion. Of the 75 subjects, $73(97 \%)$ had admitted to low mood on at least one of the cross-sectional mental state assessments in 1982 and 1989.

B. schizophrenia - Case definition for the schizophrenia cases has been described in our previous publication (Jones et al, 1994a).

\section{Risk set}

A. chronic affective disorder - The risk set (those individuals at risk of being identified as suffering with chronic affective disorder) was defined as the individuals who, in the 1982 interview, had answered the question on persistent depression, and had had valid PSE interview results $(n=3293)$. All non-cases in the risk set, including survey members with schizophrenia, were included as controls $(n=3218)$.

B. schizophrenia - The risk set used for the schizophrenia cases consisted of 4746 individuals at risk of being identified as schizophrenic in adulthood (Jones et al, 1994a).

\section{Exposure variables}

Three categories of variables from the period between birth and 16 years were included.

- Neuro-developmental and physical - for example, data on the timing of language (saying words other than 'Mumma' or 'Dadda' as recalled by the mother at age two), motor milestones (sitting, walking and standing unaided). Observations made during medical examinations at age $6,7,11$ and 15 years such as speech abnormalities.

- Cognitive ability - as measured by non-verbal, verbal and reading ability tests administered at ages 8,11 , and 15 years, vocabulary at ages 8 and 11 years, arithmetic tests at ages 11 and 15 years (Pidgeon, 1964; 1968). Test scores of survey members had been normalised (Andrews et al, 1973), adjusting for sex and social class. 
- Socio-behavioural - These included a range of behaviours and characteristics. At age 26, survey members were asked about lifetime "nervous trouble", and its age of first onset. At ages 13 and 15, a list of 17 teacher ratings on behaviour was available, scored on a three point scale. For example "making friends" was scored 1 "easily", 2 "average", 3 "unable". In the analyses, category 3 was compared with the first two categories. At ages 4,7 and 11 , comments from the examining doctor included "apathetic", and at age 2 and 4 mothers and teachers commented on preference for solitary play. Health visitor's comments on the home, child and mother were obtained in early childhood.

\section{Analyses}

The main analyses used odds ratios (OR) which, in view of the relative rarity of CAD, estimated the desired incidence rate ratio. OR's and 95\% confidence intervals (Cl) were calculated using the logistic regression (Breslow \& Day, 1987) procedure of the computer program STATA (STATA corp. 1993). Interaction between independent variables was assessed using the likelihood ratio test (LRS). Composite exposure variables used for some of the analyses, had been defined at the time of data collection, unrelated to the present investigation. Normally distributed, continuous exposure variables were divided by their tertiles so as to assess linear trend (Breslow \& Day, 1987); these analyses yielded similar results as those using the continuous distributions. For highly correlated continuous exposure variables, principal component (PC) analysis was used to summarize the data, by reducing a larger number of original variables to a smaller number of transformed variables that accounted for most of the variation within the data (Johnson \& Wichem, 1988). The presence of a risk factor may be considered as a diagnostic test for later occurrence of CAD; the positive predictive value (PPV) of the test is the probability that a person will develop the disease given a positive test, and was calculated for developmental risk factors.

\section{Confounders}

The possible confounding effect of gender and social class as defined by father"s occupation at birth was examined, as well as some other variables that might conceivably be associated with developmental, cognitive, and behavioural variables as well as CAD. For example, we wished to examine whether any associations between motor development and later CAD could be explained by i) having been separated from the mother for more than one week before the age of 6 (for reasons of, amongst others, severe physical illness), ii) death of a parent, iii) mental illness in the parent and iv) the health visitor's opinion on mother's "management and understanding of the child", or the child being "among the least clean", and v) domestic crowding, all these factors possibly resulting in delayed development through under-stimulation or maybe even neglect of the child. Presence of parental history of psychiatric disorder was based on information whether either parent had ever suffered from any "nervous trouble," while health visitors commented on cleanliness of the house and the child and the quality of motherchild interaction at age 4 years. The measure of crowding was based on the number of people per room at age 2 years. Similarly, in examining associations between cognitive performance and later CAD we adjusted for family history of nervous disorder, parental death, teacher's ratings of parental interest in the child's progress at school, the teacher's opinion as to whether the 
child's school work was affected adversely by factors outside the school, as well as for teachers' ratings of anxiety/depression and anti-social behaviour, as all these factors may be associated both with cognitive performance and, independently, with CAD.

\section{RESULTS}

\section{Hospital, out-patient and GP contacts for CAD cases}

In order to obtain more details of illness in CAD cases; we studied all available hospital clinical details, which are collected systematically for all survey members (Kuh et al, 1994). Details include clinical diagnosis, treatment and clinical synopsis. Twenty-five CAD cases (33\%) were known to have had out-patient or in-patient contact with psychiatric services; 23 of these 25 CAD cases (92\%) had a confirmed clinical diagnosis of affective disorder, the majority of neurotic/reactive/anxiety depression. Twenty-one of the $25(84 \%)$ had been a psychiatric inpatient; three patients had received lithium, five had received the antipsychotic trifluoperazine, four had been treated with ECT.

No systematic details on lifetime treatment for psychiatric disorder by the GP were available, but; in the 1982 survey, 37 CAD cases $(49.3 \%)$ had had contact with their GP for their "nerves" in the year before.

\section{Demographic characteristics and family antecedents in CAD cases.}

The prevalence of CAD in the risk set was $2.3 \%$ in 1989 , which is in agreement with published findings (Weissmann \& Myers, 1978; Weismann et al, 1988; Favarelli et al, 1990; Howard, 1993). Female gender was associated with increased risk of CAD (controls: $1600(49.7 \%)$; cases: $54(72 \%)$; OR: $2.6 ; 95 \% \mathrm{CI}: 1.6-4.3)$, and there was a trend for an increased risk with lower childhood social class $\left(\chi^{2}\right.$ trend: $\left.2.5 ; p=0.1\right)$. A family history of nervous disorder was also associated with an increase in risk ( $\mathrm{OR}=2.4 ; 95 \% \mathrm{CI}: 1.5-3.8)$ regardless of whether the mother was affected $(\mathrm{OR}=2.5 ; 95 \% \mathrm{CI}: 1.5-4.0)$, or the father $(\mathrm{OR}=2.9 ; 95 \% \mathrm{CI}: 1.6-5.3)$, the highest risk being conferred by a history in both parents ( $O R=7.7 ; 95 \% \mathrm{CI}: 3.6-16.5$ ).

\section{Physical development in CAD cases}

Age in months at reaching motor milestones as recalled by the mother at age two (sitting, standing, walking) were significantly correlated (all: $r \geq 0.5 ; \mathrm{p}<0.001$ ). A general motor attainment measure; explaining $73 \%$ of the variance, was obtained by using scores of the first principal component of the educational test variables at the three ages. Subsequently, the number of cases and controls were compared in each of the tertiles of the population distribution. There was no significant association between motor attainment and later CAD (OR=1.2; 95\% CI: 0.91.7). However, there was effect modification by gender (test for interaction: $L$ RS: $5.2, p=0.03$ ), such that in women, but not in men, the probability of being a case increased progressively with age at motor attainment (OR for linear trend: 1.5; 95\% CI: 1.1-2.2), with an excess of cases in the highest tertile (Table 1). 
Table 1. Association between motor millestones attainment and later $C A D$

\begin{tabular}{|c|c|c|c|c|c|c|}
\hline & & Tert & He poppanio & Bustoont & & \\
\hline & & $\begin{array}{l}\text { horer } \\
\text { ages }\end{array}$ & fintiolie wge & lingher & $\begin{array}{l}\text { on for } \\
\text { lingar } \\
\text { trend }\end{array}$ & $\begin{array}{l}\text { Ad dusted } \\
\text { onit }\end{array}$ \\
\hline & number of & 11 & 14 & 23 & & \\
\hline Wonten & 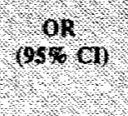 & $1 \dagger$ & $\begin{array}{c}1: 2 \\
(0.5-2.6)\end{array}$ & $\begin{array}{c}2.2 \\
(1.1-4.6)\end{array}$ & $\begin{array}{c}1.5 \\
(1.1-2.2) \\
p=0.02\end{array}$ & $\begin{array}{c}1.5 \\
(1.0-2.3) \\
p=0.06\end{array}$ \\
\hline & number of & 8 & 5 & 4 & & \\
\hline Men & $(0 \mathrm{or}) \mathrm{cn}$ & $1 \dagger$ & $\begin{array}{c}0.6 \\
(0.2-1.8)\end{array}$ & $\begin{array}{c}0.5 \\
(0.2-1.7)\end{array}$ & $\begin{array}{c}0.7 \\
(0.4-1.3) \\
p=0.3\end{array}$ & $\begin{array}{c}0.7 \\
(0.3-1.4) \\
p=0.3\end{array}$ \\
\hline
\end{tabular}

* divided such that one third of controls is in each tertile

** the summary OR for shifting one tertile

$\dagger$ baseline

\# risk set restricted to those whose parents were alive, adjusted for health visitor's opinion on mother's understanding and management of the child and cleanliness of the child, crowding in the household, and fanily history of nervous disorder.

The association was not reduced after restriction of the risk set to those girls whose parents were alive, and adjustment for family history of nervous disorder, household crowding the health visitor's opinion on quality of child-mother interaction ( $\mathrm{eg}$ being worse than average in management and understanding of the child) and cleanliness of the ehild (OR linear trend: 1.6; 95\% CI: 1.02 2.5; Table 1). Further restriction of the risk set to those girls who had never been separated from their mother (ie excluding cases with severe physical illness requiring hospital admission) increased the association (OR linear trend: $2.1 ; 95 \% \mathrm{CI}: 1.1-4.0$ ). Of the three motor milestones, later walking differentiated somewhat better between female cases and controls (sitting in months OR: $1.10 ; 95 \%$ CI: 0.91-1.32; standing OR: 1.10; 95\% CI (0.97-1.25); walking OR: $1.13 ; 95 \%$ CI: $1.02-1.25$ ).

No significant associations were apparent with age at first speech, although in female cases this again tended to be later compared to controls (OR: 1.05; 95\% CI: 0.98-1.13). At the medical examinations at ages $6,7,11$ and 15 years, 11 CAD cases (14.7\%) had shown speech defects on at least one occasion (yielding 17 reports of such defects in cases in total), versus 263 controls (8.2\%; OR $=1.9 ; 95 \% \mathrm{CI} 1.01-3.7)$. Only one of the 11 cases with speech defects was male, and was not noted until the examination at age 15 years. Thus, the logistic regression analysis revealed a significant gender-speech defect interaction (LRS: $5.8, p=0.02$ ), with effect confined to women. For women, the association with speech defects was apparent at all four examinations, but was more conspicuous at age 15 years (Table 2). These associations were not entirely attributable to multiple reports of the same female cases over the four periods of examination, as there were only 16 reports of speech defects from 10 different cases. 
Table 2. Associations between speech defects and later CAD in women

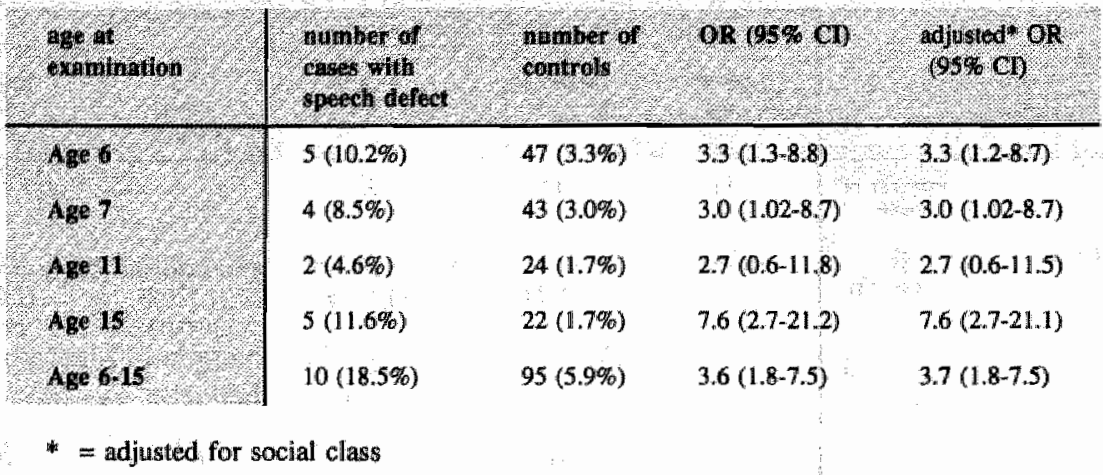

\section{Cognitive ability in CAD cases}

The educational test scores at ages 8,11 and 15 were, in each age group, highly correlated (all: $1>0.5 ; p<0.001$ ). The first PC of the scores at each age was a general measure of cognitive ability, explaining around $75 \%$ of the variance. At all ages, cases were more likely than controls to have cognitive performance in the group below the lowest tertile. Similarly, there were significant "dose-response relationships" between decreasing childhood cognitive ability and the probability of later CAD (Table 3).

Table 3. Associations berween cognitive ability test scores\# and later $C A D$ at ages 8,11 and 15

\begin{tabular}{|c|c|c|c|c|c|c|}
\hline & & \multicolumn{3}{|c|}{ Terther of he popula ilon ditributioge } & \multicolumn{2}{|c|}{ OR for then rendr (os\% C) } \\
\hline & & bowert & $\begin{array}{l}\text { adihalir } \\
\text { abulis }\end{array}$ & 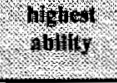 & & idjosted oRt \\
\hline Age 8 & $\begin{array}{l}\text { number } \\
\text { of casere }\end{array}$ & 32 & 21 & 15 & & \\
\hline & $(8,4)$ & $1 \dagger$ & $\begin{array}{c}0.7 \\
(0.4-1.1)\end{array}$ & $\begin{array}{c}0.5 \\
(0.3-0.9)\end{array}$ & $\begin{array}{c}0.7(0.5-0.9) \\
p=0.01\end{array}$ & $\begin{array}{c}0.7(0.5-1.1) \\
p=0.09\end{array}$ \\
\hline Age 11 & $\begin{array}{l}\text { number } \\
\text { or caber }\end{array}$ & 29 & 19 & 14 & & 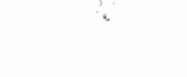 \\
\hline & $\begin{array}{l}\text { on } \\
\text { (oss } c h)\end{array}$ & $1 \dagger$ & $\begin{array}{c}0.7 \\
(0.4-1.2)\end{array}$ & $\begin{array}{c}0.5 \\
(0.3-0.9)\end{array}$ & $\begin{array}{c}0.7(0.5-0.9) \\
\mathrm{p}=0.02\end{array}$ & $\begin{array}{c}0.7(0.5-1.1) \\
p=0.1\end{array}$ \\
\hline$A p e=15$ & of cos? & 32 & 13 & 15 & & \\
\hline & $605 \%$ & $1 \dagger$ & $\begin{array}{c}0.4 \\
(0.2-0.8)\end{array}$ & $\begin{array}{c}0.5 \\
(0.3-0.9)\end{array}$ & $\begin{array}{c}0.7(0.5-0.9) \\
p=0.009\end{array}$ & $\begin{array}{c}0.7(0.4-0.97) \\
p=0.04\end{array}$ \\
\hline
\end{tabular}

\# all test scores adjusted for gender and social class using multiple classification analysis

* divided such that one thind of controls in each tertile

** the summary OR for moving one tertile

$\dagger$ baseline

\# risk set confined to those whose parents were alive and adjusted for gender, social class, parental interest in school progress, teacher ratings of anxiety and antisocial behaviour at ages 13 and 15 , and teacher's opinion on whether school work was adversely affected by factors outside the school 
Adjusting for a range of confounding factors and restriction of the analyses to those whose parents were alive did not reduce the parameters (Table 3 ).

At age 15 years, there was evidence of a significant gender-cognitive ability interaction (LRS $=5.3 ; p=0.02$ ). Stratified analyses revealed stronger associations for female cases at all ages. In women, the odds ratios for linear trend for cognitive ability at ages 8,11 and 15 years were $0.6,0.6$ and 0.5 respectively $[p=0.007, p=0.008, p=0.001]$; in men, however, the odds ratios for linear trend were $0.8,1.0$, and $1.2[\mathrm{p}=0.7, p=0.8, p=0.6]$.

\section{Early socio-behavioural characteristics in $\mathrm{CAD}$ cases}

No association with preference for solitary play as reported by the mother at ages 4 and 6 was demonstrated (OR age 4: 1.1; 95\% CI: 0.6-2.1; OR age 6: 1.4; 95\% CI: 0.6-2.9).

Cases were more likely to be described as "apathetic" by the medical examiners at ages 6,7 and 11 (no measure available at age 15); these were different cases on each occasion, although the number was small (total cases ages 6-11: $\mathrm{n}=4,5.3 \%$; total controls ages $6-11: \mathrm{n}=36,1.1 \%$; $\mathrm{OR}=5.0,95 \% \mathrm{CI}: 1.7-14.4)$.

Analysis of teachers' comments at ages 13 and 15 years (when teachers usually would have been different) denoted a pattern of anxious, avoidant and dejected behavioural characteristics (Table 4).

At both ages, CAD cases were persistently sad and gloomy, and unable to make friends. At age 13 years, CAD cases were also described as avoiding rough games, fearful, timid and avoiding teacher's attention; and at age 15 years as tired and washed out. Items such as disobedience, problems with discipline, truanting and lying did not discriminate between cases and controlls.

There was no evidence of excess of physical handicap amongst $\mathrm{CAD}$ cases. At age 13, for example, $16 \%$ of cases and $15 \%$ of controls were estimated to be "below average" in their ability at sports ( $\mathrm{OR}=1.1 ; 95 \% \mathrm{CI}: 0.6=2.2$; women: $\mathrm{OR}=1.1$; $95 \% \mathrm{CI}: 0.5-2.5)$.

There was no statistical evidence of effect modification by gender for any of the above measures, although again the raw effects tended to be larger for female cases.

Fourty-one CAD cases (63.1\%) versus 533 controls $(18 \%)$ had admitted to lifetime "nervous trouble" at the age of $26(\mathrm{OR}=7.3 ; 95 \%$ CI: 4.7-13.0). The mean age of onset for CAD cases was 17.9 years (SD 5.8), on average two years earlier than onset of such troubles in controls (mean age of onset: 19.7, SD 5.6; $t=1.9, \mathrm{p}=0.05$ ).

\section{Manic symptoms}

The 1982 PSE interview included three items on manic symptomatology. While the reliability of these symptoms could not be adequately established because of their relative rarity (Rogers \& Mann, 1986), we conservatively rated manic symptoms as present if subjects had non-zero scores on at least two of the three items. Presence of manic symptoms were strongly associated 
with later $\mathrm{CAD}(\mathrm{OR}=10.9 ; 95 \% \mathrm{Cl}: 4.0-29.7)$. Howewer, adjusting for presence of manic symptoms in the associations with developmental, cognitive and behavioural wariables as described above, changed the regression coefficients by only a tiny amount (equally often in either direction), and all the OR's previously reported significant remained so. Thus, it is unlikely that associations between developmental risk factors and later CAD reflect a high prevalence of early deficits in a subgroup of bipolar patients.

Table 4. Associations between adult chranic affective disorder and behavioural ratings by teachers at ages 13 and 15 years.

\begin{tabular}{|c|c|c|}
\hline Teacher's comment on & $\begin{array}{l}\text { Age } 13 \\
\text { Adjusted ORt } \\
(95 \% \mathrm{CI})\end{array}$ & 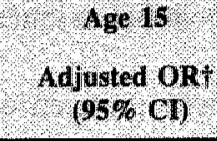 \\
\hline fired and washed out & $1.2(0.4-3.5)$ & $2.4(1.2-4.9)^{* * *}$ \\
\hline marked anxiety in class & $2.4(0.6-10.4)$ & $2.3(0.6-10.1)$ \\
\hline truants ofiten & nonet - $\mathrm{p}=1.0$ & $3.0(0.3-23.7)$ \\
\hline avolds rough games & $1.9(1.1-3.3)^{* * *}$ & $1.1(0.6-2.0)$ \\
\hline aroids teacher's attention & $1.7(0.9-3.2)^{*}$ & $11.3(0.7-2.5)$ \\
\hline fearful, not a dare-devil & $4.1(1.6-10.8)^{* *}$ & $1.3(0.3-1.6)$ \\
\hline avolids competition & $1.6(0.9-2.9)$ & $1.1(0.6-2.0)$ \\
\hline sad and gloomy & $2.7(1.0-6.9)^{* *}$ & $2.5(1.1-5.6)^{* *}$ \\
\hline timid in class, not quarrelsome & $2.1(1.1 .3 .9)^{* *}$ & $0.9(0.4-2.1)$ \\
\hline unduly resents criticisin & $0.8(0.2-3.3)$ & $1.3(0.5-3.6)$ \\
\hline persistently late & $1.8(0.2-13.5$ & none $-p=1.0$ H \\
\hline problems with discipline & nonet $-p=1.0$ & $1.6(0.2-12.1)$ \\
\hline restless in dass & $1.2(0.4-3.2)$ & $2.0(0.8-5.2)$ \\
\hline frequently day dreams & $0.5(0.1-2.2)$ & $0.8(0.2-2.5)$ \\
\hline unable to make friends & $2.2(0.9-5.6)^{*}$ & $2.6(1-6.7)^{3 *}$ \\
\hline Mrequently disobedient & none $-\mathrm{p}=0.6$ & $1.1(0.2-8.3)$ \\
\hline frequenty lying & none\# - $p=1.0$ & $0.8(0.1-6.2)$ \\
\hline
\end{tabular}

\section{Chronic versus less severe forms of depression}

In order to examine whether the reported associations were specific to the group of severe and chronic disorders we had identified, we compared, using the same exposures, cases of CAD with subjects who had either complained of persistent depression in 1982 or in 1989, or who had met the severity criterion in 1982 or in 1989 , but who had not been complaining of persistent 
depression both in 1982 and in 1989. These cases will be referred to as non-chronic affective disorder (NCAD, $n=521$; prevalence $15.8 \%$ ).NCAD cases did not differ from those with CAD in the proportion who had admitted to low mood at either the 1982 or the 1989 interview (NCAD: 95\%; CAD: 97\%). CAD cases were slightlly more likely to be female than their NCAD counterparts ( $\mathrm{OR}=1.6 ; 95 \% \mathrm{CI} 0.9-2.7$ ), and family history did not differentiate between the two groups (OR=0.9; $95 \%$ CI: $0.5-1.7$ ). However, a family history in both parents was significantly more common in CAD cases (OR=3.6; 95\% CI: 1.5-8.5). The median PSE index of severity at the 1982 interview was 5 for $\mathrm{CAD}, 3$ for NCAD, and 1 for the other individuals in the risk set. In 1982, 37 CAD cases $(49.3 \%)$ versus 93 NCAD cases $(17.9 \%)$ had seen their GP for their "merves" in the previous year ( $\mathrm{OR}=4.5 ; 95 \% \mathrm{CI}$ 2.6-7.7). These findings suggest that $\mathrm{CAD}$ and NCAD were broadly similar in type, but not severity of illness.

In view of the effect modification by gender, only female cases of CAD were compared with their NCAD counterparts. This revealed that developmental and socio-behavioural variables differentiated between the two groups, similar to the CAD-control comparisons (Table 5).

Table 5. Camparison between women with CAD (n=54) and less severe forms of affective disorder ( $N C A D ; n=323$ ), adjusted for social class

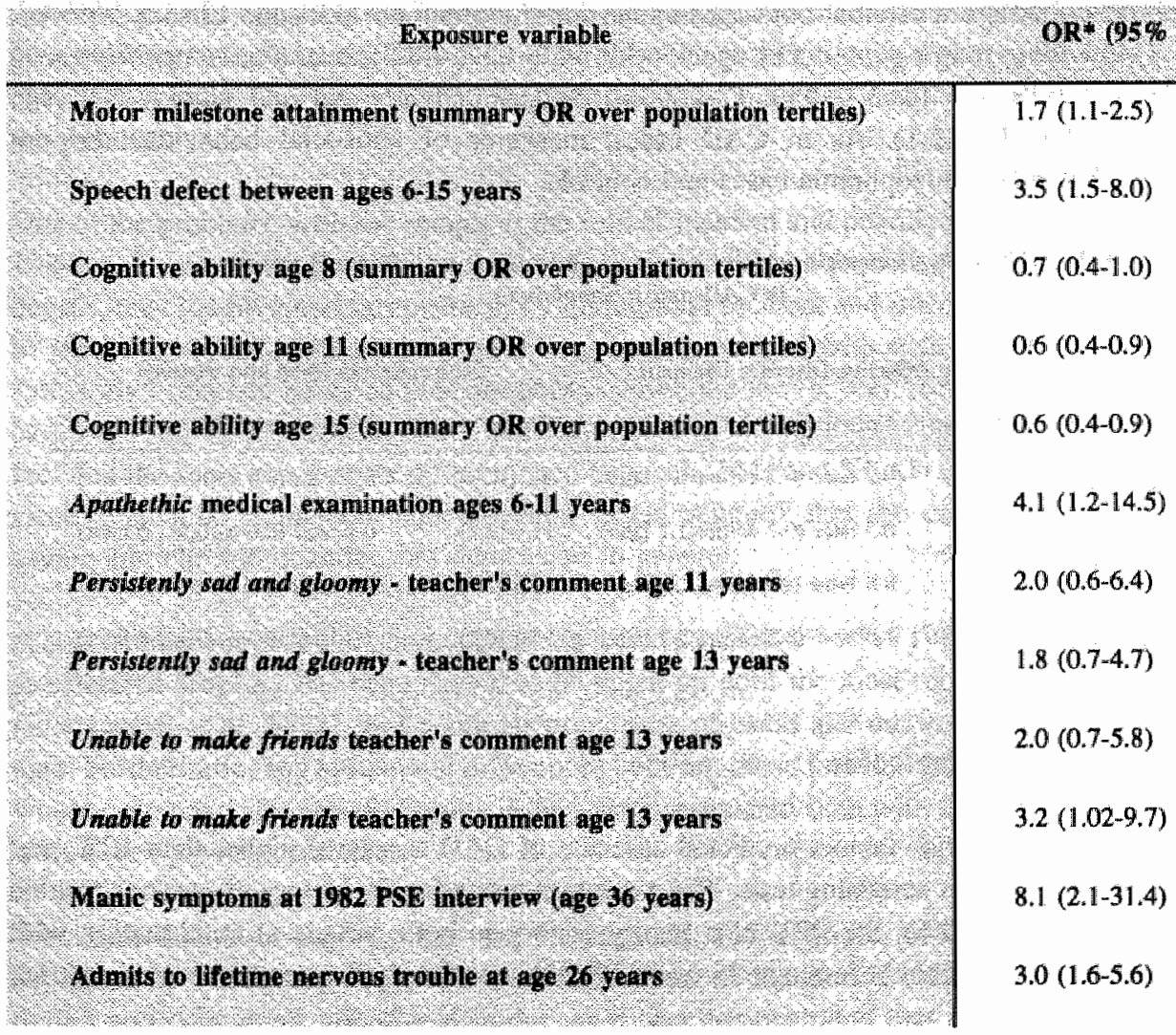

* NCAD is baseline; $O R>1$ means higher levels of exposure increase risk of $C A D$, $\mathrm{OR}<1$ means lower levels of exposure increase risk of CAD 


\section{CAD cases compared with schizophrenia cases}

Contrary to $C A D$ cases, the risk for schizophrenia by age 43 was higher for men (OR=1.8; $95 \%$ C1: $0.9-3.9)$.

Schizophrenia cases were similarly delayed in attaining milestones; for both groups differences with controls were especially evident for walking (Table 6).

Lower educational test scones at ages 8,11 and 15 years were associated with increased risk of becoming both a CAD case and a schizophrenia case. The OR for linear trend for schizophrenia cases, adjusted for sex and social class, were of similar magnitude: age 8 years: $O R=0.7$ (95\% $\mathrm{CI}: 0.4-1.2)$, age 11 years: $\mathrm{OR}=0.6(95 \% \mathrm{CI}: 0.4-0.99)$; age 13 years: $\mathrm{OR}=0.5$ (95\% CI: $0.3-0.9)$.

Contrary to CAD cases, solitary play at ages 4 and 6 predicted later schizophrenia (OR age 4 : $2.1 ; 95 \%$ CI: $0.9-4.7$ ); OR age $6: 2.5 ; 95 \%$ CI: $0.8-6.9$ ).

Teacher ratings at ages 13 and 15 had a pattern of discriminating between schizophrenia cases and controls which was similar to that between CAD cases and controls. Schizophrenia cases had also been described more often at both ages as tired and washed out (OR age $15=3.5 ; 95 \% \mathrm{CI}$ : 1.01-11.7), avolding rough games (OR age $15=3.7 ; 95 \%$ CI 1.4-8.8), avoiding competition with other children (OR age $15=3.1 ; 95 \% \mathrm{CI}: 1.2-7.4)$, and persistently sad and gloomy (OR age 15=3.8; 95\% $\mathrm{Cl}: 1.1-12.1$ ). As in CAD cases, measures of antisocial behaviour did not discriminate between schizophrenia cases and controls.

Table 6. Age (months) at developmental milestones (recall at age 2 years) in CAD and schizophrenia

\begin{tabular}{|c|c|c|c|c|}
\hline \multirow{2}{*}{ Whostone } & \multicolumn{2}{|c|}{ Ghronic A fiective Disorder (romien) } & \multicolumn{2}{|c|}{ Sehluphrenia (men and younen) } \\
\hline & Comtrol uneai & Casoscontrol dimateroes & Control inean & Case-conimul difitrence \\
\hline Wing & $6.5(1.4)$ & 0.2 later ( 0.2 earlier- 0.6 later) & $6.5(1.5)$ & 0.1 later $(0.5$ earlier 0.8 later $)$ \\
\hline Nronding & $11.3(2.1)$ & 0.5 later (0.2 earlier- 1.1 later $)$ & $11.4(2.2)$ & 0.2 later (0.6 earlier -1.0 later $)$ \\
\hline Wolkng & $13.5(2.4)$ & 0.8 later (0.1 later-1.5 later) & $13.5(2.4)$ & 1.2 later (0.1 later 2.3 later) \\
\hline Talking & $13.8(4.2)$ & 1.0 later ( 0.3 earlier 2.2 later) & $14.3(4.2)$ & 1.2 later $(0.4$ earlier -2.8 later $)$ \\
\hline
\end{tabular}

\section{Positive predictive value}

In women, none of the risk factors predicted caseness of CAD at a level higher than $10 \%$, and are therefore of no use as screening tests. The exception was a family history of nervous trouble in both parents (PPV 20\%); the PPV of a family history in both parents in combination with reporting of nervous trouble before age 26 was $47 \%$. However, only 8 cases had both these risk factors. 


\section{Missing data}

Cases and controls did not show large or significant differences in the proportion that had missing values on any of the exposure variables (mean difference: $1.7 \%$, range: $-4.4 \%-6.5 \%$ ).

\section{DISCUSSION}

Our findings suggest that later CAD is associated with early, subtle, developmental and behavioural deficits, remarkably similar to those seen in schizophrenia in this sample (Jomes et al, 1994a).

\section{Methodological considerations}

This was a prospective cohort study with standardized exposure assessments at different ages, collected prior to any knowledge of psychiatric outcome. Inclusion of cases on the basis of interviews in a general population sample, rather than on the basis of contacts with medical services, greatly enhances the validity and generalizability of the findings, as only a selected group of depressed patients will ever come to medical attention (Kessler et al, 1994). Indeed, only $33 \%$ of our CAD cases had attended hospital and only half recalled contacting their GP in the previous year.

One of the problems with the sample is the mix of incident and prevalent cases in both 1982 and 1989, and the resulting confusion over whether risk factors predict onset or chronicity. To a degree, we circumvented this problem by giving both incident and prevalent depressive subjects in 1982 a "chance" to become a case if they again presented with such symptoms in 1989, thus "allowing" incident cases to become chronic over the seven year period. However, prevalent cases in 1982 may still have had a greater probability of reporting depression in 1989, and the fact that the same risk factors differentiated between CAD and NCAD (the latter group from the same $1982 \mathrm{mix}$ of incident and prevalent cases) suggests that the exposures mediate risk of severe and chronic disonder, not depression in general.

A substantial proportion of survey members were not interviewed in 1982 and 1989, but bias due to selective drop-out has been shown to be slight up until the most recent data collection point (Wadsworth et al, 1992), and a similar proportion of cases and controls had missing exposure data. Furthermore, any systematic differences between those lost to follow-up and those assessed would only have affected the results if they were associated both with CAD and independently, with devellopmental variables.

We think that it is unlikely that the findings on, for example, motor development can be explained by a subgroup of cases with chronic physical disorders associated with developmental deficit and concurrent affective disorder, as it was demonstrated that risk was not confined to a subgroup with, for example, very low educational test scores or very high age at motor attainment; there were significant linear trends across the population distributions. Furthermore, exclusion of those who had been separated from their mothers before the age of 6 effectively excluded those with severe childhood illnesses needing hospital admission, whom in a previous 
investigation in this cohort (Rogers, 1990a) were shown to be at higher risk of developing mental health problems. At later ages, there was no evidence of an excess of physical ill-health among cases. At age 13 , for example, $16 \%$ of cases and $15 \%$ of controls were estimated to be "below average" in their ability at sports.

Although we can be fairly certain that there were no cases with schizophrenia or schizo-affective disorder among our CAD cases, it is likely that at least a proportion of cases had experienced psychotic symptoms at some time during their illness. Indeed, we know that 5 had received the antipsychotic trifluoperazine. As presence of psychotic symptoms considerably worsens the prognosis of unipolar and bipolar affective states (Coryell et al, 1990b; Coryell et al, 1990c; Lee \& Murray, 1988), patients with delusions and hallucinations may have been over-represented in the CAD group, compared to both NCAD patients and controls. A conservative interpretation is therefore that CAD cases represented affective disorders towards the severest, psychotic end of the spectrum.

\section{The findings}

Later motor development in CAD is compatible with a report that motor abnormalities in the functional psychoses are not confined to patients with typicall schizophrenic illness (Crow et al, 1994). Moreover, we were able to show that these results were not attributable to a subgroup of cases with markedly delayed motor development, as there was a linear trend across the population distribution in the association between motor attainment and risk of CAD: the later the motor milestones, the higher the risk of CAD. These findings were echoed in the elevated risk for speech abnormalities at later ages. The number of cases with speech defects was small, but the binary risk factor would only have been able to pick up the small proportion of cases in the upper end of the population distribution; an ordinal measure would possibly have revealed more clearly the association between exposure and risk, given that many cases may present with significant deviation, yet without exceeding the population normal range.

In a previous study using a longitudinal design, childhood intellectual performance has been found to differentiate between controls and cases with affective disorder selected through medical contacts (Done et al, 1994). As with motor attainment, the evidence indicates a dose-response relationship between educational test performance and risk of CAD. There was no evidence that other factors such as mood or behavioural abnormalities, lack of parental stimulation, parental loss, or other factors interfering with school work were confounding our results.

The evidence from the medical examinations and teacher ratings suggests $\mathrm{CAD}$ cases were more likely to be described as anxious and sad. The evidence for significant sadness and anxiety in these individuals, in the absence of an association with anti-social behaviour, is all the more compelling as teachers usually have a much lower threshold for reporting behavioural deviance than for appearance of sadness and anxiety (Watt, 1978),

Within the group of affective disorders as a whole, patients with CAD were much more likely to present with premorbid developmental deficits, after adjustment for social factors. This is in agreement with previous investigations in this cohort, which showed that mainly childhood social 
factors were predictive of a cross-sectional measure of affective and neurotic symptomatology at age 36 (Rogers, 1990a).

\section{Schizophrenia and Chronic Affective Disorder}

The same neuromotor, cognitive and socio-behavioural risk factors differentiated between controls and cases of CAD and cases of schizophrenia. Differences were few and quantitative rather qualitative: For example, the differences in age at walking was larger between schizophrenia cases and controls, and behavioural abnormalities in childhood were more conspicuous in schizophrenia cases. Indeed, the wide confidence intervals for most measures are compatible with there being large quantitative differences between CAD and schizophrenia cases. The presence of quantitative rather than qualitative differences suggests that the degree of developmental attenuation progressively increases along a continuum from severe affective to non-affective illness, similar to the finding that risk of chronicity in psychotic illness progressively increases from affective psychosis, through to schizo-affective-psychosis and schizophrenia (Tsuang \& Dempsey, 1979; Grossman et al, 1991).

The most notable qualitative difference was i) that risk for CAD was greater for women; whereas the reverse held for schizophrenia cases, and ii) schizophrenia cases, but not CAD cases, had a preference for solitary play at an early age. While the sample of schizophrenic subjects was too small to detect anything other than large effect modification by gender, there was evidence that attenuated development increased risk for CAD only in women. This may indicate that female CAD cases were a more homogeneous group of affective disorder, but a gender-specific increase in risk of later CAD conferred by attenuation of development cannot be excluded (Emst et $a l_{\text {* }}$ 1992), similar to reports of a gender-specific effect of exposure to prenatal influenza in women (Takei et al, 1994).

The significance of the lack of more widespread qualitative differences in the developmental risk profile for CAD and schizophrenia depends on the postulated role of premorbid developmental deviance in mediating risk of later illness. We consider five main possibilities:

- i) Premorbid developmental deviance is an early illness manifestation - If early abnormalities were early indicators of illness, schizophrenifa and CAD might be related, with considerable overlap of prepubertal psychopathologicall manifestations that deviate progressively in adulthood, men showing more schizophrenic symptoms, and women more affective symptomatology, but both followed by a chronic illness course. The excess of affective symptomatology in women even within the diagnostic construct of schizophrenia is an accepted finding (Flor Henry, 1990, Van Os et al, 1995b), and would support this theory. However, deviant measures of development as an early illness manifestation does not really fit the combination of their high population prevalence and the rarity of schizophrenia and CAD. Therefore, one would have to assume that the measures of development used are crude and only proxies of more specific deficits that await discovery. 
- ii) Premorbid deficils are an early manifestation of a predisposition to illness.

Premorbid deficits may be non-specific indicators of "vulnerability" to severe mental disorder. Genetic predisposition may be such an area of vulnerability. For example, studies have demonstrated subtle differences in neurological and cognitive development in the (as yet) unaffected offspring of schizophrenia patients (Fish et al, 1992). Furthermore, in schizophrenia cases, the presence of a family history has been reported to predict poor premorbid functioning (Foerster et al, 1991). Thus, there may be overlapping genetic predisposition between CAD and schizophrenia, whereas differences in adult psychopathology are determined by other (genetic and/or environental) factors interacting with the genetic predisposition. Family studies have shown considerable overlap in the familial risk for (schizo)depression and schizophrenia, although the evidence for an overlap in genetic risk between bipolar disorder and schizophrenia is much weaker (review by Taylor, 1994).

- iii) Premorbid developmental deviance is a risk factor interacting with a pre-existing (genetic) predisposition to increase the risk of psychiatric disorder.

Under a slightly modified version of model two above, premorbid developmental deviance is not merely an indicator of underlying vulnerability, but itself modifies risk of illness in (genetically) predisposed individuals, either additively or interactively to produce schizophrenic symptoms. However, the above cited findings that the (as yet) unaffected offspring of schizophrenic individuals also show such childhood abnormalities is more in favour of scenario two.

- iv) Premorbid developmental deviance is a non-specific risk factor for chronicity in CAD and schizophrenia, and does not affect the likelihood of emergence of disorder.

This would explain the excess of identical premorbid developmental deficits in both CAD and schizophrenia. However, in all likelihood premorbid developmental deviance is a risk factor for both onset and chronicity of disorder, as premorbid deficits have also been demonstrated in first-onset cases of both affective disorder and schizophrenia (Ernst et al, 1992, Jones et al, 1994a). Furthermore, most other established risk factors for onset of psychosis such as life events, cerebral ventricle dimensions, familial morbid risk and ethnic group, also predict subsequent illness course (Jones et al, 1994a; Van Os et al, 1994; Van Os et al, 1995c; Verdoux et al, 1995; McKenzie et al, 1995).

- v) The measures of early development used are too crude and tangential to be able to discriminate between affective disorder and schizophrenia. This is the least parsimonious solution, but cannot be excluded.

Therefore, on balance, our findings point towards overlap in either illness manifestation or illness predisposition between chronic affective disorder and schizophrenia. Overlapping early illness manifestation would suggest a disorder of possible neurodevelopmental origin with uniform poor outcome and gender-related pathoplastic effects on psychopathology, rather than of multiple disorders with homogeneous cross-sectional manifestations but varying degrees of chronicity within each diagnostic category. Overlapping predisposition suggests the existence of other factors that determine which type of disorder, given a common vulnerability, will emerge in adulthood. 


\section{REFERENCES}

Andrews F, Morgan I, Sonquist J \& Klein L (1973) Multiple Classification Analysis. 2nd Edition. Ann Arbor: University of Michigan.

Atkins E, Cherry N, Douglas JWB, Kieman KE \& Wadsworth MEJ (1981), The 1946 birth cohort: An account of the origins, progress and results of the National Survey of Health and Development. In: SA Mednick \&

$\mathrm{AE}$ Baerts (eds.) "Prospective Longitudinal Research: an Empirical Basis for the Primary Prevention of Psychosocial Disorders London OUP.

Breslow NE \& Day NE (1987) Statistical Methods in Cancer Research, Vol 2, pp 192-246. Lyon: WHO.

Brodaty, H., Harris, L., Peters, K., Wilhelm, K., Hickie, I. \& Boyce, P. et al (1993) Prognosis of depression in the elderly. British Journal of Psychiary, 163, 589-596.

Coryell, W., Endicott, j. \& Keller, m. (1990a) Outcome of patients with chronic affective disorder: a five year follow-up American Journal of Psychiatry, 147, 1627-1633.

- Keller M, Lavori $P$, et al (1990b) Affective syndromes, psychotic features, and prognosis. I: depression. Archives of General Psychiatry, 47, 651-657.

am.- Keller M, Lavori $P_{\text {s }}$ et al (1990) Affective syndromes, psychotic features, and prognosis: II: mania. Archives of General Psychiatry, 47, 658-662.

Crow, T., Done, D.J. \& Sacker, A. (1994) Neurological abnormality in children who develop psychosis in adulthood. Schizophremia Research, 11, 96.

Deister, A. \& Marneros, A. (1993) Predicting the long-term outcome of affective disorders. Acta Psychiatrica Scandinavica, 88, 174-177.

Done I, Sacker A \& Crow TJ (1994) Childhood antecedemts of schizophrenia and affective illness: intellectual performance at ages 7 and 11. Schizophrenic Research, 11, 96-97

Duggan, C.F., Lee, A.S. \& Murray, R.M. (1990) Does personality predict long-term outcome in depression? British Jourmal of Psychiatry, 157, 19-24,

Emst, C., Schmid, G. \& Angst, J. (1992) The Zurich Study. XVI. Early antecedents of depression. A longitudinal study on incidence in young adults. European Archives of Psychiatry and Clinical Neuroscience, 242,142151.

Favarelli C, Degl'Innocenti BG, Aiazzi L, et al (1990) Epidemiology of mood disorders: a community survey in Florence. Journal of Affective Disorders, 20, 135-141.

Fish, B., Marcus, J., Hans, S.L., Anerbach, J. \& Perdue, S. (1992) Infants at risk for schizophrenia: Sequelae of a genetic neurointegrative defect. Archives of General Psychiatry, 49, 221-235.

Flor-Henry, P. (1990) Influence of gender in schizophrenia as related to other psychopathological syndromes. Schizophrenia Bulletin, 16, 211-227.

Foerster, A. Lewis, S.W., Owen, M. \& Murray, R.M. (1991) Low birth weight and a family history of schizophrenia predict poor premorbid functioning in psychosis. Schizophrenia Research, 5, 13-20.

Grossman, L., Harrow, M., Goldberg, J. \& Fichtner, C. (1991) Outcome of schizoaffective disorder at two longterm follow-ups: comparisons with outcome of schizophrenia and affective disorders. American Journal of Psychiatry, 148, 1359-1365.

Harder, D., Greenwald, D., Ritzler, B., Strauss, J., Kokes, R. \& Gift, T. (1990). Prediction of outcome among adult psychiatric admissions. Journal of Clinical Psychology, 46, 119-129.

Howard H (1993) Chronic depression. Hospital and Community Psychiatry , 44, 633-639.

Johnson RA \& Wichem DW (1988) Applied Multivariate Statistical Analysis, 2nd edition. London: Prentice Hall.

Jones, P., Rodgers B, Murray R, \& Marmot M (1994a) Child developmental risk factors for adult schizophrenia in the British 1946 birth cohort. Lancet, 344, 1398-402.

-_..., Harvey, I. Lewis, S. Toone, B., Van Os, J., Williams, M. \& Murray, R.M. (1994b). Cerebral ventricle dimensions as risk factors for the functional psychoses. Psychological Medicine, 24, 995-1011.

-...- Murray, R. \& Rodgers, B. (1995) Childhood risk factors for adult schizoprenia in a general population birth cohort at age 43 years. In: Neural Development in Schizophrenia: Theory and Research (ed S.A. Mednick).

Kessler RC, McGonagle KA, Zhao S et al (1994) Lifetime and 12-month prevalence of DSM-IM-R psychiatric disorders in the United States. Results from the National Comorbidity Survey. Archives of General Psychiatry, 51, 8-19.

Kuh, D., Wadsworth, M. \& Yusuf, E. (1994) Burden of disability in a post war cohort in the UK. Journal of Epidemiology and Community Health, 48, 262-269.

Lee A, \& Murray R (1988) The long term outcome of Maudsley depressives. British Journal of Psychiatry, 153, 741-751.

McKenzie, K., Van Os, J., Fahy, T., Jones, P., Harvey, I., Toone, B. \& Murray, R. (1995). Evidence for good prognosis psychosis among UK Afro-Caribbeans. British Medical Journal, in press. 
Pidgeon, D.A. (1964) Tests used in the 1954 and 1957 surveys. In JWB Douglas The Home and the School pp 129-132. London: MacGibbon \& Kee.

_._. (1968) Details of the fifteen year tests. In JWB Douglas, JM Ross \& HR Simpson. All Our Futures pp 194-197. London: Peter Davies

Rogers B. (1990a) Bethaviour and personality in childhood as predictors of adult psychiatric disorder. Journal of Child Psychology and Psychiatry, 3, 393-414.

-...., (19906) Adult affective disorder and early enwironment British Journal of P sychiarn, $157,539.550$.

Scott, J., Eccleston, D. Boys, R. (1992) Can we predict the persistence of depression? British Journal of Psychiatry, 161, 633-637:

STATA corporation (1993) STATA Reference Manual: Release 3.1, 6th Ed. College Station:TX.

Stoffelmayr, B., Dillavou, D. \& Hunter, J. (1983) Premorbid functioning and outcone in schizophrenia: a cumulative analysis. Journal of Conculting and Clinical Psychology, 51, 338-352.

Takel, N., Sham, P., O'Callaghan, E., Glover, G. \& Murray, R. (1994) Prenatal exposure to influenza and the development of schizophtrenia: Is the effect confined to females? American Journal of $P_{\text {sychiatry, }} 151,117$ 119.

Taylor, M. (1992) Are schizophrenia and affective disonder related? A selective literature review. American Journal of Psychiatry, 149, 22-32.

Tsuang, M.T. \& Dempsey, G.M. (1979) Long term outcome of Major Psychoses II. Schizoaffective disorder, compared with schizophrenia, affective disorders, and a surgical control group. Archives of General Psychiatry, 36, $1302-1304$.

Van Os, J., Fahy, T., Bebbington, P., Jones, P., Wilkins, $S_{n}$, Sham, P, et al. (1994) The influence of life events on the subsequent course of psychotic illness. Psychological Medicine, 24, 503-513.

---, Takei, N., Castle, D., Wessely, S, Der, G. \& R. Murray (1995a). Premorbid abnormalities in mania, schizomania, acute schizophrenia and chronic schizophrenia. Sacial Psychiatry \& Psychiatric Epidemiology, in press.

2....., Fahy T.A., Jones P., Harvey I., Sham P., Lewis S., Bebbington P., Toone B., Williams M., Murray R. (1995a) Psychopathological syndromes in the functional psychoses" associations with course and outcome. Psychological Medicine, in press.

-.n-_.-. Fahy, T. Jones, P. Harvey, I, Lewis, S., Williams, M., Toone, B. \& Murray R. (1995b) Increased intra-cerebral CSF spaces predict unemployment and negative symptoms in psychotic illness: a prospective study. British Journal of Psychiatry, 166, 750-759.

Verdoux, H., Van Os, I., Sham, P., Jones, P., Gilvarry, K. \& Murray, R. Does familiality predict both onset and persistence of illness in the functional psychoses' A prospective study. (submitted paper).

Wadsworth MEJ (1991) The Imprint of Time. Childhood History and Adult Life. Oxford: Clarendon Press.

..... Mann SL, Rodgers B, Kuh DL, Hilder WS \& Yusuf EJ (1992) Loss and representativeness in a 43 year follow-up of a national birth cohort. Journal of Epidemiology and Community Health, 46, 300-304.

Watt, N.F. (1978) Patterns of childhood social development in adult schizophrenics. Archives of General Psychiatry, 35, 160-165.

Weissman MM \& Myers JK (1978) Affective disorder in a US urban community. Archives of General Psychiatry, 35, 1304-1311.

- Leaf PJ, Bruce ML et al (1988) The epidemiology of dysthymia in five communities: rates, risks, comorbidity and tieatment. American Journal of Psychiatry, 145, 815-819.

Wing JK, Cooper JE Sartorius N (1974) Present State Examination. Cambridge University Press: London. 


\subsubsection{Does familiality predispose to both emergence and persistence of psychosis?}

\section{A prospective study}

\section{SUMMARY}

It has been suggested that, in schizophrenia, an association exists between family history of schizophrenia and poor outcome on the one hand, and family history of affective disorders and good outcome on the other. We tested for associations between four-year outcome and familial loading for psychotic disorders in a sample of 150 consecutively admitted patients with functional psychosis of recent onset. For each proband, a familial loading score for i) broadly defined psychotic disorder, ii) schizophrenia, and iii) affective disorder was calculated using information on relatives obtained through the Family History-Research Diagnostic Criteria method and direct interviews of relatives with SADS-LA. In our sample of psychotic patients, familial loading for psychotic disorder predicted persistent negative symptoms over the follow-up period (OR: 1.5; 95\% CI: 1-2.2), and was also associated with more time hospitalised (p<0.02), and more social disability at follow-up $(p<0.06)$, especially in schizophrenia. Greater familial loading for schizophrenia predicted a lower likelihood to recover $(\mathrm{OR}=2.2 ; 95 \% \mathrm{CT}: 1.1-4.4)$ and a greater likelihood to have had persistent negative symptoms over the follow-up period $(\mathrm{OR}=1.7 ; 95 \% \mathrm{CI}: 0.9-3,1)$. No association was found between outcome and familial loading for affective disorder. We conclude that familial loading is a continuous risk factor for clinical outcome in the functional psychoses, especially in schizophrenia. This suggests that there is a continuum of genetic liability not only to the emergence of psychotic illness, but also the subsequent chronicity of the disorder.

\section{INTRODUCTION}

Robins \& Guze (1970) suggested that family history might be used to subdivide good and poor prognosis schizophrenia, the former being more likely to be associated with a family history of affective disorder, and the latter with a family history of schizophrenia. Kendler \& Tsuang (1988) have reviewed other studies on this topic published between 1931 and 1986. Some showed an assiociation between family history of schizophrenia and poor outcome, and many showed an association between family history of affective disorder and good outcome. However, these studies suffered from many methodological limitations, such as obtaining family history data from case notes or probands, and failure to take into account confounding factors such as family size, age and sex of the relatives in assessing the probands' familial loading for psychiatric disorders (Roy \& Crowe, 1994). Weiss et al (1982) have shown how such methodological problems can lead to biased results. Furthermore, many studies have considered outcome as an unidimensional, global variable, and have not measured outcome in several distinct clinical and social areas of functioning (Strauss \& Carpenter, 1974). Samples frequently consisted of a mixture of both recent onset patients and chronic patients, making the outcome findings more difficult to interpret than if homogeneous groups of subjects were studied (McGlashan, 1986b). Finally, as most of the variability in the course of schizophrenia occurs in

\footnotetext{
'Hélène Verdowx, Jim van Os, Pak Sham. Peter Jones, Karyna Gilvarry \& Robin Murray (submitted for publication)
} 
the first five years (Ciompil, 1980), association with any predictor should be more readily' detectable over this period.

Given these methodological limitations, especially as regards assessment and classification of family history, it is not surprising that the literature is inconsistent, some studies demonstrating an association between a positive family history for schizophrenia and poor outcome (McGlashan 1986a, 1986b; Keefe et al, 1987, Sautter \& Mc Dermot, 1994), and others reporting inconclusive findings (Bleuler, 1978, Ciompi, 1980; Huber et al, 1980; Alda et al, 1991).

The largest study to date was by Kendler \& Tsuang (1988), who examined outcome and familial psychopathology in a sample of 253 DSM-III schizophrenics and their 723 first-degree relatives. Family history was viewed from a cohort perspective, where relatives were regarded as the subjects at risk of developing mental illness, and good or poor outcome in the proband was regarded as an exposure variable. Morbid risk of non-affective psychotic disorder was higher in the relatives of poor-outcome probands, more so at short-term outcome than at long-term outcome, although for both periods this failed to reach statistical significance. Morbid risk of affective illness was significantly higher in relatives of good outcome patients both at short term and long term follow-up. A major problem with this study was that information on outcome was lost by dichotomizing outcome into good and poor outcome categories, and that short term outcome (arguably the most sensitive measure) was assessed retrospectively from very old casenote material. Also, the sample was already defined in terms of chronicity at baseline, as the investigators used DSM criteria. This has been shown to be particullarly problematic in follow-up studies (Angst, 1988): if there is little variation in course, prediction of course is difficult to demonstrate.

The Camberwell Collaborative Psychosis follow-up study was designed to investigate four-year risk of chronicity in the functional psychoses in relation to social, demographic and biological variables collected as baseline (Jones et al, 1993; Van Os et al, 1995 a \& b). The main questions addressed in the present extension of the study are i) does a positive family history for schizophrenia or for broadly defined psychotic disorder predict a poorer outcome in psychotic patients? ii) is a positive family history for affective disorder associated with a better outcome? iii) how does the association between familiality and outcome vary as a function of a) the proband's diagnosis, b) other important risk factors for course and outcome including premorbid social adjustment, negative symptoms and cerebral ventricle size?

\section{METHOD}

\section{Index cases and baseline assessment}

Detaills on the method and sampling of the Camberwell Collaborative Psychosis Study have been more fully described previously (Harvey et al, 1991; Jones et al 1993; Van Os et al, 1995 a \& b). Briefly, consecutive admissions to two South London Hospitals, with at least one psychotic symptom according to the RDC (Spitzer et al, 1978) occurring in clear consciousness, and whose first psychotic symptoms had appeared within 5 years of index assessment were included in a 4-year follow-up study. Baseline assessments were generally completed within three days of admission, and included detailed demographic and historical information. Ethnicity was classified 
as white European, Afro-Caribbean, or other ethnic group, Age at onset was defined as appearance of first psychotic symptoms. Duration of ilness was defined as the difference between age at index assessment and age of onset. $\mathrm{A}$ measure of premorbid social adjustment was obtained through matemal interview, using the Philips' premorbid social adjustment scale (Philips, 1953).

Patients were interviewed using the Present State Examination (PSE, Wing et al, 1974). PSE data and case note material were used to complete the Operational Criteria Checklist for Psychotic Illness (OCCPI, McGuffin et al, 1991). The computer program OPCRIT (McGuffin et al, 1991) was used to analyse the OCCPI data, ylelding DSM-III-R index diagnoses.

The method used for CT-scanning and establishing inter-rater reliability has been described in detail elsewhere (Jones et al, 1994; Van Os et al, 1995b). In summary; a lateral ventricular volume was constructed by adding together all the area measurements of its body, frontal horns, occipital homs and anterior homs. Volumes for the third ventricle and sylvian fissures were also constructed, and an estimate of the intracranial volume was made.

\section{Information on relatives}

The method used to collect information on first degree relatives has been described elsewhere (Sham et al, 1994; Davies el al in preparation). Information was obtained from four sources: personal interviews with relatives and the probands' mothers, medical records of relatives with a psychiatric diagnosis, and probands' case notes. With permission from the probands, the mothers were interviewed according to the Family-History-Research Diagnostic Criteria (FHRDC, Andreasen et $a l, 1986)$. The FH-RDC were applied blind to information on the proband: Whenever possible, relatives were personally interviewed using the Schedulle for Affective Disorders and Schizophrenia (SADS) (Endicott \& Spitzer, 1978) to obtain RDC diagnoses blind to information on the proband. For those latter relatives, data provided by SADS were preferentially used to give diagnoses.

\section{Assessment of familial loading scores for psychiatric disorders}

Instead of Kendler's \& Tsuang's cohort approach, family history was considered as an attribute of the individual cases themselves, which might be related to their outcome. The method of calculating familial loading scores for the probands is based on the relative likelihoods of

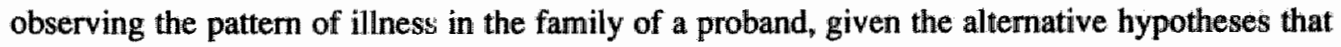
the proband is a famillial or sporadic case of psychosis. Details of the method and its assumptions are described in the Appendix I.

Three familial loading scores for psychiatric disorder were calculated for each proband. First, a familial loading score for schizophrenia was calculated. Secondly, a familial loading score for affective disorders was calcullated. A narrow definition of family history of affective disorder was used (owing to the common and non-specific nature of broadly defined depression), including only relatives with a diagnosis of bipolar disorder or with a diagnosis of major depression requiring psychiatric admission. It was assumed that only the most severe depressions required 
admission, and that those depressed hospitalized relatives were more prone to have displayed a psychotic depression. Thirdly, a familial loading score for "psychotic disorders" was callculated, including schizophrenia, schizoaffective disorder, delusional disorder, unspecified psychosis, bipolar disorder and major depression with a history of psychiatric admission. In order to be able to present means and frequencies of the outcome dimensions by familial loading, the familial loading score for psychotic disorder was transformed in some analyses as a binary variable (positive/negative family history according whether the score was $>0$ or $\leq 0$, respectively - see Appendix I): There were no large or significant differences in the number of relatives between any of the familial loading groups dichotomized in this way; the largest difference for family history of affective disorder being very small ( $s$ core $>0$ : mean number of relatives $=4.6, \mathrm{SD}=1.8$; score $\leq$ : mean number of relatives $=4.2, \mathrm{SD}=2.7 ; \mathrm{t}=-0.7 ; \mathrm{p}=0.5$ ).

\section{Follow-up}

The method used has been described previously (Van Os et al, 1995 a \& b). The rater (JVO) was blind to all data collected at index assessment, including family history data. Most patients were interviewed in the community. Multiple sources of information were used for follow-up assessment where possible, the median number of informants being 2 (range: 0-3). Crosssectional outcome measures were provided by the following instruments: WHO/disability Assessment Schedule (DAS, Jablensky et al, 1980), Iager Negative Symptom Rating Scale (Iager, 1985), and the PSE (Wing et $a l_{*}$ 1974). The longitudinal assessment of course of illness and associated disabilities was made with a slightly modified version of the Life Chart instrument from the WHO Multi-Center Study on the Course and Outcome of schizophrenia (WHO, 1992). The Life Chart allows assessment of course of illness in a multidimensional way, using clear definitions for all ratings (Appendix II). Course type was rated as "continuous" or "non-continuous". A "usual severity of symptoms" rating was made to indicate the symptomatic level of the patient during most of the follow-up period. This rating was modelled as a binary variable (recovered vs non-recovered). Evidence of persistent negative symptoms over the follow-up period was rated according to the Iager scale and modellled as a binary variable (presence/absence). The proportions of the length of the individual follow-up period spent unemployed (time in institutions excluded; full time students and housewives were rated as employed), living independently (i.e., not in an institution or supervised residence), or in hospital, were also rated.

Four main outcome dimensions were identified using factor analysis (Negative symptoms/social disability; Severity of illness; Time living independently; Unemployment - Van Os et al, 1995a), with 9 variables covering these four dimensions as described previously (Vam Os et al; 1995 a and $b$; see Table I).

\section{Analyses}

Multiple regression yielding regression coefficients (B) and $95 \%$ confidence intervals was used to examine the associations between continuous outcome variables and family history while allowing for potential confounding variables. Dependent variables were transformed to remove skewness whene appropriate, and residuals were examined for evidence of non-normality. 
Logistic regression was used to analyse binary dependent variables. Odds ratios (OR) and $95 \%$ confidence intervals (CI) were calculated from the results of the logistic regression.

\section{RESULTS}

One hundred and ninety-one patients met the inclusion criteria for the follow-up study. Followup data were available on $166(87 \%)$ of these. Seven patients had committed suicide, and two had died of other causes. One-hundred and fifty-three patients were personally interviewed, 4 of whom by telephone; of the patients not interviewed, high-quality information was available on a further 13. Family history information was available for 150 of the 166 subjects traced. There were no significant or large differences on age, gender, social class, ethnicity, age of onset and familly history between the 150 follow-up subjects with family history data compared to the 41 not followed-up or without family history information.

\section{Characteristics of the sample}

Of the 150 probands, $100(66.7 \%)$ were male. The mean age at index assessment was 26.1 years (range 16-50, SD 6.3). White Europeans, Afro-Caribbeans, and other ethnic groups comprised $54 \%, 37.3 \%$, and $8.7 \%$ of the sample respectively. The mean length of follow-up was 47 months (range $3-77$, SD 13.5, range with death excluded 26-77).

At index admission, 69 subjects (46\%) had a DSM-III-R diagnosis of schizophrenia, 41 subjects $(27.3 \%)$ of affective disorder (bipolar disorder or major depression), 13 subjects (8.7\%) of delusional disorder, 11 subjects $(7.4 \%)$ of schizoaffective disorder, 10 subjects $(6.7 \%)$ of schizophreniform disorder, and 6 subjects (4\%) of psychosis not otherwise specified. The median duration of illness prior to baseline assessment was 2.5 years (IQR 1.5-4.5), and the mean age at onset was 23.2 years (SD 6.2). Sixty subjects (40\%) were first admissions. Men and women showed the expected (Castle and Murray, 1991) difference in age at onset (men: 22.4 years; women: 24.9 years, $t=-2.3, p=0.02$ ). The mean age at onset was highest for the affective psychoses (26.2 years), and lowest for schizophrenia (20.7 years), with schizoaffective, schizophreniform disorder and other psychoses somewhere in between $(22.9,24.9$ and 25.2 years respectively).

A total of 636 first degree relatives (16 years old and above) of the 150 probands were identified. All mothers had had FH-RDC interviews, and an additional 172 relatives other than the mother were personally interviewed. Diagnoses of schizophrenia, affective disorder, and psychotic disorder (as previously defined) were made for 17,22 and 49 relatives, respectivelly. The range of the familial loading scores were: -1.2 to 3.7 for psychotic disorders (figure 1 ) ; -1.2 to 2.6 for schizophrenia; and -1.2 to 3.6 for affective disorders. A familial loading score of 0 means that there is equal support for the proband to be "famillial" or "sporadic", a positive score means that there is greater support for the proband to be "familial", while a negative score means that there is greater support for the proband to be "sporadic" (for a full explanation, see Appendix 1). 
Hg. I Distribution of proband-wise calculated famitial loading score for psychosis

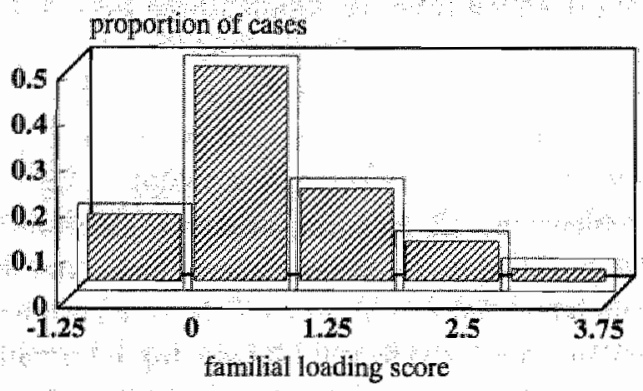

\section{Outcome and family history}

Associations between the scores of family history of schizophrenia, affective disonders and psychotic disorders on the one hand, and measures of course and outcome on the other, were examined in the total sample through regression analyses i) unadjusted for potentiall confounding variables and iii) controlled for diagnosis, age (strongly correlated with age at onset; $r=0.92, p$ $<0.001$ ), sex, ethnicity, and duration of illness, as we felt these could be associated independently with both family history and outcome. Diagnosis was modeled as a binary variable (schizophrenic psychoses/non-schizophrenic psychoses); adjustment using more diagnostic categories yielded similar results.

\section{Familial loading score for psychotic disorder}

No significant or large associations were found between the familial loading score for psychotic disorder, and sociodemographic (sex, ethnicity) and anamnestic (previous duration of illness) characteristics of the probands, with the exception of age and age of onset, which were both associated with family loading score for psychotic illness (Spearman's rank correlation $=0.3$; $\mathrm{p}=0.01$ for both variables). To maximise the stringency of the analyses, all the sociodemographic and anamnestic variables were adjusted for in the multiple regression analyses, regardless of statistical significance with either of the main variables.

Greater familial loading for psychotic disorder predicted persistent negative symptoms over the follow-up period as well as more negative symptoms at follow-up; more social disability as assessed by the DAS; having spent more time in hospital, and less time living independently (Table 1). In terms of risk this meant, for example, that one unit increase in the familial loading score for psychotic disorder predicted being 1.5 times more likely to thave been rated as having negative symptoms over the follow-up period. Given a range of around four units $(-1.2-3.7)$, this means a difference in risk of $1.5^{4}=5.1$ times between the lowest and the highest score of familiall loading. Greater familial loading for psychotic disorder also predicted, at trend level, more time spent unemployed, and increased likelihood of non-recovery. Associations with the other measures were not significant, but in the same direction.

In order to examine effect modification by diagnostic category, "diagnosis-familial loading" interaction terms were included in the regression models, with diagnostic categories collapsed into schizophrenic and non-schizophrenic psychoses to increase statistical power of the 
interaction terms. The model significantly improved $(\mathrm{p}=0.04)$ when this "diagnosis-familial loading" interaction term was entered in the equation with "negatiwe symptoms over the followup period" as the dependent variable, stratified analyses indicated that the association was much stronger in the schizophrenic psychoses $(O R=3.5,95 \% \mathrm{CI}: 1.2-10.2)$ than in the nonschizophrenic psychoses (OR=1.2,95\%CI: 0,7-1.9).

\section{Familial loading for schizophrenia}

Greater "familial loading score for schizophrenia" was associated with non-recovery (adjusted $\mathrm{OR}=2.2 ; 95 \% \mathrm{CI}: 1.1-4.4)$ and with more negative symptoms at the end of the follow-up (adjusted $\mathrm{B}=0.13, \mathrm{p}=0.01$ ). There were trends for greater "familial loading score for schizophrenia" to predict presence of negative symptoms over the follow-up period (adjusted $\mathrm{OR}=1.7,95 \% \mathrm{CI}: 0.9-3.1$ ), having spent less time living independently (adjusted $\mathrm{B}=-6.2, \mathrm{p}=0.11$ ) and having more social disability (adjusted $\mathrm{B}=0.1, \mathrm{p}=0.12$ ).

When "diagnosis-familial loading" interaction terms were entered in the equation with "nonrecovery" as dependent variable, significant improvement in the model $(p=0.03)$ was obtained. Stratified analyses indicated that the association with being "non-recovered" was stronger, although not significant, in the schizophrenic psychoses $(\mathrm{OR}>99,95 \% \mathrm{CI}: 0.2-99)$ than in the non-schizophrenic psychoses $(\mathrm{OR}=1.4,95 \% \mathrm{CI}: 0.65-3)$.

"Familial loading score-gender" interaction terms were included in all the above regressions models. There was no evidence for effect modification by gender.

\section{Familial loading for affective disorders}

Associations between outcome measures and the familial loading score for affective disorders were neither large nor significant, nor in any consistent direction.

\section{Relationship to other important predictors of outcome}

We previously showed that poor outcome, especially presence of negative symptoms over the follow-up period, was associated with cerebral ventricle dimensions as assessed by CT, and that negative symptoms at baseline were also predictive of poor outcome (Van Os et al, 1995 a \& b). In addition, it is known that premorbid social adjustment is also a powerful predictor of outcome (reviewed by Stoffelmayr, 1983). The association between poor premorbid adjustment as assessed by the Philips scale and measures of course and outcome was also apparent in our data: Philips scalle scores were significantly associated with continuous illness course, nomrecovery, more negative symptoms and more social disability at follow-up, and more time spent unemployed over the follow-up period (all $\mathbf{p} \leq 0.01$, also after adjustment for gender, diagnosis and age of onset; data not shown). 
Table 1, Genetic diathesis for psychonic disorders and outcome

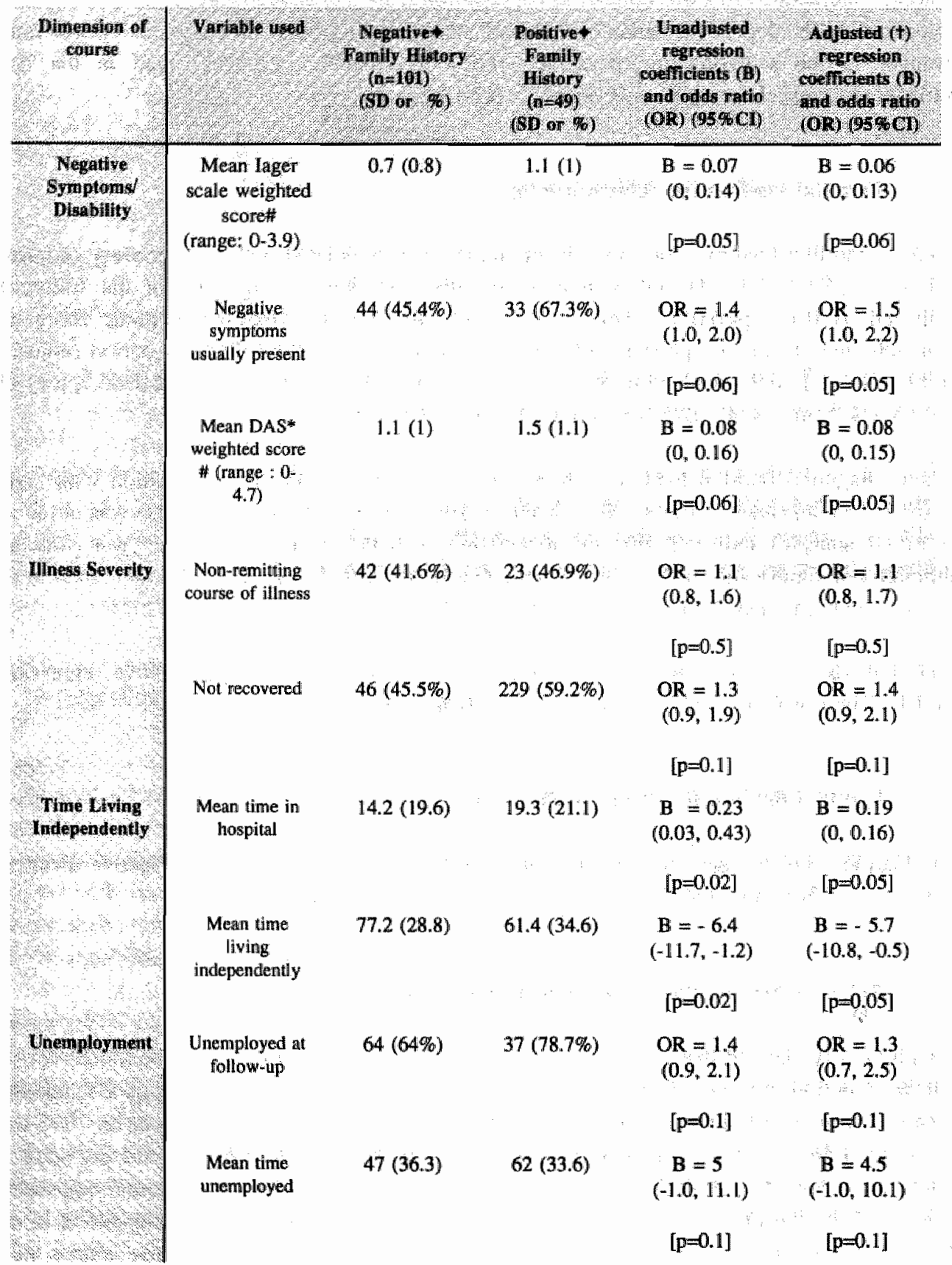

- Positive family history $=$ familial loading score for psychotic disorders $>0$, Negative family history $\leq$ 0 (see appendix. I)

+ Adjustment for age, sex, ethnicity, diagnosis and duration of illness (see text)

* Disability Assessment Schedule \#= Higher scores indicate greater severity 
Not only were these other predictors associated with outcome, but further analyses revealed that the familial loading score of psychotic illness was associated with i) premorbid social adjustment as measured by the Philips scale (Spearman's tho: $0.2, p=0.04$ - indicating poor premorbid adjustment was associated with higher family loading for psychosis); ii) cerebral ventricle size as measured by lateral ventricular volume (Spearman's tho: $-0.15, p=0.08$ ), especially in male patients (Spearman's rho: $-0.22, p=0.04$ - indicating large ventricular volume was associated: with lower familial loading), but not in female patients (Spearman's rho $=0.10, p=0.5$ ); nii) negative symptoms at baseline measured as the sum of the PSE syndromes "affective flattening"; "slowing" and "self-neglect" (Spearman's tho: 0.2, $p=0.02$ - indicating that more negative symptoms was associated with a higher degree of family loading for psychosis). All these associations remained significant after adjusting for age, sex, ethnicity, diagnosis and (for lateral ventricular volume) total intracranial volume (see Van Os et al, 1995b).

As these three predictors were both associated with the exposure (family history) and the outcome (illness course), they could well confound the associations between family loading for psychosis and course and outcome. To examine whether the associations between familial loading for psychosis and measures of course and outcome were independent of these other important predictors, we adjusted, in the analyses on familial loading for psychosis and outcome, for i) cerebral ventricle dimensions (adjusting for age, sex ethnicity and total intracranial volume), ii) negative symptoms at baseline (the sum of the PSE syndromes "affective flattening", "slowing" and "self-neglect"), iii) the Philips' scale score. These analyses revealed that the effect of familial loading was independent of other predictors, with the exception of the negative symptoms/disability dimensions of outcome, where adjustment for negative symptoms at baseline reduced the parameters of familial loading by more than $50 \%$.

\section{DISCUSSION}

Greater familial loading for broadly defined psychotic disorders and for marrowly defined schizophrenia were associated with poorer outcome in psychotic probands, especially with poor clinical outcome. Probands with greater familial loading for psychotic disorder had spent more time hospitalized, less time living independently, had displayed more prominent negative symptoms over the follow-up period and at the cross-sectional evaluation, and had more social disability. The association between greater familial loading for psychotic disorders and negative symptoms over the follow-up period was especially marked in schizophrenia. Greater familial loading for narrowly defined schizophrenia was associated with more prominent negative symptoms at the cross-sectional evaluation, and fajlure to recover over the follow-up period. No significant relationship was found between dimensions of outcome and familly history of affective disorder.

\section{Methodological issues}

The most accurate method for family history assessment, i.e., personal interview of the relatives with structured interview schedules, was used for more than $50 \%$ of the relatives. The accuracy of the family history method is lower than the personal interview method (Andreasen et al, 1986). However, information provided by the FH-RDC method was complemented by 
examination of hospital medical records, and it has been demonstrated previously (Sham et al, 1994, Davies et al, in preparation) that a high level of reliability in the psychiatric diagnoses for relatives was obtained for this sample of patients. More importantly, as outcome was rated blind to family history, a systematic bias could not occur.

There is no consensus on what constitutes the most relevant method to subdivide family history in relation to relatives' diagnoses. Therefore, it is difficult to avoid some degree of arbitrariness. The definition of family history of affective disorders we used was relatively stringent, owing to the exclusion of non-hospitalized major depression. This implies that the most severe types of affective disorders were selected for the calculation of the affective familial loading score; the group of depressed relatives selected by this method was probably more homogeneous, with more reliable depression diagnoses for the relatives who were not personally interviewed.

The "familial loading score" approach described above was chosen because we were primarily interested in predicting outcome by family history. This method proved very convenient in the comparative analyses, especially in models examining associations between famillial loading and both binary and continuous measures of course and outcome, and in models adjusting for confounding.

The longitudinal assessments of clinical outcome measures over the follow-up period (i.e., presence of negative symptoms, symptom severity, etc.) were made retrospectively, with the possibility of misclassification. However, this information was collected blind to family history, so that it is unlikely to have lead to a systematic bias in the relationship between outcome and family history.

\section{Interpretation of findings}

Our results suggest that a greater familial loading for broadly defined psychotic disorder or narrowly defined schizophrenia is associated with a poorer clinical outcome in psychotic patients. For some measures, this relationship appeared stronger in, but was not specific to, schizophrenic probands compared to non-schizophrenic probands, especially with regard to clinical outcome dimensions such as likelihood to recover and chronicity of negative symptoms.

As mentioned before, Kendler \& Tsuang (1988) reported findings in a similar direction, although failing to reach statistical significance. In their study, differences in morbid risk for non-affective psychosis were more pronounced at short-term outcome (mean length of follow-up: 2.5 years), which would fit with our prediction that any association would be more liable to be detected early in the course of psychotic illness, when there is substantial variability. The fact that their findings failed to reach statistical significance may be related to inaccuracies in classifying outcome on the basis of case note information, leading to random error and statistical imprecision. In an earlier report on the same sample (Coryell \& Tsuang, 1982), simillar findings emerged in that morbid risk of schizophrenia in the relatives of (good-outcome) schizophreniform disorder was lower $(1.8 \%$ ) than in relatives of probands with (poor outcome) schizophrenia $(2.8 \% ; \mathrm{p}=0.1)$. 
In Kendler \& Tsuang's (1988) study, the morbid risk for unipolar affective disorder was higher in the relatives of schizophrenics with global good (short and long term) outcome. Our failure to replicate this finding may be related to our narrow definition of (severe) affective disorder. On the other hand, the suggestion that family history of affective disorder predicts good outcome in schizophrenia sits uneasy with the findings reported recently by Winokur and colleagues (1993), who found that bipolar patients with a positive family history of mania or schizomania (assessed through interviews with all available first degree relatives) had a poorer clinical outcome, as assessed by the number of episodes. In other words, higher genetic loading may predict poorer outcome in any functional psychotic disorder.

To our knowledge, no previous study has looked, without an a priori stratification into diagnostic categories of questionable validity, at the relationship between outcome and family history in a sample of patients presenting with a functional psychosis. The association between chronic negative symptoms and family history of psychosis was especially marked in schizophrenia, but no evidence of interaction was evident for the other outcome measures. However, our interaction analyses were lacking in statistical power, and only analysis in a larger sample can resolve this issue.

The association between familial diathesis and poor outcome may be linked in part to sociofamilial factors. It is possible that being brought up by, or living with, psychotic relatives might have some deleterious impact on social adjustment and outcome. Although we cannot exclude this possibility, it is unlikely that living with an affected relative would create, for example, the kind of low-stimulus environment that has been shown to induce negative symptoms.

Our results suggest that familial loading is associated with outcome independent of other risk factors for chronicity such as premorbid social adjustment, cerebral ventricle dimensions, gender, ethnicity and age of onset, as all were controlled for in the analyses. The only exception was negative symptoms at baseline in the association between familial loading and the negative symptoms/disability dimension of outcome: here adjustment for negative symptoms at baseline greatly reduced the strength of the association. One explanation is that negative symptoms are on the causal pathway between genetic risk and poor outcome in terms of negative symptoms and disability; such an explanation is compatible with i) reports that negative symptoms themselves might be familial (Dworkin \& Lenzenweger, 1984; Dworkin et al, 1988; McGuffin et al, 1984; Walker \& Lewine, 1988) which is also supported by our own data, and ii) reports on associations between negative symptoms and poor outcome in schizophrenia (reviewed by Pogue-Geile, 1989).

Of interest is our finding that premorbid adjustment, negative symptoms and cerebral ventricle dimensions in men were all associated with family loading for psychosis. Negative symptoms and premorbid adjustment were positively associated with genetic risk, which may indicate, for example, that they are the manifestations of a genetically induced illness, or, alternatively, that they interact with genetic predisposition to increase the risk of psychosis. The negative association between cerebral ventricle size and family loading for psychosis in males echoes the recent findings by Vita and colleagues (1994) who reported that in men, but not in women, the ventricle-brain-ratio (VBR) was significantly larger in sporadic cases $(n=325)$, compared to familial cases $(n=122)$. These issues will be further explored in a later report. 
Familial history of psychosis is well established as a risk factor for emergence of psychotic illness. Although further replication is needed, our report suggests that the familial diathesis to psychosis is also a risk factor for persistence or prognosis of illness. Were the relationship between hyperlipidaemia and cardiovascular disease still unknown, then a similar relationship would be evident for emergence of cardiovascular disease and poor subsequent prognosis (early onset, rapid onset of complications and high mortality) in relation to family history of cardiovascular disorder (Wang et al, 1994). It is possible that such a general mechanism also exists in the genetic predisposition for psychosis, either through the direct action of the genes, or by making individuals more susceptible to a contributory environmental causal factor. 


\section{APPENDIX $\mid$}

\section{Familial loading scores}

For the purpose of calculating a simple score summarising the extent of psychiatric morbidity in the family of a proband, each proband is regarded conceptually as either a "familial" or a "sporadic" case. It is then assumed that the lifetime risk of illness in a first degree relative is $10 \%$ for "familial" probands, and $0.5 \%$ for "sporadic" probands. It is also assumed that the agerange at risk is $15-50$, and that in this range risk increases linearly with age from 0 to the lifetime risk. The probability that a relative of age $x$ is affected if the proband is "familial" is therefore $(0.1)(x-15) /(50-15)$ and the probability that such a relative is unaffected is simply 1 minus this. Similarly, the probability that a relative of age $\mathrm{x}$ is affected if the proband is "sporadic" is $(0.005)(x-15) /(50-15)$. The likelihood ratio for whether the proband is familial or sporadic, given that a relative of age $x$ is affected is therefore $[(0.1)(x-15) /(50-15)] /[(0.005)(x-$ $15) /(50-15)]=20$, and a similar ratio can be defined for an unaffected relative, although this ratio will be dependent on age. Such a likelihood ratio is calculated for every relative of a proband, and an overall likelihood ratio for whether the proband is "familial" or "sporadic" is obtained by multiplying together these individual likelihood ratios. Because this overall likelihood ratio is likely to be highly skewed, we take its common logathrithm and define it as the familial loading score. A familial loading score of 0 means that there is equal support for the proband to be "familial" or "sporadic", a positive score means that there is greater support for the proband to be "familial", while a negative score means that there is greater support for the proband to be "sporadic". Because of the many simplifying assumptions that are made in its construction, this score has no precise biological interpretation except as an index of the familial loading of an illness which takes into account family size and age structure. 


\section{APPENDIX \|}

\section{COUASE TYPE N THIS PERIOD.}

In estimating duration of an episode that began before this period but extended into the period, include the time before this period

- eplodic - discrete epispdes (none longer than 6 MONTHS) with clear periods of remission an between At least one remission latsted 6 months or more.

4 conthuous - psychotic over most of the period If amy nemissions, these were brief (none longer than 6 monthy).

- neither eptsodic nor continuous - e g. longest psychotic episode was 12 months and llongest remission was 9 months.

- never actively psychotic in this period.

\section{USUAL SEVERITY OF SYMPTOMS, (Categeries are modified from M. Bleuler.)}

Select the rating that best describes MOST of the period if no rating applies to more than half of the period, select the one with the longest duration.

- mild - bethavior was generally nomal and llmess was not immediately obvious in conversation, although definite psychotic symptoms persisted.

- moderate consistently showed signs of health in spite of marked psychotic symptons, eg, could speak with reasonable clarity on some occasions; could work or care for others to some extent, marked psychotic symptoms may. be overt or suggestiwe (see definitions).

- severe - could not carry on a coherent conversation, could not work without close supervision, required constant care.

- recovered - only residual or no psychotic symptoms.

\section{PSYCHOTIC}

\section{A OVERT SIGNS AND SYMPTOMS}

- hallucinations or pseudohallucinations (in any modality);

- delusions:

- : marked thought and speech disorder ( $\mathrm{e}, \mathrm{g}$, incoherence, irretevance, thought bloeking, neologisms, incomprehensibility of speech) other than simple retardation or accelleration;

- marked qualitative psychomotor disorder ( $\mathrm{e}$ g., negativism, mutism or stupor, catatonic excitement; constrained attitudes or unnatural postures maintained for long periods) other than retardation or acceleration of movements;

- bizarre or grossly inappropriate behavior (e.g., talking to self or giggling to self, acts incomprehensible to others, loss of social constraints, etc.).

\section{B. SUGGESTIVE SIGNS AND SYMPTOMS}

- extreme reduction or loss of interests, initiative and drive, leading to serious deterioration of the performance of usual activities and tasks;

- extreme social withdrawal;

- severe excitement, purposeless destructiveness or aggression (frequent episodes or continuous):

- episodes or persistent states of owerwhelming fear or severe anxiety;

- gross and persistent self-neglect.

\section{REMISSION}

A state following a psychotic episode, in which none of the symptoms and signs required for rating a psychotic episode are present. The absence of psychotie symptoms and signs qualifies as a renission only if it lasts for four weeks or more. Two varieties of remission will be distinguished:

(A) COMPLETE REMISSION: the subject is virtually symptom-free and shows his/her usual premorbid personality;

(B) INCOMPLETE REMISSION: the subject is no longer psychotic (in the sense defined above) but shows any combination of: (i) residual symptoms (i.e., "traces" of the symptoms and signs which were present in the psychotic episode but which in no way would justify a rating of a continuing psychotic episode); (ii) non-psychotic symptoms, e.g., non-specific neurotic or mild affective disturbances which are not traits of the premorbid personality; (iii) personality change, e.g., accentuation of premorbid personality traits or emergence of new traits; change or loss of interests; stable aiterations of expressive and communicative behavior; decline of intellectual performace.

\section{COMMUNITY LIVING INDEPENDENT OF PROFESSIONAL SUPERVISION}

Independent community living includes all living arangements other than an institution (e.g., hospital, jail), a supervised restdence, and homelessfvagrant. Thus, it includes living alone, living with friends, living with family, and: any other non-institutional domicile. 


\section{REFERENCES}

Alda, M., Zvolsky, P, Dvorakova, M, Paperova, H. (1991) Study of chronic schizophrenics with positive and negative family histories of psychosis. Acta Psychiatrica Scandinavica, 83, 334-337.

Andreasen, N.C., Rice, J.P., Endicott, J, Reich, T. Coryell, W. (1986a) The familial history approach to diagnosis. How useful is it? Arehives of General Psychiatry $43,421-429$

Angst, J. (1988) European long-term follow-up studies of schizophrenila. Schizophrenia Bulletin, 14, 501-513.

Bleuler, M. (1978) The schizophrenic disorders. long term patient and fomily studies, Clemens S. (translator). New Haven: Yale University:

Ciompi, L. (1980) Catamnestic long-term study on the course of life and aging of sehizophrenics. Schizophrenia Bulletin, 6, 606-618.

Coryell, W. \& Tsuang, M.T. (1982): DSM-III schizophrenifom disorder: Comparisons with schizoplrenia and affective disorder. Archives of Gemeral Psychiatry, 39, 66-69.

Davies, N., Sham. P., Gilvarry, $\mathbb{K}$, et al. Comparison of methods of family history data collection: a report from the Camberwell Collaborative Psychosis Study. In preparation.

Dworkin , R.H., Lenzenweger, M.F. (1984) Symptoms and the genetics of schizophrenia : implications for diagnosis. American Journal of Psychiatry 141, 1541-1546.

Moldin, S.O., Skillings, G.F. L Levick, S.E. (1988) A multidimensional approach to the genetics of schizophrenia. American Journal of Psychiatry, 145, 1077-83.

Endicott, J. \& Spitzer, R. (1978) A diagnostic interwiew: the Schedule for Affective Disorders and Schizophrenia. Archives of General Psychiatry, 35, 837-844.

Huber, G., Gross, G., Schüttler, R., \& Linz, M. (1980) Longitudinal studies of schizophrenic patients. Schizophremia Bulletin, 6, 592-605.

Iager, A.C.. Kirch D.G., Wyatt, R.J. (1985) A negative symptom rating Scale. Psychiatry Res. 16, 27-36.

Jablensky, A., Schwartz, R. Tomow, T. (1980) WHO collaborative study of impairments and disabilities associated with schizophrenia disorders. A preliminary communication. Objective and methods. Acta Psychiatrica Scandinavica suppl. 285, 152-163.

Jones, P.B., Bebbington, P., Foerster, A., Lewis, S., Murray "R.M., Russel, A., Sham, P., Toone, B., Wilkins, S. (1993) Premorbid social underachievement in schizophrenia. Results from the Camberwell Collaborative Psychosis Study. British Journal of Psychiatry 162, 65-71.

-...-. Harvey, I., Lewis, S., Toone, B.n Van Os, J., Williams, M. \& Murray, R. (1994) Cerebral ventricle dimensions as risk factors for schizophrenia and affective psychosis. An epidemiological approach to analysis. Psychological Medicine 24, 995-1011.

Keefe, R.S.E., Mohs, R.C., Losonczy, M.F., Davidson, M., Silverman, J.M., Kendller, K.S., Horvath, T.B., Nora, R., Davis, K.L. (1987) Characterisitics of very poor outcome schizophrenia. Am. J. Psychiatry 144, 889-895.

Kendler, K.S., Tsuang, M.T. (1988) Outcome and familial psychopathology in schizoplurenia. Arch. Gen. Psychianry 45, 338-346.

McGlashan T.H. The prediction of outcome in chronic schizophrenia. IV (1986a) The Chesnut Lodge Follow-up study. Archives of General Psychiarry 43, 167-176.

-.-.-, (1986b) Predictors of shorter-, medium- and longer-term outcome in schizophrenia. Am. J. Psychiatry 143, 50-55.

McGuffin, P., Farmer, A.E, Gottesman, L.I., Murray, R.M., Reveley, A.M. (1984) Twin concordance for operationally defined schizophrenia, Confirmation of familiality and heritability. Arch. Gen. Psychiatry 41 : 541-545.

McGuffin, P., Farmer, A.E., Harvey, I. (1991) A polydiagnostic application of operational criteria in psychotic illness: development and reliability of the OPCRIT system. Archives of Gerteral Psychiatry 48, 50-55.

Pogue-Geile, M.F. (1989) The prognostic significance of negative symptoms in schizophrenia. British Journal of Psychiatry 7 (suppl.), 123-127.

Robins, E. , Guze, S.B. (1970) Establishment of diagnostic validity in psychiatric illness: its application to schizophrenia. American Journal of Psychiatry 126, 983-987.

Roy, M.A., Crowe, R.R. (1994) Validity of the familial and sporadic subtypes of schizophrenia. American Journal of Psychiatry 151, 805-814.

Sautter, F.J, McDermott, B.E. (1994) The short-term course of familial and nonfamilial schizophrenic spectrum disorder. Journal of Psychiary Research 28, 97-106.

Sham P., Jones P. Russell A., Gillvarry K. , Bebbington P., Lewis S., Toone B., Murray R. (1994) Age at onset, sex, and familial psychiatric morbidity in schizophrenia. Camberwell Collaborative Psychosis Study. British Journal of Psychiatry 165, 466-473.

Stoffelmayr, R. (1983) Premorbid functioning and outcome in schizophrenia. A cumulative analysis. Journal of Consultant and Clinical Psychology, 51, 338-352. 
Strauss, $1 . S_{., 3}$ Carpenter, W.T. (1974) The prediction of outcome in schizophreria. Arch. Gen. Psychiatry 31, 3742 .

Spitzer R.L, Gibbon M., Endicott J. The Global Assessment Scale (GAS). New York Psychiatric Institute : New York. 1975.

Van Os I, Fahy T.A., Jones P., Harvey I, Sham P., Lewis S., Bebbington P, Toone B., Williams M., Murray R. (1995a) Psychopathological syndromes in the functional psychoses: associations with counse and outcome. Psychological Medicine, in press.

_..., Fahy TA, Jones D, Harvey I, Lewis S, Givarry K, Willams M., Toone B., Murray R. (1995b) Increased intra-cerebral CSF spaces predict unemployment and negative symptoms in psychotic illness: a prospective study, British Jownal of Psychiatry, 166,750-758.

Vita, A, Deicl, M., Gtobbo, G.M., et al (1994) A reconsideration of the relationship between cerebral structural abnomalities and family histony of schizophrenia: Psychiatry Research, 53, 41-55.

Walker E., Lewine, E.J. (1988) The positive/negative symptom distinction in schizophrenia. Validity and etiological relevance. Schizophrenia Research $1,315-328$

Wang, X.L., Tam, C., McCredie, R.M. \& Wilcken, D.E. (1994) Determinants of severity of coronary artery disease in Australian men and women Circulation, 89. 1974-81.

Weiss, K.M., Chakraborty, R., Majumder, P.P. \& Smouse, P. (1982) Problems in the assessment of relative risks of chronic disease among biological relatives of affected individuals. $\int$ Chron Diseases, 35, 539-551

Wing JK., Cooper, J.E, Sartorius, N. (1974) The measurement and classification of psychiatric symptoms. Cambridge University Press: Cambridge.

Winokur, G., Coryell,W., Keller, M. Endicott, J., Akiskal, H. (1993) A prospective follow-up study of patients with bipolar and primary unipolan affective disorder. Archives of General Psychiatry, 50, 457-465. 
Chapter 5: Risk Factors for the Tardive Dyskinesia Outcome V. 


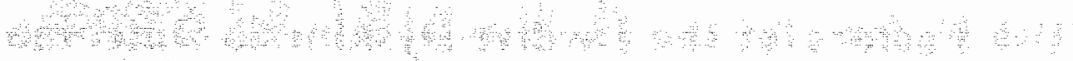

(8) 


\subsection{Tardive dyskinesia: Effect of medication or illness deterioration?}

\section{SUMMARY}

There is controversy over whether tardive dyskinesia (TD) is associated with female gender; affective symptoms and good outcome, or, altematively, with negative symptoms, cognitive deterioration and deteriorated illness course. Furthermore, antipsychotic medication is thought to be an important risk factor, yet TD also occurs in never-medicated patients. In order to clarify these issues, we followed 166 subjects with recent onset of psychotic illness and brief previous exposure to antipsychotic medication. Information on 17 previously reported risk factors was collected at baseline and, for factors that vary over time, again at follow-up 4 years later. Movement disorder was assessed at follow-up with the AIMS. Only six, non-interacting; variables were independently associated with the four-year risk of TD: male sex $(O R=2.5 ; 95 \%$ $\mathrm{CI}=1.1-5.0$ ), age (OR over quartiles at baseline: 1.6; 1.1-2.2), lack of insight at baseline (OR over four categories: 2.0; 1.2-3.2), time on antipsychotics over the follow-up period (OR over quartiles: $2.3 ; 1.5-3.4$ ), an increase in negative symptoms over the follow-up period (OR over quartiles: $1.7 ; 1.2-2.5)$, and alcohol/drug misuse at follow-up $(O R=3.0 ; 1.3-7.4)$.

The results suggest that two discrete effects may operate to increase risk of TD: an exogenous factor (medication, drugs), and an illness-related factor, the highest risk being conferred by deteriorating illness in male patients. Presence of individual risk factors was found to be of little use as a screening test for later clinically relevant TD. Given absence of a risk factor, however, the probability that an individual would not develop TD was high.

\section{INTRODUCTION}

Clinicians need accurate predictors of TD to guide them in estimating the risk-benefit ratio in individual cases. Unfortunately, however, the literature is controversial in this regard. For example, a number of reports indicate that affective symptoms and female sex (eg Kane et al, 1986; Yassa \& Jeste, 1992), both associated with good outcome in the functional psychoses, increase the risk for TD, whereas other reports find negative symptoms, cognitive deterioration and poor prognosis are associated with TD (Edwards, 1970; Wegner et al, 1985; Waddington et al, 1985; Liddle $e t a l, 1993$ ). In addition, the positive predictive value for risk factors (the probability of developing TD given the presence of a risk factor) is not known, yet this is essential for clinicians. Another controversy involves the role of antipsychotic medication. There is evidence that antipsychotic drugs have a major role in the aetiology of tardive dyskinesia (TD; Jeste \& Wyatt, 1981; Kane \& Smith, 1982), but other findings suggest that the primary risk factor is psychiatric disease, rather than the interventions associated with it (Fenton et al, 1994; Waddington et al, 1990). It is also possible, however, that there is an interaction between the disease and its interventions (Liddle et al, 1993).

Some of the controversy is likely to be related to the following issues: 
1) Most samples are selected for their continuing exposure to antipsychotic medication, and therefore for their chronicity. This will lead to biased estimates of risk, as individuals with a less deteriorated course of illness are likely to be excluded. ii) TD is most often defined as a discrete condition by more or less arbitrary dichotomization of continuous abnormal movement scores (Jeste \& Caligitu, 1993, Kane et al, 1986, Chouinard et al, 1986; Glazer et al, 1991; Waddington \& Youssef, 1986), the Abnormal Involuntary Movement Scale (AIMS; Guy, 1976) being most frequently used (Ieste \& Caligiuri, 1993). Such an approach ignores the fact that there is important variation in the phenomenon along an unbroken scale of severity; iii) Unless the focus is on a comprehensive set of risk factors, the possibility that results are confounded cannot be excluded, iv) Reversed causality may operate in the case of some putative mediators of risk. For example, severe TD may lead to affective symptoms, or to increased alcohol intake, rather than the other way round. Waddington and colleagues drew attention to the importance of establishing the temporal order of ewents with regard to experience of risk and outcome of TD. They found there was no difference in cognitive function between patients who did and did not go on to develop TD. However, "those who went on to devellop TD showed a selective deterioration in their cognitive function over the time period in which their involuntary movements emerged" (Waddington el al, 1990).

In this investigation, we distinguished the following exposures in a sample of patients with a functional psychotic illness of recent onset, little previous antipsychotic medication, and followed up for a period of four years: i) fixed exposures, such as gender, ethnic group, etc; and ii) variable exposures, ie factors that may vary over time such as affective and negative symptoms, diagnosis, alcohol use, and medication. These exposures were investigated at two time points in relation to the outcome of TD; i) at baseline and over the follow-up period - ie a prospective design; and ii) at follow-up - ie a cross-sectional design. Our analyses included measures indicative of the magnitude of the effect of many of the risk factors reported in the literature, while taking into account the severity of movement disorder along a continuous scale.

The following issues were addressed: i) which variables are independently associated with the four-year cumulative risk of TD, and, as screening tests; is their predictive value high enough to be of use in clinical practice? Do variables that vary over time (eg cognitive functioning, negative symptoms) show similar associations with the TD outcome at baseline and at follow-up, and at which time point -antecedent or cross-sectional- is the association strongest? iii) what can we conclude about the roles of illness-related risk factors, such as cognitive deterioration ${ }_{4}$ and exogenous risk factors, such as medication?

\section{METHOD}

\section{Baseline assessments}

This investigation forms part of a large cohort study investigating predictors of social and clinical outcome and tardive dyskinesia in a sample of recent onset psychotic patients. The sample and study rationale have been described in detail elsewhere (Jones et al, 1993; Van Os et al, 1995a \& 1995b). Basically, 191 patients aged 16-60 years with recent onset of at least one psychotic symptom according to the RDC (Spitzer et al, 1978), occurring in clear consciousness, and who had been extensively investigated, were followed up after an average of four years 
(median: 50 months; interquartile range: 29 -70). Baseline assessments were generally completed within three days of admission and included the Present State Examination (PSE; Wing et al, 1974), and demographic ratings. Ethnicity was classified as white European; Afro-Caribbean, or other ethnic group, based on appearance and country of birth of both the subject and his parents.

Age at onset was defined by the appearance of the first psychotic symptoms. Length of previous contact with services (a marker of length of previous treatment) was defined as the difference between age at index assessment and age at first treatment for psychosis.

The Operational Criteria Checklist for Psychotic Illness (OCCPI, MeGuffin et $a l$, 1991) was completed for all patients for the time up to index assessment to give DSM-III-R index diagnoses. The computer program OPCRIT (McGuffin et al, 1991) was used to analyze the OCCPI data. Psychopathological data were derived from detailed cross-sectional mental states at index assessment, on the basis of the PSE, and from the patients' case records.

The following psychopathological variables were obtained from the PSE/Catege syndromes: i) a measure of negative symptoms, being the sum of the score on the syndromes "affective flattening", "self-neglect" and "slowing", ii) a measure of affective symptoms, composed of the syndromes "simple depression" and "hypomania",;iii) the insight item (No 104) into psychotic symptoms, scored from 0 to 3 (zero corresponding to "perfect insight").

Sixty-two percent of the sample were assessed by three different raters on abnormal movements at index assessment, using the AIMS global judgement measure of "severity of abnormal movements" (five-point scale). Inter-rater relliability was not assessed, but all raters were experienced psychiatrists, trained in the same institution. The baseline AIMS assessment was discontinued for the remaining subjects because of the low yield of cases with abnormal movements (see below).

Individuals who had used illegal substances more than once in the month before baseline assessment, or alcohol in excess of 50 units per week were classiffed as "misusing alcohol or drugs".

An estimation of verbal intelligence was derived from the NART (Nelson, 1982), and in some cases a comparable measure was obtained from the verbal subset of the WAIS (Wechsler, 1955). Standardized values of normally distributed NART and WAIS scores were used to ensure comparability.

The method used for CT-scanning and establishing inter-rater reliability, has been described in detail elsewhere (Jones et al, 1994; Van Os et al, 1995b). In summary, a lateral ventricular" volume was constructed by adding together all the area measurements of its body, frontal horns, occipital horns and anterior horns. An estimate of the intracranial volume was made, and the Ventricle Brain Ratio (VBR) was obtained by dividing lateral ventricle volume by total intracranial volume.

Information on psychiatric history of first degree relatives was obtained from three sources: i) the proband's medical records, ii) the proband's mother using the FH-RDC (Andreasen et al, 
1986) and iii) the medical records of relatives with a psychiatric history. The FH-RDC category of chronic schizophrenia was used in the analyses.

Information on obstetric complications (OCs) was obtained through maternal interview by a rater who was blind to proband characteristics. OCs were rated as absent or definitively present according to the scale of Lew is et al (1989), except that rapid labour was no longer classified as an OC; this scale has been validated by O'Callaghan et al (1990).

\section{Follow-up assessments}

All follow-up ratings were completed by an experienced psychiatrist ( $\mathrm{JvO}$ ), blind to information collected at baseline (with the exception of gender and ethnicity). The modal number of informants used for all assessments was 2 (range 0-3).

A measure of overall severity of illness was taken from the WHO Life Chart which assesses course of illness longitudinally, using clear definitions for all ratings (WHO, 1992). Course type was rated as episodic (no episode longer than 6 months), continuous (no remission longer than six months), neither episodic nor continuous, and not psychotic in this period.

The following data on pharmacological interventions over the follow-up period were obtained: i) number of months on antipsychotic medication, anticholinergics, antidepressants and lithium, and ii) whether or not the subject was taking antipsychotics, anticholinergics, antidepressants or lithium at follow-up.

Total length of contact with services at follow-up was defined as the difference between age at follow-up and age at first treatment for psychosis, minus the time patients had not been in contact with services over the follow-up period.

A measure of alcohol and drug misuse was also completed at follow-up, similar to the one used at index assessment.

A further PSE interview at follow-up yielded another measure of affective symptoms and insight. Instead of the PSE-measures of negative symptoms described above, the Iager scale for negative symptoms (Iager, 1985) was used at follow-up, as the results for all the analyses with either measure were similar and the Iager scale is the more valid measure. A second OCCPI checklist was completed to include all psychopathological information up to and including follow-up assessment, and analysed with the OPCRIT programme, to generate DSM-III-R follow-up diagnoses.

A measure of cognitive functioning was also obtained: the Trail making test A and B (Reitan, 1958).

Abnormal movements at follow-up were assessed using the AIMS, which identifies seven areas of the body and their most common abnormal movements, which are rated on an 0 to 4 severity scale (none, minimal-extreme normal, mild, moderate, severe; maximum score:28). 


\section{Analyses and exposure variables}

The aim of the analyses was to avoid arbitrary dichotomization while retaining the possibility of calculating readily comparable measures of effect. Tertiles of the total AIMS score (hereafter referred to as the "TD outcome") were analysed using the ordered polytomous logistic regression procedure of the STATA program (STATA, 1993). Ordered logistic regression yields odds ratios (OR) which are a single test statistic that summarizes the differences between the groups. The "proportional odds assumption" in ordered logistic regression is that the OR is the same for all choices of cut-off between ordered categories (McCullagh, 1980). To show the results were robust, we also conducted analyses using ordinary multiple regression with the log-transformed total AIMS score as the dependent variable. The results with these two methods concurred for all risk factors.

Based on recent reviews and studies of the topic (Waddington et al, 1990; Liddle et al, 1993; Jeste \& Caligiuri, 1993; Bames, 1987; O'Hara et al, 1993; Miller \& Chouinard, 1993; Zaretsky et al, 1993; Caracci et al, 1990; O'Callaghan et al, 1990), the following list of exposure variables was considered: i) at index assessment: age, gender, ethnic group, diagnosis, affective symptoms, negative symptoms, attitude to illness, misuse of alcohol and drugs, measure of cognitive functioning (verbal IQ), VBR, family history of schizophrenia ${ }_{*}$ obstetric complications, length of contact with services; ii) over the follow-up period: type of illness course, months on antipsychotics, anticholinergics, antidepressants and/or lithium iii) at follow-up: on antipsychotics, anticholinergics, antidepressants and/or lithium, misuse of alcohol or drugs, affective symptoms, measure of cognitive functioning (Trails test), negative symptoms, diagnosis, length of contact with services.

Guided by published findings (Yassa \& Jeste, 1992), we included an age by gender interaction term in the anallyses, as well as an age by negative symptoms interaction (Liddle et al, 1993). We also included a diagnosis by affective symptoms interaction and a diagnosis by family history of schizophrenia interaction (Waddington et al, 1990; Jeste \& Caligiuri, 1993).

In the case of normally distributed continuous variables (age, verbal IQ, months on antipsychotics, negative symptoms at follow-up) quartiles of distributions were entered in the equations. Odds ratios were examined in each quartile with the last quartile as the baseline, before calculating the summary OR of linear trend. Some severely skewed continuous variables, such as time on antidepressants and time on lithium (for both variables, around $70 \%$ of subjects scored zero) were entered as a binary variable in the equations. In order to select the primary, independent, risk factors, we used an analysis with step-up criteria (probability of F-to-enter< 0.05).

The presence of a risk factor may be considered as a diagnostic screening test for later occurrence of TD; the positive predictive value (PPV) of the test is the probability that a person will develop the disease given a positive test (calculated as the number of individuals with both the risk factor and the disease, divided by the total number of individuals with the risk factor). Analogously, the negative predictive value (NPV) is the probability that an individual is truly disease-free given a negative screening. Given the high prevalence of TD in treated patients, both PPV and NPV were calculated for independent risk factors, using the dichotomous definition for 
TD of both the research diagnostic criteria of Schooler \& Kane (1982, narrow definition), and a definition using a score of zero on the AMS as the cut-off (broad definition).

\section{RESULTS}

Of the 191 subjects, adequate follow-up data were available on $166(87 \%)$. Seven patients had committed suicide, and two had died of other causes. Complete data on each risk factor and the TD outcome was avallable for 125 of the 1.66 subjects; all further analyses relate to this group only, with the exception of the VBR measure, data for which were available only on 110 subjects. As there were no large or significant associations with VBR, analyses for the other risk factors were conducted with all 125 subjects.

The sample composition is described in Table 1.

\section{Table 1. Sample characteristics}

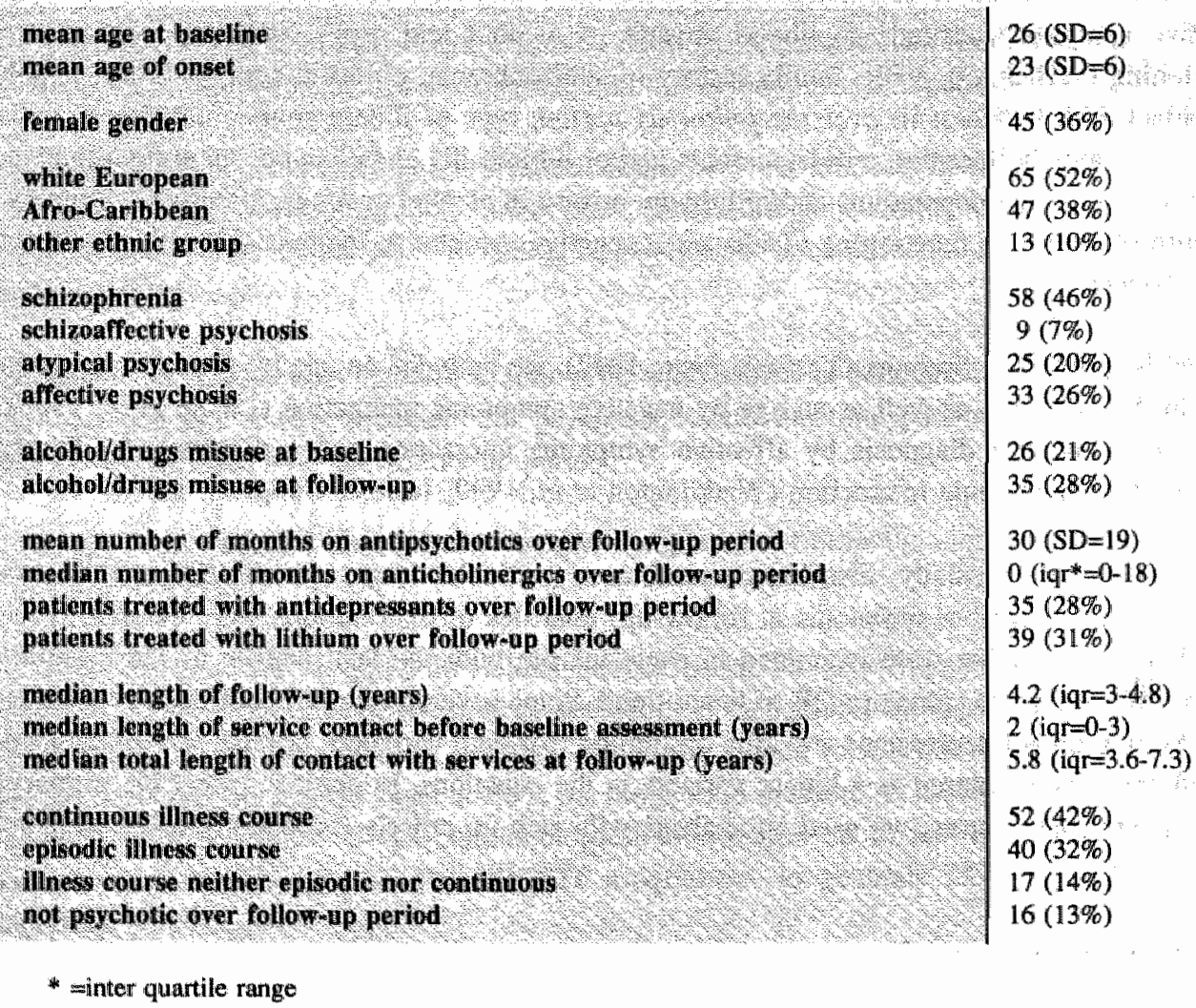

Length of follow-up (in months) was not even marginally associated with occurrence of abnormal movements ( $\mathrm{OR}=1.0 ; 95 \% \mathrm{CI}$ : 0.98-1.03), ruling out confounding by variation in length of follow-up for other variables (length of contact with services is considered below). 
Of the 78 subjects who had been rated on the AIMS severity item at index assessment, $68(87 \%)$ had shown no evidence of abnormal movements, and $8(10 \%)$ had shown minimal movement disorder, leaving only 1 subject with a rating of "mild". and 1 with a rating of "moderate"

At follow-up, the median AIMS score was 2 (interquartile range: 0-4). Twenty-one subjects (17\%) had a score of 3 (moderate) or more on at least one of the items. There were no significant associations between the TD outcome and the PSE syndromes of agitation $(\mathrm{OR}=1,7$, $95 \% \mathrm{CI}=0.5-5.8)$ and general anxiety $(\mathrm{OR}=1 ; 95 \% \mathrm{CI}=0.6-1.4)$ at follow-up.

\section{Analysis of risk factors separately}

In the first instance, each of the risk factors was entered separately in the ordered logistic regression equation (Table 2). Thus analysed, many risk factors were significant predictors of the TD outcome at follow-up, though not necessarily in the expected direction. For example, female gender and affective psychosis were associated with decreased risk. The only evidence of effect modification was an age by gender interaction ( $\mathrm{p}=0.05)$, indicating that increasing age was a risk factor for TD in women, but not in men. In general, the risk appeared to increase linearly with the following variables: age, length of service contact, negative symptoms at baseline and follow-up, months on antipsychotics, insight at baseline, and Trails score A and B at follow-up. For example, compared to the baseline OR of 1 in the highest quartile of months on antipsychotics, OR's in the quartiles 1 to 3 were, respectively, $0.1,0.6$, and 0.9 , indicating a progressively increasing risk with increasing exposure to antipsychotics.

\section{Strength of variable exposures at baseline and at follow-up}

In order to gain more insight into the temporal order of events, we repeated, for variable exposures with a measure both at baseline and at follow-up, the analyses for statistically significant predictors at either baseline or follow-up (Table 3), entering the baseline and followup variables jointly in the equations (ie adjusting one for the other). The baseline measures were, of course, strongly correlated with their analogous follow-up measures. For example, verbal IQ at baseline was strongly correlated with trails test $B$ at follow-up ( $r=0.6 ; p<0.01$ ), and the same applied to negative symptoms, alcohol/drug use, diagnosis of affective psychosis, etc (Table 3 ). In spite of these high correlations, however, one of the two variables was, in general, a much stronger predictor than the other, and in most cases this was the follow-up variable rather than the baseline variable (Table 3). The exceptions were PSE insight, where the predictive effect was confined to the baseline variable, and alcohol and drug misuse, where baseline and follow-up measures predicted the TD outcome equally strongly. 


\section{Independence of risk factors}

We conducted a stepwise analysis, entering those variables that had shown significant associations with increased abnormal movements in the previous analyses. A sex by age interaction term was also entered. Six of the 20 variables entered were selected in the final model (Table 4). Instead of the age by sex interaction, age entered the equation as an independent variable. Further analyses revealed that, after controlling for gender and months on antipsychotics, age was significantly associated with the TD outcome, suggesting that the sex by age interaction was mediated by the effect of time on antipsychotics. Neither Trails test A nor $B$ were selected for the final model. Separate analyses indicated this was because the OR for the Trails test dropped by more than 55\% after adjustment for negative symptoms at follow-up (ie was confounded by negative symptoms). The association with negative symptoms at follow-up was not reduced after adjusting for all indices of antipsychotic and other medication, and there was no evidence for an interaction with current exposure to antipsychotic medication $(p=0.6)$. Alcohol/drugs misuse at follow-up was selected in the final model, but if only alcohol/drugs misuse at baseline was entered, this was selected as well with an OR of similar magnitude. Length of exposure to antipsychotic medication may be a "proxy" of illness severity. However, adjustment for negative symptoms at baseline, PSE total symptom score at baseline, insight at baseline, course type and cognitive functioning at follow-up changed the parameter of the association with antipsychotic medication by only $4 \%$.

\section{Table 2. Risk of abnormal movements analysed separately for each expasure}

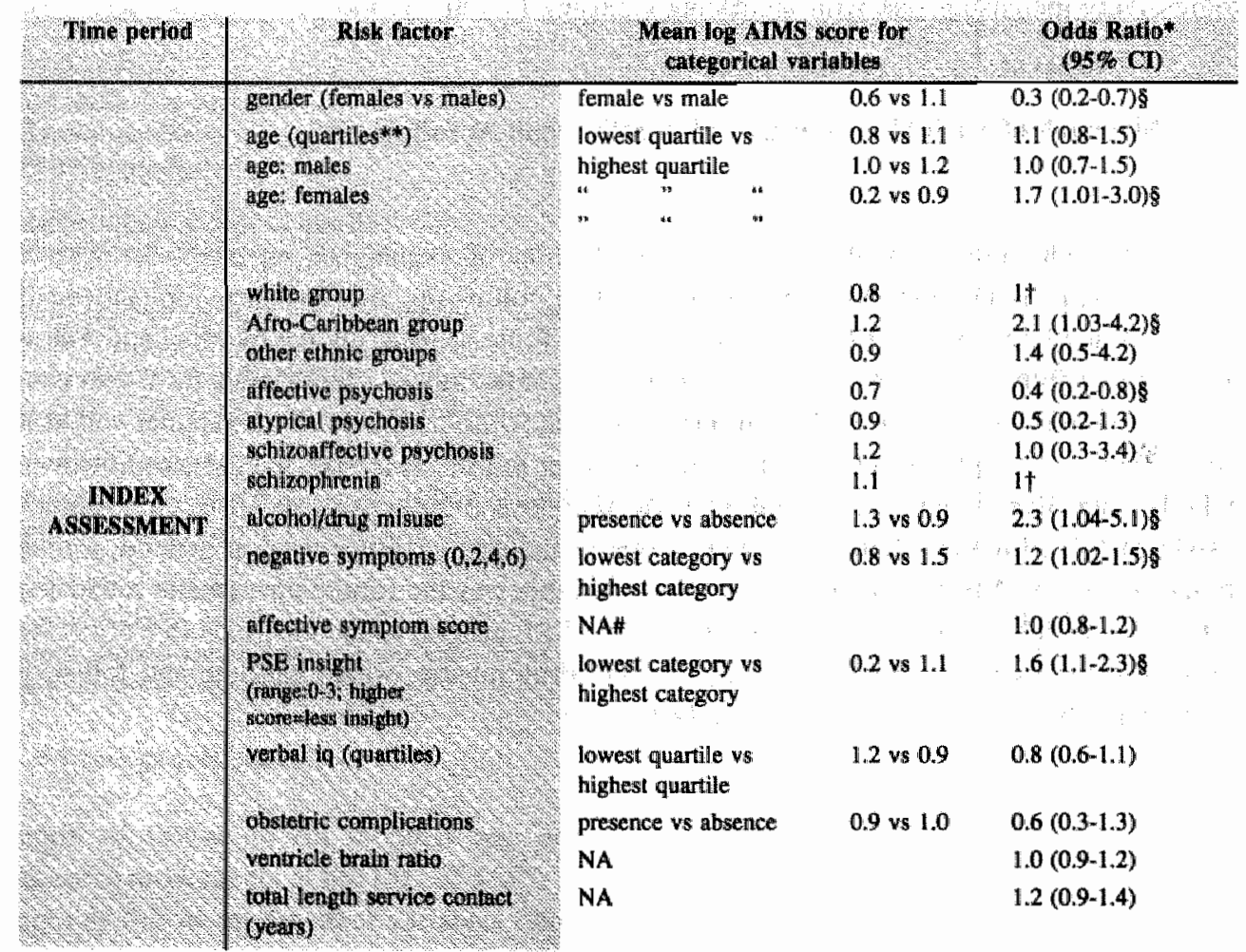


Table 2

(continued)

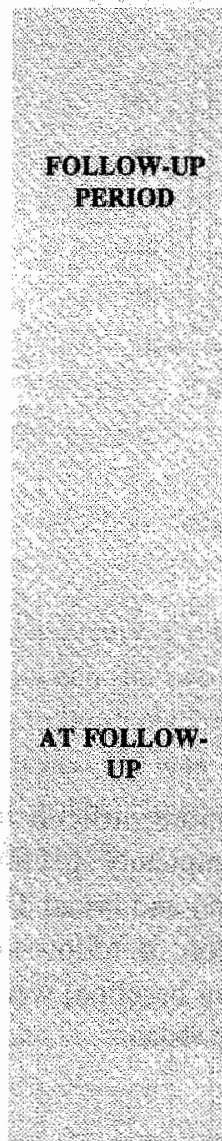

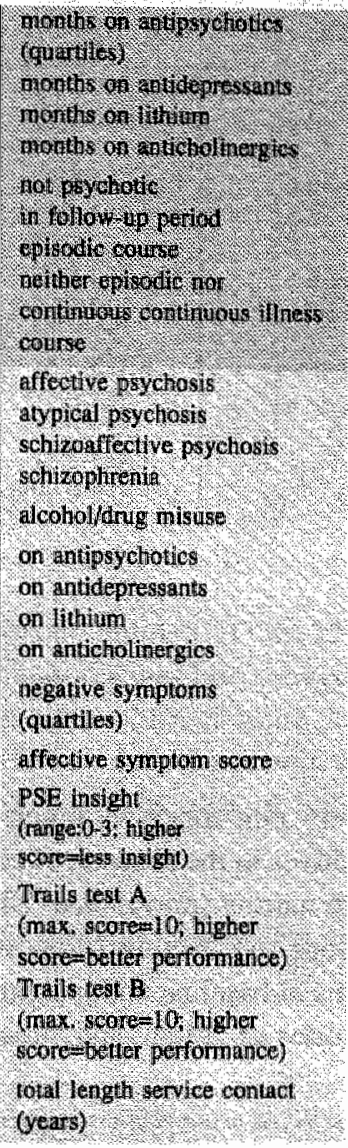

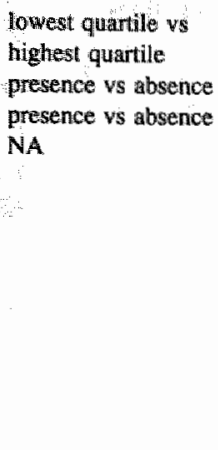

presence vs absence

presence ws absence presence vs absence presence us absence presence vs absence llowest quartille ws highest quartile

NA

lowest category ws highesi category

NA

0.3 vs 1.2

0.7 vs 1.1

$2.1(1.5-3.0)$

1.0 v 1.0

$0.4(0.2-0.95)$

$0.9(0.5-1.8)$

$1.05(1.02-1.07)$

0.7

0.6

1.0

1.3

$0.2(0.1-0.7) 8$

$0.3(0.1-0.7) 8$

$0.7(0.3-1.8)$

$1+$

$0.2(0.1-0.5) 8$

$0.3(0.1-1.0)$

$0.7(0.3-1.7)$

11

1.2 ws 0.8

2. $1(1,1-4.4) 8$

1. I. v 0.4

0.5 ws 1.0

$6.7(2.7-16.4)$

$0.4(0.1-0.9) 8$

I. 1 vs 0.9

$1.4(0.6-3.1)$

1.4 vs $0.8 \quad 3.3(1.6-6.7) 8$

0.7 ws 1.6

$2.1(1.5-2.9) 8$

$1.0(0.8-1.3)$

$1.3 \mathrm{vs} 1.3 \quad 1.2(0.8-1.8)$

$0.9(0.8-0.98)$

$0.8(0.7-0.98)$

NA

$1.2(1.041 .4)$

* $=\mathrm{OR}<1$ indicates decreased risk; $\mathrm{OR}>1$ indicates increased risk

** $=$ age by gender interaction: $\mathrm{p}=0.05$

\# = continuous variable

$t=$ baseline

$\S=p \leq 0.05$

Furthermore, there was evidence that the association between medication and TD was extralinear, in that addition of a squared exposure term significantly improved the model $(p=0.03)$. Thus, the risk of TD increased exponentially with length of exposure to antipsychotic medication. There was no evidence for statistical interaction between antipsychotic medication and other independent risk variables. We did not find an interaction with site of abnormal movement (orofacial dyskinesia and trunk and limb dyskinesia subsyndromes). 
Table 3. Risk of TD for variable exposures: values at follow-ip are entered together with baseline wallues (see text for interpretation of values).

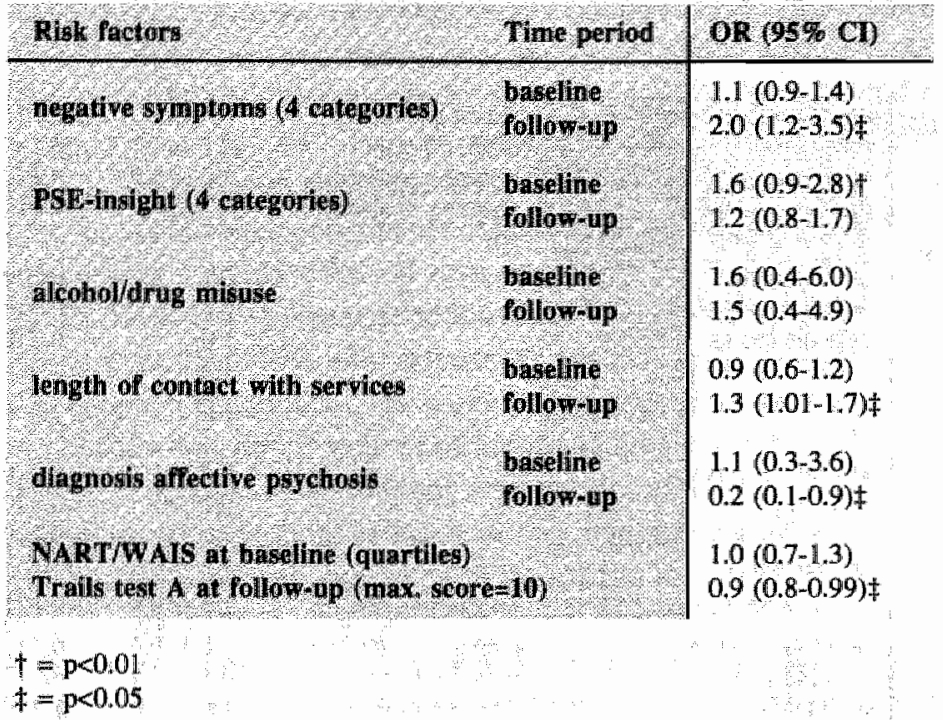

\section{Positive and negative predictive values}

As the PPV and NPV are prevalence dependent (very rare illness is much more difficult to predict because of the inevitably high rate of false positives in the absence of completely specific risk factors), we calculated the PPV and NPV for both narrow (prevalence: 28\%) and broad TD (prevalence: $57 \%$ ) as defined earlier. Non-dichotomous risk factors (proportion of follow-up period on antipsychotic medication, negative symptoms at baseline and insight at baseline) were divided by their median values, so as to create categories of "high" and "low" risk.

Table 4. Independent risk factors for TD, selected using step-up criteria

\begin{tabular}{|c|c|}
\hline Risk Factor & $0 R 05 \% 01$ \\
\hline age at baseline (quartiles) & $1.6(1.1-2.2) \dagger$ \\
\hline femile gender & $0.4(0.2-0.9) \S$ \\
\hline PSt lnight at basellie $(03)$ & $2.0(1.2-3.2) \ddagger$ \\
\hline time on antipsychatics over follow- ip period (quartiles) & $2.3(1.5-3.4) \neq$ \\
\hline megative sknptong at follow-up (quarties) & $1.7(1.2-2.5) *$ \\
\hline Alcoholdaribe mibuse at follow up & $3.0(1.3-7.4) \dagger$ \\
\hline
\end{tabular}


For narnowly defined TD, the possibility to accurately predict later TD on the basis of high values of individual risk factors was low, whereas the possibility to predict absence of TD on the basis of low values of risk factors was quite high (fig. 1 and fig. 2).

Fig. 1 Positive and negative predictive values for six independent risk factors for "narrow" $T D^{\text {" }}$ high NPV and low PPV.

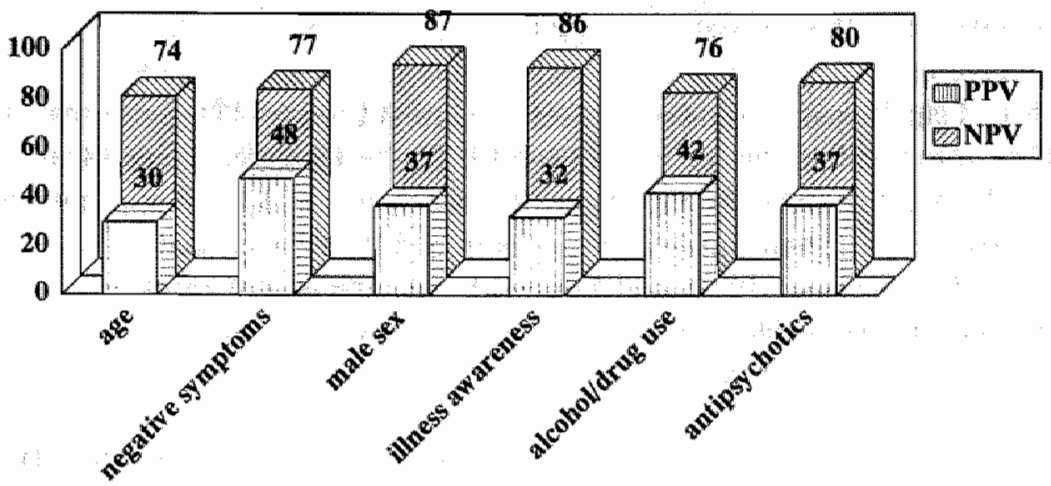

Conversely, for broadly defined TD the PPV was higher than the NPV, although the difference between the two measures was less marked than for narrowly defined TD. Prediction was better in individuals with high values on more than one risk factor. For example, the PPV for broadly defined TD was $100 \%$ in individuals with both negative symptoms at baseline and use of alcohol or drugs. However, the proportion of individuals with both these risk factors was very small $(\mathrm{n}=8 ; 6 \%)$.

Fig. 2 Positive and negative predictive values for six independent risk factors for "broad" TD: high PPV and low NPV

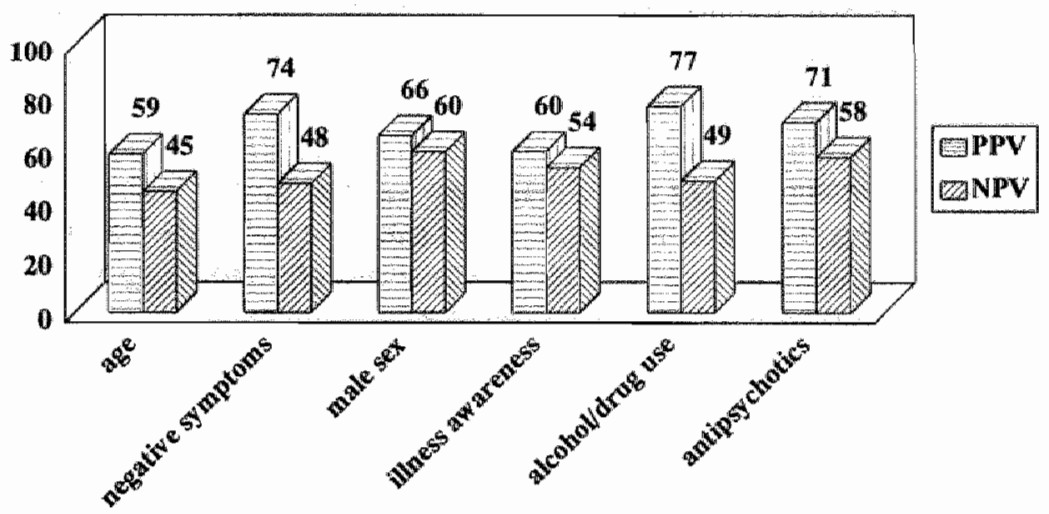

\section{DISCUSSION}

Length of prior exposure to antipsychotic drugs, older age, poor insight at baseline, alcohol/drug misuse (at baseline and at follow-up), and negative symptoms at follow-up, were found to be independently associated with the TD outcome. In addition, risk was higher for men than for women. 


\section{Methodologicall issues}

The AMS is the most frequently used TD scale (Jeste \& Caligiuru,1993), and although it is not specific for TD (Khot \& Wyatt, 1991), the prevalence of dyskinesias due to, for example, senile chorea and Huntington's chorea will have been negligible. Furthermore, the use of this scale facilitates comparison with numerous other studies.

The prevalence of significant movement disorder was low at baseline, but some cases at baseline will have been misclassified due to suppression of TD by increased doses of antipsychotic medication in the acute admission wards. However, our figures of $17 \%$ of moderate movement disorder at follow-up are in agreement with figures from TD incidence studies, with 4-year risk estimates of $18.5 \%$ (Kane $e t$ al, 1986), and 5-year risk estimates of $20 \%$ (Morgenstern \& Glazer, 1993). Therefore, it is reasonable to assume that most cases of movement disorder identified at follow-up were new-onset cases, and not persistent cases from the time of baseline assessment.

Misclassification may have occurred, firstly because some cases could have developed movement disorder over the follow-up period but spontaneously remitted before follow-up assessment; secondly because some cases would have had movement disorder at follow-up which was suppressed by antipsychotic medication. The effect of both types of misclassification, however, would have been to dilute the reported associations. Nonetheless, we do not dispute that a future study should include both baseline and, preferably, serial follow-up TD assessments, which would allow us to evaluate the absolute risk of TD and its risk factors, instead of the cumulative four-year risk reported in this study.

A limitation of this study is that we were not able to include all reported risk factors for TD. For example, acute extrapyramidal side effects (EPS) was not rated at baseline, even though they have been reported to increase the risk of TD (Kane et al, 1986). Measurement of some variables was crude, such as use of drugs and alcohol, but this was necessary to have a comparable measurement at both baseline and follow-up.

Our time window was appropriate, as risk of TD onset is much greater in the first 5 years of exposure to antipsychotics (Glazer et $a l, 1993$ ). Other strengths include the prospective study design and systematic sample collection which was independent of continuing psychiatric service contact at the time of assessment of movement disorder.

\section{Predicting TD}

The sample consisted of individuals who were: young, in the beginning of their psychotic illness and admitted to hospital. This limits comparability with many other studies, which focussed on chronic patients (eg Liddle et al, 1993; O'Hara et al, 1993), and may explain why we did not find effect modification with site of abnormal movement, as reported by other authors (Waddington et al, 1990; Duke et al, 1994). 
- age - Most other prospective studies have also reported an increased risk of emergence or severity of TD with older age (Waddington et al, 1990; Kane et al, 1986; Morgenstern \& Glazer, 1993; Gibson, 1981; Kane et al, 1982), although some did not (Jeste \& Caligiuri, 1993; Chouinard et $a l, 1986$ ). Only one specifically tested for an age-by-sex interaction, which was not significant (Kane et $a l, 1986$ ). Most of these studies reported an inereased risk with duration of antipsychotic exposure, as in our study. Furthermore, our results are indicative of a linear increase with duration of antipsychotic treatment. Selection of patients regardless of outcome and continuing contact, and therefore with widely varying exposures to antipsychotics, is likely to have facilitated the demonstration of such a dose-response relationship (Baldessarini et al, 1988). No association with length of anticholinergic treatment was found, as in the majority of other prospective studies.

- gender - None of the cited prospective studies reported an association with gender, although the authors of one reported a decreased risk in females in another study (Morgenstern et al, 1987), which concurs with our own finding. However, many of the cross-sectional studies reported higher risk for female patients (Kane et al, 1986). The most obvious reason for this discrepancy is selection bias. In samples of chronic patients, especially in-patients, individuals who are not in contact with services because of good outcome and/or recovery, will not be selected. As females generally fare better than males (Chouinard et al, 1986), only relatively unrepresentative individuals with the severest and most chronic illnesses will be included in these samples, while in our sample all the recent-onset, good-outcome cases were included, more of whom were female (Van Os et al, 1995a). Even in the prospective studies cited above, illness duration at baseline was much longer than for our sample, and mean age much higher than in our study: ie these were also patients with established need for long-term intervention, with the exception of the study by Kane and colleagues (1986). Therefore, our finding suggests that male sex is a risk factor for TD in more recent onset patients.

- psychopathology: negative symptoms and cognitive deterioration - Of the above cited prospective studies, only Kane et al (1986) reported an increased risk for individuals with a diagnosis of affective disorder, but they also commented on the generally poor prognosis of such patients, which is in contrast with the follow-up findings in our own sample (Van Os et al, 1995a). In agreement with most of the prospective studies, we did not find an independent effect for affective diagnosis or affective symptoms, and there was no evidence for an interaction with diagnostic category.

In studies where it was examined, the association between negative symptoms and decreased cognitive function on the one hand, and TD on the other was often significant (Liddle et al, 1993; Edwards, 1970; Wegner et al, 1985; Waddington et al, 1985). As adjustment for baseline values in prospective studies is in fact the best way to assess the significance of change in values in relation to the outcome (Frison \& Pockock, 1992), our results indicate that an increase in negative symptoms is associated with onset of TD. Our results echo those of Waddington and colleagues (Waddington et al, 1990), that cognitive deteriaration over the follow-up period predicted the emergence of TD. However, of the two reported associations (cognitive deterioration and increase in negative symptoms) only negative symptomatology was an independent risk factor. The absence of an independent association with cognitive deterioration, however, may be 


\section{REFERENCES}

Andreasen NC, Rice JP, Endicott J, Reich T, and Coryell W. (1986) The family history approach to diagnosis. How useful is it? Archives of General Psychiarry, 43, 421-429.

Baldessarini RJ, Cohen BM and Teichner MH, (1988) Significance of neuroleptic dose and plasma level in the pharmacological treatment of psychosis. Archives of Gemeral Psychiatiny, 45, 79-91.

Bames TRE. (1987) The present status of tardive dyskinesia and akathisia in the treatment of schizophrenia. P pychiatric Developments, 4, 301-319.

Buchanan A. (1992) A two year prospective study of treatment compliance in patients with schizophrenia. Psychological Medicine, 22, 787-797

Caracci G, Mukherjee S, Roth SD et al (1990). Subjective awaneness of abnormal irwoluntary movernents in chronic schizophrenic patients. Amercian Journal of Psychiatry, 147, 295-298.

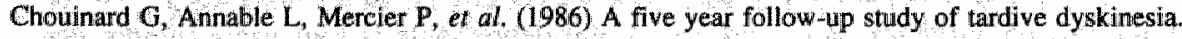
Psychopharnacological Bultetin, 22, 259-263.

Crane GE. (1972) Pseudoparkinson and tardive dyskinesia Archives of Neurology, 27, 426-430.

Cuesta MJ and Peralta V. (1994) Lack of insight in schizophrenia: Schizophrenia Bulletin, 20, 359-366.

Dixon L, Weiden PJ, Haas G et al (1992). Increased Tardive dyskinesia in alcohol-abusing schizophrenic patients. Comprehensive Psychiatry, 33, 121-122.

Duke, PI., Pantelis, C., Barnes, T.R. (1994) South Westminster schizophrenia survey. Alcohol use and its relationship to symptoms, tardive dyskinesia and illness onset. British Journal of Psychiatry, 164, 630-6.

Edwards H. (1970) The significance of brain damage in persistent oral dyskinesia. British Journal of Psychiatry, $116,271-285$.

Fenton WS, Wyatt RJ and McGlashan TH. (1994) Risk factors for spontaneous dyskinesia in schizophrenia. Archives of General Psychiatry, 51, 643-650.

Frison L and Pocock S (1992) Repeated measures in clinical trials, analysis usimg mean summary statistics and its implications for design. Statistics in Medicine, $11_{*}$ 1685-1704.

Gibson AC (1981). Incidence of tardive dyskinesia in patients receiving depot neuroleptic medication. Acta Psychiatsica Scandinavica, 63, $111-116$.

Glazer WM, Morgenstern H, Doncette JT (1993). Predieting the long-term risk of tardive dyskinesia in outpatients maintained on neuroleptic medications. Journal of Clinical Psychiatry, 54, 133-9.

- w.... Morgenstern H and Doucette JT (1991). The prediction of chronic persistent versus intermittent tardive dyskinesia. British Journal of Psychiatry, 158, 822-828.

Guy W. (1976) ECDEU Manual for Psychopharmacalogy, pp 534-537. Washingten, DC, DHEW.

Iager $\mathrm{AC}$, Kirch DG, \& Wyatt R (1985) A negative symptom rating scale. Psychiatry Research, 16, 27-36.

Jeste DV and Caligiuri MP (1993). Tardive dyskinesia. Schizophrenia Bulletin, 19, 303-315.

-.....-. Wyatt RJournal Changing epidemiology of tardive dyskinesia (1981). An overwiew. American Journal of Psychiatry, 138, 297-309.

Johnson D A W, Pasterski G, Ludlow J M, Dtreet K, \& Taylor R D W (1983). The discontinuance of maintenance neuroleptic therapy in chronic schizophrenic patients, drug and social consequences. Acta Psychiatrica Scandinavica, 67, 339-352.

Jones, P.B., Bebbington, P., Foerster, A., Lewis, S., Murray, R., Russel, A., Sham, P., Toone, B. \& Wilkins, S. (1993) Premorbid social underachievement in schizophrenia. Results from the Camberwell Collaborative Psychosis Study. British Journal of Psychiarry, 162, 65-71.

-..n..., Harvey, I. Lewis, S, Toone, B., Van Os, J., Willams, M. Murray, R.M. (1994a). Cerebral ventricle dimensions as risk factors for the functional psychoses. Psychological Medicine, 24, 995-1011.

Kane $\mathrm{JM}$, Woermer M, Weinhold $\mathrm{P}$ et al (1982). A prospective study of tardive dyskinesia development. preliminary results. Journal Clinical Psychopharmacology, 2, 345-349.

........., Woemer M, Bonenstein $M_{n}$ ef al (1986), Integrating incidence and prevalence of tardive dyskinesia. Psychopharmacological Bulletin, 22, 254-258.

-an-.-.-, and Smith JM (1982). Tardive dyskinesia, prevalence and risk factors, 1959 to 1979. Archives of General Psychiatry, 39, 473-481.

Khot, V. \& Wyatt, R.J. (1991) Not all that moves is tardive dyskinesia. American Journal of Psychiatry, 148, $661-666$.

Levin, S. (1984) Frontal lobe dysfunctions in schizophrenia--II. Impaiments of psychological and brain functions. Journal of Psychiatry Research, 18, 57-72.

Lewis, S.W., Owen, M.I., \& Murray, R.M. (1989) Obstetric complications and schizophrenia: methodology and mechanisms. In Schizophrenia - A Scientific Focus (Ed S.C. Schulz \& C.A. Tamminga), pp56-58. Oxford University Press: New York. 
Liddle PF, Barnes TRE, Speller J and Kibel D (1993). Negative symptoms as a risk factor for tardive dyskinesia.

British Joumal of Psychiatiry, 163, 776-780.

McCullagh P (1980). Regression models for ordinal data. Journal of the Royal Statistical Society, 42, 109-142.

McGlashan T (1981), Does attitude toward psychosis relate to outcome? American Journal of Psychiatry. 138 , 797-801.

McGuffin P, Farmer AE, and Harvey I (1991). A polydiagnostic application of operational criteria in psychotic illness, development and reliability of the OPCRIT system. Archives of General Psychiatry, 48, 764-770.

Miller $\mathbf{R}$ and Chouinard G (1993). Loss of striatal cholinergic neurons as a basis for tardive and I-dopa-induced dyskinesias, neuroleptic-induced supersensitivity psychosis and refractory schizophrenia. Biological Psychiatry, 34,713-738.

Morgenstern $\mathrm{H}_{i}$ Glazer WM (1993). Identifying risk factors for tardive dyskinesia among chronic outpatients maintained on neuroleptic medications, mesults of the Yale Tardive Dyskinesia Study. Archives of General Psychiarry, 50, 723-733.

Morgenstern $\mathrm{H}_{4}$ Glazer WM, Gibowski LD, Holmberg S. (1987) Predictors of tardive dyskinesia results of a cross-sectional study in an outpatient population. Journal of Chronic Diseases; 40, 319-27

Nelson HE (1982) National Adult Reading Test (NART), Test Manual. NFER-Nelson, Windsor.

O'Callaghan, E., Larkin, C. \& Waddingtom, J.L. (1990a). Obstetric complications in schizophrenia and the validity of maternal recall. Psychological Medicine, 20, 89-94.

-........, Larkin, C., Kinsella, A. et al (1990b) Obstetric complications, the putative familial-sporadic distinetion, and tardive dyskinesia in schizophrenia. British Journal of Psychiatry, 157, 578-584.

O'Hara P, Brughe T, Lesage A and Wing J. (1993) New findings on tardive dyskinesia in a community sample. Psychological Medicine, 23, 453-465.

Oliveira AA, Kiefer MW and Manley NK (1990). Tardive dyskinesia in psychiatric patients with substance use disorders. Amercan Journal of Drug and Alcontol Abuse, 16, 57-66.

Reitan RM (1958). Validity of the trail making test as an indicator of organic brain damage. Perceptual and Motor Skills, 8, 271-276

Schooler, N.R. \& Kane, JiM. (1982) Research diagnoses for tardive dyskinesia. Archives of General Psychiatry, $39,486-487$.

Spitzer $R_{\text {. Endicott }}$; and Robins E (1978) Research diagnostic criteria, rationale and reliability. Archives of General Psychiatry, 35, 773-782.

STATA corporation (1993) STATA Reference Manual, Release 3.1, 6th Ed. College Station,TX.

Swayze VW, Yates WR, Andreasen NC et al (1988). CT abnomalities in tardive dyskinesia. Psychiatry Research, 26, 51-58.

Van Os J, Fahy T, Jones P. Harvey I, Lewis S, Sham P, Toone BK, Murray RM (1995a). Psychopathological syndromes in the functional psychoses, associations with course and outcome, Psychological Medicine, in press.

-...-, Fahy T, Jones P, Harwey I, Lewis S, Williams M, Toone B, Murray RM (1995b), Increased intracerebral CSF spaces predict unemployment and negative symptoms in psychotic illness, a prospective study. British Journal of Psychiatry, in press.

Waddington JL, Youssef HA, Molloy AG et al (1985). Association of intellectual impairment, negative symptoms, and aging with tardive dyskinesia, clinical and animal studies. Journal of Clinical Psychiatry, 46, 29-33.

-_.-- O'Callaghan, E., Buckley, P. et al (1995) Tardive dyskinesia in schizophrenia. Relationship to minor physical anomalies, frontal lobe dysfunction and cerebral structure on magnetic resonance imaging. British Journal of Psychiatry, in press.

-..-.-. Youssef HA, Kinsella A et al (1990). Cognitive dysfunction in schizophrenia followed up over 5 years, and its longitudinal relationship to the emergence of tardive dyskinesia. Psychological Medicine, $20,835-842$.

-.-.-.-, and Youssef HA (1986). An unusual cluster of tardive dyskinesia in schizophrenia, association with cognitive dysfunction and negative symptoms. American Journal of Psychiatry, 143, 1162-1165.

Wechsler, D (1955) Wechsler Adult Intelligence Scale, Manual. Psychological Corporation, New York.

Wegner JT, Kane JM, Weinhold P et al (1985). Cognitive impairment in tardive dyskinesia. Psychiatry Research, $16,331-337$.

WHO (1992). WHO coordinated multi-center study on the course and outcome of schizophrenia. WHO, Genewa. Williamson, P., Pelz,D., Merskey, H. ef al (1991) Correlation of negative symptoms in schizophrenia with frontal lobe parameters on magnetic resonance imaging. British Journal of Psychiatry, 159, 130-4

Wing JK, Cooper JE, and Sartorius N (11974). The Measurement and Classification of Psychiatric Symptoms. Cambridge University Press, Cambridge.

Wolkin, A., Sanfilipo, M.., Wolf, A.P., et al (1992) Negative symptoms and hypofrontality in chronic 
schizophirenia. Archives of General Psychiatry, 49,959-65.

Yassa R, Jeste DV (1992). Gender differences in Tardive Dyskinesia, a Critical review of the literature. Schizophrenia Bulletin, 18, 701-715.

Zaretsky A, Rector NA, Seeman MV and Fornazzari X (1993). Current carnabis abuse and tardive dyskinesia. Schizophrenia Research, 11, 3-8. 
We examined risk factors for emergence and persistence of psychotic illness, relating these to issues of case definition. We started out from an international perspective, and derived our hypotheses on nosological and aetiological issues from the diversity of views and underlying assumptions that we encountered in European psychiatry. A need was identified for epidemiological groundwork, and we conducted a series of studies trying to clarify issues of case definition and areas of risk in the functional psychoses. We cautioned against adding more thesis to the already hypertrophic collection. However, it is customary, and indeed tempting, to venture to integrate one's findings into a wider framework, from which further testable hypotheses and clinical implications can be derived. We will build further on the extensive literature review in chapter one, (which will not be discussed again in detail here) and provide a tentative empirical model of heterogeneity in risk, psychopathology and outcome in the functional psychoses. "Bottom up" empirical models necessarily lack the acumen of more comprehensive "top-down" models that seek to explain the phenomenon of psychosis from beginning to end. However, they are more flexible, yield more refutable predictions (Murray et al, 1988), and, perhaps most importantly, reduce the risk of selective, self-fulfilling reporting, (Paneth \& Susser, 1995), and selective and/or defensive interpretation of the literature.

\section{General summary and implications of the findings}

- A neurodevelopmental mechanism in the young, and a neurodegenerative mechanism in the elderly best fits our epidemiological findings concerning ageing and the onset of psychosis (chapter 4.1). This would be compatible with evidence of clear differences in the psychopathological profiles of early- and late onset patients (Howard \& Castle, 1993). Thus, to consider the two groups as separate may facilitate our understanding of their aetiology (Castle \& Murray, 1993; Murray et al, 1992), analogous to, for example, the distinction between type I and type II diabetes mellitus on the basis of similar epidemiological and clinical characteristics such as age of onset and familial clustering.

- Dimensional representations of psychopathology have greater predictive validity than categorical representations. In other words, there is better evidence that overlapping psychopathological dimensions, rather than mutually exclusive traditional categories, constitute fundamentally different concepts in the functional psychoses. These results reflect clinical practice in mental health settings, where rigid boundaries in pharmacological and psychosocial treatments do not exist (Johnstone et al, 1988).

Diagnostic categories, when ordered along a continuous scale ranging from affective, acute onset psychosis to insidious onset, non-affective psychosis, are predictive of progressively poorer outcome; we showed that DSM-III-R categories, when ordered in this way, in fact tended to "summarize" underlying psychopathological dimensions, even after adjustment for essential confounding factors. This is shown for three major dimensions in figure 1 . What appears to be most valid is not the individual diagnostic categories, but the order in which they best reflect underlying psychopathological dimensions. Thus, while retaining the typologies, their predictive validity is enhanced by having them occupy different positions on a single illness continuum. Such a single illness continuum has the advantage that it is much easier to work with then with multiple axes requiring complicated statistical procedures, "...straining the resources of a largely 
innumerate and conservative profession" (Kendell, 1993). However, such a summary is imperfect at best, for example, subjects with affective illness can score high on the negative symptom dimension and vice versa (chapter 3.1 ).

Fig. T Ordered DSMAIT $R$ categorles as a crude "summary" of three psychopathological dimensions. Shown are three psychopathological axes ar dimensions (negative symptoms positive symptoms affective simptoms). Poor oucome is associated with high scores on positive and negative axes, and with low scores on The affective aris, DSM $M$-R categories may be viewed as combining elements of the three axtes on one single axis along which the caregonies are ordered according to their association with outcome.

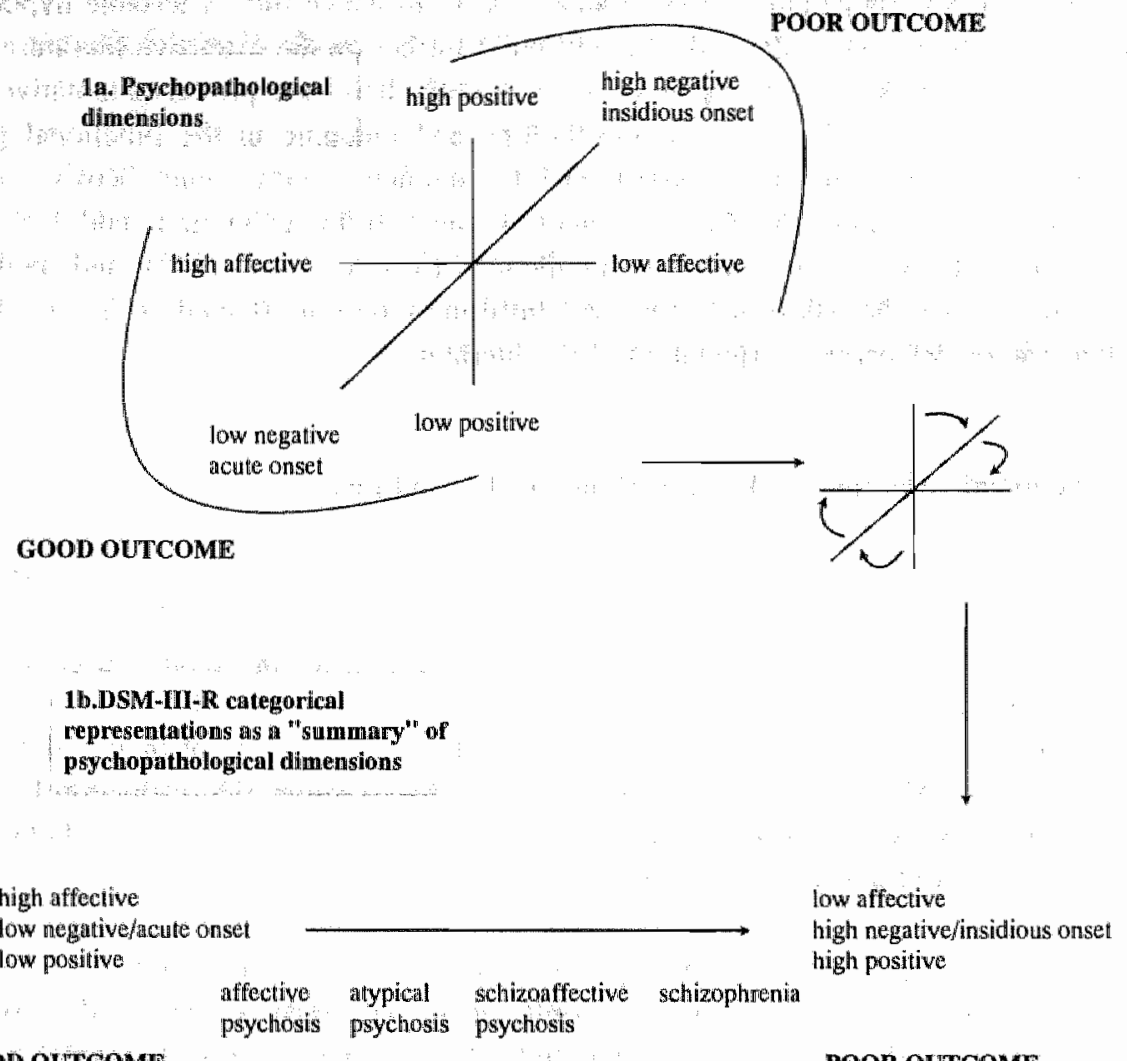

GOOD OUTCOME:

POOR OUTCOME

Randomised clinical trials may prove to be more precise if treatment response on several clinical dimensions is taken into account. Indeed, dimensional representations are increasingly being included in the outcome measures of such trials, as evidenced by claims that certain novel antipsychotics are effective for both positive and negative symptoms.

Similarly, risk factor research may yield more valid results focusing on (overlapping) symptom dimensions rather than categories, along the lines explained below. This approach has already proved its usefullness in biological reserach, and it likely to continue to gain in importance (Liddle et al, 1992; McGuire \& Murray, 1994; Dworkin et al, 1988). In fact, we have shown that it is profitable to examine several diagnostic categories together (the "lumping approach"), and to derive data and interpretations from a group of individuals with psychosis, without adopting unproven taxonomic assumptions (the "splitting approach"). Sadly, the politics surrounding 
categorical representations may be more important than scientific reasoning. For example entire uniwersity departments and grant-giving bodies have been split up along the lines of popular taxonomic systems, with the result that a self-perpetuating machine chums out ever more "evidence" justifying its own existence. Furthermore, the economical interests served by, for example, the DSM system of jointly exhaustive and mutually exclusive diagnostic categories in an insurance-based health care system, may leave room for frequent; increasingly complicated, minor "boundary" changes, but not for any conceptual change.

- Risk factors for emergence of psychosis are also risk factors for persistence of psychosis (figure 2). In other words, risk factors do not only mediate risk for psychosis in general, but for poor outcome psychosis (neurodevelopment, cerebral ventricle dimensions, genetic diathesis; chapter 4.4, 4.5\& 4.6), and good outcome psychosis (life events, ethnic group; chapter 4.1 \& 4.2).

Fig. 2 Risk factors for emergence of illness also predict persistence of illtess

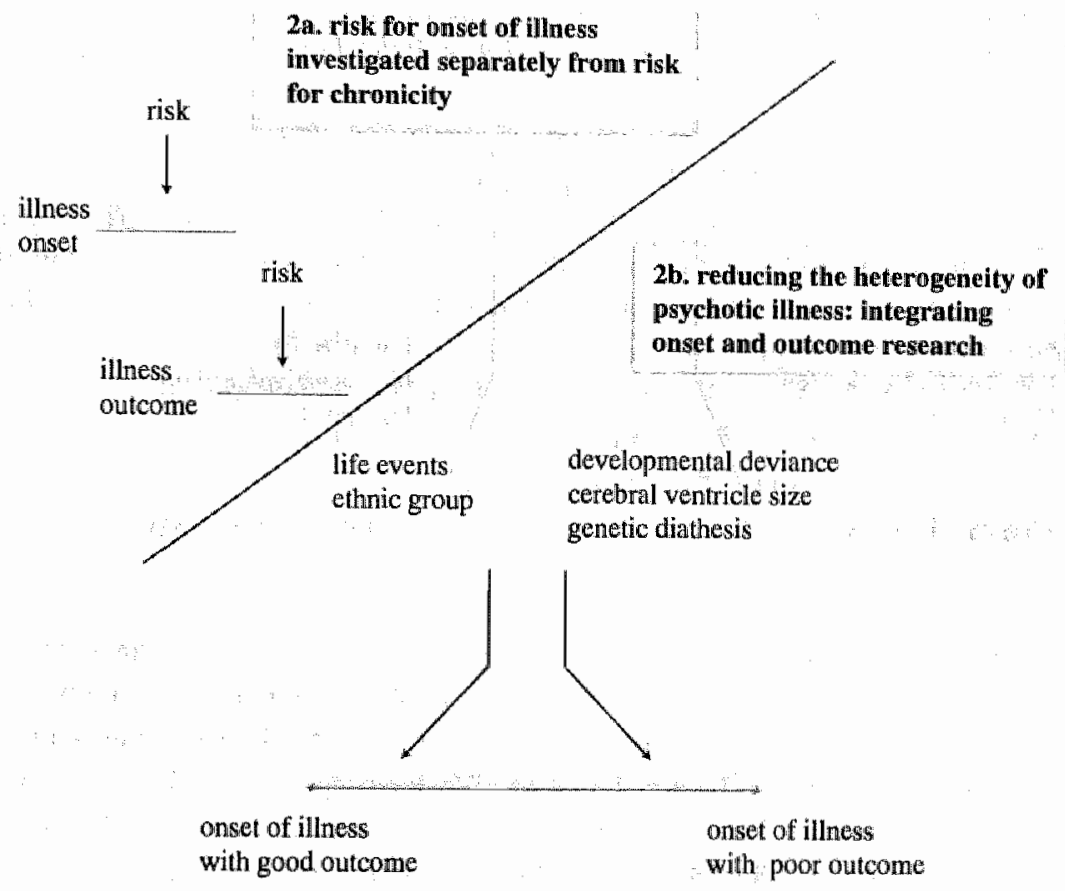

"Good outcome psychosis" should not be interpreted as not in need of preventive strategies. On the contrary, we have shown that, were it not for their poorer social circumstances, the outcome of psychotic illness in ethic minority groups would be much better. Furthermore, the same social adversity may play a role in the increased incidence of psychosis in certain ethnic minority groups (see below), raising an important public health problem which is likely to increase even further in the years to come.

- The effect of some risk factors may be madified by prevailing psychopathological dimension: eg associations between life events and, to a lesser degree, ethnic group on the one hand, and onset and persistence of psychosis on the other, were more prominent in affective illness, but 
for cerebral ventricle dimensions, familial morbid risk and, to a lesser extent, neurodevelopment, such associations were more conspicuous in non-affective illness (figure 3 ; chapter 4 ). Thus, rather than the confusing strategy of reporting effects for risk factor $A$ in category $X$ in one study for readers specilalized in category $X$, and in category $Y$ in another study for a readership specialised in category $Y$, we would argue that more is to be gained from looking for variation in the strength of the association at different ends of a (valid) continuous psychopathological scale. Our strategy has yielded new quantitative and qualitative information, which can be exploited further to address therapeutical and aetiological questions. For example, in chapter 4.5 .2 we suggested that early social, cognitive and motor deficits were possibly the early manifestation of a common predisposition to severe psychiatric illness, with other factors determining the type of aduilt disorder. Our findings suggest that discrete effects may indeed be operating at different ends of a psychopathological continuum, and could "tip" a general predisposition to severe psychiatric illness in a certain psychopathological direction.

Fig. 3 Risk factors for emergence and persistence of illness

interact with psychopathological dimensions. Three Levels are cansidered in the context of a longitudinal perspective. The level of antecedent risk refers to risk factors for emergence of psychosis. These risk factors preferentially operate at one end of a psychopathological continum (level 2), corresponding to a severity or chrovicity continuum (level 3 )

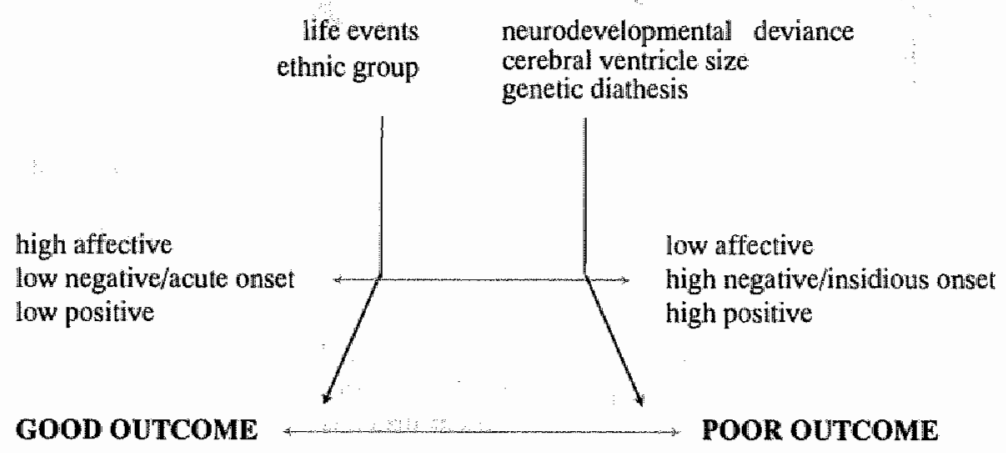

- There is some (preliminary) evidence that risk factors for emergence and persistence of psychosis are associated with genetic predisposition: familial morbid risk is positively associated with premorbid developmental deviance and life events, and negatively associated with cerebral ventricle size (but only in men; chapter $4.1,4.6$ ). We have speculated that the most likely interpretation of these findings is, respectively: i) for premorbid developmental deviance and genetic risk: behavioural endophenotype (ie developmental deficits are markers of genetic transmission); ii) for life events and genetic risk: interactive genetic and environmental effects (genetic and environmental influences separately are not sufficient causes, but in interaction with each other they are); iii) for cerebral ventricle size (in men) and genetic risk: independent genetic and environmental effects (both genetic and non-genetic factors are sufficient causes; for a full discussion on gene-environment interaction, see chapter 1).

The putative relationships between risk factors are summarised in figure 4; we acknowledge that the model of heterogeneity is necessarily simplistic, but has the advantage of being empirical and allowing further investigations of predictions derived from it. 
Of course, this model needs further refinement We have assumed, in accordance with the evidence discussed in chapter 1 and evidence provided in chapter 4.5 .2 , an overlapping genetic predisposition between affective and non-affective psychosis, with the possibility of homotypia in families at the extreme ends of the continuum. As explained in chapter 1, what we refer to here as "genetic predisposition" may best be seen as a pool of polygenes, rather than a single gene. For example, it is unlikely that the (hypothetical) genetic factors involved in gene-life event interaction and neurodevelopmental dewiance as an indicator of genetic risk would involve the same genetic pathways.

One prediction from the model inwolves the mechanism whereby certain ethnic minority groups are at increased risk of psychosis. As life event-related illness is associated with better outcome, and excess of good outcome psychosis associated with ethnic minority membership suggests that the exposure rate to adverse (racial) life events may be higher in such individuals, and account for (at least) part of the reported excess of psychosis (chapter 4.2.1 \& 4.2.2). This is also illustrated is figure 4.

As life events are common and may act interactively with genetic predisposition to increase the risk of psychosis, one would expect, as demonstrated in chapter one, higher rates of illness in the relatives of ethnic minority individuals, compared to the general population. There is already some evidence for such a proposition (although interpreted quite differently - Sugarman \& Craufurd, 1994), and our own work is also suggestive of increased familial morbid risk in ethnic minority individuals with psychotic illness (Hutchinson, personal communication). Although the idea of increased disadvantage resulting in deteriorating mental health certainly has face validity, the actual measurement of stress and life events in ethnic minorities is for anthropological reasons fraught with methodological problems (Creed, 1995), and new, culturally appropriate techniques need to be developed to further examine these issues. The importance of such research lies in the scope for prevention. The existence of gene-environment interaction in the sense that without environmental exposure the increased risk conferred by genes alone is minimal, implies that prevention of environmental exposures is enough to "neutral ise" the genetic risk. Such a scenario is considerably less pessimistic in its preventive scope than assuming the existence of genes that will inevitably lead to illness.

The joint examination of life events and cerebral ventricle dimensions should clarify the relative independence of these risk factors in their relationship with both onset and course and outcome." There is a strong suggestion from the model, however, that this is the case. Firstly, their associations with outcome differed qualitatively as described above: cerebral ventricle size was associated with poor outcome, and life events with comparatively good outcome. Secondly, the effect of both risk factors was modified by diagnostic category, but in opposite directions: the association between life events and illness course was more evident in the non-schizophrenic category, whereas the reverse held for cerebral ventricle dimensions. Thus, the divergent pattern of associations between life events and cerebral ventircle size on the one hand, and psychopathology and outcome on the other, provides evidence for the "discreteness" of their effects.

Similarly, further joint examination of genetic risk and cerebral ventricle size should clarify whether "sporadic" non-affective psychosis in men really exists. 
Fig. 4 Rish factors for emergence and persistence of illness interact with each other. Possible relationships

between antecedent risk factors at level one are depicted geme environutent interaction for life events and genetic risk, premarbid developmental attenuation as an indicator of genetic vulnerability, and, in men, cerebral ventricle size as an indeperdent, enwironmental risk factor. Life events may act to increase the risk of psychosis in certain ethnic groups.

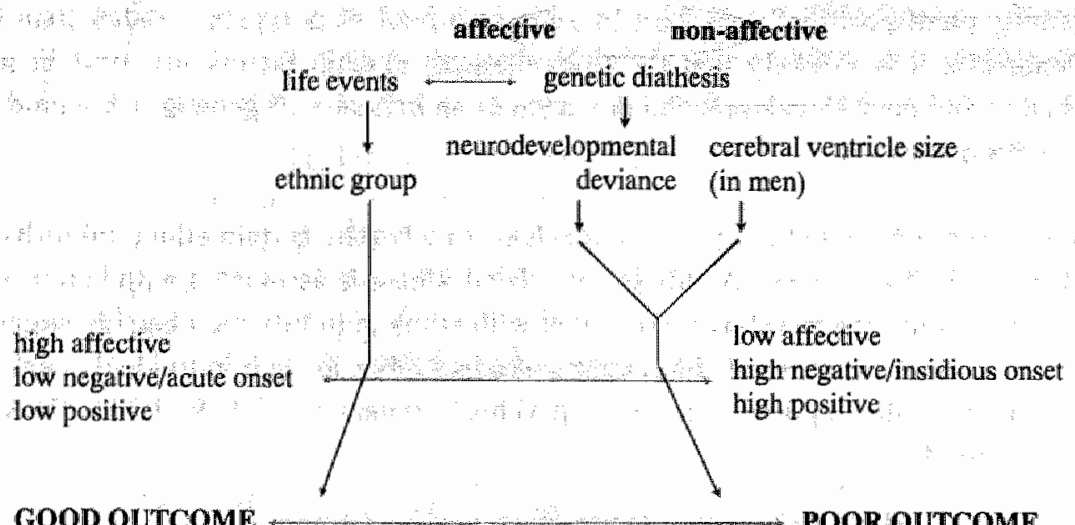

It should be borne in mind that we have shown in chapter one that a negative association between cerebral ventricle size and genetic predisposition does not exclude (weak) interaction between the two risk factors, and while our findings are compatible with the large study of Vita and colleagues (Vita et al 1994,) recent evidence has suggested a positive association between cerebral ventricle dimensions and genetic risk (Cannon et al, 1993). The fact that women may be at greater familial risk than men (Pulver et al, 1992; Goldstein et al, 1992; Sham et al, 1994) would tie in well with the hypothesis of a higher frequency of sporadic form of illness in males. Also, the possibility of "sporadic" illness suggests the possibility of intervention. Thus, the correlates of cerebral ventricle size need to be investigated further, as there is little to suggest any consistent finding in the literature (Jones et al, 1994). On the other hand more specific brain measures may provide better cues between genetic risk and cerebral structure, although as yet the conflicting neuroimaging literature has not provided us with much to go on (Chua \& McKenna, 1995).

- Neurological abnormalities occur both before onset of psychotic illness (eg delayed milestones - chapter 4.5) and after onset of illness (tardive dyskinesia - chapter 5.1). The risk for both types of neurological abnormality is highest in the more severe, chronic form of illness. The occurrence of tardive dyskinesia is likely to involve the interaction between a developmentally compromised central nervous system, and other factors such as ageing and D2 receptor blockade. In other words, tardive dyskinesia is a clinical illness outcome, as well as a medication side-effect. These findings are compatible with the reports discussed in chapter one, describing tardive dyskinesia in unmedicated patients. This has important implications. Tardive dyskinesia is the only outcome in the functional psychoses where the clinician has direct evidence of organic brain dysfunction without the need to perform ancillary investigations. Although there is no well circumscribed neuropathology of this neurological disorder, it is more common in the elderly and the brain damaged, which suggests that (eg degenerative) lesions can be identified (Rogers, 1992). Thus, if the occurrence of tardive dyskinesia can be related to specific illness characteristics within the functional psychoses, then this has direct consequences for both primary prevention as well as for elucidating the underlying mechanisms of psychosis. 
In chapter 5.1 , we reponted that tardive dyskinesia was associated with both cognitive deterioration and deterioration in terms of negative symptoms, which were not independent risk factors and were equally well represented by negative symptoms alone:

One prediction from the model would be that early and later neurological abnomalities are i) related, and ii) in part genetically mediated (figure 5 ).

Fig. 5 The tardive dyskinesia (TD) autcome. TD is more often foumd in those patiens whose allness is characterised by negative symptoms and poor outcome preceded by risk factors such as early neurodevelopmental attenuation and cerebral ventricle enlorgenent This suggests that TD is the expression of a developmentally compromised central nervous system, interacting with the effects of antipsyohotic medication.

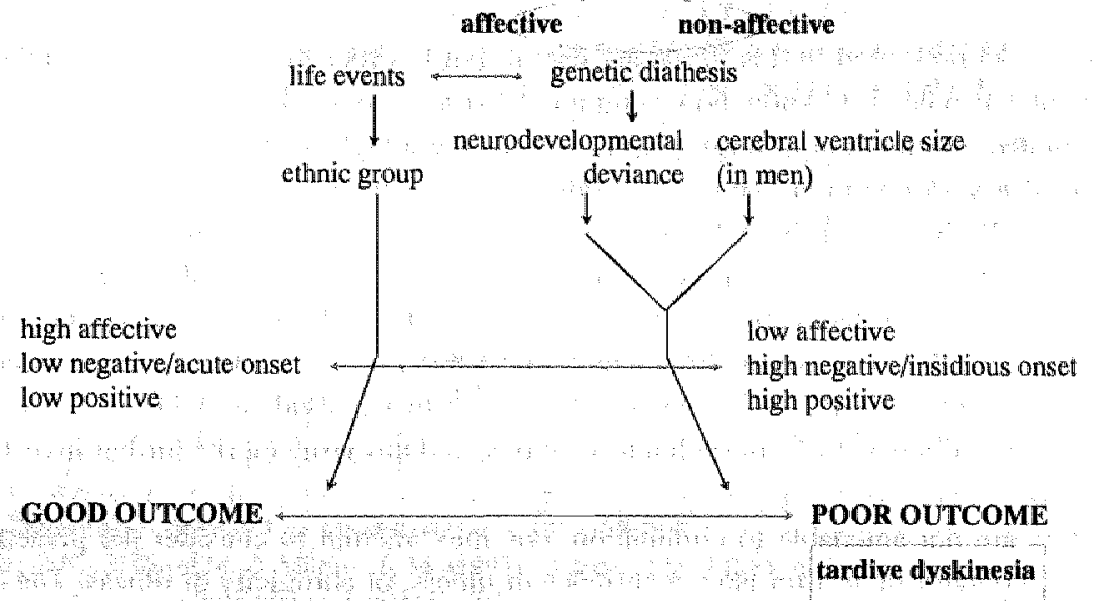

Although we failed to find an association between family history and cumulative incidence of tardive dyskinesia (chapter 5,1), our measure of genetic diathesis was crude (see chapter 4.6.1), and we discussed other work which did show an association between familial morbid risk and outcome of TD.

- We have demonstrated, within the realm of the functional psychoses, i) a continuum of risk for some exposures such as familial morbid risk and cerebral ventricle dimension (continuous risk factors for onset and persistence of psychosis), ii) a continuous illness psychopathology (scored along different normally distributed dimensions), and iii) a continum of severity (continuous outcome measures" showing linear relationships with continuous exposures). These notions are far removed from current thinking in clinical psychiatry, yet they are entirely compatible with nature presenting us virtually everything as a process or continuum rather than a dichotomy. The relationship between blood pressure and occurrence of stroke is linear, and infectious diseases can range from subclinical phenomena to severe disease (Rose, 1992). Studies of "psychotic-proneness" in the general population suggest a continuum from mild abnormality to overt and disabling psychotic symptoms (Claridge \& Brocks, 1984). In other words, risk and severity come in all sizes, not just in two classes. Rose (1992) has discussed the possible implications of the convention of referring to patients as cases of illness. What is really meant in terms of "caseness" is a case for treatment: the yes-no decision is about treatment, not of illness, since illness occurs in all grades of severity and not all individuals with illness manifestations seek medical help, especially in psychiatry. Of course, future investigations may 
find definitive proof for a cut-off somewhere along the psychopathological continuum, which to date has gone undetected because of the crude measures used. The irony is, however, that such a cut-off can never be found unless we start out sampling subjects using a "lumping" approach, ignoring the "splitting" approach dictated by current taxonomic rules.

Thus, dichotomous thinking has its place in clinical practice, but the familiar sight of psychiatrists having sometimes lengthy discussions about a patient's diagnosis according to the latest revision of the prevailing diagnostic system, may just as well make way for discussions about a patient's treatment given certain symptomatic dimensions of a certain severity, ie a more problem-oriented approach as advocated by some institutions (Bosman \& Arends, 1989).

The relative risks presented in this thesis are useful, but in order to evaluate their public health importance one also needs to know how common the risk is, so that the effect of its modification can be evaluated: elimination of a rare exposure carrying a high relative risk is less useful than elimination of a very common exposure carrying a small relative risk. It is difficult, however, to think about elimination of the risk factors we have presented. For example, we do not know enough about the determinants of cerebral ventricle size to start thinking about ways of "eliminating" it as a risk factor for onset and chronicity of psychosis. However, life events are more promising as they are extremely common and a even a small reduction may reduce rates of illness in susceptible individuals; our results suggest that individuals with evidence of high genetic risk especially benefit from such interventions, and this issue merits further investigation.

If risk factors are not amenable to elimination, one may attempt to consider the presence of a risk factor as a diagnostic test for later occurrence of illness, or chronicity of illness. The positive predictive value (PPV) of the test is the probability that a person will develop the disease given a positive test. In chapter 4.5 .2 , we saw that this strategy was not successful in predicting chronic affective disorder from indices of developmental deviance (highest PPV around 50\%). However, these attempts at prediction involved remote exposures of low specificity and widespread overlap with normal population values, and we may be more successful in predicting chronicity within groups of patients, which is the focus of a current investigation. Showing an association is the first step towards enabling clinicians to make a prognostic prediction with a certain degree of certainty, resulting in rational secondary prevention (Van Os, 1995). 


\section{REFERENCES}

Bosman, F.T. \& Arends, J.W. (1989) Teaching pathology in a problem-oriented curriculum: the Maastricht experience. Journal of Pathology, 159, 175-178.

Cannon, T.D., Mednück, S., Parnas, J., Schulsinger, F. Praestolm, J. Vestergaard, A. (1993) Developmental brain abnomalities in the offspring of schizophrenic mothers. I coniributions of genetic and perinatal factors. Archives of General Psychiatry, 50, 551-564.

Chua, S.E., \& McKenna, P.J. (1995) Schizophrena-a brain disease? A critical review of structural and functional cerebral abnornalitiy in the disonder. Britist Journal of Psychiatry, 563-583.

Claridge, G.S. \& Brocks, P. (1984). Schizotypy and hemisphere function - theoretical considerations and the measurement of schizotypy. Persomatity and Individual Differences, 5, 633-648.

Creed, F. (1995) Ethnic minorties and life events. Paper presented at "Culture, Ethnicity and Mental Health", Institute of Psychiatry, 15-17 February 1995.

Dworkin, R.H., Lenzenweger, M.F., Moldim, S.O., Skillings, G.F. \& Levick, S.E. (1988) A multidimensional approach to the genetics of schizophenia. American Journal of Psychiatry $145,1077-83$.

Goldstein, J.M., Faraone, S., Chen, W.J. \& Tsuang, M.T. (1992) Gender and the familial risk of schizophrenia. Disentangling the confounding factors. Schizophrenia Research, 7, 135-140.

Howard, R., Castle, D. Wessely, S. and Murray $\mathbb{R}$. (1993) A comparative study of 470 cases of early and lateonset schizophrenia. British Joumal of Psychiary, 163, 352-357.

Johnstone, E.C., Crow, T.J., Frith, C.D. \& Owens, D.G. (1988) The Northwick Park "functional" psychosis study: diagnosis and treatment response. Lancet, July 19th, 1988.

Murray, $\mathbb{R}$., O'Callaghan, $\mathbb{E}$, Castle, D., et al (1992) a neurodevelopmental approach to the classification of schizophrenia. Schizophrenia Bulletin, 18, 319.332.

-www-... Lewis, S.W., Owen, M.J., et al (1988) The neurodevelopmental origens of dementia praecox. In Schizophrenia: the Major Issues (eds P. Bebbington \& P. McGuffin) pp 90-107. Oxford: Heinemann.

Kendell, R.E., Z Zealley, A. K. (Eds) Diagnosis and classification. In; Companion to Psychiatric Studies, fifth edition, pp 277-295. London: Churchill Livingstone.

Liddle, P., Friston, K., Frith, C., Hirsch, S., Jones T. \& Frackowiak, R. (1992) Patterns of cerebral blood flow in schizophrenia British Jownal of Psychiatry, 160, 179-186.

McGuire, P.K. Shah, G.M. \& Murray, R.M. (1993) Increased blood flow in Broca's area during auditory hallucinations in schizophremia. Lancet ii, $703-6$.

Paneth, N. \& Susser, M. (1995) Early origin of coronary weart disease (the "Barker hypothesis") Hypotheses, no matter how intriguing, need rigorous attempts at refutation. British Medical Journal, 310, 411-412.

Pulwer, A., Brown, C., Wolyniec, P., MoGrath, J., Tam, D., Adler, L., Carpenter, W., and Childs, B. (1990) Schizophrenia: age at onset, gender and familial risk. Acta Psychiamica Scandinavica, 82, 344-351.

Rogers, D. (1992) Motor disorder in psychiatry: towards a neurological psychiatry. New York: Wiley \& sons.

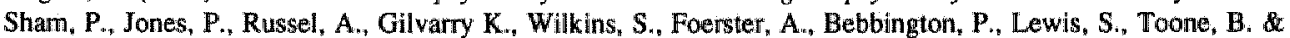
Murray, R. (1994) Age at onset, gender, and familial psychiatric morbidity in schizophrenia. Report from the Camberwell Collaborative Psychosis Study, British Journal of Psychiatry 165, 466-473.

Sugarman, P.A. \& Craufurd, I. (1994) Schizophrenia in the Afro-Caribbean communily. British Journal of Psychiary, $164,474-480$.

Van Os, J. (1995) Een eeuw en meer van psychosemonderzoek. Relevant voor de klinische praktijk? (submitted).

Vita, A, Deici, M., Giobbo, G.M., et al (1994) A reconsideration of the relationship between cereloral structural abnomalities and family history of schizophrenia. Psychiatry Research, 53, 41-55. 


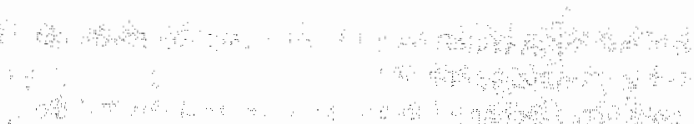

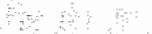

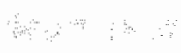

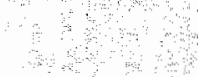

का a 


\section{Summary}

We examined risk factors for i) emergence and ii) persistence of psychotic illness, and related these to the clinical heterogeneity of the functional psychoses. We started out from an international perspective, and derived our hypotheses on aetiological, prognostic and nosological issues from the diversity of views and underlying assumptions that we encountered in European psychiatry. The international psychiatric community appears to disagree about what are the underlying causes of psychotic disorders, what constitutes a case for treatment, and what are the prognostic implications of diagnosing illness. A need was identified for epidemiological groundwork, and we conducted a series of studies trying to clarify areas of risk and prognosis in the functional psychoses (chapter 1).

The following issues were addressed:

- what are the risk factors for emergence of psychosis;

- what are the risk factors for persistence of psychosis;

- how specific are these risk factors with regard to our current nosology of categorical representations of psychopathological phenomena, which generate impressive reliability statistics but remain of questionable validity; how does the predictive vallidity of categorical representations compares with the validity of dimensional representations?

\section{Risk factors for emergence of psychosis}

The following putative risk factors for onset and persistence of psychosis were considered in our review of the literature: age, ethnic group, life events, cerebral ventricle dimensions, childhood neurodevelopmental deviance, and familial morbid risk of psychotic illness. All these factors have been implicated in the onset of illness. However, despite speculation that i) a neurodevelopmental mechanism underlies the emergence of psychosis in younger individuals; and ii) a neurodegenerative mechanism operates to induce psychosis in the elderly, there is little epidemiological evidence to support such opposite mechanisms at the two extremes of the age-atrisk period. Also, reports of increased rates of psychosis in certain ethnic minority groups are subject to serious methodological limitations.

Ageing and ethnic group were therefore further examined with regard to onset of psychotic illiness.

\section{Risk factors for persistence of psychosis}

Our literature review suggests that all of the above risk factors for emergence of psychosis are also predictive of illness chronicity. However, this area remains underresearched, in spite of its possible importance. For example, the finding that the same risk factor is implicated in both onset and outcome of a disease, in such a way that it can be differentiated from other high-risk groups, is suggestive of the existence of discrete major effects and may constitute an important contribution towards elucidating the heterogeneity of underlying mechanisms or possibly even aetiology of functional psychotic illmess. 
as ethnic group, life events neurodevelopment, cerebral ventricle dimensions and genetic diathesis on the one hand, and course and outcome of psychotic illness on the other. We reviewed comprehensively what factors are of particular importance in carrying out and interpreting follow-up studies (chapter 2).

\section{Categorical and dimensional representations of psychopathology}

Current nosological thinking centers on largely a priori systems of categories that are jointly exhaustive and mutually exclusive. Our literature review indicates that studies attempting to establish the predictive validity of categorical representations have been generally unsuccessful. Furthermore, there is little to suggest that risk factors for onset and persistence of illness are entirely specific to any diagnostic category, although there is some interesting evidence that the strength of the associations is modified by the prevailing psychopathology of the particular category.

We wished to compare the predictive validities of dimensional and categorical representations, ie how well they would do in predicting illness course. We also investigated whether a dimensional representation could help clarify the possibility of effect modification by psychopathology in the association between risk factors and emergence and persistence of psychotic illness.

We attempted to find support for the following hypotheses:

\section{- Categorical and dimensional representations of psychopathology}

Hi) The predictive validity of (overlapping) dimensional representations of psychopathology in the functional psychoses is greater than for (mutually exclusive) traditional categorical representations. Categorical representations of psychopathology, when ordered approximately along the axes of these dimensions, in fact show significant linear trends in their associations with measures of course and outcome, indicating that thus ordered they occupy different positions on an illness continuum corresponding to progressively more severe illness.

- Risk factors for emergence of psychosis

Hii) high rates of psychotic illness observed in UK Afro-Caribbeans cannot be entirely attributed to serious methodological shortcomings in epidemiological investigations. However, the surplus risk may be explained, at least in part, by an excess of good-outcome, possibly reactive, psychosis.

Hiii) the relationship between ageing and onset of psychosis supports a neurodevelopmental model in the young, and a neurodegenerative model in the elderly.

\section{- Risk factors for persistence of psychosis}

Hiv) contrasting outcomes exist in high risk groups. good outcome in life-event related illness and illness in high-risk ethnic minority groups, and poor outcome in illness associated with attenuated neurodevelopment, cerebral ventricle size, and familial morbid risk of psychosis. The organic outcome of tardive dyskinesia is also associated with poor outcome illness.

Hv) We further hypothesized that these associations would vary as a function of baseline 
psychopathology: for example, we predicted that the association between life events aind illness onset and illness outcome would be stronger at the affective end of the continuum, whereas the association between cerebral ventricle dimensions and illness onset and outcome would be more apparent at the non-affective end of the continuum.

In the third chapter, we compared the predictive power of categorical and dimensional representations of psychopathology. Factor analysis of the psychopathological features of 166 consecutively admitted patients with functional psychosis of recent onser revealed seven psychopathological dimensions, explaining $63 \%$ of the variance. Five of these seven syndromes bore differential associations with subsequent treatment and illness course, independent of i) associations with DSM-IIR diagnosis, ii) associations with other prognostic factors and ii) associations with the baseline values of outcome variables. The most striking associations were shown for an early and insidious onset syndrome with affective flattening, which predicted a more disabled course of illness on 3 of 4 outcome dimensions, and which was commoner in males and single individuals. A second syndrome, characterised by bizarre behaviour; inappropriate affect, catatonia, and poor rapport showed similar, slightly less striking, associations with illness course as well as with poor childhood sociall functioning. A third syndrome, characterised by positive psychotic symptoms was to a lesser degree associated with poorer outcome, whereas a fourth syndrome distinguished by manic symptomatology predicted a more benign illness course. A fifth syndrome identified by lack of insight predicted more time in hospital and admission under a section of the Mental Health Act over the follow-up period.

It was demonstrated that dimensional representations of psychopathological features were considerably more useful than categorical representations (DSM-II-R and ICD-10) as predictors of illness course and treatment decisions.

The associations between dimensions and outcome were compatible with the consistent finding in the literature that diagnostic categories, when ordered along a continuum ranging from affective, acute onset psychosis to insidious onset, non-affective psychosis, are predictive of progressively poorer outcome. We replicated this finding, and showed that diagnostic categories, ordered in this way, effectively "summarized" underlying psychopathological dimensions. For example, individuals in the schizophrenic category tended to score high on "positive" and "negative" syndromes, and low on affective syndromes; the reverse held for the affective psychoses, whereas the other categories presented with values somewhere in between.

In chapter 4.1.1 We examined the prediction of the neurodewelopmental hypothesis of schizophrenia, that maturational events in the brain at puberty interact with congenital defects to produce psychotic symptoms. As girls reach puberty at a younger age than boys, we predicted that (i) females would show earlier onset of psychotic illness arising around puberty, and (ii) onset of psychosis in females would be rellated to menarche. Analysis of epidlemiological data regarding admission to psychiatric units in a) England over the period 1973 - 1986, b) France over the period 1975-1980, as well as examination of 97 psychotic adolescents referred to an adolescent unit over a 14 year period, supported both these propositions.

We subsequently (chapter 4.1.2) tested the related hypothesis that the expression of schizophrenic psychopathology is dependent on the stage of adolescent development. The study had a retrospective design, using high-quality case-note material of cases of schizophrenia at first 
admission. One-hundred-and-eighty patients with onset of illness between age 11 and age 21 years were included. There was evidence that the age at which positive symptoms first appeared differed between males and females. The frequency of typical, "first rank", schizophrenic symptoms such as auditory hallucinations, passivity phenomena and thought interference, increased linearly with age in male patients, but did not vary with age in their female counterparts. The likelihood of displaying delusional beliefs such as persecutory delusions, explanatory delusions, delusions of reference and grandiose delusions increased with age in both sexes, but the association was stronger in males.

The observation that typical schizophrenic symptoms in male patients are relatively uncommon during early adolescence, but increase in frequency as they grow older, could be explained by the later manifestation of puberty and associated maturational processes in boys.

In chapter 4.1 .3 we focussed on the other end of the age range, examining the association between ageing and administrative incidence rate of late onset (after age 59) non-organic, nonaffective psychosis in two samples of patients aged 60 years or older, who were first admitted to hospital in i) the Netherlands between 1978 and $1992(n=8010)$, and ii) nine Regional Health Authorities in England and Wales ( $n=1777)$ between 1976 and 1978. There was a linear trend in the association between increasing age and first admission rates for non-organic, non-affective psychosis in the elderly, after adjustment for the possible confounding effects of time trend and gender, corresponding to an $11 \%$ increase in the incidence with each five year increase in age.

These observations support a connection between degenerative brain processes and onset of nonaffective psychosis in the elderly.

In chapter 4.2.1, we investigated whether reports of high rates of severe mental illness among ethnic minority groups could be explained by underenumeration in census data. Age and sexadjusted first admission rates for operationally-defined schizophrenia and other non-affective psychosis in different ethnic groups were calculated over the period 1988-1992, in a defined catchment area in South London. Standardized rates for schizophrenia, corrected for age- and gender-related under-reporting in the 1991 census, and a $20 \%$ underestimate of the size of the ethnic minority populations in the area, were not only higher in the Afro-Caribbean group (SMR: 3.1; 95\% CI: 2.0-4.7), but also in the African group (SMR: 4.2; 95\% CI: 2.8-6.2). It was further found that higher rates were not specific to schizophrenia among the non-affective psychoses.

These findings suggest that underenumeration cannot explain the observed excess risk of nonaffective psychotic illness in ethnic minority groups.

In chapter 4.2 .2 , the specificity of the association between ethnic minority membership and nonaffective psychosis was examined. First contact rates for mania in the defined area of Camberwell, South London, between 1965 and 1984 were established. It was shown that individuals of Afro-Caribbean origin showed significantly higher rates than the White group (rate ratio: 3.2; $95 \% \mathrm{CI}$ 1.5-7.4), and more often displayed mixed manic and schizophrenic symptomatology (risk ratio: $2.2 ; 95 \% \mathrm{CI} 1.1-4.3$ ). 
High rates of mental illness among members of ethnic minorities are not specific to schizophrenia, suggesting that a risk factor common to both manic and schizophrenic illness is more prevalent among these groups.

In chapter 4.2 .3 we compared course and outcome of psychotic iliness between two groups of Afro-Caribbean $(n=53)$ and British-born White individuals $(n=60)$, in a cohort study of consecutive admissions fulfilling study criteria, followed-up for 4 years. Patients who were admitted to two South-London hospitals with recent-onset psychotic illness were assessed prospectively on severity of course of illness, self-harm, social disabillity, hospital use and treatment variables, adjusted for socio-economic origin. Our results showed that the AfroCaribbean group spent more time in a recovered state over the follow-up period, (adjusted $\mathrm{OR}=7.2 ; 95 \% \mathrm{CI}: 1.7-14.5$ ), were less likely to have had a continuous illness course (adjusted $\mathrm{OR}=0.3 ; 95 \% \mathrm{CI}: 0.1-0.8$ ), were less at risk of self-harm (adjusted $\mathrm{OR}=0.2 ; 95 \% \mathrm{Cl}: 0.1-0.7$ ), and less likely to have been preseribed antidepressant treatment (adjusted $\mathrm{OR}=0.3 ; 95 \% \mathrm{CI}: 0.1$ 0.9). There were no differences in use of hospital, but the Afro-Caribbean group had more involuntary admissions (adjusted $\mathrm{OR}=8.9 ; 95 \% \mathrm{CI}: 2.1-35.6$ ), and more imprisonments (adjusted $\mathrm{OR}=9.2 ; 95 \% \mathrm{CI}: 1.6-52.3)$.

The observed excess risk of psychosis among the UK Afro-Caribbean group can in part be explained by an excess of good-outcome illness, possibly associated with a high level of exposure to social stress.

In chapter 4.3.1, we examined the relationship between stressful life events and illness course. Fifty-nine psychotic patients with acute onset of illness, who had been interviewed about their experience of stressful life events before the episode, were followed up for an average of 42 months. Thirty patients $(51 \%)$ had experienced a stressful life event in the three months immediately before onset $(\mathrm{EV}+), 29$ had not (EV-).

In patients with an RDC diagnosis of affective disorder or unspecified functional psychosis, the presence of stressful life events was associated subsequently with milder symptom severity, less time spent in hospital, more treatment for depressive symptoms and less for psychotic symptoms. In the schizophrenia category, differences were less apparent, but patients with event-associated episodes had less need of anti-psychotic maintenance medication over the follow-up period and tended to have spent more time in complete remission.

Examination of familial morbid risks of illness in the relatives of probands with and without life events, yielded evidence compatible with gene-environment interaction. There was no evidence of discrete subtypes of independent environmentally and genetically mediated illnesses.

In chapter 4.4.I, the proposal that cerebral ventricle dimensions are a risk factor for poor outcome in psychotic illness was scrutinized. A cohort of 140 patients with functional psychoses of recent onset who had undergone CT-scanning, were followed-up for an average of 46 months and assessed on six dimensions of course and outcome of illness. Taking into account multiple testing procedures and the possible effect of outliers, we were able to show that left and right sylvian fissure volumes and, to a lesser extent, third ventricular volume predicted negative symptoms and unemployment over the course of the follow-up period, the latter association being mediated by poor cognitive functioning. There was a significant linear trend in risk over 
the sylvian fissure volume distribution in the cohort, and associations were especially evident in schizophrenic patients.

These findings support the notion that cerebral ventricle dimensions are a continuous risk factor for some dimensions of outcome in the functional psychoses, especially in schizophrenia.

In chapter 4.5 .1 , we wished to examine the hypothesis that differences in outcome among affective and non-affective psychoses are associated with differences in the degree of developmental deviance. We studied the case notes of 545 patients representing $88 \%$ of all patients with non-depressive functional psychoses, residing in a South London catchment area, who received their first psychiatric treatment between 1965-1984. Cases were classified according to the relative chronicity of their illness into four non-overlapping groups: mania, schizomania, acute schizophrenia, chronic schizophrenia.

There was a linear trend in the association between illness chronicity and proxy measures of developmental deviance, such as pre-psychotic unemployment, single status and poor academic achievement. Compared to individuals with mania, schizophrenic patients had a $3-6$ times increased risk of pre-psychotic deviance. For patients with schizomania and acute schizophrenia, the risk was 1.5-3 times greater than for manic subjects.

We conclude that the prevalence of developmental attenuation is highest among chronic schizophrenia, but similar disturbances also occur, to a lesser degree, in less disabling affective and non-affective psychotic disorders.

In chapter 4.5.2, we took the issue of childhood development and outcome further and predicted that a group of patients with chronic, severe affective disorder would show similar developmental attenuation as schizophrenic patients. The study examined associations between prospectively collected childhood data on the one hand, and chronic affective disorder and schizophrenia on the other, in a prospectively studied national British birth cohort of 5262 individuals born in the week March 3-9th 1946.

Seventy-five cases (prevalence: $2.3 \%$ ) with chronic affective disorder (CAD) were identified using a mental health survey at ages 36 and 43 . Thirty cases with schizophrenia or schizoaffective disorder (SZ), had been identified previously.

SZ cases and CAD cases showed the same developmental deficits, but significant interaction with gender was present in the latter. Attainment of motor milestones was later in female CAD cases, with a significant linear trend over the population distribution ( $\mathrm{OR}=1.5 ; 95 \% \mathrm{CI}: 1.1-2.2)$, followed by greater risk of speech defects between the ages of 6 and 15 years (OR=3.6; 1.8-7.5). At ages 8,11 and 15 years, educational test scores differentiated between CAD cases and controls, such that the lower the test scores, the more likely children were to develop later CAD: Again effects were more evident in women. At ages 13 and 15, CAD cases were more likely to be rated "persistently sad and gloomy" by their teachers (OR's $2.7 \& 2.5 ; \mathrm{p}<0.05$ ), and as "apathetic" by the medical examiner between the ages of 6 and 11 years (OR=5.0; 95\% CI:1.714.4).

By age 43 years, men were more likely to develop schizophrenia $(O R=1.8 ; 0.9-3.9)$, whereas 
women were more at risk of developing $\mathrm{CAD}(\mathrm{OR}=2.6 ; 1.6-4.3)$. The were few qualitative differences between $C A D$ and $S Z$ cases in the associations with developmental variables and later psychiatric disorder, some quantitative differences, however, were evident.

Unless the measures used are too crude and tangential to differentiate between severe affective disorder and schizophrenia, the results suggest that early social, cognitive and motor deficits are either the early manifestation of a unitary syndrome with poor outcome and gender-related pathoplastic effects on symptomatology, or the early manifestation of a common predisposition to severe psychiatric illness, with other factors determining the type of adult disorder.

In chapter 4.6.1, we examined the suggestion that, in psychotic illness, an association exists between family history of schizophrenia and poor outcome on the one hand, and family history of affective disorders and good outcome on the other. We tested for associations between fouryear outcome and famillial loading for psychotic disorders in a sample of 150 consecutively admitted patients with functional psychosis of recent onset. For each proband, a familial loading score for i) broadly defined psychotic disorder, ii) schizophrenia, and iii) affective disorder was calculated using information on relatives obtained through the Family History-Research Diagnostic Criteria method and direct interviews of relatives with SADS-LA.

In our sample of psychotic patients, famillial loading for psychotic disonder predicted persistent negative symptoms over the follow-up period (OR: $1.5 ; 95 \% \mathrm{CI}: 1-2.2$ ), and was also associated with more time hospitalised $(p<0.02)$, and more social disability at follow-up $(p<0.06)$, especially in schizophrenia. Greater familial loading for schizophrenia predicted a lower likelihood to recover $(\mathrm{OR}=2.2 ; 95 \% \mathrm{CI}: 1.14 .4)$ and a greater likelihood to have had persistent negative symptorns over the follow-up period ( $\mathrm{OR}=1.7 ; 95 \% \mathrm{CI}: 0.9-3.1)$. No association was found between outcome and familial loading for affective disorder.. Familial loading was associated with other, independent, predictors of outcome such as childhood social development (positive association), but controlling for these did not affect the results.

We conclude that familial loading is a continuous risk factor for clinical outcome in the functional psychoses, especially in schizophrenia.

In chapter 5, we wished to clarify prospectively the relationship between tardive dyskinesia (TD) and its many reported risk factors, especially for risk factors that vary over time, such as aspects of psychopathology and cognitive functioning, and their temporal relationship with regard to the emergence of TD. The sample consisted of 166 subjects with recent onset of psychotic illness and brief previous exposure to antipsychotic medication. Information on 17 reported risk factors was collected at baseline and, for factors that vary over time, again at follow-up 4 years later. Movement disorder was assessed at follow-up with the Abnormal Involuntary Movements Scale. Analysis using step-up criteria revealed that only six variables were independently associated with TD outcome: male sex $(\mathrm{OR}=2.5 ; 95 \% \mathrm{CI}$ : 1.1-5.0), age (OR over quartiles at baseline: 1.6; 95\% CI: 1.1-2.2), lack of insight at baseline (OR over four categories: 2.0 ; 95\% CI: 1.2-3.2), time on antipsychotics over the follow-up period (OR over quartiles: 2.3 ; 95\% CI: 1.5-3.4), an increase in negative symptoms over the follow-up period (OR over quartiles: $1.7 ; 95 \%$ CI: 1.2 2.5 ), and alcohol/drug misuse at follow-up ( $\mathrm{OR}=3.0 ; 95 \% \mathrm{CI}: 1.3-7.4)$.

The results indicate that apart from age, antipsychotic medication and use of alcohol and drugs, 
risk for abnomal movements is also mediated independently by male sex, lack of illness awareness, and illness deterioration. These latter three factors suggest that, among the functional psychoses, illness at the severe end of the spectrum carries a higher risk of "organic" outcomes, providing support for the notion of a developmentally comprised central nervous system in such disorders,

In the epilogue, our findings are reviewed. We conclude that the epidemiological evidence supports a neurodevelopmental mechanism in the young and a neurodegenerative mechanism for onset of psychosis in the elderly. Within the former group, however, there remains considerable heterogeneity at the level of antecedent risk, psychopathology, and outcome. We provide a tentative, empirical model for the heterogeneity at the three levels involving risk factors for good outcome psychosis (eg life events) which operate especially, but not exclusively, at the affective end of an illness continuum, and risk factors for poor outcome illness (eg cerebral ventricle dimensions, genetic diathesis), which are more evident at the non-affective end of an illness continuum. The model allows for interaction between risk factors; three types of relationship between genetic risk and other risk factors are considered, such as interactive effects of genetic predisposition and life events, and early developmental deviance as a marker of genetic transmission. 


\section{Samenvatting}

Onderzocht werd welke factoren een mogelijk risico inhouden op i) het optreden, ii) het voortduren van psychotische stoomissen; tevens werd getracht na te gaan of statistische verbanden met risicofactoren variatie vertonen al naar gelang het klinische beeld wisselt. We gingen uit van een internationaal perspectief, en onze hypothesen omtrent de aetiologie, nosologie en prognose van psychotische stoornissen werden ontleend aan de opzienbarende diversiteit aan gezichtspunten en onderliggende veronderstellingen die in de Europese psychiatrie te vinden zijn. Daar vele van deze overtuigingen niet op een epidemiologische basis stoelen, werd een reeks onderzoeken uitgevoerd in een poging om opheldering te verschaffen omtrent de risicofactoren en de klinische wariabiliteit in de functionele psychosen,

We trachtten antwoord te geven op de volgende vragen:

- Wat zijn de risicofactoren voor het ontstaan van psychose?

- wat zijn de risicofactoren voor het voortduren van psychose?

- hoe specifiek zijn deze risicofactoren met betrekking tot de modeme nosologie van categorische weergaven wan psychopathologische verschijnselen; wat is de voorspellende validiteit van de categorische indeling in vergelijking met een dimensionale weergave van de psychopathologie?

\section{Risicofactoren met betrekking tot het ontstaan van psychose}

De volgende mogelijke risicofactoren voor het ontstaan en voortduren van psychose werden bij ons literatuuronderzoek betrokken: leeftijd, etnische groep, life events, afmetingen van de cerebrale ventrikels, vroege stoomissen in de neuro-ontwikkeling en familiair woorkomen van psychotische stoornissen. Al deze factoren worden verondersteld het risico op psychotische stoomissen te verhogen, maar er blijven onduidelijkheden bestaan. Zo ontbreekt bijvoorbeeld elk epidemiologisch bewijs voor de veronderstellingen dat i) een stoomis in de neuro-ontwikkeling in jonge individuen, en ii) een neurodegeneratieve stoornis in oudere mensen ten grondslag ligt aan het ontstaan van psychotische stoomissen. Ook rapporten over frequenter vórkomen van psychose bij bepaalde etnische minderheden vertonen ernstige methodologische tekortkomingen.

De factoren 'ouder worden' en 'etnische groep' worden daarom nader onderzocht met betrekking tot het ontstaan van psychotische stoomissen.

\section{Risicofactoren met betrekking tot het voortduren van psychose.}

Ons literatuuronderzoek (eerste hoofdstuk) suggereert dat alle bovengenoemde risicofactoren op het gebied van het ontstaan van psychose ook wijzen op een chronisch verloop van de ziekte. Dit gebied is echter nog steeds te weinig geëxploreerd, ondanks het mogelijke belang ervan. Zo kan bijvoorbeeld de conclusie dat eenzelfde risicofactor werkzaam is bij zowel het ontstaan als bij het verloop van een ziekte, en wel op zo'n manier dat het onderscheiden kan worden van andere factoren, wijzen op belangrijke, onafhankelijke effecten, en aldus een bijdrage leveren aan pogingen klaarheid te brengen in de heterogeniteit van de onderliggende mechanismen of aetiologie van de functionele psychosen. 
Daarom concentreren de meeste onderzoeken van dit proefschrift zich op het verband tussen risicofactoren als etnische groep, life events, afmetingen wan de cerebrale ventrikels, neurologische ontwikkeling en genetische diathese enerzijds, en verloop van psychotische ziekte anderzijds. Daarbij besteden wij uitgebreid aandacht aan de methodologische factoren die voor de uitvoering en de interpretatie van beloopsonderzoek van belang zijn (tweede hoofdstuk).

\section{Categorische en dimensionale weergaven van de psychopathologie.}

Het moderne nosologische denken richt zich op een goeddeels a priori systeem van elkaar wederzijds uitsluitende en tezamen het gehele terrein bestrijkende categorieën. Een literatuurstudie van de relatie tussen deze a priori psychiatrisch-diagnostische constructies enerzijds en het ziekteverloop in de functionele psychosen anderzijds suggereerde dat i) het patroon van verbanden tussen de klassieke typologische constructies en het ziekteverloop niet in overeenstemming is met de opvatting dat diagnostische categorieên syndromale discontinuitteit vertegenwoordigen. Zo is bijyoorbeeld de prognose van de affectieve psychose beter dan die van de schizofrenie, terwijl de zogenoemde schizoaffectieve stoornis daar ergens tussenin ligt, hetgeen suggereert dat er kwalitatieve, maar geen kwantitatieve verschillen bestaan. Het is mogelijk, als men het ziektebeloop als validerend criterium neemt, dat deze stoomissen onderdeel zijn van een ziektecontinuüm, en geen separate ziekteëntiteiten vertegenwoordigen. Pogingen om psychotische stoornissen te valideren zouden zich dus wellicht kunnen richten op de validatie van een systeem, waar individuele patiënten een positie wordt toebedeeld op én of meer ononderbroken assen of dimensies.

Er is verder weinig dat er op wijst dat de risicofactoren voor ontstaan en voortduren van psychotische ziekte geheel specifiek zijn voor een bepaallde diagnostische categorie, hoewel er een interessante aanwijzing is dat de sterkte van het verband verandert met de overheersende psychopathologie van die bepaalde categorie.

We wilden de voorspellende waarde van de dimensionale en categorische voorstellingen vergelijken, dat wil zeggen hun bruikbaarheid om het verloop van de ziekte te voorspellen. Ook onderzochten we of een dimensionale weergave meer licht kan werpen op de observatie dat het verband tussen risicofactoren en het ontstaan en het voortduren van psychose niet specifiek is voor een bepaalde diagnostische categorie, maar wel kan variëren in sterkte al naar gelang de belangrijkste psychopathologische component van de diagnostische categorie.

We trachtten bewijs te vinden voor de volgende hypothesen:

\section{- Categorische en dimensionale weergaven van de psychopathologie}

Hi) De voorspellende waarde van (elkaar overlappende) dimensionale weergaven van de psychopathologie van de functionele psychose is groter dan die van de traditionele (elkaar wederzijds uitsluitende) categorische voorstellingen. Als de categorische voorstellingen van de psychopathologie ruwweg langs de assen van én of meer van deze dimensies worden gerangschikt, tonen ze wat het ziektebeloop betreft feitelijk een lineaire trend, waarbij hogere posities aan de assen bepalend zijn voor toenemend ongunstige prognose. Zo'n lineaire relatie wijst op een ziektecontinuüm, dat overeenkomt met een progressief ernstiger ziekteverloop. 


\section{- Risicofactoren voor het ontstaan van psychose}

Hii) De hoge incidentie van psychotische stoornissen onder de etnische groep van Afro-Caribięrs in het Verenigd Koninkrijk zijn niet alleen het resultaat van methodologische gebreken in epidemiologische onderzoekingen en is een authentieke bevinding. Het verhoogde risico betreft echter vooral de acute, wellicht reactieve, psychosen met een gunstige prognose.

\section{- Risicofactoren met betrekking tot het voortduren van psychose}

Hiii) In sommige zogenaamde "high-risk" groepen is er sprake van een karakteristiek ziekteverloop: goede prognose bij ziekte voorafgegaan door life events en in etnische minderheden met en hoog risico, slechte prognase bij ziekte voorafgegaan door ontwikkelingsstoornissen, vergrote hersenventrikels en genetische predispositie. Centralle neurologische complicaties als de tardieve dyskinesie gaan samen met net als ventrikeldimensies, een ongunstig ziekteverloop.

Hiv) We onderzochten verder de hypothese dat deze effecten zouden worden gemodificeerd al naar gelang de overheersende psychopathologie: we veronderstelden bijvoorbeeld dat de associatie tussen life events en ziekteverloop vooral merkbaar zou zijn aan het affectieve einde van een psychopathologisch continuüm, terwijl het verband tussen afmetingen van de cerebrale ventrikels en ziekteverloop duidelijker zou zijn aan het niet-affectieve uiteinde.

In het derde hoodstuk werden de predictieve waarden van dimensionale en categoriale weergaven van psychopathologisehe verschijnselen met elkaar vergeleken. Een factorièle analyse van de psychopathologische karakteristieken wan 166 suecessievelijk opgenomen patiënten suggereerde zeven onderliggende psychopathologische dimensies, die $63 \%$ van de variantie verklaarden. Vijf van de zeven syndromen waren differentieel geassocieerd met latere behandeling en ziekteverloop, onafhankelijk van i) verbanden met $\mathbb{D S M}$-III- $\mathbb{R}$ diagnose, ii) verbanden met andere prognostische factoren, en ii) verbanden met de beginwarde van ziekteverloopwariabelen. De meest opvallende verbanden werden aangetoond voor een syndroom gekarakteriseerd door een sluipend ontstaan en affectieve vervlakking, dat voorspellende waarde had voor drie van de vier dimensies van het ziekteverloop, en dat vaker voorkwam by mannen en alleenstaanden. Een tweede syndroom werd gekarakteriseerd door bizar gedrag, catatonie, incongruent affect en distantie in het interpersoonlijk contact. Dit syndroom vertoonde vergelijkbare, maar minder sterke verbanden met het ziekteverloop alsmede met het premorbide sociale functioneren. Het derde syndroom bestond vooral uit zogenaamde positieve psychotische symptomen, en was in mindere mate geassocieerd met een ongunstig ziekteverloop, terwijl een vierde, manisch, syndroom een prognostisch relatief gunstig voorspellende waarde had. Een vijfde syndroom werd gekarakteriseerd door afwezigheid van ziektebesef, en was geassocieerd met langere opnameduur en gedwongen opname gedurende de studieperiode.

De voorspellende waarde van de dimensionalle weergave van psychopathologische verschijnselen bleek, vergeleken met haar (ICD-10 en DSM-III-R) categoriale tegenhanger, aanzienlijk beter; zowel wat het ziekteverloop als wat de klinische behandelingsvormen gedurende de studieperiode betrof.

De samenhang tussen deze psychopathologische dimensies en het ziekteverloop zijn compatibel met de consistente bevinding in de literatuur, dat de categoriale weergave van psychopathologische verschijnselen, indien gerangschikt langs een continuüm reikend van 
acuut/affectieve en sluipend/niet affectieve ziekte, overeenkomt met een progressief ermstiger ziekteverloop. Deze bewindingen werden gerepliceerd, en tevens werd aangetoond dat deze wijze wan categoriale rangschikking in feite neerkomt op een globale "samenvatting" van de onderliggende psychopathologische dimensies. Bijvoorbeeld, patiënten in de DSM-III-R categorie "schizofrenie" scoorden hoog in "positieve" en "negatieve" dimensies, maar laag in affectieve dimensies; het omgekeerde was het geval voor de categorie van de affectieve psychosen, terwijl de andere cattegorieën waarden ergens tussenin vertoonden.

In het hoofdstuk 4.1.1 werd getracht de "neurodevelopmental hypothesis" van de schizofrenie te toetsen volgens deze theorie zouden ontwikkelingsprocessen in de hersenen tijdens de puberteit in interactie met congenitale defecten psychotische symptomen veroorzaken. Aangezien de puberteit bij meisjes op jongere leeftijd begint dan bij jongens, stelden wij dat i) bij vrouwen psychotische ziekten rond de puberteit eerder zou ontstaan, en dat ii) het ontstaan van psychosen bij vrouwen zou samenhangen met de menarche. Analyse van epidemiologische gegevens met betrekking tot opname in psychiatrische afdelingen in a) Engeland gedurende de periode 1973 1986, b) Frankrijk in de periode $1975-1980$, en een klinisch onderzoek van 97 psychotische adolescenten, bevestigden beide stellingen.

Getest werd tevens de verwante hypothese dat de vorm waarin de schizofrene psychopathologie zich uit afhankelijk is van de graad van volwassenheid van de patiënt (hoofdstuk 4.1.2). Het onderzoek was retrospectief van opzet. $\mathrm{Er}$ werd gebruikt gemaakt van betrouwbaar dossiermateriaal van schizofreniegevallen bij de eerste opname; het betrof 180 patiënten bij wie de ziekte zich openbaarde op een leeftijd tussen 11 en 21 jaar.

Er werden man/vrouw-verschillen geconstateerd in de leeftijden waarop de eerste positieve symptomen zich voordeden. De frequentie van typische schizofreniesymptomen als gehoorshallucinaties en beïnvloedingswaan namen bij de mannen lineair met de leeftijd toe, maar toonden bij de vrouwen geen leeftijdsvariaties. De kans op voorkomen van wanen als vervolgingswaan, betrekkingswaan en grootheidswaan namen bij beide geslachten toe met de leeftijd; maar bij mannen was dit verband duidelijker.

De waarneming dat typisch schizofrene symptomen bij mannelijke patiënten in de vroege adolescentie relatief weinig voorkomen en toenemen met het klimmen der jaren, hangt wellicht samen met de latere inzet van de puberteit en daarmee samenhangende rijpingsprocessen bij jongens.

In het hoofdstuk 4.I.3 werd aandacht geschonken aan mogelijke verbanden tussen ouder worden en psychose aan het andere einde van de leeftijdsschaal: Onderzocht werd het mogelijke verband tussen veroudering en de administratieve incidentie van op latere leeftijd optredende (na 59 jaar) niet-organische, niet-affectieve psychosen in twee groepen patiënten van 60 jaar en ouder, die voor het eerst werden opgenomen i) in Nederland tussen 1978 en $1992(n=8010)$, en ii) in negen Regional Health Authorities in Engeland en Wales ( $n=1777)$ tussen 1976 en 1978.

Er bleek een lineair verband te bestaan tussen het toenemen van de leeftijd en het aantal eerste opnamen voor niet-organische, niet-affectieve psychosen bij ouderen, na correctie voor mogelijke confounders als geslacht en periode-effecten. De toename was zodanig dat met ellke vijf jaar toename van de leeftijd de incidentie toenam met $11 \%$. 
Deze waamemingen wijzen op een verband tussen degenerative hersenprocessen en het ontstaan van niet-organische, niet-affectieve psychosen bij oudere mensen,

In het hoofdstuk 3.2.1 onderzochten wij of de berichten over de hoge incidentie van psychotische aandoeningen in sommige etnische minderheden in Engeland en Nederland konden worden verklaard door een te lage schatting van het gedeelte van de popullatie dat tot zulke minderheden behoort. De administratieve incidentie van operationeel gedefinieerde schizofrenie en andere nietaffectieve psychosen, gecorrigeerd voor leeftijd en geslacht, werd berekend voor verschillende etnische groeperingen gedurende de periode 1988-1992, in een begrensd gebied in Londen. Uit de gestandaardiseerde incidentiecijfers, gecorrigeerd voor i) leeftijd-en geslachtgebondien variatie in de medewerking aan de volkstelling, en ii) cen $20 \%$ te lage schatting van het aantal mensen behorend tot etnische minderheden, bleek dat niet alleen Afro-Caribiërs (standard "mortality" ratio $=3.1 ; 95 \%$ Cl: 2.0-4.7), maar ook Afrikanen $(S M R=4.2 ; 95 \%$ Cl: 2.8-6.2) in London een verhoogd risico lopen op schizofrenie.

Een verdere bevinding was dat hogere incidentiecijfers in deze groepen niet specifiek waren voor de schizofrenie.

Deze resultaten suggereren dat een te lage schatting van het bevolkingsdeel dat tot een etnische minderheid behoort een onwaarschijnlijke verklaring is voor de hoge incidentiecijfers in sommige van deze groepen.

In het hoofdstuk 4.2 .2 werd de specificiteit van het verband tussen hoge incidentie van nietaffectieve psychose en bepaalde etnische groepering onderzocht. Incidentiecijfers voor manische stoornis, gebaseerd op eerste contacten met de psychiatrische hulpverlening, werden berekend voor een geografisch begrensd gebied in Londen in de periode 1965-1984. De Afro-Caribische groep had een hoger incidentiecijfer dan de blanke groep (rate ratio $=3.2 ; 95 \% \mathrm{CI}$ : $1.5-7.4$ ), en vertoonde vaker een combinatie van manische en typisch schizofrene symptomatologie (risk ratio $=2.2 ; 95 \% \mathrm{CI}: 1.1-4,3$ ).

Hoge incidentie van psychiatrische stoomissen in bepaalde etnische groepen in Engeland is miet specifiek voor de schizofrenie, hetgeen suggereert dat een risicofactor voor zowel manische als schizofrene stoomissen verhoogd aanwezig is in deze groepen.

In het hoofdstuk 3.23 vergeleken wij het ziekteverloop van twee etnische groepen van AfroCaribbean $(\mathrm{AC}, \mathrm{n}=53$ ) en British-born White $(\mathrm{BW}, \mathrm{n}=60)$ origine, in een cohortstudie van psychotische patiënten, achtereenvolgens opgenomen en gevolgd gedurende een periode van gemiddeld vier jaar. Patiënten werden prospectief geèvalueerd met betrekking tot klinische ernst van het ziekteverloop, (para)suicide, sociale handicap, ziekenhuisopname en behandeling; gecorrigeerd voor sociaal-economische status. De bevinding was dat de AC groep langere perioden van remissie had gedurende de studieperiode (gecorrigeerde odds ratio $=7.2 ; 95 \% \mathrm{CI}$ : 1.7-14.5), minder kans op een ononderbroken ziektepatroon (gecorrigeerde OR:0.3; 95\% CI: 0.10.8 ), en minder kans op (para)suïcide (gecorrigeerde $\mathrm{OR}=0.2 ; 95 \% \mathrm{Cl}: 0.1-0.9$ ). Er waren geen verschillen in opnameduur, maar de $\mathrm{AC}$ groep was vaker gedwongen opgenomen (gecorrigeerde $\mathrm{OR}=8.9 ; 95 \% \mathrm{CI}: 2.1-35.6$ ), en valker in de gevangenis tijdens de studieperiode (gecorrigeerde $\mathrm{OR}=9.2 ; 95 \% \mathrm{CI}: 1.6-52.3)$. 
De hoge incidentie van psychotische aandoeningen onder Afro-Caribiërs in Engeland hangt gedeeltelijk samen met een werhoogd risico op klinisch minder emstige ziekte met een gunstige prognose, en is mogelijk het resultaat van de relatief hoge blootstelling aan sociale stress in deze groep. Deze bevinding was niet beperktt tot de patièntengroep met affectieve psychosen, maar het effect was groter dan aan het niet-affectieve einde van het psychopathologische continuium.

In het hoofdstuk 4.3.I werd de relatie tussen stressvolle life events en ziekteverloop onderzocht. Negenenvijftig patienten met acute psychosen, wier ervaring met life events voor het begin van de ziekte was vastgesteld, werden gevolgd gedurende gemiddeld 42 maanden. Eenendertig patienten hadden een life event meegemaakt in de drie maanden voor het begin van de ziekte

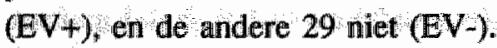

Patiënten met een affectieve psychose of een "unspecified functional psychosis" volgens de RDC criteria, die een life event hadden meegemaakt voor het begin van de ziekte, haddlen een betere prognose: hun symptomen waren minder emstig, ze brachten minder tijd door in het ziekenhuis, werden meer behandeld voor depressieve symptomen, en minder voor psychotische symptomen. In de patiëntengroep met schizofrenie waren de verschillen minder duidelijk, hoewel patiënten met life events voor het begin van de ziekte minder antipsychotische behandelingen ondergingen, en er een tendens bestond tot langere remissies.

Patiënten bij wie de relatie tussen life events en ontstaan van ziekte niet kon worden vastgesteld wegens een sluipend begin van de ziekte, leden vaker aan schizofrenie, en hadden een ongunstiger ziekteverloop, ook in de categorie van de schizofrenie.

In het hoofdstuk 4.4.I werd de theorie dat omvang van de hersenventrikells een risicofactor is voor een ongunstig ziekteverloop nader onderzocht. Een cohort van 140 patiënten met een recente functionele psychose, die allen hadden ingestemd met een CT-scan onderzoek, werd gevolgd gedurende gemiddeld 46 maanden, en zes dimensies van het ziekteverloop werden geëvallueerd. Wij konden aantonen, rekening houdend met de mogelijke effecten van veelvoudige statistische vergelijkingen en van statistische randwaarden, dat het volume van linker- en rechter fissura sylvii, en, in mindere mate, het volume van de derde hersenventrikel, waren geassocieerd met latere negatieve symptomen en cognitieve stoomis, resulterend in werkloosheid.

Er was sprake van een lineaire trend in het verband tussen fissura sylvii en slechte prognose: hoe groter de fissura sylvii, des te groter het risico op ongunstig ziekteverloop. De gevonden verbanden waren voorall evident in de groep van schizofrene patiènten. Er waren geen verbanden met de emst van het longitudinale ziektebeeld, opnameduur, dakloosheid, of affectieve symptomatologie.

Deze bevindingen steunen de theorie dat omvang van de hersenventrikels kan worden opgevat als een continue risicofactor voor aspecten van het ziekteverloop, vooral in de schizofrenie.

In het hoofdstuk 4.5.1 onderzochten wij de hypothese dat verschillen in het ziekteverloop tussen affectieve en niet-affectieve psychosen samenhangen met verschillen in de vroege ontwikkeling. We onderzochten daartoe de dossiers van alle patiënten met niet-depressieve functionele psychose in een gebied in Zuid-London, die hun eerste psychiatrische behandeling kregen tussen 1965 en 1984. De gevallen werden volgens het relatief chronische karakter van hun ziekte 
onderscheiden in vier, elkaar niet overlappende groepen: manie, schizomanie, acute schizofrenie en chronische schizofrenie.

Er was een lineaire trend in het verband tussen het chronisehe karakter van de ziekte en plaatsvervangende maatstaven voor afwijkingen in de vroege ontwikkeling als premorbide werkeloosheid, ongehuwde status en slechte studieresultaten. Bij de chronisch schizofrene patiènten werd drie- tot zesmaal zo veel premorbide abnormaliteit geconstateerd als bij de manische, en bij patiënten met schizomanie en acute schizofrenie anderhalf- tot driemaal zo veel als bij de manische individuen.

Wij concluderen dat premorbide abnormaliteiten weliswaar het meest voorkomen bij chronische schizofrenie, maar niet specifiek zijn voor dit ziektebeeld; in patiènten met minder invaliderende affectieve en niet-affectieve psychotische stoornissen kan de vroege ontwikkeling ook afwijkend zijn, zij het in mindere mate.

Dit thema werd verder uitgewerkt met prospectieve gegevens in hoofdstuk 4.5.2. Het onderzoek betrof de mogelijke verbanden tussen prospectief vergaarde gegevens in de kindertijd enerzijds en chronische, ernstige affectieve stoornissen en schizofrenie anderzijds, in een nationaal Brits cohort van 5262 individuen, geboren in de week 3-9 maart 1946 .

Vijfenzeventig gevallen (prevalentie $2,3 \%$ ) met chronische, ernstige affectieve stoomissen (CAD) werden geïdentificeerd door middel van sequentieel onderzoek naar de geestelijke gezondheid van de individuen in het cohort op 36-en 43-jarige leeftijd. Dertig gevaller met schizofrenie of schizo-affectieve stoornissen $(\mathrm{SZ})$ waren reeds eerder vastgesteld.

Patiënten met SZ en CAD vertoonden dezelfde ontwikkelingsstoomissen, maar in de CADgevallen was er een significante interactie met het geslacht aanwezig. Bij de vrouwelijke gevallen met CAD werden de motorische mijlpalen later bereikt, met een significant lineair verloop over de distributie van waarden in het cohort ( $\mathrm{OR}=1.5 ; 95 \% \mathrm{CI}: 1.1-2.2)$, gevolgd door een groter risico op spraakdefecten tussen 6 en 15 jaar $(\mathrm{OR}=3.6 ; 95 \% \mathrm{CI}$ : $1.8-7.5)$. Op de leeftijden van 8 , 11 en 15 differentieerden schooltests tussen CAD-gevallen en controlekinderen, in die zin dat naarmate de testscores lager uitvielen, de kans op toekomstige chronisch affectieve stoornissen groter was. De effecten bleken evidenter bij wrouwen. Op 13- en 15-jarige leeftijj werden de CAD-gevallen eerder "constant bedrukt en somber" genoemd door hun leraren (OR=2.7 en 2.5; p<0.05), en tussen 6 en 11 jaar werden ze door de medische onderzoeker vaker als "apatisch" beoordeeld ( $\mathrm{OR}=5.0 ; 95 \% \mathrm{CI}: 1.7-14,4)$.

Op 43-jarige leeftijd hadden de mannen meer de neiging getoond om schizofrenie te ontwikkelen $(\mathrm{OR}=1.8 ; 95 \% \mathrm{CI}: 0.9-3.9)$, terwijl de vrouwen eerder $\mathrm{CAD}$ hadden gekregen $(\mathrm{OR}=2.6 ; 95 \% \mathrm{CI}$ : 1.6-4.3). De onderzochte verbanden tussen ontwikkelingsvariabelen en latere psychiatrische stoomissen toonden overigens zeer weinig verschillen tussen $\mathrm{CAD}$ - en SZ-gevallen.

De resultaten suggereren dat vroege sociale, cognitieve en motorische deficienties ofwel de vroege manifestaties zijn van een syndroom met een gesllachtsafhankelijke variatie in de symptomatologie en een slechte prognose, ofwel de vroege manifestaties van een algemene predispositie voor chronische ziekte, met andere bepalende factoren van thet type ziekte bij volwassenheid. 
In het hoofdstuk 4.6.1 werd getracht het eventuele verband tussen genetische predispositie en ziekteverloop te onderzoeken. Er is geopperd dat er bij schizofrenie verband bestaat tussen het voorkoinen van schizofrenie in eerstegraads familieleden en een slechte prognose enerzijds, en voorkomen van affectieve stoomissen in familieleden en gunstig ziekteveriloop anderzijds.

We onderzochten mogelijke verbanden tussen het ziekteverloop over vier jaar en voorkomen van psychose in de familie in een groep van 150 successievelijk opgenomen patiënten met recent ontstane functionele psychose. Voor elke persoon werd de familiaire belasting berekend wat betreft i) psychotische stoornis in ruime zin, ii) schizofrenie en iii) affectieve stoomis. Hiervoor werd gebruik gemaakt van informatie over familieleden, verkregen door middel van de methode van de Family-History-Research Diagnostic Criteria en directe gesprekken met de familieleden met SADS-LA. In onze groep van psychotische patiënten bleek de familiaire belasting op het gebied van psychotische stoomis samen te gaan met aanhoudend negatieve symptomen (OR=1.5; 95\% CI: $1.0-2.2$ ) gedurende de verwolgperiode en ook verband te houden met langere opnamen $(p<0.02)$, en meer sociaal onvermogen gedurende de follow-up $(p<0.06)$, speciaal bij de schizofrenie. Een grotere familiaire predispositie voor schizofrenie bleek samen te gaan met een lagere kans op herstel $(\mathrm{OR}=2.2 ; 95 \% \mathrm{Cl}, 1.1-4.4)$ en een grotere waarschijnlijkheid dat er in de vervolgperiodle negatieve symptomen waren blijwen bestaan (OR=1.7; 95\% CE 0.9-3.1). Er werden geen verbanden gevonden tussen familiaire predispositie voor affectieve stoornis en ziekteverloop.

Wij concluderen dat familiaire belasting een continue risicofactor is voor zowel het ontstaan als het verloop van de functionele psychosen, vooral die van de schizofrenie.

In het vijfde hoofdstuk werd getracht door middell van een prospectieve studie duidelijkheid te scheppen in de relatie tussen de tardieve dyskinesie (TD) en haar vele vermeende risicofactoren, vooral wat betreft blootstellingen die aan variatie onderhevig zijn, zoals aspecten van de psychopathologie en het cognitieve functioneren, en hun temporele relatie met betrekking tot het ontstaan van TD. De patiëntengroep bestond uit 166 personen met een recente psychotische ziekte, en korte voorafgaande blootstelling aan antipsychotische medicatie. Informatie over 17 vermeende risicofactoren werd verzameld aan het begin van de studieperiode, en, bij veranderlijke blootstellingen, opnieuw aan het einde van de studie vier jaar later. De aanwezigheid van dyskinesie werd vastgesteld met behulp van de Abnormal Involuntary Movements Scale. Uit anälyse met step up-criteria bleek dat slechts zes van de 17 vermeende factoren onafhankelijk waren geassocieerd met de dyskinetische complicatie, namelijk: mannelijk geslacht (odds ratio= 2.5; 95\% CI: 1.1-5.0); leeftijd (OR over kwartielen=1.6; 95\% CI: 1.1-2.2); afwezigheid van ziektebesef aan het begin van de studie (OR over vier categoriëen $=2.0 ; 95 \%$ CI: 1.2-3.2); lengte wan behandeling met antipsychotica tijdens de studieperiode (OR over kwartielen $=2.3 ; 95 \% \mathrm{CI}: 1.5-3.4$ ), een toename van "negatieve" symptomatologie tijdens de studieperiode (OR over kwartielen $=1.7 ; 95 \%$ CI: 1.2-2.5), en het gebruik van alcohol en drugs (OR=3.0; 95\% CI: $1.3-7.4)$.

De resultaten wijzen erop dat behalve "traditionele" risicoverhogende factoren, zoals antipsychotische medicatie, leeftijd, en het gebruik van alcohol en drugs, tevens mannelijk geslacht, afwezig ziektebesef en progressieve verslechtering van het klinisch ziektebeeld een onafhankelijke bijdrage leveren aan het ontstaan van neurologische complicaties in de schizofrenie. Vooral deze laatste drie factoren wijzen erop dat in de functionele psychosen, 
ziekten met het klinisch $\mathbb{k}$ waadaardigste karakter tevens het hoogste risico meebrengen op organische complicaties. Deze observaties steunen de opvatting dat de enstigste psychosen mede het resultaat zijn van een ontwikkelingsstoomis tijdens de hersenmaturatie.

In de epiloog worden de resultaten besproken. We concluderen dat onze epidemiologiische studies het bestaan bevestigen van twee discrete effecten, waarvan de kracht varieert al naar gelang de positie op het door ons prospectief gevalideerde psychopathologische continuim: Life events zijn geassocieerd met het ontstaan van ziekte vooral aan het acute/affectieve einde van het continutim, en "verwijdering" van de risicofactor wordt gevolgd door een gunstig ziekteverloop. De omvang van de hersenventrikels is geassocieerd met ontstaan en verloop van de ziekte aan juist het sluipende/niet-affectieve einde van het psychopathologische continuüm. Het belang van deze bevindingen voor de gezondheidszorg lijkt weerspiegeld in, onder andere, de hoge incidentie van psychotische ziektebeelden met een gunstige prognose in bepaalde etnische minderheden, en de hoge incidentie van dyskinetische complicaties in psychosen met een ongunstig ziekteverloop. Verdere methoden en procedures om deze zaken te onderzoeken worden besproken. 
s.

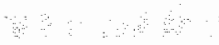

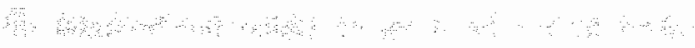

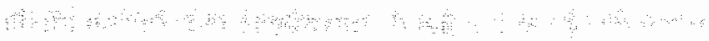

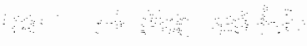
as,,$\cdots$ क्ष

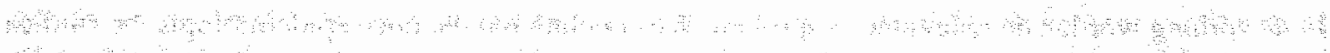
क s. अ,

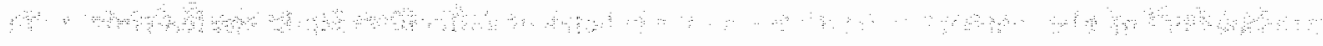
अन

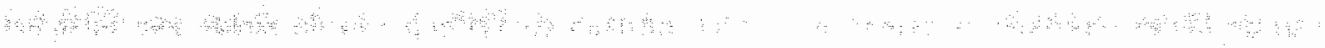

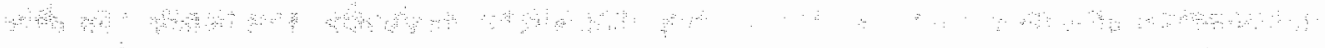
अ.

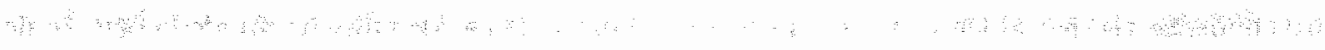
a b a

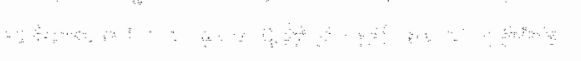




\section{Acknowledgements}

This thesis was the result of the ongoing collaboration of the members of the Camberwell Functional Psychosis study, and the kind cooperation of a large group of patients and their families. Nobody could have been a more enthusing and uniquely enabling supervisor than Professor Robin Murray, with whom it was a true privilege to work. His enthusiasm and ideas continue to inspire an ever increasing group of clinicians and researchers to undertake a large investigation in a coordinated fashion. Prof. de Vries generously provided many helpful comments on earlier versions of this work, and provided an ecological perspective. Dr Tom Fahy introduced the longitudinal perspective, and was instrumental in the organization of the follow-up study. Dr Peter Jones, Dr. llan Harvey, Dr. Paul Bebbington, and Prof. Shôn Lewis were the main researchers at the baseline side of the longitudinal study, and the high quality of their and their colleagues' work at index assessment, as well as their many helpful comments on earlier versions of my work, is gratefully acknowledged. Dr. Nori Takei and Dr. David Castle were my companions in the medical records department, where we worked our way through seemingly endless amounts of notes and papers. Dr Pak Sham kindly (and patiently) supervised my efforts to come to grips with issues to do with gene-environment interaction. I am very grateful to $\mathrm{Dr}$ Paloma Galdos for her help, scientific excellence, and for our heated arguments that not infrequently resulted in testable hypotheses. The collaborative efforts of two research workers who worked under my supervision, Dr. Kwame McKenzie of the Institute of Psychiatry and Dr. Hélène Verdoux of the University of Bordeaux are gratefully acknowledged. Dr. Kwame McKenzie brought a fresh view on the issue of ethnic group and the feasibility of including it in medical reserach, Dr Hélène Verdoux worked tirelessly to devellop the theme of familial morbid risk and outcome. Prof. Mike Wadsworth and the staff at the MRC National Survey of Health and Development provided me with the opportunity to examine hypotheses relating to premorbid adjustment and illness chonicity in the uniquely rich database of the 1946 UK birth cohort. Prof. Sir Michael Rutter provided many helpful comments on our work with the 1946 UK birth cohort. The help of the staff of the medical records department and the CT-scanning department in the Maudsley Hospital is also gratefully acknowledged, as is the help of Mr. Ullrich Cox. Drs. Geeske Menalda generously provided hard-needed editorial and linguistic advice, and Dr Peter Woodruff greatly facilitated the lengthy process of acculturisation to the English social climate. Dr. WAA van Os showed the academic way, and unselfishly organised logistic support from different corners of the world. I am very grateful to the U.K. Medical Research Council, which generously supported my full-time training in Epidemiology at the London School of Hygiene and Tropical Medicine. Trees Soute, Marie-José Duchateau and Sara Legg very kindly helped overcome some of the difficulties associated with organising a PhD from the other side of the Channel, and Dr Philippe Delespaul provided expert advice on lay-out and other technical issues. Finally, a special word of thanks to those patients who, in spite of continuing severe disability, kindly helped me establish in detail aspects about their lives in the previous years in sometimes lengthy interviews. 
अa: : : :

a

अं:

es as a

40 मैब

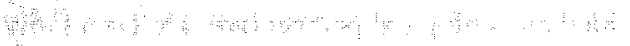

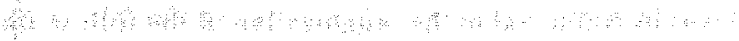

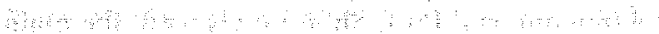

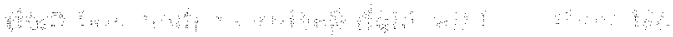

औ. is

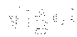

$\therefore \quad: \quad$,

औ:

$3=$

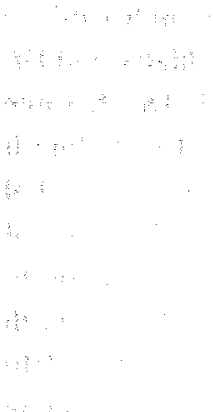




\section{Curriculum vitae}

The author of this thesis was born the 8th April 1960 in Utrecht. In 1978 he obtained his gymnasium beta diploma, and in 1987 the cum laude artsdiploma at the University of Amsterdam, having spent one year studying psychiatry in Indonesia and Morocco. He continued his training in psychiatry between 1987 and 1991, first in Bordeaux, and later in London at the Institute of Psychiatry and the Maudsley and Bethlem Royal Hospital. In 1992 he became research psychiatrist, and in 1993 Lecturer and MRC training fellow at the department of Psychological Medicine, Institute of Psychiatry and King's College Hospital. In 1993, he trained in epidemiology at the London School of Hygiene and Tropical Medicine. Apart from his work on risk factors for onset and persistence of psychotic illness, he was clinically attached to the Brixton Community Team in South London, where he specialised in the developnent and evaluation of casemanagement treatments in the community. He is academic coordinator of the UK-800 casemanagement clinical trial group, funded by the UK department of health. In 1995 , he moved to the University of Limburg, the Netherlands, to take up the position of Senior Lecturer in psychiatric epidemiology. His current clinical attachment is as team psychiatrist with the RIAGG at the "gate" of the Maastricht catchment area referral system. 


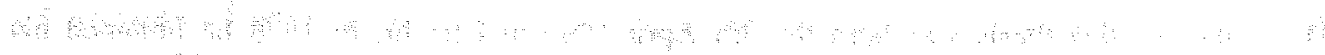

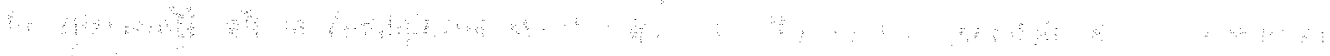

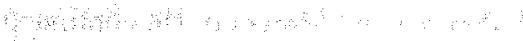

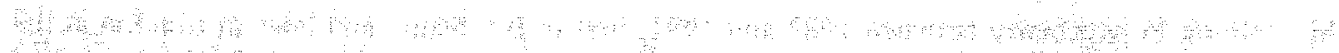

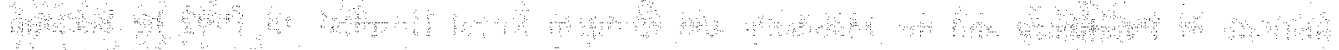

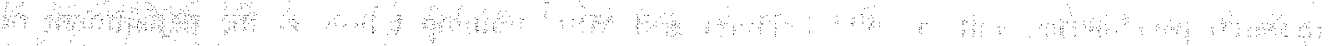

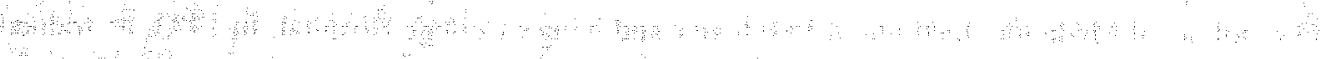

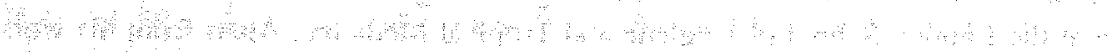

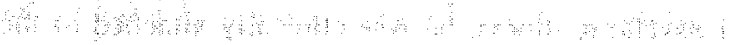

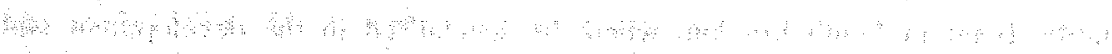

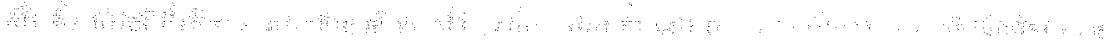

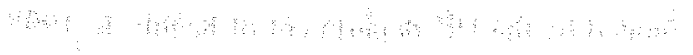

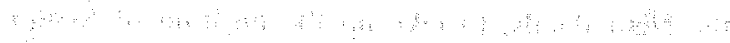
: 


\section{List of peer-refereed publications}

J. van Os, P. Galdos, G. Lewis, A. Mann, M. Bourgeois (1993). Schizophrenia sans frontieres British Medical Journal, 307, 489-492.

J. van Os, T. Fahy, P. Bebbington, S. Wilkins, P. Jones, K. Gilvarry, S. Lewis, B. Toone \& R. Murray (1994), The influence of life events on the subsequent course of psychotic illiness: a follow-up of the Camberwell Collaborative Psychosis study. Psychological Medicine, 24, 503-513.

J. van Os \& J. Neeleman (1994) Mental iliness in Europe. British Medical Joumal, 309, 1218-1222.

J. van Os \& J. Neeleman (1995). Europe and psychiatric ethics. Psychiatric Bulletin, 19. 1-3;

J. van Os, T. Fahy, P. Jones, I. Harvey, S. Lewis, M. Williams, B. Toone, R. Murray. Increased intra-cerebral CSF spaces predict unemployment and negative symptoms in psychotic Hiness. a prospective study. Brivish Journal of Psychiatry, 166, 750-758.

J. van Os, D. Castle, N. Takei, G. Der, R. Murray. Schizophrenia in ethnic minorities: clarification from the 1991 census. Psychological Medicine, in press.

J. van Os, R. Howard, N. Takei \& R. Murray. Increasing age is a risk factor for psychosis in the elderly. Sacial Psychiatry \& Psychiatric Epidemiology, in press.

J. yan Os, N. Takei, D. Castle, S. Wessely, G. Der \& R. Murray. Premorbid abnormalities in mania, schizomania, acute schizophrenia and chronic schizophrenia. Social Psychiarry \& Psychiatric Epidemiology, in press.

J. van Os, T. Fahy, P. Jones I Haryey, S, Lewis, P Sham, B. Toone, R. Murray Psychopathological syadromes in the functional psychoses: associations with course and outcome. Psychological Medicine, in press.

J. van Os, N. Takei, D. Castle, S. Wessely, G. Der, A. MacDonald \& R. Murray. The incidence of mania: time trends in relation to gender and ethnicity. Social Psychiatry \& Psychiatric Epidemiology, in press.

A. S. David, J. wan Os, P. Jones, II. Harvey, T. Fahy, \& R. Murray. Insight and psychotic illness: cross-sectional and longitudinal associations. British Journal of Psychiatry, in press.

J. van Os \& J. Neeleman (1994). European Union Government legislation affecting psychiatric prattice. Psychiatric Bulletin, 18, 390-394.P.M.

J. Neeleman \& J. van Os. Ethical issues in European psychiatry, European Psychiatry, in press.

P. Galdos \& J. van Os (1995) Gender, development and psychopathology: from puberty to early adulthood. Schizophrenia Research, 14, 105-113.

J. Neeleman \& J. van OS (1994). The feasability of a psychiatric common market - British and continental approaches to postgraduate psychiatric training. Psychiatric Bulletin, 18, 193-196.

Galdos, J. van Os \& R.M. Murray (1993). Puberty and the onset of psychosis. Schizophrenia Research, 10, 7 14.

J. McGrath, J. van Os, C. Hoyos, P.B. Jones, I. Harvey, R.M. Murray. Minor physical anomalies in the functional psychoses: associations with clinical and putative aetiological variables. Schizophrenia Research, in press.

K. McKenzie, J. van Os, T. Fahy, P. Jones, I. Harvey, B. Toone, R. Murray. Evidence for good prognosis psychosis among UK Afro-Caribbeans. British Medical Journal, in press. 
MJ. Owen, R Mant, E, Partit, P. Asherson, G,O. Mahoney, J, van Ox, D.A Llewellyn, D. Collier, M. Gill and P. MoGuffin (1992). A study of asisociation between RULS at the porphobilinogen deaminase gene and schizophremia. Human Genetics, $90,131-1,12$

P. Jones, 1. Harvey, 5, Lewis, B. Toone, $J$ van Os, M. Whllams \& $\mathbb{R}$ M. Murray (1994), Cerebral ventricle dimensions as isk factors for the functional psychoses. Psychological Medicine, 24,995 -1011.

S. Sthaikh, S. Hodgkinson, L, Pllowsky, J, van Os, D. Coller \& M. Gil. Analysis of the conserved ASP (14) residue of the dopamine D2 receptor in schizophrenic patients. Psychatric Genetics, in press.

N. Takei, J. van Os, R, Murny. Maternal exposure to influenza and risk of schizophrenia: a 23 year study from the Netherlands Journal of Prychatry Research, in press.

E. Dawson, J. Powell, M. Nơthen, M. Crocg, M, Lanczilk, J, Körner, M. Ruetschel, J, Van Os, P. Wright, \& M Gill. (1994) An associastion study of debrisoquine hydroxylase (CYP2D6) polymorphisms in schizophrenia. Psychiatric Gienefics, $4,215-218$.

J. van Os, A. Osacar \& M. Segovia (1992). Rehabilitacion de los manicomios: una valoracion critica de los servicios comunitarios de salud mental en Gran Bretaña Anales de Psiquiatria, 8, 383-388, 1992.

S. Achard, J.M. Chignon, M.F. Poinier-Litte, A. Galinowsk, eter D. Pringuey, J, van Os, F. Lemonnter Adaptation sociale et dêpression: intêtêt de léchelle SAS-SR. Encéphale, 21, 107. 116.

B. Guterrez, M. arranz, L. Fañanas, V. Valles, R. Guillamat, M. Campillo, J. wan Os, R. Kerwin, D. Coller (1995) No allelic associaiton between the $5 \mathrm{HT}_{2 \mathrm{~A}}$ receptor gene and bipolar affective disorder. Lancet, in press.

J. Neeleman, P. Jones, I. van Os, R.M. Murray. Parasuicide in Camberwell - ethnic differences. Sacial Psychiatry \& Pychiamic Epidemiology, un press.

S. Shaikh, S. Hodgkinson, L. Pilowsky, J. wan Os, D. Collier \& M. Gill. Analysis of the conserved ASP (114) residue of the dopamine D2 receptor in schizophrenic patients. Psychatric Genetics, in press. 